Steven Engler, Julia Janik, Matthias Wolf (Hg.)
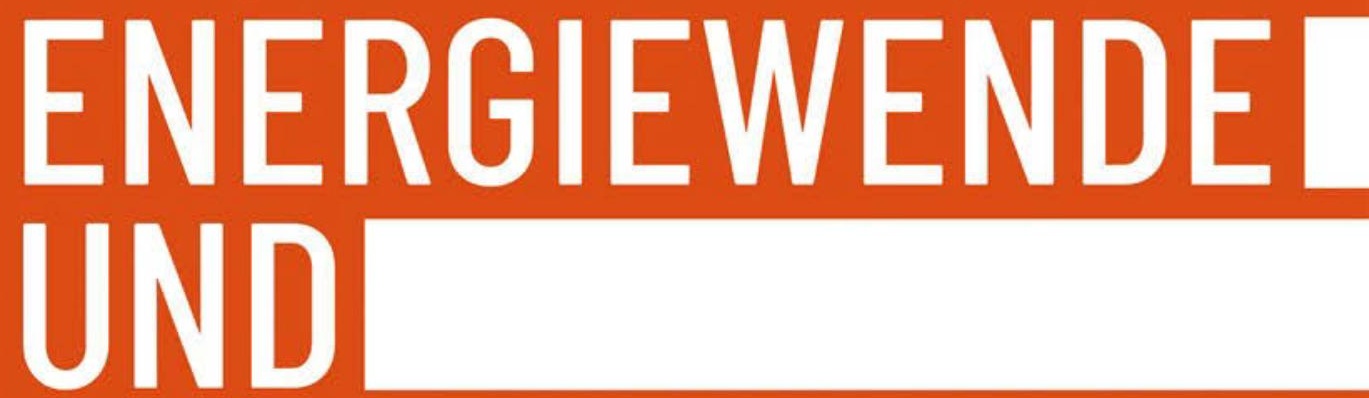

MEGATRENDS \title{
entwicklung und Nachhaltigkeit
}

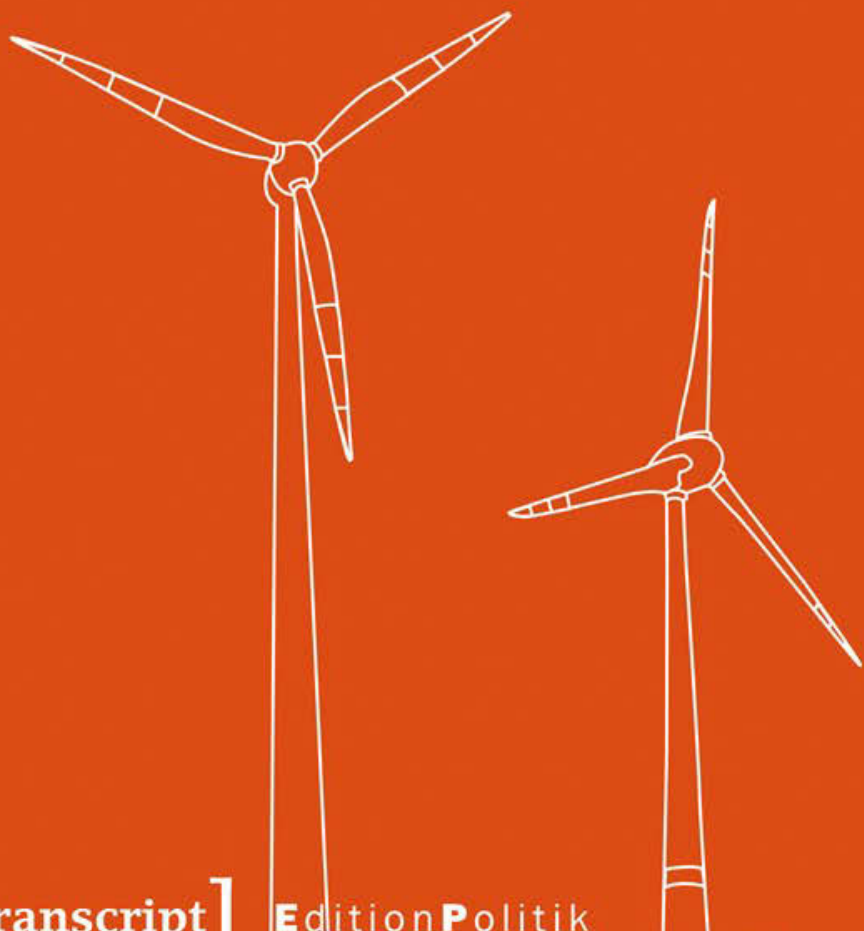


Steven Engler, Julia Janik, Matthias Wolf (Hg.)

Energiewende und Megatrends

Edition Politik | Band 93 
Die freie Verfügbarkeit der E-Book-Ausgabe dieser Publikation wurde ermöglicht durch den Fachinformationsdienst Politikwissenschaft POLLUX

\section{POLLUXX Informationsdienst}

und ein Netzwerk wissenschaftlicher Bibliotheken zur Förderung von Open Access in den Sozial- und Geisteswissenschaften (transcript, Politikwissenschaft 2020)

Die Publikation beachtet die Qualitätsstandards für die Open-Access-Publikation von Büchern (Nationaler Open-Access-Kontaktpunkt et al. 2018), Phase 1

https://oa2020-de.org/blog/2018/07/31/empfehlungen_qualitätsstandards_oabücher/

Karl-Franzens-Universität Graz | Universität

Wien Bibliotheks- und Archivwesen |

Bergische Universität Wuppertal | Carl von Ossietzky-Universität (University of

Oldenburg) | Freie Universität Berlin (FU)

(Free University of Berlin) | Georg-August-

Universität Göttingen | Goethe-Universität-

Frankfurt/M (University of Frankfurt am Main) | Gottfried Wilhelm Leibniz Bibliothek

- Niedersächsische Landesbibliothek |

Gottfried Wilhelm Leibniz Universität

Hannover | Humboldt-Universität zu Berlin | Justus-Liebig-Universität Gießen (University of Giessen) | Ludwig-Maximilians-Universität München (LMU) | Martin-Luther-Universität Halle-Wittenberg | Max Planck Digital Library | Ruhr-Universität Bochum (RUB) | Sächsische Landesbibliothek Staats- und Universitätsbibliothek Dresden (SLUB) | Staatsbibliothek zu Berlin (Berlin State Library) | ULB Darmstadt | Universität Bayreuth | Universität Duisburg-Essen | Universität Hamburg (UHH) | Universität Potsdam (University of Potsdam) | Universität Vechta | Universität zu Köln| Universitätsund Landesbibliothek Düsseldorf (University and State Library Düsseldorf) | Universitäts- und Landesbibliothek

\begin{abstract}
Münster (University of Munster) | Universitätsbibliothek Bielefeld (University of Bielefeld) | Universitätsbibliothek der Bauhaus-Universität Weimar (University of Weimar) | Universitätsbibliothek
\end{abstract} Erlangen-Nürnberg (FAU University Erlangen-Nürnberg) | Universitätsbibliothek Hagen (Fernuni Hagen) (University of Hagen) | Universitätsbibliothek Kassel | Universitätsbibliothek Koblenz-Landau | Universitätsbibliothek Konstanz (University of Konstanz) | Universitätsbibliothek Leipzig (University of Leipzig) | Universitätsbibliothek Mainz (University of Mainz) | Universitätsbibliothek Marburg | Universitätsbibliothek Osnabrück (University of Osnabrück) | Universitätsbibliothek Passau | Universitätsbibliothek Siegen | Universitätsbibliothek Würzburg | Zentral- und Hochschulbibliothek Luzern (ZHB) (Central and University Library of Lucerne) | Zentralbibliothek Zürich (Central Library of Zurich) | Bundesministerium der Verteidigung | Landesbibliothek Oldenburg (State Library of Oldenburg) | Leibniz-Institut für Europäische Geschichte | Stiftung Wissenschaft und Politik

Steven Engler (Dr.), geb. 1985, ist promovierter Geograph und Projektleiter der Arbeitsgruppe »Gesellschaft und Nachhaltigkeit im Wandel« am Geographischen Institut der Ruhr-Universität Bochum (RUB) und des Virtuellen Instituts »Transformation - Energiewende NRW«.

Julia Janik, geb. 1993, studiert Wirtschaft und Politik Ostasiens mit Schwerpunkt Politik und Chinesisch an der Ruhr-Universität Bochum (RUB) und ist dort Mitarbeiterin am Geographischen Institut in der Arbeitsgruppe »Gesellschaft und Nachhaltigkeit im Wandel«.

Matthias Wolf, geb. 1993, studiert Anglistik und Philosophie an der Ruhr-Universität Bochum (RUB) und ist dort Mitarbeiter am Geographischen Institut in der Arbeitsgruppe »Gesellschaft und Nachhaltigkeit im Wandel«. 
Steven Engler, Julia Janik, Matthias Wolf (Hg.)

\section{Energiewende und Megatrends}

Wechselwirkungen von globaler Gesellschaftsentwicklung und Nachhaltigkeit 
Ministerium für Wirtschaft, Innovation,

Digitalisierung und Energie

des Landes Nordrhein-Westfalen
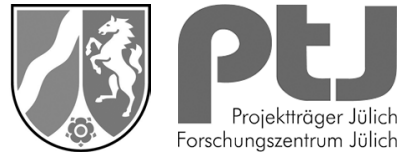

\section{Bibliografische Information der Deutschen Nationalbibliothek}

Die Deutsche Nationalbibliothek verzeichnet diese Publikation in der Deutschen Nationalbibliografie; detaillierte bibliografische Daten sind im Internet über http://dnb.d-nb.de abrufbar.

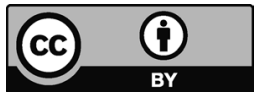

Dieses Werk ist lizenziert unter der Creative Commons Attribution 4. - Lizenz (BY). Diese Lizenz erlaubt unter Voraussetzung der Namensnennung des Urhebers die Bearbeitung, Vervielfältigung und Verbreitung des Materials in jedem Format oder Medium für beliebige Zwecke, auch kommerziell. (Lizenztext: https://creativecommons.org/licenses/ by/4.o/deed.de)

Die Bedingungen der Creative-Commons-Lizenz gelten nur für Originalmaterial. Die Wiederverwendung von Material aus anderen Quellen (gekennzeichnet mit Quellenangabe) wie z.B. Schaubilder, Abbildungen, Fotos und Textauszüge erfordert ggf. weitere Nutzungsgenehmigungen durch den jeweiligen Rechteinhaber.

\section{Erschienen 2020 im transcript Verlag, Bielefeld (c) Steven Engler, Julia Janik, Matthias Wolf (Hg.)}

Umschlaggestaltung: Maria Arndt, Bielefeld

Druck: Majuskel Medienproduktion $\mathrm{GmbH}$, Wetzlar

Print-ISBN 978-3-8376-5071-6

PDF-ISBN 978-3-8394-5071-0

EPUB-ISBN 978-3-7328-5071-6

https://doi.org/10.14361/9783839450710

Gedruckt auf alterungsbeständigem Papier mit chlorfrei gebleichtem Zellstoff. Besuchen Sie uns im Internet: https://www.transcript-verlag.de

Unsere aktuelle Vorschau finden Sie unter www.transcript-verlag.de/vorschau-download 


\section{Inhalt}

Mit dem Wissen von heute für eine Welt von morgen

Ein Vorwort

Claus Leggewie

9

Einleitung: Energiewende und Megatrends

Für ein integriertes Verständnis in Wissenschaft und Praxis

Steven Engler, Julia Janik und Matthias Wolf 13

\section{Megatrends: Entwicklung, Konzept, Kritik}

\section{Kontext bitte!}

Einblicke in die Geschichte der Zukunftsforschung und ihre Relevanz für die Erfindung der Megatrends

Jenny Zorn und Stefan Schweiger

Trends und Megatrends als Ansatz

der modernen Zukunftsforschung

Entwicklung und Praxis

Edgar Göll

45

Irrwege und Entwicklungspfade

Eine konstruktive Kritik der Trend- und Zukunftsforschung

Holger Rust 
Quid agis, Megatrend?

Von anekdotisch deduzierten Zukunftserzählungen

und ihren Wirkungen

Stefan Schweiger und Nora Schecke

81

\title{
Megatrends im Kontext von Energiewende und Nachhaltigkeit
}

\author{
Was nützt die Wende in Gedanken? \\ Überlegungen zur Steuerbarkeit von Transformationen am Beispiel \\ der deutschen Energiewende \\ Jörg Radtke und Emily Drewing
} 119

Umbrüche und Verunsicherungen

Der Windkraft- und der Stromnetzausbau in Deutschland

Florian Weber und Olaf Kühne 167

"Einfach zu viele Autos»: Neue Antriebe alleine reichen nicht.

Weert Canzler und Andreas Knie. 193

\section{Die Post-Landwirtschaftliche Revolution}

Oliver Stengel 215

\section{Empowerment in Transformations-Arenen}

Berücksichtigung sozialer Disparitäten im Energiewendeprozess

Anna Nora Freier, Stephanie Lübke, Katharina Schrot und Jenny Zorn.

Die Energiewende als Werk ausgewählter Gemeinschaften?

Zur sozialen Exklusivität von Energiegenossenschaften

Emily Drewing und Sabrina Glanz

Zwischen Wettbewerbsfähigkeit und Versorgungssicherheit

Wie wird der Megatrend »Digitalisierung « im Lichte

einer umzusetzenden Energiewende in der Industrie

diskursiv verhandelt?

Sonja Knobbe, Marius Beckamp und Ann-Kristin Götz.... 
Megatrends und die Energiewende in NRW aus Sicht kommunaler Akteure

Einblicke in eine NRW-Gemeindestudie

Juliane Lunge, Benjamin Best und Katja Witte....

Urbane nachhaltige Entwicklung am Beispiel von Initiativen in Gießen und Essen

Abeer Abdulnabi Ali.

Transformation von unten gestalten

Das Fachgeschäft für Stadtwandel als Ort

des sozial-ökologischen Wandels im Quartier

Martina Nies und Björn Ahaus

Biographische Angaben zu den Beiträger*innen 385 



\section{Mit dem Wissen von heute für eine Welt von morgen \\ Ein Vorwort}

Claus Leggewie

Seriöse Trendforschung versucht, gesellschaftlichen und kulturellen Wandel zu erfassen und dessen mögliche Richtungen zu bestimmen, dabei auch disruptive Entwicklungen ins Auge zu fassen. Gerade überlagern sich wenigstens drei Trends der letzten Jahrzehnte, die auch medial immer mehr Aufmerksamkeit erhalten:

- das Artenstreben und der Klimawandel sind nachweislich an Kipppunkte gestoßen oder haben sie bereits überschritten;

- nicht nur in den reichen Ländern haben sich die Werthierarchien "postmaterialistisch« verändert;

- in den politischen Systemen machen sich nach einer langen Welle der Demokratisierung autokratische Gegenströmungen bemerkbar.

Vor diesem Hintergrund haben sich neue soziale Bewegungen entwickelt, zuletzt ökologische Protestbewegungen wie »Fridays for Future« und »Extinction Rebellion«. Vor allem junge Menschen, die in Zukunft existenziell von Artensterben und Klimawandel betroffen sein werden, sind nicht untätig geblieben. Wenn die globale Erwärmung gebremst werden soll, wofür die erforderlichen Technologien und Investitionsmittel zur Verfügung stehen und ein deutlicher Mentalitätswandel stattgefunden hat, müssen die politischen Entscheider*innen Verantwortung übernehmen und die Große Transformation zur Nachhaltigkeit endlich entschlossen auf den Weg bringen. 
Derzeit laufen die beschriebenen Trends noch auseinander:

- Die Erkenntnis der planetaren Grenzen führte zu Verleugnung, Panik und Indifferenz, statt zu rationalem Kalkül, was mit der rasch zur Neige gehenden Restzeit zu tun hat;

- man bringt ökologische und soziale Gerechtigkeit gegeneinander in Stellung, statt die Chancen einer nachhaltigen Wirtschaft für ein besseres Leben, auch eine bessere Arbeitswelt, zu ergreifen. Die Widerstände gegen die Energiewende sind ein Beispiel dafür;

- statt einen konsensfähigen ökologischen Pol in der Mitte der Gesellschaft zu bilden, führt der völkisch-autoritäre Nationalismus zu Stagnation und Regression und es werden multilaterale Lösungen durchkreuzt.

Die Jugendlichen, die in den Schulstreik für Klimaschutz getreten sind, wehren sich dagegen - wenn nötig mit zivilem Ungehorsam. Hier liegt das Potential einer großen Weltbürgerbewegung - planetar denkend und lokal handelnd, kosmopolitisch und in der eigenen Region verankert. Das war ein Weckruf auch an »die«Wissenschaft, die sich zum Teil als »Scientists for Future« betätigen.

Zwei Botschaften sind von dieser Jugendbewegung zu vernehmen: dass sie ihr Vertrauen in »die Politik « verloren hat und dass sie Erkenntnissen der Wissenschaft $\mathrm{zu}$ folgen bereit ist. Ersteres ist durchaus verständlich, dabei entsteht in dieser Bewegung aber gerade eine neue Politik und jede Protestbewegung auf den Straßen und in Klassenzimmern und Seminarräumen ist letztlich auf gute Gesetzgebung, wirtschaftliche Anreize und technische Innovation angewiesen. Der Appell zur Selbstveränderung der eigenen Lebenswelt ist wichtig, aber er wird für sich allein nicht ausreichen. Die neue Klimabewegung hat gezeigt, dass sie etwas verändern will. Was genau geändert werden muss, um im Sinne der Großen Transformation tätig zu werden, dazu können wir als Wissenschaftler*innen beitragen. Wie es geändert werden soll, entscheiden Gesellschaften in einem partizipativen Prozess, in dem jede"r Einzelne sich als politische"r Akteur*in einbringt und Verantwortung gegenüber der Zukunft an den Tag legt.

Dieser Sammelband, der noch zur Zeit meiner Tätigkeit als Direktor des Kulturwissenschaftlichen Instituts in Essen (KWI) und der Anstellung des Projektteams bei eben jenem begonnen wurde, unternimmt eine wichtige interdisziplinäre Bestandsaufnahme der aktuellen Wechselwirkungen der Energiewende mit gesellschaftlichen Großentwicklungen unserer Zeit. Die oben 
aufgezeigten politischen und gesellschaftlichen, oft in Gegnerschaft stehenden Trends können als Rahmen und gleichzeitig als eine Auswirkung auch dieser Wechselwirkungen verstanden werden. Thematisiert werden diverse Bereiche, wie beispielsweise die oft ominöse »Digitalisierung ", die in die Energiewende hineinspielen, nachhaltige Entwicklungen beeinflussen oder davon beeinflusst werden. Die Zusammenschau meiner geschätzten Kolleg*innen macht deutlich: Die Große Transformation bleibt nicht bei thematisch abgesteckten Bereichen wie Naturschutz oder Energiewende stehen, sie muss mit anderen Großentwicklungen in Verbindung gebracht werden. 



\section{Einleitung: Energiewende und Megatrends ${ }^{1}$ Für ein integriertes Verständnis in Wissenschaft und Praxis}

Steven Engler, Julia Janik und Matthias Wolf

Vor fast einer Dekade erklärte die Ethik-Kommission »Sichere Energieversorgung « die Energiewende zum Gemeinschaftswerk, das »nur mit einer gemeinsamen Anstrengung auf allen Ebenen der Politik, der Wirtschaft und der Gesellschaft gelingen wird« (Ethik-Kommission Sichere Energieversorgung 2011:11). Die »Große Transformation« zu einer Gesellschaft, in der nachhaltige Lebens- und Wirtschaftsweisen vorherrschen, schwingt darin unüberhörbar mit. In diesem Sinne ist die Energiewende mehr als nur der Ausbau Erneuerbarer Energien und die Abkehr von Kohle und Kernkraft und damit definitiv aber mit hohen Anforderungen versehen. Diverse Akteure mit unterschiedlichsten Handlungslogiken und Motivlagen sollen - von der Bundesregierung bis zur Grünstromkundin, vom multinational operierenden Energiekonzern bis zum mittelständischen Handwerksbetrieb mit photovoltaikgeeigneter Dachfläche - beteiligt werden und zum Gelingen des Gemeinschaftswerks beitragen. So lassen sich mühelos der neue Green Deal der Europäischen Union, das Klimapaket der Bundesregierung und der RadEntscheid in Essen in den Kontext der Energiewende stellen (was die wissenschaftliche Befassung mit ihr freilich nicht einfacher macht - geschweige denn ihre Umsetzung).

1 Dieser Sammelband ist im Rahmen des Forschungsprojektes »EnerTrend - Systemische Analyse von Wechselwirkungen der Energiewende in NRW mit zentralen Megatrends « entstanden. Die Zuwendung für dieses Projekt stammt vom Ministerium für Wirtschaft, Innovation, Digitalisierung und Energie des Landes Nordrhein-Westfalen (MWIDE). Wir bedanken uns beim Fördermittelgeber sowie dem Projektträger ]ülich, die diesen Band ermöglicht haben. 
Nun geschieht die vielgestaltige, mitunter widersprüchliche und nicht selten mit Konflikten einhergehende Realisierung der Energiewende keineswegs im luftleeren Raum. Sie bleibt naturgemäß nicht unberührt von Großentwicklungen unserer Zeit, wird davon teils stark beeinflusst und steht auch in mancher Wechselbeziehung zu ihnen. Solche Meta-Dynamiken gesellschaftlichen Wandels lassen sich mit Hilfe der Methoden der $\mathrm{Zu}$ kunftsforschung bestimmen, die den berühmten Blick in die Kristallkugel verschmäht und stattdessen von der sorgfältigen Beobachtung aktueller Entwicklungen ausgehend Möglichkeitshorizonte aufspannt. Wenn im Titel sowie im Folgenden also die Rede von sogenannten Megatrends ist, so sind damit tiefgreifende, globale Entwicklungen gemeint, deren Wirkungen sich heute schon beobachten lassen und deren Fortsetzung wahrscheinlich ist.

Die Relevanz des Megatrends Digitalisierung für die Energiewende etwa scheint angesichts der medienwirksam geführten Debatte um fluktuierende Einspeisung erneuerbar erzeugten Stroms und den sich daraus ergebenden Bedarf der Anlagenregulation in Echtzeit, der flexiblen Speicher, intelligenten Stromnetze, automatisierten Ladevorgänge und ferngesteuerten Haushaltsgeräte evident. Indes wirkt die Digitalisierung nicht nur als der essentielle Ermöglichungsfaktor für die Energiewende, als der sie weithin gelesen wird, sondern bringt ihrerseits erheblichen Mehrbedarf an Energie mit sich. Auch einem Megatrend Mobilität kommt eingedenk der wiederholt geforderten und bis dato weithin vermissten Verkehrswende eine zentrale Bedeutung für die Energiewende zu. Forderungen nach mehr öffentlichen Verkehrsmitteln und Radwegen stehen Beliebtheit, Komfort und Verbreitung des (ungleich emissionsintensiveren) motorisierten Individualverkehrs entgegen. Zwischen sozialen Milieus divergierende ökologische Fußabdrücke, aber auch Strompreiserhöhungen, Stellenabbau in Unternehmen der konventionellen Energieerzeugung und Konflikte um die Verortung von Windparks verweisen auf Fragen sozialer Gerechtigkeit und Ungleichheit, deren Aushandlung im Kontext der Energiewende schon ob der zu ihrer Umsetzung erforderlichen gesellschaftlichen Akzeptanz ansteht. Entsprechend kommt auch dem Megatrend (zunehmender) sozialer Disparitäten eine wesentliche Rolle zu.

Große Transformationen wie die Energiewende sind demnach in eine Reihe von zueinander in Wechselwirkung stehenden sozialen, technischen, ökonomischen, politischen und kulturellen Bedingungen und Entwicklungen eingebettet. Um diese Komplexität analytisch zu erfassen und somit möglicherweise konstruktiv nutzbar zu machen, bedarf es einer wissenschaftlichinterdisziplinären und transdisziplinär informierten Perspektive, die es er- 
laubt, Schnittstellen der Energiewende mit gesellschaftlichen Großentwicklungen - eben: Megatrends - zu identifizieren. Unter der Prämisse, dass die Energiewende sowohl ökologisch notwendig als auch politisch gewollt ist, gilt es, die Wirkungen zeitgenössischer Megatrends auf den Fortgang der Energiewende zu verstehen und weiterhin auch zu ergründen, inwiefern die Energiewende selbst diese Großentwicklungen beeinflusst.

Ziel dieses Sammelbandes ist es, aus inter- und transdisziplinären Perspektiven globale Gesellschaftsentwicklungen unter Berücksichtigung von Konzepten der wissenschaftlichen Zukunftsforschung in den Blick zu nehmen und in Bezug zu (teils lokalen) energiewenderelevanten Entwicklungen zu setzen. Wichtige Anregungen und Ideen lieferte eine Konferenz mit dem Titel »Forschungstrend Trendforschung: Ist Nachhaltigkeit ein Megatrend?«, die 2019 im Rahmen des Projekts »EnerTrend - Systemische Analyse von Wechselwirkungen der Energiewende mit zentralen Megatrends « ${ }^{2}$ am Kulturwissenschaftlichen Institut Essen stattgefunden hat.

Der Sammelband gliedert sich in zwei Teile. Im ersten Teil mit dem Titel Megatrends: Entwicklung, Konzept, Kritik finden sich Überlegungen zu Konzepten, Methoden und Strategien der wissenschaftlichen und unwissenschaftlichen Befassung mit Megatrends. So werden in vier Beiträgen die historische Entwicklung des Konzeptes »Megatrend« sowie die der Zukunftsforschung näher beleuchtet und Kriterien entwickelt, an denen sich wissenschaftliche Zukunftsforschung messen lassen muss, um sich von den anekdotischen Erzählungen der feuilletonistischen Trendforschung abzugrenzen. Kritische Überlegungen schließen den ersten Teil des Bands $a b$.

Den Auftakt machen Jenny Zorn und Stefan Schweiger in ihrem Beitrag Kontext bitte! -Einblicke in die Geschichte der Zukunftsforschung und ihre Relevanz für die Erfindung der Megatrends. Darin widmen sie sich der historischen Entwicklung der Zukunftsforschung und legen einen Fokus auf die Grabenkämpfe zwischen den Vertreter"innen der wissenschaftlichen und der feuilletonistischen Herangehensweise. Die akademische und diskursive Randexistenz der wissenschaftlichen Zukunftsforschung ermöglichte es feuilletonistischen Ansätzen, öffentliche Aufmerksamkeit und Deutungshoheit zu erlangen. Dadurch entsteht womöglich ein falscher Eindruck der Kontrollierbarkeit zu- 
künftiger Entwicklungen, dessen bedenkliches Resultat in Zeiten tiefgreifender anthropogener Umweltveränderungen unangebrachte Gelassenheit und Zögerlichkeit bei der Adressierung dringender Handlungsbedarfe sein kann.

Edgar Göll liefert in seinem Beitrag Trends und Megatrends als Ansatz der modernen Zukunftsforschung - Entwicklung und Praxis Beispiele für populäre Megatrends und diskutiert mögliche Kriterien zu ihrer Identifikation. Ausgehend von der Beobachtung, dass die Fragestellungen, zu deren Beantwortung Megatrends herangezogen werden, teils stark divergieren, zieht er zur eindeutigen Feststellung eines Megatrends die Merkmale Dauer, Ubiquität, Universalität, Robustheit, Richtung und Wechselwirkung heran. Auf dieser Grundlage behandelt Göll abschließend die Frage, ob Nachhaltigkeit ein Megatrend ist.

Im Anschluss befasst sich Holger Rust in seinem Aufsatz Irrwege und Entwicklungspfade - Eine konstruktive Kritik der Trend-und Zukunftsforschung mit zeitgenössischen Ausprägungen der feuilletonistischen Trendforschung. Ausgehend von ihrer historischen Entwicklung charakterisiert er jene Form der Zukunftsforschung als Dienstleistung und analysiert ihre Betriebslogik. Aktuelle Konzepte der Foresight Research werden schlaglichtartig beleuchtet und dem interdisziplinären, wissenschaftlichen Ansatz des Superforecastings vergleichend gegenübergestellt.

Ebenfalls kritisch setzen sich Stefan Schweiger und Nora Schecke in ihrem Beitrag Quid agis, Megatrend? - Von anekdotisch deduzierten Zukunftserzählungen und ihren Wirkungen mit den Narrativstrukturen des Zukunftsinstituts auseinander. Die Autor*innen stellen spezifische Erzählweisen fest, die vornehmlich dazu dienen, Komplexität zu reduzieren, Optimismus zu verbreiten und gesellschaftliche Großentwicklungen allein marktorientiert zu begreifen.

Die Beiträge im zweiten Teil - Megatrends in Kontext von Energiewende und Nachhaltigkeit - widmen sich konkreten Berührungspunkten und Wechselwirkungen zwischen der Energiewende und Megatrends.

Eröffnend beschäftigen sich Jörg Radtke und Emily Drewing in Was nützt die Wende in Gedanken? - Überlegungen zur Steuerbarkeit von Transformationen am Beispiel der deutschen Energiewende mit der Entwicklung und dem aktuellen Stand der Energiewende zwischen normativem Anspruch und tatsächlicher Umsetzung. Dabei gehen sie insbesondere auf die Dimensionen Bewusstsein (der Bevölkerung für die Energiegewinnung), Partizipation (im Sinne eines »Gemeinschaftswerks«), Diffusion (von »bottom-up«-Innovationen für mehr Nachhaltigkeit) und Steuerung (der Energiewende als bundespolitisches Projekt, das in den Ländern, Regionen und Kommunen umgesetzt wird) ein. Damit bieten Radtke/Drewing einen Einblick in aktuelle Energiewende- 
Debatten, der als Rahmen für die Analyse von Wechselbeziehungen zwischen der Energiewende und aktuellen Megatrends dienen kann.

Florian Weber und Olaf Kühne beleuchten in ihrem Aufsatz Umbrüche und Verunsicherungen - Der Windkraft- und der Stromnetzausbau in Deutschland aktuelle gesellschaftliche Aushandlungsprozesse und Konfliktfelder im Kontext der Energiewende. Sie legen ein besonderes Augenmerk auf kritische Bürgerinitiativen. Sie lesen Windkraft als »flottierende Signifikante« (Laclau), die sich kontext- und standpunktabhängig unterschiedlich werten und argumentativ in Stellung bringen lässt. So sind Stromtrassen und Windräder zugleich als eine »Verschandelung der Landschaft « und Weg in eine nachhaltige $\mathrm{Zu}$ kunft lesbar. Anhand ihrer grundlegenden Ausrichtung sowie den von ihnen kritisierten Aspekten entwickeln Weber/Kühne eine Typisierung von Bürgerinitiativen, die gegen Energiewendeprojekte protestieren.

Für veränderte gesetzliche Rahmenbedingungen und neue Narrative der Mobilitätswende in Deutschland plädieren Andreas Knie und Weert Canzler in »Einfach zu viele Autos«: Neue Antriebe alleine reichen nicht. Die Dekarbonisierung des Automobilverkehrs, so die Autoren, lässt sich im aktuellen Geflecht aus Innovationsarmut seitens der Hersteller, technischen Pfadabhängigkeiten, Gewohnheiten der Konsument*innen sowie der den Automobilverkehr privilegierenden gesetzlichen Regelungen nur schwerlich voranbringen. Vor diesem Hintergrund empfehlen die Autoren die Nutzung der Experimentierklauseln diverser Gesetze und die Durchführung umfassender, lokaler Experimente.

In Die Post-Landwirtschaftliche Revolution beleuchtet Oliver Stengel die Notwendigkeit, Herausforderungen und Chancen einer Ernährungswende. Ausgehend von gegenwärtigen Problemen der Landwirtschaft wie dem immensen Bedarf an Fläche, dem hohen Verbrauch von Wasser sowie diversen Umweltbelastungen wird evident, dass sich im Rahmen einer globalen Transformation zu mehr Nachhaltigkeit auch die Produktionsweise unserer Nahrungsmittel ändern muss. Einen wesentlichen Beitrag dazu könnten in Zukunft die Bioreaktoren von Start-ups leisten, in denen heute schon Fisch, Fleisch, Milch oder sogar pflanzliche Produkte unter Laborbedingungen aus Stammzellen herangezüchtet werden.

Anna Nora Freier, Stephanie Lübke, Katharina Schrot und Jenny Zorn diskutieren in ihrem Beitrag Empowerment in Transformations-Arenen - Berücksichtigung sozialer Disparitäten im Energiewendeprozess den Megatrend Soziale Disparitäten als Gefahr für die Energiewende. Um sie im Sinne eines Gemeinschaftswerks gerecht zu gestalten, bedarf es Partizipationsmöglichkeiten, de- 
rer sich insbesondere die Transformative Forschung bedienen sollte. Die Autorinnen stellen zwei im Rahmen verschiedener Projekte erprobte Konzepte des sogenannten Empowerments vor - das Partizipative Reallabor und den Citizen Engagement Prozess - sowie ein Instrument, das als Beispiel für einen erweiternden Baustein bei der Umsetzung eines EmpowermentKonzepts dient: ein Escape Room zum Thema "Nachhaltigkeit im Alltag«.

Ebenfalls vor dem Hintergrund des Megatrends Soziale Disparitäten sowie zeitgenössischen Befunden zur sozialen Gerechtigkeit und dem partizipatorischen Vermögen der Energiewende explorieren Emily Drewing und Sabrina Glanz in Die Energiewende als Werk ausgewählter Gemeinschaften? - Zur sozialen Exklusivität von Energiegenossenschaften mögliche Gründen für die empirisch erwiesene Überrepräsentation älterer, wohlhabender Männer ohne Migrationsgeschichte in Energiegenossenschaften. Theoretisch informiert durch Joan Ackers Gendered Organizations sowie das auf Kimberlé Crenshaw zurückgehende Konzept der Intersektionalität werten sie Interviews mit Personen aus, die in Energiegenossenschaften im Ruhrgebiet eine leitende Funktion erfüllen.

Sonja Knobbe, Marius Beckamp und Ann-Kristin Götz untersuchen in Zwischen Wettbewerbsfähigkeit und Versorgungssicherheit - Wie wird der Megatrend "Digitalisierung" im Lichte einer umzusetzenden Energiewende in der Industrie diskursiv verhandelt? die brancheneigenen Pressemitteilungen energieintensiver Industriezweige. Die Autor*innen fokussieren ihre Inhaltsanalyse auf die Schlagworte Energiewende und Digitalisierung sowie etwaige Verbindungen der beiden Themen. Darauf aufbauend werden Forderungen und Argumentationslinien der Branchenverbände identifiziert und eingeordnet.

In Megatrends und die Energiewende in NRW aus Sicht kommunaler Akteure Einblicke in eine NRW-Gemeindestudie ergründen Juliane Lunge, Benjamin Best und Katja Witte, inwiefern die Megatrends Digitalisierung, Soziale Disparitäten, Übernutzung natürlicher Ressourcen, Urbanisierung und Demographischer Wandel bereits auf kommunaler Ebene verhandelt werden. Basierend auf Ergebnissen aus einer Onlinebefragung und darauf aufbauenden explorativen Interviews mit kommunalen Akteuren werden die Dimensionen Wahrnehmung, Wissen, Einstellungen, Verhalten und Handlungskapazitäten betrachtet. Aus dieser Analyse leiten die Autor*innen Treiber und Hemmnisfaktoren des kommunalen Umgangs mit Megatrends und der Energiewende ab. Abschließend diskutieren sie die Notwendigkeit neuer Formen der Governance. 
Der Band schließt mit zwei anwendungsnahen Beiträgen, die zivilgesellschaftliche Initiativen im städtischen Kontext in den Blick nehmen. In Urbane nachhaltige Entwicklung am Beispiel von Initiativen in Gießen und Essen betrachtet Abeer Abdulnabi Ali die Realisierung von Nachhaltigkeit durch bürgerschaftliches Engagement auf der lokalen Ebene. Ausgehend von der Frage, wie unterschiedliche Initiativen nachhaltige Entwicklung in urbanen Räumen verbessern können, wurden Experteninterviews durchgeführt. Dabei wurden neben Wissenschaftler*innen und Politiker*innen auch Repräsentant*innen von vier Initiativen befragt. Aus der Analyse ließen sich akteursspezifische Handlungsperspektiven ableiten.

Martina Nies und Björn Ahaus nehmen in Transformation von unten gestalten - Das Fachgeschäft für Stadtwandel als Ort des sozial-ökologischen Wandels im Quartier die Perspektive wissenschaftlich geschulter Praktiker"innen ein. Sie liefern einen lebendigen Bericht aus dem Fachgeschäft für Stadtwandel im Stadtteil Essen-Holsterhausen, das Bürger"innen vielfältige Möglichkeiten bietet, sich einzubringen und zu engagieren. So fungiert das Stadtgeschäft als Dreh- und Angelpunkt für die lebendige Nachbarschaft, den sozialökonomischen Wandel und die interkulturelle Gemeinschaft im Quartier.

Mit diesem Band hoffen wir, einige Anregungen zu liefern, um die Energiewende, der es - zumal im weiteren Sinne einer sozialökologischen Transformation - an Komplexität gewiss nicht mangelt, mit zeitgenössischen Megatrends zusammenzudenken. Daraus ergeben sich gleichermaßen spannende Forschungsperspektiven und wertvolle Erkenntnisse zur Optimierung ihrer Umsetzung in der Praxis.

Wir bedanken uns in erster Linie bei den Autor*innen der einzelnen Beiträge. Weiterhin danken wir den Mitgliedern der Arbeitsgruppe Gesellschaft und Nachhaltigkeit im Wandel des Geographischen Instituts der RuhrUniversität Bochum, die uns im Verlauf der Genese dieses Sammelbands stets zur Seite standen.

\section{Literatur}

Ethik-Kommission Sichere Energieversorgung (2011): Deutschlands Energiewende - Ein Gemeinschaftswerk für die Zukunft. Online verfügbar unter: https://archiv.bundesregierung.de/resource/blob/656922/ 394384/962bafo9452793c8a87402c9ee347379/2011-07-28-abschlussberichtethikkommission-data.pdf. Zuletzt aufgerufen am 01.03.2020. 

Megatrends: Entwicklung, Konzept, Kritik 



\section{Kontext bitte! \\ Einblicke in die Geschichte der Zukunftsforschung und ihre Relevanz für die Erfindung der Megatrends}

Jenny Zorn und Stefan Schweiger

Es scheint so simpel, wie selbstverständlich: Wir Menschen können kein gesichertes Wissen von der Zukunft haben, weil es die Zukunft per definitionem (noch) nicht gibt. Der Wissenschaft fehlt jeglicher Bezug zu ihr: Zukunft liegt nicht als Ereignis, Gegebenheit oder Gegenstand vor. Der Forschungsgegenstand »Zukunft« ist schlichtweg inexistent. Es gibt nur höhere oder geringere Wahrscheinlichkeiten, dass bestimmte Ereignisse eintreffen werden. Ereignisse, die bestimmten Kausalitäten oder Naturgesetzen unterliegen »etwa in welche Richtung ein Gegenstand fällt, wenn ich ihn fallen lasse, oder wann die nächsten zwanzig Sonnenfinsternisse sein werden [...] (Gransche 2015: 40) - können mit sehr hoher Wahrscheinlichkeit als wiederholbar gelten. Solche Annahmen über die Zukunft im Rahmen von experimentell eng begrenzten Situationen lassen sich jedoch nicht auf individuelle Erfahrungen oder gar gesellschaftliche Prozesse übertragen. Je komplexer ein System, je mehr Variablen, Einflüsse, Zusammenhänge und Wechselwirkungen gegeben sind, desto schwieriger wird es, die Zukunft zutreffend vorhersagen zu können. Ein weiteres Problem ist, dass [m] it fortschreitender Entwicklung der modernen Gesellschaft [...] die Prognostizierbarkeit ihrer Entwicklung ständig ab[nimmt] « (Jischa 2009: 46), weil durch die Beschleunigung von Forschungs- und Entwicklungsprozessen - unter anderem durch die Nutzung digitaler Infrastrukturen - die Kenntnisse und Informationen, die wir

1 Dieser Beitrag ist im Rahmen des Forschungsprojektes »EnerTrend: Systemische Analyse von Wechselwirkungen der Energiewende in NRW mit zentralen Megatrends « entstanden. Die Zuwendung für dieses Projekt stammt vom Ministerium für Wirtschaft, Innovation, Digitalisierung und Energie des Landes Nordrhein-Westfalen (MWIDE). 
uns aneignen, wesentlich schneller veralten. ${ }^{2}$ Dadurch vermehren sich zwar die Informationen im Allgemeinen, aber die Möglichkeit der Vorhersage der Zukunft nimmt ab (vgl. Jischa 2009: 37). Das Verhältnis von Information und Zukunft ist also nicht linear: Mehr Information ermöglicht sowohl bessere Kenntnis von Bedingungen, Chancen und Risiken als auch die Nutzung dadurch ermöglichter Chancen, wodurch sich jedoch wieder neue Bedingungen, Chancen und Risiken ergeben. Diese Nichtlinearität im Verhältnis von gegenwärtigem Wissen über zukünftige Ereignisse macht die Komplexität aus, mit der man sich insbesondere in den Bereichen auseinandersetzten muss, die auf eine möglichst genaue Einschätzung zukünftiger Entwicklungen angewiesen sind. Diese besondere Form gilt entsprechend und erst recht auch für diejenige wissenschaftliche Disziplin, deren Gegenstand die Zukunft ist.

Fragt man Zukunftsforscher*innen, wie die Welt in 10 Jahren aussehen wird - ob wir beispielsweise mit dem Auto zur Arbeit fliegen, statt zu fahren; ob wir smarte Implantate tragen, statt Smartphones zu nutzen; ob ein Krieg aus- oder das Internet zusammenbrechen wird - so werden sie antworten: "Zukunftsforschung macht keine Vorhersagen " (Gransche 2015: 40). Das mag irritieren, da »[d]er Begriff Zukunftsforschung suggeriert, dass es eine Forschungsrichtung gibt, die >die Zukunft< erforschen kann.« (Popp 2012: V, zitiert nach Gransche 2015: 98) Zukunftsforscher"innen wissen um dieses Problem nicht nur aus einer abstrakt erkenntnistheoretischen Perspektive. Es bringt sie auch in ihrem eigenen Forschungsbereich immer wieder in Erklärungsnöte und erschwert damit die symbolische Anerkennung und Sicherung der Finanzierung.

Die Angst vor der Zukunft ist ein einträgliches Geschäft. Wer die Zukunft kennt oder andere glauben machen kann, dass sie sich vorhersagen lässt, bietet Herrschaftswissen an. Es ermöglicht, so die Erwartung, den Konkurrent"innen im politischen oder wirtschaftlichen Ringen um Deutungshoheit, Einfluss und Profit, die eine entscheidende Nasenlänge voraus zu sein. Entsprechend sorgt der unterschiedliche Umgang mit dem Problem der Erforschung einer Zukunft, die es noch nicht gibt, für Spannungen, die den Wissenschaftlichkeitsanspruch der Zukunftsforschung - sich bei der Analyse in nachvollziehbarer Weise auf vorliegende und überprüfbare Datenkorpora zu

2 Jischa nimmt hier u.a. Bezug auf Hermann Lübbe (1994), der in Bezug zum Internet von einer »Cegenwartsschrumpfung «spricht und damit meint, dass Informationen wie Technologien und Innovationen immer schneller veralten und damit in die Vergangenheit rücken. 
beziehen - selbst betreffen: Je expliziter seriöse Zukunftsforscher*innen darauf hinweisen, dass es in ihrer Forschung »um ein besseres Verständnis systemischer Entwicklungen, also um relevante Dynamiken innerhalb von Gesellschaften, Forschungslandschaften, Wirtschaftssystemen etc. [geht]« (Gransche 2015: 40) und darum, »[...] Zukunft als Reflexionsbegriff für gegenwärtige Einschätzungen eines zukünftig Möglichen zu konzeptualisieren [...]« (Grunwald 2009: 33, zitiert nach Gransche 2015: 97), desto unattraktiver sind ihre Ergebnisse für die wirtschaftliche und politische Sekundärverwertung. Begriffe wie »systemische Entwicklungen«, »relevante Dynamiken« oder »Reflexionsbegriff« suggerieren abstrakte, praxisferne Forschung, die die Erwartungen an eine nützliche, zweckgerichtet anwendbare Zukunftsforschung gerade nicht erfüllt.

Erfüllt die Zukunftsforschung wiederum die Erwartungen, die an sie herangetragen werden, kann man nicht mehr von seriöser Forschung sprechen: Zukunftsforscher"innen, die de facto Wahrsagerei im Gewand wissenschaftlicher Forschung anbieten, verkaufen - im wahrsten Sinne des Wortes ${ }^{3}$ - anderen ihre Erkenntnisse als sdie Zukunft،, auf die man sich einstellen sollte. Dieses Dilemma lässt sich auf eine Formel bringen: Je wissenschaftlicher, desto nutzloser erscheint die Erforschung der Zukunft; je nützlicher sie aber erscheint, desto unwissenschaftlicher ist ihre Herangehensweise.

Der wesentliche Unterschied zwischen wissenschaftlicher Zukunftsforschung und feuilletonistischer Trendforschung ${ }^{4}$ besteht vor allem in der Überprüfbarkeit ihrer Herangehensweisen. Während die wissenschaftliche Zukunftsforschung sich der komplexen Problematik bewusst ist, die sich aus dem Zusammenhang von gegenwärtigem Wissen und zukünftigen Möglichkeiten ergibt, betrachtet die Trendforschung die Zukunft zumeist als lineare Fortführung bekannter Entwicklungen. Wissenschaftliche Forschung muss ihre Kriterien, Bedingungen und Daten offenlegen, um überzeugen zu können. Die feuilletonistische Trendforschung erfindet Namen für Methoden, die sie nicht näher darlegt (vgl. Rust 2009: 3-4, 11). Statt Überprüfbarkeit und

3 Die Megatrend-Dokumentation des Zukunftsinstituts kostet immerhin über 600 Euro (vgl. https://onlineshop.zukunftsinstitut.de/shop/megatrend-dokumentation/). Vgl. dazu außerdem den Beitrag »Quid agis, Megatrend? - Von anekdotisch deduzierten Zukunftserzählungen und ihren Wirkungen« von Stefan Schweiger und Nora Schecke in diesem Band.

4 Diese Unterscheidung ist angelehnt an Rust (2008: 64). Rust selbst nennt Zweitere jedoch meist boulevardeske Trendforschung. 
Transparenz - die Grundlagen wissenschaftlicher Forschung - soll die Eigenbezeichnung als Zukunftsforscher*in, die Unternehmensbezeichnung als Institut oder allgemein formulierte Methodentitel die Wissenschaftlichkeit der Herangehensweise belegen. Der Unterschied ist ein Unterschied ums Ganze: hier wissenschaftliche Methode, dort Wissenschaftssimulation, um Marktinteressen zu befriedigen und damit Kapital zu vermehren.

Dass es für Nichtwissenschaftler*innen schwierig ist, seriöse Zukunftsforschung von feuilletonistischer Trendforschung $\mathrm{zu}$ unterscheiden, liegt unter anderem daran, dass ähnliche Begrifflichkeiten verwendet werden. Aus der feuilletonistischen Richtung stammt das Konzept der Megatrends, das aber heute auch im wissenschaftlichen und wissenschaftsnahen Kontext verwendet wird. Für die feuilletonistische Trendforschung ist die Erforschung sogenannter Megatrends in den letzten Jahrzehnten zum wesentlichen Marktsegment geworden. Sie prophezeien Großentwicklungen mit Buzzwords wie »Digitalisierung«, »Silver Society«, »Mobilität« oder »New Work« und umschreiben damit aktuelle Themen, über die sie glauben sagen $\mathrm{zu}$ können, dass es nicht nur die aktuellen, sondern die Zukunftsthemen seien, die die Gesellschaft noch lange beschäftigen werden. Eine bekannte deutsche $\mathrm{GmbH}$, die nach diesem Muster agiert, ist das Zukunftsinstitut. Sie definieren Megatrends als Großentwicklungen, die unser aller Leben bereits beeinflussen und es in den nächsten Jahrzehnten auch noch weiter beeinflussen werden. Sie wirken - so ihre Annahme - global und allgegenwärtig und sind robust gegenüber Veränderungen, die in die entgegengesetzte Richtung laufen (vgl. Horx 2007: 1). Das Konstrukt Megatrend stammt von John Naisbitt, der es 1982 mit dem Bestseller »Megatrends« im gesellschaftlichen Diskurs etablierte. Aber auch in heutigen wissenschaftsnahen Kontexten wird eine ähnliche Definition gebraucht, wie etwa bei der Europäischen Umweltagentur:

»Megatrends are those trends visible today that are expected to extend over decades, changing slowly and exerting considerable force that will influence a wide array of areas, including social, technological, economic, environmental and political dimensions. « (European Environment Agency 2007, zitiert nach Lorenz/Haraldsson 2014: 16).

Bei der Commonwealth Scientific and Industrial Research Organisation, Australiens staatliche Behörde für Wissenschaft und industrielle Forschung, wird der Gegenwartsbezug nur noch implizit erwähnt: 
»A megatrend is a significant shift in environmental, economic and social conditions that will play out over the coming decades. The indicative time frame for the analysis is 20 years. «(Hajkowic/Cook/Littleboy 2012: 2)

Megatrends sind also nicht nur Teil feuilletonistischer Trendforschung, sondern der Begriff spielt auch in wissenschaftsnahen Forschungen eine Rolle. Insofern kann man davon sprechen, dass die Behauptungen, dass es Megatrends gibt und die Festlegung, welche Megatrends das konkret sind, einen nicht unerheblichen gesellschaftlichen Impact haben. Die Entstehungsbedingungen des Konstrukts Megatrend sollen im weiteren Verlauf aufgezeigt werden.

\section{Gestaltung von Zukunft(-svorhersagen)}

Eine adäquate theatralische Inszenierung von Vorhersagen verspricht seit jeher erzählerische Legitimation und einen Zugewinn an Plausibilität. Das gilt nicht erst für Trendforscher*innen, sondern für alle Arten von Prophet*innen und Wahrsager*innen:

»Kassandra, die Seherin von Troja, die u.a. die List des hölzernen Pferdes und die fatale Rolle ihres Bruders Paris für den Untergang der Stadt vorhersah, kann als Gegenteil der Delphi-Priesterinnen gelten: Sie konnte tatsächlich die Zukunft (selbstverständlich im antiken Singular) vorhersehen, nur hatte sie, wie man es heute nennen würde, kein Showtalent, sie konnte in der Präsentation ihrer Voraussagen nicht reüssieren, niemand glaubte ihr. Im Gegensatz dazu konnten die Priesterinnen von Delphi nicht tatsächlich vorhersehen, hatten aber gute Inszenierungskünste und konnten so mit ihrer fiktiven Vorhersage überzeugen.«(Gransche 2015: 103)

Die Plausibilität von Zukunftsvorhersagen war so schon immer gekoppelt an eine gelungene Inszenierung, die Kunst, die Vorhersage gut in Szene zu setzen. Die Vorhersage gilt es zu gestalten, damit sie - über die Ereignisbehauptung hinaus - narrativ wirksam werden kann.

Das ist für die heutige Zukunftsforschung insofern von Interesse, da sich diese Performanz auf der einen Seite als Gefahr für den Seriositäts- und Wissenschaftsanspruch der wissenschaftlichen Zukunftsforschung darstellt. Auf der anderen Seite betrifft aber die Gestaltungsmöglichkeit der Zukunft - und nicht nur die Vorhersage der einen möglichen Zukunft - die Zukunftsfor- 
schung im Kern: Bereits die Ereignisbehauptungen der Zukunftsforschung sind immer schon in einen narrativen Kontext eingebettet, implizieren utopische oder dystopische Szenarien und gestalten so die Zukunft, die sie ermöglichen sollen, mit.

Zukunftsforschung entwickelt Szenarien möglicher Zukünfte - strukturiert diese durch die Angabe von Wahrscheinlichkeiten, impliziert aber gerade dadurch Handlungspotential, die Möglichkeit also, selbst einzugreifen und einen Teil der Zukunft aktiv mitzugestalten. Inszenierungen von Zukunftsvorstellungen formen die Zukunft insofern mit, als dass diese Inszenierungen ein hohes Glaubwürdigkeitspotential haben, weil sie eindeutig erscheinen und Handlungsanweisungen beinhalten. Interessant ist daran, dass die Gestaltungsmöglichkeit der Zukunft und nicht das ihr bloße Ausgeliefertsein ein Grund für die Ausdifferenzierung der Zukunftsforschung war. Gestaltungsmöglichkeit geht aber selbst mit Unsicherheit einher: Die Zukunft liegt in den Händen der handelnden Menschen und ist insofern nicht von einer orakelähnlichen Instanz vorhersehbar.

\section{Geschichte der Zukunftsforschung}

Die Zukunftsforschung ist eine vergleichsweise junge Disziplin. Sie hat es entsprechend schwer, sich als Wissenschaft im universitären Kanon zu etablieren. Trotzdem kann die Zukunftsforschung auf eine bisher wechselvolle Entwicklungsgeschichte zurückblicken, deren Verstehen es leichter macht, das Konzept der Megatrends und den Kampf um die Deutungshoheit der Zukunft zu verstehen. So zeigt sich bei näherer Betrachtung, dass der eingangs geschilderte Zwist zwischen wissenschaftlicher Zukunfts- und feuilletonistischer Trendforschung kein für die Zukunftsforschung neues Phänomen ist. Bereits innerhalb der wissenschaftlichen Zukunftsforschung zeigen sich Macht- und Anerkennungskämpfe darum, wie Zukunftsforschung betrieben werden sollte.

Diese Geschichte der Zukunftsforschung wurde bereits ausführlich dargelegt; beispielsweise von Steinmüller (2012, 2013, 2014), Gransche (2015) oder Seefried (2015). Eine schlaglichtartige Rekapitulation der historischen Entwicklung der Zukunftsforschung dient dazu, im Anschluss kurz den Begriff Megatrend zu kontextualisieren und den historischen Hintergrund besser zu verstehen, in dem und für den dieser Begriff entwickelt wurde. Je nachdem, was man unter Zukunftsforschung versteht, ergeben sich unterschiedliche 
Gründungsdaten. Hinzu kommt, dass sich die Zukunftsforschung in den USA und in Europa, insbesondere aber in Deutschland und Frankreich, nicht zeitgleich entwickelte, obwohl sie einen ähnlichen Verlauf nahm. Außerdem fanden wechselseitige Rezeptionen statt, durch die etwa die US-amerikanische die europäische Zukunftsforschung beeinflusste und umgekehrt. Das macht die Rede von »der« Zukunftsforschung problematisch. Daher können hier nur holzschnittartig diejenigen Konfliktlinien aufgezeigt werden, die sich innerhalb verschiedener Schulen der Zukunftsforschung sowie zwischen der wissenschaftlichen Erforschung von Zukunft und der diese Forschung rezipierenden Öffentlichkeit entwickelt haben. Die eingangs geschilderte Unterscheidung von wissenschaftlicher Zukunftsforschung und feuilletonistischer Trendforschung ist, so die These, auf diese Konfliktlinien zurückzuführen.

\section{Zukunft vorhersagen und Zukunft gestalten}

In den späten 1940er Jahren galt es, zumal im Kontext des Kalten Krieges, als besonders produktiv, wenn man die nächsten Schritte des Gegners vorhersagen konnte. Die Förderung des technischen Fortschritts, mit dem die Grenzen der Verarbeitung von Informationen erweitert werden konnten (vgl. Flechtheim 1949: 207-208 in Gransche 2015: 47), war daher einerseits besonders geeignet für die Cold War Science (vgl. Seefried 2015: 49), deren Denken andererseits von den Herangehensweisen des Kalten Krieges selbst geprägt waren: »Wissenschaftler ordneten sich der Politik nicht unter, sondern setzten initiativ Themen und machten sich so in ambivalenter Weise auch die Denkstrukturen des Kalten Krieges zu eigen.«(Ebd.) Zu den daraus entstandenen wissenschaftlichen Tätigkeitsfeldern gehören das Manhattan Project (zur Entwicklung der Atombombe) sowie die ab 1948 vom Militär geförderte RAND Corporation (Research and Development Corporation), die »aus dem Kalkül hoher US-Militärs der Air Force [entstanden ist, um] wissenschaftliche Expertise für Militär und Wirtschaft auch nach Kriegsende zu sichern.« (Seefried 2015: 52) Ihre Methoden, wie die später in der Zukunftsforschung weit verbreitete Szenarioanalyse, entwickelten sich aus einer Mischung von »Kybernetik, der Spieltheorie und der Rational-Choice-Theorie« (Seefried 2015: 53).

Auch Ossip Kurt Flechtheim, der als Begründer der Zukunftsforschung und Erfinder der Bezeichnung Futurologie gilt (vgl. Gransche 2015: 55), die er im US-amerikanischen Exil entwickelte, sieht die Zukunftsforschung als Aus- 
prägung des Kalten Krieges. Genauer sieht er den Einfluss der Sowjetunion im Planungsparadigma, den Einfluss der USA in der Prognostik und stellt diesen beiden Aspekten einen dritten, den Aspekt der »Zukunftsphilosophie«, gegenüber:

»Der Aspekt der Zukunftsgestaltung als der Bereich konkreten Planens und (planwirtschaftlichen) Umsetzens beruht [...] wesentlich auf den Realexperimenten der Sowjetunion. Beide Aspekte kritisiert Flechtheim [...] als neokonservativ, bürokratisch, technokratisch und ergänzt Zukunftsforschung (Prognosewissenschaft) und Zukunftsgestaltung (Planungswissenschaft) um einen methodologisch-reflexiven, ethisch-humanistischen Aspekt, den der Zukunftsphilosophie.«(Gransche, 2015: 49)

Während Flechtheim Zukunftsforschung paradigmatisch den USA und Zukunftsgestaltung der Sowjetunion zuordnet, geht Gransche mit Flechtheim in seiner Einordnung so weit $\mathrm{zu}$ sagen, dass die Zukunftsphilosophie in Deutschland verortet werden könne (vgl. Gransche 2015: 49-50). Am Beispiel Deutschland kann man allerdings ebenfalls aufzeigen, dass auch hier die drei Dimensionen Zukunftsforschung, Zukunftsgestaltung und Zukunftsphilosophie eine Rolle spielten und in ihrer wechselwirkenden Dynamik dazu führten, dass sich die Fronten der Zukunftsforschung verhärteten. Diese beiden Fronten wiederum nennt Flechtheim im Rückblick »kritischhumanistisch« und »konservativ-technokratisch» (Flechtheim 1972: 17-18; zitiert nach Steinmüller 2012: 15).

Im öffentlichen Diskurs in Westdeutschland wurde die Zukunftsforschung das erste Mal 1952 durch die Arbeit von Robert Jungk wahrgenommen. Mit dem Sachbuch »Die Zukunft hat schon begonnen«, in dem er die aktuelle amerikanische Zukunftsforschung beschreibt, begann die Beschäftigung mit ihr jedoch zunächst im öffentlichen Diskurs und nicht innerhalb der scientific community (vgl. Steinmüller 2012: 12). Der Tenor von Jungks Buch lautet:

»Es geht den Amerikanern nicht, wie den meisten Zukunftsdenkern anderer Länder, darum, über die Zukunft zu philosophieren, sondern etwas mitzutun: sie zu erobern und ihr, soweit das menschenmöglich ist, Richtung und Marschtritt vorzuschreiben.く(Jungk 1952, S. 290) ( (Steinmüller 2012: 12).

Jungk verband in seinem einflussreichen Buch die Zukunftsforschung mit dem utopischen Bild des American Dream und bildete diese Kombination zu einer politischen Praxis um: Die Zukunft ist eine (unterschätzte) Ressource, die durch den Menschen gestaltet werden kann. Damit erscheint Jungks Darstel- 
lung bereits als Verbindung von Zukunftsforschung und Zukunftsgestaltung: Die offensive Betonung der Machbarkeit, in der das Omnipotenzversprechen der Kriegspartei des Kalten Krieges mitschwingt, wird verbunden mit einer teils politisch-pragmatisch, teils humanistischen Vorstellung von der Zukunft als einem Möglichkeitshorizont für den Selbstentwurf des neuen Menschen.

Im wissenschaftlichen Diskurs kam die Zukunftsforschung dagegen in der klassischen Form der wissenschaftlichen Rezeption an: Deutsche Wissenschaftler, die ihre ersten Gehversuche im Bereich der Zukunftsforschung unternahmen, kombinierten Prognosemethoden aus dem Dritten Reich - »Trendextrapolationen, Zyklen-Analysen, Analogiebetrachtungen, Nutzung von Expertenmeinungen « - mit den neuen Methoden der amerikanischen Zukunftsforschung und den statistischen Herangehensweisen und methodologischen Reflexionen der Franzosen (Steinmüller 2012: 13). Dennoch kann man die Zukunftsforschung erst ab den 1960er Jahren in Deutschland als wissenschaftlich etabliert bezeichnen, als Institute und Zeitschriften für Zukunftsforschung gegründet wurden. Die Gesellschaft war vor dem utopisch-dystopisch gefärbten Hintergrund dieser Zeit sehr an Zukunftsfragen interessiert, so dass im öffentlichen Diskurs nicht nur Wissenschaftler*innen, sondern auch Politiker*innen und Prominente zu Wort kamen (vgl. Steinmüller 2012: 14). Die Gründung eines Vereins (Gesellschaft für Zukunftsfragen e.V., im Folgenden: GfZ) und die Gründung eines außeruniversitären, interdisziplinären Think-Tanks (Zentrum Berlin für Zukunftsforschung e.V.) waren die Folgeschritte dieser Entwicklung Ende der 1960er Jahre.

Zugleich bildeten sich in dieser Zeit die beiden Fronten heraus, die Flechtheim »kritisch-humanistisch « und »konservativ-technokratisch" nennt und die Steinmüller (2012: 15) wie folgt charakterisiert:

»Einerseits eine sozialkritische, wenn nicht sogar sozialistische, emanzipatorische und utopisch inspirierte Zukunftsforschung, die auf soziale Fantasie setzte [...] [u]nd andererseits eine eher systemtechnisch orientierte, von der Kybernetik inspirierte Zukunftsforschung, die nahe am Markt operierte [...]."

Im wissenschaftlichen Verteilungskampf um Diskursmacht und Forschungsgelder zog der kritisch-humanistische Ansatz, assoziiert u.a. mit Robert Jungk, gegen den konservativ-technokratischen Ansatz, verbunden u.a. mit Karl Steinbuch, einem Informatiker und Fortschrittsbefürworter, den Kürzeren: »Steinbuch forderte die GfZ auf, Jungk, einen sweltfremden Phantasten`, aus ihren Gremien zu samputieren«, um ihren seriösen Ruf zu 
wahren; Jungk zog sich daraufhin selbst zurück, damit der GfZ nicht >der Geldhahn abgedreht « würde (N.N./Der Spiegel 13/1970, S. 195)« (Steinmüller 2012: 16).

Mit dem Sieg der technokratischen Seite und dem Einfluss der Informatik und der mehr mathematisch-naturwissenschaftlich orientierten Methoden verstärkte sich auch der Einfluss der Kybernetik in der Zukunftsforschung. Weil kybernetische Methoden vor allem mit dem Ziel entwickelt wurden, komplexe Systeme zu erfassen, erschienen sie für Zukunftsfragen mehr als geeignet, um »eine objektive und neutrale Politikberatung durch Experten, eine optimale Steuerung sozialer Prozesse, verlässliche Prognosen über Folgen politischer Entscheidungen und verlässliche politische Langfristplanungen [zu] ermöglichen [...] « (Steinmüller 2013: 6). ${ }^{5}$

In den Folgejahren erreichte die Zukunftsforschung zunächst in den USA einen Höhepunkt im Rahmen der Ressourcenkrisen der 1970er Jahre, insbesondere der Ölkrise. Dieser Höhepunkt zeigte jedoch zugleich auf, dass die Zukunftsforschung ihre Versprechen auf eine berechenbare Zukunft nicht erfüllen konnte. Das führte zu einem weiteren Paradigmenwechsel der $\mathrm{Zu}$ kunftsforschung. In »The Limits of Growth« stellten die Autor*innen des Club of Rome 1972 ihre Studie mit Hochrechnungen vor, die durch exponentielles Wachstum in verschiedenen Bereichen Wachstums- und Ressourcengrenzen des Planeten in nur wenigen Jahrzehnten voraussagte. Die Studie war so erschreckend wie kritikanfällig: Die Wachstumsgrenzen seien in den nächsten hundert Jahren erreicht, wenn das Wachstum und die Nutzung von Ressourcen in bestimmten Bereichen genauso ansteigen, wie in den letzten hundert Jahren. In den Berechnungen wurde aber technischer Fortschritt linear bewertet, Bevölkerungswachstum jedoch exponentiell. Die Begründungen für diese methodologischen Entscheidungen sind in »The Limits of Growth « nicht hinreichend, die Vorhersagen sind in ihrer Radikalität nicht eingetreten. Aber auch andere Zukunftsmodelle stellten sich als nicht tragfähig in ihrem Anspruch bzw. im Anspruch der Auftraggeber heraus, die Zukunft vorhersagen zu können; zumindest nicht so, dass man langfristige Planungen auf ihnen

5 Die Kybernetik als Wissenschaft von Steuerungs- und Regelungsmechanismen selbst ging über technokratische Fragestellungen mit beispielsweise dem Methodenideal der Organizität (Maturana/Varela 1980), eklektisch-ganzheitliche Perspektiven (Bateson 1985) oder ethischen Reflexionen (von Förster 1993) hinaus. Dank des Anathemas der »weltfremden Phantasten« wurden diese Aspekte der Kybernetik in der Zukunftsforschung jedoch wenig rezipiert. 
aufbauen konnte (Steinmüller 2013: 7). Die Zukunftsforschung geriet so in eine Krise, die ausgerechnet vom technokratischen Methodenapparat ausgelöst wurde. 1972 diskutierten deshalb die Vertreter*innen der Zukunftsforschung eine Neuausrichtung ihrer Disziplin.

»Die Mehrheit der Teilnehmer wandte sich gegen eine nur von Experten betriebene, aus ihrer Sicht konservative, auf Erhaltung des 'Systems< ausgerichtete >Establishment-Futurologie< mit ihren technokratischen Langfristplanungen im Auftrag der Industrie. Sie setzten ihr eine partizipatorische >kritische Futurologie « entgegen, die als `Theorie und Wille zur Praxis (van Steenbergen 1973, S. 90) zu den gesellschaftlichen Veränderungen beitragen sollte [...]«(Steinmüller 2013: 8).

Dafür müsse die Zukunftsforschung aber ihre Eigenständigkeit als Disziplin aufgeben und sowohl inter- als auch transdisziplinär arbeiten, als Kompetenzfeld verschiedener Disziplinen. Zukunftsforschung und Friedensforschung verbanden sich auf diese Weise in Zentren und Gemeinschaften, die so Zukunftsforschung und Friedensideal mit einer dezidierten Partizipationskultur kombinierten. Bürger*innen wurden als kritischer Gegenpart zur technokratischen Zukunftsforschung eingebunden.

»Die partizipativen Verfahren entsprachen dem emanzipatorischen Anspruch, sie öffneten die Zukunftsforschung zur Breite der Cesellschaft und zugleich sind sie der wichtigste Beitrag, den die sneue Phase< zur Entwicklung der Zukunftsforschung leistete. Dagegen aber taugten die globalen Cesellschaftsentwürfe und phantasievollen Utopien der kritischen Futurologen nicht zur Umsetzung in der realen Welt (Kreibich 1991, S. 136)《 (Steinmüller 2013: 9-10).

So verlor die derart popularisierte Zukunftsforschung ihren wissenschaftlichen Anspruch und das Kriterium der Überprüfbarkeit von Methode und Vorhersage sowie ihre Relevanz im transformativen Sinn. Sie konnte weder dem Anspruch einer Prognose gerecht werden, noch dem der Zukunftsgestaltung. Damit hatte sie der Gesellschaft nichts mehr zu bieten - keine Vorhersagen, keine Handlungsempfehlungen oder Handlungsmöglichkeiten. Wo die Zukunftsforschung in den 1960er und 1970er Jahren auf Popularität setzte, geriet sie schnell in den Ruch einer weltfremden Harmonieideologie. Wo sie dagegen als pragmatisches Instrument des militärisch-industriellen und zunehmend auch des wirtschaftlichen Bereichs der Gesellschaft firmierte, wurde sie der Instrumentalisierung menschlicher Möglichkeitsräume verdächtigt. 
Die Tendenz der beiden wissenschaftlichen Paradigmen zu einem immer mehr ideologisch aufgeladenen Kampf um die Zukunft radikalisierte Alvin Toffler bereits 1970 in seinem Sachbuch »Future Shock« zu einem Geschäftsmodell, indem er

»als umfassendes gesellschaftliches Leitmotiv den >Disease of Change $<$, die Krankheit durch den Wandel oder die am Wandel diagnostizierte Krankheit und damit das Grundmotiv des Bedrohungs-Szenarios formulierte. Cestützt durch anekdotische Evidenz und opportune Belegketten aus Einzelbeobachtungen, die als Markierungen der Zukunft charakterisiert wurden, entwickelte Toffler einen neuen Stil, der den öffentlichen Bedürfnissen entgegenkam und die News Values der unmittelbaren Betroffenheit und der Sensationslust durch die Prophezeiung revolutionärer Veränderungen befriedigte.« (Rust 2009: 10)

Das Buch wurde zu einem Bestseller. Die wissenschaftliche Zukunftsforschung hatte dieser Analyse nichts entgegenzusetzen. Der Streit, der sich innerhalb der Disziplin entwickelt hatte, war nach außen gedrungen und hatte den öffentlichen Diskurs erreicht. Von nun an saßen die Konkurrenten um die Deutung der Zukunft nicht mehr in der Wissenschaft, sondern kamen aus der Öffentlichkeit selbst. Die feuilletonistische Trendforschung war geboren, die sich demagogischer Mittel bediente, um kurzgreifende Prognosen möglichst wirksam zu verkaufen.

Anfang der 1980er Jahre wurde es still um die wissenschaftlich geprägte Zukunftsforschung, während sich auf dem Sachbuchmarkt Zukunftsdeutungen gut verkauften. ${ }^{6}$ Die beiden Lager der kritischen bzw. pragmatischen Forschung standen sich unversöhnlich gegenüber; die adaptierten gesellschaftspolitischen Themen beispielsweise der Umwelt- und Energiepolitik differenzierten sich im öffentlichen und wissenschaftlichen Diskurs aus und wurden Gegenstand selbstständiger Institute. Damit schien der Zukunftsforschung auch ihr Gegenstandsbereich abhanden zu kommen:

»Diskreditiert durch gravierende Fehlprognosen über Wirtschaftswachstum, Energiebedarf und Verkehr sowie durch medial verbreitete unrealistische Hightech-Phantasien, hatte die Zukunftsforschung ihre Attraktivität 
für ökologisch orientierte und sozialkritische Wissenschaftler verloren (Kreibich 1991, S. 107)« (Steinmüller 2013: 15).

Als Folge wurde 1981 eine der wichtigsten deutschen Zeitschriften der $\mathrm{Zu}$ kunftsforschung - »analysen und prognosen - über die Welt von morgen« - eingestellt. 1982 wurde das IFZ aufgrund von personellen, konzeptionellen und finanziellen Schwierigkeiten aufgelöst, wobei Letzteres nicht an fehlenden Projektmitteln lag, sondern an der Schwierigkeit der Vorfinanzierung im Institut (vgl. Steinmüller 2013: 14). Vor dem Verlust aller Zukunftsforschung im universitären und öffentlichen Bereich, gründete Kreibich 1981 das Institut für Zukunftsstudien und Technologiebewertung (IZT). »Als das einzige unabhängige und gemeinnützige, auf Zukunftsforschung spezialisierte Institut in der Bundesrepublik hatte das IZT über Jahre eine exzeptionelle Stellung inne.« (Steinmüller 2013: 16) Sie war lange die letzte Bastion dieser Forschungsrichtung und hat bis heute in einem kleinen wohnhausähnlichen Gebäude in Berlin überlebt.

Eine Weiterführung des normativ-gesellschaftlichen Zweiges der $\mathrm{Zu}$ kunftsforschung lässt sich erst in der in den 1990ern aufkommenden Nachhaltigkeitsforschung nachweisen (vgl. Steinmüller 2014: 8), die sich explizit argumentativ auf die kommenden Generationen richtet und verschiedene Dimensionen der Entwicklung (ökonomisch, ökologisch, politisch usw.) mit einbezieht. War Nachhaltigkeitsforschung einst Teil der normativen Zukunftsforschung, so ist diese heute umgekehrt ein wichtiger Teil der Nachhaltigkeitsforschung. In gewisser Weise verschiebt sich damit in der Wissenschaft der Fokus weg von einem prognostischen Entwurf der Zukunft auf eine Erhaltung der Vergangenheit.

Für den prognostischen Bereich der Zukunftsforschung interessierten sich vor allem Unternehmen. Aufgrund der intrinsischen Motivation des Kapitalismus, den Markt beherrschbar zu machen (Vogl 2010) ${ }^{7}$, wurde die Zukunftsforschung im konservativ-technokratischen Sinne durch die $\mathrm{Ab}$ spaltung der normativen Ebene für Unternehmer*innen interessant. Sie richteten Bereiche für "Strategische Planung « und Forschungsgruppen ein; Unternehmensberatungen spezialisierten sich auf Szenarien-Bildung (vgl. Steinmüller 2013: 16-17) und strategisches Management.

Während also in einzelnen Bereichen Zukunftsforschung im Kleinen betrieben wurde, jeweils bezogen auf klar begrenzte Systeme wie die eines ein-

$7 \quad$ Vgl. dazu im Besonderen Kapitel 5 und 6. 
zelnen Unternehmens, einer Branche oder einer thematisch eingegrenzten Entwicklung, gab es für die Entwicklungen, die das Gesellschaftssystem im Ganzen betrafen, keine großen Leitlinien. Die Nische der ökonomisch orientierten Zukunftsforschung entwickelte sich zeitgleich mit der Ratlosigkeit der Wissenschaft im Umgang mit der Zukunft zu einem immer lukrativeren Geschäftsmodell. Nach den unbefriedigenden Ergebnissen sowohl der konservativ-technokratischen als auch der kritisch-humanistischen Zukunftsforschung war die Erforschung zukünftiger Modelle damit auf dem Dienstleistungsmarkt der Unternehmensberatung angekommen.

Die Zukunft erschien so ungewiss wie nie zuvor, solange man nicht im Besitz des privatwirtschaftlich erzeugten Herrschaftswissens der ökonomischen Trendforschung war. Die Krise der wissenschaftlichen Zukunftsforschung und ihre Unfähigkeit zur nützlichen Vorhersage nährte die Angst vor einer ungewissen Zukunft, den von Toffler diagnostizierten »Disease of Change«. In diese Lücke spielte die feuilletonistische Trendforschung. Sie

»nutzte die zunehmende Desillusionierung der wissenschaftlichen $\mathrm{Zu}$ kunftsforschung, die unter dem Druck ihrer Einsichten in die unausweichlich wachsende Komplexität der Wirklichkeit, die Kontingenz der soziokulturellen und wirtschaftspolitischen Entwicklung und die wechselseitigen Wirkungen einer großen Zahl von Komponenten in unüberschaubaren Umwelten ihren Optimismus dämpfte.« (Rust 2008: 64)

Die ökonomisch orientierte Trendforschung ist damit ein Nutznießer des Ringens der Wissenschaft um eine unabhängige Zukunftsforschung. Der Streit innerhalb der Wissenschaft um einen allzu idealistischen oder allzu pragmatischen Umgang mit der Zukunft führte zu einer Delegitimierung beider Ansätze und führte in eins mit dem technologischen Aufstieg am Ende des 20. Jahrhunderts und dem damit einhergehenden Anwachsen von Komplexität zu einer paradoxen Situation: Eine immer ungewisser erscheinende Zukunft stand immer selbstsicherer wirkenden Vorhersagen gegenüber.

\section{Megatrends und Trendforschung}

Das Bedürfnis nach Orientierung und der fehlende Optimismus für die $\mathrm{Zu}$ kunft waren entscheidende Faktoren für den Erfolg des Konzepts der Megatrends. John Naisbitt - der Erfinder des Begriffs und Konzepts Megatrend schließt mit der Aufzählung der Entwicklungen, mit denen die Gesellschaft 
in den kommenden Jahrzehnten konfrontiert sei, nicht nur die Lücke der Orientierungslosigkeit, er macht sie auch noch am Kapitalismus fest, indem er die ökonomischen Entwicklungen als entscheidenden Einflussfaktor begreift:»Das Geschäft ersetzt die Politik als Weltstimmungsbarometer!« (Naisbitt 1984: 353, zitiert nach Opaschowski 2015: 42) Dieser Slogan misst dem ökonomischen Bereich den höchsten Stellenwert zu: Geht es der Wirtschaft gut, so geht es der Gesellschaft gut, weil sich die Wirtschaft gut an die Megatrends anpasst und die Gesellschaft dadurch an Orientierung gewinnt. Die Adressat"innen sind also in erster Linie die Unternehmer*innen, die es zum megatrendorientierten Handeln anzuleiten gilt. Dieses System hat das Potential dazu, selbstreferentiell zu werden: Die Megatrends beziehen sich auf die Nachfrage der Unternehmen, die durch diese Megatrends wiederum bestätigt und gelenkt werden. Die kulturbildende Funktion dieses Kreislaufsystems ist nicht zu unterschätzen. Sie ermöglicht den Unternehmen über das Einfallstor der Zukunft als Thema - Zukunftsangst, Anlegerinteressen, Kreditversprechen, politische Weltanschauungen als gestaltete Zukunft usw. auf einzigartige Weise auf die Gesellschaft Einfluss zu nehmen.

Das zugrunde liegende zirkuläre Muster - nur in Hinblick auf die Vergangenheit und nicht auf die Zukunft - findet sich idealtypisch in Naisbitts Ausgangsüberlegung zur Identifikation von »Megatrends« in der Gesellschaft. Wie kam er auf die Idee, die Megatrends, die er noch nicht mit Buzzwords belegte, sondern mit »von ... zu ...«-Entwicklungen, also genetisch, beschreibt (beispielsweise »Von der Nationalökonomie zur Weltwirtschaft«, »Von Norden nach Süden« oder »Von kurzfristig zu langfristig«), als solche zu identifizieren? Naisbitt erzählt dazu folgende Geschichte:

»Es gab tatsächlich so etwas wie einen auslösenden Funken, der mich dazu inspirierte, das Buch »Megatrends «zu schreiben. Ich kaufte gerade eine Ausgabe der sSeattle Times an einem etwas abgelegenen Kiosk in Chicago. Als ich da so stand und auf mein Wechselgeld wartete, wanderte mein Blick über die verschiedenen Schlagzeilen der vielen lokalen Blätter aus ganz Amerika, die dort verkauft wurden. Beim Anblick der diversen Artikel wurde mir plötzlich bewusst, dass man ganz neue Entwicklungsmuster des Landes erkennen könnte, wenn man all diese lokalen Zeitungen jeden Tag gleichzeitig lesen würde. Man könnte wirklich verstehen, was in den Vereinigten Staaten los ist. Das war der Schlüssel.« (Naisbitt 2015: 3)

Naisbitt, der zuvor in politischen und unternehmerischen Kontexten gearbeitet hat und Erfahrungen in der Trendforschung zum Thema Stadt und In- 
tegration mit seinem eigenen Unternehmen sammelte (vgl. Rust 2009: 10), stellte also in seinem Buch Megatrends die gesellschaftlichen Großentwicklungen zusammen, die er durch den Vergleich lokaler Zeitschriften - durch "content analysis«, wie er diese "Methode« bezeichnet - entdecken zu können glaubte. Er geht davon aus, dass diese Entwicklungen größer seien als einfache Trends, langlebiger, stärker im Einfluss auf die Gesellschaft. »Das Buch erlebte einen gigantischen Erfolg - erstaunlicherweise, denn es enthält nichts, was nicht längst bekannt, diagnostiziert und in der wissenschaftlichen Foresightforschung fundiert ausgebreitet war. «(Rust 2008: 81) ${ }^{8}$

Erfolgreich war Naisbitts Buch nicht zuletzt deswegen, weil die vergleichsweise trivialen Erkenntnisse - eine simple Zusammenfassung und Zuspitzung dessen, was Ergebnis einer recht einseitigen und sogar interessengeleiteten Medienanalyse ist - nicht als Beschreibungen des aktuellen Diskurses, sondern als sich daraus ergebende Prophezeiungen für die Zukunft deklariert wurden. Die Bestimmung der einen Zukunft, die Orientierung versprach und die im Gegensatz zum Bericht des Club of Rome in Optimismus gekleidet war, war der Schlüssel für die feuilletonistische Zukunftsforschung. Sie ermöglichte es, die Zukunft so darzustellen, dass sich die Rezipient"innen dieser Forschung auf diese Zukunft einrichteten und die Vorhersage so nachträglich bestätigten. Die fehlende wissenschaftliche Belegbarkeit wurde durch Showtalent und Kreativität - der Erfindung eigener, wissenschaftlich klingender, aber letztlich zirkulärer »Methoden« - wettgemacht und der Zugriff der Öffentlichkeit im Konkurrenzgeschäft der Zukunftsforschung beschränkt. Dadurch war man in der Lage, den Wissenschaftlichkeitsanspruch immer weiter auszubauen, ohne je in die Gefahr einer unabhängigen Überprüfung zu geraten. Die so etablierte feuilletonistische Trendforschung erhielt mit dieser Strategie und ihrem Erfolg in Wirtschaft und Politik irgendwann auch das Vertrauen der Öffentlichkeit. Die Rollen waren verkehrt: Die Öffentlichkeit, die eigentlich die kritische Prüfinstanz wissenschaftlicher Ansprüche ist, wurde zur Konsumentin einer

Ähnliche Befunde, allerdings gekennzeichnet als Rahmenbedingungen für Trendentwicklungen und selbst nur Systembeschreibung ohne Prognosekompetenz, wurden bereits 1979 von Harry Igor Ansoff in seinem Buch Strategic Management dargelegt (vgl. Rust 2008: 71-72). Inwiefern sich Naisbitt hat davon inspirieren lassen, ist unklar. Auch Naisbitts spätere Analysen sind geprägt von offensichtlichen Beschreibungen der Cegenwart, die er als Zukunft verkauft, wie 1990 für 2000 »den Eintritt in ein Zeitalter der Bio- und Centechnologie, den Triumph des Individuums über den Wohlfahrtsstaat und das Ende des Sozialismus (ein Jahr nach dem Fall der Mauer)«(Rust 2008: 82). 
»Wissenschaft«, die sich selbst überprüfte, die Prüfkriterien aber niemals offenlegte.

Die wissenschaftliche Zukunftsforschung geriet durch diese unerwartete Konkurrenz massiv unter Druck. Obwohl die feuilletonistische Trendforschung gar keine Wissenschaft betrieb, wurde sie durch ihren scheinbaren Erfolg maßgeblich auch für die öffentliche Unterstützung wissenschaftlicher Forschung. Zum einen wurde es schwer, für den Forschungszweig der $\mathrm{Zu}$ kunftsforschung Drittmittel zu akquirieren, zum anderen war die Zukunftsforschung selbst für Wissenschaftler*innen, die die Methoden der Zukunftsforschung beherrschten, oft wenig attraktiv, weil sie die Gefahr sahen, mit den feuilletonistischen Trendforscher*innen in einen Topf geworfen zu werden (vgl. Gransche 2015: 100). »Somit ergibt sich derzeit die paradoxe Situation, dass wissenschaftlich fundierte zukunftsorientierte Forschung zum allergrößten Teil außerhalb der Szene der so genannten Zukunftsforschung geleistet wird.«(Popp 2012: VI, zitiert nach Gransche 2015: 100)

\section{Eine Geschichte des Scheiterns?}

Die Geschichte der Zukunftsforschung ist geprägt vom Scheitern. Zunächst scheiterte sie daran, eine einheitliche Vorgehensweise für ihre Disziplin zu finden; sie rieb sich auf in Grabenkämpfen zwischen der kritisch-humanistischen und der konservativ-technokratischen Herangehensweise der Wissenschaftler*innen und dem fehlenden Austausch sowie der fehlenden Bereicherung für beide Seiten. Dieser Kampf und die bis dahin verzeichneten Misserfolge in der Prognostik und der Gestaltung der Zukunft sorgten dafür, dass die Zukunftsforschung gesellschaftlich irrelevant wurde und auch in weiten Teilen der Politik und Ökonomie keinen Platz mehr fand. Die daraus entstandene Lücke nutzte die feuilletonistische Trendforschung und rief nicht nur einzelne, kurzlebige Moden auf, sondern gestaltete aktiv Megatrends, gesellschaftliche Entwicklungen also, die die globale Zukunft langfristig und in allen Lebensbereichen beeinflussen würden.

Wie diese Megatrends konkret erfasst werden und aus welchen Gründen sie dem Anspruch genügen sollen, weit in die Zukunft zu wirken, bleibt bis auf Weiteres unklar. Doch warum sollte man abstrakt Methoden reflektieren, wenn der Erfolg der Trendforschung für sich selbst spricht? Das zirkuläre Schema - ein oft genanntes Thema aufzugreifen, zu einem »Megatrend « auszugestalten, dadurch Entscheider*innen zu beeinflussen, die im besten Fall 
für das Eintreten der »Vorhersage« sorgen - verhilft der feuilletonistischen Trendforschung gemeinsam mit dem Abstraktionsgrad der Begrifflichkeiten und dem Hintertürchen des »noch nicht eingetroffen « $\mathrm{zu}$ einem steten Fluss der korrekten Annahmen, einer Leistung also, die die wissenschaftliche $\mathrm{Zu}$ kunftsforschung nie erreicht hat.

Das gleiche Schema ist auch der Grund für die Schwierigkeiten, mit denen wissenschaftliche Zukunftsforschung heute zu kämpfen hat: Durch den Erfolg der de facto unwissenschaftlichen Methoden der Trendforschung, die sich als wissenschaftliche maskieren, um gesellschaftliches Prestige zu akkumulieren, spielt die de facto wissenschaftliche Zukunftsforschung keine Rolle mehr, weil ihre Herangehensweise als wenig erfolgversprechend gilt. Die wissenschaftliche Methode wird durch die zirkuläre Produktion von Ergebnissen ausgehebelt und Wissenschaftlichkeit wird zu einem Markenprädikat, das nur noch an das Behaupten, nicht mehr an den Vollzug wissenschaftlicher Methodik gebunden ist. Durch diese verquere Entwicklung ist wissenschaftliche Zukunftsforschung heutzutage eher ein Nischenbetrieb, der in anderen Disziplinen mit zum Tragen kommt, dort allerdings pragmatisch klingende Titel wie »Nachhaltigkeitsforschung« oder »Technikfolgenabschätzung« trägt.

Stellt man sich die Frage, für welches Problem die feuilletonistische Trendforschung die Lösung war ${ }^{9}$, so wäre eine mögliche Antwort: Es beruhigt die Gesellschaft zu wissen, dass es ein Wissen um die Zukunft gibt, ganz gleich, ob es wissenschaftlich belegt werden kann oder nicht ${ }^{10}$. Der Streit der Zukunftsforschung um eine ideologische oder pragmatische Ausrichtung wurde von der Trendforschung für die erstere Option entschieden. Die Gesellschaft braucht die Vorhersagen der Trendforschung nicht, um Handlungsempfehlungen abzuwägen, sondern um sich ein beruhigendes Bild von der Zukunft zu machen: Es ist alles unter Kontrolle.

In Zeiten des Klimawandels können solche optimistischen Zukunftserzählungen gefährlich sein - nicht nur für die wissenschaftliche Zukunftsforschung, sondern für die Menschen, die damit beruhigt werden, dass die Zukunft ausschließlich Positives für sie bereithält. Eine solche ideologische Funktion der Zukunftsforschung ${ }^{11}$ ersetzt planvolles Handeln in unübersicht-

9 Angelehnt an Nassehi 2019: 12: »Für welches Problem ist die Digitalisierung eine Lösung?«

10 Vgl. den Beitrag von Schecke/Schweiger in diesem Band.

11 Vgl. den Beitrag von Schecke/Schweiger in diesem Band. 
lichen Situationen durch Herrschaftswissen über den tatsächlichen Verlauf der Geschichte. Das Gegenmittel wäre eine Rückkehr der wissenschaftlichen Methode in das Bewusstsein der Gesellschaft: Ambiguitätstoleranz (Bauer 2018), Pluralismus und Skepsis, hypothetisches statt setzendes Denken müssten auf dem Lehrplan stehen, um zukünftige Generationen gegen diese Entwicklung fit zu machen.

\section{Literatur}

Bateson, Gregory (1985): Ökologie des Geistes. Anthropologische, psychologische, biologische und epistemologische Perspektiven, Frankfurt a.M.: Suhrkamp.

Bauer, Thomas (2018): Die Vereindeutigung der Welt. Über den Verlust an Mehrdeutigkeit und Vielfalt, Stuttgart: Reclam.

Flechtheim, Ossip K. (1949): »Futurology: The New Science?«, in: The Forum, April 1949, S. 206-209.

Flechtheim, Ossip K. (1972): Futurologie. Der Kampf um die Zukunft, Frankfurt a.M.: Fischer.

Förster, Heinz von (1993): KybernEthik, Berlin: Merve Verlag.

Gransche, Bruno (2015): Vorausschauendes Denken. Philosophie und Zukunftsforschung jenseits von Statistik und Kalkül, Bielefeld: transcript. https://doi.org/10.14361/9783839430385

Grunwald, Armin (2009): »Wovon ist Zukunftsforschung eine Wissenschaft?«, in: Reinhold Popp/Elmar Schüll (Hg.), Zukunftsforschung und Zukunftsgestaltung, Beiträge aus Wissenschaft und Praxis, Berlin: Springer, S. 2535. https://doi.org/10.1007/978-3-540-78564-4_3

Hajkowicz, Stefan/Cook, Hannah/Littleboy, Anna (2012): Our future world. Global megatrends that will change the way we live, The 2012 Revision, Australia: CSIRO.

Horx, Matthias (2007): Die Macht der Megatrends. Wie Globalisierung, Individualisierung und Alterung unsere Welt verändern werden, Kelkheim: Zukunftsinstitut Horx GmbH. Online verfügbar unter: www.horx.com/Reden/Macht-der-Megatrends.aspx. Zuletzt aufgerufen am 20.05.2019.

Jischa, Michael F. (2009): »Gedanken zur Wahrnehmung der Zukunft«, in: Reinhold Popp/Elmar Schüll (Hg.), Zukunftsforschung und Zukunftsge- 
staltung, Beiträge aus Wissenschaft und Praxis, Berlin: Springer, S. 37-50. https://doi.org/10.1007/978-3-540-78564-4_4

Lorenz, Ullrich/Haraldsson, Hördur V. (2014): Impact assessment of global megatrends. Two case studies connecting global megatrends to regional topics, Swedish Environmental Protection Agency Report 6602, Bromma: Swedish EPA. Online verfügbar unter: https://www.naturvardsverket.se/ Documents/publikationer6400/978-91-620-6602-4.pdf?pid=11739. Zuletzt aufgerufen am 13.01.2020.

Lübbe, Hermann (1994): Im Zug der Zeit. Verkürzter Aufenthalt in der Gegenwart, Berlin: Springer. https://doi.org/10.1007/978-3-662-07852-5

Maturana, Humberto R./Varela, Francisco J. (1980): Autopoiesis and cognition. The realization of the living, Boston: D. Reidel. https://doi.org/10. 1007/978-94-009-8947-4

Meadows, Donella H./Meadows, Dennis L./Randers, Jorgen/Behrens III, William W. (1974): The Limits of Growth. A Report for the Club of Rome's Project on the Predicament of Mankind, New York: Universe Books.

Naisbitt, John (1982): Megatrends. Ten New Directions Transforming Our Lives, New York: Warner Books.

Naisbitt, John (2015): »Der Horizont reicht meist nur bis zum nächsten Wahltag.«, Interview mit John Naisbitt, in: Aus Politik und Zeitgeschichte 65, Heft 31-32: Megatrends?, S. 3-5.

Naisbitt, John/Aburdene, Patricia (1985): Re-Inventing the Corporation. Transforming Your Job and Your Company for the New Information Society, New York: Warner Books.

Nassehi, Armin (2019): Muster. Theorie der digitalen Gesellschaft, München: C. H. Beck. https://doi.org/10.17104/9783406740251

Opaschowski, Horst W. (2015): »Mode, Hype, Megatrend? Vom Nutzen wissenschaftlicher Zukunftsforschung«, in: Aus Politik und Zeitgeschichte (APuZ) 65, Heft 31-32: Megatrends?, S. 40-45.

Popp, Reinhold (Hg.) (2012): Zukunft und Wissenschaft. Wege und Irrwege der Zukunftsforschung, Berlin: Springer. https://doi.org/10.1007/9783-642-28954-5

Rust, Holger (2008): Zukunftsillusionen. Kritik der Trendforschung, Wiesbaden: VS Verlag für Sozialwissenschaften.

Rust, Holger (2009): »Verkaufte Zeit. Strategien und Inhalte der kommerziellen >Trendforscher« «, in: Reinhold Popp/Elmar Schüll (Hg.), Zukunftsforschung und Zukunftsgestaltung, Beiträge aus Wissenschaft und Praxis, Berlin: Springer, S. 3-16. https://doi.org/10.1007/978-3-540-78564-4_1 
Schweiger, Stefan/Schecke, Nora (im Erscheinen): „Quid agis, Megatrend? Von anekdotisch deduzierten Zukunftserzählungen und ihren Wirkungen«, in: Steven Engler/Julia Janik/Matthias Wolf (Hg.), Energiewende und Megatrends. Wechselwirkungen von globaler Gesellschaftsentwicklung und Nachhaltigkeit, Bielefeld: transcript, S. 81-116.

Seefried, Elke (2015): Zukünfte. Aufstieg und Krise der Zukunftsforschung 1945-1980, Berlin: de Gruyter Oldenbourg. https://doi.org/10.1515/ 9783110349122

Steinmüller, Karlheinz (2012): Zukunftsforschung in Deutschland. Versuch eines historischen Abrisses (Teil 1), in: Zeitschrift für Zukunftsforschung, 1, 1, S. 6-19.

Steinmüller, Karlheinz (2013): Zukunftsforschung in Deutschland. Versuch eines historischen Abrisses (Teil 2), in: Zeitschrift für Zukunftsforschung, 2, 1, S. 5-21.

Steinmüller, Karlheinz (2014): Zukunftsforschung in Deutschland. Versuch eines historischen Abrisses (Teil 3), in: Zeitschrift für Zukunftsforschung, 3, 1, S. 5-23.

Taylor, Paula (1982): Kids' Whole Future Catalog, New York: Random House. Toffler, Alvin (1970): Future Shock, New York: Random House.

Toffler, Alvin (1980): The Third Wave, New York: Morrow.

Vogl, Josef (2010): Das Gespenst des Kapitals, Zürich: Diaphanes Verlag.

Zukunftsinstitut GmbH (o.J.): Megatrend-Dokumentation. Online verfügbar unter: https://onlineshop.zukunftsinstitut.de/shop/megatrend-dokumentation/. Zuletzt aufgerufen am 13.01.2020. 



\section{Trends und Megatrends als Ansatz der modernen Zukunftsforschung Entwicklung und Praxis}

Edgar Göll

Menschliche Gesellschaften befinden sich in permanenter Veränderung, es sind lebendige Systeme. Das wird bereits in alten Weisheiten zum Ausdruck gebracht, etwa durch die Heraklit von Ephesos zugeschriebene Erkenntnis: »Nichts ist so beständig wie der Wandel.« Auch im »I Ging - Buch der Wendungen« oder in Ovids »Metamorphosen« wird dies meisterhaft artikuliert. Und der arabische Gelehrte Ibn Khaldun hat schon vor über sechshundert Jahren in seinem »Buch der Beispiele. Die Einführung in die al-Muqaddima« die Machtwechsel seiner Ära und Region genau beobachtet und so etwas wie »historische Muster« und Zyklen beschrieben.

Seither haben sich Veränderungsprozesse in den meisten Gesellschaften hinsichtlich Dynamik und Verbreitung sehr verstärkt, insbesondere in hochindustrialisierten und sich industrialisierenden Ländern ist dies zu beobachten (vgl. Rosa 2005). Zugleich nimmt die Komplexität von Gesellschaften zu und damit die Schwierigkeit, die jeweilige Gegenwart hinreichend zu beschreiben. Umso größer ist daher die Herausforderung, die als wesentlich einzustufenden Entwicklungslinien und maßgeblichen Veränderungsmuster $\mathrm{zu}$ benennen und fundiert zu beschreiben.

\section{Das menschliche Bewusstsein für die Zukunft}

Voraussetzung für die Wahrnehmung und für das mehr oder weniger zutreffende Verständnis von gesellschaftlichem Wandel ist das menschliche Zeitempfinden. Das in stetiger Entwicklung sich befindliche Selbstbewusstsein des Menschen bringt auch ein Bewusstsein für die Zukunft mit sich. 
Menschen sind sich darüber bewusst, dass sie in der Zeit leben, dass es ein Gestern, ein Heute und Morgen gibt. Hierbei kann zwischen individueller und kollektiver Zukunft unterschieden werden. Zum einen interessieren sich Menschen vor allem für die eigene, die persönliche Zukunft. Aber schon früh in der Menschheitsgeschichte gibt es offensichtlich auch ein Interesse für Fragen, die über die individuelle Zukunft hinausgehen. Als vergesellschaftetes Wesen kommt für den Menschen die Zukunft der eigenen Gruppe ins Spiel, die sich im Laufe der Entwicklung sozial und geographisch ausweitet. Das Interesse an der Zukunft kann demnach als anthropologische Konstante betrachtet werden. Während jedoch die Vergangenheit mit dem Gedächtnis eine Zugangsmöglichkeit besitzt und die Gegenwart durch das Bewusstsein zugänglich ist, verbleibt die Beschäftigung mit Zukunft in prinzipieller Ungewissheit (vgl. Wersig 2009).

Im historischen Verlauf des Umgangs mit Zukunft ergaben sich im westlichen Kulturkreis unterschiedliche Muster. Ab etwa dem 17. Jahrhundert ergab sich die schrittweise Herausbildung und Verbreitung des an Naturgesetzen orientierten, durch exakte Voraussagen legitimierten neuzeitlichen Wissenschaftsbegriffes. Zunehmend verbreiteten sich empirisch-analytische und formalisierend-mathematische Wissenschaftsmethoden. Daraus ergab sich die Sichtweise, dass Prognosen zum »wissenschaftlichen Geschäft « gehören. Zukunft wurde als Objekt angesehen, welches methodisch aus der Gegenwart abgeleitet werden könne. Andererseits verbreitete sich spätestens gegen Ende des 19. Jahrhunderts in Europa ein starkes Interesse für die Zukunft, das sich auch in der Literatur und Diskussion der damaligen Zeit niederschlug. Demnach muss sich die Zukunft nicht vollständig aus den Zwängen der Gegenwart ableiten, denn es kommt auch Neues hinzu (hierfür stehen exemplarisch C. H. de Saint-Simone, Charles Fourier und schließlich auch Karl Marx). Im 20. Jahrhundert wiederum lassen sich zwei Verständnisse von Zukunft erkennen. So dominierte vom Ende des Zweiten Weltkriegs bis in die 1970er Jahren in der entstehenden Zukunftsforschung ein vornehmlich quantitatives und naturwissenschaftliches Wissenschaftsverständnis. Und so wurde auch die Zukunft als etwas angesehen, dass "ausgerechnet « werden kann. Anfang der 1970er Jahren fand in thematischer, methodischer und organisatorischer Hinsicht nach und nach eine Relativierung dieser affirmativen und technokratischen Ausrichtung statt und die Zukunft wurde als prinzipiell offen angesehen. Eine zeitgenössische Definition: 
»Man kann die Zukunft nicht vorhersagen. Man kann allerdings solides wissenschaftliches Zukunftswissen erarbeiten und nutzen, um Zukünfte besser zu erfassen (wahrscheinliche, mögliche, wünschbare), und in einem partizipativ-demokratischen Prozess darauf hinarbeiten, dass Katastrophen vermieden und die besten zukunftsfähigen Zukünfte realisiert werden « (Kreibich 2013: 381).

Kurz gefasst soll Zukunftsforschung im Anschluss an diese Definition hier als die wissenschaftliche Befassung mit möglichen, wahrscheinlichen und wünschbaren Zukunftsentwicklungen (»Zukünften«) und Gestaltungsoptionen sowie deren Voraussetzungen in Vergangenheit und Gegenwart verstanden werden.

\section{Zukunftsforschung und Megatrends}

Vor dem Hintergrund großer, historisch einmaliger Herausforderungen und bedrohlicher Entwicklungen kommt der Zukunftsforschung eine besondere Rolle $\mathrm{zu}$, denn sie versucht, nicht nur intendierte, sondern auch unintendierte und langfristige Effekte von Verhaltensweisen, Maßnahmen und Strategien in den Blick zu nehmen, zu sondieren und einzuschätzen. Einer der wichtigsten Ansätze moderner Zukunftsforschung und Zukunftsgestaltung ist die Trend- und die neuere Megatrendforschung.

Der Begriff »Megatrend« wurde 1982 vom US-amerikanischen Futurologen John Naisbitt geprägt. Der Politologe Naisbitt, der auch den Begriff "Globalisierung « bekannt machte, ist einer der bekanntesten Trend- und Zukunftsforscher, beriet US-Präsidenten. John Naisbitt definierte Megatrends als »large social, economic, political, and technological changes (...) [which] influence us for some time - between seven and ten years, or longer (Naisbitt/Aburdene 1990: xvii, ähnlich Naisbitt 1982). Mit diesem Ansatz beschrieb er die damaligen umfangreichen Umwälzungen in den USA. Die zehn Megatrends waren Veränderungsprozesse...

1 von der Industriegesellschaft zur Informationsgesellschaft

2 von niedriger $\mathrm{zu}$ höherer Technologie

3 von der Nationalökonomie zur Weltwirtschaft

4 von kurzfristig zu langfristig

5 von Zentralisation zu Dezentralisation 
6 von der institutionalisierten Amtshilfe zur Selbsthilfe

7 von der repräsentativen zur partizipatorischen Demokratie

8 von Hierarchien zu Verbundenheit, Verflechtung und gegenseitiger Abhängigkeit

9 von Norden nach Süden

10 vom »Entweder-Oder« $\mathrm{zu} »$ multiplen Optionen« (Naisbitt 1984: 7f.)

Eine wichtige Erkenntnis bei der Beschreibung und Einschätzung von Megatrends ist, dass Megatrends in sich nicht homogen, monolithisch und gleichförmig sind, sondern auch Widersprüche und Gegentrends beinhalten und diese teilweise provozieren. Zum Beispiel hat sich gemäß Naisbitt angesichts der zunehmenden Durchtechnisierung des Alltags- und Arbeitslebens moderner Menschen auch das Bedürfnis nach unmittelbarer körperlicher Berührung intensiviert. Daher spricht er von einer gewissen Gleichzeitigkeit von »High-tech« und »High-touch« (Naisbitt 1982: 35ff.).

Im Zuge der Weiterentwicklung des Ansatzes Megatrend ging es unter anderem darum, dass er sich insbesondere von kurzfristigen Trends und Modetrends abheben sollte, die keinen tiefergehenden gesellschaftlichen Einfluss haben. In einer Publikation aus dem Jahr 1990 formulierte Naisbitt: »By identifying the forces pushing the future, rather than those that have contained the past, you possess the power to engage with your reality.« (Naisbitt/Aburdene 1990: 335)

Seit dem Aufkommen des Zukunftsdenkens mithilfe von Megatrends wurden diese in sehr unterschiedlicher Weise definiert und beschrieben. So ergab sich im Laufe der Zeit eine große Vielfalt an gesellschaftlichen Veränderungsprozessen, die als Megatrends bezeichnet worden sind. Hier eine kleine Liste von Beispielen häufig angewendeter Megatrends:

- Zunahme und Ausbreitung des Klimawandels

- Shift zu Wissensgesellschaft und weg von der produzierenden Industriegesellschaft

- Feminisierung: Frauen finden eine neue Identität und gewinnen an Bedeutung in der öffentlichen Politik und Wirtschaft

- Demographische Schere: durch Geburtenkontrolle und aufgrund des steigenden Wohlstandes fällt die Geburtenrate

- Zunahme der Geschwindigkeit von Veränderungen in allen Bereichen des Lebens - vom Verkehr über die technische Innovation bis hin zum Kommunikationsverhalten in den sozialen Netzwerken 
- Verbesserte Gesundheit wird insbesondere in Mitteleuropa zum höchsten gesellschaftlichen Wert

- Verstärkte Globalisierung in fast allen Lebensbereichen, intensivierte Verflechtung, Wachstum von Mittelschichten

- Zunehmende Individualisierung, auch in bislang kollektiv agierenden Gesellschaften

- Globale Verbreitung von Informationstechnologie und deren Ubiquität

- Alterung der Gesellschaften

- Kommerzialisierung von immer mehr Lebensbereichen (Marktförmigkeit, Verwertbarkeit)

- Zunahme an Mobilität (Bedarfe, Formen)

- Zunahme von Umweltbewusstsein, ökologische Sensibilisierung

- Peak Everything: Ressourcenverknappung, insbesondere im Bereich fossiler Brennstoffe, zunehmend aber auch im Bereich der Edelmetalle

- Wachsende Vernetzung der Gesellschaften (z.B. Netzwerkgesellschaft, Industrie 4.0)

- Verbreitete Urbanisierung: Verstädterung des Lebensraumes bis hin zu Megacities

- Virtualisierung zahlreicher alltäglicher Abläufe in Freizeit und Arbeitswelt

- Wertewandel: Verbreitung eines Wertepluralismus

Bei genauerer Betrachtung werden die unterschiedliche Qualität, Plausibilität und Begründetheit der jeweiligen Megatrends deutlich. Zugleich lassen sich für jeden der Megatrends auch Kritiken und Gegentrends benennen.

Megatrends werden in der Literatur und Öffentlichkeit dann mehr oder weniger genau und fundiert beschrieben und mit Fakten oder quantitativen Werten und Daten sowie sozialwissenschaftlichen Theorien beschrieben.

Eine allgemeingültige Definition von Megatrends aufzustellen ist schwierig, unter anderem deshalb, weil Megatrends in sehr unterschiedlichen Kontexten und $\mathrm{zu}$ unterschiedlichen Zwecken (und für unterschiedliche Forschungsfragen) herangezogen bzw. geschaffen werden. Gleichwohl lassen sich, basierend auf bisherigen konzeptionellen Diskussionen sowie auf Erfahrungen und Arbeiten des Instituts für Zukunftsstudien und Technologiebewertung (IZT), basale Merkmale für Megatrends formulieren. Die sechs Merkmale sind:

- Dauer: Ein Megatrend sollte mindestens über 15 Jahre andauern (manche sagen: 30 Jahre), bis er seinen Zenit erreicht. $\mathrm{Zu}$ beachten ist hierbei, dass 
einige Megatrends eine überaus lange Dauer haben können, und zwar in Abhängigkeit von der Definition und der zugrunde gelegten Daten und Kontexte.

- Ubiquität: Ein Megatrend und dessen Effekte sind in allen bzw. vielen Lebensbereichen wirksam und spürbar, er ist »allgegenwärtig« und entwickelt Signifikanten in Konsum, Ökonomie, Lebenswelt, Technologie usw.

- Universalität: Ein Megatrend hat einen grundlegend globalen Charakter, auch wenn er sich in den verschiedenen Regionen und Kulturen unterschiedlich schnell und in unterschiedlicher Weise und verschiedenen Formen durchsetzt.

- Robustheit: Ein echter Megatrend verträgt auch Backlashs, also vorübergehende Rückschläge, ohne seine Dynamik zu verlieren.

- Richtung: Megatrends beschreiben gesellschaftlichen Wandel, eine gewisse Dynamik und eine Richtung.

- Wechselwirkung: Megatrends beeinflussen sich gegenseitig verstärkend oder abschwächend.

So ist das gesellschaftliche Phänomen »Urbanisierung « im gesamten Zivilisationsprozess vieler Kulturen zu beobachten, beginnend mit Mesopotamien und dem Nahen Osten vor mehr als fünftausend Jahren. Allerdings geschah dies in relativ wenigen Regionen. Der in den letzten zwei bis drei Jahrzehnten zu beobachtende Urbanisierungsprozess hingegen ist hinsichtlich globaler Verbreitung und Stärke seiner Dynamik von besonderer Qualität - sowie den immensen Folgen - und kann daher als Megatrend bezeichnet werden. Ähnliches lässt sich über Globalisierung oder Individualisierung sagen. Daher ist es unabdingbar, dass Auswahl, Definition und genaue qualitative und quantitative Beschreibung von Megatrends möglichst fundiert, transparent und nachvollziehbar dargestellt werden.

Das ursprüngliche, von Naisbitt praktizierte Vorgehen, um Megatrends $» z$ finden«, bestand vor allem in den auf »content analysis« basierenden Medienanalysen von Paul Lazarsfeld und Harold Lasswell (Naisbitt 1982: xxiv). Die in den Medien zu findenden Informationen über Neuerungen und den Wandel in Gesellschaften wurde als hinreichend angesehen, wirkmächtige Veränderungsprozesse zu erkennen und $z u$ beschreiben. So griff Naisbitt in den 1980er Jahren auf Printmedien und Fernsehen zurück, sammelte mehr oder weniger umfassend alle Daten und Informationen über einen bestimmten Zeitraum. Dabei wurde beispielsweise auch die Anzahl der Artikel und Beiträge zu einem Thema gezählt und bewertet. Wenngleich dieses empiri- 
sche Vorgehen sehr simpel war, geht doch eine Erfahrung daraus hervor, die wie folgt ausgedrückt worden ist: »The most reliable way to anticipate the future is by understanding the present.« (Naisbitt 1982: 2)

Der Schwerpunkt der Arbeit mit Megatrends besteht in der Klärung von deren Wirkungen auf ausgewählte Strukturen und Organisationen. Der konkrete und praktische Nutzen von Megatrends ergibt sich durch die Analyse seiner Einflüsse auf die Gesellschaft und für eine bestimmte Forschungsfrage oder politische Frage ausgewählte Bereiche. Denn nicht der Megatrend selbst ist letztendlich von Interesse, sondern dessen Relevanz für Akteure sowie die strategischen Implikationen und Konsequenzen.

Aufgrund der prinzipiellen Unsicherheit, künftige Entwicklungen treffend absehen und einschätzen zu können, aber der Notwendigkeit, sich Entscheidungsgrundlagen zu schaffen und sich auf Eventualitäten einstellen zu können, dienen Megatrends dazu, relativ plausible Veränderungspfade beschreiben zu können. Megatrends können als eine Art von »Schlaglichter in die Zukunft hinein« angesehen und genutzt werden. Sehr häufig werden Megatrends als Grundlage zur Ausarbeitung von Szenarien genutzt, also zur Herstellung von schlüssigen und nachvollziehbaren Zukunftsbildern, von Panoramen künftiger Zustände. Damit wird es möglich, unterschiedliche Richtungen künftiger Entwicklungen zu beschreiben und damit den Möglichkeitsraum und letztlich den Entwicklungs- und Handlungsspielraum von Akteur*innen, insbesondere von Entscheidungsträger*innen auszuweiten, eine Vielfalt an Optionen zu skizzieren.

Ein wesentlicher Arbeitsschritt im Umgang mit Megatrends bezieht sich dabei auf die oben bereits angesprochenen Wechselwirkungen zwischen Megatrends. Eine passende Methode hierfür ist die Cross-Impact-Analyse. Dieser Arbeitsschritt kann unterschiedlich intensiv durchgeführt werden. Im Grundsatz geht es bei der Cross-Impact-Analyse darum, die gegenseitigen Wechselwirkungen der Megatrend-Themen zueinander weitgehend $\mathrm{zu}$ quantifizieren und die Struktur der Wechselwirkungen zu beschreiben. Die Wechselwirkungen zwischen Megatrends lassen sich wie folgt unterscheiden:

- Der direkte Einfluss (direct influence) eines Megatrend-Themas auf die anderen Themen,

- die direkte Beeinflussung (direct dependence) eines jeden MegatrendThemas durch die anderen Themen,

- der indirekte Einfluss (indirect influence) eines Megatrend-Themas auf die anderen Themen und 
- die indirekte Beeinflussung (indirect dependence) eines jeden MegatrendThemas durch die anderen Themen.

Hierbei wird häufig die MICMAC-Methodik verwendet, die eine Strukturanalyse von Wechselbeziehungen ermöglicht. Mit dieser Methodik werden die in einem Forschungs- oder Beratungsvorhaben jeweils ausgewählten Megatrend-Themen in einer Matrix zueinander angeordnet und ihre gegenseitige Einflussnahme mithilfe von Experteneinschätzungen quantifiziert. Häufig wird hierfür ein kleines Ranking genutzt, also beispielsweise $0=$ kein Einfluss, $1=$ schwacher Einfluss, $2=$ moderater Einfluss sowie $3=$ starker Einfluss. Meist erfolgt dies in einem multidisziplinär zusammengesetzten Forscherteam. Je nach Aufgabenstellung und den zeitlichen und personellen Ressourcen kann eine Cross-Impact-Analyse auch im Rahmen spezieller Workshops mit externen Expert*innen und Stakeholder*innen erfolgen. Auch hier ist darauf zu achten, dass das Spektrum der involvierten Personen möglichst breit und der Thematik angemessen ist.

Die Summen der Einflussnahmen und Beeinflussungen pro MegatrendThema ergeben die kumulative Höhe der direkten Einflussnahmen und Beeinflussungen jedes Megatrend-Themas und erlauben ein Ranking aller Megatrend-Themen gemäß der jeweiligen Höhe ihrer direkten Wechselwirkung mit den anderen Megatrend-Themen. Ferner lässt sich so jedes Megatrend-Thema in einem Koordinatensystem hinsichtlich ihrer direkten Einflussnahme und ihrer Beeinflussung verordnen (Mapping) sowie die Struktur der direkten Wechselwirkungen in Netzdiagramme visualisieren.

Eine weitere Art von Wechselwirkungen sind die indirekten Einflussnahmen und Beeinflussungen. Dies sind diejenigen Wechselwirkungen, die ein Megatrend-Thema entweder über ein anderes Megatrend-Thema auf ein weiteres ausübt (indirekte Einflussnahme) oder aber an Beeinflussung erfährt (indirekte Beeinflussung). Um die indirekten Wechselwirkungen zu erfassen, werden die direkten Wechselwirkungen per Matrixoperation iterativ miteinander verknüpft. Analog zu den direkten Wechselwirkungen liegen im Ergebnis ein Ranking aller Megatrend-Themen gemäß der Höhe ihrer indirekten Einflussnahme und indirekten Beeinflussung, ein Mapping sowie Netzdiagramme vor. Mithilfe von Graphiksoftware lassen sich diese sehr anschaulich sowie dynamisch dadurch darstellen, dass einzelne Parameter, also hier Megatrends in varianten Ausprägungen und unterschiedlichen Zeitverläufen, eingegeben werden. 
Wenn es um die Frage geht, inwiefern Megatrends einzeln oder in ihren Wechselbeziehungen besonders beachtenswerte Wirkungen auf bestimmte Strukturen und Organisationen oder ganze Gesellschaften haben, können auch zusätzliche Methoden angewendet werden, wie z.B. eine SWOT-Analyse oder der STEEP-Ansatz, mit denen Stärken, Schwächen, Potentiale und Gefahren von Trends oder Effekte für verschiedene Bereiche wie Soziales, Technologie, Wirtschaft usw. genauer analysiert und im Zeitablauf abgeschätzt werden.

Eine besondere Herausforderung für das 21. Jahrhundert stellt nun das Leitbild >nachhaltige Entwicklung< dar, das 1987 und 1992 durch internationale Gremien und die UN proklamiert wurde und von immer mehr Regierungen zumindest symbolisch anerkannt wird. In Staaten wie der Bundesrepublik Deutschland werden langsam auch konkrete Politiken und Maßnahmen angekündigt und teilweise umgesetzt, die z.B. auf den Klimawandel und weitere problematische und existenzbedrohende Entwicklungen (»planetary boundaries" (Steffen et al. 2015)) gerichtet sind. Vor dem Hintergrund der Darlegungen über Megatrends kann hier nun gefragt und erörtert werden, ob und inwieweit »nachhaltige Entwicklung« als Megatrend angesehen werden kann.

\section{Nachhaltige Entwicklung als existenzielles globales Leitbild: ein Megatrend?}

Nach dem Zweiten Weltkrieg ergab sich - von den USA ausgehend - in den westlichen Staaten und Japan eine historisch einmalige Entwicklungsphase, die mit rapiden Wachstumsprozessen in zahlreichen gesellschaftlichen Bereich einherging (z.B. Rohstoffverbrauch, Bruttoinlandsprodukt, Mittelschichten). Abgesehen von früheren Warnungen bezüglich sozialer, kultureller und ökologischer Negativfolgen kapitalistischer Industrialisierung kamen in 1960er Jahren deutliche Probleme und Krisenphänomene zu Bewusstsein (z.B. ,Ölkrise«, »Grenzen des Wachstums« (Meadows 1974)), führten beispielweise zu Beginn der 1970er Jahre zur Etablierung von Umweltministerien und zur wissenschaftlichen Untersuchung globaler Problemzusammenhänge. Dieser umfangreiche Klärungsprozess führte zu einem systemischen Verständnis planetaren Wandels, der Wechselwirkung zwischen Umweltund Entwicklungsprozessen, und kulminierte gewissermaßen in der UNGipfelkonferenz für Umwelt und Entwicklung im Jahr 1992 in Rio de Janeiro. 
Dort wurde Nachhaltige Entwicklung als globales Leitbild künftiger Entwicklung in die Öffentlichkeit gebracht und findet seither, wenn auch langsam, immer breitere Anerkennung und positive Resonanz.

Die unintendierten Folgen des westlichen Entwicklungsmodells industriell-kapitalistischer Entwicklung, also der dynamisierenden Kopplung von Massenproduktion und Massenkonsum im Rahmen einer profitorientierten Wirtschaftsordnung, werden immer offensichtlicher und spürbarer - allerdings höchst ungleich verteilt. In manchen Regionen des Planeten versinken Küstengebiete und ganze Inselgruppen aufgrund des von der Klimaforschung vorhergesagten Anstiegs des Meeresspiegels. Die "planetaren Grenzen « (Rockström et al. 2009; Steffen et al. 2015) sind in wichtigen Bereichen bereits überschritten und zerstören allmählich (und fast unmerklich!) die Existenzgrundlage menschlicher Zivilisation. Ein zusammenfassender Index, der seit 1987 vom Global Footprint Network erstellt wird, macht diesen Verfall deutlich und erhält in letzter Zeit mehr Aufmerksamkeit: der »Earth Overshoot Day«. Mit ihm wird angegeben, in welchem Zeitraum binnen eines Jahres die Erdbevölkerung die von der Natur produzierten Rohstoffe und Materien verbraucht. Im Jahr 2019 fiel der Welterschöpfungstag auf den 29. Juli. In den Jahren zuvor sah dies wie folgt aus:
2018: 1. August
2017: 2. August
2016: 8. August
2015: 13. August
2014: 19. August

Im ersten Jahr dieser Berechnung fiel der Welterschöpfungstag noch auf den 19. Dezember 1987!

Das heißt, dass derzeit die Menschheit alle Ressourcen in nur 8 Monaten aufgebraucht hat, die der Planet Erde für ein Jahr liefert. Die Menschheit hat dann für 2019 das nachhaltige Natur-Budget unseres Planeten so früh und schnell aufgebraucht wie nie zuvor. Die Phase der ökologischen Verschuldung wird heute früher erreicht als in allen Jahren zuvor. Und dies ist ein globaler Durchschnittswert. Wenn die gesamte Menschheit die natürlichen Ressourcen so verbrauchen würde wie Deutschland, so wäre der Welterschöpfungstag im Jahr 2018 bereits am 2. Mai erreicht gewesen! Selbst wenn methodische Probleme und Annahmen anheimgestellt werden: diese fatalen Veränderungen sind alarmierend (vgl. Utopia Team 2019). Wenn sich selbst die vorsich- 
tigen und konservativen Prognosen vor allem der Klimafolgenforschung weiterhin bewahrheiten sollten, kann mit exponentiell zunehmenden Problemen und sogar Katastrophen gerechnet werden (vgl. Schellnhuber 2015). Und dann ist nicht gewährleistet, dass demokratische Politik die dann auftretenden gesellschaftlichen Konflikte und Verteilungsprobleme angemessen und human lösen kann. Vielmehr besteht dann wohl die Gefahr technokratischer Reaktionsformen und zunehmend autoritärer, nationalistischer, rassistischer und faschistoider Herrschaftsmechanismen (vgl. Fischer 2017).

Vor mittlerweile fast drei Jahrzehnten hat die internationale Staatengemeinschaft auf dem UN-Erdgipfel von Rio de Janeiro 1992 das Konzept "sustainable development« als Leitbild für das 21. Jahrhundert angenommen, um den wachsenden Herausforderungen der Menschheit zu begegnen. Die UN legte mit der »Agenda 21《 einen weltweiten Aktionsplan zur Umsetzung einer solchen nachhaltigen Entwicklung vor, der von 174 Regierungen unterzeichnet wurde. Bereits fünf Jahre zuvor hatte die World Commission on Environment and Development, die sogenannte »Brundtland Kommission", in ihrer häufig zitierten Formulierung Sustainable Development als eine Entwicklung definiert "that meets the needs of the present without compromising the ability of future generations to meet their own needs." (United Nations 1987: 154). Daraus lässt sich der folgende Handlungsimperativ ableiten: "Die Menschen sollen ihr Handeln so organisieren, dass sie nicht auf Kosten der Natur, nicht auf Kosten anderer Menschen, nicht auf Kosten anderer Regionen, nicht auf Kosten anderer Generationen leben.« (Göll 2001: 10)

Die politische und gesellschaftliche Diskussion zur Nachhaltigen Entwicklung hat sich in den letzten Jahren stark weiterentwickelt. Insbesondere im Hinblick auf den erstarkenden Transformationsdiskurs, aber auch im Hinblick auf wichtige Strategieprozesse im Bereich von Umwelt- und Nachhaltigkeitspolitik wurden bzw. werden gegenwärtig wichtige Fortschritte in Richtung eines gesellschaftlichen Wandels zu mehr Nachhaltigkeit erzielt (u.a. Agenda 2030, Fortschreibung der nationalen Nachhaltigkeitsstrategie sowie zahlreiche sektorale Strategien). Gleichzeitig ist eine Trendumkehr hin zu einer langfristig Nachhaltigen Entwicklung sowohl national als auch international in vielen Bereichen noch nicht in Sicht (z.B. bei der Artenvielfalt oder dem Anteil des Schienenverkehrs am Gütertransport) oder der Wandel vollzieht sich nicht schnell genug, um selbstgesetzte Ziele zu erreichen (z.B. beim Ausbau des ökologischen Landbaus und der Reduktion des Stickstoffüberschusses, aber auch bei sozialen Themen wie der Gleichstellung von 
Männern und Frauen; vgl. Indikatorenbericht der Nachhaltigkeitsstrategie, Destatis 2017). Mit dem politischen Leitbild der Nachhaltigen Entwicklung der Nachhaltigkeitsstrategie des Bundes wurde vor über eineinhalb Jahrzehnten eine Grundlage für die strategische Ausrichtung deutscher Nachhaltigkeitspolitik geschaffen. Im Rahmen regelmäßiger Fortschrittsberichte fanden zudem wichtige Ergänzungen, wie z.B. die Anerkennung absoluter Belastbarkeitsgrenzen des Ökosystems, Eingang in die Strategie.

Wenn es nun darum geht, einzuschätzen, ob nachhaltige Entwicklung als Megatrend verstanden werden kann, müssen vor allem die oben angeführten sechs Merkmale von Megatrends angewendet werden. In Bezug auf die Dauer kann Nachhaltige Entwicklung als Megatrend gelten. Auch Ubiquität trifft zu, denn die Effekte sind in vielen Lebensbereichen wirksam und spürbar. Universalität ist ebenfalls gegeben, weil Nachhaltige Entwicklung einen grundlegend globalen Charakter besitzt, auch wenn sie sich in den verschiedenen Regionen und Kulturen unterschiedlich schnell und in unterschiedlicher Weise und verschiedenen Formen zeigt. Das Merkmal Robustheit trifft ebenfalls $\mathrm{zu}$, weil Nachhaltigkeit auf sehr viel Trägheit und sogar Widerstände und Hemmnisse stößt, und dennoch seine Dynamik nicht verliert. Richtung ist auch zutreffend, insofern, dass Nachhaltige Entwicklung auf gewissen Standards und Kriterien basiert und mit der Agenda 2030 und den 17 Nachhaltigkeitszielen (>Sustainable Development Goals (SDGs)<) dezidiert Orientierung zu bieten versucht. Und schließlich steht Nachhaltigkeit in Wechselwirkung mit zahlreichen anderen Megatrends, wobei die meisten dieser Megatrends die Durchsetzung und Realisierung eher negativ beeinflussen und den Einfluss abschwächen (siehe Destatis 2017).

Hierbei gilt es allerdings zu betonen, dass es sich bei der Einordnung von Nachhaltiger Entwicklung als Megatrend um eine Besonderheit handelt. Denn während so gut wie alle anderen Megatrends aus der komplexen Interaktion von Menschen miteinander und mit der natürlichen Umwelt (Natur) entstehen, handelt es sich doch um Entwicklungspfade, die auf atomisierten, fragmentierten, häufig nur impliziten Entscheidungen in kleineren privaten und familiären, organisationalen und lokalen Zusammenhängen basieren. Beim Megatrend Nachhaltige Entwicklung hingegen handelt es sich um einen Entwicklungsprozess, der auf einem weitgehenden internationalen, multilateralen, mehr oder weniger rational geführten reflexiven Diskurs basiert.

In dieser Hinsicht besteht eine Parallele zum Konzept der >Transformation<, das seit dem Jahresgutachten des WBGU von 2011 verbreitet und in- 
tensiv diskutiert wird. Dieses Konzept setzt auch an einer früher entstandenen Quelle an, modifiziert diese aber in entscheidender Weise. Während der ungarisch-österreichische Wirtschaftshistoriker Karl Polanyi in seinem Werk rückblickend von historischen Transformationen spricht, also beispielsweise vom Übergang von Agrargesellschaften zu Industriegesellschaften mit einer Vielzahl von Veränderungen in allen gesellschaftlichen Bereichen, so versteht die aktuelle Debatte über ('große`) Transformation unter diesem Konzept eine explizit intendierte, strategisch gemeinte Veränderung ganzer Gesellschaften (Kahlenborn et al. 2019). Diese aktiv-gestaltende Absicht trifft auch auf Nachhaltige Entwicklung zu. Insofern wäre Nachhaltige Entwicklung ein unüblicher Megatrend, weil es sich dabei um eine intendierte Entwicklung handelt, ein im Umfang einmaliges historisches Ansinnen.

Ergänzt und flankiert wird die hier konstatierte "Einmaligkeit« des Megatrends Nachhaltige Entwicklung durch den Umstand, dass es sich um eine Entwicklung handelt, die zum einen auf einem globalen strategischen Konzept bzw. einem Leitbild beruht, und dass es sich zum anderen auf eine systemische, allumfassende Problematik bezieht, die sich absehbar weiter verschärfen und zunehmend spürbarer werden wird und die Existenzgrundlagen menschlicher Zivilisation bedroht. Daher ist es nicht verwunderlich, dass aus zivilgesellschaftlichen Bereichen radikale Haltungen und Akteur*innen wie beispielsweise »Ende Gelände«, Extinction Rebellion oder auch Fridays for Future bzw. Scientists for Future entstehen und sich artikulieren. Mit zunehmender Spürbarkeit und Betroffenheit wird der Handlungsdruck für die politischen und gesellschaftlichen Akteure weiter steigen.

In einem ähnlichen historischen Kontext wie dem heutigen hat der Politikwissenschaftler und Zukunftsforscher Ossip K. Flechtheim eine provozierende, herausfordernde Idee formuliert:

»Deshalb müsste eigentlich, wohl ähnlich wie im Kriege auf Jahre hinaus ein universaler Notstand der gesamten Menschheit proklamiert werden, um alle Kräfte auf den weltweiten Kampf gegen die die Menschheit in ihrer Existenz oder zumindest in ihrer Kultur bedrohenden Cefahren zu konzentrieren.«(Flechtheim 1973: 25)

Wie auch immer die Frage beantwortet werden sollte, ob Nachhaltige Entwicklung ein Megatrend sei oder nicht: maßgeblich und letztlich relevant wird sein, dass mit den Prinzipien und dem Leitbild Nachhaltiger Entwicklung ganz praktisch gehandelt wird. 


\section{Literatur}

Destatis - Statistisches Bundesamt (2017): Nachhaltige Entwicklung in Deutschland. Indikatorenbericht 2016, Wiesbaden. Online verfügbar unter: https://www.destatis.de/DE/Themen/Gesellschaft-Umwelt/Nach haltigkeitsindikatoren/Publikationen/Downloads-Nachhaltigkeit/ indikatoren-pdf-5850013.pdf;jsessionid=ED47904275273D389040B8A761 E43737.internet722?_blob=publicationFile. Zuletzt aufgerufen am 12.02.2020.

Fischer, Frank (2017): Climate Crisis and the Democratic Prospect: Participatory Governance in Sustainable Communities, Oxford: Oxford University Press. https://doi.org/10.1093/oso/9780199594917.001.0001

Flechtheim, Ossip K. (1973): Futurologie in der zweiten Phase?«, in: Dietger Pforte/Olaf Schwencke (Hg.), Ansichten einer künftigen Futurologie. Zukunftsforschung in der zweiten Phase, München: Reihe Hanser, S. 17-25.

Göll, Edgar (2001): Sozialindikatoren für eine Nachhaltig-zukunftsfähige Wasserwirtschaft, IZT WerkstattBericht Nr. 47, Berlin. Online verfügbar unter: https://www.izt.de/fileadmin/downloads/pdf/IZT_WB47.pdf. Zuletzt aufgerufen am 11.02.2020.

Kahlenborn, Walter/Clausen, Jens/Behrendt, Siegfried/Göll, Edgar (Hg.) (2019): Auf dem Weg zu einer Green Economy: Wie die sozialökologische Transformation gelingen kann, Bielefeld: transcript.

Kreibich, Rolf (2013): »Zukunftsforschung für Gesellschaft und Wirtschaft«, in: Reinhold Popp/Axel Zweck (Hg.), Zukunftsforschung im Praxistest, Wiesbaden: Springer VS, S. 353-384. https://doi.org/10.1007/978-3-53119837-8_15

Meadows, Donella H./Meadows, Dennis L./Randers, Jorgen/Behrens III, William W. (1974): The Limits of Growth. A Report for the Club of Rome's Project on the Predicament of Mankind, New York: Universe Books.

Naisbitt, John (1982): Megatrends: Ten New Directions Transforming Our Lives, New York: Warner Books, Inc.

Naisbitt, John (1984): Megatrends. 10 Perspektiven, die unser Leben verändern werden, Bayreuth: Hestia.

Naisbitt, John/Aburdene, Patricia (1990): Megatrends 2000: Ten New Directions For the 1990's, New York: Avon Books.

Rockström, Johan/Steffen, Will/Noone, Kevin/Persson, Åsa/Chapin, F. Stuart III/Lambin, Eric/Lenton, Timothy M./Scheffer, Marten/Folke, Carl/Schellnhuber, Hans Joachim/Nykvist, Björn/Wit, Cynthia A. 
de/Hughes, Terry/Leeuw, Sander van der/Rodhe, Henning/Sörlin, Sverker/Snyder, Peter K./Costanza, Robert/Svedin, Uno/Falkenmark, Malin/Karlberg, Louise/Corell, Robert W./Fabry, Victoria J./Hansen, James/Walker, Brian/Liverman, Diana/Richardson, Katherine/Crutzen, Paul/Foley, Jonathan (2009): »Planetary boundaries: exploring the safe operating space for humanity«, in: Ecology and Society 14 (2), 32. https://doi.org/10.5751/ES-03180-140232

Rosa, Hartmut (2005): Beschleunigung. Die Veränderung der Zeitstrukturen in der Moderne, Frankfurt a.M.: Suhrkamp.

Steffen, Will/Richardson, Katherine/Rockströmn, Johan/Cornell, Sarah/Fetzer, Ingo/Bennet, Elena/Biggs, Reinette/Carpenter, Stephen/Vries, Wim/Witt, Cynthia de/Folke, Carl/Gerten, Dieter/Heinke, Jens/Persson, Linn/Ramanathan, Veerabhadran/Reyers, Belinda/Sörlin, Sverker (2015): »Planetary Boundaries: Guiding human development on a changing Planet«, in: Science 347, 6223. https://doi.org/10.1126/science.1259855

Utopia Team (2019): »Earth Overshoot Day 2019 schon am 29. Juli - das früheste Datum in der Geschichte der Menschheit«, 29.07.2019. Online verfügbar unter: https://utopia.de/ratgeber/earth-overshoot-day. Zuletzt aufgerufen am 13.02.2019.

United Nations (UN) (1987): Report of the World Commission on Environment and Development. General Assembly Resolution 42/187, 11 December 1987. Online verfügbar unter: https://undocs.org/pdf?symbol=en/A/ RES/42/187. Zuletzt aufgerufen am 12.02.2020.

Wersig, Gernot (2009): »Zukunftsforschung aus postmoderner Perspektive«, in: Reinhold Popp/Elmar Schüll (Hg.), Zukunftsforschung und Zukunftsgestaltung, Berlin/Heidelberg: Springer-Verlag, S. 51-75. https:// doi.org/10.1007/978-3-540-78564-4_5 



\section{Irrwege und Entwicklungspfade Eine konstruktive Kritik der Trend- und Zukunftsforschung}

Holger Rust

\section{Vorbemerkung}

Wenn wir heute die Geschichte der Zukunftsforschung seit dem Zweiten Weltkrieg studieren, zeichnet sich ein bemerkenswerter Trend ab: In der Zeit der Hochkonjunktur der einschlägigen Methoden um die späten 1940er und die 1950er Jahre lag der prognostische Horizont der Szenarioforschung bei 40, der der Foresight Research noch bei etwa 30 Jahren. Im Laufe der Jahrzehnte verringerten sich diese Reichweiten merklich. Die aktuellen Variationen, die derzeit in den USA erprobt werden, nehmen mit wenigen Ausnahmen noch maximal fünf Jahre in den Blick.

Im gleichen Zeitraum, in dem sich die prognostischen Reichweiten verringerten, erweiterte sich das Spektrum der Informationen durch die technische Entwicklung von Big Data-Analysen und künstlicher Intelligenz. Gleichzeitig wuchs die Masse an Informationen über diese Innovationsflut. Als einer der wichtigen Befunde eines Projekts über die Folgenabschätzung nichttechnischer Konsequenzen der Digitalisierung (Rust 2019) stellt sich die unsystematische mediale Informationspraxis über die Zukunft heraus. Die in diesem Projekt durchgeführte Medienanalyse von 1.035 Beiträgen meinungsführender Tageszeitungen und Magazine in Deutschland und im englischsprachigen Ausland bietet den Eindruck einer unüberschaubaren Anekdotik und großer thematischer Beliebigkeit. Durch tausenderlei Beispiele digitaler Transformationen in Unternehmen, Alltag, Politik, Kultur, Kunst, Medizin, Gartenpflege oder Mobilität und natürlich auch in der Energiewende entsteht der Eindruck einer unkontrollierbaren Flut von Neuerungen. Nur in Ausnahmefällen wird eine ganzheitliche Idee von Zukunft formuliert - das, was viele 
Kommentatoren in Wirtschaft und Politik als »Vision« anmahnen. Ein Fokus liegt dabei auf allgegenwärtiger künstlicher Intelligenz, die im Kontext einer frankensteinartigen Erzählung insbesondere als Bedrohungspotential inszeniert wird. Das ist zu Teilen auch darauf zurückzuführen, dass den großen ITKonzernen eine "Hidden Agenda« vorgeworfen wird, die sich aus Allmachtsund Übernahmefantasien zur Kolonisierung der analogen Welt nach dem Modell algorithmischer Modellierung ergibt. Diese Kritik am Mangel einer übergreifenden Sicht auf die Chancen und Folgen der Transformationen und an der einseitigen Ausbeutung der Chancen stammt keineswegs nur aus den Reihen der Technikskeptiker, sondern wird vehement auch von prominenten Vertretern der Wirtschaft und vor allem von Dissidenten der kritisierten Konzerne formuliert, die ebendort zu Gründern oder Spitzenmanagern gehörten (ausführlich dazu Rust 2019, Kapitel »Techlash«).

Traditionell wird eine Situation, in der für eine erforderliche Prognose zunächst nur unzureichende Daten verfügbar sind, als »Fermi-Problem« bezeichnet - benannt nach dem berühmten Kernphysiker Enrico Fermi, dem die Fähigkeit nachgesagt wurde, trotz mangelnder Informationen spontan den Ausgang einer Versuchssituation treffend prognostizieren zu können. Gegenwärtig nun stellt sich das Problem in einer umgekehrten Variante, als Fermi-Problem 4.0: Es stehen zu viele Informationen zur Verfügung, die sich dazu noch oft widersprechen. In dieser Situation wächst auch die Vielfalt der oft inkompatiblen Deutungen dieser Informationen. Ein solches Defizit an produktiver Zukunftssicherheit erzeugt, den gängigen Theorien zufolge, eine »erlernte Hilflosigkeit« (Seligmann 1975). Die große Frage, die sich in dieser Lage aufdrängt, ist die nach der Rolle der so genannten »Zukunftsforschung«. Die Problematik, die damit angesprochen ist, soll hier in drei Schritten erörtert werden, die einem zeitlichen Kontinuum folgen.

Erstens: In der Betrachtung globaler Zukunftsforschung der zweiten Hälfte des 20. Jahrhunderts (die man damals meist Futurologie nannte) und einer schleichenden Desillusionierung, die in den 1970er und 1980er Jahren einsetzte, soll eine Erklärung entwickelt werden, warum die heute so lautstark sich als Königsdisziplin titulierende »Trendforschung entstand und die klassische Zukunftsforschung aus dem Fokus der öffentlichen Aufmerksamkeit verdrängen und durch verkaufsorientierten strategischen Opportunismus verfremden konnte.

Zweitens: Die Betriebslogik dieser selbst ernannten Alternative wird erörtert und beispielhaft vorgeführt. Dabei ist der Fokus auf die konstitutive Phase dieser Dienstleistung zwischen 1980 und 2010 gerichtet. Da in diesem 
Beitrag der Raum sehr begrenzt ist, kann eine Reihe von kritischen Aspekten nicht näher beleuchtet werden, daher werden Hinweise auf Studien und Projekte des Autors eingefügt, die als Supportive Material Zugänge zu tiefergehenden Informationen, illustrativen Einzelheiten und Belegen bieten.

Drittens: Schließlich werden die sich seit 2010 abzeichnenden Konzepte einer künftigen Zukunftsforschung (die sich nun meist Foresight Research nennt) skizziert - jener Ansätze, die sowohl in der Lage sind, das Problem des Information Overchoice in den Griff zu nehmen als auch pragmatische Lösungen $\mathrm{zu}$ entwerfen. Exemplarisch steht dabei der Ansatz des so genannten »Superforecastings « im Zentrum, ein intellektuelles System mit interdisziplinärem Hintergrund, ebenso mathematisch fundiert wie sozialwissenschaftlich inspiriert und vor allem pragmatisch auf individuelle Lösungen ausgerichtet.

\section{Teil 1:}

\section{Die Vergangenheit: Globaler Optimismus und Desillusionierung}

Noch zur Mitte des 20. Jahrhunderts dominierte der optimistische Geist einer wissenschaftlich begründeten Prognostik, die schon in den 6oer Jahren als globale intellektuelle Kongregation organisiert war. Der renommierte Soziologe Daniel Bell hatte bereits 1963 im einflussreichen Essay »Douze modes de prévision en science sociale. Enumeration préliminaire« die Idee einer solchen Zukunftsforschung umrissen, als Fortschreibung des bereits 1954 vom Wohlfahrtsökonomen Bertrand de Jouvenel entworfenen »Projet futuribles«. Diese Initiative war eine Kooperation der Ford Foundation und der Fondation pour l'étude des relations internationales en Suisse (F.E.R.I.S.), eine Art Konföderation internationaler Geistesgrößen.

Der Vorschlag, ein solches Projekt auch praktisch zu initiieren, wurde von de Jouvenel seit 1960 ausgearbeitet. Das Ziel definierte sich durch die kontinuierliche Diskussion über zukunftsträchtige Entwicklungen, als Begründung einer »art de la conjecture«. Eine Übersicht über die Publikationen de Jouvenels zeigen, dass bereits Anfang der 6oer Jahre nicht nur von Daniel Bell intensive methodologische Bemühungen unternommen wurden, die fast alles vorwegnehmen, was von der Trendforschung als große Entdeckung beansprucht wird. »Les prévisions de croissance erschien im Bulletin SEDEIS im Juni 1960. »De la conjecture« im selben Jahr. 1962 veröffentlichte de Jouvenel seine Gedanken über »La prévision économique à court terme«. Es folgte der Aufsatz »La prévision des idées« in Futuribles 68 am 1. Dezember 1963, dann das be- 
rühmt gewordene wissenschafts- und erkenntnistheoretische Werk »Lart de la conjecture« (Bensaïd/de Jouvenel 1965). Eine Spielerei mit der wünschenswerten Zukunft wurde unter dem Titel »Utopia 1980« in der Zeitschrift The Spectator vom 14. Februar 1964 publiziert. Die breite Wirkung Jouvenels zeigte sich auch in Publikationen in amerikanischen Zeitschriften, etwa Political Science and Prevision in der American Political Science Review 59, 1965. "Tâche de la prévision« erschien als programmatischer Beitrag in Économie et Humanisme in der Winterausgabe 1967 (vgl. zur weiteren Geschichte Jouvenel 1986).

Dieses einflussreiche Netzwerk unternahm den Versuch, durch die pluralistische Nutzung der unterschiedlichsten Disziplinen jene 1959 in einem weltberühmten Vortrag des Physikers und Romanciers Charles Percy Snow angemahnte »Third Culture « zu realisieren - was nichts anderes hieß, als die Grenzen der disziplinären Arroganz zu überwinden, die eine technokratischingenieursgetriebene Wissenschaftskultur und die eher hermeneutisch-geisteswissenschaftlichen Weltdeutungen (bzw. ihre Protagonisten) voneinander trennten. Snow führte diese Gedanken erstmals in einem Vortrag aus, den die britische Financial Times 50 Jahre später, im Mai 2009, zu einem der wichtigsten Vorträge des vergangenen Jahrhunderts zählte: »The Two Cultures and the Scientific Revolution«(Snow 1959, Neuauflage 2012). Mit einer solchen Grenzüberschreitung sollten die sichtlichen Defizite der bisherigen Zukunftsforschung kompensiert werden: zum Beispiel das Scheitern der mathematischminutiösen Berechnungen des Instituts von Hermann Kahn oder die zwar luziden aber praktisch kaum umsetzbaren Ideen einer Futurologie Flechtheims (Flechtheim 1973).

Doch schon 1969 deutet sich in einem Beitrag des renommierten Ökonomen und Politikwissenschaftlers Andrew Schonfield vom British Science Research Council eine gewisse Desillusionierung an (Schonfield 1969). Die Futurologie bliebe »an die Phantasie gebunden«. Sie könne nicht einfach »in eine respektable sharte Wissenschaft« verwandelt werden. Bereits in dieser frühen Relativierung der pragmatischen Potentiale der Zukunftsforschung setzte sich Schonfield äußerst kritisch mit dem raumgreifenden Interesse der Industrie und der damit einhergehenden Verfremdung des bis dahin von Partikularinteressen unbeeinflussbaren Recherchevorbehalts auseinander.

Damit waren zwei wichtige Motive umrissen, wenngleich noch als Gegensatzpaar formuliert - sozusagen als Beitrag zu einer Nutzwert-Debatte der Zukunftsforschung: Fantasie und Kommerz. Sie sollten zu den integrierten Triebkräften der bald einsetzenden Absage an eine vorbehaltlose Wissenschaft und Hinwendung zum bereits angedeuteten strategischen Opportu- 
nismus der Auftragsforschung werden. Ein drittes Motiv wurde 1978 formuliert: der schon besagte Information Overload. In diesem Jahr erschien ein von den Politikberatern Alain Minc und Simon Nora im Auftrag des französischen Staates formuliertes umfangreiches Gutachten, das sich mit der »Informatisierung der Gesellschaft « befasste (Nora/Minc 1978). Es ist ein sperriges, komplexes Werk mit einem ungeheuren Anhang, das dennoch großen öffentlichen Anklang fand und zum Bestseller avancierte. Seine Komplexität rührte vor allem daher, dass sich die Autoren mit allen denkbaren Konsequenzen der, wie sie es damals noch nannten, »Telematik« beschäftigten - den wirtschafts- und beschäftigungspolitischen, kulturellen und gesellschaftlichen Verflechtungen dieser fundamentalen technologischen Innovation. Am Ende ihrer Analyse stand die Diagnose, dass trotz oder gerade wegen der Vielfalt an Informationen konkrete Voraussagen des sich in seiner Komplexität unvorhersehbar entwickelnden Ganzen unmöglich seien.

Das heißt nicht, dass die wissenschaftlich fundierte Zukunftsforschung nicht weiterentwickelt worden wäre. Doch verblasste die Idee einer umfassenden Vision von der - wie Niklas Luhmann es in seiner Systemtheorie entwarf - »Weltgesellschaft«. An ihre Stelle traten pragmatisch disziplinäre Forschungsansätze, etwa der Entwicklung von digitalen Technologien, medizinischen Fortschritten, Gentechnik und Biogenetik, Raumfahrttechnik, Materialwirtschaft, soziologisch inspirierter Organisationstheorien und Managementkonzepten wie etwa der betriebswirtschaftlich inspirierte Ansatz der »Weak Signal Research « von Igor Ansoff (Ansoff 1976) - all jenen und vielen weiteren Spezialgebieten also, denen sich einzelne Disziplinen der weltweit führenden Technischen Universitäten und nicht-akademischen Forschungseinrichtungen widmeten.

Im Zuge dieser disziplinären und thematischen Spezialisierung entstand insbesondere in Deutschland eine höchst produktive Forschungslandschaft: zum Beispiel abzulesen an den vielfältigen intellektuellen Filialbetrieben der Fraunhofer- oder Max Planck-Gesellschaft oder an den Forschungseinrichtungen der Branchen des Deutschen Mittelstandes, in denen Milliardensummen aufgewendet werden, um zukunftsfähige Prozesse und Produkte zu generieren; an Forschungsinstitutionen von Unternehmensberatungen und schließlich den Abteilungen für Corporate Foresight der Konzerne, die ganz sicher nicht in die Öffentlichkeit gehen mit ihren Ideen.

Die komplexen Ergebnisse dieser Bemühungen entsprechen allerdings kaum den News Values und Talkshow-Dramaturgien der Medien und den Erwartungen der Öffentlichkeit an die »Zukunftsforschung«. Wesentliche Ziele 
der gegenwärtigen Forschung sind zudem abstrakt: Sie konzentrieren sich eher auf die Möglichkeiten, unplanbare Folgen der großen Entwicklungen strategisch schnell bewältigen zu können als darauf, diese Folgen bereits heute mit lärmenden und irritierend inhaltsleeren, aber medientauglichen Anglizismen zu definieren. Anders ausgedrückt: Sie konzentrieren sich auf Möglichkeiten, destruktive Emergenzen zu bewältigen und modifikatorische oder auch konstruktive zu nutzen (dazu Tichy 2001). Die Wortwahl zeigt schon, dass derartige Arbeiten schwer verständlich sind.

In diese Austastlücken der Verständlichkeit zunehmend komplexer $\mathrm{Zu}$ sammenhänge und der emergenten Folgen zukunftsoptimistischer Planungen stießen nun jene Feuilletonisten vor, die zunächst einmal umfassende Diagnosen, dann gleichzeitig eine Art intellektueller Lebenshilfe entwarfen, indem sie alles zu ihrem Thema erklärten und für alles Erklärungen bereithielten. Drei publizistische Vorreiter ebneten den Weg in diese enzyklopädische Anmaßung: Alvin Toffler, John Naisbitt und die Betreiberin einer New Yorker Werbeagentur namens Faith Plotkin, die sich kokett »Popcorn« nennt und damit neben Fantasie und Kommerz ein weiteres Motiv inthronisierte, das sie Naming nannte - den mutmaßlichen Zukunftstendenzen einen medial tauglichen Begriff zu verpassen, der sich viral in der Öffentlichkeit verbreitete, sich auf diese Weise verselbstständigte und den Eindruck einer Realität erzeugte. Das wurde unterfüttert mit einer nicht selten aggressiven Diskreditierung der überkommenen wissenschaftlichen Praxis, mit der Zukunft umzugehen - eine Kritik, die bis heute durch den Vorwurf legitimiert wird, Wissenschaft der klassischen Sorte sei purer Alarmismus. Denn auch dies wurde schnell und bleibt bis heute ein Kernmotiv der Trendforschung: strategischer Optimismus als kommerzielles Argument gegen die zersetzenden oder doch zumindest weltfremden Theorien zum Beispiel »der« Soziologen (Horx/Wippermann 1996; Wenzel 2019). Dabei beginnt die Geschichte der »Trendforschung« selber mit einem Alarmruf, nämlich mit der Diagnose eines »Future Shock .

\section{Teil 2: \\ Die Gegenwart: Pseudowissenschaftliche Ersatzreserve und Kommerzialisierung}

Das war der Titel eines 700 Seiten umfassenden Buches von Alvin Toffler, einem Futurologen. Das Buch verkaufte sich weltweit mehr als 8 Millionen Mal. 
Es setzte Maßstäbe. Keine inhaltlichen, denn es ist, wie fast alle Rezensenten damals anmerkten, nicht zu klar zu umreißen, worüber Toffler eigentlich schreibt, weil er über alles schreibt. Auch dieses Motiv einer enzyklopädischen Anmaßung wird in der Folge die Protagonisten der Trendforschung inspirieren. Interessanter noch war aber vor allem, wie er es tat: anekdotisch statt wissenschaftlich. Sensationelle Beispiele wurden zu Belegen, weil sie einfach als Ansatz künftiger Entwicklungen identifiziert, dann durch Formulierungen wie »heute schon ...« oder »immer mehr ...« und ähnliche Formulierungen einer nebulösen Mengenlehre dieses Genres von Zukunftsforschung validiert wurden (Toffler 1972).

Der nächste Protagonist dieser neuen Entwicklung, John Naisbitt, sortierte dann die anekdotische Unordnung in einem Dekalog vorgeblicher Grundströmungen, identifiziert durch einen semantisch eigentlich unsinnigen Begriff: »Megatrends«. Dieses Wort wurde zum Titelbegriff des nächsten Weltbestsellers (Naisbitt 1982). Er führt eine weitere Praxis in das Narrativ vom seherischen Trendforscher ein: die biographische Politur.

Welchen Berufsweg Naisbitt nun genau absolvierte, lässt sich so einfach nicht recherchieren. Sicher ist nur, dass er bis 1977 eine Trendforschungsagentur (die Urban Research Corporation) leitete, eine "publishing company", die einen »Urban Crisis Monitor « betrieb. Das Unternehmen geriet 1977 in einen umstrittenen Konkurs. Dieses Kapitel wird allerdings aus verschiedenen Gründen, die hier nichts zur Sache tun, in seinen Selbstdarstellungen verschwiegen. Naisbitt, so die weiteren Informationen, die man der Selbstbeschreibung (und dann natürlich den biographischen Informationen der Auftraggeber) entnehmen konnte, gibt an, an der Harvard University, der Cornell University und der University of Utah studiert zu haben. Was, wann, wo genau wird nicht klar. Dazu bedarf es komplizierter Recherchen. Ebenso wie $\mathrm{zu}$ anderen biographischen Angaben: Vorstandsmitglied von IBM und Kodak, später Assistent des CEO von IBM, Tom Watson jr., Berater von Präsident Johnson. Im Vorwort zur deutschen Ausgabe von >Mind-Set!< (Naisbitt 2007) bezeichnet er sich erstaunlicherweise als "stellvertretender Erziehungsminister« unter John F. Kennedy. Eine Recherche zeigt, dass dies zumindest eine sehr weitläufige Auslegung einer Assistententätigkeit in dem damals von Anthony Celebrezze geleiteten Erziehungsministerium als Assistent des US Commissioners of Education Francis Keppel war.

Wie auch immer: In der Regel wurden die Angaben als Exzellenzbeweis von den Auftraggeber*innen unrecherchiert übernommen. Diese druckten auch die Behauptung des Autors gerne nach, dass über 95 Prozent seiner Pro- 
gnosen eingetroffen seien, was bei der weitläufigen Formulierung auch nicht wundert, vor allem, weil die meisten der zehn Megatrends (eigentlich naturgemäß) längst bekannt waren, etwa der von Daniel Bell bereits Jahre zuvor prognostizierte Übergang zur "postindustrial society" (Bell 1976). Immerhin aber befriedigte Naisbitt das Gefühl der Millionen von Leser*innen, dass sie es immer schon gewusst hatten.

Auch die New Yorker Werberin Faith (Popcorn) Plotkin (Namensgeberin des Trends zum »Cocooning«) antwortete in einem Interview der New York Times auf die Frage, ob sie schon einmal einen Trend übersehen habe: »I'm trying to think of a trend that I've missed - it would be more credible if I could think of one« (zitiert nach Sherden 1998: 221).

Diese Vorbilder inspirierten zwei deutsche Interessenten, die in der Folge zu den umtriebigsten Protagonisten der nationalen »Trendforschung « wurden: Peter Wippermann und Mathias Horx. 1993 gründeten sie das so genannte »Trendbüro«. Die in den vorangehenden Absätzen identifizierten Motive lassen sich leicht erkennen, vor allem eine geradezu aggressive Absage an die herkömmliche Wissenschaft. In einem Pamphlet mit dem Titel »Was ist Trendforschung?«, das die Geschäftsidee umreißt, wurde als Unique Selling Proposition (USP) die Begründung einer neuen Universalwissenschaft gefeiert. Die erstand aus den Trümmern der behäbigen akademischen Disziplinen (Horx/Wippermann 1996).

Die klassischen Disziplinen werden auf sieben Seiten (33-40) im besten Falle als Hilfsinstrumente dieser neuen integrativen Praxis charakterisiert, meist aber gleich als gescheiterte Versuche der Welterklärung auf den erkenntnistheoretischen Müllhaufen expediert: Die Geschichtswissenschaften seien »das kleine Einmaleins der Trendforschung «, die Markt- und Meinungsforschung indes wegen des Gegenwartsbezugs »zwangsläufig ein stumpfes Instrument«; die Psychologie wird immerhin als »unverzichtbar" gewertet, weil sie erklären könne, warum das Auto so faszinierend ist und warum Menschen gern im Stau stehen. Semiotik sei »Trendforschung pur«, die Futurologie ihr »integraler Bestandteik. Die Evolutionswissenschaften »bieten außerordentlich wertvolle Impulse«, Kulturanthropologie (»oder auch Kulturethnologie«) sei eine hilfreiche »Analogiewissenschaft«. Die Komplexitätstheorie »hat enorm spannende Teilbereiche. "Offensichtlich unvermeidlich wurde wieder einmal auch und insbesondere die Soziologie ins Feuer genommen: Sie leide »unter dem >Komplexitätsschock « .

Die Idee, die klassischen Wissenschaften seien nichts Anderes als Vorstufen zu der von Wippermann und Horx, nach der Trennung der Partner des 
Trendbüros dann von Horx allein betriebenen Trendforschung, entspringt keineswegs dem stürmischen Enthusiasmus junger Unternehmensgründer. Sie wird sogar zu einem zentralen Motiv des nach eigenen Aussagen »wohl renommiertesten « Trendforschers Europas, Horx: »Ich nutze die Erkenntnisse der neuen interdisziplinären Wissenschaften wie Neurobiologie, Ethnopsychologie, Kognitionswissenschaft, Systemtheorie und Soziobiologie für einen ganzheitlichen Ansatz « (zahlreiche Selbstbeschreibungen dieser Art mit weiteren Disziplinen von der Statistik über die Chaostheorie bis zur Hirnforschung in Rust 2008, Kapitel 8). Auf der Website heißt es aktuell: »Inspiriert von den universalistischen Denkern kombiniert er Evolutionstheorie, Systemwissenschaften, kognitive Psychologie und Komplexitätstheorie« (Horx 2020a). Zudem lässt er sich in seinen biographischen Hinweisen heute wahlweise als Wissenschaftler, Soziologe oder auch als "studierter Soziologe « bezeichnen, als Absolvent eines Studiums also, das just zu den Zeiten absolviert sein müsste, in der diese Disziplin vorgeblich unter »Komplexitätsschock« stand.

Dieses akademische Selbstbewusstsein kulminiert dann 2014 und 2015 im Angebot eines selbst erdachten Abschlusses, der akademisch klingt und in Ein-Tages-Seminaren des Horxschen Zukunftsinstituts erworben werden konnte: »Master of Future Administration«. Der Link zum damaligen Veranstalter »Euroforum « und der Bewerbung für dieses Event und den »einmaligen Abschluss« ist leider nicht mehr aktiv. Der MFA wurde aber dann nach zwei Durchgängen wieder aufgegeben, und der Erfinder kommentierte auf Youtube, dies sei kein Titel, sondern eine humorvolle Auseinandersetzung mit der klassischen Wissenschaft (Horx 2020b). Dennoch findet man im Internet ausreichend Personen, die diesen Titel angeben (ergänzende Beispiele zu dieser Strategie: Schwertfeger 2018; Schrep 2019).

Dass es sich, wie schon Schonfield 1965 argwöhnte, bei derartigen wissenschaftlichen Ansprüchen und der mit ihnen verbundenen enzyklopädischen Anmaßung um die schlichte Flankierung rein kommerzieller Dienstleistungen handelt, dass also »Zukunft« zu einem Handelsobjekt und die Ansprüche wissenschaftlicher Qualifikationen zu einem verkaufsfördernden USP degradiert werden, räumen Konkurrent"innen von Horx freimütig ein. David Bosshart zum Beispiel, zum Zeitpunkt der im Folgenden zitierten Aussage Direktor des GDI in der Schweiz: Etwas lerne man von den Amerikanern

»auf harte Weise: Informationen haben einen Cash Value. Geht man unvorsichtig damit um, begibt man sich auf eine Ebene, wo man von jedermann 
kopiert werden kann. [...] In den USA geht es einzig darum, ob man aus der Trendforschung ein Produkt machen kann, mit dem man Geld verdient. [...] Das Entscheidende ist doch, Informationen aufzubereiten, so dass sie einen Käufer finden, der Nutzen daraus ziehen kann« (Prange 1998).

Bei dieser Auseinandersetzung geht es um Positionen in der neuerdings so genannten »Ökonomie der Aufmerksamkeit« durch Begriffe, die es in die Medien schaffen. "Der Wettbewerb um diese Begriffe ist hart«, kommentierte das Wirtschaftsmagazin >brand eins in seiner Ausgabe 3/2007. »Denn ihre Besetzung schlägt sich in Form von mehr Zitaten, mehr Aufmerksamkeit und mehr Rednerhonorar nieder. Manchmal werden die Begriffe daher auch gekapert.«

Dieser Wettbewerb ist beträchtlich und vollzieht sich nicht nur unter Trendforschern. Auf den Podien der Managementberatung tummeln sich TV-Wettermoderatorinnen, die etwa dem Wissensforum der >Süddeutschen Zeitung über Charisma plaudern, Pop-Philosophen, die über alles reden von der Digitalisierung über die Reformunwilligkeit der deutschen Pädagogik bis zur Ethik; es werden Speaker Slams veranstaltet, auf denen sich die unterhaltsamsten Repräsentanten dieses Gewerbes profilieren, und die Kataloge der Referentenagenturen bieten Tausende von 5-Sterne-Speakers an, die alle das gleiche Versprechen intonieren: Selbstoptimierung als Weg zum Erfolg im Sinne eines "Smart Capitalism« (Horx 2001). Interessanterweise liest offensichtlich niemand den Subtext dieser Dienstleistungsbeschreibungen: uneingestandene Lebenshilfe für partielle Versager, die weder in der Lage sind, ihr Erfolgspotential zu erkennen, noch dazu, zukunftsrelevante Entscheidungen zu treffen.

\section{Teil 3:}

\section{Die Zukunft: Dekonstruktion und Rekonstruktion}

Nun muss also eine Frage gestellt werden: Wer sind eigentlich die zahlenden Adressaten derartiger Dienstleistungen? Man kann mit einiger Begründung annehmen, dass die Coachings und die Vorträge vor allem als eine Art Incentive für das mittlere oder untere Management und die operativen $\mathrm{Mi}$ lieus von Unternehmen darstellen, dass die Hauptklientel vor allem kleinere Agenturen und Ein-Personen-Betriebe sind, die sich mit Coachings oder speziellen Trendentwicklungen beschäftigen. Wenn man beispielsweise, um 
irgendwo anzufangen, die Liste all jener Dienstleister im Internet aufsucht, die in ihrem Portfolio den bereits erwähnten Titel des »Master of Future Administration« führen, ist darunter keine einzige Person des gehobenen Managements. Wenn man, wie das in einem Projekt 2012, also zur Hochkonjunktur der Trendforschung, geschehen ist, nachfragt, welche Vordenker die Managerinnen und Manager von 310 repräsentativ ausgewählten mittelständischen Konzernen (Rust 2012) schätzen, sucht man vergebens nach den medial flächendeckend vermarkteten Namen wie Janszky, Bolz, Wenzel, Opaschowski oder gar denen der selbst ernannten Gurus wie Horx und der gefeierten Excellence-Speakers. Dabei wurden von 126 Personen, die auf diese Frage eine Antwort hatten, insgesamt 78 unterschiedliche Namen genannt auffallend viele Vorgesetzte und Kollegen. Es sind Personen, von denen man fachlich oder menschlich profitieren konnte, die man bewundert hat - und zwar vor allem für ihre Fähigkeit, unerwartete Herausforderungen mit Ruhe, Erfahrung und innovativer Fantasie zu bewältigen und dies vor dem Hintergrund der Unverwechselbarkeit ihres Unternehmens und der Strategien, die im Wettbewerb mit anderen exklusiv entwickelt worden waren.

Denn diese Herausforderungen sind nicht weniger geworden. Schon im eben erwähnten Projekt 2012 wurden auf die Frage nach Entwicklungen der letzten Jahre, mit denen man in keiner Weise gerechnet hatte, mehr als 700 Antworten von 310 Befragten gegeben. Natürlich auch die erstaunliche Entwicklung des Internets. Die Rasanz, die dieses Thema kurz darauf entwickelte, insbesondere die Big Data-Analytics, war allerdings noch kein Thema. Dieses des Daten-Überangebots, der Schwemme an unstrukturierten durch Tausenderlei Quellen generierten Datenbestände geriet allerdings schon zwei Jahre später in den Fokus und motivierte eine Menge Experten zu sehr unterschiedlichen, durchaus auch kritischen Einschätzungen des digitalistischen Zukunfts-Optimismus. Nur einige Beispiele aus einer Analyse von 260 Dokumenten zu Big Data, die mithilfe algorithmisch fundierter Techniken einer Contentanalyse unterzogen wurden: Data Garbage durch irrelevante internettypische Geschwätzigkeit; Echo Chambers und affirmative Zirkel; Sample-Bias durch ungleiche Verteilung der Kommunikationschancen; interessengeleitete Präferenzverfälschungen durch selektive Datensammlungen; das Echtzeit-Dilemma durch unablässig in laufende Entscheidungsprozesse einsickernde Datenbestände, die jede Entscheidung obsolet machen; schließlich die Kontamination der Datenbestände durch Pornographie und politische Irrationalitäten. 
Zusammengefasst entsteht das Problem, dass weder Repräsentativität noch Relevanz von Daten objektiviert ohne Vorentscheidungen überprüft werden können. Das wiederum führt zur systematischen Ausblendung eventuell wichtiger "schwacher Signale«, die nicht in den algorithmisch erarbeiten Korrelationen auftauchen - oder zur Fehlinterpretation von Koinzidenzen als Kausalzusammenhänge. Es ist also richtig, was Schonfield 1969 schon schrieb: Fantasie sei erforderlich. Nur ist damit eine andere Fantasie gemeint, als die der Erfindung verkäuflicher Trend-Slogans.

Und hier liegt der tatsächlich gepflegte Ansatz der nächsten Stufe einer heute vorsichtig so genannten Corporate Foresight Research, die sich in den vergangenen Jahren ohne viel Aufsehen im internationalen und interdisziplinären Diskurs entwickelt hat. Sie stellt sich Fragen: Wie ist diese Fantasie so zu gestalten, dass sie valide Ergebnisse und tragbare Handlungsoptionen ermöglicht? Die Kernthese: Gerade die Unvorhersagbarkeit »der« Zukunft bietet eine Möglichkeit zur Entwicklung unkonventioneller und damit wettbewerbsfähiger Handlungsoptionen im Rahmen einer pluralistischen Innovationskultur. Interessant ist dabei die wachsende Forderung nach einer neuen »Interpretation Science« auf der Grundlage einer »Dualen Agenda«, die alle Potentiale der natürlichen wie der artefiziellen Intelligenz in klassisch dialektischer Manier nutzen. Diese »domestizierte« Fantasie, die sich von allen strategischen Opportunismen freihält, weil sie einen unbeeinflussten Recherchevorbehalt pflegt, wird die Alternative zur arbiträren Trendforschung sein. Sie lässt sich gebündelt im Ansatz des sogenannten "Superforecasting« illustrieren, entwickelt in einem hoffnungsfroh so genannten »Good Judgement Project $«$.

Das fand wiederum im Rahmen eines Wettbewerbs der IARPA (»Intelligent Advanced Research Project Activity«) statt. In einem Experiment wurden mehr als 20.000 Freiwillige rekrutiert, die unter wechselnden Versuchsbedingungen über mehrere Jahre hinweg in einem Feldexperiment Zukunftsprognosen zu vorweg bestimmten Fragen erstellten, die sich auf wenig bekannte komplexe Entwicklungen bezogen. Aus den beteiligten Personen rekrutierten die Wissenschaftler jährlich die 60 Prognostiker, die der Realität am nächsten gekommen waren. Sie wurden anschließend in fünf Elite-Gruppen versammelt. Insgesamt traf diese Gruppe rund 66 Prozent zuverlässigere Vorhersagen als der Durchschnitt der Befragten. Im Experiment wurden sie als »Superforecaster« bezeichnet.

Zwar räumen auch diese Forscher ein, dass Zukunft im Großen und Ganzen grundsätzlich nicht vorhersagbar sei und dass so genannte »Megatrends«, 
die 50 Jahre umfassen sollen, schlichter Unsinn sind. Doch greifen sie auch eine von Nassim Taleb formulierte strategische Differenzierung des Problems auf: dass sich in bestimmten Bereichen sehr wohl klare Vorhersagen treffen lassen, in anderen sehr wahrscheinliche, in wieder anderen nur noch mutmaßliche und schließlich in einem vierten Bereich keine. Diese Hierarchie ist abhängig von der Möglichkeit binärer Entscheidungen (ja/nein) oder den zur Verfügung stehenden Informationen und ihrer zeitlichen und räumlichen Reichweite (Taleb o.J.).

Im unübersehbaren Datenwust gebe es also "Pockets of Predictability« oder auch Regionen der »Local Predictability«. Die Entdeckung gelingt aber nur dann, wenn Methodenvielfalt herrscht und gleichzeitig eine Vielfalt von Perspektiven auf einen Gegenstand gerichtet sind, die im Diskurs, in der Diskussion und in einer Debatte die Möglichkeiten der Deutung ausloten. Das Magazin Wired erläuterte in einer Rezension der Superforecasting-Ansätze: "If there are pockets of predictability, they will surely be buried under a haystack of gross unpredictability. The signal of local predictability can be masked by a swirling mess of noise from a thousand other variables (Kelly 2009: o.S.).

Aus der Sicht eines methodologisch engagierten Sozialwissenschaftlers ist die Beschreibung des Vorgehens vor dem Hintergrund der Big DataDiskussion auch deshalb interessant, weil sie das gesamte klassische Repertoire der quantitativen und der qualitativen Instrumentarien reaktiviert:

- Konzentration auf evidenzbasierte Prognosen;

- Konfrontation ressort- oder themenspezifischer Kennzahlen mit diesen Prognosen;

- Diversifizierte Teams mit Personen und Sachkundigen breiter Qualifikation und Herkunft;

- Individuen mit differenzierten Forschungserfahrungen, die in der Lage sind, Szenarien zu entwickeln und gleichzeitig die Disziplin besitzen, sich selbst jederzeit zu relativeren und ihre Annahmen der Prüfung durch andere auszusetzen;

- Nutzung von Algorithmen, die komplexe, aber auch subjektive Begründungen produzieren;

- Integration der Data Scientists in alle Ressorts der unternehmerischen oder institutionellen Praxis;

- klare Definitionen aller Begriffe, die in diesem Diskurs benutzt werden, hier vor allem »Megatrends« und »Trends«, die im Trubel der kommerzi- 
ellen Konkurrenz um Vorträge, Aufträge und Aufmerksamkeit zu semantischer Unschärfe abgestumpft sind.

Dabei gilt eine fundamentale Regel: Intelligente Vereinfachung. Die Autoren und Betreiber des Projekts konzentrieren sich also auf erfahrbare Bereiche im Wust der ansonsten chaotisch anmutenden Daten (ausführlicher zum Prozedere siehe Tetlock 2015).

Im Prozess und in der Routine der alltäglichen Forschung in Unternehmen und Institutionen sind nur geringe Adaptionen nötig. Um die themenaktuelle Sekundärauswertung aller anfallenden Daten und Befunde zu gewährleisten, ist vor allem die Modularisierung von bereits validierten Einzelbefunden und Erfahrungen in kleinste Einheiten sinnvoll, also die Erfassung von binären Korrelationen. Diese Modularisierung erlaubt algorithmische Zugrif$\mathrm{fe}$, die es ermöglichen, jedes einzelne mit allen anderen in Beziehung zu setzen.

Ein solcher Forschungsansatz nutzt also die digitale Betriebslogik von Hypertext-Konstruktionen sowohl für die analoge Vorbereitung sinnvoller Algorithmen als auch für die Nachbereitung der von diesen Algorithmen gelieferten Informationen. Prinzipiell also handelt es sich um die Realisierung eines Prinzips, das von ungezählten Trendforschern als »Megatrend« gehandelt wird: »Connectivity«. Interessanterweise ist diese Konnektivität, wie es eingedeutscht heißt, weniger ein Trend als ein klassisches erkenntnistheoretisches Prinzip des »Kritischen Rationalismus«.

Technisch ausgedrückt: Es geht darum, Einzelbefunde durch eine thematisch inspirierte Vernetzung in bestimmte überprüfbare Relationen zu überführen, die auf diese Weise neue Zusammenhänge bilden.

Inhaltlich ausgedrückt: Ziel ist die Kontextualisierung und somit die Konstruktion eines Zusammenhangs mit größerer Aussagekraft, weil auf diese Weise ein besserer Zugang etwa zu den Handlungsoptionen einer Zielgruppe eröffnet wird, die zu bestimmten Themen angesprochen werden soll.

Nur eines dieser Beispiele kann hier kurz skizziert werden: Die Vernetzung des Einzelbefunds zu den sichtlichen Handlungsoptionen junger Nutzerinnen auf den Bilder-Plattformen des Internets, die eine starke kontextuelle Einbettung von Produkten dokumentieren, mit inhaltlichen Befunden, in denen sie sich neuen Inhalten gegenüber aufgeschlossener zeigen als gleichaltrige männliche Personen, und mit weiteren Informationen, in denen sich eine ausgeprägtere biographische Kalkulation andeutet (Familiengründung), sowie mit der sichtlichen Bevorzugung bestimmter ästhetischer und sozialer Muster, führt zu der 
Erkenntnis, wie diese weiblichen Nutzerinnen als Early Adopters beispielsweise bei der Umsetzung ökologischer Transformationen angesprochen werden können. Allerdings müssen Widersprüche in die Interpretation einbezogen werden. Denn es sind auch diese Nutzerinnen, die die größte Faszination für SUVs zeigen, wiederum in Kombination mit dem Modul der biographischen Kalkulation.

Abstrakt ausgedrückt: Auf diese Weise können so genannte »kulturelle Algorithmen« identifiziert werden, die als grundständige Muster in vielen Ausprägungen variiert den Alltag bestimmen. Diese Idee ist nicht neu: Sie erscheint schon 1972 in der Konzeption der struktur-funktionalen Erklärung gesellschaftlicher Prozesse durch den Soziologen Talcott Parsons, heißt damals aber natürlich noch nicht Megatrend, sondern »Pattern Variables«. Weitere inhaltliche Beispiele für die Umsetzung dieser Methodologie sind im Projekt »Geschmackskulturen im Internet « dargestellt (Rust 2017). Insgesamt hat sich in diesem Projekt gezeigt, dass die Betrachtung eines Internet-Phänomens durch eine pluralistisch besetzte Gruppe weit differenziertere und fantasievollere Ausgangsbedingungen für Interpretationen und weitere Forschungsschritte offenbart, als die mechanische Anwendung von Trendvorgaben.

\section{Schluss und Folgerung}

Am Ende deutet sich eine Lösung des Fermi-Problems 4.0 in den Kategorien und Theorien und Methoden der klassischen Soziologie sowie der Wirtschafts- und Geisteswissenschaften an, im Zusammenspiel von Empirie und Hermeneutik, kurz: im Pool jenes Wissens, das vor Jahrzehnten aus der Bemühung um systematische Lösungen des damals wie heute dominierenden Problems entstand, unüberschaubare Komplexität zu bewältigen und die daraus erwachsenden Herausforderungen für die Interpretation und Gestaltung des Alltags zu entwickeln. Eine Leistung der "Klassiker« liegt darin, dass sie dieses Unterfangen ohne digitale Hilfsmittel im heutigen Sinne bewältigen mussten. Man sollte nicht vergessen, dass aber gerade diese heute im Prozess der Digitalisierung und der Entwicklung künstlicher Intelligenz verdrängte und durch Nutznießer der Popularisierung von komplexen Antworten diskreditierte intellektuelle Kultur eine maßgebliche Voraussetzung für die Entstehung der digitalen Transformation darstellte. Sie führte schon Ende der 1940er Jahre zu ersten Ideen, aus denen im Santa Ana Valley jene heute weltbeherrschenden Tech-Firmen entstanden. 
Daraus folgt eine klare Lektion: Zukunft ist Produkt willentlicher Gestaltung unter Einbeziehung äußerer Einflüsse und der aus dieser Kombination resultierenden destruktiven, modifikatorischen und konstruktiv nutzbaren Emergenzen. Jede generelle Vorhersage über die Entwicklung dieser Logik ist arbiträr, vor allem sind es die in lärmenden Anglizismen inflationär verbreiteten Trenderfindungen, die ja letztlich nichts anderes darstellen, als Raster der Interpretation und somit auch eine Antwort auf die Frage nach den Interessen an ihren Trenderfindungen bieten: »Trendforscher« sind im Prinzip nur die Vorläufer von Influencern mit der Funktion der Marktbeeinflussung.

Was über die Zukunft bekannt sein kann, ist, wenn diese Begriffe auf den Markt kommen, längst in den erwähnten Forschungseinrichtungen erdacht (und zwar ohne den strategischen Opportunismus der Verkaufsorientierung) und in innovativen Unternehmen umgesetzt. Dort also entwickeln sich Trends, dort sind die Trendsetter, die die Pattern Variables in den Handlungsmustern ihrer Klientel längst identifiziert haben, lange bevor sich die Erfinder der Anglizismen als Entdecker feiern.

Verantwortungsvolle Foresight-Research, die sich zwangsläufig kompliziert ausdrückt, um unfassbar komplizierte Dinge nicht zu vernebeln, sollte nicht länger als Elfenbeinturm diskreditiert werden, wie es "führende» Trendforscher gern tun. Und die sollten sich vielleicht daran erinnern, dass, um nun doch zumindest einen Prognose-Flop anzufügen, manche von ihnen vor Jahren noch zu aggressiven Kritikern der Befunde der Klimaforschung zählten und einen heftigen Verbalkrieg gegen Klimaforscher führten (dazu Horx 2007; Rahmstorf 2008). Es ist der Beharrlichkeit komplexer wissenschaftlicher Zukunftsforschung zu verdanken, dass auf der Grundlage vieler kleinteiliger Arbeiten an den »Pockets of Predictibility« heute selbst in der Trendforschung ein anderes Meinungsklima herrscht - als, sei's drum, Megatrend.

\section{Literatur}

Ansoff, Igor H. (1976): »Managing Surprise and Discontinuity. Strategic Response to Weak Signals«, in: Zeitschrift für betriebswirtschaftliche Forschung 28, S. 129-152.

Bell, Daniel (1963): »Douze modes des Prévision«, in: Bulletin Sedeis 863, Supplement Futuribles 64. 
Bell, Daniel (1976): The Coming of the Postindustrial Society, New York: Basic Books.

Bensaïd, Georges/Jouvenel, Bertrand de (1965): »Lart de la conjecture«, in: Tiers-Monde 6 (23), S. 798-799.

Flechtheim, Ossip K. (1973): Futurologie. Der Kampf um die Zukunft, Frankfurt a.M.: Fischer Taschenbuch.

Horx, Matthias (2001): Smart Capitalism, Frankfurt a.M.: Eichborn.

Horx, Matthias (2007): „Warum ich nicht an die Klimakatastrophe glaube«, in: Welt Online vom 13.03.2007. Online verfügbar unter: https://www.welt.de/politik/article759237/Warum-ich-nicht-an-dieKlimakatastrophe-glaube.html. Zuletzt aufgerufen am 13.02.2020.

Horx, Matthias (2020a): Über uns - Die Zukunftsfamilie. Online verfügbar unter: https://www.horx.com/ueber-uns-about-us/. Zuletzt aufgerufen am 13.02.2020.

Horx, Matthias (2020b): Matthias Horx über den »Master of Future Administration« am 23.10.2014. Online verfügbar unter: https://www.youtube. com/watch?v=DJzDW_yGXMQ. Zuletzt aufgerufen am 18.02.2020

Horx, Matthias/Wippermann, Peter (1996): Was ist Trendforschung?, München: Econ.

Jouvenel, Hugues de (1986): »Cent Futuribles, et après?«, in: Futuribles 100, S. 3-6.

Kelly, Kevin (2009): Out Of Control: The New Biology of Machines, Social Systems And The Economic World. Online verfügbar unter: https://kk.org/ mt-files/books-mt/ooc-mf.pdf. Zuletzt aufgerufen am 27.02.2020.

Naisbitt, John (1982): Megatrends. Ten New Directions Transforming Our Lives, New York: Warner Books.

Naisbitt, John (2007): Mind-Set! Wie wir die Zukunft entschlüsseln, München: Hanser.

Nora, Simon/Minc, Alain (1978): L'Informatisation de la Societe, Paris: La Documentation Française.

Prange, Oliver (1998): Interview mit: Bosshart. Online verfügbar unter: https://www.persoenlich.com/interview/bosshart-david-gottliebduttweiler-institut-dezember-1998. Zuletzt aufgerufen am 13.02.2020.

Rahmstorf, Stefan (2008): »Klimawandel - Deutsche Medien betreiben Desinformation«. Online verfügbar unter: https://www.faz.net/ aktuell/wissen/klima/klimawandel-deutsche-medien-betreibendesinformation-1463241-p4.html. Zuletzt aufgerufen am: 13.02.2020. 
Rust, Holger (2008): Zukunftsillusionen. Kritik der Trendforschung, Wiesbaden: VS Verlag für Sozialwissenschaften.

Rust, Holger (2012): Strategie? Genie? Oder Zufall? Was wirklich hinter Managementerfolgen steckt, Wiesbaden: Gabler. https://doi.org/10.1007/ 978-3-8349-6922-4

Rust, Holger (2017): Virtuelle Bilderwolken. Eine qualitative Big Data-Analyse der Geschmackskulturen im Internet, Wiesbaden: Springer VS.

Rust, Holger (2019): Rettung der Digitalisierung vor dem Digitalismus. Der >Europäische Weg` in eine nicht nur künstlich intelligente Zukunft, Wiesbaden: Springer Gabler. https://doi.org/10.1007/978-3-658-26998-2

Schonfield, Andrew (1969): »Thinking about the Future«, in: Encounter 32 (2), S. $15-26$.

Schrep, Bruno (2019): »Der doppelte Professor«, in: Der Spiegel 42 vom 12.10.2019.

Schwertfeger, Bärbel (2018): »Eine zweifelhafte Expertin fürs »CharakterProfiling «, in: Übermedien vom 03.05.2018. Online verfügbar unter: https:// uebermedien.de/27497/eine-zweifelhafte-expertin-fuers-charakterprofiling. Zuletzt aufgerufen am 13.02.2020.

Seligman, Martin E.P. (1975): Helplessness: On Depression, Development and Death. Freeman and Comp: San Francisco.

Sherden, William A. (1998): The Fortune Sellers. The Big Business of Buying and Selling Predictions, New York: John Wiley \& Sons.

Snow, Charles P. (2012): The Two Cultures. Cambridge: Cambridge University Press.

Taleb, Nassim N. (2008): The Fourth Quadrant: A Map of the Limits of Statistics. Online verfügbar unter: https://www.edge.org/conversation/nassim_ nicholas_taleb-the-fourth-quadrant-a-map-of-the-limits-of-statistics.

Zuletzt aufgerufen am 13.02.2020.

Tetlock, Philip (2015): A Short Course in Superforecasting. Online verfügbar unter: https://www.edge.org/conversation/philip_tetlock-a-short-coursein-superforecasting. Zuletzt aufgerufen am 13.02.2020.

Tichy, Gunther (2001): »Erfordert die Informationsgesellschaft flexiblere Arbeitszeiten?«, in: Perspektiven der Wirtschaftspolitik 4 (1), S. 29-41. Online verfügbar unter: https://core.ac.uk/download/pdf/33821466.pdf. Zuletzt aufgerufen am 13.02.2020.

Toffler, Alvin (1972): Future Shock, London: Pan Books. 
Wenzel, Eike (2019): »Meisterdenker verfallen in einen toxischen Klimablues«, in: Handelsblatt vom 09.09.2019. Online verfügbar unter: https://www. handelsblatt.com/meinung/kolumnen/expertenrat/wenzel/expertenrateike-wenzel-meisterdenker-verfallen-in-einen-toxischen-klimablues/ 24994680.html?ticket=ST-2445209-cmaDWN7t1Z5melJdssya-ap6. Zuletzt aufgerufen am 13.02.2020. 



\title{
Quid agis, Megatrend?' \\ Von anekdotisch deduzierten Zukunftserzählungen und ihren Wirkungen
}

Stefan Schweiger und Nora Schecke

\begin{abstract}
»Er hat zwei Cegner: Der erste bedrängt ihn von hinten, vom Ursprung her. Der zweite verwehrt ihm den Weg nach vorn. Er kämpft mit beiden.«
\end{abstract}

(Kafka 2008: 1359)

»Doch es kam alles ganz anders.«

(Kinderzimmer Productions: Back)

\section{Einleitung}

Die Vergangenheit besteht aus unveränderlichen Fakten, deren Geltung in Raum und Zeit variieren (Eagelton 1997: 1ff.). Die Zukunft erscheint uns hingegen als fiktional-visionäres Reich utopischer oder dystopischer Möglichkeiten, wenngleich pfadabhängig an Entwicklungen und Bedingungen in der Vergangenheit geknüpft (Neuhaus/Steinmüller 2015: 17). Zwischen diesen beiden Reichen bewegt sich - wie im Eingangszitat von Franz Kafka - der um

1 Dieser Beitrag ist im Rahmen des Forschungsprojektes »EnerTrend: Systemische Analyse von Wechselwirkungen der Energiewende in NRW mit zentralen Megatrends« entstanden. Die Zuwendung für dieses Projekt stammt vom Ministerium für Wirtschaft, Innovation, Digitalisierung und Energie des Landes Nordrhein-Westfalen (MWIDE). 
Handlungsmacht ringende Mensch in seiner Gegenwart ${ }^{2}$. Die unabänderliche Vergangenheit belastet ihn, die Zukunft, die er in der Gegenwart zu entschlüsseln sucht, droht, ganz anders zu verlaufen als von ihm erwartet. Das Reich der Zukunft als nahezu unendlich erscheinende Potentialität entzieht sich durch seine inhärente Fiktionalität jedweder Interpretationsmöglichkeit. Zukunft lässt sich nicht interpretieren, da Daten aus der Zukunft schlicht nicht vorliegen können. Analysiert werden kann nur das bereits vergangene Erzählen möglicher Zukünfte (Beckert 2018: 294), das sich wiederum nur aus Daten aus der Vergangenheit speisen kann (Gigerenzer 2013: 55f.; Russel 1950: 64).

Eine besondere Form der Zukunftsnarration ist der seit Mitte der Achtziger Jahre des vergangenen Jahrhunderts aufgekommene "Diskursstrang» (Jäger 2015: 8of.) zu so genannten »Megatrends«, dem in außerakademischen Bereichen hohe Plausibilität zugeschrieben wird (Rust 2008: 140ff.). Der Begriff kam nicht zufällig in die Welt. Das Ende der großen Erzählungen, mit der Jean-François Lyotard (1999) die Postmoderne einläutete ${ }^{3}$, die Zunahme an verfügbarem Wissen und Erklärmustern via Internet und die Etablierung von Englisch als lingua franca (Fiedler 2009) und nicht nur lingua scientia, ermöglichte ein über die Elite hinausgehendes, transkontinental und -national wechselseitiges Verstehen. Dies sind die zentralen Prämissen, um Großentwicklungen in situ beobachtbar $\mathrm{zu}$ machen und Megatrends identifizieren oder zumindest assertive Aussagen über zukünftige Großentwicklungen tätigen zu können. Zuvor konnten sie ausreichend datengesättigt nahezu ausschließlich von Historikern ex post rekonstruiert werden.

Der zunehmend demokratisierte Zugang zu Wissen und die damit verbundenen Möglichkeiten des Verstehens bedeuteten das Ende einer Elitarisierung von Wissen durch einen politisch, sozial und ökonomisch eingeschränkten Zugang zu Bildung (Schultz/Hurrelmann 2013) und machte im Grundsatz die Komplexität der Welt zugänglicher und damit wirksame politische Mitbestimmung wahrscheinlicher (Hadjar/Becker 2009: 195, 204). Die

2 Der Frage danach, ob Gegenwart existiert und falls ja, sie beobachtbar sein kann und wie lange dieses Zeitintervall zwischen bereits Verändertem und noch nicht Verändertem andauert, weichen wir hier aus, sind uns jedoch der Problemstellung bewusst. Dabei handelt es sich in der Lesart Lyotards durch Wolfgang Welsch (1997: 232) um die Konstatierung des Fehlens einer »Metaregel (eines obersten Prinzips, eines Cottes, eines Königs, eines jüngsten [sic!] Cerichts oder auch nur einer respektablen Diskurspolizei)«. 
Zukunft zeigt sich daher in einer bis dato unbekannten diskursiven Wirksamkeit in der Gegenwart (Jischa 2009: 37), was sich u.a. an der Inflation von zum Teil paradoxen Leitbildern zeigt (Behrens 2003: 126; Giesel 2007: 38). Doch für plausible Schlüsse von vergangener Welt auf zukünftige und herrschende Großentwicklungen in der Gesellschaft braucht es (Medien-)Kompetenz zur Priorisierung sowie Ein- und Zuordnung von Informationen (Schauerte/Vehlken 2018: 10f.). Unabhängig davon, ob solch ein Umgang mit Information ge- oder misslingt, wird dem Einzelnen sein geringer Einfluss auf solche Entwicklungen und die damit verbundenen (gesellschafts-)politischen Entscheidungen kenntlich (Jörke 2019: 7-13). Folgerichtig beschreibt die »neue Unübersichtlichkeit«, die Jürgen Habermas (1985) zu einer Zeit des Aufkommens des Megatrendbegriffs konstatierte, nicht in erster Linie eine Unübersichtlichkeit durch Pluralisierung und Individualisierung. Vielmehr ist die Unübersichtlichkeit Ausdruck fehlenden Vertrauens in die individuelle und kollektive Handlungsfähigkeit (Voigt 2015: 31f.). Die geographische Ausdehnung komplexer supranationaler Gebilde, von Märkten und Kommunikationskanälen sowie die angewachsene Zahl aktiv beteiligter und passiv betroffener Personen bedeuten auch faktisch eine Verringerung der Wahrnehmbarkeit und "Relevanz der einzelnen Stimme« (Jörke 2019: 145). Eine nicht nur empfundene Hilflosigkeit gegenüber ökonomischen und politischen Entwicklungen, wie Finanz- und Staatsschuldenkrisen sowie die Entkoppelung des Individuums von politischen Entscheidungsprozessen mit der Begründung durch so genannte Sachzwänge (Dörre 2019: 43f.; Séville 2017: 425f.) als auch eine immer schnellere Abfolge technischer Innovationen schüren eine »Denormalisierungsangst vor einer völlig unbekannten Zukunft« (Link 2018: 272). Jene Angst resultiert aus einem Gefühl einer von Habermas, Nida-Rümelin und Bofinger (2012) konstatierten »Ohnmacht«. Das Individuum bemerkt im 21. Jahrhundert, dass es sich in einem Strudel unterschiedlicher und zum Teil paradoxer "grundlegende[r] und tief greifende[r] Veränderungen, die zwar relativ langsam vor sich gehen, sich dafür aber über eine große Zeitspanne hinziehen und den Rahmen bilden für viele andere, davon beeinflusste Entwicklungen« (Piepenbrink 2015: 2) befindet. Begriffe wie »Postdemokratie« (Crouch $2004^{4}$ ) und der Bundeskanzlerin Angela Merkel nachgesagte Begriff 
der »marktkonformen Demokratie« (von Altenbockum 2012) sind sowohl Interpretation als auch Treiber der Wahrnehmung der Bedeutungs- und Machtlosigkeit des/der Einzelnen. Solch ein angstbesetzter Kontrollverlust (Bude 2014: 9f.) weckt bei Individuen und Institutionen gleichermaßen eine Nachfrage an Handlungsempfehlungen, die gelingende Anpassungsleistungen beschreiben und die Zukunft als feststehende Größe, zu der es sich bloß noch angemessen bis gewinnbringend zu verhalten gilt, erscheinen lassen.

Eine als unsicher wahrgenommene ${ }^{5}$ Welt schafft eine Nachfrage nach Handlungssicherheit. Diese Marktchancen werden von Beratungsfirmen, wie z.B. dem Zukunftsinstitut (ZI), genutzt. In diesem Beitrag setzen wir uns insbesondere mit den vom ZI konstruierten Erzählungen über die Zukunft auseinander. Als Untersuchungsgegenstand wurde das ZI gewählt, da dieses sich nicht allein auf Unternehmensberatung konzentriert, sondern sich darüber hinaus in viele öffentliche Debatten in der Bundesrepublik Deutschland publizistisch (Horx 2017) als auch medial (Horx 2007) einbringt, vom Evangelischen Pressedienst (Rust 2008: 138) ebenso wie vom GreenpeaceMagazin (2019) zitiert und auch in wissenschaftlichen Journals als Quelle angegeben wird (Burel 2017: 124f., 128). Dies unterstellt keine hegemoniale Stellung für die Definition des Megatrendbegriffs. Das Privatunternehmen Zukunftsinstitut $\mathrm{GmbH}$ ist jedoch eine laut vernehmbare und durch Matthias Horx charismatisch personalisierte Stimme innerhalb des Diskurses. Insbesondere die starke Fixierung auf die Person Matthias Horx zeichnet das ZI vor anderen Unternehmensberatungen wie KPMG oder PWC aus, die sich viel mehr auf das Kerngeschäft der Unternehmensberatung fokussieren. $\mathrm{Ob}$ auf der Holzschutztagung (Knauf 2012), bei der ökologischen Strategieentwicklung von Industrie 4.0 (Kampker/Deutskens/Marks 2015: 77), der Feuerwehr (Klamert 2019), der Schuhindustrie (Sauerbier 2019), der Kosmetik (Hinterkörner 2019), in Fragen zukünftiger Ernährung (Glogowski 2019; Usleber 2019), der Architektur (Boße 2019) oder des nachhaltigen Konsums im

tiert, dass diejenigen, die in liberalen Demokratien Mitbestimmungsrechte erhalten, diese gegen andere verteidigen. Er sieht dabei die vom politischen Entscheidungsprozess exkludierenden Konfliktlinien zwischen a. Besitzenden und Nichtbesitzenden, b. der Konkurrenz unter den Nicht-Besitzenden und c. Staatsbürger*innen und NichtStaatsbürger*innen verlaufen.

Obgleich in der westlichen Welt zahlreiche Quellen der Unsicherheit nahezu beseitigt worden sind: So ist die Kriegsgefahr in Europa als niedrig einzuschätzen, ausreichende medizinische Versorgung ist in Westeuropa nahezu für alle Bevölkerungsschichten zugänglich und Missernten stellen kaum eine Gefahr für das Individuum dar. 
Allgemeinen (Dziemba/Wenzel 2014: 118f., 120, 130); das Megatrendnarrativ des Zukunftsinstituts findet sich in vielen Bereichen, die Nachhaltigkeit betreffen und entfaltet dort eine bestimmte und bestimmbare Wirkung. Unsere These besagt, dass dieses Narrativ Nachhaltigkeit nicht systematisch befördert, sondern bestenfalls akzidentiell hervorzubringen vermag.

Einfach lesbare Fahrpläne (Zukunftsinstitut 2018a) machen Zukunft auf den Schwingen des Megatrendbegriffs des ZIs versteh-, erklär-, berechenund damit auch potentiell planbar. So entfaltete und entfaltet der Begriff $\mathrm{Me}$ gatrend Attraktivität und Wirkmächtigkeit im außerakademischen Diskurs. Der Begriff dient - wie hier in diesem Beitrag gezeigt wird - erstens der Reduktion von Komplexität, zweitens der Bewältigung tiefgreifender Verunsicherungen in Wirtschaft (Opaschowski 2015: 42), Politik und Gesellschaft, drittens als kategorischer Imperativ ${ }^{6}$ zur Anpassung an Veränderungen, die als unausweichlich gerahmt werden, viertens als Mittel zur Produktwerbung sowie fünftens dem Schüren von Ängsten, Hoffnungen und der Bestätigung sowie Reproduktion eines Kulturpessimismus bzw. -optimismus. Der wissenschaftliche Nutzen des Begriffs kann in Frage gestellt werden (Rust 2008), die diskursive Wirkmächtigkeit jedoch kaum (Simon 2011: 184). Sowohl die Formen des Erzählens über Megatrends als auch deren Inhalte beeinflussen Denken und Handeln an entscheidenden Stellen in Wirtschaft, Verwaltung, aktiver wie passiver Zivilgesellschaft und Politik. Der Megatrendbegriff wurde zum Buzzword in Drittmittelanträgen, zum sozial akzeptierten Muster der Problembetrachtung, fand Eingang in das, was als Common Sense bezeichnet werden kann und spielt eine zentrale Rolle bei der Legitimierung von Lösungsvorschlägen sowie folgenreicher Entscheidungen auf allen Diskursebenen. Vor diesem Hintergrund erscheint ein Blick auf die vielfältigen Implikationen und Wirkungen des Diskursstrangs zu Megatrends vielversprechend. Zur Rekonstruktion des Diskursstranges wurden Webauftritte und frei verfügbare Publikationen des ZIs und die Webpräsenz der Foresight Company Z_punkt (als Vergleichsfolie) kursorisch ausgewertet. Ziel ist es, einen Beitrag zur Aufhellung von Nutzen und Fallstricken des Begriffs im »Interdiskurs $\aleph^{7}$ (Link 1986: 5f.) und dessen dortiger diskursiver Architektur zu leisten.

\footnotetext{
6 Handle stets gemäß dem allgemeinen Trend.

7 Link unterscheidet zwischen naturwissenschaftlichen, humanwissenschaftlichen und interdiskursiv dominierten Spezialdiskursen (z.B. Theologie und Philosophie). Aus diesen Spezialdiskursen »sammelt sich nun in den Redeformen mit totalisierendem und integrierendem Charakter (z.B. Journalismus, z.B. Populärwissenschaft und Populärphilosophie) ein stark selektives kulturelles Allgemein-Wissen [sic!], dessen Ce-
} 
Aus diesem zugrunde liegenden Datenkorpus können die Megatrends des ZIs als Erzählungen von Zukünften, die aus anekdotischen Episoden der jüngsten Vergangenheit abgeleitet wurden, verstanden und aus dieser Warte heraus interpretiert werden. Vom ZI werden Aussagen über Skalierungspotentiale aus anekdotischen Erzählungen abgeleitet, die sich ausschließlich auf marktorientierte Bewertungskriterien beziehen und keinen gedanklichen Raum für Reversibilität zulassen, um das als komödiantisch zu verstehende Emplotment nicht zu gefährden. Im Gegensatz zum ZI steht die mit wissenschaftlichen Methoden arbeitende Zukunftsforschung ${ }^{8}$, die von Beginn an von »einer Fülle möglicher Zukünfte« (Jungk 1968: 4-7, zitiert nach Seefried 2015:356) ausgegangen ist, die in Bezug stehen zu gegenwärtigen Entscheidungen und stets mit Unsicherheiten behaftet sind. Bei Horx hingegen bleibt der zu Beratende in einer passiven Position gegenüber irreversiblen Entwicklungen, die als nahezu eindeutig gerahmt werden. In drei Dimensionen wollen wir den Megatrendbegriff des ZIs beleuchten. Wir betrachten diese Auflistung nicht als abgeschlossen und hoffen, mit diesem Aufsatz weitere Beschäftigung mit der diskursiven Funktion des Begriffs anzustoßen.

\section{Die Orientierungsdimension des Megatrendbegriffs}

Diskurse um Megatrends verschränken (Jäger 2015: 80f., 86f.) sich in erzählerischer Form mit anderen Diskursen, wobei lediglich semipermeable Einflussnahme in den Veröffentlichungen des ZIs angenommen wird. Der jeweilige Megatrend beeinflusst (vermeintlich) nur die nach Beratung und Weisung suchende Organisation, mit deren zukünftiger Entwicklung ein bestimmter Megatrend in Verbindung gebracht wird. Die zu beratende Organisation bleibt hingegen (vermeintlich) unbedeutend für die zunehmende Manifestation des Megatrends, da derselbe als Teilaspekt der Umwelt der jeweiligen Organisation angesehen wird. Zweifelsfrei aber stützt die jeweilige Organisation den Megatrend ebenso durch Affirmation, ihrer Eigenlogik entsprechenden

samtheit hier Interdiskurs genannt wird. Der Interdiskurs ist nicht wie die Spezialdiskurse explizit geregelt und systematisiert, ihm werden keine Definitionen abgefordert, keine Widerspruchsfreiheit usw. [...] bildlich haben wir den Interdiskurs als >fluktuierendes Gewimmel zu kennzeichnen gesucht. «(Link 1986: 5f.). 
Reaktionen und diskursive Reproduktion. Die Megatrend-Erzählungen handeln von unumgänglichen Veränderungen auf allen Diskursebenen - vom Alltag über Wirtschaft, Politik, Wissenschaft bis hin zu religiösem Glauben und Praxis des Geschlechtsverkehrs (Horx 2017). Ohne Anpassungsleistungen an die als unumgänglich dargestellten Veränderungen - so die Botschaft des ZIs - gerät der jeweilig diskursiv mit Megatrend-Erzählungen in Zusammenhang gebrachte soziale Ort in Gefahr der Destabilisierung oder versäumt zumindest die Realisierung von Wachstumschancen.

Die Verschränkung mit dem Megatrend-Diskurs ist ein Tanz um als fragil gerahmte Organisationen. Stabilität erreicht die Organisation bei Adaption an die erwarteten Veränderungen durch den oder die Megatrends, fragil werden sie durch Beibehaltung des Status quo. Die Waage zwischen Fragilität und Stabilität wird in den Organisationen eigentlich geeicht auf die Leitdifferenz des jeweiligen Funktionssystems (z.B. Politik, Familie, Wirtschaft) (Luhmann 2009: 241). Doch das ZI setzt allein die ökonomische Leitdifferenz an, auch wenn Megatrends durch das ZI (o.J. a) holistisch beschrieben werden. Dies kann - wenn die Leitdifferenz der zu Beratenden nicht durch Gewinn/Verlust beschrieben wird - in »eine Art dezentrale Nervosität, die sich in unentwegtes Suchen nach Problemlösungen umsetzt, ohne Garantie für integrierbare Gesamtlösungen« (Luhmann 2009: 245) führen. Hierdurch ergibt sich in den öffentlichen Beiträgen des ZIs ein Highlighting bestimmter Anekdoten bei gleichzeitiger Vernachlässigung (Hiding) anderer - zum Teil gegenläufiger - ebenfalls anekdotischer Erzählungen, um einen möglichst hohen Grad von Persuasivität zu erreichen. Daraus resultierend werden Bewertungskriterien nahegelegt, welche nicht allen Handlungsmotiven gerecht werden. Die Problematik inadäquater Leitdifferenz lässt sich an zwei Beispielen verdeutlichen:

a. Das ZI als ein erfolgreiches Modell halböffentlicher ${ }^{9}$ Unternehmensberatung bewertet gesellschaftliche Veränderungen, die Unsicherheit mit sich bringen, aus der Sicht potentieller betriebswirtschaftlicher Kapitalmehrung oder -einbußen. Matthias Horx (2001: 12) macht die Orientierungsdimension seines Instituts explizit: »Der Markt: Er ist [...] das verletzliche Herz der menschlichen Kultur, die erste Sphäre der Öffentlichkeit und des Sozialen.« 
So wird die Sammlung umfangreicher Patientendaten im Rahmen des Megatrends Gesundheit »branchenübergreifend [zum] Quell neuer Wertschöpfung« (Zukunftsinstitut o.J. b), und selbst in der Beschreibung des Megatrends Sicherheit bleibt Big Data ein "Quell der Wertschöpfung« (Zukunftsinstitut o.J. c). Die Ereignisse der Welt werden auf die Möglichkeit der Erwirtschaftung von Gewinnen reduziert und die durch das ZI identifizierten Megatrends bestimmen, auf welche Weise Unternehmen zukünftige Gewinne realisieren können. Dabei wird jeder Trend als Marktchance interpretiert, ohne dabei auf Gefahren, Probleme und Antagonismen hinzuweisen.

Die Reaktion auf Umweltprobleme, die das ZI als Megatrend Neo-Ökologie (Zukunftsinstitut o.J. d) identifiziert, kann auf diese Weise gar keine Kritik am Wirtschaftssystem beinhalten, da dieser nicht als Mittel zum Zweck des Erhalts lebensfreundlicher Bedingungen auf dem Planeten Erde gerahmt wird, sondern als Mittel zur erfolgreichen wettbewerblichen Durchsetzung im bestehende System der Produktion und Distribution von Waren. So heißt es auch im ZI Österreich: »Effizienz ist der Weg zu grünem Wohlstand und wirtschaftlicher Stabilität.« (Gatterer/Schuldt/Varga 2014: 32) und unterbelichtet auf diese Weise die Nachhaltigkeitsstrategien der Suffizienz und Konsistenz. Dies wäre im Grundsatz kaum problematisch, wenn nicht der Begriff des Megatrends erstens von Naisbitt (1984) weiter gefasst worden wäre und damit andere Leitdifferenzen miteinschlösse und zweitens der Megatrendbegriff im »Interdiskurs« (Link 1986: 5f.) auch auf andere Systeme übertragen werden würde. So wird z.B. auch Gemeinnützigkeit (Gatterer/Schuldt/Varga 2014) unter diesen Vorzeichen behandelt.

b. Die im Sinne einer staatlichen Regulierung und im diskursiven Umgang (Fuchs 2019: 12; Pille 2016) immer noch als neu zu bezeichnenden sozialen Medien strahlen stark auf den Interdiskurs aus. Auch wenn das ZI keine hohen Klickzahlen auf YouTube erreicht, wird mit der stark fokussierten Marktorientierung wohl Breitenwirkung angestrebt. Dies kann problematische Auswirkungen auf den Nachhaltigkeitsbegriff innerhalb des öffentlichen Diskurses haben. Die Orientierung des Megatrendbegriffs auf eine kunden- und gewinnorientierten Leitdifferenz wird auch in diesen Medien unreflektiert auf andere Teilsysteme übertragen. So behält etwa Lena Papasabbas, Mitarbeiterin des ZIs, die Marktorientierung ihrer Megatrendforschung auch dann bei, wenn sie anekdotenhaft ein soziologisches Bild ihrer eigenen Generation $\mathrm{zu}$ zeichnen anstrebt. Sie konstruiert ihre Generation paradox als eine besonders an Nachhaltigkeit orientierte Generation mit Reisedrang (Papasabbas/Horx 2018: 06:00). So wird das vorerst gescheiterte Vorhaben »The Ocean 
Cleanup« des jungen niederländischen Erfinders Boyan Slat allein durch den hohen Betrag, der durch Crowdfunding eingeworben werden konnte, von ihr als »unheimlich erfolgreich « (ebd.: 12:22) beschrieben. Auch in anekdotischen Erzählungen über andere Projekte, die als Beispiel für die Nachhaltigkeitsbestrebungen einer konstruierten »Generation Global« (ebd.: 7:30) angeführt werden, erfolgt keine Bewertung etwa hinsichtlich des Erreichens von Nachhaltigkeitszielen, sondern eine Elaboration darüber, wie viel Aufmerksamkeit den Projekten international geschenkt wurde und, damit einhergehend, welche Marktchancen sie haben könnten. Die Anwendung der binären Codierung Markterfolg/-misserfolg auf Nachhaltigkeit transformiert Nachhaltigkeit vom Zweck zum Mittel, wie etwa folgendes Zitat illustriert: »Nachhaltigkeit boomt wie nie zuvor. [...] Wer sich offensiv einer nachhaltigkeitsorientierten Wertschöpfungskette stellt, kann enorme Chancen für neues Wachstum realisieren.« (Henkel 2015). Diese Zweck-Mittel-Umkehrung führt - wenn überhaupt - nur zufällig zu einer Beförderung ökologischer Nachhaltigkeit. Ein funktionierendes Zusammenspiel von ökonomischem Wachstum und Nachhaltigkeit wird indes im Inter- als auch Spezialdiskurs heftig diskutiert (z.B.: Adler/Schachtschneider 2017, Adloff 2015: 82, Best 2019: 33, Latouche 2015, Miegel 2010, Paech 2012, Schneidewind/Palzkill-Vorbeck 2011, Seidl/Zahrnt 2010, Stengel 2011, Stengel 2016, Welzer/Wiegandt 2014). Die Orientierung an betriebswirtschaftlichen Zielen bleibt dabei freilich durch die Spielregeln des Kapitalismus bzw. der Marktwirtschaft legitimiert. Allerdings gibt es in der Wissenschaft eine breite Diskussion darüber, ob ökonomisches Wachstum nicht eng an $\mathrm{CO}_{2}$-Emissionen gekoppelt ist (z.B. Mardani/Streimikiene/Cavallaro et al. 2019, Schröder/Storm 2018).

\section{Die Reversibilitätsdimension von Megatrends}

Trotz vor allem anekdotischer Beweisführung rahmt das ZI Megatrends als irreversibel, indem sie als »Entwicklungskonstanten« (Zukunftsinstitut o.J. a) bezeichnet werden. Tristan Horx, der Sohn des Gründers des ZIs, sagt in einem Vortrag über den Megatrend Urbanisierung: »[...] [die Urbanisierung] wird immer so weitergehen « (Papasabbas/Horx 2018: 12:43). Damit wird die Nachfrage nach Handlungssicherheit bedient, allerdings auch jedweder Fallibilismus, und damit der Anspruch auf Wissenschaftlichkeit aufgegeben und die Realität negiert. Mitte der Siebziger wurde Stadtflucht im Sinne einer Suburbanisierung beklagt (Heuer 1977). Dies kann durchaus wieder der Fall 
werden. ${ }^{10}$ Abhängig ist dies von »diskursive[n] Ereignisse[n] ${ }^{11}$. Diskursive Ereignisse können sich beschleunigend oder verlangsamend auf eine identifizierte Großentwicklung auswirken. Diskursive Ereignisse können Megatrends sogar umkehren. Um die Nachfrage nach Handlungssicherheit bedienen und gleichzeitig auf solche Veränderungen reagieren zu können, braucht das ZI eine intellektuell ansprechende Absicherung. Zur rhetorischen Absicherung und Verargumentierung baut Horx die Geschichtsphilosophie des 19. Jahrhunderts in seine Methodenerzählung ein. Dafür bedient sich Horx beim deutschen Idealisten Georg Wilhelm Friedrich Hegel. Horx spricht von "Gegentrends« (Horx 2015), die sich jedoch bei ihm dialektisch in von ihm definierten Megatrend auflösen bzw. synthetisieren. Dies ist ein instrumenteller Einsatz von Hegels Geschichtsphilosophie, der unterkomplex und »strategisches Handeln« (Habermas 1995: 130ff.) bleibt, da er nur dazu dient, die eigenen Thesen vor Falsifikation zu schützen.

Um sich der Reversibilitätsdimension nähern zu können, müssen die unterschiedlichen von Horx identifizierten Megatrends in vier Kategorien seziert werden, deren Konstruktion unterschiedliche Stabilität aufweist. Wir unterscheiden erstens Konstrukte, die entweder zur Simplifizierung der Wirklichkeit dienen oder Hilfskonstruktionen für Narrative und/oder Ideologien sind, zweitens immer wieder neu auszuhandelnde Interpretationen »sozialer Totalphänomene [Hervorhebung durch die Autor*innen] (Mauss 1999: 17f.) mit ebenfalls der Funktion von Simplifizierungen oder Hilfskonstruktionen sowie drittens die Interpretation von Wissensbeständen, denen durch methodische Falsifikationsversuche mehr oder minder hohe Robustheit zugeschrieben werden kann. Viertens lassen sich bereits heute weitestgehend funktionsfähige und gesellschaftlich angenommene (Rogers 1983: 241-270) technische Neuerungen als letzte Kategorie nennen. Mithilfe dieser Heuristik wird im Folgenden geklärt werden, mit welcher Wahrscheinlichkeit sich die von Horx identifizierten Megatrends überhaupt umkehren können. Dabei gilt, dass sich gesell-

10 Nach Analysen des BBSR (Milbert 2017: 8) sind neben dem evidenten Erstarken von Städten und Agglomerationsräumen ebenso gleichzeitig verlaufende Suburbanisierungstendenzen zu beobachten.

11 »Als diskursive Ereignisse sind [...] nur solche Ereignisse zu fassen, die medial groß herausgestellt werden und [...] die Richtung und Qualität des Diskursstrangs, zu dem sie gehören, mehr oder minder stark beeinflussen. (Jäger 2004: 162). So war der SuperGAU in einem Atomkraftwerk im japanischen Fukushima ein Ereignis, das starken Einfluss auf den Diskurs hatte, während der CAU in Harrisburg/PA im Jahre 1979 kein diskursives Ereignis war. 
schaftliche Konstrukte potentiell immer ändern können, auch wenn diese sich um soziale Totalphänomene herum strukturieren. Auch wissenschaftlich robustes Wissen ist nicht apodiktisch, jedoch nur unter großem Aufwand allein erzählerisch umzuformen, wie man das z.B. bei Klimawandelleugner"Innen findet (Schweiger/Trost 2020). Technische Errungenschaften können aufgegeben werden, doch dazu braucht es wirksame diskursive Ereignisse. Potentiell jedoch ist es möglich, dass sich technische Großentwicklungen mitsamt ihren sozialen Implikationen umkehren. Beispiele aus diesen vier Ebenen mit unterschiedlichem Regressionspotential werden im Folgenden betrachtet.

\section{a. (Soziale) Konstrukte}

Einer unzulässigen Simplifizierung geschuldet sind dabei unter anderem Beschreibungen der Jugend als Generation X, ein Begriff aus der Feder des Romanautors Douglas Coupland (2004), und die ohne Couplands schriftstellerisches Zutun fortgeschriebene Genealogie, Generationen Y und Z. Auch die Vorstellungen von Papasabbas und Tristan Horx' Generation Global (Papasabbas/Horx 2018) gehen in diese Richtung. Dabei hat Papasabbas weder den globalen Süden ${ }^{12}$ im Blick, noch die Lebenswirklichkeit der Jugend in Deutschland, von der sie behauptet, sie hätte "neue Werte, neue Haltung« (Papasabbas/Horx 2018: 03:00) durch gesteigerten Wohlstand, Reisen und die Benutzung von digitalen und vernetzten Endgeräten erworben. Der Soziologe Martin Schröder (2018) zeigt dieser These widersprechend auf, dass eine relativ stabile Haltung und Lebenszielsetzung zwischen den Generationen zu verzeichnen ist - und dies von der als skeptisch bezeichneten Nachkriegsgeneration bis zur »Generation Schneeflöckchen« (Fox 2017: 57-144). Die breit angelegte Shell-Studie von 2015 bestätigt dies in Teilen ebenfalls. Mit Blick auf Erwartungen an die Berufstätigkeit rangiert der Wunsch nach einem sicheren Arbeitsplatz weiterhin vor dem Ziel, etwas Nützliches für die Gesellschaft zu

12 Papasabbas meint in dem hier genauer beleuchteten Vortrag auf YouTube: »Fast jeder auf der Welt hat heutzutage ein Smartphone (Papasabbas/Horx 2018: 02:17), was nicht mal auf die Hälfte der Menschen dieses Planeten zutrifft (Silver 2018). Daran zeigt die westliche Zentrierung des Megatrend-Begriffs des Zukunftsinstituts. Die Vorstellung, dass es überall so zugehe, wie in den boomenden westlichen Metropolen lässt dann auch zu Aussagen hinreißen wie: »Es gibt zum Beispiel in jeder Stadt, egal ob in Europa oder in den USA oder auch Asien, Urban-Gardening-Projekte. (Papasabbas/Horx 2018: 09:59). 
tun (Albert/Hurrelmann/Quenzel 2015: 81). Mehr als doppelt so viele Jugendliche sehen politischen Handlungsbedarf für die Familie als dringlicher an als für den Bereich Umwelt- und Naturschutz (ebd.: 171). Daran ändern Phänomene wie »Fridays for Future«, Urban Gardening-Projekte oder »Occupy Wall-Street « kaum etwas und solche Bewegungen passen auch kaum zur Konstruktion einer Generation Global. Tristan Horx ergänzt Papasabbas auf der auf YouTube abrufbaren Veranstaltung, indem er die These äußert, dass die Individuen der Generation Global »wissen und verstehen, dass sie im globalen Netzwerk auch funktionieren müssen« (Papasabbas/Horx 2018: 05:20), was nicht nach Protest und der Durchsetzung »neuer Werte« (ebd.: 03:00) klingen mag, sondern nach Anpassung an die vom ZI erkannten, postulierten und als unumkehrbar identifizierten Megatrends. Die Einzeltrends der Generation Global sind bei Papasabbas der Blick auf Nischenphänomene, von denen behauptet wird, sie breiteten sich überall auf dem Globus aus (ebd.: 06:38), was ohne Beleg bleibt. Abgeleitet wird aus dieser Behauptung, die Übernahme von Verantwortung zum Schutz des Klimas und der Umwelt durch die Generation Global. Die anekdotenhafte Beweisführung beschränkt sich in ihren Protagonisten auf Mitglieder einer Diskurselite bzw. verliert dadurch eine sich in postindustriellen Zeiten verkleinernde Mittelschicht aus dem Blick. Dennoch gilt hier, dass das, was die Menschen als real konstruieren, real in seinen Auswirkungen ist (Thomas 1928: 572), jedoch nur so lange die dafür notwendigen materiellen Bedingungen vorhanden sind diese Auswirkungen tatsächlich zu zeitigen. Das macht die Megatrends, die von Medien und Beratungsinstitutionen konstruiert werden, voraussetzungsvoll und damit zur fragilsten Kategorie im Sinne einer Reversibilität. Eine durchgehende ökonomische Boomphase ist notwendig, um einen freiwilligen Postmaterialismus (Papasabbas/Horx 2018: 11:12) aufrechtzuerhalten. Die globale Wirtschaftskrise, die in der Immobilienkrise in den USA im Jahre 2007 ihren Anfang nahm, hat sich in den Nationalökonomien unterschiedlich ausgewirkt, aber eins zeigte sich global: Erst durch diesen unfreiwilligen Postmaterialismus gingen die Emissionen von Treibhausgasen merklich und nachweislich zurück (Peters/Marland/Le Quéré et al. 2012). Die postmateriellen Nachhaltigkeitsbestrebungen der Generation Y (Huber/Rauch 2013: 30, 36) haben hingegen kaum Impact auf die Menge der klimawandeltreibenden $\mathrm{CO}_{2}$-Emissionen (World Meteorological Organization 2019). Auf Megatrends, die ohne Anbindung an Natur, Wissenschaft oder technischen Innovationen bleiben, ist nur geringer Verlass. Trotzdem werden sie vom ZI (2018a) als Fahrplan dargestellt, obgleich sie mehr einem Pogo-Tanz auf dünnem Eis gleichen. Diese Megatrends kön- 
nen sich bei Änderungen von Randbedingungen schnell sogar in ihr Gegenteil verkehren, wohingegen die Sehnsucht der Menschen nach psychischer und physischer Stabilität einen durchgehend hohen Stellenwert hat. Dies jedoch als Megatrend verkaufen zu wollen, befriedigt kaum eine Nachfrage nach $\mathrm{Zu}$ kunftswissen und Handlungssicherheit in unsicher erscheinenden Zeiten.

\section{b. Soziale Totalphänomene}

Megatrends haben jedoch nicht nur imaginierte Simplifizierungen von kaum eingegrenzten Personengruppen zum Gegenstand. Sie behandeln auch »soziale Totalphänomene« (Mauss 1999: 17f.), also basale anthropologische Konstanten, mit der sich jede Kultur an jedem Ort und zu jeder Zeit auseinandersetzen muss. Diese Totalphänomene umfassen u.a. Geburt, biologische Reproduktion, Nahrung, Tod, Wetter wie auch selbst- oder fremdbestimmte Zuordnung eines Menschen zu einem oder mehreren Geschlechtern. Horx (2011: 96-110) rief den "Megatrend Frauen $\aleph^{13}$ aus und dieser Megatrend war in bestimmten Sektoren auch stabil, auch wenn sich die von Horx prognostizierten Zahlenwerte nicht in Gänze bewahrheiteten und auch seine Wortwahl 2020 nicht mehr opportun erscheinen würde. Die Präsenz von Frauen hat sich in historisch vornehmlich Männern vorbehaltenen gesellschaftlichen Sphären zum Teil sogar überproportional ${ }^{14}$ erhöht. Diskursiv hat sich die Erwerbsarbeit von Frauen sogar in konservativ orientierten Medien als auch aus so-

In diesem Text erklärt Horx seinen Leser*innen, Unterschiede zwischen Männern und Frauen evolutionsbiologisch (Horx 2011: 100f.).

14 Frauenanteil in ausgewählten Berufen (Stand: 30.06.2017): Medizinische Gesundheitsberufe: 82,7\%; Berufe in Recht und Verwaltung: 75,7\%; Berufe in Unternehmensführung: 64,3\%; Berufe in den Geistes-, Gesellschafts- und Wirtschaftswissenschaften: $63,3 \%$; Berufe in der Finanzdienstleistung, Rechnungswesen und Steuerberatung: $61,7 \%$; Lehrende und ausbildende Berufe: 55,3\%; Werbung, Marketing und redaktionelle Medienberufe: 54,1 \% und sogar die weiblichen Angehörigen der Streitkräfte betragen bereits 41,5\% (Statista 2019). Der Frauenanteil an deutschen Hochschulen lag 2017 mit 39 \% so hoch wie noch nie. Allein von 2016 auf 2017 stieg der Frauenanteil um 2,5\% (Forschung und Lehre 2018). Selbst in den stark männlich dominierten MINTBerufen steigen mindestens seit 2013 die Anteile der Frauen kontinuierlich an (Statistik der Bundesagentur für Arbeit 2019: 38). In Deutschland stieg auch der Anteil weiblicher Führungskräfte an (Statistisches Bundesamt 2011); dieser lag 2017 bei 29,4 \%. Im EU-Vergleich bewegt sich Deutschland damit im unteren Drittel: Lettland weist beispielsweise den höchsten Anteil von 46,3\% weiblicher Führungskräfte auf (Eurostat 2020). 
ziologischer Sicht in weiten Teilen der Mittelschicht (Reckwitz 2019: 91, 110ff.) normalisiert und die Unterrepräsentation von Frauen befindet sich in nahezu allen Sektoren in der Bundesrepublik Deutschland auf dem Rückmarsch (Berner/Vogel/Wettstein/Tesch-Römer 2019: 140; Faust/Assmann 2014: 145; Meyn 2019; Reckwitz 2019: 111; Wagner 2017: 25).

Die kommerzielle Megatrendforschung nimmt in Anekdoten und verallgemeinernden Aussagen Bezug auf solche Prozesse und skaliert diese für die westliche Welt, wobei sie sich auch dort vor allem auf eine akademisch gebildete, relativ einkommensstarke weibliche Diskurselite bezieht. Dies zeigt sich zum Beispiel bei Papasabbas (2018) darin, dass jene, die nicht ins optimistisch-feministisch konnotierte Selbstbild der eigenen Konstruktion der eigenen Generation passen wollen, pejorativ als "Prolls« (ebd.) bezeichnet werden und bei Horx in einer als hegelianisch gerahmten Synthese (Horx 2015) aufgehen sollen. Diese »Prolls« haben dann - im konstruierten Gegensatz zum eigenen ebenfalls wenig komplexen und widersprüchlichen Sittengemälde, das Papasabbas malt - nicht eine andere, sondern »eine einfache [...] Rollenaufteilung (Papasabbas 2018). Negativ konnotierte und zur eigenen These gegenläufige Trends werden durch Papasabbas (ebd.) zwar beachtet, sind jedoch kein Grund zur Modifikation der eigenen These. Sofern Megatrends globale Veränderungen markieren sollen (Zukunftsinstitut o.J. a), sollte zur Konstituierung eines umfänglichen Gender Shift jedoch nicht allein in die Filterblase ökonomisch prosperierender westdeutscher Großstädte geblickt werden: Die Re-Islamisierung der Türkei (Somer 2015) und Tunesiens (Aldailami/Zapf 2018: 328f.), das Missverhältnis von Hochschulabsolventinnen und beruflichem Erfolg von Frauen (Gorges 2019: 72) ${ }^{15}$, die immer noch nicht eingedämmte Beschneidung von Frauen in ihrer schrecklichen Tragweite in nicht unerheblichen Teilen Afrikas (WomanStats Project 2015), die Aussagen des amerikanischen Präsidenten Donald Trump über Frauen (Wiesner 2016), die Erfolge der Rechtspopulisten und ihr patriarchales Familienbild nicht nur (Hentges/Nottbohm 2017: 167-208) in den europäischen Viségrad-Staaten (Amjahid 2019), sondern ebenfalls im Osten der Bundesrepublik Deutschland, wie die Landtagswahlen im Jahr 2019 zeigten: Trotz eines

15 Darüber hinaus geben die aufgezeigten Entwicklungen zum steigenden Frauenanteil in den dargestellten Berufsgruppen (siehe Fußnote 14) keine Einsicht in die konkret ausgeübten Berufe und Tätigkeiten. Somit können hierbei auch keine Aussagen zum Inhalt, zur Position, zum Verdienst, zur Voll- oder Teilzeitbeschäftigung sowie zu Karrieremöglichkeiten getätigt werden. 
regressiven Frauen- und Familienbildes konnte die Alternative für Deutschland (AfD) dort horrende Zugewinne an Wählerstimmen verzeichnen. Die Wähler*innen hielten diese Haltung zu Genderaspekten zumindest nicht von der Wahl der AfD ab. Die Lebenspraxis von muslimischen Frauen in Europa (Krause 2018: 80f.) zeigt zudem vom ZI Megatrend Gender Shift bzw. dem Megatrend Frauen gegenläufige Tendenzen, die nicht dazu führen, dass »die Tatsache, ob jemand als Mann oder Frau geboren wird und aufwächst, weniger darüber aus[...]sagt, wie Biographien verlaufen werden« (Zukunftsinstitut o.J. e). Es zeigt sich hier keine hegelianische Synthetisierung. Megatrends, die »soziale Totalphänomene« (Mauss 1999: 17f.) zum Gegenstand haben, sind zwar deutlich weniger fragil, jedoch ist auch deren Verlauf nicht wie auf einem Fahrplan (Zukunftsinstitut 2018a) vorgezeichnet. Die Haltestellen sozialer Totalphänomene müssen bedient werden, aber sowohl Fahrtrichtung als auch - um im Sprachbild zu bleiben - Fahrzeug sind weniger eindeutig, als das ZI glauben machen will. Dies ist strategisch jedoch nachvollziehbar. Wen das ZI als Protagonist*innen vorstellig machen will, sind Konsument*innen und nicht ausgebeutete, misshandelte und unterdrückte Menschen. Die Orientierungsdimension bestimmt hier die Auswahl der beweisführenden Anekdoten und bestimmt auch das agierende Personal und deren charakterliche Eigenschaften. Emanzipierte Frauen gelten als einkommensstärker und eignen sich so besser als Marktteilnehmerinnen, was der eigentliche Grund zu sein scheint, sie in den Mittelpunkt zu rücken, auch wenn nicht nur in globaler Hinsicht gegenläufige Tendenzen auf eine Weise unleugbar sind.

\section{c. Robuste wissenschaftliche Erkenntnisse}

Als dritte Kategorie benennen wir robuste wissenschaftliche Erkenntnisse. Ihre Robustheit erhalten diese Erkenntnisse durch erstens Peer-ReviewVerfahren in wissenschaftlichen Journals und zweitens durch ihre grundsätzliche Falsifizierbarkeit (Popper 1989, 2005). Besonders robust sind neben den Naturgesetzen auch das Wissen um den Zusammenhang von Treibhausgasen und der Erderwärmung (IPCC 2014), der die Bewohnbarkeit des Planeten für Teile der Menschheit gefährden könnte und als Anstoß zur Konstruktion des Megatrends Neo-Ökologie durch das ZI gewesen sein könnte. Das ZI führt Veränderungen in der natürlichen Umwelt gar nicht in ihren Megatrends aus. Die Foresight Company Z_punkt, die unter anderem die Autofirma AUDI beraten hat (Glockner o.J.), nimmt hingegen auch Anthropogene Umweltbelastung (Z_punkt o.J.) als Megatrend auf. Darunter fasst Z_punkt 
nicht nur Klimawandel, sondern auch andere anthropogen verursachte Umweltschäden, wie z.B. abnehmende Biodiversität (ebd.). Das ZI beschäftigt sich mit solchen auf wissenschaftlich begründeten Wahrscheinlichkeiten beruhenden Zukunftsvorhersagen nicht. Mehr noch: Horx sagt deutlich, dass er an den Klimawandel nicht glaube (Horx 2007). Daran zeigt sich ganz, dass alles, was erzählerisch kein Happy End suggeriert, nicht in die Erzählung des ZIs passt und notfalls durch Horx selbst oder einem seiner Mitarbeiter*innen unterbelichtet bleibt bzw. bleiben muss/soll.

\section{d. Produkte technischen Fortschritts}

Viertens sind sehr häufig Produkte technischen Fortschritts Gegenstand von Megatrends. Vernetzung, Konnektivität, Mobilität und vor allem Digitalisierung $^{16}$, die »den Menschen auf sein Menschsein« (Zukunftsinstitut o.J. f, vgl. auch Zukunftsinstitut o.J. g) zurückwerfen soll, sind die aufgerufenen Megatrends, die das Leben der Menschen dauerhaft ändern sollen. Technische Innovationen, wenn sie erstens funktionabel, also über den Status als Prototyp hinausgelangt sind, zweitens vom Markt absorbiert oder aber drittens jenseits von Markt und Staat vertrieben werden unterscheiden sich in der Frage der Reversibilität deutlich von den drei zuvor genannten Gegenständen der Megatrends. Grundsätzlich ist es möglich von einer DSL-Verbindung zurück auf den Stand eines 56K-Modems zu wechseln. Grundsätzlich irreversibel ist der technische Fortschritt also nicht. Die sprichwörtliche Unaufhaltsamkeit technischen Fortschritts ist nicht gegeben. Dies zeigt sich auch empirisch. Es werden aus politischen Gründen technische Entwicklungszweige nicht weiterverfolgt. Die Energiegewinnung aus Uran und Plutonium wurde - zumindest in der Bundesrepublik Deutschland - nicht weitergeführt, obgleich die Kernenergie mindestens bis zum Reaktorunglück in Tschernobyl

16 Digitalisierung gefährdet die Bestrebungen, die Energieversorgung zu einem Großteil auf Erneuerbare Energieträger umzustellen. Bereits jetzt verursachen digitale Anwendungen $4 \%$ der Treibhausgaremissionen. Im Jahre 2018 verursachte Video-Streaming weltweit so viel Treibhausgasemissionen wie Spanien in einem Jahr, davon entfallen $27 \%$ auf pornographische Inhalte (The Shift Project 2019). Durch eine Erhöhung der Übertragungsgeschwindigkeiten und flächenmäßigen Ausbreitung von Endgeräten und Empfangbarkeit ist damit zu rechnen, dass sich die Treibhausgasemissionen noch erhöhen. Flächendeckender Empfang mag ein ökonomisch und politisch anstrebenswertes Ziel sein, jedoch ist kein Mittel, das zur Umsetzung von Suffizienzstrategien beiträgt. 
im April 1986 als ein Megatrend der Energiegewinnung bezeichnet werden konnte. Technische Innovationen sind im Grundsatz reversibel, nicht immer jedoch ihre Folgen. Mit Exnovationen (Kropp 2015), wie beim Atom- und Kohleausstieg, als auch bei Verboten von Kunststoffprodukten mit kurzer Nutzungsdauer (Richtlinie (EU) 2019/904), aber in der Umwelt langlebigen oder gar umweltschädlichen Materialien, ist immer zu rechnen. Innerhalb der Entwicklung von künstlicher Intelligenz (KI), autonom gesteuerten Fahrzeugen oder Software, die gegen Datenschutz verstößt, könnte der technische Fortschritt ebenfalls aus politischen Gründen gedrosselt oder gar ausgebremst werden. Solchen demokratisch legitimierten Vorgängen schenkt das ZI keine Aufmerksamkeit. Das Unternehmen bleibt in der Fortschrittsgläubigkeit längst vergangener Jahrzehnte verhaftet (Rust 2008: 69-72).

\section{e. Zwischenfazit}

Wie in einem Trichter engen sich die Möglichkeiten sozialer Konstruktion in dieser Betrachtungsdimension immer stärker ein. Das robuste Wissen um hinreichende Entwicklungen und Zusammenhänge in der Natur lassen weniger Spielraum, um sie in unterschiedliche Narrative mit unterschiedlichem Personal einzuflechten. Die Besonderheit liegt im Gegensatz zu Konstrukten mit oder ohne Anbindung an soziale Totalphänomene darin, dass kausal begründeten Vorgänge sich nicht allein über den Gradmesser der Plausibilität messen lassen, sondern im Sinne Bruno Latours, die lange Zeit als allein durch den Menschen konstruierte Welt »um die Welt der Dinge erweitert" (Krauss 2011: 595). Der Klimawandel vollzieht sich auch, wenn man ihn leugnet oder gesellschaftlich wirksame Konstrukte um diesen spinnt oder anders ausgedrückt: »Diskurse über das Klima sind nicht nur Gerede« (Passoth 2010: 49). »Anthropogene Umweltbelastung« (Z_punkt o.J.) kann als ein Megatrend mit Auswirkungen auf die gesamte Menschheit bezeichnet werden.

\section{Die Erzählstrukturen der Megatrends des Zukunftsinstituts}

$\mathrm{Zu}$ unterscheiden sind a. normativ-ontologische Megatrends, also Entwicklungen, die postuliert werden (z.B. Neoökologie), b. erfahrungswissenschaftlich nachweisbare Megatrends, die konstatiert werden (z.B. soziodemographischer Wandel), c. tragisch-dystopische Megatrends (z.B. anthropogene Umweltbelastung), deren Ausweitung verhindert werden soll und d. Rah- 
mentrends (z.B. Demokratie, Kapitalismus), welche die Spielregeln des Sprechens und Handelns vorgeben, da sie innerhalb des Diskurses hegemonial sind. Normativ-ontologische Megatrends tauchen beim ZI nur in einer komödiantisch motivierenden Form auf. Komödie definieren wir nach Hayden White:

»In der Komödie erscheint die Hoffnung als befristeter Triumph des Menschen über seine Welt durch die Aussicht auf gelegentliche Versöhnungen der in Gesellschaft und Natur wirkenden Kräfte. [...] Die Versöhnungen [...] sind Versöhnungen zwischen Menschen, der Menschen mit ihrer Welt; der Zustand der Cesellschaft erscheint nun reiner, vernünftiger und verbessert, als Ergebnis des Konflikts zwischen scheinbar unveränderlich widerstreitenden Kräften, von denen sich jetzt herausstellt, dass sie auf lange Sicht miteinander vereinbar [...] sind.«(White 1991: 23)

Der Historiker Hayden White hat sich gefragt, wie die Historiker ${ }^{17}$ des 19. Jahrhunderts bei nahezu gleicher Datenlage auf solch unterschiedliche Interpretationen der menschlichen Geschichte kommen. White hat herausgearbeitet, dass die Historiker verschiedene Formen der Erzählung benutzen, welche die Inhalte konfigurieren. Idealtypisch sind dies Romanze, Satire, Tragödie und Komödie. Diese verknüpft White mit den vorherrschenden Ideologien des 19. Jahrhunderts. Diese Überlegungen passen auch für den zur Beratung vorgesehenen Umgang mit Megatrends. Das ZI inszeniert seine Vorträge und Erzählungen vorwiegend als Komödien. Dies erscheint auch adäquat zu einer neoliberalen Ideologie. Im Kapitalismus soll Geld investiert werden, sollen Kredite aufgenommen und mit Zinsen zurückgezahlt werden, sollen Produkte erdacht, entwickelt und mit monetärem Gewinn distribuiert werden. Dazu passen Komödien wesentlich besser als Tragödien, Romanzen oder Satiren. Der Grund liegt offen: Wer nicht an ein positives Ende der Megatrenderzählungen glaubt, der ist auch nicht bereit zu investieren, zu entwickeln, zu produzieren und Waren und Dienstleistungen mit dem Ziele des Verkaufs und dem nachgeordnet, der Kapitalmehrung, zu bewerben. Normativ-ontologische Megatrends sind (soziale) Konstrukte und damit fragil und tendenziell reversibel. Sie sind Postulate, die sich aus gesellschaftlich als bereits beantwortet angesehen ethischen Fragestellungen ergeben und deren Umsetzung

17 Hier wurde nur die männliche Form gewählt, da es sich bei den vier durch White untersuchten Historikern ausschließlich um Männer handelt. 
in den Augen der Angestellten und Führungskräfte des ZIs neue Handlungswelten eröffnen. Das ZI konzentriert sich auf optimistische Szenarien und bettet dies in eine zeitgenössische Kultur- und Gesellschaftskritik: »ZukunftsVisionen werden heute dominiert von Dystopien und nicht mehr vom Glauben an eine bessere Welt.« (Zukunftsinstitut 2018b). Alles ist als Chance zu begreifen (Seitz 2015), jede Bedrohung ist eine Herausforderung, die man mit Elan und Optimismus angehen soll. Das Bedürfnis der Menschen nach angstfreien Räumen wird durch Unternehmen und eigenverantwortlich durch den Menschen befriedigt werden (ebd.). Solcherart »Flexicurity (ebd.) wird anekdotisch im Bereich der "Überall-Erreichbarkeit« (ebd.) innerhalb der New Work beschrieben. Diese Flexicurity findet man bei »sinnorientierten Unternehmen« (Li 2016) wie "Netflix« (ebd.) und wird natürlich als Herausforderung begriffen, also als Hürde, die man als Held oder Heldin nehmen muss, um am Schluss wie einst zu Beginn der Erzählung einen harmonischen Endpunkt zu haben, nur eben noch angenehmer, noch harmonischer. Der Schluss spiegelt dann die Rendite der Investition oder den Zins des Fonds wieder. Sogar der "Shitstorm« (Wieneke 2015) wird zur "Chance« (ebd.) promoviert, dessen Schluss ein harmonisch-versöhnliches Ende bildet. Bei Matthias Horx (2019) wird in wenigen Sätzen die komödiantische Ausrichtung ersichtlich:

»In den goldenen Jahren des naiven Fortschritts, als die ersten Menschen ihren Fuß auf den Mond setzten, wies die Linie der Zukunft in eine eindeutige Richtung: nach oben! Man glaubte, die Menschheit würde sich in die Tiefen des Weltraums ausdehnen. Als Neil Armstrong am 20. Juli 1969 seinen Schuh in den grauen Staub des Trabanten setzte, sollte das nur der kleine Schritt am Anfang eines vorgezeichneten Weges voller Wunder, Entdeckungen und Eroberungen sein.

Die Wunder gab es - allerdings nur im Kino. Die Eroberung der Himmelskörper endete nach sechs Mondlandungen. Die Weltlage wurde düster: Umweltverschmutzung, Kriege, soziale Probleme. Und so ist es bis heute geblieben.

Doch nun scheint sich der Wind wieder zu drehen. Eine alte Euphorie, eine nicht gestillte Sehnsucht kehrt zurück. Plötzlich treten wieder sichtbare Astronauten-Stars auf.

$[\ldots]$

Im Flug in die Vertikale ordnen wir die Ebenen unserer Existenz neu, die Ordnungen von Familie, Gruppe bis zu 'Kultur<, 'Land und `Nation<. So konstruieren wir uns erst als Spezies, als Menschheit, selbst. 
Und vielleicht ist es auch genau das, was uns das Stocken der Weltraumfahrt in den vergangenen Jahrzehnten sagen will: Die Rakete steigt in einer ewigen Parabel auf-um immer zu uns zurückkehren. Der eigentliche Grund für den Lift-off ist das Heimweh.

WE HAVE A LIFT-OFF! «

Zur Zeit der ersten Mondlandung waren es zwar goldene Jahre, aber der Fortschritt war noch »naiv«. Dies enthält das Implikat, dass der heutige Fortschritt nicht mehr so negativ konnotiert ist, sondern ein reifer, überlegter Fortschritt. Der von Horx prognostizierte Endzustand erscheint also harmonischer als der Ausgangszustand. Dazwischen lagen viele Irrungen und Wirrungen. Doch das Ende erscheint klar: Es wird sich rentieren. Ein anderes Ende ist in den Megatrenderzählungen des ZIs nicht vorgesehen. Dabei ergibt sich nicht nur ein verqueres Weltbild für die Leser*innen des Outputs des ZIs, sondern auch Gefahren für die Unternehmen, die sich durch das ZI beraten lassen. Negative Entwicklungen werden als Gegentrend wegerzählt. Dies führt dazu, dass Unternehmen für die Zukunft nicht richtig auf volatile Märkte oder Unsicherheiten durch den Klimawandel, den Horx (2007) leugnet, vorbereitet sind.

Ein besonders nachdrücklich empirisch-analytischer Megatrend nennt sich beim Zukunftsinstitut »Silver Society« und meint den demographischen Wandel hinsichtlich der Altersstruktur. Dieser Megatrend wird nicht als ein Soll-Zustand propagiert. Das Zukunftsinstitut rahmt die sicheren Aussichten auf eine überalterte Gesellschaft sehr positiv und versucht sich in Neologismen wie Downaging (Papasabbas 2016), Free-Ager (Muntschick/Hertle 2017, von Boch 2016), Silverpreneure (Steinle/Seitz/Schuldt et al. 2014), Pro-Aging (Dettling 2016) oder Multigraphien (Zukunftsinstitut o.J. h). Auf diese Weise erhält der Kunde des Zukunftsinstituts ein konstruiertes Bild von möglichen Zielgruppen, denen Waren, Dienstleistungen und Daten verkauft werden können. Heute bereits stattfindende Probleme wie Pflegenotstand bleiben weitestgehend ausgeblendet, ein Free-Ager steht rüstig mit Trekking-Bike und Tablet am See.

Tragisch-dystopische Megatrends kennt das Zukunftsinstitut nicht; bzw. nur als Gegenstand einer Kritik. Dies ist dann jedoch keine Kritik des Zustands der Welt, sondern nur eine Kritik der Haltung zu diesem Zustand. Dabei wird nicht Ignoranz oder eine ähnliche Haltung kritisiert, sondern den Menschen die Aufforderung »Think Positive« entgegengebracht. Nun ist es jedoch so, dass kaum ein Megatrend nicht auch Schattenseiten in sich birgt und 
es immer wieder Irrwege und Fehlentwicklungen geben wird. Mit dem Impetus des Zukunftsinstituts bleibt man für negative Zukunftsszenarien blind. Anders agiert die Foresight Company Z_punkt (o.J.), die mit Blick auf den Megatrend gesellschaftliche Disparitäten "politische[...] Radikalisierung«, »soziale[...] Exklusion « und »terroristische[...] Aktivitäten« wahrnimmt, warnend thematisiert und nicht als Chance rahmt. Bei Z_punkt sind tragische Ausgänge möglich. Nach White ist die Tragödie wie folgt definiert:

»In der Tragödie [...] gibt es Zeichen für einen Zustand der Gespaltenheit, der schrecklicher ist, als das, was den Agon zu Beginn des Dramas anstiftete. Doch erscheinen Untergang des Protagonisten und die Erschütterung der von ihm bewohnten Welt am Schluss des tragischen Schauspiels nicht als schlechthin bedrohlich für die, die den Kampf auf Leben und Tod überstehen. [...] Die Versöhnungen am Schluss der Tragödie [...] sind düster und bedeuten eher, dass sich die Menschen in die Bedingungen ihres mühseligen irdischen Daseins schicken müssen.«(White 1991: 23)

Für White ist die Tragödie das Emplotment, dass den Menschen die Möglichkeit der Katharsis und damit der Selbsterkenntnis bietet. Bei Z_punkt klingt dies dann in Bezug auf anthropogene Umweltbelastungen als Megatrend so:

»Eine Trendwende bei den Treibhausgasemissionen ist noch nicht erreicht. Kraftwerke, Industrieanlagen, Verkehrssysteme und Landwirtschaft sind die Hauptemittenten. Im Zuge des anthropogenen Klimawandels steigen die Oberflächen- und Wassertemperaturen, die Polkappen beginnen abzuschmelzen, der Meeresspiegel steigt, extreme Wetterereignisse nehmen zu. Auch die Verschmutzung durch Lärm und Licht nimmt weiter zu, Müllberge wachsen, Böden werden verseucht. Eine Flut von Gesetzen, Regularien und Initiativen versucht gleichzeitig, den Menschen davor zu bewahren, seine eigene Lebensgrundlage zu zerstören.«(Z_punkt o.J.)

Hier bleibt offen, ob der Held >Menschheit< mit seinen Versuchen ein glückliches Ende herbeizuführen vermag. Z_punkt lässt einen tragischen Schluss zu. Die Zukunft bleibt ungewiss. Zu einer Tragödie, die strukturell konservativen Ideologien entspricht, lässt sich allerdings auch Z_punkt nicht hinreißen. Unternehmensberatung kann sich auch nicht darin erschöpfen, den entscheidenden Stellen den Rat: »Lassen Sie alles so wie gehabt« mit auf dem Weg zu geben. Die Romanze zielt darauf ab, die äußeren Umstände zu ändern. Dies ist weder für $Z_{-}$punkt noch für das $\mathrm{ZI}$ eine Option. 
Die äußeren Umstände, in denen sich die Megatrends durch eine Vielzahl konkreter Ereignisse abstrakt zusammenfassen lassen, werden weder von Z_punkt noch vom ZI als Trend angesehen. Wir fügen dies hier dennoch an. Dies ist notwendig, weil beim ZI die Rahmentrends die Spielregeln vorgeben, die jedoch dethematisiert werden. Rahmentrends können in politische, ökonomische und gesellschaftliche Rahmentrends differenziert werden. Ein politischer Rahmentrend in der westlichen Welt sind die unterschiedlichen Ausformungen liberaler Demokratie in den unterschiedlichen Nationalstaaten. Ein ökonomischer Rahmentrend ist die Wirtschaftsform Kapitalismus bzw. die freie/soziale Marktwirtschaft. Gesellschaftliche Rahmentrends sind anerkannte Institutionen wie die Reproduktion in der Kleinfamilie, Konzepte von ausgleichender Gerechtigkeit, positiv konnotierte Werte wie Fleiß und sexuelle Tabus wie Pädophilie. Rahmentrends bleiben bei Z_punkt als auch beim Zukunftsinstitut implizit. Trotzdem schwingen sie bei allen Megatrends mit. Rahmentrends bestimmen den Horizont der Handlungsmöglichkeiten. Sie geben die Spielregeln vor, wie auf Megatrends reagiert werden kann und was sagbar ist und was nicht. Besonders deutlich wird dies, wenn etwas außerhalb des Sagbarkeitsraums geäußert wird, d.h., wenn ein*e Diskursteilnehmer*in gegen die Spielregeln verstoßen möchte. Ein Beispiel aus der jüngsten Zeit war der Kollektivierungsvorschlag des SPDMitglieds und Juso-Vorsitzenden Kevin Kühnert (Bittner/Hildebrandt 2019) als Reaktion auf die negativen Externalitäten des Megatrends Urbanisierung. Der Schutz des Eigentums ist in den Spielregeln des Kapitalismus nahezu unverletzlich, wohingegen der demokratische Megatrend Demokratie in der deutschen Verfasstheit des Grundgesetzes dafür Spielräume lässt ${ }^{18}$. In solchen Widersprüchlichkeiten treten Rahmentrends erst sichtbar hervor. Ansonsten sind Rahmentrends das Grundrauschen langer historischer Linien.

18 Art. 14 (3) GG:»Eine Enteignung ist nur zum Wohle der Allgemeinheit zulässig. Sie darf nur durch Gesetz oder aufgrund eines Cesetzes erfolgen, das Art und Ausmaß der Entschädigung regelt. Die Entschädigung ist unter gerechter Abwägung der Interessen der Allgemeinheit und der Beteiligten zu bestimmen. Wegen der Höhe der Entschädigung steht im Streitfalle der Rechtsweg vor den ordentlichen Gerichten offen.« 


\section{Schlussbemerkungen}

Die Zukunft ist das Noch-Nicht-Angekommene (lat.: advenere), was jedoch auch bedeutet, dass sie kommen wird. Unaufhaltsam. Eine Werbung für ein Bauunternehmen, die 2017 auf einem Betonmischer zu finden war und wahrscheinlich auch noch heute zu finden ist, lautet: »Damit Zukunft möglich wird.« Dafür braucht man jedoch keinen Betonmischer. Wenn etwas mit Sicherheit eintritt, dann ist es die Zukunft. Sie wird immer in der Gegenwart ankommen, egal in welcher Form. Doch das Unwissen über die konkrete Ausgestaltung macht unruhig und unsicher. Die Megatrendforschung möchte diese Unsicherheit verringern. Allerdings bleibt die kommerziell orientierte Forschung stark in neoliberaler Ideologie verfangen. Dies macht sie erstens blind für Entwicklungen, die nicht auf Märkten stattfinden und/oder nicht durch Angebot und Nachfrage erklärt werden können. Dies zeigt sich in der Bewertung, wie in der Orientierungsdimension gezeigt wurde. Außerdem ist die kommerziell orientierte Megatrendforschung allzu überzeugt von ihren identifizierten Megatrends. Möglichkeiten einer Reversibilität werden nicht oder nur kaum in Betracht gezogen. Dies verwundert arg, da doch z.B. in der Stadtforschung lange Zeit das Thema der Entvölkerung den Diskurs bestimmt hatte. Es ging um Städte wie »Wittenberge« (Bude/Medicus/Willisch 2011) und die dortige Aussichtslosigkeit wegen des Fortzugs. Gerade bei der Urbanisierung hat man auch immer das Negativbild des Megatrends: Wenn irgendwo Zuzug vorherrscht, dann muss an einem anderen Ende ein Problem mit Fortzug existieren. Dies bleibt bei den Publikationen des ZIs unterbelichtet. Eine Angabe über die Festigkeit eines Megatrends bleibt aus, auch in relativer Sichtweise. So steht Globalisierung in gleicher Größe zum Megatrend New Work. Schlussendlich haben nahezu alle Zukunftserzählungen zumindest des ZIs von Matthias Horx einen komödiantischen Plot, was sie ideologisch verdächtig macht. Die Vorstellung, dass sich alle Großentwicklungen, die heute schon ablesbar sind, in Harmonie auflösen, kann bestenfalls als naiver Optimismus gewertet werden, schlechtestenfalls bereitet es die Unternehmen, die das ZI berät, nicht vollständig auf mögliche zukünftige Entwicklungen vor. Unternehmen, Stiftungen, Parteien, Glaubensgemeinschaften und andere Organisationen wären gut beraten, verließen sie sich auf akademische Zukunftsforschung. Dafür sprechen drei Gründe. Erstens überprüft sich die scientific community durch Review-Verfahren und durch die Methode der Falsifikation wechselseitig. Zweitens wird in wissenschaftlichen Verfahren die Methodik offengelegt und es kann nachvollzogen werden, wie man zu einem 
Schluss kam. Dabei gilt eine willkürlich getroffene Sammlung von Anekdoten nicht als Methodik. Drittens sind Kräfte aus der Wissenschaft nicht in gleichem Maße auf öffentliche Aufmerksamkeit angewiesen. So klingen die Ergebnisse vielleicht nicht so spektakulär und es finden sich weit weniger Neologismen wie z.B. »Silverpreneure«, aber dafür stehen diese auf festem Boden wissenschaftlicher Methoden und verzichten auf Glamour. Auch die Wissenschaft liegt mit ihren Prognosen nicht immer richtig. Jedoch eröffnet die Wissenschaft den Zugang zu unterschiedlichen Szenarien und scheut auch nicht ohne ideologische Scheuklappen, negative Entwicklungen zu benennen, ohne dabei über Angst Handlungsdruck zu erzeugen. Setzt man sich Nachhaltigkeit, sprich den Erhalt eines lebenswerten Planeten für zukünftige Generation, als Handlungsziel, ist man mit dem ZI schlecht beraten: Nachhaltigkeit wird Mittel zum Zweck der Kapitalmehrung. Der Klimawandel wird - wenn nicht geleugnet (Horx 2007) - dann doch ignoriert. Möglichkeiten zu einer Trendumkehr werden banalhegelianisch abgeschmettert, ökologische Innovationen werden nur dann positiv bewertet, wenn sie Geld einspielen, Privatreisen werden positiv beurteilt und Exnovationen finden keine Betrachtung. Mit solch einer Form der Beratung kann sicherlich durch die komödiantische Formgebung eine gute Mitarbeitermotivation gelingen, durch witzige Anekdoten für Unterhaltung gesorgt werden, aber eine Hilfe oder gar ein Treiber einer nachhaltigeren Gesellschaft liegt im Falle der ZI GmbH nicht vor.

\section{Literatur}

Adler, Frank/Schachtschneider, Ulrich (2017): Wege zur wachstumsunabhängigen Gesellschaft, München: oekom.

Adloff, Frank (2015): »Immer im Takt bleiben. Zu einer konvivialistischen Affektpolitik«, in: Frank Adloff/Volker M. Heins (Hg.), Konvivialismus. Eine Debatte, Bielefeld: transcript, S. 71-84. https://doi.org/10.14361/ 9783839431849

Albert, Mathias/Hurrelmann, Klaus/Quenzel, Gudrun (2015): 17. Shell Jugendstudie. Jugend 2015, Frankfurt a.M.: Fischer.

AlDailami, Said/Zapf, Holger (2018): »Narrative von der Rückkehr des Islam in der Öffentlichkeit. Der postrevolutionäre Diskurs in Tunesien«, in: Holger Zapf/Oliver Hidalgo/Philipp W. Hildmann (Hg.), Das Narrativ von der Wiederkehr der Religion, Wiesbaden: Springer VS, S. 315-342. https://doi. org/10.1007/978-3-658-18451-3_13 
Altenbockum, Jasper von (2012): »Marktkonforme Demokratie? Oder demokratiekonformer Markt? «, in: Frankfurter Allgemeine Zeitung vom 15.04.2012. Online verfügbar unter: https://www.faz.net/aktuell/politik/ harte-bretter/marktkonforme-demokratie-oder-demokratiekonformermarkt-11712359.html. Zuletzt aufgerufen am 09.12.2019.

Amjahid, Mohamed (2019): »Gebär-Mütter", in: Zeit Online vom 13.02.2019. Online verfügbar unter: https://www.zeit.de/2019/08/ungarnmutterschaft-subvention-viktor-orban-emanzipation-westen. Zuletzt aufgerufen am 10.12.2019.

Beckert, Jens (2018): Imaginierte Zukunft: Fiktionale Erwartungen und die Dynamik des Kapitalismus, Berlin: Suhrkamp.

Behrens, Roger (2003): »Pop Mythos Pop. Zur Konstruktion von Subversiven«, in: Roger Behrens, Die Diktatur der Angepassten. Texte zur kritischen Theorie der Popkultur, Bielefeld: transcript, S. 101-131. https://doi.org/10. 14361/9783839401156

Berner, Frank/Vogel, Claudia/Wettstein, Markus/Tesch-Römer, Clemens (2019): »Frauen und Männer: Eine Gesamtbetrachtung der empirischen Befunde«, in: Claudia Vogel/Markus Wettstein/Clemens Tesch-Römer (Hg.), Frauen und Männer in der zweiten Lebenshälfte. Älter werden im sozialen Wandel, Wiesbaden: Springer VS, S. 133-143. https://doi.org/10. 1007/978-3-658-25079-9_8

Best, Benjamin (2019): Energiewende und Bürgerbeteiligung. Multi-LevelKonstellationsanalysen der InnovationCity Ruhr - Modellstadt Bottrop, Wiesbaden: Springer VS. https://doi.org/10.1007/978-3-658-26184-9

Bittner, Jochen/Hildebrandt, Tina (2019): »Was heißt Sozialismus für Sie, Kevin Kühnert?«, in Zeit Online vom 01.05.2019. Online verfügbar unter: https://www.zeit.de/politik/deutschland/2019-05/kevin-kuehnertspd-jugendorganisation-sozialismus. Zuletzt aufgerufen am 11.02.2020.

Boch, Rosalie von (2016): Carpe diem: Die Achtsamkeit des Alters. Online verfügbar unter: https://www.zukunftsinstitut.de/artikel/carpe-diem-dieachtsamkeit-des-alters/. Zuletzt aufgerufen am 10.12.2019.

Bofinger, Peter/Habermas, Jürgen/Nida-Rümelin, Julian (2012):»Kurswechsel für Europa. Einspruch gegen die Fassadendemokratie«, in: Frankfurter Allgemeine Zeitung vom 03.08.2012. Online verfügbar unter: https:// www.faz.net/aktuell/feuilleton/debatten/europas-zukunft/kurswechselfuer-europa-einspruch-gegen-die-fassadendemokratie-11842820-p4. html. Zuletzt aufgerufen am 09.12.2019. 
Boße, André (2019): "Bauvisionen für Mensch und Klima«, in: karriereführer bauingenieure 2019.2020 27, S. 13-14. Online verfügbar unter: https:// www.karrierefuehrer.de/magazin/bauingenieure-19-20.pdf. Zuletzt aufgerufen am 10.12.2019.

Bude, Heinz (2014): Gesellschaft der Angst, Hamburg: Hamburger Edition.

Bude, Heinz/Medicus, Thomas/Willisch, Andreas (Hg.) (2011): ÜberLeben im Umbruch. Am Beispiel Wittenberge: Ansichten einer fragmentierten Gesellschaft, Hamburg: Hamburger Edition.

Burel, Simone (2017): Vorständin und Allround-Papa: Geschlechterstereotype in der Online-Kommunikation in beruflichem Kontext «, in: 1oplusı: Living Linguistics 3, S. 121-139.

Coupland, Douglas (2004): Generation X. Geschichten für eine immer schneller werdende Kultur, München: Goldmann.

Crouch, Colin (2004): Post-Democracy, Cambridge: Polity Press.

Dettling, Daniel (2016): Pro-Aging: Altersreichtum statt Altersarmut. Online verfügbar unter: https://www.zukunftsinstitut.de/artikel/pro-agingaltersreichtum-statt-altersarmut/. Zuletzt aufgerufen am 10.12.2019.

Dörre, Klaus (2019): »Demokratie statt Kapitalismus oder: Enteignet Zuckerberg! «, in: Hanna Ketterer/Karina Becker (Hg.), Was stimmt nicht mit der Demokratie? Eine Debatte mit Klaus Dörre, Nancy Fraser, Stephan Lessenich und Hartmut Rosa, Berlin: Suhrkamp, S. 21-51.

Dziemba, Oliver/Wenzel, Eike (2014): \#Wir. Wie Digitalisierung unseren Alltag verändert, München: Redline.

Eagelton, Terry (1997): Einführung in die Literaturtheorie, 4. Auflage, Stuttgart: Metzler. https://doi.org/10.1007/978-3-476-04139-5

Eurostat (2020): Beschäftigung nach Geschlecht, Alter, Stellung im Beruf und Beruf, Eurostat Datenbank, Luxemburg.

Faust, Friederike/Assmann, Corinna (2014): »Queering Football - Körperpraktiken im Frauenfußball zwischen Normierung und Destabilisierung der Geschlechterordnung«, in: Body Politics 2 (3), S. 145-177.

Fiedler, Sabine (2009): »Lingua-Franca-Kommunikation. Eine vergleichende Studie Englisch - Esperanto«, in: Detlev Blanke/Jürgen Scharnhorst (Hg.), Sprachenpolitik und Sprachkultur (= Sprache, System und Tätigkeit, Band 572), 2. Auflage, Frankfurt a.M.: Peter Lang.

Fox, Claire (2017): >I Find That Offensive! ‘, London: Biteback Publishing.

Fuchs, Christian (2019): Soziale Medien und Kritische Theorie. Eine Einführung, München: UVK/utb. 
Gatterer, Harry/Schuldt, Christian/Varga, Christiane (2014): Die Zukunft der Gemeinnützigkeit, Wien: Zukunftsinstitut. Online verfügbar unter: https://www.zukunftsinstitut.de/fileadmin/user_upload/Publikationen/ Auftragsstudien/Studie_Gemeinnuetzigkeit_Internet_2.pdf. Zuletzt aufgerufen am 10.12.2019.

Giesel, Katharina D. (2007): Leitbilder in den Sozialwissenschaften. Begriffe, Theorien und Forschungskonzepte, Wiesbaden: Springer VS.

Gigerenzer, Gerd (2013): Risiko, München: C. Bertelsmann.

Glockner, Holger (o.J.): Wie Audi in die zukünftigen Lebenswelten seiner Kunden eintaucht. Online verfügbar unter: https:/www.z-punkt.de/de/ themen/artikel/wie-audi-in-die-zukuenftigen-lebenswelten-seinerkunden-eintaucht1/535. Zuletzt aufgerufen am 22.01.2020.

Glogowski, Stella (2019): »Food Report 2020: Minimahlzeiten, Urban Food und Beyond Plastic«, in: ERNÄHRUNGS UMSCHAU vom 11.06.2019. Online verfügbar unter: https://www.ernaehrungs-umschau.de/news/11-062019-minimahlzeiten-urban-food-und-beyond-plastic/. Zuletzt aufgerufen am 10.12.2019.

Gorges, Michael (2019): Kleines Iran-Lexikon. Hintergrundwissen für das erfolgreiche Iran-Geschäft, Wiesbaden: Springer Gabler. https://doi.org/10. 1007/978-3-658-23698-4

Greenpeace (2019): »Zukunftsforscher Horx: Welt wird »mit Milde« auf Trump zurückblicken«, in: Greenpeace-Magazin vom 12.09.2019. Online verfügbar unter: https://www.greenpeace-magazin.de/ticker/zukunftsforscherhorx-welt-wird-mit-milde-auf-trump-zurueckblicken. Zuletzt aufgerufen am 15.10.2019.

Habermas, Jürgen (1985): „Die neue Unübersichtlichkeit. Die Krise des Wohlfahrtsstaates und die Erschöpfung utopischer Energien«, in: Merkur 39, S. 1-14.

Habermas, Jürgen (1995): Theorie des kommunikativen Handelns, Band 1, Handlungsrationalität und gesellschaftliche Rationalisierung, Frankfurt a.M.: Suhrkamp.

Hadjar, Andreas/Becker, Rolf (2009): »Erwartete und unerwartete Folgen der Bildungsexpansion in Deutschland«, in: Rolf Becker (Hg.), Lehrbuch der Bildungssoziologie, Wiesbaden: Springer VS. https://doi.org/10.1007/9783-531-91711-5_7

Henkel, Simon (2015): Authentisch und ästhetisch: Nachhaltigkeit 2.0. Online verfügbar unter: https://www.zukunftsinstitut.de/artikel/nachhaltigkeit20/. Zuletzt aufgerufen am 10.12.2019. 
Hentges, Gudrun/Nottbohm, Kristina (2017): »Die Verbindung von Antifeminismus und Europakritik. Positionen der Parteien "Alternative für Deutschland « und »Front National«, in: Gudrun Hentges/Kristina Nottbohm/Hans-Wolfgang Platzer (Hg.), Europäische Identität in der Krise. Europäische Identitätsforschung und Rechtspopulismusforschung im Dialog, Wiesbaden: Springer VS, S. 167-208. https://doi.org/10.1007/9783-658-14951-2_8

Heuer, Hans (1977): »Ist die Stadtflucht zu bremsen?«, in: Wirtschaftsdienst Zeitschrift für Wirtschaftspolitik 57, S. 205-210.

Hinterkörner, Ines (2019): "Schöne neue Beautywelt«, in: Salzburger Nachrichten vom 19.11.2019. Online verfügbar unter: https://www.sn.at/ leben/lifestyle/schoene-neue-beautywelt-79446592. Zuletzt aufgerufen am 10.12.2019.

Horx, Matthias (2001): Smart Capitalism: Das Ende der Ausbeutung, Frankfurt a.M.: Eichborn.

Horx, Matthias (2007): »Warum ich nicht an die Klimakatastrophe glaube«, in: Welt vom 13.03.2007. Online verfügbar unter: https://www.welt.de/ politik/article759237/Warum-ich-nicht-an-die-Klimakatastrophe-glaube. html. Zuletzt aufgerufen am 09.12.2019.

Horx, Matthias (2011): Das Megatrend-Prinzip. Wie die Welt von morgen entsteht, Stuttgart: Deutsche Verlags-Anstalt.

Horx, Matthias (2015): Metatrends: Wie Komplexität entsteht. Online verfügbar unter: https://www.zukunftsinstitut.de/artikel/future-forecast/

metatrends-wie-komplexitaet-entsteht/. Zuletzt aufgerufen am 12.12.2019.

Horx, Matthias (2017): Future Love: Die Zukunft von Liebe, Sex und Familie, München: Deutsche Verlags-Anstalt.

Horx, Matthias (2019): Countdown zum neuen Space Age. Online verfügbar unter: https://www.zukunftsinstitut.de/artikel/zukunftsreport/ countdown-zum-neuen-space-age/. Zuletzt aufgerufen am 10.12.2019.

Huber, Thomas/Rauch, Christian (2013): Generation Y. Das Selbstverständnis der Manager von morgen, Düsseldorf: Signium International (Hg.). Online verfügbar unter: https:/www.zukunftsinstitut.de/fileadmin/user_ upload/Publikationen/Auftragsstudien/studie_generation_y_signium. pdf. Zuletzt aufgerufen am 15.10.2019.

Intergovernmental Panel on Climate Change (IPCC) (2014): Klimaänderung 2014: Synthesebericht. Beitrag der Arbeitsgruppen I, II und III zum Fünften Sachstandsbericht des Zwischenstaatlichen Ausschusses für Klimaän- 
derungen, Genf. Online verfügbar unter: https://www.de-ipcc.de/128.php. Zuletzt aufgerufen am 10.12.2019.

Jäger, Siegfried (2015): Kritische Diskursanalyse. Eine Einführung, 7. Auflage, Münster: Unrast.

Jischa, Michael F. (2009): »Gedanken zur Wahrnehmung von Zukunft«, in: Reinhold Popp/Elmar Schüll (Hg.), Zukunftsforschung und Zukunftsgestaltung. Beiträge aus Wissenschaft und Praxis (= Wissenschaftliche Schriftenreihe "Zukunft und Forschung« des Zentrums für Zukunftsstudien Salzburg, Band 1), Berlin/Heidelberg: Springer, S. 37-50. https://doi. org/10.1007/978-3-540-78564-4_4

Jörke, Dirk (2019): Die Größe der Demokratie. Über die räumliche Dimension von Herrschaft und Partizipation, Berlin: Suhrkamp.

Kafka, Franz (2008): Sämtliche Werke, Frankfurt a.M.: Suhrkamp.

Kampker, Achim/Deutskens, Christoph/Marks, Alexander (2015): »Die Rolle von lernenden Fabriken für Industrie 4.0«, in: Alfons Botthoff/Ernst Andreas Hartmann (Hg.), Zukunft der Arbeit für Industrie 4.0, Berlin/Heidelberg: Springer Vieweg, S. 77-85. https://doi.org/10.1007/978-3662-45915-7_9

Kinderzimmer Productions (1998): Back - Ich bin nur ein Remix!!!, in: Die Erste (Album).

Klamert, Lukas (2019): »Plug-in-Hybrid. Der Feuerwehr-Truck der Zukunft«, in: traktuell vom 11.12.2019. Online verfügbar unter: https://traktuell.at/a/ der-feuerwehr-truck-der-zukunft. Zuletzt aufgerufen am 10.12.2019.

Knauf, Marcus (2012): $\gg$ Trends und Perspektiven in der Forst- und Holzwirtschaft«, in: Holger Militz (Hg.), Deutsche Holzschutztagung. Trends und Chancen, Göttingen: Cuvillier.

Krause, Halima (2018): »Islam und Geschlechtergerechtigkeit«, in: Zekirija Sejdini (Hg.), Islam in Europa. Begegnungen, Konflikte und Lösungen, Münster: Waxmann.

Krauss, Werner (2011): »Bruno Latour: Making things public«, in: Stephan Moebius/Dirk Quadflieg (Hg.), Kultur. Theorien der Gegenwart, 2. Auflage, Wiesbaden: Springer VS, S. 595-612. https://doi.org/10.1007/978-3-53192056-6_48

Kropp, Cordula (2015): »Exnovation - Nachhaltige Innovation als Prozesse der Abschaffung ", in: Annika Arnold/Martin David/Gerolf Hanke/Marco Sonnberger (Hg.), Innovation - Exnovation. Über Prozesse des Abschaffens und Erneuerns in der Nachhaltigkeitstransformation, Marburg: Me- 
tropolis, S. 13-34. Online verfügbar unter: https://www.researchgate.net/ publication/283725652Z. Zuletzt aufgerufen am 15.01.2020.

Latouche, Serge (2015): Es reicht! Abrechnung mit dem Wachstumswahn, München: oekom.

Lessenich, Stephan (2019): Grenzen der Demokratie. Teilhabe als Verteilungsproblem, Stuttgart: Reclam.

Li, Linda (2016): Der Sinn des Unternehmens. Online verfügbar unter: https:// www.zukunftsinstitut.de/artikel/rezensionen/sinn-des-unternehmenveken/. Zuletzt aufgerufen am 10.12.2019.

Link, Jürgen (1986): »Noch einmal: Diskurs. Interdiskurs. Macht«, in: kultuRRevolution 11, S. 4-7. https://doi.org/10.13109/9783666370724

Link, Jürgen (2018): Normalismus und Antagonismus in der Postmoderne. Krise, New Normal, Populismus, Göttingen: Vandenhoeck \& Ruprecht.

Luhmann, Niklas (2009): Soziologische Aufklärung 3. Soziale Systeme, Gesellschaft, Organisation, 5. Auflage, Wiesbaden: VS.

Lyotard, Jean-François (1999): Das postmoderne Wissen. Ein Bericht, Wien: Passagen.

Mardani, Abbas/Streimikiene, Dalia/Cavallaro, Fausto/Loganathan, Nanthakumar/Khoshnoudi, Masoumeh (2019): "Carbon dioxide $\left(\mathrm{CO}_{2}\right)$ emissions and economic growth: A systematic review of two decades of research from 1995 to 2017 «, in: Science of the Total Environment 649 (1), S. 31-49. https://doi.org/10.1016/j.scitotenv.2018.08.229

Mauss, Marcel (1999): Die Gabe. Form und Funktion des Austausches in archaischen Gesellschaften, Frankfurt a.M.: Suhrkamp.

Meyn, Jörn (2019): »Erste Schiedsrichterin in der Ligue 1. Frankreichs Bibiana Steinhaus", in: Spiegel Online vom 23.04.2019. Online verfügbar unter: https://www.spiegel.de/sport/fussball/stephanie-frappartwird-erste-schiedsrichterin-in-der-ligue-1-a-1264102.html. Zuletzt aufgerufen am 10.12.2019.

Miegel, Meinhardt (2010): Exit. Wohlstand ohne Wachstum, Berlin: Propyläen.

Milbert, Antonia (2017): Wie viel (Re-)Urbanisierung durchzieht das Land?, BBSR-Analysen KOMPAKT 07/2017, Bonn. Online verfügbar unter: https://www.bbsr.bund.de/BBSR/DE/Veroeffentlichungen/Analysen Kompakt/2017/ak-07-2017-dl.pdf?_blob=publicationFile\&v=2. Zuletzt aufgerufen am 15.01.2020.

Muntschick, Verena/Hertle, Diana (2017): Free-Aging: Jenseits des Rentendaseins. Online verfügbar unter: https://www.zukunftsinstitut.de/ 
artikel/lebensstile/free-aging-jenseits-des-rentendaseins/. Zuletzt aufgerufen am 10.12.2019.

Naisbitt, John (1984): Megatrends. 10 Perspektiven, die unser Leben verändern werden, Bayreuth: Hestia.

Neuhaus, Christian/Steinmüller, Karlheinz (2015): »Grundlagen der Standards Gruppe 1", in: Lars Gerhold/Dirk Holtmannspötter/Christian Neuhaus/Elmar Schüll/Beate Schulz-Montag/Karlheinz Steinmüller/Axel Zweck (Hg.), Standards und Gütekriterien der Zukunftsforschung. Ein Handbuch für Wissenschaft und Praxis, Wiesbaden: Springer VS, S. 1781. https://doi.org/10.1007/978-3-658-07363-3_2

Opaschowski, Horst W. (2015): »Mode, Hype, Megatrend. Vom Nutzen wissenschaftlicher Zukunftsforschung «, in: Aus Politik und Zeitgeschichte 65, 31-32, S. 40-45.

Paech, Niko (2012): Befreiung vom Überfluss: Auf dem Weg in die Postwachstumsökonomie, Wiesbaden: Springer VS.

Papasabbas, Lena (2016): Downaging: Die Erneuerung des Alters. Online verfügbar unter: https://www.zukunftsinstitut.de/artikel/downaging-dieerneuerung-des-alters/. Zuletzt aufgerufen am 10.12.2019.

Papasabbas, Lena (2018): Rückschläge für den Megatrend Gender Shift. Online verfügbar unter: https://www.zukunftsinstitut.de/artikel/rueckschlaegefuer-den-megatrend-gender-shift/. Zuletzt aufgerufen am 10.12.2019.

Papasabbas, Linda/Horx, Tristan (2018): »Generation Global: Wie sie lebt und wie sie tickt«, in: YouTube vom 08.01.2018. Online verfügbar unter: https://www.youtube.com/watch?v=Nmi5s_oMpog. Zuletzt aufgerufen am 15.10.2019.

Passoth, Jan-Hendrik (2010): »Diskurse, Eisbären, Eisberge: MaterialSemiotische Verwicklungen und der Klimawandel«, in: Martin Voss (Hg.), Der Klimawandel. Sozialwissenschaftliche Perspektiven, Wiesbaden: Springer VS, S. 49-60. https://doi.org/10.1007/978-3-531-92258-4_3

Peters, Glen P./Marland, Gregg/Le Quéré, Corinne/Boden, Thomas/Canadell, Josep G./Raupach, Michael R. (2012): »Rapid growth in $\mathrm{CO}_{2}$ emissions after the 2008-2009 global financial crisis«, in: Nature Climate Change 2, S. 2-4. https://doi.org/10.1038/nclimate1332

Piepenbrink, Johannes (2015): „Editorial«, in: Aus Politik und Zeitgeschichte 65, 31-32, S. 2.

Pille, Jens-Ulrich (2016): Meinungsmacht sozialer Netzwerke. Hamburger Schriften zum Medien-, Urheber- und Telekommunikationsrecht, BadenBaden: Nomos. https://doi.org/10.5771/9783845272559 
Popper, Karl R. (1989): »Zwei Bedeutungen von Falsifizierbarkeit«, in: Helmut Seiffert/Gerard Radnitzky (Hg.), Handlexikon zur Wissenschaftstheorie, München: Ehrenwirth, S. 82-86.

Popper, Karl R. (2005): Logik der Forschung, 11. Auflage, Tübingen: Mohr Siebeck.

Reckwitz, Andreas (2019): Das Ende der Illusionen. Politik, Ökonomie und Kultur in der Spätmoderne, Berlin: Suhrkamp.

Richtlinie (EU) 2019/904 des Europäischen Parlaments und des Rates vom 5. Juni 2019 über die Verringerung der Auswirkungen bestimmter Kunststoffprodukte auf die Umwelt. Online verfügbar unter: https://eur-lex. europa.eu/legal-content/DE/TXT/PDF/?uri=CELEX:32019L0904\& from=DE. Zuletzt aufgerufen am 10.12.2019.

Rogers, Everett M. (1983): Diffusion of innovations, 3. Auflage, New York: Free Press.

Russell, Bertrand (1950): Probleme der Philosophie, Wien: Humboldt.

Rust, Holger (2008): Zukunftsillusionen. Kritik der Trendforschung, Wiesbaden: VS. https://doi.org/10.1007/978-3-531-91778-8

Sauerbier, Ilona (2019): »Was bringen die 2020er?«, in: SchuhMarkt vom 03.12.2019. Online verfügbar unter: https://www.schuhmarkt-news.de/ trends/nachhaltigkeit/03-12-2019-was-bringen-die-2020er/. Zuletzt aufgerufen am 10.12.2019.

Schauerte, Eva/Vehlken, Sebastian (2018): »Faktizitäten. Einleitung in den Schwerpunkt«, in: Zeitschrift für Medienwissenschaft 10, 19, S. 10-20. https://doi.org/10.14361/zfmw-2018-100204

Schneidewind, Uwe/Palzkill-Vorbeck, Alexandra (2011): »Suffizienz als Business Case. Nachhaltiges Ressourcenmanagement als Gegenstand einer transdisziplinären Betriebswirtschaftslehre«, in: Wuppertal Institut für Klima, Umwelt, Energie gGmbH (Hg.), Impulse zur WachstumsWende, Wuppertal. Online verfügbar unter: https://www.econstor.eu/obitstream/ 10419/59296/1/716107074.pdf. Zuletzt aufgerufen am 12.12.2019.

Schröder, Enno/Storm, Servaas (2018): Economic Growth and Carbon Emissions: The Road to >Hothouse Earth is Paved with Good Intentions, Delft. Online verfügbar unter: https://www.boeckler.de/pdf/v_2018_10_ 27_schroeder.pdf. Zuletzt aufgerufen am 12.12.2019.

Schröder, Martin (2018): »Der Generationenmythos «, in: Kölner Zeitschrift für Soziologie und Sozialpsychologie 70, S. 469-494. https://doi.org/10.1007/ s11577-018-0570-6 
Schultz, Tanjev/Hurrelmann, Klaus (2013): Die Akademiker-Gesellschaft. Müssen in Zukunft alle studieren?, Weinheim: Beltz Juventa.

Schweiger, Stefan/Trost, Esther/Engler, Steven (2020): »Neue Perspektiven auf die Energiewende. Sprachbilder und Bildsprache«, in: Steven Engler/Jan-Hendrik Kamlage (Hg.), Dezentral, partizipativ und kommunikativ - Energiewende als Teil der Großen Transformation, Nordhausen: Traugott Bautz.

Seefried, Elke (2015): Zukünfte. Aufstieg und Krise der Zukunftsforschung 1945-1980 (= Quellen und Darstellungen zur Zeitgeschichte, Band 106), Berlin: De Gruyter Oldenbourg. https://doi.org/10.1515/9783110349122

Seidl, Irmi/Zahrnt, Angelika (Hg.) (2010): Postwachstumsgesellschaft. Konzepte für die Zukunft, Marburg: Metropolis. https://doi.org/10.14512/oew. v25i3.1066

Seitz, Janine (2015): Sicherheit - ein Megatrend. Online verfügbar unter: https://www.zukunftsinstitut.de/artikel/sicherheit-ein-megatrend/. Zuletzt aufgerufen am 10.12.2019).

Séville, Astrid (2017): »There is no alternative«. Politik zwischen Demokratie und Sachzwang, Frankfurt a.M.: campus.

Silver, Laura (2018): »Smartphone Ownership Is Growing Rapidly Around the World, but Not Always Equally«, in: Pew Research Center vom 05.02.2019. Online verfügbar unter: https://www.pewresearch.org/global/2019/02/05/ smartphone-ownership-is-growing-rapidly-around-the-world-butnot-always-equally/. Zuletzt aufgerufen am 15.10.2019.

Simon, Walter (2011): GABALs großer Methodenkoffer Zukunft. Grundlagen und Trends, Offenbach a.M.: GABAL.

Somer, Murat (2015): »Wither with secularism or just undemocratic Laiklik? The evolution and future of secularism under the AKP«, in: Valeria Talbot (Hg.), The uncertain path of the New Turkey, Milan: Instituto per gli Studi di Politica Internazionale, S. 23-48.

Statista (2019): Frauenanteil in verschiedenen Berufsgruppen in Deutschland am 30. Juni 2018. Online verfügbar unter: https://de.statista.com/ statistik/daten/studie/167555/umfrage/frauenanteil-in-verschiedenenberufsgruppen-in-deutschland/. Zuletzt aufgerufen am 10.12.2019.

Statistik der Bundesagentur für Arbeit (2019): Berichte: Blickpunkt Arbeitsmarkt - MINT-Berufe, Nürnberg. Online verfügbar unter: https:// statistik.arbeitsagentur.de/Statischer-Content/Arbeitsmarktberichte/ Berufe/generische-Publikationen/Broschuere-MINT.pdf. Zuletzt aufgerufen am 10.12.2019. 
Statistisches Bundesamt (2011): Anteil von Frauen an Führungskräften in Deutschland 1996 und 2010, Pressemitteilung Nr.215 vom 08.06.2011, zitiert nach de.statista.com. Online verfügbar unter: https://de. statista.com/statistik/daten/studie/190521/umfrage/frauenanteilfuehrungskraefte-in-deutschland/. Zuletzt aufgerufen am 13.01.2020.

Steinle, Andreas/Seitz, Janine/Schuldt, Christian/Rauch, Christian/Huber, Thomas (2014): Silverpreneure: Vom Beruf zur Berufung. Online verfügbar unter: https://www.zukunftsinstitut.de/artikel/silverpreneure-vomberuf-zur-berufung/. Zuletzt aufgerufen am 10.12.2019.

Stengel, Oliver (2011): Suffizienz. Die Konsumgesellschaft in der ökologischen Krise, München: oekom.

Stengel, Oliver (2016): Jenseits der Marktwirtschaft. Ökonomie im 21. Jahrhundert, Wiesbaden: Springer VS. https://doi.org/10.1007/978-3-65811759-7

The Shift Project (2019): Climate crisis: The unsustainable use of online video. The practical case study of online video. Online verfügbar unter: https://theshiftproject.org/wp-content/uploads/2019/07/ExcutiveSummary_EN_The-unsustainable-use-of-online-video.pdf. Zuletzt aufgerufen am 13.01.2020.

Thomas, William I. (1928): The Child in America: Behavior Problems and Programs, New York: Alfred A. Knopf, S. 553-576.

Usleber, Verena (2019): »Snackification. Wie der Megatrend Minimahlzeit die Gastronomie verändert«, in: tophotel.de vom 07.10.2019. Online verfügbar unter: https://www.tophotel.de/snackificationwie-der-megatrendminimahlzeit-die-gastronomie-veraendert-44901/. Zuletzt aufgerufen am 10.12.2019.

Voigt, Rüdiger (2015): Der moderne Staat. Zur Genese des heutigen Staatsverständnisses, Wiesbaden: Springer VS.

Wagner, Leonie (2017): »Erfolgsgeschichte oder »Sündenfall«?«, in: Sozial Extra, Praxis aktuell Sabine Hering - Soziale Arbeit, Frauenbewegung, Internationales 41, S. 22-25. https://doi.org/10.1007/s12054-017-0036-5

Welsch, Wolfgang (1997): Unsere postmoderne Moderne, 5. Auflage, Berlin: Akademie. https://doi.org/10.1515/9783050074511

Welzer, Harald/Wiegandt, Klaus (Hg.) (2014): Wege aus der Wachstumsgesellschaft, Frankfurt a.M.: S. Fischer.

White, Hayden (1991): Metahistory. Die historische Einbildungskraft im 19. Jahrhundert in Europa, Frankfurt a.M.: S. Fischer. 
Wieneke, Patrick (2015): Der Shitstorm als Chance. Online verfügbar unter: https://www.zukunftsinstitut.de/artikel/der-shitstorm-als-chance/. Zuletzt aufgerufen am 10.12.2019.

Wiesner, Maria (2016): »Trumps sexuelle Übergriffe. »Wenn Du ein Star bist, lassen sie es $\mathrm{zu}$ «, in: FAZ vom 08.10.2016. Online verfügbar unter: https:/www.faz.net/aktuell/politik/trumps-praesidentschaft/trumpssexuelle-uebergriffe-wenn-du-ein-star-bist-lassen-sie-es-zu-14471698. html. Zuletzt aufgerufen am 10.12.2019.

WomanStats Project (2015): WomanStats Maps. Female Genital Cutting. Online verfügbar unter: www.womanstats.org/maps.html. Zuletzt aufgerufen am 10.12.2019.

World Meteorological Organization (2019): The State of Greenhouse Gases in the Atmosphere Based on Global Observations through 2018, WMO Greenhouse Gas Bulletin No. 15. Online verfügbar unter: https:// library.wmo.int/doc_num.php?explnum_id=10100. Zuletzt aufgerufen am 10.12.2019.

Z_punkt (o.J.): Der neue Blick auf die globale Dynamik des Wandels. Online verfügbar unter: https:/www.z-punkt.de/de/themen/artikel/ megatrends. Zuletzt aufgerufen am 10.12.2019.

Zukunftsinstitut (2018a): Die Megatrend-Map. Online verfügbar unter: https://www.zukunftsinstitut.de/index.php?id=1532. Zuletzt aufgerufen am 15.10.2019.

Zukunftsinstitut (2018b): »Impressionen//Future Day 18«, in: YouTube vom 30.10.2018. Online verfügbar unter: https://www.youtube.com/ watch?time_continue $=182 \& v=$ mbZRw6Y7AQA. Zuletzt aufgerufen am 10.12.2019.

Zukunftsinstitut (o.J. a): Megatrends. Online verfügbar unter: https:// www.zukunftsinstitut.de/dossier/megatrends/. Zuletzt aufgerufen am 10.12.2019.

Zukunftsinstitut (o.J. b): Gesundheit Glossar. Online verfügbar unter: https:// www.zukunftsinstitut.de/artikel/mtglossar/gesundheit-glossar/. Zuletzt aufgerufen am 10.12.2019.

Zukunftsinstitut (o.J. c): Sicherheit Glossar. Online verfügbar unter: https:// www.zukunftsinstitut.de/artikel/mtglossar/sicherheit-glossar/. Zuletzt aufgerufen am 10.12.2019.

Zukunftsinstitut (o.J. d): Neo-Ökologie Glossar. Online verfügbar unter: https:/www.zukunftsinstitut.de/artikel/mtglossar/neo-oekologieglossar/. Zuletzt aufgerufen am 10.12.2019. 
Zukunftsinstitut (o.J. e): Gender Shift Glossar. Online verfügbar unter: https:// www.zukunftsinstitut.de/artikel/mtglossar/gender-shift-glossar/. Zuletzt aufgerufen am 10.12.2019.

Zukunftsinstitut (o.J. f): New Work Glossar. Online verfügbar unter: https:// www.zukunftsinstitut.de/artikel/mtglossar/new-work-glossar/. Zuletzt aufgerufen am 10.12.2019.

Zukunftsinstitut (o.J. g): Die Pioniere der neuen Arbeitswelt. Online verfügbar unter: https://www.zukunftsinstitut.de/artikel/lebensstile/diepioniere-der-neuen-arbeitswelt/. Zuletzt aufgerufen am 10.12.2019.

Zukunftsinstitut (o.J. h): Individualisierung Glossar. Online verfügbar unter: https://www.zukunftsinstitut.de/artikel/mtglossar/individualisierungglossar/. Zuletzt aufgerufen am 22.01.2020. 
Megatrends im Kontext von Energiewende und Nachhaltigkeit 



\section{Was nützt die Wende in Gedanken? Überlegungen zur Steuerbarkeit von Transformationen am Beispiel der deutschen Energiewende}

Jörg Radtke und Emily Drewing

\section{Einleitung}

Im eigentlichen Wortsinn verweist die Energiewende zunächst auf eine Abkehr von angestammten Pfaden der Energieversorgung. Im alltäglichen Sprachgebrauch ist zumeist eine Stromwende gemeint; mitgedacht werden müssen aber auch die Wärmewende und die Verkehrswende. Das alte dualistische Denken, sprich: die Unterscheidung von Strom aus der Steckdose und Kraftstoff von der Tankstelle, scheint passé. Die Stromwende wird zur Verkehrswende, wenn batterieelektrische Fahrzeuge zur Ladung Strom benötigen oder Wasserstoff mithilfe von elektrischer Energie erzeugt wird. Damit kommt der schon älteren Idee der Sektorkopplung im gesamten Produktionsgewerbe zusehends größere Bedeutung zu. Immer wieder wird indes darauf hingewiesen, dass die Zahlen für den Anteil erneuerbarer Energien am Strommix zwar eindrucksvoll, jedoch gemessen am Primärenergiebrauch ernüchternd sind, zumal der Energieverbrauch ungebrochen hoch ist (vgl. UBA 2020, 2019 sowie IEA 2018 für internationale Entwicklungen). Offenbar gibt es einfachere Formen und Wege, die Energiewende umzusetzen (z.B. Solaranlage installieren), und solche, die ungleich komplizierter und weitaus anspruchsvoller sind (z.B. umfassender Ausstieg aus fossilen Brennstoffen), die vermutlich langfristig eine zweite Phase im begonnenen Transformationsprozess einläuten werden. Im Folgenden skizzieren wir vier Perspektiven, die uns eine Annäherung an die Energiewende in ihrer aktuellen Gestalt im Spannungsfeld zwischen übergeordneten, teils normativen Erwartungen und Zielsetzungen sowie empirisch feststellbaren Entwicklungen erlauben. 
Schon aufgrund der Emissionsintensität konventioneller Energieerzeugung lässt sich die Energiewende mühelos dem Megatrend ${ }^{1}$ Nachhaltigkeit (verstanden als gesamtgesellschaftliches Bemühen um die Verhinderung bzw. Abschwächung des Klimawandels und seiner Folgen) zuordnen. Und doch hat die Energiewende eine etwas eigenartige Anmutung, die zu der eher alltagsund individualbezogenen Vorstellung von Nachhaltigkeit, wie sie im Diskurs aktuell populär ist (vgl. Schad 2017: 58ff.), nicht gänzlich passen will. Wenn wir an Nachhaltigkeit denken, dann assoziieren wir das vielleicht mit ökologisch verträglicher Ernährung (vgl. Stengel in diesem Band), weniger Autofahren (vgl. Canzler/Knie in diesem Band) und Flugreisen sowie dem Verzicht auf Plastiktüten beim Einkaufen (vgl. Schweiger 2020). Die Energiewende taucht - wiederum als Stromwende gedacht - im konsumfokussierten Alltagserleben von Nachhaltigkeit am ehesten auf, wenn es um den Bezug von Öko-Strom geht. Die damit verbundene Vorstellung von Energie, die »aus der Steckdose« kommt, wird in der einschlägigen Literatur häufig beschrieben. Dahinter steht die Annahme: So lange der Strom verlässlich fließt, interessiert die (meisten) Verbraucher*innen nicht, welchen Erzeugungsquellen er entstammt. Eine designierte Wirkung der Energiewende besteht daher in der Schaffung eines neuen Bewusstseins der Verbraucher*innen für die Energieversorgung, das in der Folge zunächst individuelle Verhaltensänderungen (z.B. Energiesparen, Investitionen in Gebäudesanierung) zeitigt und schließlich in einem gesamtgesellschaftlichen Umdenken und Umsteuern gipfelt, das alle Sektoren erfasst.

Mit dem öffentlichen Nachdenken über Gewinnung, Verteilung und Verbrauch von Energie geht freilich nicht nur die Schärfung individuellen und gesellschaftlichen Bewusstseins, sondern auch eine weitere zentrale Frage einher: nämlich - da das alte System zur Disposition steht - jene nach der konkreten Gestaltung des zukünftigen Energieregimes. Gemäß der Idee des Gemeinschaftswerks meint der Wandel im Energiesystem nicht nur den Austausch einer bestimmten Technologie, sondern es sollten - im Einklang mit zeitgenössischen Ansprüchen an Demokratie, Politik, Wirtschaft und Gesellschaft - hieran viele (z.B. Bürger*innen, zivilgesellschaftliche Organisationen und Unternehmen) mitwirken, mitentscheiden und neue Impulse geben. Mitunter wurde die Energiewende gar als Jahrhundertchance charakterisiert,

$1 \quad$ Wir verzichten an dieser Stelle auf eine kritische Einordnung des Megatrend-Begriffs und verweisen insbesondere auf die Beiträge von Göll, Rust, Schweiger/Schecke und Zorn/Schweiger in diesem Band. 
in einem besonderen Moment der Geschichte einen entscheidenden Einfluss geltend zu machen - gleichsam, als würde man James Watt oder Rudolf Diesel an ihren Arbeitsplätzen besuchen und sie motivieren, alternative Vorschläge zu entwickeln.

Mit Blick auf die angestrebte Partizipation an der Energiewende ergeben sich zwei Fragen: Wer setzt die Transformation um und wer entscheidet darüber? Zu beobachten ist ein vermehrter Einbezug der Bevölkerung sowie neuer Akteure jenseits traditioneller Energieversorger. Eine partizipative Energiewende ist jedoch von einer übergeordneten Steuerung abhängig, die Einzelakteure oder -personen nicht vornehmen können und für die maßgeblich Politik und Staat verantwortlich zeichnen. Betreibung der Energiewende im ökonomischen Sinne und Steuerung der Energiewende im politischen Sinne können bei der Re-Definition des Energieregimes grundsätzlich hinterfragt und neubestimmt werden, wobei starke Pfadabhängigkeiten und Beharrungskräfte berücksichtigt werden müssen.

Schon in den 1960er und 1970er Jahren experimentierten »Pioniere des Wandels« mit den heute weit verbreiteten und für die Energiewende essentiellen Technologien der Stromerzeugung durch Photovoltaik und Windenergieanlagen. In ihrem Geiste sollten Small-Scale-Zukunftszellen - etwa in Form von Energiedörfern - die Blaupause für die (Energie-)Zukunft liefern. Fragen der Übertragbarkeit, Adaption und Diffusion von Technologien, Praktiken und Modi Operandi begleiteten die Energiewende als Transformationsprozess von Anfang an und zeichnen auch heute noch ausschlaggebend für die Skepsis hinsichtlich ihrer »Machbarkeit«: Ein paar Windräder machten doch nicht die zahlreichen Kraftwerkparks wett, die erforderliche Anzahl an Windenergieanlagen ließe sich jedoch kaum gegen den Widerstand der Bevölkerung bauen - und wie sollte die Industrie zuverlässig versorgt werden?

Hinsichtlich der Steuerung der Energiewende lässt sich ein Rückgriff auf das State-of-the-Art-Instrumentarium der Governance konstatieren (analog zum Paradigma der Partizipation). Eigentlich würde man ob der Betrachtung der Energiewende-Governance im Sinne einer modernen Toolbox, aus der sich Politik und Verwaltung zur Steuerung und Umsetzung bedienen, zu Beginn des 21. Jahrhunderts keine besonderen Erkenntnisse erwarten, wenn nicht ein ganz bestimmter historisch gewachsener Stil im Form einer engen und fest fixierten Assemblage zwischen staatlicher Überwachung bzw. Steuerung und Praxis der Energieunternehmen vorläge. Diese Form der Steuerung wurde in Teilen hinfällig und brauchte neue Formen der Zusammenarbeit. Auf Seiten der Unternehmen und Verbände, des Staates 
und der Politik entstanden unversehens heterogene, widersprüchliche, teils antagonistische Interessenlagen, die Verteilungsfragen neu aufbringen, traditionelle Allianzen erodieren und so zu einer Zersplitterung des vormals über viele Jahrzehnte stabilen Energieregimes führen. Um indes zur Umsetzung der Energiewende zu gelangen, bedarf es eines Konsenses für die neue Energie-Policy, der angesichts einer pluralistischen und asymmetrischen Akteurslandschaft nur schwerlich zu erreichen ist und dessen Fehlen Politik und Verwaltung tendenziell unter Druck setzt.

Fassen wir die kurz umrissenen Beobachtungen einmal zusammen: Die Energiewende versinnbildlicht und spiegelt wie kein anderes zeitgenössisches Unterfangen ein nachhaltiges, ein partizipatives und ein reflexives Zeitalter wider. In Meta-Debatten zu gesellschaftlichen Zuständen und zur Charakterisierung der Moderne finden sich zahlreiche kritische Stimmen, die Ansprüche und Prinzipien der Nachhaltigkeit fundamental grundsätzlich in Frage stellen. Im Mittelpunkt stehen Fragen der Authentizität und Faktizität: Wird wirklich Nachhaltigkeit erreicht, handelt es sich um echte (d.h. authentische) Partizipation und sind vorherrschende Entscheidungs- und Handlungsformen tatsächlich reflexiv? Es wird eine Simulation erwünschter Aktivitäten und Maßnahmen bei gleichzeitiger Verschleierung der tatsächlichen Zustände, die nicht den Desideraten entsprechen, vermutet (vgl. Blühdorn 2013; Ekardt 2018; Paech 2014; Swyngedouw 2011). Wenngleich es sich um wichtige, kontroverse Impulse im Nachhaltigkeitsdiskurs handelt, lassen sich jene Thesen - wie für zeitdiagnostische Überlegungen üblich - kaum falsifizieren. Und selbst wenn die empirische Überprüfung von Teilaspekten gelänge, kann immer eingewendet werden, dass die Empirie gleich einem Potemkinschen Dorf nur eine verzerrte Form der Wirklichkeit abbilde. Gewiss ist die Kritik zutreffend, dass die Energiewende bezogen auf den Primärenergieverbrauch bislang wenig erreicht hat - zumal im globalen Maßstab. Gleichzeitig ist jede Handlung, welche die Energiewende befördert, ein Schritt in Richtung mehr Nachhaltigkeit, obgleich etwa Windräder natürlich nicht ohne Ressourceneinsatz produziert, errichtet und betrieben werden können. Ähnlich verhält es sich mit der Kritik simulierter Partizipation und technokratischer Governance. Die tatsächliche Nachhaltigkeit der Energiewende ist kaum seriös zu bewerten, jedoch können einzelne Aspekte im Spannungsfeld zwischen Anspruch und Status quo reflektiert werden. Im Folgenden werfen wir einen näheren Blick auf die genannten Dimensionen Bewusstsein, Partizipation, Diffusion und Steuerung. 


\section{Die Geschichte der Energiewende und das Bewusstsein für (nachhaltige) Energiegewinnung}

Die Energiepolitik stand lange unter der Prämisse des berühmten Zieldreiecks der Versorgungssicherheit, Wirtschaftlichkeit und Umweltverträglichkeit. Heute - in Zeiten der Energiewende - wäre dieses Dreieck verzerrt, da Umweltverträglichkeit nicht mehr als gleichberechtigt, sondern als vorrangig angesehen würde. Energie kann historisch betrachtet insbesondere seit der Industriellen Revolution als ein Stoff angesehen werden, auf dem die gesamte moderne Gesellschaft ruht und der Fortschritt erst möglich machte (vgl. Mitchell 2011). In der Anfangszeit der Industrialisierung stand die Frage im Mittelpunkt, wie sich der Bedarf an Energie für die Güterproduktion, Beheizung von Gebäuden und neue Verkehrsmittel decken ließe. Die erste EnergieRevolution der Neuzeit vollzog sich mit der Kohle; lange dominierte das Prinzip der Dampfmaschine (vgl. Brüggemeier 2015; Rifkin 2011). Knapp fünfzehn Jahre nach der Fahrt der kohlegetriebenen Titanic stellten die Transatlantikdampfer auf Öl als Treibstoff um. Die zweite Revolution rückte flüssiges Öl und daraus gewonnene Treibstoffe in den Mittelpunkt und machte so Berufe mit unmenschlichen Arbeitsbedingungen wie die der Heizer und Kohlentrimmer überflüssig. Aus Sicht der Energiepolitik war mit dem immer schnelleren Fortschritt in Industrie, Wirtschaft und Verkehr ein ständiger Druck verbunden, den wachsenden Energiehunger zu stillen und Wege zu finden, die Energieproduktion effizienter zu machen. Es entstanden mehr und mehr zentralisierte Strukturen mit konzentrierten Kraftwerksparks und einer Überlandversorgung (vgl. Renn/Marshall 2016). Nur so konnte eine komplette Elektrifizierung erreicht werden, die Dörfer und Städte auch jenseits der Industriestandorte erfasste. Über moderne Straßen und motorisierte Verkehrsmittel ließ sich eine nie gekannte Mobilität erreichen. Die »Politik der hohen Schornsteine« sowie der Kraftwerke, die von den Wohnsiedlungen der wohlhabenderen Mittelschichten immer weiter abrückten, sollte die graue Kehrseite des Fortschritts kaschieren (vgl. Schulz-Walden 2013). Europa war lange Zeit durch Fabriken, Schornsteine, Schmutz und Staub geprägt; die heutigen grünen Landschaften sind ein Produkt des 20. Jahrhunderts. In filmischen Darstellungen ist es an Deck der Titanic romantisch - tatsächlich rieselte aus gewaltigen schwarzen Rauchwolken der Kohlenstaub aus den Schornsteinen herab. Dennoch blieb die Begeisterung für Geschwindigkeit, Technik und Fortschritt ungebrochen: Die Welt rückte zusammen, Distanzen schrumpften, und der Lebenskomfort erhöhte sich für immer mehr Menschen - et- 
wa durch Zentralheizungen und Zugang zu fließend Warmwasser. Die Vorteile der Modernisierung schienen die Nachteile stets zu überwiegen. Alles schien möglich, die rasanten Innovationen, Rekorde und Weiterentwicklungen überholten sich fast täglich. Mit der Etablierung moderner Standards im Sinne der Leitkriterien Effizienz und Effektivität beim Bauen und Wohnen, für Infrastruktur und Verkehr sowie in der Industrie in Kombination mit einer aufkommenden Sozialpolitik und Wohlfahrtsstaatlichkeit konnten die Industrienationen die für die meisten Menschen elende Ära ein für alle Mal hinter sich lassen. Zuletzt war große Fortschrittseuphorie in Deutschland und Europa nach dem Zweiten Weltkrieg zu spüren; sie sollte sich in den folgenden Jahrzehnten nach und nach abschwächen (vgl. Herbert 2017). Im Energiesektor änderte sich in der zweiten Hälfte des 20. Jahrhunderts vergleichsweise wenig: Neben die Kohle trat die Atomenergie als vermeintlich saubere, umweltfreundliche und endgültige Lösung für die Energiefrage bzw. -krise (vgl. Hake et al. 2015). Der Siegeszug des Benzinmotors setzte sich fort, Flugzeuge lösten den Schiffsverkehr für Passagier*innen ab, die Eisenbahn wechselte rasch von der Kohle zu Öl und Diesel und zur Elektrifizierung. Auch heute steht das System noch relativ unverändert da: Flugzeuge sind kerosin- und Straßenverkehr ist vornehmlich mineralölbetrieben, Atomund Kohlekraft prägen international überwiegend die Kraftwerkslandschaft, Heizungen basieren meist auf Gas- oder Ölfeuerung. Die Ölkrisen der 1970er Jahre führten zwar die immense Abhängigkeit von den Rohstoffen unmittelbar vor Augen, doch über die Jahrzehnte konnte mehr und mehr das Bild eines sicheren, stabilen und vergleichsweise günstigen Systems vermittelt werden, das dem Prinzip des Erhard'schen »Wohlstands für alle« entsprach: Energie steht immer im ausreichenden Maße bereit, sie ist günstig und gehört zur basalen Grundversorgung, auf die jeder Mensch in der modernen Gesellschaft einen Anspruch hat (vgl. Czada 2019). Strom, Wärmeenergie und moderner Verkehr werden $\mathrm{zu}$ einer Selbstverständlichkeit: Jede* $\mathrm{r}$ profitiert davon, jede* $r$ kann darauf zurückgreifen, eine moderne Gesellschaft ist schließlich gar nicht mehr ohne allzeit verfügbare Energie denkbar. Die Sicherstellung der Versorgung steht nicht zur Disposition, Stromausfälle werden selten und verlieren ihren Schrecken. Gleichzeitig geraten durch das fossil-atomare System unmittelbar verursachte Umweltprobleme stärker aus dem Blick. Zwar weisen Hauptstraßen und Innenstädte nach wie vor schlechte Luftwerte auf, doch das $\mathrm{CO}_{2}$ der Kraftwerke ist unsichtbar und geruchsneutral, »schmutzige« Industrien wurden dezimiert oder in andere Weltregionen externalisiert (vgl. Brand/Wissen 2017; Lessenich 2016) - im Ruhrgebiet etwa ist es im Ver- 
gleich zur Nachkriegszeit dank Strukturwandel und Renaturierung wieder grün und sauber.

Die Energiewende bricht insofern mit zentralen Leitlinien der Energiepolitik des 19. und 20. Jahrhunderts, als sie keine Reaktion auf steigende Energiebedarfe darstellt oder die größere Umweltfreundlichkeit der Erzeugung durch die faktische Unsichtbarmachung der Schädigungen nur suggeriert. Sie folgt allerdings denselben Prämissen des technischen Fortschritts. Die Energiewende antwortet primär auf die Bedrohung durch den Klimawandel und steht in Deutschland in einer dialektischen Beziehung zum Atomausstieg (vgl. Roose 2010). Seit Aufkommen der Anti-Atomkraft-Bewegung in den 1970er und 1980er Jahren stellte die Kernenergie ein latentes Konfliktpotential dar, das man durch die Förderung erneuerbaren Energien zu adressieren suchte (vgl. Hermville 2016; Mez 2012). Auf Atomausstieg und die flächendeckende Einführung erneuerbarer Energien folgen Kohleausstieg und das Vorantreiben der Elektromobilität (Energieregime der Erneuerbaren, vgl. Elliott 2020; Strunz 2013). Die derzeitige politische Vision der Energiewende sieht die großmaßstäbliche Nutzbarmachung der Onshore- und OffshoreWindkraft in Verbindung mit der Errichtung neuer sowie dem Ausbau bestehender Überlandstromtrassen vor, die durch energieeffizientes Bauen inklusive Biomasse- und Gasheizsystemen sowie verstärkter Bahn- und Radverkehrsnutzung und elektrisiertem Kraftverkehr komplettiert werden ${ }^{2}$. Dieses System stellt zu Beginn des 21. Jahrhunderts einen Fortschritt hinsichtlich der mit ihm einhergehenden (deutlich verminderten) $\mathrm{CO}_{2}$-Emissionen dar, ist allerdings weit davon entfernt, tatsächlich ressourcenschonend zu sein.

Nach Kohle und Öl konstituiert die flächendeckende Einführung erneuerbarer Energien eine dritte Energierevolution und erschließt damit Möglichkeitsräume für die Gestaltung des zukünftigen Energiesystems. Schon einige Jahrzehnte zuvor wurde die Idee eines dezentralen, kleinteiligen Energiesystems entwickelt, das auf erneuerbaren Energien basiert (vgl. z.B. Lovins 1977). Die Vorteile, so die Befürworter*innen, lägen auf der Hand: Abhängigkeiten würden vermieden und Überlandleitungen weitgehend verzichtbar, jede www.bundesregierung.de/resource/blob/997532/778196/8c6acc2c59597103d1ff9a437 acf27bd/infografik-energie-textversion-data.pdf?download=1, zuletzt aufgerufen am: 26.02.2020) sowie das Energiekonzept von 2010 (online verfügbar unter: https:// archiv.bundesregierung.de/resource/blob/656922/779770/794fdoc40425acd7f46afacbe62600f6/energiekonzept-final-data.pdf?download=1, zuletzt aufgerufen am: 26.02.2020). 
Kommune könnte die Energieversorgung selbstbestimmt gestalten und etwaige Profite vor Ort halten (vgl. Eiselt 2012; Gailing/Röhring 2015). Viele derzeit gegen neue Manifestierungen von Energieinfrastruktur protestierende Menschen argumentieren mit dieser Vision einer kleinteiligen Energiewende, die schon Hermann Scheer vertrat (vgl. Scheer 2010) und die auch heute noch teilweise radikal im Sinne einer Energieautarkie gefordert wird (Offthe-grid-Bewegung). Tatsächlich lässt sich ein immer größeres Bewusstsein der Bevölkerung für die Energiewende beobachten, eine dezentralere Ausgestaltung würde womöglich auch höhere Zustimmung finden (vgl. AEE 2019). Gleichzeitig hält sich die traditionelle Vorstellung, der zufolge die Energieversorgung durch einschlägig professionalisierte (Groß-)Unternehmen und den Staat sichergestellt wird, hartnäckig (vgl. Sonnberger/Ruddat 2016: 61). Allgemein hat sich zunehmend ein Dualismus entwickelt, der in der öffentlichen Debatte wenig abgebildet wird: Die von der Umsetzung der Energiewende vor Ort »betroffene« Politik, Verwaltung und Bevölkerung stehen der Energiepolitik auf Bundesebene gegenüber, welche durch Regierung, Parlament und Parteien und damit mannigfaltige Eigenheiten unserer repräsentativen, oft konsensorientierten Verhandlungsdemokratie geprägt ist. Sowohl Narrative, Leitbilder und Ansprüche als auch Kommunikation, Diskursformate und Rhetoriken fallen immer häufiger auseinander (vgl. Kühne/Weber 2018; Mast/Stehle 2016; Renn et al. 2020). So gelingt es bundespolitischen Akteuren kaum, die heterogenen Konstellationen an den vielfältigen Umsetzungsorten der Energiewende aufzunehmen, adäquat abzubilden und als Interessenaggregation in den Diskurs und die Entscheidungsfindung einzuspeisen.

Mit Blick auf die Vielfalt politischer Einstellungen in der Bevölkerung wurde die Energiewende in die neue Ordnung der Weltbilder übersetzt: Das konservative Lager stellt die Energiewende-Politik mitunter grundsätzlich in Frage, während die stärker sozialökologisch orientierte Seite großskalierte Energiewende-Maßnahmen zunehmend pauschal verteidigt. Studien zeigen, dass die Energiewende allgemein zwar als wünschenswert gilt, aber erhebliche Kritik an der Umsetzung besteht - vor allem hinsichtlich lokaler Projekte (vgl. Hübner et al. 2019: 19f.; Setton 2019). Offenbar gerät die Gesellschaft nach dem grundsätzlichen Entschluss zur Energiewende nun an einen Punkt, an dem der Frage nach dem Wie eine immer größere Bedeutung zukommt. Zwar spielt das Ideal einer nachhaltige(re)n Gesellschaft bei jüngeren Generationen eine zunehmend wichtigere Rolle, allerdings offenbart sich gleichzeitig eine wachsende Zerrissenheit zwischen normativen Ansprüchen und der individuellen Lebenspraxis, die ihnen kaum entsprechen kann (vgl. Gossen et 
al. 2016). Die Vorstellung des "guten Lebens« ist historisch betrachtet heute nachhaltigkeitsbezogener denn je, von einem breiten gesellschaftlichen Konsens kann jenseits sozialer Erwünschtheiten jedoch keine Rede sein. Womöglich befindet sich eine »erschöpfte« Gesellschaft (Rosa 2018) in einer ungünstigen Ausgangslage für eine »Große Transformation« (WBGU 2011) - sofern nicht Suffizienz noch zum Leitbild wird.

\section{Das Gemeinschaftswerk zwischen Akzeptanz, Partizipation und Exklusivität}

Die grundsätzlich hohe Zustimmung der Bevölkerung zur Energiewende variiert erheblich, wenn man Teilaspekte - wie etwa einzelne Energieträger - in den Blick nimmt. Es überrascht nicht, dass die allgemein mit wenigen Beeinträchtigungen assoziierte Photovoltaik besonders beliebt ist (vgl. AEE 2019). Windkraft und Bioenergie, die visuelle Beeinträchtigungen sowie Schall- bzw. olfaktorische Emissionen mit sich bringen, finden weniger $\mathrm{Zu}$ stimmung. Insbesondere Störungen des Landschaftsbilds, (wahrgenommene) potentielle Gefahren für Flora und Fauna und die menschliche Gesundheit stellen Faktoren dar, die für die Akzeptanz maßgeblich zeichnen (vgl. Batel et al. 2015; Bertsch et al. 2016; Schweizer-Ries et al. 2016; Sonnberger/Ruddat 2017). Hinzu kommen die Dimensionen der Energiegerechtigkeit: Verteilungsfragen (etwa hinsichtlich der räumlichen Verteilung von EnergieInfrastrukturen), prozessuale Aspekte (z.B. Ablauf der Planung und Realisierung neuer Anlagen) sowie die Anerkennung lokaler Gegebenheiten (spezifische Ansprüche und Kontexte) (vgl. Jenkins et al. 2016). Der Akzeptanz wäre es zum Beispiel wenig zuträglich, wenn eine Region mit deutlich mehr Energieanlagen »belastet « wäre als andere, die Bevölkerung von Planungen ausgeschlossen würde und lokale Besonderheiten - zum Beispiel besondere naturräumliche Gegebenheiten oder historische Deprivationserfahrungen - keine Berücksichtigung fänden. Das Konzept der Energiegerechtigkeit berücksichtigt zeitliche, ökonomische, soziopolitische, räumliche und technologische Faktoren (vgl. Bickerstaff et al. 2013; Pesch et al. 2017; Sovacool/Dworkin 2014; Williams/Doyon 2019). Diverse Aspekte werden im Kontext der Verfahren zur Öffentlichkeitsbeteiligung und in lokalen Diskursen abgebildet.

Neben konzeptionellen Arbeiten zur Energiegerechtigkeit finden sich in der Literatur auch Elaborationen zur Energiedemokratie. Zum einen findet ein Rückgriff auf Bausteine der Demokratietheorie statt: So lassen sich par- 
tizipatorische (politische Beteiligung an Entscheidungsfindungsprozessen), assoziative (Verbandslandschaft der Energiewende) als auch deliberative Elemente (Diskurse und Legitimation der Energiewende) identifizieren. Zum anderen werden zwei neue Dimensionen materieller Partizipation erfasst, welche die Bedeutung von Objekten und dem Zugang zu Ressourcen hervorheben (vgl. Veelen/Horst 2018: 22). Ziele der Energiedemokratie mit revolutionärem oder reformatorischem Charakter sind Widerstand gegen die dominante energiepolitische Agenda sowie die Re-Aneignung und Restrukturierung des Energiesektors. Veränderungsbedarfe zur Erreichung der Energiedemokratie betreffen die Steuerung der Energiewende, Finanzierungsund Fördermechanismen und etablierte Energieversorgungsunternehmen sowie das Zusammenwirken neuer Akteure und Institutionen, räumlicher Netzwerke und Kommunen, Städten und Regionen (vgl. Burke/Stephens 2017: 38,43 ).

Aus der Perspektive der Energiegerechtigkeits- und Energiedemokratieforschung fällt mit Blick auf die Entwicklung der Energiewende in Deutschland auf, dass diese einen stark regionalen Charakter aufweist, der sich durch einen spezifischen Mix der politischen Förderung erklären lässt (vgl. Morris/Jungjohann 2016). Als wichtige Grundlage der deutschen Energiewende-Dynamik gilt die finanzielle Förderung, die im ErneuerbareEnergien-Gesetz (EEG) festgeschrieben ist. Sie motivierte kleinere und größere Akteure zu zahlreichen Investitionen in Anlagen zur regenerativen Energieerzeugung. Parallel realisierten Kommunen, Städte und Regionen eine Fülle ambitionierter Vorhaben, denen nicht selten Best-PracticeCharakter attestiert wurde (vgl. Schönberger/Reiche 2016). So entstanden Energiedörfer und $100 \%$-Erneuerbare-Energien-Regionen, die ihrerseits auf spezifische Förderprogramme zurückgreifen konnten. Die Idee dahinter: Die Pionierarbeit in den Kommunen und Regionen zur Erprobung möglicher Implementierungen einer ganzheitlichen Energiewende würde im Sinne des Upscaling Blaupausen für das Gelingen der Energiewende im ganzen Land liefern (vgl. Graf/Kern 2018). Im Ergebnis wurde eine starke Heterogenisierung des Energiewende-Geschehens in der Bundesrepublik in Gang gesetzt - und es setzte sich zunehmend die Erkenntnis durch, dass erfolgreiche Umsetzungen an einem Ort nicht automatisch anderswo adaptiert werden können (vgl. Nagorny-Koring 2018). Im Sinne der Energiedemokratie lassen sich bei der Planung der Energiewende durchaus Erfolge konstatieren: Im Rahmen einer partizipativen und kooperativen Governance unter Einbezug von Akteuren aus Wirtschaft, Zivilgesellschaft und Politik sowie der Bevöl- 
kerung konnten zahlreiche Energiekonzepte und Maßnahmen entwickelt, erprobt und umgesetzt werden (vgl. Holstenkamp/Radtke 2018).

Wenn von Beteiligung die Rede ist, wird gemeinhin zunächst Bürgerbeteiligung und nicht Akteursbeteiligung assoziiert. Die Bürgerbeteiligung an der Energiewende lässt sich, orientiert an den oben bereits angeführten Demokratiekonzepten, grob unterscheiden in politische Beteiligung, wie sie im Rahmen von Öffentlichkeitsbeteiligung bei Planungsverfahren stattfindet, assoziativ-materielle Formen (z.B. Bürgerenergie, s.u.) sowie assoziativ-deliberative Ausprägungen (z.B. Mitgestaltung eines Energiekonzeptes durch einen Umweltverband). Die Öffentlichkeitsbeteiligung ist sowohl bei der überregionalen Planung (z.B. beim Netzausbau), der Aufstellung von Regionalplänen (die sämtliche Energiewendemaßnahmen in einer Region umfassen) als auch der kommunalen Planung (z.B. Windkraft) mittlerweile Standard, von Politik und Bevölkerung gewünscht und auch teilweise gesetzlich vorgeschrieben. Ihre Bilanz fällt nicht allzu positiv aus, sollte allerdings auch nicht zu kritisch beurteilt werden (vgl. Huge/Roßnagel 2018; Renn et al. 2017; Schroeter et al. 2016; Schweizer et al. 2016). Insbesondere bei Stromnetz- und Windkraftausbau besteht das grundsätzliche Problem, dass das Ergebnis in Gestalt des geplanten Baus von Anfang an feststeht. Zwar sind Fälle bekannt, in denen die Planungen nicht in Realisierungen mündeten - sie ändern aber wenig an der grundsätzlichen Ausrichtung von Planungsverfahren und den Intentionen der beteiligten Stakeholder (vgl. Hänlein/El Alaoui 2015; Kamlage et al. 2014; Roßnagel et al. 2016). Wenn Bürger*innen sich etwa an der Planung von Windenergieanlagen bzw. festgelegten Zonen zum Bau derselben beteiligen, stehen die Windräder perspektivisch bereits. Wichtige Fragen bei diesen Verfahren betreffen die Umwelt- und gesundheitliche Verträglichkeit des jeweiligen Vorhabens. Hierzu werden zahlreiche Stellungnahmen von Behörden und verschiedene Gutachten eingeholt. Immer wieder werden indes Aspekte des Naturschutzes durch Gegner"innen des Projekts instrumentalisiert, um das Vorhaben zu verhindern. Zahlreiche Untersuchungen haben gezeigt, dass es stark auf die Prozessdimension, also die konkrete Ausgestaltung des Beteiligungsverfahrens ankommt (vgl. u.a. Gross 2007; Langer et al. 2017; Liebe et al. 2017; Lienhoop 2018; Zaunbrecher/Ziefle 2016). Dabei spielen Fairness, Transparenz, die vorurteilsfreie Anhörung unterschiedlicher Standpunkte, Resonanz bzw. Responsivität sowie ein Diskurs »auf Augenhöhe« entscheidende Rollen (vgl. Hildebrand et al. 2015). Schließlich finden sich Entscheidungsspielräume auch beim Stromnetzausbau und der Errichtung neuer Windkraftanlagen: So können Standorte bzw. Verläufe leicht vari- 
iert, Abschaltungen zu bestimmten Zeitpunkten oder Erdverkabelung vereinbart und ausgleichende Maßnahmen für die lokale Gemeinschaft entwickelt werden. Im Zuge der Energiewende wurden diverse Beteiligungsinstrumente eingesetzt bzw. erprobt - von Dialogverfahren über Werkstattarbeit bis hin zu Begehungen. Aus der Beteiligungsforschung ist schon lange bekannt, dass kein Königsweg existiert, sondern eine situativ angepasste Kombination diverser Instrumente am vielversprechendsten ist. Studien zu einschlägigen Verfahren zeigen, dass es häufig zu Problemen zwischen Fachverwaltungen/Vorhabenträgern und der Bevölkerung kommt, weil unterschiedliche Rationalitäten und Erwartungshaltungen vorliegen und es an einer gemeinsamen Sprache mangelt (vgl. Bauer 2015; Kamlage et al. 2018; Schweizer/Bovet 2016). Eine flächendeckende Lösung konnte bislang nicht gefunden werden, da die effektive Adressierung der beschriebenen Problemlagen Änderungen bei der politischen Steuerung sowie von Zuständigkeiten erfordern würde. So greifen bei Energiewende-Planungen zahlreiche Bestimmungen, die lokale Verwaltungen und Politik lediglich befolgen, aber nicht verändern können, da darüber auf höheren Ebenen entschieden wird. Es zeichnet sich ein Mehrebenen-Dilemma der politischen Steuerung ab, das im Folgenden noch näher erläutert wird. Öffentlichkeitsbeteiligung im Zuge der Energiewende stellt eine immense Herausforderung dar, die auch in Zukunft eine große Rolle spielen wird. Hierbei ist ein verstärkter Einsatz digitaler Formate zu erwarten, die Beteiligung in Teilen erleichtern, den Trade-off zwischen Komplexität, Pluralität und Interessenaggregation sowie Konsensfindung aber durchaus auch verschärfen könnten.

Neben Akteurs- bzw. Öffentlichkeitsbeteiligung spielt die materielle Bürgerbeteiligung in Gestalt der Bürgerenergie ${ }^{3}$ eine wesentliche Rolle für die Energiewende. Eine Bürgerenergie-Bewegung, deren verstärktes Aufkommen nicht zufällig zusammenfiel mit Höchstsätzen der für Photovoltaikstrom gesetzlich garantierten Einspeisevergütungen, konnte zwar etwa 1.000 Energiegenossenschaften bzw. rund 1.700 Bürgerenergiegesellschaften

3 Unter Bürgerenergie werden »kollektive Investitionen von Bürgerinnen und Bürgern in Anlagen in ihrer Region « (Holstenkamp et al. 2018: 1061) verstanden. Es handelt sich um einen Sammelbegriff für unterschiedliche Bürgerinitiativen und Organisationen. Als zentrale Merkmale von Bürgerenergiegesellschaften gelten Regionalität bzw. Lokalität, nicht-monetäre Zielsetzungen, die Offenheit der Mitgliedschaft sowie Einfluss der Bürger*innen auf Entscheidungen der Organisation (vgl. Kahla et al. 2017: 6). 
(vgl. Kahla et al. 2017: 13, 25) ins Leben rufen - eine flächendeckende Revolution blieb aber aus. Zunächst wurden die zahlreichen Projekte vielfach als Indiz für eine Energiewende, die genuin durch Bürger*innen getragen wird, gewertet: Vor Ort, in Dörfern und Quartieren, formierten sich unzählige »Mighty Davids« (Debor 2018), um dem schwerfälligen Goliath der fossilen Energieversorgung entgegenzutreten. Tatsächlich befand sich ein großer Anteil der installierten Leistung zur erneuerbaren Stromerzeugung zunächst in der Hand von Privatpersonen (wobei auf Bürgerenergiegesellschaften nur ein kleiner Teil entfiel), während die großen Energieversorgungsunternehmen die Wende $\mathrm{zu}$ verschlafen schienen (vgl. trend:research/Leuphana Universität Lüneburg 2013). Weiterhin wurde hier, so schien es vielen, eine altehrwürdige, zuletzt ein wenig angestaubte Organisationsform wiederentdeckt: Energiegenossenschaften belebten demnach nicht nur das wenig dynamische Gründungsgeschehen im deutschen Genossenschaftswesen (vgl. Stappel 2016: 66ff.), sie fungierten auch als »Vehikel par excellence für die Bürgerbeteiligung an der Energiewende« (Müller et al. 2015: 97), waren Vorboten und Erprobungsräume für ein anderes Energiesystemdesign, das im Sinne Hermann Scheers dezentraler, demokratischer, verteilungsgerechter und umweltfreundlicher sein sollte als das alte (vgl. Elsen 2012; Fischer et al. 2015). Und mehr noch: Bürger*innen, die ihre Energie selbst produzierten, würden darüber womöglich $\mathrm{zu}$ einem bewussteren Umgang mit derselben finden (vgl. Leggewie 2013: 19ff.). Kritisch-differenziertere Stimmen bemerkten die meist fehlende Umsetzung des genossenschaftlichen Identitätsprinzips (vgl. Herbes et al. 2017: 89), den simplen Modus ${ }^{4}$, in dem sich dank Einspeisevergütung für Photovoltaikstrom verlässliche und durchaus attraktive Renditen erzielen ließen (die über die EEG-Umlage und damit den Strompreis finanziert wurden) (vgl. Chatalova/Valentinov 2014; Martens 2015) und die zur Mitgliedschaft obligatorische Zeichnung von Genossenschaftsanteilen, die für materiell schlechter gestellte Personen unerschwinglich sein kann (vgl. Heindl et al. 2014: 509). Studien zu Mitgliedern von Bürgerenergiegesellschaften zeigen, dass es sich vornehmlich um ältere, wohlhabende,

4 Die Gründung von Cenossenschaften ist auch nach der Novellierung des Cenossenschaftsgesetzes 2006 für kleine Initiativen keineswegs unkompliziert. Zur Entstehung der zahlreichen Energiegenossenschaften trugen vergleichsweise einfache Geschäftsmodelle, die teils zur Nachahmung aufbereitet kostenlos zur Verfügung gestellt wurden, sowie Beratungen durch öffentliche Stellen - in Nordrhein-Westfalen etwa die EnergieAgentur - bei (vgl. Blome-Drees et al. 2016: 576; Stappel 2016: 75). 
höhergebildete Männer handelt (vgl. Holstenkamp et al. 2018: 1068ff.; Radtke 2016; Yildiz et al. 2015). Der Mitgliedschaft stehen verschiedenste Hürden entgegen, darunter Unkenntnis über die Existenz von Energiegenossenschaften (vgl. Theurl/Wendler 2011: 76), Lebensrealitäten, die andere Themen in den Vordergrund rücken (vgl. Breukers et al. 2017) und die Indifferenz der Führungsriegen mit Blick auf soziale Diversität sowie Stereotype hinsichtlich der Eignung und des Interesses bestimmter Bevölkerungsgruppen für die bzw. an der Mitgliedschaft (vgl. Łapniewska 2019; Drewing/Glanz in diesem Band). Zudem sind Energiegenossenschaften ungleichmäßig über das Bundesgebiet verteilt, was auf unterschiedliche Potentiale der regenerativen Energieerzeugung sowie Förderprogramme und Netzwerke zurückgeht, die in manchen Bundesländern stärker ausgeprägt sind (vgl. Poppen 2015; Kahla et al. 2017). Empirisch lassen sich die Potentialzuschreibungen also bislang kaum bestätigen. In der Folge vermag die Bürgerenergie auch im inkrementellen Sinne jenseits basisdemokratischer Aneignungsprozesse kaum zum Fortschritt der Energiewende beitragen, beträfen die ihr zugeschriebenen Wirkungen auf die Umsetzung einer »top-down« implementierten Energiewendepolitik - Akzeptanzsicherung für neue Anlagen sowie Erhöhung der sozialen Gerechtigkeit der Energiewende - doch nur einen kleinen Teil der Bürgerschaft. Mehr noch: Gegenteilige Effekte sind nicht ausgeschlossen, da deprivierte Milieus kaum in Bürgerenergiegesellschaften vertreten sind und die Beteiligung einiger (aber eben nicht aller) Bürger*innen an lokalen Anlagen auch zur Spaltung und zur Verhärtung der Fronten beitragen kann (vgl. Radtke 2018a). Die Aussichten für eine partizipative Trendwende sind nicht eben ungetrübt: Die Energiewende in einer fortgeschrittenen Phase bedeutet massiven Zubau von Erneuerbare-Energien-Anlagen sowie radikale Umstellung des Verkehrs und von Heizsystemen. So kommt es fast unweigerlich zu mehr Zweifeln, mehr Kritik und mehr Konflikten - mit der lokalen Bevölkerung sowie gesamtgesellschaftlich, hinsichtlich Fragen des Naturschutzes, des Aufwands und der Kosten.

\section{Eine Frage der Diffusion}

Man kann neben Bürgerenergiegesellschaften sowie Dörfern und Regionen, deren Energieversorgung vollständig oder in großen Teilen auf erneuerbaren Energien basiert, vielfältige weitere zivilgesellschaftliche Aktivitäten und Initiativen beobachten, die im Sinne einer ganzheitlichen Energiewende $\mathrm{Zu}$ - 
kunftsvisionen eines nachhaltigen, weitgehend auf regenerativen Ressourcen beruhenden Lebensstils schon heute umsetzen. Hinter dem Enthusiasmus für urbane Mobilitätslösungen jenseits des Verbrennungsmotors, neue Antriebstechnologien und Sharing-Plattformen sowie dem Bemühen, Akzep$\operatorname{tanz}$ durch verständigere Beteiligungsverfahren zu sichern und der Idee einer bürgergetriebenen Energiewende, stehen zahlreiche wohlerwogene Überlegungen. Sie sind nicht unrealistisch in dem Sinne, dass sich solche Phänomene der Innovativität und Kreativität, gleichsam bereits verwirklichten Visionen, sozialen bzw. nachhaltigen Innovationen - oder eben: realen Utopien ${ }^{5}$ (Wright 2017) - nicht in der Praxis finden ließen und ihre Analyse nicht erhellend wäre. Als gelebte Erprobung von Variationen alltäglicher energie- und emissionsintensiver Praktiken markiert ihre bloße Existenz den schillernden Beweis dafür, dass es anders geht. Das macht sie gesellschaftlich wertvoll und wissenschaftlich interessant. Nicht selten wird jedoch nicht oder nur unzureichend unterschieden zwischen normativ wünschenswerten und empirisch belegbaren Potentialentfaltungen. Dies gilt für die Verhandlung der Energiewende im täglichen Diskurs, mitunter aber auch für transformativ orientierte wissenschaftliche Befassungen und dabei insbesondere für Arbeiten zu Nachhaltigkeit, Innovation und gesellschaftlichem Wandel. So befinden JaegerErben und Kolleg*innen (2017: 247):»Das Versprechen einer besseren Zukunft durch (soziale) Innovationen kann dazu verführen, bei der Untersuchung der deskriptiven und visionären Elemente von Innovationsprozessen das Visionäre mit allzu unreflektierter Begeisterung zu betrachten« und Kropp (2017a: 312) ergänzt, dass "auch Steuerungseliten ihre Einsicht in die Notwendigkeit fundamentaler Transformationen einerseits beteuern, dann aber deren Last und insbesondere die Verantwortung für die damit verbundenen Unsicherheiten, Verluste und Konsequenzen an das amorphe Feld innovativer Nischenexperimente delegieren«. Die Verortung der Energiewende als Teilaufgabe des Klimaschutzes, also als inhärenter Bestandteil des Megatrends Nachhaltigkeit in normativer Auslegung sowie einschlägiger Politiken, führt jedoch weiter: nämlich zu einer grundlegenden und tiefgreifenden Veränderung zeitgenössischer Modi des Lebens und Wirtschaftens, welche die Lebensgrundlagen zukünftiger Generationen nicht länger gefährdet (vgl. Aykut genwärtig beschaffen ist, entwickelt werden können, die dabei aber die Welt, wie sie sein könnte, vorwegnehmen und dazu beitragen, dass wir uns in dieser Richtung voranbewegen.«(Wright 2017: 11). 
et al. 2019). Dazu genügt es nicht, auf bestehende Best-Practice-Beispiele, nachhaltige und soziale Innovationen hinzuweisen, die in ihrem Auftreten und Erfolg in erster Linie kontingent sind. Sie werden nicht die erwünschten gesamtgesellschaftlichen Veränderungen zeitigen, sofern sie nicht weitreichend diffundieren, nicht die Gesellschaft in ihrer Breite, in unterschiedlichen sozialen Milieus, Organisationen der Zivilgesellschaft und der öffentlichen Hand sowie Unternehmen erfassen (vgl. Geels et al. 2016). Wir können zum gegenwärtigen Zeitpunkt nicht sagen, $o b$ die Große Transformation möglich ist und wie sie aussähe. Sicher ist jedoch: Es würde sich nicht um eine Gesellschaft der heutigen Best Practices handeln, da diese erstens auf Grundlage nicht-nachhaltiger Bedingungen existieren und zweitens in ihrer Identität, ihren Strukturen und Eigenschaften fundamental auf der Differenz zum Bestehenden basieren (vgl. Star 2020). Der Knackpunkt liegt in der Diffusion, die wiederum Innovationen, die sich möglichst nahtlos in bestehende Strukturen, Systeme und Logiken einfügen, weitaus leichter fällt als Ideen, die jene grundlegend in Frage stellen. Das ist insofern problematisch, als dass letzteren ungleich größeres Potential innewohnt, grundlegende Veränderungen im Sinne einer genuinen Nachhaltigkeitstransformation mit sich zu bringen (vgl. Kropp 2018). Dies beschreibt ein zentrales Dilemma der nachhaltigen Gesellschaft. Konträr zu den Intentionen vieler Initiativen kann ein Nachhaltigkeitsverständnis, das primär auf Nischeninnovationen und einen Bewusstseinswandel (der sich nur bei einem Bruchteil der Bevölkerung in tatsächliche Verhaltensänderungen übersetzt) abhebt, zur Legitimierung (und damit Konsolidierung) eines gesellschaftlichen und politischen Status quo beitragen, der zwar einige Aktivitäten und Maßnahmen zur Verminderung der Umweltschädlichkeit zeitgenössischer westlicher Lebens- und Wirtschaftsweisen kennt, die indes in Qualität und Verbreitung kaum ausreichen, um Nachhaltigkeit in jenem Ausmaß zu implementieren, das weithin als obligatorisch erachtet wird (etwa zur Erreichung der Klimaschutzziele von Paris). In der Folge kann es zur Verschleppung der Suche nach Lösungen für drängende, hochkomplexe Problemlagen kommen, die, sobald sie in der öffentlichen Wahrnehmung virulent werden, unter akutem Handlungsdruck womöglich weit unüberlegter angegangen werden, als bei früherer Thematisierung notwendig gewesen wäre (vgl. Blühdorn 2013; Blythe et al. 2018; Kropp 2017b). Zudem besteht die Gefahr, durch die Nicht- bzw. einseitige Befassung mit komplexen Gegenständen potentiell kontroverser Aushandlungsprozesse die öffentlichkeitswirksame Verhandlung derselben undemokratischen Kräften zu überlassen, deren vereinfachend-polarisierende Diskurspraxis einen inklusiven, 
zwischen verschiedenen Interessen sorgfältig abwägenden, lösungsorientierten Entscheidungsprozess erschwert. So werten etwa Blühdorn und Butzlaff (2019: 204) den Rechtspopulismus als »indicative of the incremental exhaustion of the old democratic project«.

Der Begriff der Energiewende wurde erstmals durch eine Veröffentlichung des Öko-Instituts aufgebracht (vgl. Krause et al. 1980). Selbiges war aus Protesten gegen den Bau eines Atomkraftwerks hervorgegangen, um der verbreiteten Auffassung, der zufolge Kernenergie eine sichere, verlässliche Art der Stromerzeugung darstellte, divergente Expertise entgegenzusetzen. So lässt sich die Idee der Energiewende in ihren Anfängen selbst als nachhaltig-alternativorientierte »bottom-up«-Innovation in distinktiver Abgrenzung charakterisieren:

»Dismissed by the state and national governments as backward-looking NIMBY protesters, activists pushed back, characterizing the development plans as unnecessary, technologically unsound, and environmentally and socially destructive. Mass mobilization against a nuclear plant near the German village of Wyhl drew broad public attention to energy issues and started a national conversation about economic growth and energy policy goals. (Hager 2017: o.S.; vgl. auch Hager 2015).

Ausschlaggebend für den Richtungswechsel der Bundespolitik Jahrzehnte später und die breite Diffusion der Energiewende-Idee war der externe Schock des Fukushima-Reaktorunglücks. $\mathrm{Zu}$ diesem Zeitpunkt war der Ausbau der Erneuerbaren längst zum Hoffnungsträger jenseits der Nische geworden (vgl. Maubach 2014). Mehr und mehr trat hinter dem simplen Modus einer eindimensional gedachten subventionierten Energiewende und dem Verharren von Politik und Staat in der angestammten Rolle des passiven, sachwaltenden Leviathans ein Steuerungsproblem zu Tage.

\section{Keine Energie für die Wende? Nachhaltigkeitspolitik und Mehrebenen-Governance}

Um die aktuelle Steuerung der Energiewende zu verstehen, hilft ein Blick in die Geschichte der deutschen Energiepolitik (vgl. Radtke 2018b; Saretzki 2001). In modernen Gesellschaften stellt die Energieversorgung einen Teil der Daseinsvorsorge dar, für die der Staat garantieren muss. Daher über- 
rascht es nicht, dass zu Beginn des 20. Jahrhunderts Überlegungen bestanden, den Energiesektor zu verstaatlichen (vgl. Stier 1999). Man entschied sich aber dafür, eine starke staatliche Aufsicht zu etablieren und den Energieversorgungsunternehmen (EVU) Gebiete zuzuteilen, auf welchen diese fortan Monopole innehatten. So war die Energieversorgung bis 1998 dem marktlichen Wettbewerb und etwaiger Einflussnahme durch Verbraucher*innen entzogen. Für die EVU war dies eine komfortable Situation, insbesondere, wenn sie auf ausgereifte Technologien und sich rasch amortisierende Kraftwerke zurückgreifen konnten. Schon Ludwig Erhard war mit dieser Lösung unzufrieden, doch der Verband der Energiewirtschaft entfaltete erhebliches Beharrungsvermögen (vgl. Stier 1999: 496). Aus Sicht der Politik bestand aber kein unmittelbarer Handlungsdruck, denn mit Blick auf das energiepolitische Dreieck gewährleisteten die Gebietsmonopole die Sicherheit und Wirtschaftlichkeit der Energieversorgung. Die Bevölkerung stellte nicht in Frage, wer die Post transportiert, das Bahn- und Telefonnetz betreibt oder den Strom liefert. All dies wurde vor der Privatisierungswelle durch staatseigene Unternehmen (Bahn, Post) oder solche sichergestellt, die im Auftrag des Staates handelten (EVU) und zunehmend in ihren Strukturen erstarrten - sie wurden zu Paradebeispielen für Resistenz gegenüber Innovationen, Langsamkeit und Verkrustung. Auch die engen Verflechtungen zwischen Bundeswirtschaftsministerium und den großen Energieversorgern gelten als eine Verbindung zwischen Staat und Privatwirtschaft, die den Anforderungen der Durchlässigkeit, Transparenz und Fairness kaum Genüge leistet(e) (vgl. Mautz 2012). Der Liberalisierung des Energiemarktes, die 1998 mit der Reform des Energiewirtschaftsgesetzes eingeläutet wurde, folgte 2000 die Einführung des Erneuerbare-Energien-Gesetzes - zusammen markieren sie den Auftakt des Zeitalters der erneuerbaren Energie. Nach zahlreichen Privatisierungen kam es zu einer Rekommunalisierungswelle im Energiesektor (vgl. Brandmeyer 2013; Grünewald 2016; Sack 2014). Die "großen Vier« der EVU gerieten unter Druck, neue Unternehmen drängten auf den Markt (vgl. Sack 2018). Heute kann Strom von unzähligen Anbietern bezogen werden. Das Stromnetz allerdings wird, aufgeteilt in Zonen, nach wie vor von lediglich vier Gesellschaften betrieben, die zum Teil Tochtergesellschaften der großen EVU sind. Die Planung des Netzausbaus übernimmt die neu geschaffene Bundesnetzagentur, die als zentralisierte Institution das gewaltige Vorhaben koordinieren soll. Dem 2011 beschlossenen und 2019 novellierten Gesetz zur Beschleunigung des Energieleitungsausbaus zum Trotz, das die Vereinfachung von Planungsverfahren (und damit einhergehend, so die Kritik, die Verringerung des Einflus- 
ses von Bevölkerung und Institutionen) vorsieht, schreitet der Netzausbau nur langsam voran (vgl. Löschel et al. 2018; Schröder 2012; Steinbach/Franke 2017). Ursprünglich sollten zur Identifikation vielversprechender Trassenverläufe schon früh Präferenzen der Bevölkerung sowie der tangierten Kommunen und Regionen eingeholt werden, um in der weiteren Planung zu Lösungen zu finden, die mit möglichst geringen Belastungen einhergehen und Kompromisse aus allen Interessenlagen darstellen. Heute herrscht weitgehend Uneinigkeit hinsichtlich der Trassenverläufe. Auch die vielfach als probate Alternative angepriesene, deutlich kostenintensivere Verlegung unterirdischer Stromleitungen birgt keine Garantie für gesellschaftliche Akzeptanz des Netzausbaus allerorten (vgl. Bertsch et al. 2016). Aus Sicht der Bundespolitik verläuft der Ausbau deutlich zu langsam und öffnet Einfallstore für grundlegende Energiewende-Kritik, wenn Strom aus dem Norden ob unzureichender Stromnetzkapazitäten womöglich nicht weitertransportiert und genutzt werden kann und in der Folge (keineswegs unumstrittene) Windparks abgeregelt werden müssen. Ähnlich schleppend geht der Windkraftausbau voran, der (v.a. an Land) insbesondere seit 2019 stark nachgelassen hat. Ursachen hierfür sind fehlende (attraktive) Flächen, Klageverfahren und Protest sowie Natur- und Artenschutz, die viele windreiche Gebiete als potentielle Standorte disqualifizieren (vgl. Roßmeier/Weber 2018; Stede/May 2019; Weber/Jenal 2018). Die jüngsten Entwicklungen gefährden das weithin angestrebte Energiewende-Regime, das stark auf Windkraft und Stromnetzausbau abhebt. Die aktuellen bundespolitischen Strategien und Überlegungen zur Beschleunigung der Energiewende - etwa Richtmarken hinsichtlich des Windkraftausbaus für die Bundesländer, Schaffung finanzieller Anreize für Bürger*innen, die in der Nähe der Anlagen wohnen, Erhöhung der gesetzlich festgeschriebenen Mindestabstände $\mathrm{zu}$ neuen Windenergieanlagen sowie schnellere Planungs- und Umsetzungsverfahren bei gleichzeitiger Einschränkung von Klagemöglichkeiten - zeigen auf, dass simple Zielvorgaben und EEG-Förderung für eine gelingende Umsetzung nicht ausreichen. Seit langem wird in der Politik die Formulierung eines Masterplans diskutiert (und insbesondere von Seiten der Bundesländer teils massiv eingefordert), der alle Bestrebungen, Aktivitäten und Zuständigkeiten in Einklang bringen und einen ausbalancierten Fahrplan beinhalten würde (vgl. Klagge/Arbach 2013). Es darf indes bezweifelt werden, ob ein solcher Masterplan erstens machbar und zweitens sinnvoll wäre, vermöchte er doch an den föderalen Prinzipien und Einzellogiken einschlägiger Gesetze und Vorgaben, an divergierenden Ambitionen und Interessenlagen sowie diversen Förderprinzipien 
nichts zu ändern. Zwischen Bund, Ländern und Kommunen zeigt sich eine Ambivalenz, die nicht untypisch für Infrastruktur-Politiken ist: Hinsichtlich der Energiewende variieren Wahrnehmung, Anspruch und die Verortung der »Bringschuld« zwischen den Ebenen (vgl. Chemnitz 2018; Müller/Kahl 2015; Radtke 2018c; Rave 2016; Scheiner 2017; Schreurs/Steuwer 2015; Wurster/Köhler 2016). Während der Bund aus der Vogelperspektive den Gesamtfortschritt des Vorhabens im Blick hat, sind die Länder, Regionen und Kommunen stärker in ihren besonderen Gegebenheiten verhaftet. Und natürlich gibt es Ungleichheiten: So sind vom Kohleausstieg nur bestimmte Regionen betroffen, Windenergie ist vor allem in den nördlichen Bundesländern ein wichtiger Wirtschaftssektor und viele Städte verfolgen eigene Energiewende-Konzepte, die etwa Effizienz, Einsparungen und den Bau-/Gebäudesektor, aber auch eigene Investitionen und Programme der Stadtwerke in den Mittelpunkt stellen (vgl. Bendlin 2020; Hehn 2015; Loorbach et al. 2016; Melica et al. 2018; Schönberger/Reiche 2016). Diese unterschiedlichen Interessenlagen, Abhängigkeiten und Prinzipien kollidieren miteinander. Im Idealfall würde nach dem Konzept moderner Governance mit allen Beteiligten eine Lösung erarbeitet, die konsensuales und gemeinsames zielgerichtetes Handeln ermöglicht - sowohl im Einzelfall als auch übergreifend zur Harmonisierung der zahlreichen heterogenen Sektorpolitiken (vgl. Benz/Czada 2019; Bößner 2020; Neukirch 2019; Saurer 2019; Wright 2020). Dieser Anspruch kann meist nicht verwirklicht werden - es herrschen Konkurrenzen zwischen Städten, Regionen und Ländern sowie Unverständnis für die Handlungslogiken, Programme und Maßnahmen der jeweils anderen Akteure und Einheiten vor (MehrebenenDilemma, vgl. Eberlein/Newman 2008; Galvin 2018; Geels 2014; Hooghe/Marks 2001; Newing/Fritsch 2009; Radtke 2018c; Smink et al. 2015). Erste Muster zunehmender Disparitäten lassen sich erkennen, da einerseits zaudernde Gemeinden versuchen, Windkraftanlagen auf ihrem Gebiet zu vermeiden und Klimaschutz »auf kleiner Flamme« betreiben, während andererseits Städte und Kommunen ihre distinkten Energiewende-Konzepte mit Verve und Vehemenz verteidigen und sich $\mathrm{zu}$ eigenen Netzwerken und Verbünden zusammenschließen (vgl. Bulling et al. 2015). Hieraus erwächst zunehmend die Herausforderung einer (Ent-)Solidarisierung. Entstehende regionale Disparitäten und Stadt-Land- bzw. auch Stadt-Stadt- und Land-Land-Differenzen zwischen ambitionierten und ressourcenstarken sowie zurückhaltenden und marginalisierten Einheiten weisen auf Effekte der Energiewende hin (vgl. Rodden 2019; Vogelgesang et al. 2018), die sich mit den Ansprüchen gleichwertiger Lebensverhältnisse und einer gerechten, d.h. materiell, sozial und räum- 
lich ausgeglichenen Energiewende schwerlich vereinbaren lassen (vgl. Holstenkamp/Radtke 2020; Stefansky/Göb 2018).

Vor 45 Jahren beschrieb Fritz Scharpf Verflechtungsfallen als zentrales Problem des Föderalismus (vgl. Scharpf et al. 1976). Für die Energiewende diagnostiziert Arthur Benz aktuell das Erfordernis einer stärkeren Verflechtung, um den auseinanderfallenden und zersplitterten regionalen Zuständen wirksam zu begegnen (vgl. Benz 2019). Womöglich fällt die stringente Umsetzung der Energiewende jedoch schlicht den ungleichzeitigen Dynamiken von Akteuren, Unternehmen, Märkten und Technologien zum Opfer, die durch ihre situativ-fluiden Teilstrukturen und Eigenlogiken einen unübersichtlichen Komplex schaffen, der sich auch mittels moderner Governance nicht so einfach zielgerichtet und harmonisierend lenken lässt. Im Sinne Luhmanns kann Komplexitätsreduktion Governance-Systeme jenseits spezifischer Problemlagen überfordern, da Governance in einer immer komplexer werdenden Welt naturgemäß komplizierter geworden ist (vgl. Schimank 2019). Daher müssen zeitgemäße Governance- bzw. Policy-Tools hochgradig lernfähig sein (vgl. Dunlop et al. 2018; Tosun et al. 2019). Hinzu kommt, dass sich eine Mehrebenen-Problematik auch supranational auf der Ebene der Europäischen Union beobachten lässt: Eine harmonisierte Energie(wende)politik ist bis dato an den heterogenen Interessenlagen der Mitgliedsstaaten gescheitert (vgl. Gawel et al. 2019). Schließlich spielt im Vergleich zur Energiepolitik vergangener Tage heute die individuelle bzw. die Ebene der Verbraucher*innen eine wichtigere Rolle. Durch die Artikulation von Präferenzen via Stromanbieterwahl, Meinungsumfragen und Bürgerentscheide (verbunden mit politischer Rezeption) wird weit mehr Einfluss genommen als früher. In Kombination mit »starken « Städten, die etwa eine vollständig erneuerbare Energieversorgung anstreben (wie aktuell in diversen deutschen Großstädten $\mathrm{zu}$ beobachten ist), entstehen wirkmächtige Konstellationen, die mögliche Optimierungspotentiale der Koordination durch mehr Verflechtung oder eine stärker »top-down«-orientierten Energiepolitik in Frage stellen.

Sehr bald werden die ersten der durch das Erneuerbare-Energien-Gesetz für 20 Jahre gesetzlich garantierten Einspeisevergütungen auslaufen (vgl. Lehmann et al. 2017). Die letzte Umstellung der Förderlogik auf ein Ausschreibungsmodell, das mehr Wettbewerb und Chancengleichheit erzeugen sollte, war wenig erfolgreich, da kleinere Unternehmen tendenziell benachteiligt werden (vgl. Fiedler 2017; Ohlhorst 2018, 2019). Nach der Subventionslogik sollen Förderquoten für Erneuerbare Energien-Anlagen abgesenkt werden, bis die Technologien Marktreife erlangt haben, also unabhängig von etwaigen 
Fördermitteln nachgefragt werden ${ }^{6}$. In der Kritik standen zuvor Einzelpersonen (wie etwa Landwirt*innen), die durch großflächige Installationen etwa von Photovoltaikanlagen sehr hohe Erträge erzielen konnten (die sich aus der EEG-Umlage als Bestandteil des Strompreises finanzieren, was ebenfalls kritisiert wird) (vgl. Andor et al. 2015; Schaefer 2017). Der mittlerweile deutlich abgesenkte Fördersatz für Photovoltaik hat $\mathrm{zu}$ einem Einbruch beim Zubau geführt. In Reaktion legen manche politische Einheiten (wie Bundesländer, Kreise und Kommunen) eigene Förderprogramme auf (so zuletzt Nordrhein-Westfalen Anfang 2020), was wiederum befördern kann.

Es kann schon heute konstatiert werden, dass ein Anreiz- und Wettbewerbsmodell, das die vermeintlich effektvollsten Klimaschutzaktivitäten belohnt, eine Energielandschaft erzeugt, die demokratischen Ansprüchen an Gerechtigkeit und Gleichheit nicht gerecht wird. Das staatliche Handeln und die Energiepolitik der vergangenen zwei Jahrzehnte wirken in ihrer Ausrichtung unklar und ohne starken Steuerungsanspruch. Mit Blick auf die historische Ausgangslage wäre - so der Anspruch besteht, ein hohes Maß an Gestaltungsmacht und Kontrolle zu entfalten - ein entschiedeneres Management wohl von Anfang an erforderlich gewesen. Weder wurde die Energiewende in Bürgerhand gelegt, noch wurde ein Gesamtkonzept erarbeitet. So ist zu vermuten, dass es in den nächsten Jahren zu einer grundlegenden Revision der Energiepolitik kommen wird (vgl. Wright 2020) - insbesondere, weil sich das gegenwärtige System vermutlich nicht in die gewünschte Richtung einer vollständig auf erneuerbaren Energieträgern basierenden Versorgung in den Sektoren Strom, Wärme und Verkehr bei gleichzeitig hoher Akzeptanz, Partizipation und Konsensbildung in Politik, Wirtschaft und Zivilgesellschaft entwickeln wird.

\section{Diesseits von Roadmap und Rebound: Plädoyer für eine reflexive Energiewende-Governance}

Die Zeiten glaubwürdiger Roadmaps sind, so es sie jemals gab, mittlerweile wohl vorüber; die Energiewende ist mit zunehmendem Fortschreiten tat-

6 Vgl. hierzu etwa die energiepolitischen Stellungnahmen in den Jahresgutachten des Sachverständigenrates zur Begutachtung der gesamtwirtschaftlichen Entwicklung (online verfügbar unter: https://www.sachverstaendigenrat-wirtschaft.de/publikationen/jahresgutachten.html, zuletzt aufgerufen am: 27.02.2020). 
sächlich anspruchsvoller und komplizierter geworden. Gleichzeitig drohen unerwünschte Nebeneffekte, Widersprüchlichkeiten und Heterogenitäten höhere Ziele der Energiewende - Klimaschutz und eine Umsetzung, die Mitbestimmung durch verschiedene Akteure und Bürger*innen zulässt, bestehende Systeme idealiter gerechter macht und so öffentliche Unterstützung und Legitimation erreicht $-\mathrm{zu}$ konterkarieren (vgl. Brand/Wissen 2011). Wenngleich die Governance der Energiewende sowie allgemein von Transformationsprozessen, die auf eine nachhaltigere Gesellschaft abzielen, gewiss kein leichtes Unterfangen ist, handelt es sich beim Staat noch am ehesten um diejenige Instanz, die einem Super Wicked Problem wie dem Klimawandel zu begegnen vermag. Obschon Nachhaltigkeit als Anforderung und Qualitätsmerkmal an Produkte und Dienstleistungen neue Wertschöpfungsquellen erschließt, ist nicht zu erwarten, dass sich Unternehmen dem Klimaschutz in einer Weise annehmen, die großmaßstäbliche Veränderungen im Sinne einer Transformation erwarten lässt und sich nicht in Rebound-Effekten erschöpft. Nur (kleine) Teile der Zivilgesellschaft können es sich materiell erlauben, sich dauerhaft emanzipatorischen Projekten zu widmen (vgl. Wright 2017: 18ff.). Ob für Aktivist*innen der Fridays for Future-Bewegung, aus Sicht von Umwelt- und Naturschutzverbänden oder in zeitgenössischen Veröffentlichungen der Transition Studies (vgl. Edmondson et al. 2018; Kern/Rogge 2018): Staat und Politik gelten als ausschlaggebend für die tatsächliche Implementation von Nachhaltigkeitstransformationen jenseits einschlägiger gesellschaftlicher Nischen und Freiräume.

Solchen Vorstellungen liegt offenkundig ein unterkomplexes Verständnis von Staat und Politik zugrunde. Zweifelsohne handelt der Staat in der Demokratie im Auftrag der Bevölkerung und muss zudem zahlreiche Akteure und Institutionen, die wesentliche Einflüsse auf Erfolg und Misslingen des Energiewende-Projekts ausüben, berücksichtigen. In Deutschland war staatliches Handeln bezogen auf die Energiepolitik immer zwischen vollständig eigenverantwortlicher Bewältigung der ihm zugeschriebenen Aufgaben und der Delegation derselben zerrissen (vgl. Radtke 2018b). In den letzten Jahrzehnten wurden staatliche Zuständigkeiten zunehmend abgebaut, der Nationalstaat in seiner pastoral-fürsorglichen Gestalt als Wohlfahrtsstaat verschwimmt (vgl. Hurrelmann et al. 2008; Möllers 2008; Obinger et al. 2010; Voßkuhle et al. 2013) und nach wie vor besteht die Frage, ob das Konzept von (National-)Staatlichkeit zwischen nationaler Identität und Authentizität und übergeordneter ideeller Verortung im europäischen und internationalen Maßstab nicht »überdehnt« und damit geschwächt zu werden droht (vgl. 
Brock 2018). Dem stehen radikale Gegenmodelle gegenüber: Dirk Jörke (2019) plädiert für kleinere politische Einheiten, die losgelöst von national- und suprastaatlichen Ambitionen und Strukturen autarker werden, und Benjamin Barber (2013) zeichnete eine Regierung der Bürgermeister im Zeitalter der Städte als einzig überzeugende und verlässliche Alternative. Demgegenüber weist Jonathan Franzen (2020) darauf hin, dass New York City als grünes Utopia nicht zielführend sei, wenn Lebenswelten und Wirtschaftsweisen in Texas wenig nachhaltig sind - der Wandel müsste demnach überall einsetzen. Andere Ansätze zielen auf die Ertüchtigung von Repräsentativorganen und staatlichen Institutionen, um ihnen zu mehr Handlungsmacht, Funktionsfähigkeit und damit auch erhöhter Legitimationskraft zu verhelfen (vgl. Meinel 2019; SRU 2019).

Wenn man die Energiewende als ein reformatorisch-transformatives Projekt begreift, das auf Planung, Aushandlung und Umsetzung basiert, geraten die Prämissen des »langfristigen Planens « und die grundsätzlichen »Grenzen der Steuerbarkeit« (vgl. Kamp 2016) in den Blick. Die kritische Planungstheorie kennt schon lange Paradoxien und Unmöglichkeiten bei der Umsetzung von Visionen, die an komplexen, verschachtelten und beständig variierenden Bedingungen in der Praxis und vor Ort scheitert (vgl. Eraydın/Frey 2019; Gualini 2015; Metzger et al. 2015; Palermo/Ponzini 2010). Großmaßstäbliche Planung und demokratische Ansprüche stehen vermutlich in einem antagonistischen Verhältnis (vgl. Römmele/Schober 2013) - wenn viel Partizipation auch viel Einfluss bedeuten soll, dann wirkt sie sich zwangsläufig auch auf den Planungsprozess aus, dann ändern sich Ziele, werden Ansprüche und Abläufe verschoben, kommt es zum Aufschub der Realisierung von Maßnahmen, deren Inhalt sich ständig verändert. Uwe Schimank stellte angesichts des aktuell dominierenden, projektorientiert-flexiblen Politikstils die Frage, inwieweit in Zeiten eines Governance- und Politikstils des Muddling Through nur noch Coping zu erwarten sei (vgl. Schimank 2011 sowie auch Czada 2019). Schon vor einigen Jahren beschrieb James Meadowcroft für die Transformation des Energiesystems einen erwartbaren »messy, conflictual, and highly disjointed process « (Meadowcroft 2009: 323). Für Erik Swyngedouw stellt die zeitgenössische Governance gar das Kennzeichen einer postpolitischen Konstellation dar, die Nicht-Nachhaltigkeit zu verantworten hat (vgl. Swyngedouw 2005, 2010; Wilson/Swyngedouw 2014) - denn Management, Effizienz und Kontrolle diktierten das Handeln und führten zur Entpolitisierung, einer Entkernung 
des politischen Moments im »Sog der Technokratie« (Habermas 2013), kurz: zu einer »unpolitischen Demokratie« (Michelsen/Walter 2013).

Solchen eher pragmatischen, teils ernüchtert wirkenden Diagnosen steht eine gewisse, dem Klimaschutz innewohnende Radikalität gegenüber. Es ist nachvollziehbar, dass junge Menschen für mehr Klimaschutz demonstrieren und dabei insbesondere von Politik, Parlamenten und Regierungen konsequenteres Handeln einfordern. Die grundsätzliche Frage, ob entschiedeneres politisches Handeln stattfände, wenn die normativen Prinzipien der gewachsenen modernen Demokratie, des Staates und der Gesellschaft nur ernst genommen würden, ist kaum zu beantworten. Häufig scheint dies auf den ersten Blick immerhin theoretisch möglich. Eingedenk der Handlungslogiken, Strukturprinzipien und Rationalitäten der Gesellschaft der Singularitäten - dem extremen Zersplittern gesellschaftlicher Lebenswelten in Einzelkonstruktionen und Peer-Group-Identitäten (vgl. Reckwitz 2017) - scheint indes nur eine Harmonisierung (genauer: eine Neuausrichtung zeitgenössischer Arbeits, Lebens-, Steuerungs- und Wirtschaftsweisen) dem Ziel einer genuin nachhaltigen Gesellschaft wirklich zuträglich. Dies mag nicht zwangsläufig das Ende des liberalen Zeitalters bedeuten, aber immerhin das Auslaufen eines ausdifferenzierten Systems der Lebensweisen, denn nicht-nachhaltige Praktiken wären dann nicht mehr opportun. Kritische Denker wie Ingolfur Blühdorn (2013) verweisen auf eine fundamentale Inkongruenz zwischen dem Gesellschaftsmodell westlicher Prägung und Nachhaltigkeit, weshalb es zu Simulationen käme, unter deren Deckmantel nicht-nachhaltige Praktiken sogar verstärkt wirkten. Tatsächlich handelt es sich bei der Energiewende um ein fortgeschrittenes Projekt der ökologischen, technikbasierten und positivistisch gedachten Modernisierung (vgl. Bemmann/Metzger/von Roderich 2014; Krüger 2015; von Prittwitz 1993) - mithilfe von Technologie und Ökonomie wird ein Lösungsansatz in Anschlag gebracht - und nicht um eine Suffizienzstrategie oder ein kreislaufhaftes Cradle-to-Cradle-Modell (vgl. McDonough/Braungart 2010), die zum Erreichen der Nachhaltigkeitsziele höchstwahrscheinlich geeigneter wären, aber der Gesellschaft Verzicht abverlangen.

In diesem Sinne kann die Energiewende durchaus als Fortsetzung des klassischen technischen Fortschrittsdenkens und der Modernisierung gelesen werden. Windenergie stellt etwa im Vergleich zu einem Kohlekraftwerk die effizientere und nützlichere, kurzum: die fortschrittlichere Technologie dar (vgl. Lenferna 2020). Der Einsatz fossiler Energieträger erscheint mit Abstand betrachtet auch im eigentlichen Wortsinne fossil, entstammt er doch noch den Anfängen der Industriellen Revolution. So stehen wir heute erst am 
Anfang eines anderen Energiezeitalters, das wohl weiterhin stark auf regenerativ erzeugten Strom setzen wird, aber durchaus auch andere Energieformen erschließen könnte und sich die künstliche Intelligenz vermutlich in erheblichem Maße zu Nutze machen wird. Die Steuerung und das Management des Energiesystems werden zukünftig stärker digitalisiert stattfinden (wenngleich zumindest von Seiten der Industrie Digitalisierung und Energiewende aktuell keineswegs zwangsläufig zusammengedacht werden, vgl. Knobbe/Beckamp/Götz in diesem Band). Dies könnte helfen, einige Steuerungsprobleme zu bewältigen, bedeutet aber auch ein Mehr an Komplexität. Politik, Staat und Gesellschaft müssen auch in Zukunft Formen der Zusammenarbeit, der Entscheidungsfindung und konzertierten Aktion finden, die im Sinne der reflexiven Governance gestaltet sind. So gibt es womöglich einen (im Titel des Beitrags lakonisch in Frage gestellten) Nutzen der Wende in Gedanken, wenn jene darin besteht, Energiewende und Nachhaltigkeit nicht mehr nur als eine Frage persönlicher Einstellungen und individuellen Verhaltens, als emanzipatorisches Nischenprojekt ökologisch orientierter Milieus oder als gewaltige Heraus- und Überforderung zeitgenössischer Governance zu betrachten - sondern als Komplex aus alledem. Im Sinne einer Just Transition und reflexiver Governance (vgl. Feindt/Weiland 2018; Healy/Barry 2017; Heffron/McCauley 2018; Voß/Bornemann 2011) besteht die Grundlage einer besseren Energiewende-Governance in der Anerkennung eines Nebeneinanders von Sachzwang und Partizipation, technischem Optimum und gesellschaftlicher Akzeptabilität, konzertierter Aktion und Freiräumen zur Aneignung, das nicht destruktiv oder neutralisierend wirken muss, sondern auch befruchtend sein kann. Beständiges Ausprobieren, Überprüfen und Nachjustieren gehören idealiter ebenso dazu wie das Zusammendenken mit gleichzeitig stattfindenden gesellschaftlichen Meta-Transformationen (Megatrends), wie es in diesem Band in einigen Beiträgen durchdekliniert wird. Zweifellos läuft die Energiewende derzeitig Gefahr, sich zwischen widerstreitenden Deutungen, Interessenlagern und politischer Zögerlichkeit zu zerreiben und darüber sowohl sachlich-technische Zielmarken zu verfehlen als auch ihr partizipatives Moment, Legitimationskraft und Offenheit einzubüßen. Indes gibt es auch Stimmen, die in den Widersprüchen existierender Systeme Chancen für die Diffusion alternativer Modi des Lebens und Wirtschaftens erkennen, sofern solche Ideen bereits erprobt werden (vgl. Wright 2017, siehe zudem Überlegungen zur Verknüpfung der Transition-Forschung mit theoretischen Arbeiten $\mathrm{zu}$ policy processes bei Kern/Rogge 2018). 
Für die Forschung erscheint die Befassung mit Nachhaltigkeitstransformationen auch weiterhin vielversprechend, da die einschlägigen Prozesse naturgemäß von hoher Komplexität sind, die hohe Anforderungen an die konzeptionelle Tiefe der Modelle stellt, mithilfe derer politisches Handeln im Wechselspiel mit anderen Entwicklungen und im Kontext der bestehenden Rahmenbedingungen verortet und analysiert wird (vgl. Kern et al. 2019). Ebenso, wie wichtige Hinweise zur Gestaltung von Transformationen erarbeitet werden können, sind Ergebnisse zu erwarten, die fruchtbar für die Weiterentwicklung der beteiligten Wissenschaften sind und auch in nichttransformativ orientierten Forschungszusammenhängen gewinnbringend zur Anwendung gebracht werden können. Unabhängig vom Selbstverständnis der Forschung bieten sich hier also Potentiale, erstens theoretische und konzeptionelle Modelle zu vertiefen sowie zweitens die (Nicht-)Steuerbarkeit grundlegender Transformationsprozesse besser zu verstehen. So ergäbe sich ein Wissen, das anzuwenden eine wissenschaftliche Forderung oder gar Motivation seiner Genese, aber auch Ergebnis eines demokratischen Aushandlungsprozesses sein kann. Die Dialektik der Energiewende zwischen dem normativen Anspruch der »weiteren Verankerung des Prinzips der Nachhaltigkeit als Grundlage für die weitere Entwicklung der Gesellschaft und der Lebensstile der Menschen « (Ethik-Kommission Sichere Energieversorgung 2011: 16) im Sinne einer demokratischen Gemeinschaftsaufgabe und der stärker umsetzungs- und steuerungsbezogenen, einst durch Angela Merkel so bezeichneten Herkulesaufgabe (SZ.de 2011), die angesichts des rapide fortschreitenden Klimawandels rasch bewältigt werden muss, wird in verschiedensten Konstellationen weiterhin prägend sein, und sie wird Wissenschaft wie Gesellschaft, Politik wie Unternehmen auch zukünftig herausfordern.

\section{Literatur}

Agentur für Erneuerbare Energien (AEE) (2019): Wichtig für den Kampf gegen den Klimawandel: Bürger*innen wollen mehr Erneuerbare Energien. Online verfügbar unter: https:/www.unendlich-viel-energie.de/themen/ akzeptanz-erneuerbarer/akzeptanz-umfrage/akzeptanzumfrage-2019. Zuletzt aufgerufen am: 25.02.2020. 
Andor, Mark A./Frondel, Manuel/Sendler, Sophie (2015): Photovoltaik-Anlagen in Deutschland: Ausgestattet mit der Lizenz zum Gelddrucken?, RWI Materialien 94, Essen: RWI.

Aykut, Stefan C./Neukirch, Mario/Zengerling, Cathrin/Engels, Anita/Suhari, Mirko/Pohlmann, Angela (2019): »Energiewende ohne gesellschaftlichen Wandel? Der blinde Fleck in der aktuellen Debatte zur »Sektorkopplung«, in: ET. Energiewirtschaftliche Tagesfragen 69 (3), S. 20-24.

Barber, Benjamin R. (2013): If Mayors Ruled the World. Dysfunctional Nations, Rising Cities, New Haven: Yale University Press.

Batel, Susana/Devine-Wright, Patrick/Wold, Line/Egeland, Helene/Jacobsen, Gerd/Aas, Oystein (2015): »The role of (de-)essentialisation within siting conflicts: An interdisciplinary approach«, in: Journal of Environmental Psychology 44, S. 149-159. https://doi.org/10.1016/j.jenvp.2015.10.004

Bauer, Christian (2015): »Stiftung von Legitimation oder Partizipationsverflechtungsfalle. Welche Folgen hat die Öffentlichkeitsbeteiligung beim Stromnetzausbau?«, in: der moderne staat 8 (2), S. 273-293. https://doi. org/10.3224/dms.v8i1.21191

Bemmann, Martin/Metzger, Birgit/Detten, Roderich von (2014): Ökologische Modernisierung: Zur Geschichte und Gegenwart eines Konzepts in Umweltpolitik und Sozialwissenschaften, Frankfurt a.M.: campus.

Bendlin, Lena (2020): Orchestrating Local Climate Policy in the European Union - Inter-Municipal Coordination and the Covenant of Mayors in Germany and France, Wiesbaden: Springer VS. https://doi.org/10.1007/ 978-3-658-26506-9

Benz, Arthur (2019): »Koordination der Energiepolitik im deutschen Bundesstaat«, in: der moderne staat 12 (2), S. 299-312. https://doi.org/10.3224/ dms.v12i2.o1

Benz, Arthur/Czada, Roland (2019): »Politische Steuerung von Transformation - das Beispiel der Energiepolitik«, in: der moderne staat 12 (2), S. 243-250. https://doi.org/10.3224/dms.v12i2.05

Bertsch, Valentin/Hall, Margeret/Weinhardt, Christof/Fichtner, Wolf (2016): "Public acceptance and preferences related to renewable energy and grid expansion policy: Empirical insights for Germany«, in: Energy 114, S. 465477. https://doi.org/10.1016/j.energy.2016.08.022

Bickerstaff, Karen/Walker, Gordon/Bulkeley, Harriet (Hg.) (2013): Energy Justice in a Changing Climate: Social Equity and Low-Carbon Energy, London: Zed Books. 
Blühdorn, Ingolfur (2013): Simulative Demokratie. Neue Politik nach der postdemokratischen Wende, Berlin: Suhrkamp.

Blühdorn, Ingolfur/Butzlaff, Felix (2019): »Rethinking Populism. Peak democracy, liquid identity and the performance of sovereignty", in: European Journal of Social Theory 22 (2), S. 191-211. https://doi.org/10.1177/ 1368431017754057

Blythe, Jessica/Silver, Jennifer/Evans, Louisa/Armitage, Derek/Bennett, Nathan J./Moore, Michelle Lee/Morrison, Tiffany H./Brown, Katrina (2018): "The Dark Side of Transformation: Latent Risks in Contemporary Sustainability Discourse«, in: Antipode 50 (5), S. 1206-1223. https://doi.org/10.1111/anti.12405

Bößner, Stefan (2020): »The Role of German Regime Actors and Trade Unions in the Energy Transition: Agency and Power«, in: Geoffrey Wood/Keith Baker (Hg.), The Palgrave Handbook of Managing Fossil Fuels and Energy Transitions, Basel: Springer International Publishing, S. 341-369. https:// doi.org/10.1007/978-3-030-28076-5_12

Brand, Ulrich/Wissen, Markus (2017): Imperiale Lebensweise: Zur Ausbeutung von Mensch und Natur im globalen Kapitalismus, München: oekom.

Brand, Ulrich/Wissen, Markus (2011): »Die Regulation der ökologischen Krise. Theorie und Empirie der Transformation gesellschaftlicher Naturverhältnisse«, in: Österreichische Zeitschrift für Soziologie 36 (2), S. 12-34.

Brandmeyer, Paula (2013): »Kommunen unter Strom: Rekommunalisierung in Zeiten der Energiewende«, in: Verwaltung \& Management 19 (6), S. 313322.

Breukers, Sylvia/Mourik, Ruth/Summeren, Luc F. M. van/Verbong, Geert (2017): »Institutional >lock-out< towards local self-governance? Environmental justice and sustainable transformations in Dutch social housing neighbourhoods«, in: Energy Research \& Social Science 23, S. 148-158. https://doi.org/10.1016/j.erss.2016.10.007

Brock, Ditmar (2018): Die überdehnte Staatlichkeit: Warum der Westen scheitern könnte, Wiesbaden: Springer. https://doi.org/10.1007/978-3658-19367-6

Brüggemeier, Franz-Josef (2015): Sonne, Wasser, Wind: Die Entwicklung der Energiewende in Deutschland, Bonn: Friedrich-Ebert-Stiftung.

Bulling, Lena/Sudhaus, Dirk/Schnittker, Daniel/Schuster, Eva/Biehl, Juliane/ Tucci, Franziska (2015): Vermeidungsmaßnahmen bei der Planung und Genehmigung von Windenergieanlagen: Bundesweiter Katalog von Maßnahmen zur Verhinderung des Eintritts von artenschutzrechtlichen 
Verbotstatbeständen nach $\$ 44$ BNatSchG, Berlin: Fachagentur Windenergie an Land. Online verfügbar unter: https://www.fachagenturwindenergie.de/fileadmin/files/Veroeffentlichungen/FA-Wind_Studie_ Vermeidungsmassnahmen_10-2015.pdf. Zuletzt aufgerufen am: 28.02.2020.

Burke, Matthew J./Stephens, Jennie C. (2017): »Energy democracy: Goals and policy instruments for sociotechnical transitions«, in: Energy Resarch \& Social Science 33, S. 35-48. https://doi.org/10.1016/j.erss.2017.09.024

Chatalova, Lioudmila/Valentinov, Vladislav (2014): "Die zwielichtige Rolle der Energie-Genossenschaften«, in: Zeit Online vom 20.04.2014. Online verfügbar unter: https://www.zeit.de/wissen/umwelt/2014-04/ energiegenossenschaften-rendite. Zuletzt aufgerufen am 30.01.2020.

Chemnitz, Christine (2018): »Der Mythos vom Energiewendekonsens. Ein Erklärungsansatz zu den bisherigen Koordinations- und Steuerungsproblemen bei der Umsetzung der Energiewende im Föderalismus«, in: Jörg Radtke/Norbert Kersting (Hg.), Energiewende. Politikwissenschaftliche Perspektiven. Schriftenreihe Energietransformation, Wiesbaden: Springer VS, S. 155-203. https://doi.org/10.1007/978-3-658-21561-3_6

Czada, Roland (2019): »Politikwenden und transformative Politik in Deutschland«, in: der moderne staat 12 (2), S. 400-417. https://doi.org/10.3224/ dms.v12i2.12

Debor, Sarah (2018): Multiplying Mighty Davids? The Influence of Energy Cooperatives on Germany's Energy Transition, Wiesbaden: Springer. https:// doi.org/10.1007/978-3-319-77628-6

Dunlop, Claire/Radaelli, Claudio M./Trein, Philipp (2018): Learning in Public Policy: Analysis, Modes and Outcomes, Cham: Palgrave Macmillan. https://doi.org/10.1007/978-3-319-76210-4

Eberlein, Burkard/Newman, Abraham L. (2008): »Escaping the International Governance Dilemma? Incorporated Transgovernmental Networks in the European Union«, in: Governance 21 (1), S. 25-52. https://doi.org/10.1111/j. 1468-0491.2007.00384.x

Edmondson, Duncan L./Kern, Florian/Rogge, Karoline S. (2018): »The CoEvolution of Policy Mixes and Socio-Technical Systems: Towards a conceptual framework of policy mix feedback in sustainability transitions «, in: Research Policy 48 (10), 103555. https://doi.org/10.1016/j.respol.2018.03. 010

Eiselt, Jürgen (2012): Dezentrale Energiewende: Chancen und Herausforderungen, Wiesbaden: Springer Vieweg+Teubner. 
Ekart, Felix (2018): Ökonomische Bewertung - Kosten-Nutzen-Analyse - ökonomische Ethik. Eine Kritik am Beispiel Klimaschutz - zugleich zu Zahlen im Nachhaltigkeitsdiskurs. Beiträge zur sozialwissenschaftlichen Nachhaltigkeitsforschung 25, Marburg: Metropolis.

Elliott, David (2020): »Carbon Capture and Renewables: Strategic Conflicts or Tactical Complementarities«, in: Geoffrey Wood/Keith Baker (Hg.), The Palgrave Handbook of Managing Fossil Fuels and Energy Transitions, Basel: Springer International Publishing, S. 25-55. https://doi.org/10.1007/ 978-3-030-28076-5_12

Elsen, Susanne (2012): »Genossenschaften als Organisationen der sozialen Innovation und nachhaltigen Entwicklung«, in: Gerald Beck/Cordula Kropp (Hg.), Gesellschaft innovativ. Wer sind die Akteure? Wiesbaden: Springer VS, S. 85-102. https://doi.org/10.1007/978-3-531-94135-6_5

Engelken, Maximilian/Römer, Benedikt/Drescher, Marcus/Welpe, Isabell (2016): »Transforming the energy system: Why municipalities strive for energy self-sufficiency«, in: Energy Policy 98, S. 365-377. https://doi. org/10.1016/j.enpol.2016.07.049

Eraydın, Ayda/Frey, Klaus (Hg.) (2019): Politics and Conflict in Governance and Planning. Theory and Practice, New York: Routledge. https://doi.org/10. 4324/9781351252881

Ethik-Kommission Sichere Energieversorgung (2011): Deutschlands Energiewende - Ein Gemeinschaftswerk für die Zukunft, Berlin. Online verfügbar unter: https://archiv.bundesregierung.de/resource/blob/ 656922/394384/962bafo9452793c8a87402c9ee347379/2011-07-28-abschluss bericht-ethikkommission-data.pdf?download=1. Zuletzt aufgerufen am 26.02.2020.

Feindt, Peter H./Weiland, Sabine (2018): »Reflexive governance: Exploring the concept and assessing its critical potential for sustainable development. Introduction to the special issue«, in: Journal of Environmental Policy \& Planning, 20 (6), S. 661-674. https://doi.org/10.1080/1523908X.2018. 1532562

Fiedler, Malte (2017): Die Umstellung von der staatlich festgelegten Vergütungshöhe auf das Ausschreibungsmodell: Risiken für Akteursvielfalt und Bürgerwindparks, Berlin: BWV Berliner Wissenschafts-Verlag.

Fischer, Beate/Moser, Peter/Schenk, Katharina (2015): Zukunftsfelder der Energieversorgung: Entwicklungsoptionen, Interessenlagen und Strukturen in drei erneuerbaren Entwicklungspfaden, Kassel: Institut dezentrale Energietechnologien. 
Franzen, Jonathan (2020): Wann hören wir auf, uns etwas vorzumachen? Gestehen wir uns ein, dass wir die Klimakatastrophe nicht verhindern können, Hamburg: Rowohlt.

Gailing, Ludger/Röhring, Andreas (2015): »Was ist dezentral an der Energiewende? Infrastrukturen erneuerbarer Energien als Herausforderungen und Chancen für ländliche Räume«, in: Raumforschung und Raumordnung 73 (1), S. 31-43.

Galvin, Ray (2018): »Them and us': Regional-national power-plays in the German energy transformation: A case study in Lower Franconia«, in: Energy Policy 113, S. 269-277. https://doi.org/10.1016/j.enpol.2017.11.016

Gawel, Erik/Strunz, Sebastian/Lehmann, Paul/Purkus, Alexandra (Hg.) (2019): The European Dimension of Germany's Energy Transition: Opportunities and Conflicts, Basel: Springer International Publishing. https://doi. org/10.1007/978-3-030-03374-3

Geels, Frank W. (2014): »Regime Resistance against Low-Carbon Transitions: Introducing Politics and Power into the Multi-Level Perspective«, in: Theory, Culture \& Society 31 (5), S. 21-40. https://doi.org/10.1177/ 0263276414531627

Geels, Frank W./Kern, Florian/Fuchs, Gerhard/Hinderer, Nele/Kungl, Gregor/Mylan, Josephine/Neukirch, Mario/Wassermann, Sandra (2016): »The enactment of socio-technical transition pathways: A reformulated typology and a comparative multi-level analysis of the German and UK low-carbon electricity transitions (1990-2014)«, in: Research Policy 45 (4), S. 896913. https://doi.org/10.1016/j.respol.2016.01.015

Gossen, Meike/Scholl, Gerd/Holzhauer, Brigitte/Schipperges, Michael (2016): Umweltbewusstsein in Deutschland 2014. Vertiefungsstudie: Umweltbewusstsein und Umweltverhalten junger Menschen, Dessau: Umweltbundesamt. Online verfügbar unter https://www.ioew.de/file admin/user_upload/BILDER_und_Downloaddateien/Publikationen/2016/ Umweltbewusstsein_in_Deutschland_2014_Vertiefungsstudie_Jugend.pdf. Zuletzt aufgerufen am 24.02.2020.

Graf, Patricia/Kern, Kristine/Scheiner, Stefan (2018): »MehrebenenDynamiken in der deutschen Energiewendepolitik. Die Rolle von Städten und Regionen am Beispiel von Baden-Württemberg«, in: Jörg Radtke/Norbert Kersting (Hg.), Energiewende. Politikwissenschaftliche Perspektiven, Wiesbaden: Springer VS, S. 211-248. https://doi.org/10. 1007/978-3-658-21561-3_7 
Gross, Catherine (2007): "Community Perspectives of Wind Energy in Australia: The Application of a Justice and Community Fairness Framework to Increase Social Acceptance«, in: Energy Policy 35 (5), S. 2727-2736. https:// doi.org/10.1016/j.enpol.2006.12.013

Grünewald, Stefanie (2016): Die (Re)Kommunalisierung in der Energieverteilung: Grenzen und Möglichkeiten kommunaler wirtschaftlicher Betätigung in der Elektrizitätsverteilung. Zugleich eine kritische Auseinandersetzung mit dem energiewirtschaftlichen Konzessionsvergaberecht, Schriften zum Deutschen und Europäischen Infrastrukturrecht 7, Berlin: Duncker \& Humblot.

Gualini, Enrico (Hg.) (2015): Planning and Conflict: Critical Perspectives on Contentious Urban Developments, London/New York: Routledge. https:// doi.org/10.4324/9780203734933

Habermas, Jürgen (2013): Im Sog der Technokratie. Kleine Politische Schriften XII, Berlin: Suhrkamp.

Hager, Carol (2017): Grassroots Protest and Innovation: A New Look at NIMBY. Items: Insights from the Social Sciences. Online verfügbar unter: https://items.ssrc.org/just-environments/grassroots-protest-andinnovation-a-new-look-at-nimby/. Zuletzt aufgerufen am 24.02.2020.

Hager, Carol (2015): »From NIMBY to Networks: Protest and Innovation in German Energy Politics«, in: Carol Hager/Mary Alice Haddad (2015): NIMBY is beautiful. Cases of Local Activism and Environmental Innovation around the World, New York: Berghahn Books, S. 33-59.

Hake, Jürgen-Friedrich/Fischer, Wolfgang/Venghaus, Sandra/Weckenbrock, Christoph (2015): »The German Energiewende - History and status quo«, in: Energy 92, S. 532-546. http://dx.doi.org/10.1016/j.energy.2015.04.027

Hänlein, Rotraud/El Alaoui, Alexander (2015): Beteiligung und Transparenz bei der Stromnetzplanung: Handlungsempfehlungen vom BESTGRIDProjekt, Berlin: Germanwatch.

Healy, Noel/Barry, John (2017): »Politicizing energy justice and energy system transitions: Fossil fuel divestment and a »just transition «, in: Energy Policy 108, S. 451-459. https://doi.org/10.1016/j.enpol.2017.06.014

Heffron, Raphael J./McCauley, Darren (2018): »What is the >Just Transition«?«, in: Geoforum 88, S. 74-77. https://doi.org/10.1016/j.geoforum.2017.11.016 Hehn, Nina (2015): Postfossile Stadtentwicklung: Rechts- und Steuerungsprobleme einer Umsetzung kommunaler Energiewende- und Klimaschutzkonzepte im Rahmen der Stadtplanung, Marburg: Metropolis. 
Heindl, Peter/Schüßler, Rudolf/Löschel, Andreas (2014): »Ist die Energiewende sozial gerecht?«, in: Wirtschaftsdienst 94 (7), S. 508-514. https://doi. org/10.1007/s10273-014-1705-7

Herbert, Ulrich (2017): Geschichte Deutschlands im 20. Jahrhundert, München: C. H. Beck.

Herbes, Carsten/Brummer, Vasco/Rognli, Judith/Blazejewski, Susanne/Gericke, Naomi (2017): »Responding to policy change: New business models for renewable energy cooperatives - Barriers perceived by cooperatives' members«, in: Energy Policy 109, S. 82-95. https://doi.org/10.1016/j.enpol. 2017.06.051

Hermwille, Lukas (2016): »The role of narratives in socio-technical transitions - Fukushima and the energy regimes of Japan, Germany, and the United Kingdom«, in: Energy Research \& Social Science 11, S. 237-246. http://dx. doi.org/10.1016/j.erss.2015.11.001

Hildebrand, Jan/Rau, Irina/Hinse, Maximilian/Rühmland, Silke/SchweizerRies, Petra (2015): Die Rolle von Gerechtigkeitswahrnehmungen und Vertrauenszuschreibungen zwischen Akteursgruppen beim Netzausbau, in: Bundesnetzagentur (Hg.), Tagungsband Wissenschaftsdialog 2015: Wirtschaft und Technologie, Kommunikation und Planung, Bonn, S. 50-59. Online verfügbar unter: https://www.netzausbau.de/ SharedDocs/Downloads/DE/Publikationen/Tagungsband_15.pdf?_blob= publicationFile. Zuletzt aufgerufen am: 26.02.2020.

Holstenkamp, Lars/Radtke, Jörg (2020): »Stadt-Land-Disparitäten in der Energiewende«, in: Jan-Hendrik Kamlage/Steven Engler (Hg.), Dezentral, partizipativ und kommunikativ: Zukunft der Energiewende, Nordhausen: Traugott Bautz, S. 137-176.

Holstenkamp, Lars/Radtke, Jörg (Hg.) (2018): Handbuch Energiewende und Partizipation, Wiesbaden: Springer VS.

Holstenkamp, Lars/Centgraf, Salina/Dorniok, Daniel/Kahla, Franziska/Masson, Torsten/Müller, Jakob/Radtke, Jörg/Yildiz, Özgür (2018): »Bürgerenergiegesellschaften in Deutschland «, in: Lars Holstenkamp/Jörg Radtke (Hg.), Handbuch Energiewende und Partizipation, Wiesbaden: Springer, S. 1061-1080. https://doi.org/10.1007/978-3-658-09416-4_62

Hooghe, Liesbet/Marks/Gary (2001): Multi-level governance and European integration. Governance in Europe, Lanham, MD: Rowman \& Littlefield.

Huge, Antonia/Roßnagel, Alexander (2018): »Möglichkeiten der Öffentlichkeitsbeteiligung in Planungs- und Genehmigungsverfahren von Wind- 
energieanlagen«, in: Lars Holstenkamp/Jörg Radtke (Hg.), Handbuch Energiewende und Partizipation, Wiesbaden: Springer, S. 613-625.

Hurrelmann, Achim/Leibfried, Stephan/Martens, Kerstin/Mayer, Peter (2008): Zerfasert der Nationalstaat? Die Internationalisierung politischer Verantwortung, Frankfurt a.M./New York: campus.

Hübner, Gundula/Pohl, Johannes/Warode, Jan/Gotchev, Boris/Nanz, Patrizia/Ohlhorst, Dörte/Krug, Michael/Salecki, Steven/Peters, Wolfgang (2019): Naturverträgliche Energiewende. Akzeptanz und Erfahrungen vor Ort, Bundesamt für Naturschutz.

International Energy Agency (IEA) (2018): Key World Energy Statistics 2019. Online verfügbar unter: https://www.iea.org/reports/key-world-energystatistics-2019. Zuletzt aufgerufen am 26.02.2020.

Jaeger-Erben, Melanie/John, René/Rückert-John, Jana (2017): »Soziale Innovation: Verheißung oder Verführung?«, in: GAIA 26 (3), S. 245-248. https:// doi.org/10.14512/gaia.26.3.7

Jenkins, Kirsten/McCauley, Darren/Heffron, Raphael/Stephan, Hannes/

Rehner, Robert (2016): »Energy Justice: A Conceptual Review«, in: Energy Research \& Social Science 11, S. 174-182. https://doi.org/10.1016/j.erss. 2015.10.004.

Jörke, Dirk (2019): Die Größe der Demokratie. Über die räumliche Dimension von Herrschaft und Partizipation, Berlin: edition suhrkamp.

Kahla, Franziska/Holstenkamp, Lars/Müller, Jakob R./Degenhart, Heinrich (2017): Entwicklung und Stand von Bürgerenergiegesellschaften und Energiegenossenschaften in Deutschland. Arbeitspapierreihe Wirtschaft \& Recht 27, Lüneburg: Leuphana Universität. Online verfügbar unter: https://www.buendnis-buergerenergie.de/fileadmin/user_ upload/wpbl27_BEG-Stand_Entwicklungen.pdf. Zuletzt aufgerufen am 04.02.2020.

Kamlage, Jan-Hendrik/Richter, Ina/Nanz, Patrizia (2018): »An den Grenzen der Bürgerbeteiligung: Informelle dialogorientierte Bürgerbeteiligung im Netzausbau der Energiewende«, in: Lars Holstenkamp/Jörg Radtke (Hg.), Handbuch Energiewende und Partizipation, Wiesbaden: Springer, S. 627642. https://doi.org/10.1007/978-3-658-09416-4_38

Kamlage, Jan-Hendrik/Nanz, Partrizia/Fleischer, Björn (2014): »Bürgerbeteiligung und Energiewende: Dialogorientierte Bürgerbeteiligung im Netzausbau«, in: Hans-Christoph Binswanger/Felix Ekardt/Anja Grothe/WolfDieter Hasenclever/Ingomar Hauchler/Martin Jänicke/Karl Kollmann/Nina V. Michaelis/Hans G. Nutzinger/Hans Rogall/Gerhard Scherhorn 
(Hg.), Jahrbuch Nachhaltige Ökonomie 2014/15. Im Brennpunkt: Die Energiewende als gesellschaftlicher Transformationsprozess, Marburg: Metropolis, S. 195-216.

Kamp, Georg (Hg.) (2016): Langfristiges Planen. Zur Bedeutung sozialer und kognitiver Ressourcen für nachhaltiges Handeln, Berlin/Heidelberg: Springer.

Kern, Florian/Rogge, Karoline S./Howlett, Michael (2019): »Policy mixes for sustainability transitions: New approaches and insights through bridging innovation and policy studies«, in: Research Policy 48, 103832. https://doi. org/10.1016/j.respol.2019.103832

Kern, Florian/Rogge, Karoline S. (2018): »Harnessing theories of the policy process for analysing the politics of sustainability transitions: A critical survey«, in: Environmental Innovation and Societal Transitions 27, S. 102117. https://doi.org/10.1016/j.eist.2017.11.001

Klagge, Britta/Arbach, Cora (Hg.) (2013): Governance-Prozesse für erneuerbare Energien, Hannover: Akademie für Raumforschung und Landesplanung (ARL).

Krause, Florentin/Bossel, Hartmut/Müller-Reißmann, Karl-Friedrich (1980): Energie-Wende. Wachstum und Wohlstand ohne Erdöl und Uran. Ein Alternativ-Bericht; Frankfurt a.M.: S. Fischer.

Kropp, Cordula (2018): Sustainable Innovations. Theories, Conflicts and Strategies, SOI Discussion Paper 2018-02. Online verfügbar unter: https://www.sowi.uni-stuttgart.de/dokumente/forschung/soi/soi_2018_ 2_Kropp.Sustainable.Innovations.pdf. Zuletzt aufgerufen am: 24.02.2020.

Kropp, Cordula (2017a): »Forschung zu sozialen Innovationen am Scheideweg«, in: GAIA 26 (4), S. 309-312. https://doi.org/10.14512/gaia.26.4.5

Kropp, Cordula (2017b): »Controversies around energy landscapes in third modernity«, in: Landscape Research 43 (4), S. 562-573. https://doi.org/10. 1080/01426397.2017.1287890

Krüger, Timmo (2015): Das Hegemonieprojekt der ökologischen Modernisierung: Die Konflikte um Carbon Capture and Storage (CCS) in der internationalen Klimapolitik, Bielefeld: transcript.

Kühne, Olaf/Weber, Florian (Hg.) (2018): Bausteine der Energiewende, Wiesbaden: Springer VS.

Langer, Katharina/Decker, Thomas/Menrad, Klaus (2017): »Public Participation in Wind Energy Projects Located in Germany. Which Form of Par- 
ticipation Is the Key to Acceptance? «, in: Renewable Energy 112, S. 63-73. https://doi.org/10.1016/j.renene.2017.05.021

Łapniewska, Zofia (2019): „Energy, equality and sustainability? European electricity cooperatives from a gender perspective«, in: Energy Research \& Social Science 57, 101247. https://doi.org/10.1016/j.erss.2019.101247

Leggewie, Claus (2013). »Die Stadt als Gemeingut?«, in: Bund Deutscher Architekten (Hg.), Stadtquartiere sanieren: Sozial Ökologisch Ästhetisch, Berlin. S. 16-21.

Lehmann, Paul/Gawel, Erik/Korte, Klaas/Purkus, Alexandra (2017): »20 Jahre EEG: Ist das Förderende für alte Anlagen ein Problem für die Energiewende? «, in: Wirtschaftsdienst 97 (10), S. 727-732.

Lenferna, Alex (2020): »Fossil Fuel Welfare Versus the Climate«, in: Geoffrey Wood/Keith Baker (Hg.), The Palgrave Handbook of Managing Fossil Fuels and Energy Transitions, Basel: Springer International Publishing, S. 551567. https://doi.org/10.1007/978-3-030-28076-5_20

Lessenich, Stephan (2016): Neben uns die Sintflut: Die Externalisierungsgesellschaft und ihr Preis, München: Hanser Berlin.

Liebe, Ulf/Bartczak, Anna/Meyerhoff, Jürgen (2017): »A Turbine Is Not Only a Turbine. The Role of Social Context and Fairness Characteristics for the Local Acceptance of Wind Power«, in: Energy Policy 107, S. 300-308. https://doi.org/10.1016/j.enpol.2017.04.043

Lienhoop, Nele (2018): "Acceptance of Wind Energy and the Role of Financial and Procedural Participation. An Investigation with Focus Groups and Choice Experiments«, in: Energy Policy 118, S. 97-105. https://doi.org/10. 1016/j.enpol.2018.03.063

Loorbach, Derk/Wittmayer, Julia M./Shiroyama, Hideaki/Fujino, Junichi/Mizuguchi, Satoru (Hg.) (2016): Governance of Urban Sustainability Transitions: European and Asian Experiences, Tokyo: Springer Japan. https://doi.org/10.1007/978-4-431-55426-4

Löschel, Andreas/Erdmann, Georg/Staiß, Frithjof/Ziesing, Hans-Joachim (2018): Stellungnahme zum sechsten Monitoring-Bericht der Bundesregierung für das Berichtsjahr 2016. Online verfügbar unter: https:// www.zsw-bw.de/fileadmin/user_upload/PDFs/Pressemitteilungen/2018/ stellungnahme-der-expertenkommission-zum-sechsten-monitoringbericht.pdf. Zuletzt aufgerufen am: 27.02.2020.

Lovins, Amory (1977): Soft Energy Paths: Toward a Durable Peace, Hamondsworth: Penguin Books. 
Martens, Holger (2015): "Anders Wirtschaften - genossenschaftliche Selbsthilfe«, in: Aus Politik und Zeitgeschichte (APuZ) 65 (35-37), S. 40-46.

Mast, Claudia/Stehle, Helena (2016): Energieprojekte im öffentlichen Diskurs: Erwartungen und Themeninteressen der Bevölkerung, Wiesbaden: Springer VS.

Maubach, Klaus-Dieter (2014): Energiewende. Wege zu einer bezahlbaren Energieversorgung, Wiesbaden: Springer VS. https://doi.org/10.1007/978-3658-05474-8_9

Mautz, Rüdiger (2012): »Atomausstieg und was dann? Probleme staatlicher Steuerung der Energiewende«, in: der moderne staat 5 (1), S. 149-168.

McDonough, William/Braungart, Michael (2010): Cradle to Cradle: Remaking the Way We Make Things, New York: Farrar, Straus and Giroux.

Meadowcroft, James (2009): »What about the politics? Sustainable development, transition management, and long term energy transitions «, in: Policy Sciences 42 (4), S. 323-340. https://doi.org/10.1007/s11077-009-9097-Z

Meinel, Florian (2019): Vertrauensfrage: Zur Krise des heutigen Parlamentarismus, München: C. H. Beck. https://doi.org/10.17104/9783406731563

Melica, Giulia/ Bertoldi, Paolo/Kona, Albana/Iancu, Andreea/Rivas, Silvia/Zancanella, Paolo (2018): »Multilevel governance of sustainable energy policies: The role of regions and provinces to support the participation of small local authorities in the Covenant of Mayors «, in: Sustainable Cities and Society 39, S. 729-739. https://doi.org/10.1016/j.scs.2018.01.013

Metzger, Jonathan/Allmendinger, Philip/Oosterlynck, Stijn (Hg.) (2015): Planning against the political: Democratic deficits in European territorial governance, New York: Routledge. https://doi.org/10.4324/9780203522141

Mez, Lutz (2012): „Germany's merger of energy and climate change policy«, in: Bulletin of the Atomic Scientists 68 (6), S. 22-29. https://doi.org/10. 1177/0096340212464358

Michelsen, Danny/Walter, Franz (2013): Unpolitische Demokratie: Zur Krise der Repräsentation, Berlin: Suhrkamp.

Mitchell, Timothy (2011): Carbon democracy: Political power in the age of oil, London/New York: Verso.

Möllers, Christoph (2008): Der vermisste Leviathan. Staatstheorie in der Bundesrepublik, Frankfurt a.M.: Suhrkamp.

Morris, Craig/Jungjohann, Arne (2016): Energy democracy: Germany's Energiewende to Renewables, Basingstoke: Palgrave Macmillan.

Müller, Thorsten/Kahl, Hartmut (Hg.) (2015): Energiewende im Föderalismus, Baden-Baden: Nomos. https://doi.org/10.5771/9783845259253 
Müller, Jakob R./Dorniok, Daniel/Flieger, Burghard/Holstenkamp, Lars/Mey, Franziska/Radtke, Jörg (2015): »Energiegenossenschaften - das Erfolgsmodell braucht neue Dynamik«, in: GAIA 24 (2), S. 96-101. https://doi. org/10.14512/gaia.24.2.7

Nagorny-Koring, Nanja (2018): Kommunen im Klimawandel: Best Practices als Chance zur grünen Transformation?, Bielefeld: transcript.

Neukirch, Mario (2019): »Transition of Energy Systems: Patterns of Stability and Change«, in: Michèle Knodt/Jörg Kemmerzell (Hg.), Handbook of Energy Governance in Europe, Cham: Springer, S. 1-29. https://doi.org/10. 1007/978-3-319-73526-9_40-1

Newig, Jens/Fritsch, Oliver (2009): »Environmental governance: participatory, multi-level - and effective?«, in: Environmental Policy and Governance 19, S. 197-214. https://doi.org/10.1002/eet.509

Oberthür, Sebastian (2019): »Hard or Soft Governance? The EU's Climate and Energy Policy Framework for 2030«, in: Politics and Governance 7, S. 17-27. https://doi.org/10.17645/pag.v7i1.1796

Obinger, Herbert/Starke, Peter/Moser, Julia/Bogedan, Claudia/Gindulis, Edith/Leibfried, Stephan (2010): Transformations of the welfare state. Small states, big lessons, Oxford: Oxford University Press. https://doi. org/10.1093/acprof:oso/9780199296323.001.0001

Ohlhorst, Dörte (2019): »Biographie der Energiewende im Stromsektor«, in: Jörg Radtke/Weert Canzler (Hg.), Energiewende: Eine sozialwissenschaftliche Einführung, Wiesbaden: Springer VS, S. 97-122.

Ohlhorst, Dörte (2018): »Akteursvielfalt und Bürgerbeteiligung im Kontext der Energiewende in Deutschland: Das EEG und seine Reform «, in: Lars Holstenkamp/Jörg Radtke (Hg.), Handbuch Energiewende und Partizipation, Wiesbaden: Springer, S. 101-124.

Paech, Niko (2014): »Nachhaltigkeit jenseits grüner Wachstumsillusionen: Skizzen einer Postwachstumsökonomie«, in: Frieder Kunz/Gerhard Roller/Karlheinz Scheffold (Hg.), Konfliktfelder und Perspektiven im Umweltschutz: Einstieg in ein interdisziplinäres Studienfeld von Ökologie bis Suffizienz, München: oekom, S. 261-275.

Palermo, Pier Carlo/Ponzini, Davide (2010): Spatial Planning and Urban Development. Critical Perspectives. Urban and Landscape Perspectives 10, Dordrecht u.a.: Springer.

Pesch, Udo/Correljé, Aad/Cuppen, Eefje/Taebi, Behnam (2017): »Energy justice and controversies: Formal and informal assessment in energy 
projects«, in: Energy Policy 109, S. 825-834. https://doi.org/10.1016/j.enpol. 2017.06.040

Poppen, Silvia (2015): Energiegenossenschaften und deren Mitglieder - Erste Ergebnisse einer empirischen Untersuchung. Arbeitspapiere des Instituts für Genossenschaftswesen der Westfälischen Wilhelms-Universität Münster 157, Münster: Westfälische Wilhelms-Universität Münster. Online verfügbar unter: https://www.econstor.eu/bitstream/10419/123711/1/ 841415013.pdf. Zuletzt aufgerufen am: 11.02.2020.

Prittwitz, Volker von (1993): Umweltpolitik als Modernisierungsprozeß: Politikwissenschaftliche Umweltforschung und -lehre in der Bundesrepublik Deutschland, Opladen: Leske + Budrich.

Radtke, Jörg (2016): Bürgerenergie in Deutschland. Partizipation zwischen Gemeinwohl und Rendite, Wiesbaden: Springer. https://doi.org/10.1007/ 978-3-658-14626-9

Radtke, Jörg (2018a): »Energiedemokratie durch Bürgerenergie? Die Grenzen finanzieller Bürgerbeteiligung«, in: GAIA 2018 (3), S. 284-286. https://doi. org/10.14512/gaia.27.3.7

Radtke, Jörg (2018b): »Energie«, in: Rüdiger Voigt (Hg.), Handbuch Staat, Wiesbaden: Springer, S. 1411-1421. https://doi.org/10.1007/978-3658-20744-1_127

Radtke, Jörg (2018c): »Die Mehrebenen-Architektur der Energiewende: Drei Modelle im Vergleich «, in: Lars Holstenkamp/Jörg Radtke (Hg.), Handbuch Energiewende und Partizipation, Wiesbaden: Springer, S. 793-814. https://doi.org/10.1007/978-3-658-09416-4_47

Rave, Tilmann (2016): Der Ausbau Erneuerbarer Energien im Föderalismus und Mehrebenensystem. Neoklassische und neo-institutionalistische Perspektiven, München: ifo Institut. Online verfügbar unter: https://www. econstor.eu/bitstream/10419/167435/1/ifo-Forschungsberichte-75.pdf. Zuletzt aufgerufen am: 26.02.2020.

Reckwitz, Andreas (2017): Die Gesellschaft der Singularitäten. Zum Strukturwandel der Moderne, Berlin: Suhrkamp. https://doi.org/10.1007/9783-658-21050-2_2

Renn, Ortwin/Marshall, Jonathan Paul (2016): »Coal, nuclear and renewable energy policies in Germany: From the 1950s to the »Energiewende«, in: Energy Policy 99 (C), S. 224-232.

Renn, Ortwin/Köck, Wolfgang/Schweizer, Pia-Johanna (2017): „Öffentlichkeitsbeteiligung bei Planungsvorhaben der Energiewende«, in: Jens Schippl/Armin Grunwald/Ortwin Renn (Hg.), Die Energiewende verste- 
hen - orientieren - gestalten: Erkenntnisse aus der Helmholtz-Allianz ENERGY-TRANS, Baden-Baden: Nomos, S. 547-568.

Renn, Ortwin/Ulmer, Frank/Deckert, Anna (Hg.) (2020): The Role of Public Participation in Energy Transitions, Cambridge, MA: Academic Press.

Rifkin, Jeremy (2011): Die dritte industrielle Revolution: Die Zukunft der Wirtschaft nach dem Atomzeitalter, Frankfurt a.M./New York: campus.

Rodden, Jonathan A. (2019): Why Cities Lose: The Deep Roots of the UrbanRural Political Divide. New York: Basic Books.

Römmele, Andrea/Schober, Henrik (2013): The Governance of Large-Scale Projects. Linking Citizens and the State, Baden-Baden: Nomos. https:// doi.org/10.5771/9783845243566

Roose, Jochen (2010): »Der endlose Streit um die Atomenergie. Konfliktsoziologische Untersuchung einer dauerhaften Auseinandersetzung«, in: Peter H. Feindt/Thomas Saretzki (Hg.), Umwelt- und Technikkonflikte, Wiesbaden: VS, S. 79-103. https://doi.org/10.1007/978-3-531-92354-3_4

Rosa, Hartmut (2018): Beschleunigung und Entfremdung: Entwurf einer kritischen Theorie spätmoderner Zeitlichkeit, 6. Auflage, Berlin: Suhrkamp. Roßmeier, Albert/Weber, Florian (2018): »Stürmische Zeiten: Bürgerschaftliches Engagement beim Windkraftausbau zwischen Befürwortung und Ablehnung«, in: Andreas Stefansky/Angelina Göb (Hg.), »Bitte wenden Sie!« - Herausforderungen und Chancen der Energiewende, Arbeitsberichte der ARL 22, Hannover: Verlag der ARL, S. 52-79.

Roßnagel, Alexander/Birzle-Harder, Barbara/Ewen, Christoph/Götz, Kon$\mathrm{rad} /$ Hentschel, Anja/Horelt, Michel-André/Huge, Antonia/Stieß, Immanuel (2016): Entscheidungen über dezentrale Energieanlagen in der Zivilgesellschaft: Vorschläge zur Verbesserung der Planungs- und Genehmigungsverfahren, Interdisciplinary Research on Climate Change Mitigation and Adaption 11, Kassel: kassel university press.

Sachverständigenrat für Umweltfragen (SRU) (2019): Demokratisch regieren in ökologischen Grenzen. Zur Legitimation von Umweltpolitik. Online verfügbar unter: https://www.umweltrat.de/SharedDocs/Downloads/DE/ O2_Sondergutachten/2016_2020/2019_06_SG_Legitimation_von_Umwelt politik.pdf?_blob=publicationFile\&v=13. Zuletzt aufgerufen am: 26.02.2020.

Sack, Detlef (2018): Zwischen europäischer Liberalisierung und Energiewende - Der Wandel der Governanceregime im Energiesektor (1990-2015), in: Lars Holstenkamp/Jörg Radtke (Hg.), Handbuch Energiewende und Partizipation, Wiesbaden: Springer VS, S. 81-99. 
Sack, Detlef (2014): »Rekommunalisierung als neuer Trend? - Zwischen Privatisierungserfahrungen, direkter Demokratie und Haushaltskonsolidierung«, in: Gesellschaft, Wirtschaft, Politik63 (3), S. 339-350. https://doi. org/10.3224/gwp.v63i3.16708

Saretzki, Thomas (2001): »Energiepolitik in der Bundesrepublik Deutschland 1949-1999. Ein Politikfeld zwischen Wirtschafts-, Technologie- und Umweltpolitik«, in: Ulrich Willems (Hg.), Demokratie und Politik in der Bundesrepublik 1949-1999, Wiesbaden: VS, S. 195-221. https://doi.org/10.1007/ 978-3-322-93261-7_10

Saurer, Johannes (2019): »Transformationspfade in Energiesystemen - Steuerungsziele und -instrumente in Deutschland, Frankreich und Großbritannien«, in: der moderne staat 12 (2), S. 282-298. https://doi.org/10.3224/ dms.v12i2.07

Schad, Miriam (2017): Über Luxus und Verzicht. Umweltaffinität und umweltrelevante Alltagspraxis in prekären Lebenslagen, München: oekom.

Schaefer, Thilo (2017): EEG-Umlage: >Verursacherprinzip geht anders!<, IW Kurzberichte 75, Köln: Institut der deutschen Wirtschaft. Online verfügbar unter: https:/www.iwkoeln.de/fileadmin/publikationen/ 2017/363735/IW-Kurzbericht_2017_75_EEG-Umlage.pdf. Zuletzt aufgerufen am: 28.02.2020.

Scharpf, Fritz W./Reissert, Bernd/Schnabel, Fritz (1976): Politikverflechtung: Theorie und Empirie des kooperativen Föderalismus in der Bundesrepublik, Kronberg/Taunus: Scriptor-Verlag.

Scheer, Hermann (2010): Der energethische Imperativ. 100 Prozent jetzt: Wie der vollständige Wechsel $\mathrm{zu}$ erneuerbaren Energien $\mathrm{zu}$ realisieren ist, München: Antje Kunstmann.

Scheiner, Stefan (2017): Interessen der Bundesländer in der deutschen Klimapolitik: Föderale Konfliktverarbeitung in drei Handlungsfeldern, BadenBaden: Nomos. https://doi.org/10.5771/9783845281506

Schimank, Uwe (2011): »Nur noch Coping: Eine Skizze postheroischer Politik «, in: Zeitschrift für Politikwissenschaft 21 (3), S. 455-463. https://doi.org/10. 5771/1430-6387-2011-3-455

Schimank, Uwe (2019): »Politische Gesellschaftsgestaltung heute - sozial- und gesellschaftstheoretische Erwägungen angesichts von Transformationsvorhaben wie der »Energiewende«, in: der moderne staat 12 (2), S. 251-265. https://doi.org/10.3224/dms.v12i2.03

Schönberger, Philipp/Reiche, Danyel (2016): Why Subnational Actors Matter: The Role of Länder and Municipalities in the German Energy Transition, 
in: Carol Hager/Christoph H. Stefes (Hg.), Germany's Energy Transition: A Comparative Perspective, New York: Palgrave Macmillan, S. 27-61.

Schreurs, Miranda/Steuwer, Sibyl (2015): »Der Koordinierungsbedarf zwischen Bund und Ländern bei der Umsetzung der Energiewende aus politikwissenschaftlicher Sicht«, in: Thorsten Müller/Hartmut Kahl (Hg.), Energiewende im Föderalismus, Baden-Baden: Nomos, S. 45-67. https://doi. org/10.5771/9783845259253-45

Schroeter, Regina/Scheel, Oliver/Renn, Ortwin/Schweizer, Pia-Johanna (2016): »Testing the value of public participation in Germany: Theory, operationalization and a case study on the evaluation of participation «, in: Energy Research \& Social Science 13, S. 116-125.

Schulz-Walden, Thorsten (2013): Anfänge globaler Umweltpolitik. Umweltsicherheit in der internationalen Politik (1969-1975), München: Oldenbourg Wissenschaftsverlag.

Schweiger, Stefan (2020): Plastik: Der große Irrtum. Vom sagenhaften Aufstieg der Kunststoffe und dem Preis, den wir heute dafür zahlen müssen, München: riva.

Schweizer, Pia-Johanna/Bovet, Jana (2016): »The potential of public participation to facilitate infrastructure decision-making: lessons from the German and European legal planning system for electricity grid expansion «, in: Utilities Policy 42, S. 64-73. http://doi.org/10.1016/j.jup.2016.06.008

Schweizer-Ries, Petra/Hildebrand, Jan/Hinse, Maximilian/Rühmland, Silke/ Rau, Irina (2016): Begleit- und Akzeptanzforschung zu aktuellen Fragen des Stromnetzausbaus in Deutschland, Saarbrücken: IZES gGmbH. Online verfügbar unter: https://www.researchgate.net/profile/Maximilian_ Hinse/publication/310330514_Begleit__und_Akzeptanzforschung_zu_ aktuellen_Fragen_des_Stromnetzausbaus_in_Deutschland_Wissen schaftliche_Begleitung_der_Planungspraxis_Akzeptanz_Netzausbau_ Projekt_Schlussbericht/links/5a65da8aaca272a158200b6c/Begleit-undAkzeptanzforschung-zu-aktuellen-Fragen-des-Stromnetzausbausin-Deutschland-Wissenschaftliche-Begleitung-der-PlanungspraxisAkzeptanz-Netzausbau-Projekt-Schlussbericht.pdf. Zuletzt aufgerufen am 26.02.2020.

Schweizer, Pia-Johanna/Renn, Ortwin/Köck, Wolfgang/Bovet, Jana/Benighaus, Christina/Scheel, Oliver/Schröter, Regina (2016): »Public participation for infrastructure planning in the context of the German »Energiewende«, in: Utilities Policy 43 (B), S. 206-209. 
Setton, Daniela (2019): Soziales Nachhaltigkeitsbarometer der Energiewende 2018, Potsdam: Institut für transformative Nachhaltigkeitsforschung (IASS). Online verfügbar unter: https://www.iass-potsdam.de/sites/ default/files/2019-02/IASS_Nachhaltigkeitsbarometer.pdf. Zuletzt aufgerufen am 24.02.2020.

Smink, Magda/Negro, Simona O./Niesten, Eva/Hekkert, Marko P. (2015): »How mismatching institutional logics hinder niche-regime interaction and how boundary spanners intervene«, in: Technological Forecasting and Social Change 100, S. 225-237. https://doi.org/10.1016/j.techfore.2015.07. 004

Sonnberger, Marco/Ruddat, Michael (2016): Die gesellschaftliche Wahrnehmung der Energiewende: Ergebnisse einer deutschlandweiten Repräsentativbefragung, Stuttgart: DIALOGIK gemeinnützige $\mathrm{GmbH}$.

Sonnberger, Marco/Ruddat, Michael (2017): »Local and socio-political acceptance of wind farms in Germany«, in: Technology in Society 51, S. 56-65. https://doi.org/10.1016/j.techsoc.2017.07.005

Sovacool, Benjamin K./Dworkin, Michael H. (2014): Global energy justice: Problems, principles, and practices, Cambridge: Cambridge University Press. https://doi.org/10.1017/CBO9781107323605

Stappel, Michael (2016): »Neugründungen von Genossenschaften in Deutschland nach der Reform des Genossenschaftsgesetzes«, in: Zeitschrift für das gesamte Genossenschaftswesen 66 (2), S. 61-78. https://doi.org/10. 1515/zfgg-2016-0009

Star, Cassandra (2020). "Re-making the Future: Transition Movements and Dismantling the Environment-Economy Dichotomy«, in: Geoffrey Wood/Keith Baker (Hg.), The Palgrave Handbook of Managing Fossil Fuels and Energy Transitions, Basel: Springer International Publishing, S. 505527. https://doi.org/10.1007/978-3-030-28076-5_18

Stede, Jan/May, Nils (2019): »Strikte Mindestabstände bremsen den Ausbau der Windenergie«, in: DIW-Wochenbericht 86 (48), S. 895-903.

Stefansky, Andreas/Göb, Angelina (Hg.) (2018): »Bitte wenden Sie! « - Herausforderungen und Chancen der Energiewende, Hannover: Verlag der ARL.

Steinbach, Armin/Franke, Peter (Hg.) (2017): Kommentar zum Netzausbau, 2. Auflage, Berlin/Boston: de Gruyter.

Stier, Bernhard (1999): Staat und Strom: Die politische Steuerung des Elektrizitätssystems in Deutschland 1890-1950. Technik + Arbeit 10, UbstadtWeiher: Verlag Regionalkultur. 
Strunz, Sebastian (2013): The German energy transition as a regime shift. UFZ Discussion Papers 10/2013, Leipzig: Helmholtz-Zentrum für Umweltforschung - UFZ.

Swyngedouw, Erik (2005): „Governance Innovation and the Citizen: The Janus Face of Governance-beyond-the-State«, in: Urban Studies 42 (11), S. 19912006. https://doi.org/10.1080/00420980500279869

Swyngedouw, Erik (2010): »Impossible Sustainability and the Post-political Condition«, in: Maria Cerreta/Grazia Concilio/Valeria Monno (Hg.), Making Strategies in Spatial Planning: Knowledge and Values, Dordrecht u.a.: Springer, S. 185-205. https://doi.org/10.1007/978-90-481-3106-8_11

Swyngedouw, Erik (2011): »Depoliticized Environments: The End of Nature, Climate Change and the Post-Political Condition «, in: Royal Institute of Philosophy Supplements 69, S. 253-274. https://doi.org/10.1017/ S1358246111000300

SZ.de (2011): "Die Atomkanzlerin erklärt ihren Ausstieg«, in: Süddeutsche Zeitung (Online-Ausgabe) vom 09.06.2011. Online verfügbar unter https:// www.sueddeutsche.de/politik/regierungserklaerung-zur-energiewendemerkel-erklaert-den-atomausstieg-zur-herkulesaufgabe-1.1106773. Zuletzt aufgerufen am: 25.02.2020.

Theurl, Theresia/Wendler, Caroline (2011): Was weiß Deutschland über Genossenschaften? Münstersche Schriften zur Kooperation 96, Aachen: Shaker.

Tosun, Jale/Francesco, Fabrizio de/Peters, B. Guy (2019): »From environmental policy concepts to practicable tools: Knowledge creation and delegation in multilevel systems«, in: Public Administration 97 (2), S. 399-412. https:// doi.org/10.1111/padm.12544

trend:research/Leuphana Universität Lüneburg (2013): Definition und Marktanalyse von Bürgerenergie in Deutschland, Bremen/Lüneburg: trend:research $\mathrm{GmbH}$, Leuphana Universität Lüneburg. Online verfügbar unter: https://www.buendnis-buergerenergie.de/fileadmin/ user_upload/downloads/Studien/Studie_Definition_und_Marktanalyse_ von_Buergerenergie_in_Deutschland_BBEn.pdf. Zuletzt aufgerufen am 20.01.2020.

Umweltbundesamt (UBA) (2019): Primärenergieverbrauch. Online verfügbar unter: https:/www.umweltbundesamt.de/daten/energie/primaeren ergieverbrauch\#definition-und-einflussfaktoren. Zuletzt aufgerufen am: 26.02.2020.

Umweltbundesamt (UBA) (2020): Energieverbrauch nach Energieträgern, Sektoren und Anwendungen. Online verfügbar unter: https:// 
www.umweltbundesamt.de/daten/energie/energieverbrauch-nachenergietraegern-sektoren. Zuletzt aufgerufen am: 26.02.2020.

Vannini, Phillip/Taggart, Jonathan (2014): Off the Grid: Re-Assembling Domestic Life, New York: Routledge.

Veelen, Bredje van/Horst, Dan van der (2018): „What is energy democracy? Connecting social science energy research and political theory«, in: Energy Research \& Social Science 46, S. 19-28. https://doi.org/10.1016/j.erss. 2018.06.010

Veum, Karina/Bauknecht, Dierk (2019):»How to reach the EU renewables target by 2030? An analysis of the governance framework «, in: Energy Policy 127, S. 299-307. https://doi.org/10.1016/j.enpol.2018.12.013

Vogelgesang, Waldemar/Kopp, Johannes/Jacob, Rüdiger/Hahn, Alois (Hg.) (2018): Stadt - Land - Fluss: Sozialer Wandel im regionalen Kontext, Wiesbaden: Springer VS.

Voßkuhle, Andreas/Bumke, Christian/Meinel, Florian (2013): Verabschiedung und Wiederentdeckung des Staates im Spannungsfeld der Disziplinen, Der Staat, Beiheft 21, Berlin: Duncker \& Humblot. https://doi.org/10.3790/ 978-3-428-53944-4

Weber, Florian/Jenal, Corinna (2018): „Gegen den Wind: Konfliktlinien beim Ausbau erneuerbarer Energien in Großschutzgebieten am Beispiel der Windenergie in den Naturparken Soonwald-Nahe und RheinWesterwald«, in: Florian Weber/Fredericke Weber/Corinna Jenal (Hg.), Wohin des Weges? Regionalentwicklung in Großschutzgebieten, Arbeitsberichte der ARL 21, Hannover: Verlag der ARL, S. 217-249.

Williams, Stephen/Doyon, Andréanne (2019): "Justice in energy transitions«, in: Environmental Innovation and Societal Transitions 31, S. 144-153. https://doi.org/10.1016/j.eist.2018.12.001

Wilson, Japhy/Swyngedouw, Erik (Hg.) (2014): The Post-Political and its Discontents: Spaces of Depoliticisation, Spectres of Radical Politics, Edinburgh: Edinburgh University Press. https://doi.org/10.3366/edinburgh/ 9780748682973.001.0001

Wissenschaftlicher Beirat der Bundesregierung Globale Umweltveränderungen (WBGU) (2011): Hauptgutachten 2011 des Wissenschaftlichen Beirats der Bundesregierung Globale Umweltveränderungen »Welt im Wandel - Gesellschaftsvertrag für eine Große Transformation, Berlin: Wissenschaftlicher Beirat der Bundesregierung Globale Umweltveränderungen (WBGU). 
Wood, Geoffrey/Baker, Keith (Hg.) (2020): The Palgrave Handbook of Managing Fossil Fuels and Energy Transitions, Basel: Springer International Publishing.

Wright, Iain (2020): »Regulation and Market Reform: The Essential Foundations for a Renewable Future«, in: Geoffrey Wood/Keith Baker (Hg.), The Palgrave Handbook of Managing Fossil Fuels and Energy Transitions, Basel: Springer International Publishing, S. 453-482. https://doi.org/10. 1007/978-3-030-28076-5_16

Wright, Erik Olin (2017): Reale Utopien. Wege aus dem Kapitalismus, Berlin: Suhrkamp.

Yildiz, Özgür/Rommel, Jens/Debor, Sarah/Holstenkamp, Lars/Mey, Franziska/Müller, Jakob R./Radtke, Jörg/Rognli, Judith (2015): Renewable energy cooperatives as gatekeepers or facilitators? Recent developments in Germany and a multidisciplinary research agenda«, in: Energy Research \& Social Science 6, S. 59-73. https://doi.org/10.1016/j.erss.2014.12.001

Zaunbrecher, Barbara/Ziefle, Martina (2016): »Integrating acceptance-relevant factors into wind power planning. A discussion", in: Sustainable Cities and Society 27, S. 307-314. https://doi.org/10.1016/j.scs.2016.08.018 



\section{Umbrüche und Verunsicherungen Der Windkraft- und der Stromnetzausbau in Deutschland}

Florian Weber und Olaf Kühne

\section{Einleitung: Die Energiewende als Herausforderung}

Wenn in den ersten beiden Jahrzehnten des 21. Jahrhunderts im Kontext der Energiewende in Deutschland von einer »Elektrisierung des Landes«, einem »öffentlichen Ärgernis«, einer »Entstellung unseres Landes«, »verhängnisvollen Folgen für das Orts- und Landschaftsbild« oder einer »Verdrahtung unserer Landschaft " geschrieben oder gesprochen wird, dürften sich vor dem Hintergrund vielfältiger bürgerschaftlicher Widerstände gegen Vorhaben des Windkraft- und des Stromnetzausbaus vermutlich nur wenige wundern, doch hierbei handelt es sich durchgehend um Zitate aus unterschiedlichen Werken, die rund 100 Jahre alt sind (nachzulesen in Bayerl 2005: 40). Bereits vor dem Ersten Weltkrieg setzte eine Diskussion ein, die in Verbindung mit Heimatund Naturschutz den Aufbau eines Elektrizitätsnetzes mit Masten und Freileitungen >leidenschaftlich kritisch< begleitete. Es ist damit in gewisser Weise zu Beginn des 21. Jahrhunderts die Wiederkehr einer bekannten >Welle aus einer grundlegenden und massiven Veränderung der Energieversorgung in Verbindung mit Verunsicherungen bei der Bevölkerung im Hinblick auf Konsequenzen für vielfältige Lebensbereiche (Kühne/Weber 2016; Weber 2018).

Während nach der Reaktorkatastrophe in Fukushima im Jahr 2011 in Deutschland der Kernkraftausstieg bis 2022 beschlossen wurde und daraus resultierende Konsequenzen für die Energieversorgung shitzig debattiert wurden (vgl. allg. beispielsweise Gailing/Leibenath 2017; Kühne/Weber/Jenal 2016), dominierten im Jahr 2019 in Gesellschaft und Medien Diskussionen

um einen zügigen Ausstieg aus der Kohleförderung bis 2038 (oder häufig gefordert: 2030) in Verbindung mit der >Fridays for Future«-Bewegung und 
der Forderung nach verstärktem Klimaschutz als oberste Priorität (allg. dazu beispielsweise Hagedorn et al. 2019; Wahlström et al. 2019). Damit stellt sich noch einmal mehr die Frage, wie künftig Energie produziert und genutzt wird. Der Ausbau erneuerbarer Energien ist zwischenzeitlich eher ins Stocken geraten (siehe u.a. Bundesverband WindEnergie 2019), da Widerstände - insbesondere durch sehr aktive Bürger*innen-Initiativen vorangetrieben - zunahmen (dazu Weber/Jenal/Roßmeier/Kühne 2017). Was ist hierbei nun letztlich höher zu gewichten: der Schutz von >Natur und Landschaft< vor Ort oder das gesamtgesellschaftliche Ziel, nachhaltige Entwicklung zu stärken und etwas gegen den Klimawandel zu tun? Die Energiewende als gesamtgesellschaftliches Projekt ist entsprechend in unterschiedliche Diskurse auf verschiedenen Ebenen und zu verschiedenen Zeiten eingebunden.

Der Diskurstheoretiker Ernesto Laclau (2007) konzeptualisierte den $>$ Kampf $\prec$ widerstreitender und konkurrierender Ziele, Erwartungen und Projekte mit dem Begriff des >flottierenden Signifikanten<, der zu einer hilfreichen Kategorie werden kann, wenn wir uns dem Themenfeld Energiewende und Megatrends annähern. Wie sich aus den Einführungen bereits andeutete, sind gesellschaftliche Umbrüche regelmäßig mit gewissen Befürchtungen, Sorgen, Verunsicherungen verbunden (Bauman 1999, 1992), wobei grundlegend und zentral immer unterschiedliche Entwicklungsmöglichkeiten denkbar scheinen, die Eindeutigkeiten unmöglich werden lassen (dazu allg. Laclau/Mouffe 2015 [engl. Orig. 1985]). Zur zentralen Frage wird daher eher, was temporär zu gewissen Zeitpunkten so hohe Wirkmächtigkeit entfaltet, dass andere Deutungsweisen ausgeschlossen scheinen. Der Begriff des >Megatrends $<$, wie populär durch John Naisbitt (1982) transportiert (kritisch dazu Rust 2008: 85), ist hieran durchaus anschlussfähig, wenn soziale, ökonomische, politische, technologische usw. Umbrüche betrachtet werden, die für eine gewisse Zeit starke Wirkung entfalten. Wird im zweiten Jahrzehnt des 21. Jahrhunderts von >Tiefenströmungen des Wandels‘ gesprochen, die unter anderem Individualisierung, Globalisierung, Mobilität, Urbanisierung und Neo-Ökologie umfassen (Zukunftsinstitut GmbH 2018: o.S.), lässt sich aus einer konstruktivistischen Grundperspektive, die wir einnehmen (einführend im Überblick Collin 2008; Flick 2007; Kühne/Weber/Jenal 2018), kritisch fragen, ob diese identifizierten >Megatrends` alternativlos und vor allem konkurrenzlos sind. Wir interessieren uns vor diesem Hintergrund im Zeitverlauf für markante Veränderungen und Aushandlungsprozesse um Deutungshoheiten und damit einhergehende Konkurrenzen und Konflikte der deutschen Energieversorgung. 
Der Stromnetz- und der Windkraftausbau stellen in Deutschland Kontexte dar, in denen sich diese sehr eingängig beobachten lassen. Zu einem gewissen >Trend - als retrospektiv beobachtetem Veränderungsmuster - werden hier Bürgerinitiativen, die sich konstituieren und für ihre Belange eintreten - und dabei insbesondere für lokale Belange, die vielfach auf einer anderen Ebene als Demonstrationen für globalen Klimaschutz liegen. Im Folgenden rücken wir Aushandlungsprozesse im Zuge der `Energiewenden in den Mittelpunkt, ausgehend von einer Rückschau sowie einer Fokussierung auf Bürgerinitiativen und deren Zielsetzungen in den 2010er Jahren. Gesellschaftliche Herausforderungen werden so manifest, die zum Ende des Beitrags eine Einordnung erfahren.

\section{Transformationsprozesse der Stromversorgung in Deutschland in der Rückschau}

Raumbezogene Veränderungsprozesse fanden und finden letztlich kontinuierlich statt, wobei sich die Frage nach der Geschwindigkeit von Umbrüchen als eine mitunter zentrale erweist. Im Laufe des Mittelalters erfolgten beispielsweise Waldrodungen nach und nach und veränderten sukzessive das, was wir als `Landschaft beschreiben. Die Industrialisierung brachte hingegen raschere Veränderungen mit sich. Das Ruhrgebiet und das Saarland stellen mit dem manifesten Bedeutungsgewinn von Kohle und Stahl und physischen Manifestationen wie Fördertürmen, Stahlwerken und Bergehalden gewisse räumliche >Hotspots` dar (dazu u.v. Helfer 2015; Wehling 2006), doch insgesamt veränderten sich raumbezogene Strukturen mit Urbanisierung und Industrieproduktionsausweitungen, speziell markant sichtbaren Schornsteinen. Gegen letztere regte sich durchaus Unmut, lassen sie sich doch als »erste >Verspargelung der Heimat" (Bayerl 2005: 38) deuten, doch in erster Linie wurden sie aufgrund ihrer Emissionen kritisch beäugt. Eine Konsequenz lag letztlich in der Entwicklung von Natur- und Landschaftsschutz jenseits der industriellen Ballungsräume unter anderem mit der Etablierung von $\mathrm{Na}-$ tur(schutz)parken (siehe Weber 2013; Weber/Weber 2019). Eine elektrische Stromversorgung hätte durchaus positiv anmuten können, da sie als ssauberer deutbar ist (Felber 1998: 105), doch genau an dieser entzündeten sich mit deren Etablierung ab Beginn des 20. Jahrhunderts in Deutschland Widerstände. Sie wurde sowohl in Diskurse um eine >zukunftsorientierte Energieversorgung als auch in solche um >Landschafts- und Heimatzerstörung، 
eingewoben, was Deutungskämpfe bedingte und sie aus diskurstheoretischer Perspektive in Anschluss an Ernesto Laclau (2007) zu einem flottierenden Signifikanten werden lässt.

Bayerl $(2005: 38,40)$ führt an, dass schon vor dem Ersten Weltkrieg »insbesondere von den Heimatschützern eine zunehmende >Verdrahtung der Landschaft« bzw. eine "Elektrisierung des Landes« beklagt wurden. "Das heimatliche Dorf werde durch hässliche Trafohäuschen, Masten und Drähte entstellt, riesige Fernleitungen überzögen zunehmend das ganze Land«, sei argumentiert worden. In der Folgezeit ergaben sich immer wieder wellenartig sich abschwächende und neu aufkommende Phasen der Kritik, einhergehend mit Umbrüchen in der Energieversorgungs(infra)struktur. Den Ausgangspunkt bildete eine Elektrifizierung der urbanen Beleuchtung ab den 1880er Jahren (Würfel 2017:3-4), gefolgt von Stromübertragungen über weitere Strecken durch Drehstromhochspannungsleitungen, insbesondere ab den 1920er Jahren (Bürger 2018: 4). Mit Verbundleitungen wurden nach und nach überregionale Stromproduktionsstandorte verknüpft (Maier 1993: 65). Insofern kann im zeitlichen Umfeld des Ersten Weltkrieges und dem sich vollziehenden Aufbau von Regionalnetzen eine erste Diskussionsphase verortet werden, wie Bayerl (2005: 40) herausarbeitet, in der >Landschaft،, >Heimat<, $>$ Natur< mit `Schönheit< relationiert wurden - und damit also einer gefühlten Unvereinbarkeit von Elektrifizierung und sschöner heimatlicher Landschaft، (argumentativ damit in Anschluss an Kühne 2006). Beispielsweise SchultzeNaumburg (1901-1917, hier 1917) betonte, die »Elektrisierung des Landes« drohe, dieses »zu entstellen«. Das Aufkommen von Natur- und Heimatschutz lässt sich vor dem Hintergrund der Industrialisierung deuten (Berr 2019; Berr et al. 2019a), steht gleichzeitig, wie sich zeigt, in Verbindung mit der Entwicklung der Stromversorgung. Besondere Kritik entzündete sich bei letzterer an landschaftlich negativ gewerteten Veränderungen - in Abwägung zu ökonomischen Begründungsmustern einer Notwendigkeit (Bayerl 2005: 41-42), was als systemlogikenbezogenes Argumentieren gelesen werden kann (Kühne 2019a; Luhmann 2017). Die Thematisierung und Problematisierung von Veränderungen der physischen Grundlagen von >Landschaft< im Kontext der Industrialisierung lässt sich dabei als Element einer Ent-Selbstverständlichung beschreiben. Das $>$ Normale $<$ wird herausgefordert und ist nicht mehr selbstverständlich, wodurch es zum Element der Kommunikation wird, hier dessen Verteidigung gegen das `Neue (vgl. u.a. Dahrendorf 1972, 1994).

In der Zeit der nationalsozialistischen Herrschaft setzten sich mit dem Ausbau großer Versorgungsnetze heimatschutzbezogene Bedenken fort. In 
einer Publikation von Schwenkel (1938: 155-158) wird für Erdverkabelungen von Stromleitungen in schwerwiegenden >landschaftsverschandelnden $<\mathrm{Si}$ tuationen plädiert, wobei gleichzeitig die Problematik hoher Kosten als Herausforderung benannt wird. Der Schutz von >Land und Volkı sah sich negativen Auswirkungen der Industrialisierung, darunter auch Freileitungen, gegenüber, aber ein einheitliches und konsequentes Agieren zugunsten von Invisibilisierungen durch Lageveränderungen oder eine sharmonische Positionierung mitunter entlang von Waldrändern wurde nicht konsequent vollzogen (Bayerl 2005: 45, 46): „Die Verkabelung blieb eine landschaftsschützerische Vision - im >Dritten Reich ‘ auch aus militärischen Gründen propagiert -, die technisch, vor allem jedoch aus ökonomischen Gründen, nicht umgesetzt werden konnte«.

Nach dem Zweiten Weltkrieg standen in Deutschland Wiederaufbau und Wirtschaftsentwicklung im Fokus politisch-gesellschaftlicher Bemühungen (allg. einführend u.v. Heineberg/Kraas/Krajewski 2017; Hewitt/Nipper/Nutz 1993). Mit dem >Wirtschaftswunder < und einem Bevölkerungszuwachs stieg der Bedarf an elektrischer Energieversorgung deutlich an, was eine neue Phase des Leitungsbaus bedingte. So wurde zugunsten des Ausbaus des Verbundnetzes gerade im Höchstspannungsbereich - 110, dann 220 und schließlich $380 \mathrm{kV}$ - agiert (Bayerl 2005: 46). Und auch hier blieb eine erneute gesamtgesellschaftliche Diskussion nicht aus. Obermair/Jarass/Gröhn (1985: 15-16) rekonstruieren eine ab Mitte der 1970er Jahre aufkommende Kritik an dem weiteren Ausbau, auf die die Energiewirtschaft unzureichend vorbereitet gewesen sei, ausgehend von einer erlebten `Sättigung « mit technischen Manifestationen der Energieversorgung in Verbindung mit einer erfüllten Bedarfslage, die einen weiteren Ausbau für Bundesbürger*innen nicht notwendig erscheinen ließ. Hier wurde nun weniger über >Landschaft und Heimat<, sondern über eine Skepsis gegenüber der grundlegenden »Notwendigkeit des technischen Ausbaus « diskutiert (Bayerl 2005: 47) - eine markante diskursive Verschiebung im Vergleich zu vorherigen Debatten. Zudem wurden ökologische Bedenken in ökonomischen Bewertungen berücksichtigt.

Mit der Jahrtausendwende und insbesondere im zweiten Jahrzehnt des 21. Jahrhunderts folgte - nach einer Zeit, in der es um das deutsche Stromnetz eher wieder >ruhiger geworden war - eine neue Ära >spannungsgeladener Aushandlungsprozesse. $\mathrm{Zu}$ mitentscheidenden Komponenten werden hier eine stärkere Einbindung in das europäische Stromnetz und die Förderung erneuerbarer Energien, die einen Ausbau des Höchstspannungsnetzes bedingten (Fromme 2018; Neukirch 2014, 2017; Monstadt 2015). Letztere erhielten be- 
reits verstärkt seit dem Erneuerbare-Energien-Gesetz aus dem Jahr 2000 eine Privilegierung (EEG 2000), doch nach der Reaktorkatastrophe von Fukushima 2011, dem beschlossenen Ausstieg aus der Kernkraft bis zum Jahr 2022 und der damit verbundenen `Energiewende < kam diesen noch höhere Bedeutung zu. Verstärkt wird deren Relevanz zusätzlich durch das politische Übereinkommen des Endes der Kohleverstromung bis - aktuell diskutiert spätestens - 2038 (BMWi 2019: 64). Dadurch wandelt sich auf zentrale Weise die Zusammensetzung der Stromerzeugung: Die historisch bedingte Pfadabhängigkeit von Stein- und Braunkohle, ergänzt um Kernkraft (Würfel 2017: 9-10), bricht auf und verschiebt sich zugunsten eines sich vollziehenden und perspektivisch weiter geplanten Ausbaus erneuerbarer Energieträger (vgl. dazu Abb. 1). Im Jahr 2017 erreichte der Anteil erneuerbarer Energien am Bruttostromverbrauch einen Anteil von 36 Prozent (BMWi 2018: 10).

Abb. 1: Bruttostromerzeugung in Deutschland nach Energieträgern seit $1991 \mathrm{im}$ Zweijahresrhythmus dargestellt, ergänzt um Ziele 2025 und 2035 in Bezug auf den Bruttostromverbrauch

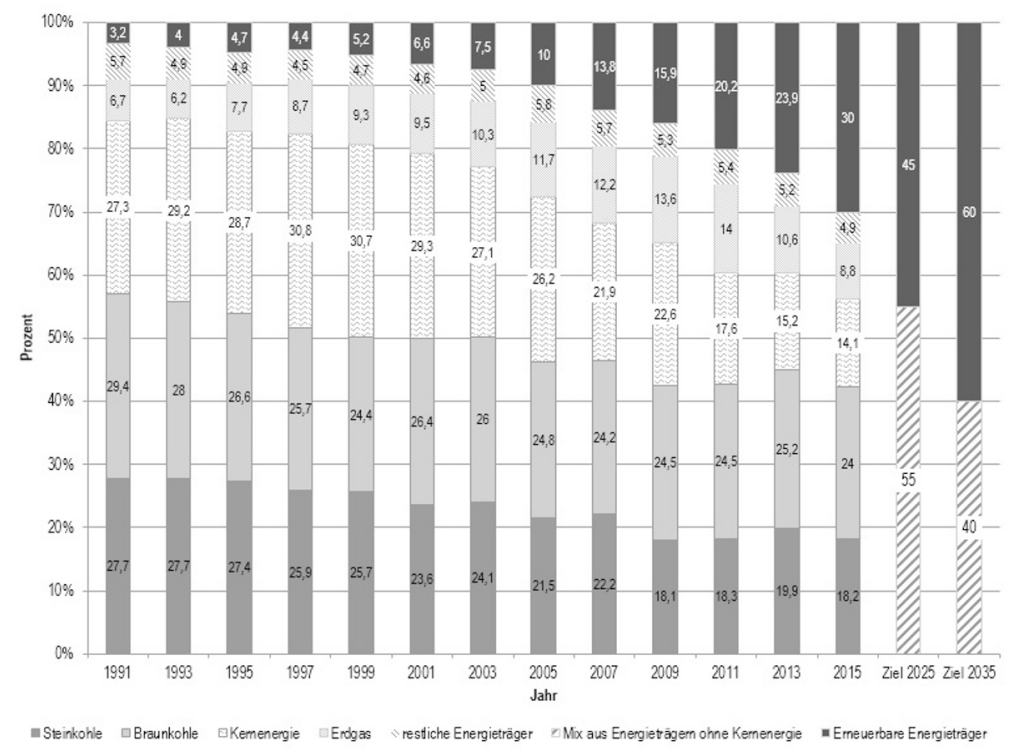

Quelle: Eigene Darstellung auf Basis von AG Energiebilanzen (2017) und BMWi (2018). 
Dies hat markante raumbezogene Auswirkungen: Während bisherige Energieproduktionsstandorte der Kohle- und Kernkraftkraftwerke eher zentralisiert ausfielen (Hook 2019: 115), verteilen sich Windkraft- und Photovoltaikanlagen süber die Fläche`(Klagge 2013; Klagge/Brocke 2015; Kühne 2011). Darüber hinaus differieren bisherige und neue Produktions- und Verbrauchszentren: Windkraft wird in hohem Maße im Norden ausgebaut, Industrieansiedlungen befinden sich in verstärktem Maße in Süddeutschland, woraus der Bedarf abgeleitet wurde, neue Übertragungswege im Übertragungsnetzbereich aufzubauen. Bereits 2009 wurde das Energieleitungsausbaugesetz (EnLAG) verabschiedet, das mit Stand aus dem ersten Quartal 2019 insgesamt 24 Vorhaben, sechs davon als Erdkabel-Pilotvorhaben, in Zuständigkeit der Länder mit einer geplanten Gesamtlänge von rund 1.800 Kilometern auflistet. Nach dem beschlossenen Kernkraft-Ausstieg wurde ein ergänzender Ausbaubedarf ermittelt. Das im Juli 2013 verabschiedete und im Dezember 2015 angepasste Bundesbedarfsplangesetz (BBPIG) umfasst nach dem ersten Quartal 2019 insgesamt 43 Vorhaben, davon acht in Höchstspannungsgleichstromübertragung (HGÜ), hierbei fünf vorrangig in Erdverkabelung, was zusammen etwa 5.900 Leitungskilometer betrifft (Bundesnetzagentur 2019: o.S; dazu auch grundlegend Bürger 2018). Während Strommasten temporär durchaus für "Fortschritt und menschlichen Triumph« stehen konnten, gehören sie nach Selman (2010: 165) heute für viele zu den »am wenigsten geliebten industriellen Artefakten ${ }^{1}$.

In den letzten Jahren entwickelte sich anwachsender Widerstand sowohl gegen den Ausbau erneuerbarer Energien in besonderer Weise in Bezug auf die Windkraft als auch den Ausbau der Stromübertragungsnetze. Mit differierenden Argumentationssträngen wird jeweils für und gegen Vorhaben agiert, womit sich neuerliche Aushandlungsprozesse um Deutungshoheiten ergeben. Diese werden nachfolgend ausführlicher beleuchtet.

\section{Bürgerschaftliches Engagement im Zuge der Energiewende zu Beginn des 21. Jahrhunderts}

In der Rückschau stehen die 1980er/1990er Jahre in Deutschland für eine gewisse Phase, in der die Stromversorgung nicht mit umfänglichen Umbrüchen verbunden war. Kohle und Kernkraft stellten zentrale Säulen dar, wo-

1 »our least loved industrial artefacts . 
bei Demonstrationen der Anti-Atomkraft-Bewegung gegen die Kernkraftnutzung und Diskussionsprozesse um die Endlagersuche eine retablierte< Regelmäßigkeit aufwiesen, die politische Entscheidungsprozesse der Regierungsparteien nicht entscheidend zu einem Umdenken brachten. Kraftwerke waren letztlich an ihren jeweiligen Standorten vorhanden und so - abgesehen von abweichenden Haltungen von Aktivist"innen - eher unhinterfragter Teil der sheimatlichen Normallandschaft‘ (allg. Kühne 2006). Die forcierte Förderung erneuerbarer Energien ab den zoooer Jahren in Verbindung mit der in Deutschland als Zäsur wirkenden Reaktorkatastrophe von Fukushima als Ausgangspunkt für den Kernkraftausstieg bedingte nun aber die bereits angerissenen raumbezogenen Veränderungsprozesse in der Form von dezentral verteilten Biomasse-, Photovoltaik- und Windkraftanlagen sowie einer Anpassung der bestehenden Stromübertragungsnetze (vgl. allg. beispielsweise Kühne/Weber 2018). Als 'normak empfundene Strukturen - räumlich wie politisch, sozial, ökonomisch usw. - wurden stark überformt und tradierte Sehmuster erneut herausgefordert (Kühne/Weber 2019). Die Forcierung der 'Energiewender mit ihren unterschiedlichen Bestandteilen lässt sich als Teil des so betitelten Megatrends $>$ Neo-Ökologie $<$ fassen (Zukunftsinstitut $\mathrm{GmbH}$ 2018: o.S.) und fügt sich auf diese Weise in Megatrends im >Weltmaßstab ein, die nach WWF Deutschland/LichtBlick SE (2015: 4-5) perspektivisch prägend sein werden und damit hegemoniale Wirkmächtigkeit entfalten (könnten): Darunter fallen ein Ende der fortschreitend kritisch betrachteten fossilen Ära, eine durchgehend massive Zunahme erneuerbarer Energien (Stromsektor, aber auch Mobilität und Wärme), eine dezentrale Versorgung und eine zunehmende Digitalisierung. Diese Umbrüche vollziehen sich nun aber keineswegs konfliktfrei - ganz im Gegenteil (Kühne 2018a; Linke 2017). Die Entstehung von Bürgerinitiativen im Windkraft- und Stromnetzausbau, mit denen wir uns in den letzten Jahren ausführlicher auseinandergesetzt haben, zeugen hiervon beispielhaft.

Ende 2015/Anfang 2016 wurden mittels einer Google-Recherche insgesamt 270 Bürgerinitiativen mit Website und/oder Facebook-Auftritt ermittelt, die sich gegen den Windkraftausbau in Deutschland engagieren. Die Auswertung zeigt, dass sich tendenziell zwei zentrale Ansprüche identifizieren lassen, zum einen eine Ablehnung verbunden mit dem Versuch einer Verhinderung lokaler Vorhaben und zum anderen eine generelle Kritik an der Förderung der Windkraft oder noch weiterreichender der Energiewende allgemein. Rund drei Viertel der ermittelten Bürgerinitiativen ziehen die Sinnhaftigkeit von Windkraft mit einem Verweis auf fehlende Speichertechnologien, erfor- 
derliche Subventionierungen, hohe Schwankungen und geringere Effizienz in Zweifel. Auf zwei Dritteln der Websites der Initiativen wird eine Profitorientierung von Politik und Anlagenbetreibern zu Lasten betroffener Bürger"innen kritisiert, knapp die Hälfte hält das politische Vorgehen zugunsten der Energiewende nicht für richtig (Abb. 2).

Abb. 2: Grundlegende Kritikpunkte von Bürgerinitiativen $(n=270)$ am Windkraftausbau (Ende 2015/Anfang 2016)

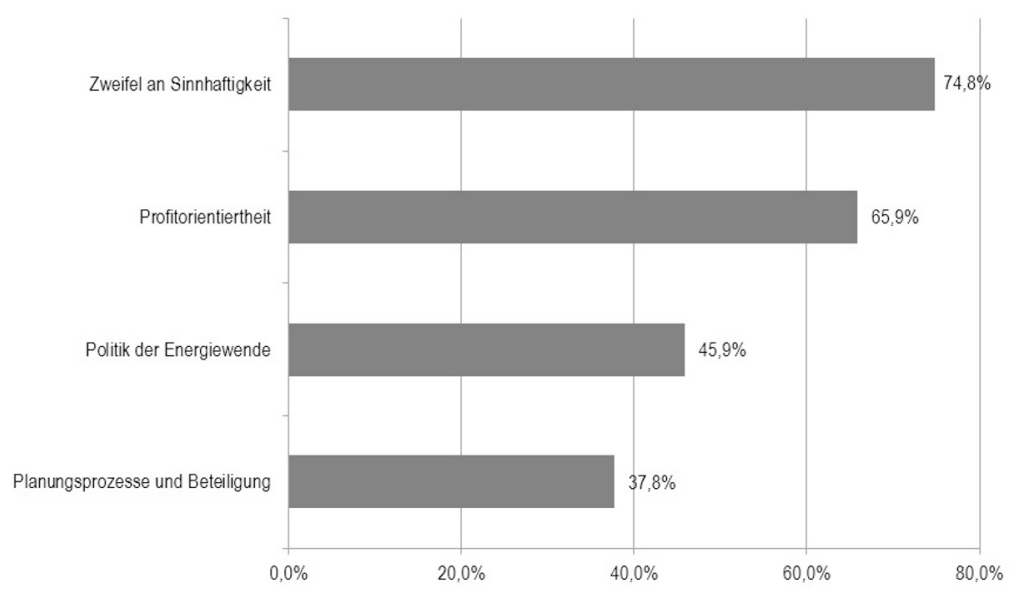

Quelle: Eigene Erhebung und Darstellung.

Durchgehend wird auf Kritikpunkte inhaltsbezogener Art rekurriert, die sich zu vier zentralen Themenkomplexen *kondensieren $<$ lassen und sich fast überall auf den Websites bzw. Facebook-Profilen finden lassen (siehe dazu Abb. 3): Naturschutz, Landschaft und Heimat, Gesundheit und wirtschaftliche Beweggründe. Diese werden entweder vorgebracht, um zu begründen, dass ein lokales Vorhaben nicht umgesetzt werden soll, oder unterstreichend als Beleg dafür angeführt, dass Windkraft bzw. die Energiewende fehlgeleitet seien (Argumentationsstränge entsprechend auch beispielsweise in Becker/Gailing/Naumann 2013; Leibenath 2013; Otto 2019). Im Hinblick auf $>\mathrm{Na}-$ tur< und `Naturschutz« wird vielfach auf Einbußen biologischer Vielfalt und Gefährdungen für Flora und Fauna rekurriert. Zudem wird Windkraft für die >Zerstörung< oder >Verschandelung< von >Landschaft< und >Heimat< verantwortlich gemacht. Hier zeigt sich markant, dass diese Argumentationslinie 
wie bei der Entwicklung des Stromnetzes im 20. Jahrhundert eine Reproduktion und Aktualisierung erfährt. Von Windmühlen zu Windkraftanlagen ergab sich in Deutschland, wie Bayerl (2005: 47) herausstellt, keine Kontinuität, da in der ersten Hälfte des letzten Jahrhunderts auf Großanlagen gesetzt wurde, die aber technisch nicht umsetzbar waren (Bayerl 2005: 47; Heymann 1995). Gewöhnungseffekte stellten sich so nicht ein, womit erste Windkraftanlagen in den 1970er Jahren in Deutschland nach der Ölkrise eine >Wiederentdeckung darstellten - und auch damals schon mit einer >Verspargelung der Landschaft< assoziiert wurden (Bayerl 2005: 47). Heutige Kritikpunkte sind damit keineswegs neu. In Bezug auf gesundheitsbezogene Sorgen wird häufig auf Störungen durch Schattenwurf und nächtliche Befeuerungen, Infraschall, Schlafstörungen sowie eine Vielzahl an potentiellen Folgen verwiesen. Unter `ökonomische Gründe` können schließlich Befürchtungen um verminderte Immobilien- und Grundstückswerte sowie Einbußen im Tourismus gefasst werden.

Abb. 3: Inhaltsbezogene Kritikpunkte von Bürgerinitiativen $(n=270)$ am Windkraftausbau (Ende 2015/Anfang 2016)

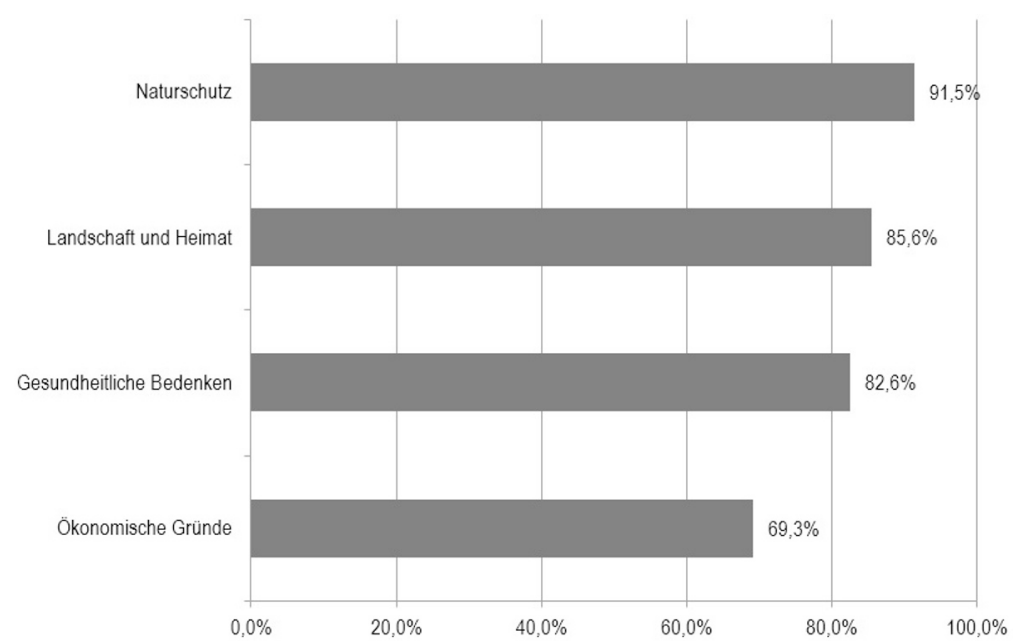

Quelle: Eigene Erhebung und Darstellung.

Alle vorgebrachten grundlegenden wie inhaltsbezogenen Argumente der Windkraftkritik lassen sich gleichzeitig durch Befürworter*innen in die 
entgegengesetzte Richtung deuten: Nur mit einer forcierten Energiewende könnten Klimaschutzziele erreicht und eine Lebensgrundlage für künftige Generationen geschaffen werden. Windkraftanlagen dienten dem Umweltund Naturschutz, fügten sich durch Gewöhnung zunehmend sunproblematisch in >Landschaft und Heimat< ein, gesundheitliche Kritikpunkte seien nicht zutreffend, ökonomische Wertverluste erfolgten höchstens als selffulfilling prophecy, wenn immer wieder hierüber in der Öffentlichkeit diskutiert würde (ausführlicher Roßmeier/Weber 2018). `Windkraft< wird damit in theoretischer Lesart zum flottierenden Signifikanten, der mit ganz unterschiedlich, durchaus diametral entgegengesetzten Argumentationslinien verknüpfbar wird. Auf Gegner*innen-Seite sind regelmäßig Verweise auf das eigene Lebensumfeld vorhanden, das nicht >zerstört « werden solle - der Megatrend >Individualisierung< kollidiert hier mit >Neo-Ökologieく mit noch ungewissem Ausgang. Die Bundesinitiative >Vernunftkraft< als Dachverband von Windkraftgegner*innen geht insgesamt von über 1000 Initiativen in Deutschland aus (Hermann 2018: o.S.), was zeigt, welche Dimension heute Widerstand erlangt hat. Im ersten Halbjahr 2019 wurden in Deutschland nur 86 Windkraftanlagen gebaut (Bundesverband WindEnergie 2019: o.S.), ausgelöst durch schleppende Genehmigungsverfahren und regelmäßig aufkommende Bürger*innen-Widerstände. $\mathrm{Zu}$ einem zentralen >Trend ist hier das Engagement gegen Vorhaben als Teil der Energiewende geworden. Dagegen fordert die Jugend-Bewegung >Fridays for Future (2019: o.S.) für Deutschland $» 100 \%$ erneuerbare Energieversorgung bis 2035«, was mit der derzeitigen (fehlenden) Geschwindigkeit des Windkraftausbaus zur Utopie wird.

Ein Vergleich mit dem eng relationierten Bereich des Stromnetzausbaus in Deutschland lässt markante Parallelen hervorscheinen. Die Planungen zum Ausbau der Übertragungsnetze riefen und rufen vielfältige Widerstände hervor, was sie ebenfalls zu einer aktuellen politischen, planerischen und gesellschaftlichen Herausforderung werden lassen (dazu u.a. auch Bräuer 2017; Hoeft/Messinger-Zimmer/Zilles 2017; Kamlage/Nanz/Fleischer 2014; Neukirch 2014, 2017). Entkomplexisiert stehen Befürworter*innen Gegner*innen gegenüber, deren Beweggründe, Argumente und Zielsetzungen deutlich unterschiedlich ausfallen. Erstere argumentieren unter anderem, wie angerissen, über die bessere Einbindung Deutschlands in das europäische Stromnetz, die Garantie von Versorgungssicherheit und die Schaffung erforderlicher Kapazitäten zur Verteilung erneuerbar produzierter Energie und letztlich einem Beitrag zu einer snachhaltigen< Umgestaltung 
des Energiesystems zur Erfüllung internationaler Verpflichtungen der Reduzierung des Ausstoßes von Treibhausgasen (Pariser Klimaabkommen, EU-Klimaschutzziele). In Teilen werden aber im Verhältnis dazu die grundlegende Notwendigkeit oder die geplante Umsetzung in Zweifel gezogen, respektive mit aktivem Widerstand belegt, der medial wie politisch zwischenzeitlich in hohem Maße Resonanz fand. 2014 und 2015 eskalierte der Streit, was sich im Freistaat Bayern in einer politischen Kehrtwende, insbesondere des damaligen Ministerpräsidenten Horst Seehofer, niederschlug: Nach anfänglicher Befürwortung stellte er sich zeitweise auf die Seite von Bürgerinitiativen, um im nächsten Schritt Erdverkabelungen statt Freileitungen zu befürworten (dazu ausführlicher Weber 2018). Wie im Kontext des Windkraftausbaus wurden, ausgehend von einem Forschungsvorhaben für das Bundesamt für Strahlenschutz, mittels einer Google-Initiative Bürgerinitiativen gegen den Stromnetzausbau mit eigener Website oder Facebook-Profil ermittelt, um deren Zielsetzungen $\mathrm{zu}$ systematisieren. 2015 konnten so 90 Initiativen identifiziert werden, 2017 sogar 123. Bräuer/Wolling (2015: 95) fanden bei ihren Recherchen zwischenzeitlich 140 Bürgerinitiativen - eine ähnlich gelagerte Zahl. Die Gegenüberstellung 2015 zu 2017 zeigt, dass die Favorisierung von Erdverkabelungen als Mittel der >Invisibilisierungく (Kühne 2013) beim so genannten SuedLink und SuedOstLink nicht $\mathrm{zu}$ einer einfachen Befriedung von Widerständen geführt hat. Zwar ist die grundlegende Ablehnung des Stromnetzausbaus von knapp zwei Drittel auf etwas mehr als die Hälfte zurückgegangen, doch heißt dies gleichzeitig, dass immer noch jede zweite Initiative den Netzausbau nicht als notwendig ansieht (siehe Abb. 4). Letztere vertreten unter anderem die Position, der Netzausbau sei überdimensioniert, diene dem Transport von Kohlestrom, einer europäischen sgrenzenlosen Marktliberalisierung und schade einer dezentral-regional auszugestaltenden Energiewende (dazu auch Jarass 2013; Neukirch 2015). Befürworter*innen von Erdverkabelungen haben zumindest $2015 \mathrm{zu} 2017$ von knapp einem Viertel auf rund ein Drittel zugenommen. Darüber hinaus werden andere Trassenverläufe, angepasste Grenzwerte oder Mindestabstände gefordert bzw. es wird gegen Konverterstationen bei Hochspannungsgleichstromübertragungstrassen agiert. Bis heute wird immer wieder eine ausgeprägte Skepsis gegenüber dem grundsätzlichen Bedarf des Übertragungsnetzausbaus manifest - in gewisser Weise also die Wiederkehr der >Kritikwelle ab Mitte der 1970er Jahre. Berechnungen der Übertragungsnetzbetreiber werden ebenso wie Prüfungen durch die zuständigen Behörden nicht anerkannt. Damit wird auch der Staat zu einer 
Konfliktpartei, die von Bürgerinitiativen nicht als neutral wahrgenommen wird. Aus deren Sicht sollten die Planungen deutlich reduziert oder sogar gestoppt werden. Aushandlungsprozesse und Deutungskämpfe sind im Gange, wobei der Netzausbau von politischer und planerischer Seite nicht in Frage gestellt wird und Genehmigungsvorhaben vorangebracht werden sollen. Da die zwischenzeitlich medial hochgradig rezeptierten Widerstände allerdings bereits Anpassungen zugunsten von Erdverkabelungen zur Folge hatten, sind künftige >Kehrtwenden keineswegs ausgeschlossen.

Abb. 4: Ziele von Bürgerinitiativen (2015: $n=90$ und 2017: $n=123$ ), ausgehend von zwei Google-Recherchen (Januar 2015 und Mai/Juni 2017)

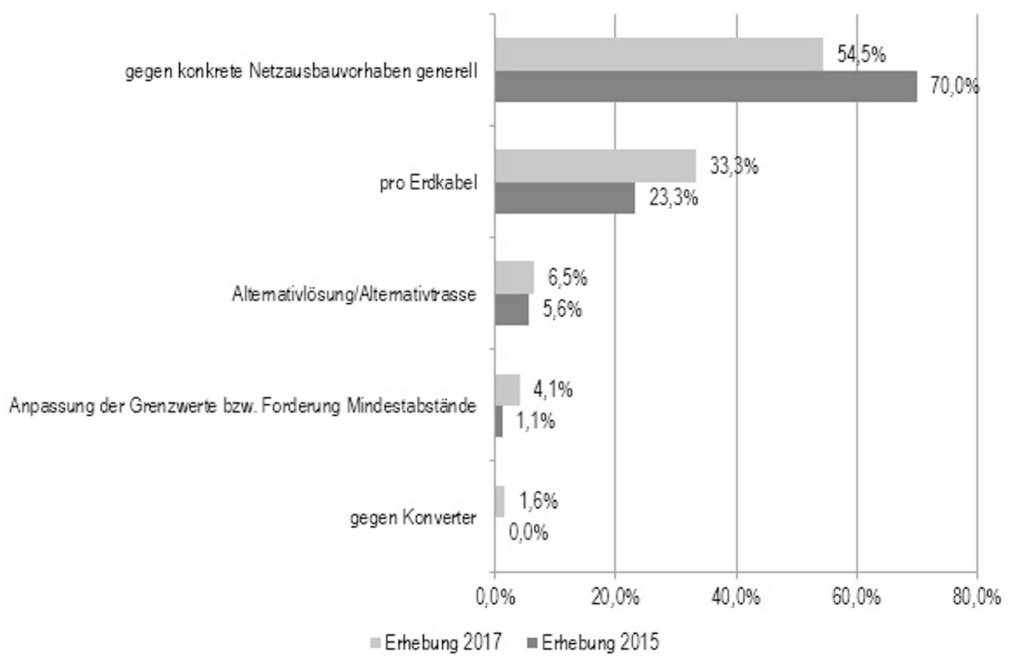

Quelle: Eigene Erhebung und Darstellung.

Der Fokus auf inhaltlich angeführte Kritikpunkte zeigt eine markante Parallele zum Windkraftausbau. Die unterschiedlichen Argumentationsstränge lassen sich unter die gleichen vier Hauptpunkte subsummieren. Durch die analysierten Bürgerinitiativen, hier in Bezug auf die Erhebung aus dem Jahr 2017, wird auf die >Zerstörung` von Landschaft und Heimat (84 \%), ökonomische Aspekte wie Verluste von Grundstückswerten oder Tourismuseinbußen (74\%), gesundheitliche Bedenken (72\%) und naturschutzbezogene Kritikpunkte (69\%) rekurriert (vgl. Abb. 5). In diesem Kontext dominieren 
Bezüge auf Landschaft, Heimat und das eigene Wohnumfeld: Der Nahbereich um das eigene Haus/die eigene Wohnung soll von Veränderungen verschont bleiben. `Zerstörungen und >Verschandelungen`von >Landschaft und Heimat d drohten, also vom Prinzip her nahezu identische Formulierungen, wie sie einhundert Jahre zuvor von Heimatschützer*innen gegen die >Elektrisierung< des Landes vorgebracht wurden.

Abb. 5: Argumentationskontexte der Bürgerinitiativen (2017 und 2015 )

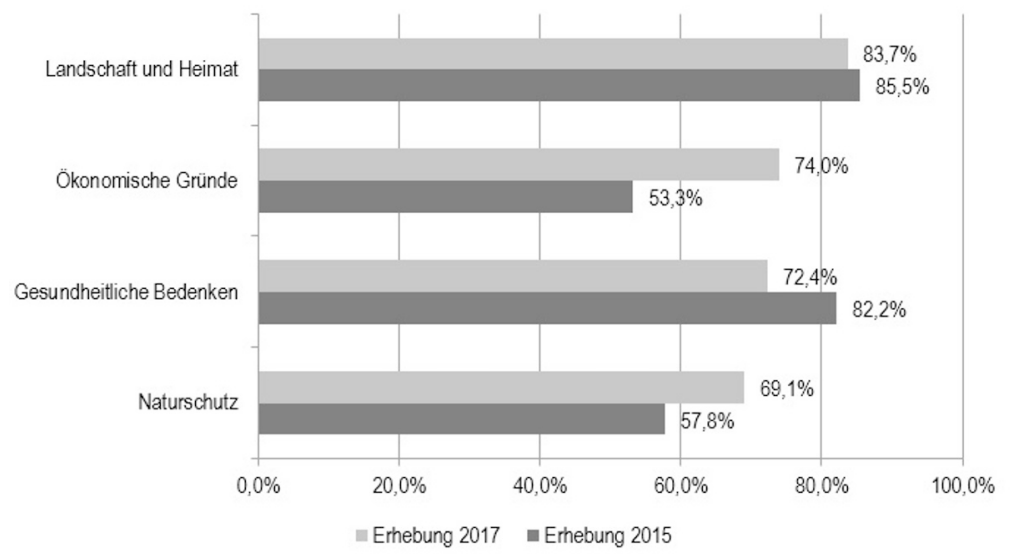

Quelle: Eigene Erhebung und Darstellung.

Ein >Trend liegt daher auch beim Stromnetzausbau zu Beginn des 21. Jahrhunderts darin, Umbrüche nicht einfach diskussionslos hinzunehmen und mit Argumenten zu arbeiten, die eine hohe lebenswirkliche Anschlussfähigkeit aufweisen, wie gerade Landschaft und Heimat. Auffällig ist, wie sehr sich Ausführungen im Hinblick auf den Windkraft- und den Stromnetzausbau ähneln (Weber et al. 2017a; Weber et al. 2017b). Wird nicht aktiv auf den einen oder anderen Kontext verwiesen, sind Ausführungen der jeweiligen Bürgerinitiativen durchaus einfach austauschbar. Und es zeigt sich eine weitere Vergleichbarkeit, die zudem mit dem ersten Drittel des 20. Jahrhunderts relationiert werden kann: Bürgerinitiativen stellen Fotographien mit und ohne Windkraftanlagen bzw. Stromtrassen gegenüber - als Positiv-NegativKontrastierungen, wie es Heimatschützer*innen Jahrzehnte zuvor taten: »Die Beiträge der Heimatschützer zeichneten sich durch reichliches Bildma- 
terial aus, da ihnen eine >Gut-Schlecht-Didaktik« zugrunde lag: Zeichnungen oder Fotos schlimmer Land- und Ortschaftsentstellungen wurden solche gelungener Beispiele gegenübergestellt« (Bayerl 2005: 43) - ein `Trend «im Zeitverlauf, der auf Herausforderungen bei markanten Umbrüchen verweist. Dies bedeutet eine Transformation einer komplexen Sachlage in eine moralische Dichotomisierung, die eine Suche nach Kompromissen erschwert, da auf Moralisierungen nur mit Gegenmoralisierungen geantwortet werden kann (Luhmann 1993), in denen die dichotom von der eigenen Position geschiedene Position einer Pathologisierung unterzogen wird (Kersting 2009). Das Ergebnis ist häufig eine Eskalation des Konfliktes, der auch über die Zeit mit einer finalen (insbesondere gerichtlichen) Entscheidung perpetuiert wird (Berr/Kühne 2019; Kühne 2018b, 2019b; Weber et al. 2018).

\section{Fazit: Megatrends und Hegemonien im Kontext der Energiewende}

Welches Resümee lässt sich abschließend auf Grundlage der Ausführungen ziehen? Die Ausrichtung des Beitrags auf die Entwicklung des deutschen Stromnetzes, der Bestandteile der Stromversorgung und der rezenten Veränderungsprozesse im Zuge der eingeläuteten Energiewende zeigt zunächst, dass gewisse Diskussionsstränge auf der Grundlage von Umbrüchen und erlebten Verunsicherungen wellenartig wiederkehren. Bereits im Laufe des 20. Jahrhunderts wurde über die >Verdrahtung< und >Verspargelung، von >Landschaft und Heimat< gestritten, was als virulente Argumentationsbestandteile im 21. Jahrhundert den Windkraft- und den Stromnetzausbau begleitet. Auch Forderungen nach Erdverkabelungen sind im Übrigen kein neues Argument: In den 1930er Jahren zu »Beginn der Verdrahtungs-Diskussion« wurden diese bereits eingefordert (Bayerl 2005: 46). Immer wieder fanden sich auch schon Phasen, in denen es um angeführte Kritikpunkte wieder >ruhiger wurdes, was durchaus in enger Verbindung mit Gewöhnungseffekten und Etablierungen steht: Bestehende Freileitungen und Kraftwerke der Energieproduktion gehören im Laufe der Zeit zur sheimatlichen Normallandschaft< dazu und werden weniger grundlegend im Hinblick auf ihre physische Manifestation hinterfragt. Es sind markante und schnelle Umbrüche, die mit Sorgen und Unsicherheiten relationiert werden. In den letzten Jahren haben hier die $>$ Megatrends` Globalisierung und Individualisierung durchaus einen nicht zu unterschätzenden Anteil: Im Zuge globaler Verflechtungen verlieren sliebgewonnene< Sicherheiten an Substanz, womit gerade in lokalen Bezügen eine 
Verankerung gesucht wird (Costadura/Ries 2016; Kühne/Spellerberg 2010; Weber/Kühne/Hülz 2019), die aber dann zu erodieren droht, wenn unter anderem Windkraftanlagen oder Stromtrassen in direkter Nähe errichtet werden sollen oder drohen könnten. Es sind gleichzeitig Erwartungen an individuelle >Lebensträume $<$, die vielleicht weniger als beispielsweise in der Nachkriegszeit ein >stilles Erdulden opportun erscheinen lassen. Insofern ist das deutsche Projekt >Energiewende< auch je nach Perspektive mehr oder weniger >nachhaltigs: Eine Vereinbarung ökologischer, ökonomischer und sozialer Komponenten gelingt nicht ohne Weiteres. Was überwiegt nun? Ist es der Klimaschutz mit einer perspektivischen Versorgung aus 100 Prozent erneuerbaren Energien oder der Schutz des Rotmilans usw. zum Naturschutz? Ist es das Wohl der Jugend und künftiger Generationen oder ist es das sungestörte Lebensumfeld? Exemplarisch zeigt sich, dass unterschiedliche Argumente aufeinandertreffen und um gesellschaftliche Hegemonie ringen (dazu allg. Glasze/Mattissek 2009; Laclau/Mouffe 2015 [engl. Orig. 1985]). Bisher konkurrieren verschiedene Diskurse im Energiewende-Kontext - in welcher Form sich dieses >Flottieren schätzbar. Genauer gesagt: Wir sind Geographen und keine Trendforscher, das heißt, wir zielen nicht auf die Vorhersage von Trends oder Megatrends ab. In der Rückschau und einer Gegenwartsanalyse erachten wir gleichzeitig die rasante Entwicklung von Bürgerinitiativen in unterschiedlichsten Gesellschaftskontexten, vernetzend forciert im Zuge der Digitalisierung, als >Trend , der sich durchaus fortführen könnte. Die Vielzahl an Initiativen illustriert, wie heute jenseits etablierter politischer Strukturen Politik beeinflusst wird (hierzu auch Berr et al. 2019b), auch wenn »die absolute Anzahl der Demonstranten und Aktivisten klein« ausfällt, wie Marg et al. (2013: 96) in ihren Analysen in Bezug auf verschiedene Bauvorhaben im Zuge der Energiewende (Windkraft, Biomasse, Pumpspeicher, Netzausbau) herausstellen. Der Ausbau erneuerbarer Energien erscheint als Bestandteil angeführter Megatrends als »Wegweiser in die energetische Zukunft« (Bayerl 2005: 47), wird derzeit aber keineswegs von allen befürwortet. Inwiefern Zielsetzungen der Energiewende und Nachhaltigkeit in Verbindung mit der neu entfachten Debatte um Klimawandel künftig assoziiert werden (können) und so vielleicht stärker (temporär) hegemonial verankert werden, gilt es für uns weitergehend $\mathrm{zu}$ analysieren. 


\section{Literatur}

AG Energiebilanzen (2017): Bruttostromerzeugung in Deutschland ab 1990 nach Energieträgern, Berlin. Online verfügbar unter: www.ag-energie bilanzen.de/index.php?article_id=29\&fileName=20161216_brd_strom erzeugung1990-2016.pdf. Zuletzt aufgerufen am 30.08.2017.

Bauman, Zygmunt (1992): Moderne und Ambivalenz. Das Ende der Eindeutigkeit, Hamburg: Junius.

Bauman, Zygmunt (1999): Unbehagen in der Postmoderne, Hamburg: Hamburger Edition.

Bayerl, Günter (2005): »Die »Verdrahtung« und »Verspargelung« der Landschaft«, in: Schriftenreihe des Deutschen Rates für Landespflege 77, S. 3849.

Becker, Sören/Gailing, Ludger/Naumann, Matthias (2013): »Die Akteure der neuen Energielandschaften - das Beispiel Brandenburg«, in: Ludger Gailing/Markus Leibenath (Hg.), Neue Energielandschaften - Neue Perspektiven der Landschaftsforschung, Wiesbaden: Springer VS, S. 19-31. https:// doi.org/10.1007/978-3-531-19795-1_2

Berr, Karsten (2019): »Heimat und Landschaft im Streit der Weltanschauungen«, in: Martina Hülz/Olaf Kühne/Florian Weber (Hg.), Heimat. Ein vielfältiges Konstrukt, Wiesbaden: Springer VS, S. 27-51. https://doi.org/10. 1007/978-3-658-24161-2_2

Berr, Karsten/Jenal, Corinna/Kindler, Hannah/Kühne, Olaf/Weber, Florian (2019a): »Heimaten gestern und heute. Von Flexibilisierungen und Hybridisierungen«, in: Informationen zur Raumentwicklung, S. 80-89.

Berr, Karsten/Jenal, Corinna/Kühne, Olaf/Weber, Florian (2019b): Landschaftsgovernance. Ein Überblick zu Theorie und Praxis, Wiesbaden: Springer VS. https://doi.org/10.1007/978-3-658-27484-9

Berr, Karsten/Kühne, Olaf (2019): "Moral und Ethik von Landschaft«, in: Olaf Kühne/Florian Weber/Karsten Berr et al. (Hg.), Handbuch Landschaft, Wiesbaden: Springer VS, S. 351-365. https://doi.org/10.1007/978-3658-25746-0_27

Bundesministerium für Wirtschaft und Energie (BMWi) (2018): Erneuerbare Energien in Zahlen. Nationale und internationale Entwicklung im Jahr 2017, Berlin. Online verfügbar unter: https://www.bmwi.de/ Redaktion/DE/Publikationen/Energie/erneuerbare-energien-in-zahlen2017.pdf?_blob=publicationFile\&v=27. Zuletzt aufgerufen am 11.09.2019. 
Bundesministerium für Wirtschaft und Energie (BMWi) (2019): Kommission »Wachstum, Strukturwandel und Beschäftigung«. Abschlussbericht, Berlin. Online verfügbar unter: https:/www.bmwi.de/Redaktion/DE/ Downloads/A/abschlussbericht-kommission-wachstum-strukturwandelund-beschaeftigung.pdf?_blob=publicationFile\&v=4. Zuletzt aufgerufen am 11.09.2019.

Bräuer, Marco (2017): Energiewende und Bürgerproteste. Eine Untersuchung der Kommunikation von Bürgerinitiativen im Themenfeld Netzausbau (= NEU - Nachhaltigkeits-, Energie- und Umweltkommunikation, Band 4), Ilmenau: Universitätsverlag Ilmenau.

Bräuer, Marco/Wolling, Jens (2015): »Protest oder Partizipation? Die Rolle der Bürgerinitiativen im Themenfeld Netzausbau«, in: Bundesnetzagentur (Hg.), Wissenschaftsdialog 2015. Wirtschaft und Technologie, Kommunikation und Planung, Bonn, S. 90-103.

Bundesnetzagentur (2019): Leitungsvorhaben, Bonn. Online verfügbar unter: https:/www.netzausbau.de/leitungsvorhaben/de.html. Zuletzt aufgerufen am 11.09.2019.

Bundesverband WindEnergie (2019): Halbjahreszahlen Windenergie an Land: Historisch niedriger Zubau trotz sehr guter Wachstumsperspektiven Genehmigungsstau dringend auflösen, Berlin. Online verfügbar unter: https://www.wind-energie.de/presse/pressemitteilungen/detail/halb jahreszahlen-windenergie-an-land-historisch-niedriger-zubau-trotzsehr-guter-wachstumsperspekti/. Zuletzt aufgerufen am 10.09.2019.

Bürger, Jakob J. (2018): Transformationsprozesse und Stromnetzausbau. Herausforderungen für die deutsche Energie-Infrastruktur, Wiesbaden: Springer Vieweg. https://doi.org/10.1007/978-3-658-23382-2

Collin, Finn (2008): Konstruktivismus für Einsteiger, Paderborn/Wien: Fink. Costadura, Edoardo/Ries, Klaus (2016): »Heimat - ein Problemaufriss«, in: Edoardo Costadura/Klaus Ries (Hg.), Heimat gestern und heute. Interdisziplinäre Perspektiven, Bielefeld: transcript, S. 7-23. https://doi.org/10. 14361/9783839435243

Dahrendorf, Ralf (1972): Konflikt und Freiheit. Auf dem Weg zur Dienstklassengesellschaft, München: Piper.

Dahrendorf, Ralf (1994): »Die Zukunft des Nationalstaats«, in: Merkur Deutsche Zeitschrift für europäisches Denken 48, 751-761.

Erneuerbare-Energien-Gesetz (EEG) (2000): Gesetz für den Vorrang Erneuerbarer Energien (Erneuerbare-Energien-Gesetz - EEG) sowie zur Änderung des Energiewirtschaftsgesetzes und des Mineralölsteuergesetzes. 
Bundesgesetzblatt Jahrgang 2000 Teil I Nr. 13 (S. 305-309). Online verfügbar unter: www.gesetze-im-internet.de/bundesrecht/eeg/gesamt.pdf. Zuletzt aufgerufen am 24.05.2017.

Felber, Ulrike (1998): » La fée électricité.くVisionen einer Technik«, in: Klaus Plitzner (Hg.), Elektrizität in der Geistesgeschichte, Bassum: GNT-Verlag, S. 105-121.

Flick, Uwe (2007): »Konstruktivismus«, in: Uwe Flick/Ernst v. Kardorff/Ines Steinke (Hg.), Qualitative Forschung. Ein Handbuch, Reinbek bei Hamburg: Rowohlt, S. 150-164.

Fridays for Future Deutschland (2019): Unsere Forderungen an die Politik, Kiel. Online verfügbar unter: https://fridaysforfuture.de/forderungen/. Zuletzt aufgerufen am 12.09.2019.

Fromme, Jörg (2018): »Transformation des Stromversorgungssystems zwischen Planung und Steuerung «, in: Olaf Kühne/Florian Weber (Hg.), Bausteine der Energiewende, Wiesbaden: Springer VS, S. 293-314. https://doi. org/10.1007/978-3-658-19509-0_14

Gailing, Ludger/Leibenath, Markus (2017): "Political landscapes between manifestations and democracy, identities and power", in: Landscape Research 42, S. 1-12. https://doi.org/10.1080/01426397.2017.1290225

Glasze, Georg/Mattissek, Annika (2009): »Die Hegemonie- und Diskurstheorie von Laclau und Mouffe«, in: Georg Glasze/Annika Mattissek (Hg.), Handbuch Diskurs und Raum. Theorien und Methoden für die Humangeographie sowie die sozial- und kulturwissenschaftliche Raumforschung, Bielefeld: transcript, S. 153-179. https://doi.org/10.14361/ 9783839411551

Hagedorn, Gregor/Kalmus, Peter/Mann, Michael/Vicca, Sara/Berge, Joke van den/Ypersele, Jean-Pascal van/Bourg, Dominique/Rotmans, Jan/Kaaronen, Roope/Rahmstorf, Stefan/Kromp-Kolb, Helga/Kirchengast, Gottfried/Knutti, Reto/Seneviratne, Sonia I./Thalmann, Philippe/Cretney, Raven/Green, Alison/Anderson, Kevin/Hedberg, Martin/Nilsson, Douglas/Kuttner, Amita/Hayhoe, Katharine (2019): „Concerns of young protesters are justified«, in: Science 364, S. 139-140. https://doi.org/10. 1126/science.aax3807

Heineberg, Heinz/Kraas, Frauke/Krajewski, Christian (2017): Stadtgeographie (= utb Geographie), Paderborn: Verlag Ferdinand Schöningh.

Helfer, Malte (2015): »Die Industrialisierung der Großregion SaarLorLux«, in: Christian Wille (Hg.), Lebenswirklichkeiten und politische Konstruktio- 
nen in Grenzregionen. Das Beispiel der Großregion SaarLorLux: Wirtschaft - Politik - Alltag - Kultur, Bielefeld: transcript, S. 3-20.

Hermann, Jonas (2018): Für manche ein Symbol des Fortschritts, für andere Teufelszeug: Deutschland hadert mit seinen Windturbinen. Neue Zürcher Zeitung. Online verfügbar unter: https://www.nzz.ch/international/ deutschland/deutsche-buergerinitiativen-gegen-windraeder-ld.1447458. Zuletzt aufgerufen am 12.09.2019.

Hewitt, Kenneth/Nipper, Josef/Nutz, Manfred (1993): »Städte nach dem Krieg. Aspekte des Wiederaufbaus in Deutschland«, in: Geographische Rundschau 45, S. 438-445.

Heymann, Matthias (1995): Die Geschichte der Windenergienutzung 18901990, Frankfurt a.M./New York: campus.

Hoeft, Christoph/Messinger-Zimmer, Sören/Zilles, Julia (Hg.) (2017): Bürgerproteste in Zeiten der Energiewende. Lokale Konflikte um Windkraft, Stromtrassen und Fracking, Bielefeld: transcript. https://doi.org/10.14361/ 9783839438152

Hook, Sandra (2019): Einführung in die Regenerative Energiewirtschaft, Wiesbaden: Springer VS. https://doi.org/10.1007/978-3-658-22416-5

Jarass, Lorenz (2013): »Netzausbau gefährdet Energiewende«, in: Energy 2.0, S. 49.

Kamlage, Jan-Hendrik/Nanz, Patrizia/Fleischer, Björn (2014): »Dialogorientierte Bürgerbeteiligung im Netzausbau«, in: Holger Rogall/HansChristoph Binswanger/Felix Ekardt et al. (Hg.), Im Brennpunkt: Die Energiewende als gesellschaftlicher Transformationsprozess, Marburg: Metropolis-Verlag, S. 195-216.

Kersting, Wolfgang (2009): Verteidigung des Liberalismus, Hamburg: Murmann.

Klagge, Britta (2013): »Governance-Prozesse für erneuerbare Energien - Akteure, Koordinations- und Steuerungsstrukturen«, in: Britta Klagge/Cora Arbach (Hg.), Governance-Prozesse für erneuerbare Energien, Hannover: Selbstverlag, S. 7-16.

Klagge, Britta/Brocke, Tobias (2015): »La transition énergétique à l'échelle locale: la production décentralisée d'électricité et le rôle des entreprises municipales et des fournisseurs régionaux«, in: Revue Géographique de l'Est 55 , S. 1-14.

Kühne, Olaf (2006): Landschaft in der Postmoderne. Das Beispiel des Saarlandes, Wiesbaden: DUV. 
Kühne, Olaf (2011): »Akzeptanz von regenerativen Energien - Überlegungen zur sozialen Definition von Landschaft und Ästhetik«, in: Stadt+Grün 60, S. 9-13.

Kühne, Olaf (2013): »Landschaftsästhetik und regenerative Energien - Grundüberlegungen zu De- und Re-Sensualisierungen und inversen Landschaften«, in: Ludger Gailing/Markus Leibenath (Hg.), Neue Energielandschaften - Neue Perspektiven der Landschaftsforschung, Wiesbaden: Springer VS, S. 101-120. https://doi.org/10.1007/978-3-531-19795-1_7

Kühne, Olaf (2018a): "»Neue Landschaftskonflikte - Überlegungen zu den physischen Manifestationen der Energiewende auf der Grundlage der Konflikttheorie Ralf Dahrendorfs«, in: Olaf Kühne/Florian Weber (Hg.), Bausteine der Energiewende, Wiesbaden: Springer VS, S. 163-186. https:// doi.org/10.1007/978-3-658-19509-0_8

Kühne, Olaf (2018b): »Die Moralisierung von Landschaft - Überlegungen zu einer problematischen Kommunikation aus Sicht der Luhmannschen Systemtheorie«, in: Stefanie Hennecke/Harald Kegler/Kirsten Klaczynski et al. (Hg.), Diedrich Bruns wird gelehrt haben. Eine Festschrift, Kassel: Kassel University Press, S. 115-121.

Kühne, Olaf (2019a): "Autopoietische Systemtheorie und Landschaft», in: Olaf Kühne/Florian Weber/Karsten Berr et al. (Hg.), Handbuch Landschaft, Wiesbaden: Springer VS, S. 91-103. https://doi.org/10.1007/978-3658-25746-0_7

Kühne, Olaf (2019b): »Vom >Bösen und >Gutenく in der Landschaft - das Problem moralischer Kommunikation im Umgang mit Landschaft und ihren Konflikten«, in: Karsten Berr/Corinna Jenal (Hg.), Landschaftskonflikte, Wiesbaden: Springer VS, S. 131-142. https://doi.org/10.1007/978-3658-22325-0_8

Kühne, Olaf/Spellerberg, Annette (2010): Heimat und Heimatbewusstsein in Zeiten erhöhter Flexibilitätsanforderungen. Empirische Untersuchungen im Saarland, Wiesbaden: VS Verlag für Sozialwissenschaften. https://doi. org/10.1007/978-3-531-92294-2

Kühne, Olaf/Weber, Florian (2016): »Zur sozialen Akzeptanz der Energiewende«, in: UmweltWirtschaftsForum 24, S. 207-213. https://doi.org/10.1007/ s00550-016-0415-6

Kühne, Olaf/Weber, Florian (2019): "Landschaft und Heimat - argumentative Verknüpfungen durch Bürgerinitiativen im Kontext des Stromnetzund des Windkraftausbaus«, in: Martina Hülz/Olaf Kühne/Florian Weber 
(Hg.), Heimat. Ein vielfältiges Konstrukt, Wiesbaden: Springer VS, S. 163178. https://doi.org/10.1007/978-3-658-24161-2_9

Kühne, Olaf/Weber, Florian (Hg.) (2018): Bausteine der Energiewende, Wiesbaden: Springer VS. https://doi.org/10.1007/978-3-658-19509-0

Kühne, Olaf/Weber, Florian/Jenal, Corinna (2016): »Der Stromnetzausbau in Deutschland: Formen und Argumente des Widerstands«, in: Geographie aktuell und Schule 38, S. 4-14.

Kühne, Olaf/Weber, Florian/Jenal, Corinna (2018): Neue Landschaftsgeographie. Ein Überblick (= Essentials), Wiesbaden: Springer VS. https://doi. org/10.1007/978-3-658-20840-0

Laclau, Ernesto (2007): On Populist Reason, London, New York: Verso.

Laclau, Ernesto/Mouffe, Chantal (2015): Hegemonie und radikale Demokratie. Zur Dekonstruktion des Marxismus, Wien: Passagen-Verlag.

Leibenath, Markus (2013): »Landschaften unter Strom«, in: Ludger Gailing/Markus Leibenath (Hg.), Neue Energielandschaften - Neue Perspektiven der Landschaftsforschung, Wiesbaden: Springer VS, S. 7-15. https:// doi.org/10.1007/978-3-531-19795-1_1

Linke, Simone (2017): »Neue Landschaften und ästhetische Akzeptanzprobleme«, in: Olaf Kühne/Heidi Megerle/Florian Weber (Hg.), Landschaftsästhetik und Landschaftswandel, Wiesbaden: Springer VS, S. 87-104. https://doi.org/10.1007/978-3-658-15848-4_6

Luhmann, Niklas (1993): »Die Moral des Risikos und das Risiko der Moral«, in: Gotthard Bechmann (Hg.), Risiko und Gesellschaft. Grundlagen und Ergebnisse interdisziplinärer Risikoforschung, Opladen: Westdeutscher Verlag, S. 327-338. https://doi.org/10.1007/978-3-322-83656-4_12

Luhmann, Niklas (2017): Systemtheorie der Gesellschaft, Berlin: Suhrkamp. Maier, Helmut (1993): Erwin Marx (1893-1980), Ingenieurwissenschaftler in Braunschweig, und die Forschung und Entwicklung auf dem Gebiet der elektrischen Energieübertragung auf weite Entfernungen zwischen 1918 und 1950, Stuttgart: GNT-Verlag.

Marg, Stine/Hermann, Christoph/Hambauer, Verena/Becké, Ana B. (2013): »Wenn man was für die Natur machen will, stellt man da keine Masten hin«. Bürgerproteste gegen Bauprojekte im Zuge der Energiewende«, in: Franz Walter/Stine Marg/Lars Geiges et al. (Hg.), Die neue Macht der Bürger. Was motiviert die Protestbewegungen? BP-Gesellschaftsstudie, Reinbek bei Hamburg: Rowohlt, S. 94-138. 
Monstadt, Jochen (2015):»European Power Grids. Raumwissenschaftliche Perspektiven zur Transformation europäischer Energienetze«, in: Nachrichten der ARL 45, S. 4-6.

Naisbitt, John (1982): Megatrends. Ten New Directions Transforming Our Lives, New York: Warner Books.

Neukirch, Mario (2014): Konflikte um den Ausbau der Stromnetze. Status und Entwicklung heterogener Protestkonstellationen. SOI Discussion Paper 2014-01, Stuttgart. Online verfügbar unter: https://www.sowi.unistuttgart.de/dokumente/forschung/soi/soi_2014_1_Neukirch_Konflikte_ um_den_Ausbau_der_Stromnetze.pdf. Zuletzt aufgerufen am 11.09.2019.

Neukirch, Mario (2015): »Mehr Netzausbau mit weniger Kohle? Zwei ökologische Perspektiven auf »Korridor D«, in: Zeitschrift für Politische Ökologie 141, S. 132-135.

Neukirch, Mario (2017): Die Dynamik des Konflikts um den Stromtrassenbau. Stabilität, Wandel oder Stagnation? SOI Discussion Paper 2017-04, Stuttgart. Online verfügbar unter: https://www.sowi.uni-stuttgart. de/dokumente/forschung/soi/soi_2017_4_Neukirch.Dynamik.Konflikt. Stromtrassenbau.pdf. Zuletzt aufgerufen am 11.09.2019.

Obermair, G. M./Jarass, L./Gröhn, D. (1985): Hochspannungsleitungen. Technische und wirtschaftliche Bewertung von Trassenführung und Verkabelung, Berlin, Heidelberg, New York, Tokyo. https://doi.org/10.1007/978-3642-93277-9

Otto, Antje (2019): "Landschaft und der Ausbau der Windenergie«, in: Olaf Kühne/Florian Weber/Karsten Berr et al. (Hg.), Handbuch Landschaft, Wiesbaden: Springer VS, S. 859-869. https://doi.org/10.1007/978-3-65825746-0_69

Roßmeier, Albert/Weber, Florian (2018): »Stürmische Zeiten. Bürgerschaftliches Engagement beim Windkraftausbau zwischen Befürwortung und Ablehnung «, in: Andreas Stefansky/Angelina Göb (Hg.), »Bitte wenden Sie! - Herausforderungen und Chancen der Energiewende, Hannover: Selbstverlag, S. 52-79.

Rust, Holger (2008): Zukunftsillusionen. Kritik der Trendforschung, Wiesbaden: VS Verlag für Sozialwissenschaften.

Schultze-Naumburg, Paul (1901-1917): Die Kulturarbeiten. 9 Bände und I Ergänzungsband, München: Callwey.

Schwenkel, Hans (1938): Grundzüge der Landschaftspflege, Berlin: Verlag J. Neumann. 
Selman, Paul (2010): "Learning to Love the Landscapes of CarbonNeutrality«, in: Landscape Research 35(2), S. 157-171. https://doi.org/10. 1080/01426390903560414

Wahlström, Mattias/Kocyba, Piotr/Vydt, Michiel de/Moor, Joost de (Hg.) (2019): Protest for a future: Composition, mobilization and motives of the participants in Fridays For Future climate protests on 15 March, 2019 in 13 European cities, Online-Ausgabe.

Weber, Florian (2018): Konflikte um die Energiewende. Vom Diskurs zur Praxis, Wiesbaden: Springer VS. https://doi.org/10.1007/978-3-658-20524-9

Weber, Florian/Jenal, Corinna/Roßmeier, Albert/Kühne, Olaf (2017a): »Conflicts around Germany's Energiewende: Discourse patterns of citizens' initiatives«, in: Quaestiones Geographicae 36, 117-130. https://oi.org/10. 1515/quageo-2017-0040

Weber, Florian/Roßmeier, Albert/Jenal, Corinna/Kühne, Olaf (2017b): »Landschaftswandel als Konflikt. Ein Vergleich von Argumentationsmustern beim Windkraft- und beim Stromnetzausbau aus diskurstheoretischer Perspektive«, in: Olaf Kühne/Heidi Megerle/Florian Weber (Hg.), Landschaftsästhetik und Landschaftswandel, Wiesbaden: Springer VS, S. 215244. https://doi.org/10.1007/978-3-658-15848-4_13

Weber, Florian/Kühne, Olaf/Jenal, Corinna/Aschenbrand, Erik/Artuković, Ante (2018): Sand im Getriebe. Aushandlungsprozesse um die Gewinnung mineralischer Rohstoffe aus konflikttheoretischer Perspektive nach Ralf Dahrendorf, Wiesbaden: Springer VS. https://doi.org/10.1007/978-3-65821526-2

Weber, Florian/Kühne, Olaf/Hülz, Martina (2019): »Zur Aktualität von >Heimat als polvalentem Konstrukt - eine Einführung«, in: Martina Hülz/Olaf Kühne/Florian Weber (Hg.), Heimat. Ein vielfältiges Konstrukt, Wiesbaden: Springer VS, S. 3-23. https://doi.org/10.1007/978-3-658-24161-2_1

Weber, Florian/Weber, Friedericke (2019): »Naturparke - Biosphärenreservate - Nationalparke: Schlaglichter auf >Großschutzgebietskonfliktlandschaften zu Beginn des 21. Jahrhunderts«, in: Karsten Berr/Corinna Jenal (Hg.), Landschaftskonflikte, Wiesbaden: Springer VS, S. 247-268. https:// doi.org/10.1007/978-3-658-22325-0_16

Weber, Friedericke (2013): Naturparke als Manager einer nachhaltigen Regionalentwicklung. Probleme, Potenziale und Lösungsansätze, Wiesbaden: Springer VS. https://doi.org/10.1007/978-3-658-01972-3 
Wehling, Hans-Werner (2006): »Aufbau, Wandel und Perspektiven der industriellen Kulturlandschaft des Ruhrgebiets«, in: Geographische Rundschau 58, S. 12-19.

Würfel, Philip (2017): Unter Strom. Die neuen Spielregeln der Stromwirtschaft, Wiesbaden: Springer. https://doi.org/10.1007/978-3-658-15164-5

WWF Deutschland/LichtBlick SE (2015): Megatrends der globalen Energiewende. Online verfügbar unter: https://mobil.wwf.de/fileadmin/fm-wwf/ lichtblick/Megatrends-der-globalen-Energiewende.pdf. Zuletzt aufgerufen am 15.07.2019.

Zukunftsinstitut GmbH (2018): Megatrends, Frankfurt a.M./Wien. Online verfügbar unter: https://www.zukunftsinstitut.de/dossier/megatrends/. Zuletzt aufgerufen am 15.07.2019. 



\title{
"Einfach zu viele Autos«: Neue Antriebe alleine reichen nicht.
}

\author{
Weert Canzler und Andreas Knie
}

Von der Steuergesetzgebung, dem Ausbau der Verkehrsinfrastruktur bis zur Straßenverkehrsordnung war über Jahrzehnte alles darauf ausgerichtet, das private Auto zu fördern. Die Entfernungspauschale, der Bundesverkehrswegeplan und das generelle Recht, auf öffentlichen Straßen das private Vehikel einfach abstellen zu dürfen, sind beeindruckende Beispiele dafür. Dahinter stand das lange mächtige Narrativ vom Privatauto als Voraussetzung und Teil eines modernen Lebensmodells. Nun sind die Grenzen des privaten Autoverkehrs allerdings längst erreicht. Zunehmend wird klar, dass es einfach zu viele Autos gibt. Dauerstaus, die Klimakrise, die schlechte Luft und der Lärm in den Städten und nicht zuletzt der Dieselskandal lassen die Zweifel an einem »Weiter-so« steigen. Das SUV wird zum Symbol für den Imageschaden, den das Auto gerade in den Städten erlebt, die Rufe nach einer Verkehrswende werden lauter. Sharing-Modelle gewinnen in Folge der persönlichen Digitalisierung an Attraktivität, der Zugang zu Autos wird wichtiger als der Besitz. Damit werden Fahrzeuge austauschbar. Für die Autohersteller bedeutet das, dass sie die Transformation zum Mobilitätsdienstleister schaffen müssen. Ansonsten drohen sie zu Zulieferern für Anbieter digitaler Plattformen abzusteigen.

Vor allem aus Gründen der Dekarbonisierung ist der Verbrennungsmotor ein Auslaufmodell. Der Antriebswechsel ist aber nur eine notwendige und keineswegs hinreichende Voraussetzung der Verkehrswende. Es braucht ebenso eine drastische Reduktion der Anzahl der Fahrzeuge. Das wird nur funktionieren, wenn intermodale Mobilitätsdienstleistungen und die emissionsfreie aktive Mobilität den erforderlichen Platz erhalten. Bisher fehlen jedoch die Rahmenbedingungen und Leitplanken für eine nachhaltige Mobilität. Starten wird die Verkehrswende im Kleinen, zunächst in den Städten, organisiert 
im Rahmen von regulatorischen Experimentierräumen, die auch eine deutlich erweiterte Form der Beteiligung vorsehen ${ }^{1}$.

\section{Das Problem: Zu viel vom Gleichen}

Das Automobil droht am eigenen Erfolg zu ersticken. In Deutschland beispielsweise sind derzeit mehr als 47 Millionen Pkw zugelassen, insgesamt sogar mehr als 65 Millionen motorisierte Fahrzeuge (KBA 2018). Gerade weil es so erfolgreich ist, ist das Auto zum Raumvernichter Nr. 1 geworden.

Das Auto hat aber nicht nur immensen Raumbedarf, es ist auch ein hochproblematischer Schadstoffemittent. Trotz vieler öffentlicher Ankündigungen der Autohersteller, auf die Elektrifizierung zu setzen, dominiert der Verbrennungsmotor unangefochten. Eine ganze Branche ist in einer tiefen Pfadabhängigkeit verfangen. Das gilt besonders für die deutsche Autoindustrie, die komplett auf den Dieselantrieb gesetzt hat. Ihre Innovationsschwäche ist umso dramatischer, weil die Kfz-Industrie zu den wichtigsten Industriezweigen in Deutschland gehört. Die Wertschöpfung ist hoch, die Exportanteile sind riesig, die Arbeitsplätze zahlreich und die Gewerkschaften stark. Nirgends gibt es so viele gut bezahlte Jobs wie in den hochmodernen Fabriken der lange überaus profitablen Premiumhersteller und ihrer Zulieferer.

Das Innovationsdilemma dieser Schlüsselindustrie ist mit Blick auf die Klimaschutzziele fatal. Kein Sektor ist so weit entfernt von dem Ziel, Treibhausgasemissionen zu senken wie der Verkehr, der ja mehr denn je vom motorisierten Straßenverkehr geprägt ist. Der Dieselskandal zeigt auch, dass mit zusätzlichen Reinigungsverfahren und einer optimierten Steuerungssoftware alleine weder die aus Klimaschutzgründen notwendigen Verbrauchsreduktionen noch die sonstigen Emissionsgrenzwerte zu schaffen sind.

Die jüngste Diskussion um den anstehenden Ausstieg aus der Verbrennungsmotortechnik und mögliche Jahreszahlen für einen Zulassungsstopp für Verbrenner ist richtig und notwendig, zugleich aber auch verkürzt. Es bedarf nicht nur neuer emissionsfreier Antriebe. Das Verkehrssystem insgesamt muss sich ändern. Der motorisierte Individualverkehr wie wir ihn kennen braucht viel zu viel Platz. Er kommt schon seit Jahren in den Metropolen der Welt an seine Grenze bzw. hat diese längst überschritten. Es stockt und 
staut sich, die Luft ist schlecht und an Platz für die Aufenthaltsqualität fehlt es auch.

Die Lage ist schizophren: Das Geschäft mit und um das Auto prosperiert, gleichzeitig sind die Grenzen des Modells deutlich. Aus Klimaschutzgründen ist die Dekarbonisierung des Verkehrs und damit die Antriebswende als Teil einer umfassenden Verkehrswende möglichst schnell umzusetzen (Agora Verkehrswende 2017). Doch lässt sich nicht einfach ein Schalter umlegen. Die sozialwissenschaftliche Mobilitätsforschung zeigt, dass der Verkehr auf Routinehandeln beruht, über das man nicht nachdenken möchte (Canzler 2016: 29ff.).

Mit dem Versagen beim Klimaschutz und der Verdrängung des Dieselskandals ist die Volatilität im Verkehr gestiegen. Zur Unsicherheit gehört auch, dass sich Einstellungen und Verhaltensweisen insbesondere in den Städten verändern und neue technische Optionen verfügbar sind, die aber nicht genutzt werden können, weil der öffentliche Verkehrsraum streng codiert und völlig verriegelt ist. Für Innovationen ist dort im wahrsten Sinne kein Platz. Diese Regulierungen haben dabei aber eine gesellschaftliche Praxis festgeschrieben, die es schon seit Jahren nicht mehr gibt. Gleichzeitig besitzen diese Regulierungen aber für den Alltagsverkehr immer noch Gültigkeit und sind Teil unserer Routinen.

Verkehr war und ist ein so träges Politikfeld, weil in dieser Komplizenschaft zwischen Autoherstellern, Konsument*innen und staatlicher Politik ein Modus Vivendi entwickelt und gelebt wurde, der heute einem Gefangenendilemma gleicht: Keiner traut sich aus dieser Grundordnung heraus, weil nicht klar ist, wie der jeweils andere reagieren wird. Es ist ein Dreiklang aus neuen technischen Optionen, veränderten Einstellungen sowie anderen Regelwerken. Alle wissen, dass es ein »Weiter-so« nicht geben kann. Dennoch ist der Weg der Transformation, die eigentliche Verkehrswende, derzeit kaum erkennbar. Technische und habituelle Pfadabhängigkeiten und in Asphalt und Beton gegossene Infrastrukturen dominieren alles. Wie konnte es dazu kommen?

\section{Wie wir überhaupt zum Auto kamen}

Ohne eine Veränderung der rechtlichen und infrastrukturellen Rahmenbedingungen wird es keine Verkehrswende geben. Dazu gehören neben einer Internalisierung der externen Kosten des Straßenverkehrs über nutzungsab- 
hängige Gebühren für die Inanspruchnahme von Straßen und Parkraum auch der Abbau der Privilegien für das Auto. Zweifelsohne müssen Subventionen wie der reduzierte Steuersatz für Dieselkraftstoff und das Dienstwagenprivileg fallen. Die Verkehrswende kann nur gelingen, wenn die Rahmenbedingungen zugunsten der Alternativen zum bisher alles beherrschenden privaten Auto verändert werden.

Die Forderung nach veränderten Rahmenbedingungen ist so alt wie die Verkehrswendediskussion (vgl. auch Rammler 2017). Bislang ist sie jedoch nicht einmal ansatzweise eingelöst worden. Warum das so ist, lässt sich nur erklären, wenn man auf die Erfolgsgeschichte des privaten Automobils schaut. Um 1900 war ihre Zahl noch auf wenige Tausend Exemplare begrenzt und es war zu diesem Zeitpunkt auch noch keineswegs klar, dass aus den knatternden, stinkenden und gefährlichen Geräten einmal eine Massenbewegung werden sollte. Deutschland steht für ein besonders krasses Entwicklungsmuster. Mit Blick auf die starke deutsche Kraftfahrzeugindustrie könnte man annehmen, dass das Auto gleichsam in die DNA der Deutschen eingeschrieben wäre. Doch so ist es nicht. Deutschland war lange das Land der Reichsbahn, das Land der Fahrräder, Mopeds und Motorräder. Bis 1957 war die schwäbische Firma NSU, die später zusammen mit der nach Ende des Zweiten Weltkrieges aus dem Osten in den Westen umgezogenen Auto-Union zur AUDI AG verschmolzen wurde, der Weltmarktführer im Zweiradgeschäft mit Fahrrädern und Mopeds. Und noch im Jahre 1938 war die Deutsche Reichsbahn mit mehr als 1,2 Mio. Beschäftigten das größte Unternehmen der Welt. Das Unternehmen war weit verzweigt, Anteile an der Lufthansa gehörten genauso dazu wie das größte deutsche Speditionsunternehmen Schenker.

Die deutsche Autoindustrie war zu dieser Zeit hingegen eine Randerscheinung. Das größte Unternehmen der Autobranche, die Adam Opel AG, war schon 1926 an den US-amerikanischen Konzern General Motors verkauft worden, die ehemals unabhängigen Unternehmen Horch, Wanderer, Audi und DKW vereinigten sich aufgrund wirtschaftlicher Zwänge unter der Regie des Finanzkapitals zur Auto-Union, Daimler war bereits kurz nach dem Ersten Weltkrieg mit Benz fusioniert worden. Im Jahr 1932 kamen in Deutschland knapp 8 Autos auf 1.000 Einwohner. Ein Mittelklassewagen kostete rund das 50-fache eines Arbeiterjahreslohnes (vgl. Canzler/Knie 2016). 


\section{Mit der »Charta von Athen« in die Moderne}

Schon in den 1920er Jahren waren es vor allen Dingen die Architekt"innen und Stadtplaner*innen, die das Auto zum Verkehrsmittel der Zukunft machten. Der private Kraftwagen kam ihnen deshalb gelegen, konnte mit ihm die Stadt der Zukunft doch aus ihrer baulichen Enge in eine gelockerte und funktional ausdifferenzierte Siedlungslandschaft überführt werden. Die Stadt der Moderne sollte hell und vor allen Dingen raumgreifend ausgestaltet sein und sich von den dichten, dunklen und oft sehr kleinteiligen Bebauungen der Altstädte komplett unterscheiden. Dafür brauchte sie ein flexibles und alle Elemente verbindendes Verkehrsmittel. Die Straßenbahnen, die U- und S-Bahnen halfen da nicht weiter, sie erschienen in ihren Strukturen zu starr. Automobile versprachen dagegen ein dynamisches und verbindendes Verkehrsmittel zu sein, dem man dann aber auch den gebührenden Raum schaffen musste. Alte Stadtanlagen waren Jahrhunderte vor dem Auto entstanden und boten daher auch wenig Platz. Dieser musste dann zunächst gedanklich, dann planerisch und schließlich auch real geschaffen werden. Pointiert brachte es der Schweizer Architekt und Stadtplaner Le Corbusier auf den Punkt: »Wohin eilen die Automobile? Ins Zentrum! Es gibt keine befahrbare Fläche im Zentrum. Man muss sie schaffen. Man muss das Zentrum abreißen! (Le Corbusier 1925: 101).

Das einflussreichste Dokument der Stadtplanung, das diese Gedanken zusammenfasst, ist die in den 1930er Jahren entwickelte und 1943 veröffentlichte »Charta von Athen «. Bis heute gelten die hier entwickelten Grundsätze in Mittel- und Südamerika und auch in weiten Teilen Asiens als die Bibel der Planung des urbanen Raumes. In West und Ost war die moderne Stadtplanung bis in die späten 1960er Jahre immer auf das Auto als das zentrale Verkehrsmittel zugeschnitten. Städte wurden primär als Transitzonen gedacht, damit die unterschiedlichen Funktionen »Arbeit«, »Wohnen« und »Freizeit« angemessen miteinander verbindbar waren. Das Auto wurde praktisch zum "Narrativ der Moderne « und verdichtete sich zugleich zum Versprechen auf Freiheit auf individuelle Lebensführung mit eigenem Haus, Garten und Kleinfamilie. Das Auto erlebte seinen Aufstieg daher nie nur als ein technisches Gerät, es war immer der Kern einer ganzen Lebensphilosophie, eines Versprechens eines selbstbestimmten und kontrollierbaren Lebens.

Doch war dieses Versprechen zunächst nur ein Traum, eine Sehnsucht, eine Idee, illustriert in vielen Bildern, Büchern und Filmen. Aber es hatte enorme Strahlkraft in viele Branchen und Sektoren hinein. Das betraf auch den schon erwähnten Reichsautobahnbau, der bereits in den späten 1920er Jahren 
vorgedacht, bis ins Detail geplant und dann ab Sommer 1933 umgesetzt wurde. Der Bau war weder militärisch noch aus arbeitsmarktpolitischen Erwägungen vordringlich. Auch verkehrspolitisch war er überhaupt nicht notwendig, weil es zu dieser Zeit in Deutschland kaum mehr als 500.000 Automobile gab. Die Signale waren jedoch eindeutig, zunehmend wurde klar, wohin die Reise gehen sollte. Es zeichnete sich ein wiederkehrendes Muster ab: Die in den deutschen Ländern sehr unterschiedlichen Verkehrsregelungen wurden ab 1934 erstmals reichseinheitlich zu einem Gesetz zusammengefasst, an dessen Gestaltungswillen eine Zukunft für den Massenverkehr einzuleiten, kein Zweifel bestand. Ein weiteres Ergebnis: Die Reichsgaragenordnung ebenfalls von 1934. Darin wurde ganz im Geiste der Charta von Athen festgelegt, dass nicht nur Straßen für den fließenden Verkehr gebaut, sondern auch Parkflächen im öffentlichen Raum zum Abstellen der künftigen Fahrzeuge vorgesehen werden müssen.

Die Nazis unternahmen weitere Anstrengungen zur Popularisierung des Automobils, der Traum vom eigenen Wagen sollte wahr werden können. Ab 1938 konnte man für einen eigenen »KdF-Wagen« ansparen, der unter 1.000 Reichsmark kosten sollte. Zwar wurde bekanntlich nichts aus der Auslieferung; die wenigen Fahrzeuge, die noch vor dem Krieg fertig wurden, blieben Parteibossen vorbehalten und alle anderen gingen als militärisches Gerät gleich in den Vernichtungskrieg. Aber der Gedanke, ein eigenes Auto zu besitzen und es sich über moderate Ansparprämien leisten zu können, war erfolgreich platziert.

Selbst die Kriegszerstörungen taten dem Traum vom Automobil keinen Abbruch, ganz im Gegenteil: Im Stab des Rüstungsministers Albert Speer begann man bereits 1942, am Wiederaufbau der Städte zu arbeiten. Die Zerstörungen kamen den Planern in zynischer Weise gerade Recht, konnte man doch gleich Platz schaffen. Als Vorbild galt den nationalsozialistischen Planern tatsächlich New York, damals die größte Metropole der Welt. Hier zeigte sich, dass dem Automobil die Zukunft gehörte - so ein Planungsdokument der Vorbereitungsgruppe. Man müsse mit einer sehr schnell steigenden Zahl von Kraftwagen rechnen. So wurde beispielsweise für Berlin vorgesehen, im Rahmen eines Generalplanes unmittelbar nach 1945 die komplette Stadt mit einem dichten Netz von Stadtautobahnen zu überziehen (vgl. Durth 1995).

Deutschland war auch nach dem Zweiten Weltkrieg noch kein Automobilland. Die Zahl der angemeldeten Kfz blieb weiterhin hinter den Erwartungen der Bundesregierung zurück. Zumal mittlerweile diese Zahl innerhalb der OECD als zentraler Indikator für die Prosperität einer Volkswirtschaft galt. 
Je mehr Autos zugelassen waren, umso besser schien es der Gesellschaft auch wirtschaftlich zu gehen. Der Grad der Wohlstandsmessung war also, wie viele Menschen sich ein eigenes Auto leisten konnten. Um hier nicht ins Hintertreffen zu gelangen und um das Auto wirklich zu einem Massenverkehrsmittel werden zu lassen, wurden die Maßnahmen zur Popularisierung des Autos weiter fortgesetzt. Der Historiker Dietmar Klenke nennt das Jahre 1957 als das Wendejahr in der (west-)deutschen Verkehrsgeschichte, in dem durch eine Reihe von fiskalischen Maßnahmen die Attraktivität des privaten Pkw nochmals zusätzlich deutlich erhöht wurde (Klenke 1995). Unter anderem konnten Privatpersonen berufsbedingte Fahrten mit einem Kilometersatz von der Einkommenssteuer absetzen, der deutlich über den eigentlichen Betriebskosten lag. Die Mineralölsteuer, die heutige Energiesteuer, die seit 1939 auf Benzin und Dieselverkäufe erhoben wird, wurde zweckgebunden, d.h. die Einnahmen durften nur noch für Bau und Erhalt von Bundesstraßen eingesetzt werden. Umfang und Wirkung dieses steuerlichen Anreizprogramms für die Massenmotorisierung sind kaum zu überschätzen. In diese Zeit fällt im Übrigen auch die Steuerbefreiung von Flugbenzin im Inland und ein reduzierter Steuersatz für Treibstoff für eine landwirtschaftliche Nutzung. Interessanterweise gab es von Anfang an Unterschiede bei den Steuersätzen für Benzinund Dieselkraftstoff. War der Steuervorteil für Diesel bis in die 1980er Jahre moderat, so änderte sich das zu Beginn der 1990er Jahre, als in zwei Steuererhöhungsrunden beim Diesel wesentlich geringere Aufschläge beschlossen wurden. Seit 1994 beträgt die Differenz ca. 18 Cent je Liter. Das Ergebnis des Dieselprivilegs ist offensichtlich, nämlich eine starke Dominanz des Dieselmotors vor allem bei hochmotorisierten Fahrzeugen wie den SUVs. Alleine im Zeitraum von 1990 bis 2015 betrugen die Steuermindereinnahmen aufgrund der geringeren Dieselbesteuerung ca. 254 Mrd. Euro (Deutscher Bundestag 2017).

Damit war ein sich selbst beschleunigender Regelkreislauf in Gang gesetzt worden: Mehr Steuern, mehr Infrastruktur, höhere Attraktivität für Autos mit steigendem Kraftstoffverbrauch, d.h. höhere Steuern, mehr Geld für Infrastrukturen. Aber auch dieser Gelddruckmechanismus für beispielsweise neue Autobahnen wurde immerfort auch programmatisch begründet: Georg Leber, Bundesverkehrsminister im ersten Kabinett Willy Brandts, wird der Ausspruch aus dem Jahre 1971 zugeschrieben, dass kein Bundesbürger mehr als 25 Kilometer zurücklegen solle, um die nächste Autobahnauffahrt zu erreichen. 
Diese Maßnahmen wurden begleitet von einer Reihe von bau- und verkehrsgesetzlichen Regelungen. Die bereits erwähnte, von den Nationalsozialisten eingeführte Reichsgaragenordnung fand sich als Stellplatzverordnung der Länder wieder. Die Verordnung besagte: Wer privat und öffentlich bauen wollte, musste genügend Flächen zum Abstellen von Pkws bereitstellen. Die Programme zur Unterstützung einer massenhaften Verbreitung von Automobilen fanden konsequenterweise auch im Straßenrecht und in der Straßenverkehrsordnung ihre Anwendung. Grundsätzlich wurde dem Auto gegenüber allen Verkehrsmitteln Vorfahrt gewährt und zwar unter dem Diktum des rollenden Verkehrs. Rechtsgrundsatz ist seither: Es muss alles fließen und was hier stört - wie Fahrradfahrer und Fußgänger - muss weichen oder wird an den Rand gedrängt. Der besondere juristische Kniff zur Förderung des privaten Besitzes von Automobilen bestand darin, die mehr und mehr herumstehenden Fahrzeuge einfach zum »ruhenden Verkehr« zu deklarieren und damit auch unter den Generalschutz des Verkehrsflusses zu stellen. Dafür musste natürlich auch der Preis des öffentlichen Raumes entsprechend reguliert werden. Mieter*innen und Wohnungseigentümer*innen müssen beispielsweise - wenn überhaupt Geld für das Parken fällig wird derzeit maximal 30 EUR pro Jahr für einen Anwohnerparkplatz zahlen. Das sind etwas mehr als 8 Cent pro Tag.

Wer sich unter solchen Bedingungen kein Auto anschaffte, der oder die war jetzt klar im Nachteil. Aber auch für die kleiner werdende Klientel der (noch) Nicht-Automobilisten wurde gesorgt. Bereits unter den Nazis war da$z u$ das bis heute noch gültige Personenbeförderungsgesetz (PBefG) eingeführt worden, durch das öffentliche Verkehre mit Bussen und Bahnen im Geltungsbereich von Entfernungen unter 50 Kilometer aber auch Taxis und Mietwagen unter eine behördliche Aufsicht gestellt werden. Der Staat deckt das durch Fahrgeldeinnahmen nicht zu finanzierende Defizit des Betriebes von Bussen und Bahnen $a b$, behält sich aber die Oberaufsicht sowie auch die Genehmigung über alle Linien, Tarife und sonstigen Bedingungen vor. Damit haben die Unternehmen, die Teil dieser staatlichen Daseinsvorsorge sind, zwar eine auskömmliche Perspektive, dafür sind aber die Kernelemente der Leistungserbringung gleichsam eingefroren. Eine wettbewerbliche Dynamik war und ist in diesem System nicht vorgesehen. Der ÖPNV erfüllte jahrzehntelang nur eine Ersatz- und Überlauffunktion für die Automobilgesellschaft. Es sollte ganz bewusst keine Konkurrenz zum eigenen Auto aufgebaut werden. Wieviel Menschen im ÖPNV unterwegs waren, galt in den 1950er und 1960er Jahren keineswegs als Ausweis eines modernen Staates, eher war das 
Gegenteil der Fall. Hohe Fahrgastzahlen wurden als Zeichen gesellschaftlicher Rückständigkeit angesehen.

\section{Der Traum vom eigenen Auto}

Nachdem hinlänglich Fernstraßen gebaut und die Innenstädte zu Transiträumen umfunktioniert, alle Neubauten mit Stellplätzen und genügend Erschließungsstraßen ausgestattet worden waren, zudem die Nutzung eines Kraftwagens steuerlich in einem Maße geltend gemacht werden konnte, die höher als die tatsächlichen Kosten lagen, stiegen die Zulassungen von Automobilen dann tatsächlich deutlich an. Mitte der 1960er Jahre erreichten sie in Westdeutschland erstmals internationales Niveau, 1965 waren knapp 9,3 Millionen Pkw zugelassen.

Hinter all diesen Maßnahmen stand immer das gleiche »Narrativ«: Der Traum vom eigenen Pkw, der das glückliche Idyll privater Lebensformen unterstützt. Dieses Leitbild fiel offenkundig auf einen fruchtbaren Boden. Nur so konnten die teilweise mit brachialer Gewalt vorgenommenen städtebaulichen Zurichtungen im Geist der »autogerechten Stadt« begründet und auch weithin akzeptiert werden. Es ging um eine offenkundig attraktive Geschichte des eigenen Autos und einer glaubhaften Hoffnung auf die materielle Hinterlegung dieser Story. Und dies nicht nur in Deutschland und Europa. Vorbild waren die USA, dort wurde schon früher der eigene Wagen $\mathrm{zu}$ einem elementaren Teil des Traumes von der Freiheit stilisiert.

In dem skizzierten Erfolgsprogramm für das private Automobil steckt auch die Blaupause für den Umbau der Automobilgesellschaft. Für eine erfolgreiche Verkehrswende braucht es ein neues Narrativ, dass ein glaubhaftes Versprechen auf eine lebenswerte Zukunft darstellt und zudem eine breite gesellschaftliche Unterstützung erlangt. Ein solches post-automobiles Leitbild zeichnet sich in ersten Konturen ab, einige Trends und Treiber sind zu erkennen.

\section{Treiber der Verkehrswende}

Einer der wichtigsten Treiber für die Verkehrswende sind die Klimaschutzziele. Dabei ist das Hauptproblem die bisher ungebrochene Dynamik des nach wie vor auf fossilen Quellen beruhenden Energiebedarfs. Der Anteil des Verkehrs am Endenergiebedarf ist von 25 Prozent im Jahr 1990 auf fast 30 Pro- 
zent im Jahr 2015 gestiegen (Agora Verkehrswende 2017: 12). Voraussichtlich wird er im Jahr 2020 bereits die Marke von 40 Prozent erreichen. Dabei befinden wir uns, global betrachtet, erst am Anfang einer nachholenden Motorisierung. In Asien, Südamerika und Afrika liegt der Ausstattungsgrad mit Autos und der Verkehrsaufwand insgesamt weit unter dem Level, den wir in unseren Breiten gewohnt sind und den wir auch vorleben.

Der verfestigte Pfad des privaten Automobilismus zeigt sich auch im individuellen Verkehrsverhalten. Gerade im Alltagsverkehr zeigt der moderne Mensch ein habitualisiertes Verhalten: Er oder sie möchte Störungen vermeiden und Verkehrsmittel »nutzen ohne nachzudenken« (vgl. Canzler 2016). Das private Auto hilft dabei, diese Bedürfnisse zu befriedigen, und macht es dadurch Alternativen schwer. So wächst weiterhin die Zahl der Autos in Deutschland, auch wenn die durchschnittliche Fahrleistung je Vehikel seit Jahren sinkt und die Pkw-Verkehrsleistung insgesamt nur leicht zunimmt (vgl. BMVI 2016: 219).

Die Dominanz des individuellen Massenverkehrsmittels Auto hält also bis heute unvermindert an. Doch sind Erosionen und mögliche Bruchlinien erkennbar. Der Erfolg des Autos, seine massenhafte Verbreitung in den früh motorisierten Regionen der Welt, ist ein Problem - es gibt einfach zu viel desselben. Das wird ganz deutlich, wenn man in die Länder schaut, wo die Massenmotorisierung gerade erst begonnen hat. In den Megacities Chinas und anderer aufstrebender Schwellenländer führt der wachsende Autoverkehr zu Stillstand und zu unerträglichen Luftverhältnissen. Zugleich ist klar, dass angesichts der unvermeidlichen Dekarbonisierung auch der Verkehrssektor seinen Beitrag leisten muss und dass der Verbrennungsmotor ein Auslaufmodell ist. Dazu kommt, dass in den gesättigten Automärkten - also in Europa, in den USA und auch in Japan und Korea - das Auto seinen Status als besonderes Konsumgut verloren hat. Es ist dort mehr und mehr zu einer Commodity geworden.

\section{Höhere Lebensqualität in den Städten und die Chancen der persönlichen Digitalisierung}

Das Erbe einer Politik der autogerechten Stadtentwicklung wirkt trotz gewandelter verkehrs- und stadtpolitischer Rhetorik bis heute. Gleichwohl verändert sich, hinter dem Rücken der Akteur*innen, die urbane Mobilität. Verschiedene politische und technische Trends wirken als Treiber des Wandels. Sie sind tiefgreifend und haben das Potential, die Spielregeln im Verkehr 
komplett zu verändern. Da ist zum einen der fortgesetzte Kampf gegen Luftverschmutzung und verbindliche Vorgaben zum Klimaschutz. Um die Luftschadstoffbelastung gerade in den Städten zu senken, werden Emissionsgrenzwerte weltweit weiter verschärft. Dahinter steht das Ziel, die Lebensqualität zu verbessern und negative Auswirkungen vor allem auf die Gesundheit zu vermindern. Ambitionierte Grenzwerte für Stickoxide und Feinstaub sind künftig von Fahrzeugen mit Verbrennungsmotoren nicht mehr zu erreichen. Viele Kommunen setzen sich außerdem in lokalen Klimaschutzplänen ambitionierte Klimaschutzziele, die nicht zuletzt auch den Verkehr betreffen. $\mathrm{CO}_{2}-$ Reduktionsziele sind im Verkehr nur mit einer Verlagerung zum Umweltverbund und mit einer verstärkten Elektrifizierung des motorisierten Verkehrs auf Grundlage Erneuerbarer Energien zu erreichen.

Die Verkehrswende wird zum anderen stark von der Digitalisierung vorangetrieben. Globale Digitalunternehmen drängen mit neuen Geschäftsmodellen in die Verkehrsmärkte. Gleichzeitig ist ein Boom an verkehrsbezogenen Apps zu beobachten. Ihre Potentiale liegen in erster Linie darin, gemäß den persönlichen Profilen optimale Verbindungen mit verschiedenen Verkehrsmitteln zu erhalten. Diese Entwicklung ist vor dem Hintergrund einer generellen Veränderung von Verhaltensmustern infolge der persönlichen Digitalisierung zu sehen. Chat-Dienste wie Whatsapp oder Facebook verändern das Mobilitätsverhalten grundsätzlich: Statt starrer Verabredungen finden in alläglichen Interaktionen vielmehr schrittweise Annäherungen via Smartphone statt (vgl. Canzler/Knie 2016). Die Digitalisierung verstärkt den gesellschaftlichen Basistrend der Individualisierung und wird ihrerseits durch sie selbst vorangetrieben. Persönliche Profile auf Verkehrs-Apps und eine transaktionskostenarme Verknüpfung verschiedener Verkehrsmöglichkeiten gehen mit differenzierten Mobilitätsmustern einher. In der Konsequenz ist eine weitere Differenzierung des Verkehrs auf der Angebotsund auf der Nachfrageseite zu erwarten. Aufgrund neuer technischer Möglichkeiten und in Folge eines real veränderten Verhaltens werden auch die individuellen Planungshorizonte kürzer und die Verkehrsteilnehmer*innen geraten in die Rolle des Prosumenten, der digital unterstützt seine eigene Mobilität organisiert.

Die Digitalisierung ermöglicht integrierte Mobilitätsdienstleistungen für die Nutzer*innen aus »einem Guss«. Damit eröffnen sich neue Chancen für den Öffentlichen Verkehr (ÖV). Ob und inwieweit der ÖV seine verkehrs- und umweltpolitischen Vorzüge ausspielen kann und spürbare Modalverschiebungen tatsächlich zu erreichen sind, hängt stark von seiner 
Attraktivitätssteigerung und insbesondere von seiner »intermodalen Passung« ab. In intermodalen Dienstleistungen ist ein moderner Bahnverkehr mit anderen Verkehrsmitteln, nicht zuletzt mit dem Auto, verbunden und die Transaktionskosten eines Wechsels der Verkehrsmittel für die Nutzer*innen niedrig. Neben den veränderten Finanzierungsstrukturen sind hier neue Wettbewerbs- und Geschäftsmodelle notwendig, um die innovativen Potentiale der Branche anzureizen. Idealerweise kommen künftig die Elektrifizierung und die geteilte Nutzung von verschiedenen Verkehrsmitteln zusammen. Die Integration zielt dann auf die elektrisch betriebene Fernbahn ebenso wie auf die Tram, die U- oder S-Bahn und das Pedelec sowie das geteilte E-Auto. So sind Netzwerkeffekte zu erzielen, die im bisherigen fahrplan- und haltestellengebundenen ÖPNV nicht $\mathrm{zu}$ erreichen waren. In vielen Städten gehören öffentliche Autos und Fahrräder bereits heute zum selbstverständlichen Teil des öffentlichen Verkehrsangebotes. Sie erlauben Haus-zu-Haus-Verbindungen und können damit einen Vorteil des privaten Autos ausgleichen, nämlich auch die »erste und letzte Meile« eines Weges schnell und bequem zu überwinden. Das sind Hinweise auf eine Konvergenz von privatem und öffentlichem Verkehr. Hinter der möglichen Konvergenz stecken nicht nur technische Entwicklungen. Gleichzeitig sind auch bei den Einstellungen und beim Verhalten insbesondere bei den jüngeren Generationen von Stadtbewohner*innen Veränderungen zu beobachten, die auf einen Bedeutungsverlust des eigenen Autos und auf eine verstärkte pragmatische Inter- und Multimodalität hinweisen (vgl. Schönduwe/Lanzendorf 2014; Deffner et al. 2014).

Vor allem in großen Städten haben sich inter- und multimodale Nutzungspraktiken etabliert, die zu eigenständigen Mobilitätstypen jenseits der klassischen Auto- oder ÖV-Affinen geführt haben (vgl. LSE/InnoZ 2015). Die hohe Verbreitung von Smartphones und Flatrates erleichtert Sharingdienste und intermodale Services. Diese Nischenmärkte zeigen seit Jahren eine große Dynamik. Die Realisierung flexibler Formen des ja bereits länger bekannten stationsgebundenen Carsharings beispielsweise ist nur möglich, weil das Auffinden der Fahrzeuge mit dem Smartphone einfach und bequem wurde. Ein Blick auf die App zeigt, welche Fahrzeuge wo stehen. Das Carsharing-Beispiel deutet eine Revolution in der Verkehrsmittelwahl an (siehe ausführlich: Canzler/Knie 2016: 13ff.). Für die Nutzer*innen von flexiblen Carsharing-Systemen ist es wichtig, hier und jetzt ein Fahrzeug zu bekommen. Die Entscheidungen werden in Sekundenbruchteilen getroffen. Weder die Marke des Fahrzeugs noch die des Carsharing-Anbieters sind dabei noch entscheidend. Der 
unmittelbare Fahrtenwunsch und dessen sofortige Ermöglichung sind vordringlich.

Es gehört zu den subtilen Wirkungen der digitalen Marktplätze, dass Wünsche und Bedürfnisse, ja das Konsumverhalten insgesamt, durch die Nutzung der Smartphones verändert werden, ohne dass dies den Einzelnen immer bewusst ist. Mit den digitalen Zugängen lassen sich alle entscheidungsrelevanten Informationen zu sämtlichen Verkehrsoptionen schnell und bequem beschaffen. Das Smartphone wird zum digitalen Generalschlüssel für den intermodalen Verkehr. Es bildet die technische Grundlage für alle Dienstleistungsoptionen, mit denen derzeit unter dem Motto »Mobility as a Service« experimentiert wird.

\section{Mehr Rad- und Fußverkehr}

Darüber hinaus erlebt der Rad- und Fußverkehr, die so genannte aktive Mobilität, vielerorts eine Renaissance. Viele - vor allem große - Städte wie Wien, Paris, London und nicht nur die Vorreiter Kopenhagen und Amsterdam investieren verstärkt in die Fahrradinfrastruktur: in Fahrradwege, Abstellanlagen, Radschnellwege usw. Die Entwicklung dürfte sich fortsetzen, da mehr und sichere Fahrradwege auch diejenigen auf das Rad bringen, die bisher ängstlich waren (vgl. Gehl 2015: 211ff.).

Zusätzlich wird das Fahrradfahren durch den Pedelec-Boom verstärkt, mit Pedelecs sind auch längere Strecken einfacher zu bewältigen. Die Verdichtung städtischer Räume erhöht schließlich die Erreichbarkeit vieler alltäglicher Ziele und erweitert damit die Spielräume für den Zufußverkehr. Umgekehrt profitiert der Zufußverkehr davon, dass der städtische Raum weniger von Autos blockiert wird - vorausgesetzt, dass es tatsächlich einen Rückbau von Autofahrbahnen und Parkflächen gibt. Es kommt zu einer positiven Feedbackschleife für den Zufußverkehr dort, wo die Bedingungen für die aktive Mobilität verbessert werden. Hinzu kommt, dass auch das steigende Gesundheitsbewusstsein der aktiven Mobilität zugutekommt: Zufußgehen, Laufen und Fahrradfahren werden zu Bestandteilen urbaner Lifestyles (vgl. Gericke/Parkin 2015). Fahrradfahren profitiert vom Fitnesshype, es wird sowohl in der Freizeit als auch für die Alltagsmobilität vor allem in großen Städten beliebter.

Bei vielen verkehrspolitischen Auseinandersetzungen der jüngsten Zeit geht es im Kern um den öffentlichen Raum und wer ihn wie nutzen darf. Die Akzeptanz auch für radikale Maßnahmen zur Neuverteilung öffentlicher Räu- 
me zulasten des privaten Pkw-Verkehrs könnte entgegen den bisherigen verkehrspolitischen Gewissheiten steigen (vgl. Andor et al. 2019; Ruhrort 2019). Parkraum gilt nicht mehr als unantastbar und selbst weitgehende Restriktionen für den Autoverkehr finden zunehmend Unterstützung (UBA 2019). Die Rückeroberung und Inwertsetzung des öffentlichen Raums ist jedoch ambivalent, denn attraktive Wohn- und Straßenräume ziehen auch ein neues Publikum an und führen oft zur Verdrängung der alteingesessenen Bewohner*innen.

\section{Chancen und Risiken des Autonomen Fahrens}

Vielfach wird derzeit angeführt, dass das Autonome Fahren die Platzprobleme gerade in der Stadt lösen könne. Gemeint sind Fahrzeuge, die nicht nur automatisch fahren, sondern von einem System gesteuert und disponiert werden und damit aus Sicht der Nutzenden »autonom « unterwegs sind. Welche Bedeutung solche Fahrzeuge in einer weiteren Zukunft haben werden, hängt neben der Bewältigung der technischen Komplexität von der Art und Weise der politischen Regulierung ab. Ob solche Systeme überhaupt im öffentlichen Straßenraum unterwegs sind und wie sich das auf den zukünftigen Verkehr auswirkt, entscheidet maßgeblich der gesetzliche Rahmen. Dieser gesetzliche Rahmen ist wiederum durch die Antwort geprägt, wie wir künftig leben wollen und wie der Verkehr von morgen aussehen soll. Viele Forschungs- und Entwicklungsprojekte der Autoindustrie sind jedoch ziemlich konventionell und weit weg vom »autonomen Fahren«. Sie zielen auf die Weiterentwicklung von Fahrerassistenzsystemen. Das elektrische "Shuttle«, das automatisch dorthin fährt, wo es gebraucht wird, hat hingegen disruptives Potential (vgl. ausführlich: Canzler et al. 2019). Es kann ein attraktives Element einer neuen integrierten Mobilitätsdienstleistung sein. Das öffentliche Auto auf Zuruf kann außerdem zum Element schlauer Stromnetze werden, wenn es nicht zum Fahren gebraucht wird. Es wäre damit zugleich auch ein »Speicher auf Rädern« für fluktuierende Erneuerbare Energien.

Die Automobilunternehmen möchten mit einer Steigerung des Automatisierungsgrades primär die Attraktivität des privaten Fahrzeuges zurückgewinnen. Private Pkw sollen in den nächsten Jahren serienmäßig mit weitgehenden Assistenzfunktionen angeboten werden. (vgl. auch Daum 2019). Der zunehmende Automatisierungsgrad von Fahrzeugen wird von den Fahrzeugherstellern als Antwort auf die Probleme des Verkehrs vermarktet: »sicherer, sauberer und optimierter Verkehrsfluss« (vgl. VDA 2015). Damit wird aber die 
Zahl der privaten Fahrzeuge weiter steigen und die Probleme der Flächenkonkurrenz mit anderen Verkehrsformen wie Busse, Bahnen und Fahrräder insbesondere in den Innenstädten werden weiter zunehmen.

Die Risiken des automatisierten Fahrens sind ohne Zweifel groß. Gleichzeitig entstehen jedoch neue Chancen. Denn (teil-)automatisierte Fahrzeuge, nicht zuletzt neue Fahrzeugformate zwischen Pkw und Bus, können helfen, den Öffentlichen Verkehr effizienter und attraktiver zu machen. Mit einem hochintegrierten intermodalen Öffentlichen Verkehr ist in weiterer Zukunft die Mobilität mit viel weniger Fahrzeugen zu gewährleisten. Eine zukünftige Regulierungspraxis könnte durch einen Mix aus Groß- und Kleinfahrzeugen, aus spurgeführten und getakteten sowie flexiblen on demand-Verkehren die Zahl der Straßenfahrzeuge zur Abwicklung sämtlicher Personenkilometer erheblich reduzieren. Gegenüber konventionellen Bussystemen können automatisch fahrende Shuttles außerdem die Kosten des operativen Betriebes drastisch senken. Urbane Mobilität mit weniger Privatautos, aber mit mehr flexiblen öffentlichen Angeboten on demand sind möglich. Hierbei müssen sich jedoch die Kapazitäten des öffentlichen Verkehrs verdoppeln, etwa ein Viertel der Angebote sind dann digital on demand verkehrende Shuttles, alle Angebote sind gegenseitig buchbar (Canzler et al. 2019).

Die positive Aussicht lautet also: Automatisierte Fahrzeuge können für die Verkehrswende und die Abkehr vom privaten Auto ein strategischer Hebel sein - zunächst in Form von automatisierten Shuttles, später in Form von vollständig autonomen Flotten. Notwendig ist jedoch eine »ermöglichende Regulierung«, die das Privileg des privaten Autos zugunsten individualisierter on demand-Verkehre aufgibt. Schon heute gilt es, die Rolle von neuen on demand-Systemen als Teil des Öffentlichen Verkehrs zu ermöglichen und zu erproben.

\section{Was passieren muss}

Zum Traum vom privaten Glück gehörten in der deutschen Nachkriegsgesellschaft eine Familie, ein eigenes Haus und auch der eigene Wagen. Eingeschrieben in ein ganzes Korsett von Rahmenbedingungen lebt dieses Bild zwar bis heute fort, doch haben sich zwischenzeitlich Wertordnungen, Selbstverständnisse und Lebensplanungen verändert. Die gesellschaftliche Differenzierung ist nicht in den 1950er Jahren stehen geblieben. Mittlerweile wird rund die Hälfte aller Ehen wieder geschieden, ein Fünftel der Kinder wird 
von Alleinerziehenden großgezogen, in Berlin und anderen Großstädten ist es sogar ein Drittel. Während das Bild der privaten Kleinfamilie nicht mehr das alleinige Maß der Dinge ist, bleibt das private Automobil allerdings noch weiter dominant. Das Normalarbeitsverhältnis ist seltener geworden, flexible Arbeitszeiten aber auch neue Beschäftigungsformate haben dazu geführt, dass die Verkehrsansprüche andere sind.

Die alte Ordnung ist jedenfalls passe. In der Soziologie wird dies gerne mit dem Übergang von der Moderne zur Post-Moderne beschrieben. Die vormaligen Gewissheiten gelten nicht mehr, die Arbeits- und Lebensformen haben sich vervielfältigt und weiter international angeglichen (vgl. Beck/BeckGernsheim 1994 und jüngst Reckwitz 2017). Aus der formierten Gesellschaft mit klaren Zeit- und Raumstrukturen ist eine plurale Gesellschaft geworden, in der auch die Verkehrsbedürfnisse vielfältiger und unübersichtlicher geworden sind. In der Verkehrspolitik findet sich hiervon jedoch wenig. Die Grundregeln für den Straßenverkehr, für die Straßenverkehrszulassung sowie auch den Betrieb von öffentlichen Verkehren gelten wie vor 80 Jahren.

Zwar ist es längst überfällig, die Privilegien für das private Auto abzuschaffen und die rechtlichen sowie steuerlichen Rahmenbedingungen zu entrümpeln sowie die heftige Schlagseite in der Bundesverkehrswegeplanung zugunsten des Straßenverkehrs zu beseitigen. Ebenso überfällig ist es, endlich Geschwindigkeitsbeschränkungen auf Autobahnen und Tempo 30 als Regelhöchstgeschwindigkeit in Städten durchzusetzen. Schließlich ist es nötig, die externen Kosten des motorisierten Individualverkehrs zu internalisieren und dafür auch die Instrumente einer streckenabhängigen Maut sowie einer City-Maut einzusetzen (siehe Becker 2016; Rammler 2017 und SRU 2017). Doch besteht derzeit kaum Aussicht auf eine Umsetzung solch weitreichender Reformen zur Unterstützung der Verkehrswende. Aus dieser realpolitischen Erkenntnis heraus plädieren wir für das Mittel des regulatorischen Experimentierraumes: Veränderungen sind vorstellbar, wenn der Staat die nötigen Reformen nur mal probeweise sowie orts- und zeitlich begrenzt vornimmt und sich dabei auch versuchsweise neue Verbündete sucht. Man hätte dann immer die Chance, wieder zum Ausgangspunkt zurückzukehren - falls gewünscht. Eine neue Kultur des Experimentierens würde es erlauben, den bereits erkennbaren neuen Praktiken auch einen entsprechenden Experimentierraum einzuräumen. Darin lässt sich testen, ob sich der von Vorreitern gelebte Alltag verallgemeinern und stabilisieren lässt und welche Folgen möglicherweise zu erwarten sind. Denn Experimente sind ja nichts anderes als von »Konsequenzen entlastetes Probehandeln« (Krohn/Weyer 1989). 
Gesetze haben oft sogenannte Experimentierklauseln, die die Gültigkeit des Gesetzes nicht einfach aushebeln können, aber doch für eine befristete Zeit und unter genau zu definierenden Umständen eine Modifikation des Regelwerkes erlauben, wenn dabei die bisherigen Schutzinteressen grundsätzlich gewahrt bleiben. Die Experimentierklausel - so heißt es in der Rechtspraxis - ist immer »eng auszulegen«. Aber sie ist ein legales Fenster in Richtung einer anderen Zukunft. Die Federführung kommt bei solchen Experimenten in aller Regel den Kommunen und regionalen Gebietskörperschaften zu. Sie sind im föderalen Deutschland zwar meist nicht in die Gesetzesarbeit eingebunden, aber sie kommen in Fragen der Auslegung und der Erteilung von Sondertatbeständen und Ausnahmegenehmigungen ins Spiel und sind hier der zentrale Akteur.

Solche regulatorischen Experimentierräume könnten - und sollten - umfassend sein. Nahezu jedes Gesetz hat die dafür passenden Klauseln, die man - unter Beachtung des Allgemeininteresses - für einen befristeten Zeitraum anwenden kann. Vorstellbar ist dabei vieles, beispielsweise: Keine privaten Fahrzeuge, nur noch E-Autos im Sharing-Modus. Menschen nehmen andere einfach mit - auch gegen Entgelt. Parkende Autos gibt es nicht mehr, dafür viel Platz für das Radfahren. Die Zustellung von Paketen und anderen Dingen wird ausschließlich mit dem Lastenrad erledigt. Die Energieversorgung erfolgt auf Basis eines zu einem »Smart Grid« zusammengefassten Netzes der vor Ort produzierten Erneuerbaren Energien (vgl. Canzler/Knie 2016).

An Ideen, technische und soziale Innovationen zu kombinieren und unter Realbedingungen zu testen, mangelt es nicht. In jedem Fall sind bestehende Regeln tangiert, müssen teilweise und temporär außer Kraft gesetzt werden. In dem skizzierten experimentellen Setting fungieren die einschlägigen Gesetze und Verordnungen als »regulatorische Schlüsselthemen«: das Personenbeförderungsgesetz (PBefG), die Straßenverkehrsordnung (StVO), die Straßenverkehrszulassung (StVZO), das Baugesetzbuch (BauGB) sowie das Energiewirtschaftsgesetz (EnWG) im Verbund mit Verordnungen wie die Ladesäulenverordnung (LSV) oder die Stromnetzzugangsverordnung (StromNZV).

Hinter der Idee regulatorischer Experimentierräume steht die Annahme, dass sich das Narrativ vom privaten Auto überlebt hat. Jedenfalls hat es keine visionäre Kraft mehr. Als alltagsstrukturierende Realität ist das private Auto jedoch nicht so ohne weiteres »umdeutbar«. Andererseits sind Narrative eng an dominante gesellschaftliche Trends geknüpft. Als ein Megatrend moderner Gesellschaften lässt sich die Individualisierung und Pluralisierung von Lebensentwürfen nicht ignorieren. Im Gegenteil: die Digitalisierung bietet 
ganz neue Optionen eines flexiblen Zuschnitts von individualisierten Alltagsabläufen. Mit dem Smartphone und dem mobilen Rechner lassen sich mehr und mehr Aktivitäten ganz individuell raum- und auch zeitunabhängig erledigen. Man kann selbst bestimmen, wann man wo und wie etwas macht. Das mag nicht für alle attraktiv sein, aber es scheint eine nicht mehr rückholbare Entwicklung der fortschreitenden Globalisierung zu sein. Es gibt immer noch die Sehnsucht nach Gemeinschaft, nach kollektiven Erlebnissen, diese werden gesucht und auch gefunden, aber bestimmen kaum individualisierte Alltagserfahrungen.

Ein erfolgreiches Narrativ braucht Begriffe, die hinlänglich allgemein sind und attraktive Bilder hervorrufen. Im Fall des vernetzten, postfossilen Verkehrs stehen wir am Anfang. Denkbar erscheint, dass der Begriff der »Elektromobilität « hier genügend Raum ließe, alle Verkehrsgeräte - vom Auto über Busse und Züge bis hin zu Pedelecs - zusammenzufassen und die weitere Prägung dieses Begriffs die Versorgung mit Strom aus Erneuerbaren Energien und die Verwendung der Verkehrsmittel nicht mehr in privaten Aneignungsformen darstellt. Für ein neues Narrativ braucht es mehr, nämlich wirkliche Erfahrungen. Es braucht eine beispielhafte gelebte Praxis.

\section{Literatur}

Agora Verkehrswende (2017): Mit der Verkehrswende die Mobilität von Morgen sichern. 12 Thesen zur Verkehrswende, Berlin. Online verfügbar unter: www.agora-verkehrswende.de/12-thesen/. Zuletzt aufgerufen am 12.02.2020.

Andor, Mark/Frondel, Manuel/Horvath, Marco/Larysch, Tobias/Ruhrort, Lisa (2019): Präferenzen und Einstellungen $\mathrm{zu}$ vieldiskutierten verkehrspolitischen Maßnahmen: Ergebnisse einer Erhebung aus dem Jahr 2018, RWI-Materialien 131, Essen. Online verfügbar unter: www.rwiessen.de/media/content/pages/publikationen/rwi-materialien/rwimaterialien_131.pdf. Zuletzt aufgerufen am 12.02.2020.

Beck, Ulrich/Beck-Gernsheim, Elisabeth (Hg.) (1994): Riskante Freiheiten. Individualisierung in modernen Gesellschaften, Frankfurt: Suhrkamp.

Becker, Udo (2016): Grundwissen Verkehrsökologie, München: oekom.

Bundesministerium für Verkehr und digitale Infrastruktur (BMVI) (2016): Verkehr in Zahlen 2016/17, Hamburg. Online verfügbar unter: https:// 
www.bmvi.de/SharedDocs/DE/Publikationen/G/verkehr-in-zahlen_2016pdf.pdf?_blob=publicationFile. Zuletzt aufgerufen am 13.02.2020.

Bundesverband CarSharing (BCS) (2018): Zahl der CarSharing-Kunden überspringt die 2 Millionen Marke. Online verfügbar unter: https:// carsharing.de/presse/pressemitteilungen/zahl-carsharing-kundenueberspringt-2-millionen-marke. Zuletzt aufgerufen am 12.02.2020.

Canzler, Weert (2016): Automobil und moderne Gesellschaft. Beiträge zur sozialwissenschaftlichen Mobilitätsforschung, Berlin: LIT.

Canzler, Weert/Knie, Andreas (2013): Schlaue Netze. Wie die Energie- und Verkehrswende gelingt, München: oekom.

Canzler, Weert/Knie, Andreas (2016): Die digitale Mobilitätsrevolution. Vom Ende des Verkehrs, wie wir ihn kannten, München: oekom.

Canzler, Weert/Knie, Andreas (2018): Taumelnde Giganten. Gelingt der Autoindustrie die Neuerfindung?, München: oekom.

Canzler, Weert/Knie, Andreas/Ruhrort, Lisa (2018). Erloschene Liebe? Das Auto in der Verkehrswende. Soziologische Deutungen, Bielefeld: transcript.

Canzler, Weert/Knie, Andreas/Ruhrort, Lisa (2019): Autonome Flotten. Mehr Mobilität mit weniger Fahrzeugen, München: oekom.

Corbusier, Le (1925): Städtebau, München: DVA.

Daum, Timo (2019): Das Auto im digitalen Kapitalismus. Wenn Algorithmen und Daten den Verkehr bestimmen, München: oekom.

Deffner, Jutta/Hefter, Tomas/Götz, Konrad (2014): »Multioptionalität auf dem Vormarsch? Veränderte Mobilitätswünsche und technische Innovationen als neue Potenziale für einen multimodalen Öffentlichen Verkehr.«, in: Oliver Schwedes (Hg.), Öffentliche Mobilität. Perspektiven für eine nachhaltige Verkehrsentwicklung, Wiesbaden: Springer VS, S. 201-227. https:// doi.org/10.1007/978-3-658-03302-6_10

Deutscher Bundestag (2017): Drucksache 18/10909 Antwort der Bundesregierung auf die Kleine Anfrage der Abgeordneten Lisa Paus, Oliver Krischer, Stephan Kühn (Dresden), weiterer Abgeordneter und der Fraktion BÜNDNIS 90/DIE GRÜNEN - Drucksache 18/10732 - Steuerliche Rahmengestaltung für Diesel-Pkw vom 19.01.2017. Online verfügbar unter: http:// dip21.bundestag.de/dip21/btd/18/109/1810909.pdf. Zuletzt aufgerufen am 12.02.2020.

Durth, Werner (1995): »Stadt und Landschaft. Kriegszerstörungen und Zukunftsentwürfe«, in: Jörn Düwel/Werner Duth/Niels Gutschow/Walter Jens (Hg.), 1945 Krieg - Zerstörung - Aufbau. Architektur und Stadtpla- 
nung 1940-1960, Schriftenreihe der Akademie der Künste 23, Berlin: Henschel, S. 127-175.

Gehl, Jan (2015): Städte für Menschen, Berlin: jovis.

Gerike, Regine/Parkin, John (Hg.) (2015): Cycling Futures - From Research into Practice, London: Routledge. https://doi.org/10.4324/9781315575742

Klenke, Dietmar (1995): »Freier Stau für freie Bürger«. Die Geschichte der bundesdeutschen Verkehrspolitik, Darmstadt: Wissenschaftliche Buchgesellschaft.

Kraftfahrtbundesamt (KBA) (2018): Jahresbilanz der Neuzulassungen, Flensburg. Online verfügbar unter: www.kba.de/DE/Statistik/Fahrzeuge/Neuzulassungen/n_jahresbilanz.html. Zuletzt aufgerufen am 20.02.2020.

Krohn, Wolfgang/Weyer, Johannes (1989): „Gesellschaft als Labor. Die Erzeugung sozialer Risiken durch experimentelle Forschung«, in: Soziale Welt 40(3), S. 349-373.

London School of Economics and Political Science Cities (LSE Cities)/Innovation Center for Mobility and Societal Change (InnoZ) (2015): Towards New urban Mobility. The Case of London and Berlin, London/Berlin. Online verfügbar unter: https://secities.net/wp-content/uploads/ 2015/09/New-Urban-Mobility-London-and-Berlin.pdf. Zuletzt aufgerufen am 30.01.2020.

Rammler, Stephan (2017): Volk ohne Wagen, Frankfurt: Fischer.

Reckwitz, Andreas (2017): Die Gesellschaft der Singularitäten. Zum Strukturwandel der Moderne, Berlin: Suhrkamp. https://doi.org/10.1007/9783-658-21050-2_2

Ruhrort, Lisa (2019): Transformation im Verkehr? Erfolgsbedingungen verkehrspolitischer Maßnahmen im Kontext veränderter Verhaltens- und Einstellungsmuster, Wiesbaden: Springer VS. https://doi.org/10.1007/ 978-3-658-28002-4

Sachverständigenrat für Umweltfragen (SRU) (2017): Umsteuern erforderlich: Klimaschutz im Verkehrssektor, Sondergutachten, Berlin.

Schönduwe, Robert/Lanzendorf, Martin (2014): Mobilitätsverhalten von Heranwachsenden und Möglichkeiten zur Bindung an den ÖPNV: eine Synthese des Forschungsstandes von deutschsprachiger und internationaler Forschungsliteratur, Arbeitspapiere zur Mobilitätsforschung 1, Frankfurt a.M. Online verfügbar unter: https://d-nb.info/1062604091/34. Zuletzt aufgerufen am 12.02.2020.

Umweltbundesamt (UBA) (2019): Umweltbewusstsein in Deutschland, Dessau. Online verfügbar unter: https:/www.umweltbundesamt.de/sites/ 
default/files/medien/1410/publikationen/ubs2018___m_3.3_basisdaten broschuere_barrierefrei-02_cps_bf.pdf. Zuletzt aufgerufen am 30.01.2020.

Verband der Deutschen Automobilindustrie (VDA) (2015): Automated Driving, Frankfurt a.M. Online verfügbar unter: www.vda.de/en/topics/innovation-and-technology/automated-driving/automated-driving.html. Zuletzt aufgerufen am 30.01.2020. 



\section{Die Post-Landwirtschaftliche Revolution}

Oliver Stengel

\section{Einleitung}

Verschiedene Megatrends gestalten gegenwärtig und in Zukunft den Planeten neu: Der anthropogene Klimawandel und andere globale Umweltveränderungen transformieren die Ökosysteme in einer Weise, wie dies zuvor die Milankovic-Zyklen getan haben - nur erheblich schneller. Aber auch die menschliche Zivilisation verändert sich: Von den rund 100 Milliarden Menschen, die nach einer Schätzung des Population Reference Bureau in der gesamten Geschichte des anatomisch modernen Menschen auf der Erde gelebt haben (Kaneda/Haub 2018), leben gegenwärtig etwa 7,5 Milliarden Menschen. Schon in wenigen Jahrzehnten werden es voraussichtlich zehn oder elf Milliarden sein. Anders als in der Vergangenheit leben sie nicht mehr vorrangig auf dem Land, sondern in Städten, deren größte, Jing-Jin-Ji in China, bald 100 Millionen Einwohner*innen haben könnte.

Ein Grund für den Umzug der Menschen von Land in die Städte ist der, dass es auf dem Land immer weniger zu tun gibt: Der Klimawandel und die Erosion einst fruchtbarer Böden verwüsten viele Felder und dort, wo sich noch Bauern/Bäuerinnen oder Viehwirt*innen halten, ersetzen immer mehr (autonome) Maschinen die Arbeit der Menschen. Außerdem ziehen nicht nur Menschen vom Land in die Stadt, auch die Landwirtschaft zieht um: In vielen Städten sprießen vertikale Farmen, in denen Nutzpflanzen nahezu vollautomatisch angebaut werden, um Lebensmittel dort zu erzeugen, wo sie letztlich auch konsumiert werden. Aber nicht nur die Landwirtschaft wandert in die Städte, sondern auch die Viehwirtschaft - und das ist eine Entwicklung, welche die globale Umwelt abermals grundlegend verändern wird. Ihr zugrunde liegen wissenschaftliche Disziplinen, die das 21. Jahrhundert mitgestalten und deutlich vom 20. Jahrhundert unterscheiden werden: die Molekularbiologie und die Biotechnologie. 


\section{Vor dem globalen Öko-Kollaps}

Einiges spricht dafür, dass sich die Menschheit in einer Art Flaschenhals befindet, in dem in der Vergangenheit bereits Kulturen wie die Maya (Kennett et al. 2012), die Anazazi (Billman et al. 2000; Kohler/Matthews 1988), das Volk der Nazca (Beresford-Jones et al. 2009, Coghlan 2009) und wohl auch die Bewohner*innen der Osterinsel (Hunt et al. 2006) stecken geblieben sind: Sie florierten, ihre Populationen nahmen zu, sie veränderten ihre lokale Umwelt vor allem durch Rodungen so sehr, dass eine Dürreperiode und anschließend ausbrechende gewalttätige Unruhen genügten, um sie teilweise oder ganz kollabieren zu lassen.

Was den Maya, Nazca, Anazazi und Osterinsulaner*innen widerfuhr, kann sich wiederholen und im 21. Jahrhundert ein globales Ausmaß annehmen. Treibende Faktoren hierfür sind die Land- und Viehwirtschaft. Zwar sind sie nicht die alleinige Ursache, aber die entscheidende. Durch sie werden Kipppunkte des globalen Ökosystems erreicht und überschritten. Sie treiben den Klimawandel voran, sind der Hauptgrund für den Verlust fruchtbarer Böden, für den Verfall von Lebensräumen und für das weltweite Artensterben, sie verbrauchen riesige Flächen, das meiste Süßwasser, sie bringen den Phosphor- und Stickstoffkreislauf durcheinander und weiten dadurch die sauerstoffarmen Todeszonen in den Meeren aus (Stramma/Schmidtko 2019).

Sind die Kipppunkte, die sog. planetaren Grenzen des globalen Ökosystems (Steffen 2015) überschritten, ist nicht vorhersehbar, was im Einzelnen passieren wird. Da sich manche von ihnen gegenseitig beeinflussen (der Klimawandel, die Versauerung der Ozeane, die Biodiversität, der Land Use Change sowie die Stoffkreisläufe von Phosphor und Stickstoff), ist zu erwarten, dass sich das globale Ökosystem innerhalb von zwei Jahrzehnten drastisch verwandeln wird - und zwar überwiegend negativ (Barnosky et al. 2012). Es ist zu erwarten, dass sich viele, vielleicht sogar die meisten Gesellschaften an die Geschwindigkeit und das Ausmaß der sich dann vollziehenden Transformation nicht werden anpassen können.

Damit kann - sofern größte ökologische und humanitäre Schäden vermieden werden sollen - die Land- und Viehwirtschaft wie sie in der Vergangenheit betrieben wurde, nicht fortgeführt werden. Seit Jahrtausenden gilt sie als selbstverständlich wie alternativlos. "Der Bauer ist der ewige Mensch, unabhängig von aller Kultur, die in den Städten nistet. Er geht ihr voraus, er überlebt sie«, schrieb der Geschichtsphilosoph Oswald Spengler 
in den 1920ern (Spengler 1995: 669). $\mathrm{Zu}$ diesem Zeitpunkt arbeiteten die meisten Menschen im westlichen Kulturkreis seit rund 10.000 Jahren als Bauern/Bäuerinnen. Hundert Jahre später waren es nur noch zwei Prozent. Zwar schwand die Zahl der Bauern/Bäuerinnen, die Fläche der von ihnen bewirtschafteten Felder jedoch nicht. Dank neuer Maschinen konnten weniger Bauern/Bäuerinnen größere Flächen beackern und eine größere Anzahl von Nutztieren halten.

Nach dem IPCC sind mittlerweile 49 Prozent der eisfreien Landmassen auf der Erde Acker- oder Weideland (IPCC 2019: 4). Aber das wird sich in den kommenden Jahrzehnten ändern und am Ende des 21. Jahrhunderts könnten es nur noch zwei Prozent sein, so sich die Postlandwirtschaftliche Revolution weltweit durchsetzt. Während Landwirt*innen und Hirt*innen Jahrtausende lang weite Flächen entwaldeten, pflügten oder abgrasen ließen, könnte sich die Natur im 21. Jahrhundert jene Flächen, oder zumindest die meisten davon, wieder zurückholen und so dazu beitragen, dass ökologische planetare Grenzen nicht (weiter) überschritten werden.

Darüber hinaus sollte die sichere Versorgung mit Lebensmitteln durch diese Transformation sogar zunehmen. Denn momentan steigt die Nachfrage nach Lebensmitteln. In Folge des Klimawandels und anderer Umweltveränderungen dürften sich die Ernteerträge künftig jedoch verringern - wenngleich ideenreiche Gentechnologen viel unternehmen, um Nutzpflanzen an die neuen Umweltbedingungen anzupassen und ertragreicher $\mathrm{zu}$ machen. Gegen Dürren, Stürme, Überschwemmungen und Bodenerosion werden sie es jedoch schwer haben und man sollte sich nicht darauf verlassen, dass sie den Wettlauf gewinnen können.

\section{Planet ohne Weiden: Teil I}

In dieser ökologisch außerordentlich dynamischen Phase läuten nicht weniger ideenreiche Biotechnologen eine neue Ära in der Menschheits- und Umweltgeschichte ein: Die Post-Landwirtschaftliche Revolution (Stengel 2020).

In ihrer Geschichte hat die Menschheit bislang drei große Etappen zurückgelegt: Mit der Landwirtschaftlichen Revolution vollzog sich der Übergang von der Steinzeit ins Agrarzeitalter und mit der Industriellen Revolution der Übergang vom Agrar- ins Industriezeitalter. Gegenwärtig befindet sich die Menschheit mit der Digitalen Revolution im Übergang zum Digitalzeitalter (Stengel et al. 2017). In diesem muss sie sich mit den Altlasten ihrer Ver- 
gangenheit beschäftigen: Mit der Energiewende sollen die atmosphärischen Veränderungen eingedämmt oder gar rückgängig gemacht werden, die durch die Verbrennung der industriellen Energieträger Öl, Gas und Kohle erfolgten. Mit der Mobilitätswende sollen die Schäden beseitigt werden, die durch das das Industriezeitalter wohl am meisten prägende Verkehrsmittel verursacht wurden: dem Auto. Die Konsumwende zielt auf die Reduktion des Ressourcenverbrauchs, der durch die industrielle Massenproduktion und den Massenkonsum angefacht wurde.

Und mit der Ernährungswende sollen schließlich jene Veränderungen eingedämmt oder gar rückgängig gemacht werden, die ihren Ursprung im Agrarzeitalter haben. Ein zentrales Element der Ernährungswende sind tierische Lebensmittel, vor allem Fleisch- und Milchprodukte. Sie schädigen die globale Umwelt, weil die Tiere Fläche zum Leben und Fläche zum Anbau ihres Futters benötigen. Weideflächen sind bereits die dominante Landschaftsform weltweit geworden (Ramankutty et al. 2010), dennoch reichen sie nicht aus, um die im Dienst der Menschen stehenden rund 25 Milliarden Rinder, Yaks, Schweine, Ziegen, Schafe, Gänse und vor allem Hühner satt zu bekommen. Zusätzlich muss Viehfutter auf einem Drittel der globalen Ackerfläche angebaut werden. Dann verbrauchen diese Tiere bemerkenswerte Mengen an Trinkwasser und ihre Gülle belastet das Grundwasser mit Nitrat. Außerdem stoßen sie ebenfalls bemerkenswerte Mengen an dem Treibhausgas Methan aus (Robinson et al. 2014).

Selbstverständlich könnte sich die Menschheit jener ökologischen und obendrein tierethischen Probleme entledigen, die durch Viehbestand und Viehhaltung zustande kommen, indem sie ihre Ernährungsweise verändert und sich überwiegend vegetarisch oder besser noch vegan ernährt. Poore und Nemecek haben die ökologischen Belastungen der carnivoren, vegetarischen und veganen Ernährungsstile miteinander verglichen und dabei festgestellt, dass von einer veganen Ernährungsweise die geringsten Umweltbelastungen ausgehen (Poore/Nemecek 2018). Aber Menschen lassen sich mehrheitlich nur schwer durch rationale Argumente überzeugen, »eingefleischte« Gewohnheiten abzuschütteln und noch weniger, wenn diese Gewohnheiten Spaß machen oder gut schmecken. Diese menschliche Neigung bietet durchaus Anlass zum Fremdschämen, weshalb jede bewusstseinsbildende Maßnahme angemessen ist. Aber selbst in einem Land wie Deutschland, in dem das Problembewusstsein vergleichsweise groß ist, dürfte es mindestens eine, wahrscheinlich eher zwei Generationen - also zwanzig bis vierzig Jahre - dauern, bis sich die kollektive Ernährungspraxis grundlegend geändert 
hat. Auf globaler Ebene wird sich dieser Prozess nicht schneller vollziehen. Global betrachtet nimmt der Pro-Kopf-Konsum tierischer Lebensmittel gegenwärtig sogar zu, die Zahl menschlicher Köpfe obendrein und ernährt sich die Menschheit zwanzig bis vierzig Jahre weiter wie bisher, wird das globale Ökosystem sehr wahrscheinlich kollabiert sein. Eine verhaltenswirksame hohe Steuer auf tierische Lebensmittel würde die Ernährungswende sicher beschleunigen, aber die meisten politischen Entscheidungsträger demokratischer Regierungen fürchten gegenwärtig noch einen kollektiven Shitstorm und ihre Abwahl. Neben der menschlichen Würde gelten den meisten Bürgern und Bürgerinnen westlicher Gesellschaften nun mal auch Steaks, Bratwürste und Milchschaum als unantastbar. Eine staatliche Kanalisierung der Ernährungspraxis würde zudem Widerstand in den Parteien entfachen: Liberale insistierten auf die Mündigkeit der Konsument*innen, Linke monierten Ungerechtigkeit und Ungleichheit, denn während Arme durch die Steuer nämlich zum Verzicht gezwungen würden, könnten Reiche ungehemmt weiter schlemmen. Konservative betonten, Menschen hätten seit biblischen Zeiten Fleisch und Milch genossen, weshalb dies natürlich und normal sei und so bleiben solle. Und ob eine außerparlamentarische Opposition wie Fridays for Future und die Extinction Rebellion eine den globalen Zeitgeist rechtzeitig wandelnde Bedeutung erlangen kann, bleibt abzuwarten.

In dieser schwierigen Situation bietet die post-landwirtschaftliche Erzeugung ehemals landwirtschaftlicher Erzeugnisse einen Ausweg. Vielleicht sogar den einzigen, der der Menschheit noch bleibt. Die Rede ist von einer biotechnologischen Bewegung, die tierische Produkte ohne Tiere, sondern mit Zellen herstellt. In der scientific community hat sich für dieses Verfahren die Bezeichnung Cellular Agriculture etabliert. Mit der klassischen Agrarwirtschaft allerdings hat die zelluläre Herstellung nichts gemein. Der einzige gemeinsame Nenner besteht darin, dass Tiere ihr eigenes Fleisch, ihre Milch und ihre Eier zellulär produzieren. Davon abgesehen macht Cellular Agriculture alles anders: Das Verfahren benötigt so gut wie keine Flächen - keine Weideflächen, keine Ackerflächen, keine Flächen für Ställe oder Schlachthöfe - und kann darum in Städten erfolgen. Das Verfahren benötigt kaum Süßwasser (sofern das eingesetzte Wasser wieder recycelt wird), es erfolgt nicht unter freiem Himmel, sondern in Laboren bzw. Anlagen mit großen »Bioreaktoren« genannten Tanks, in denen Zellmassen gerührt werden.

Bei ihnen handelt es sich zunächst um Muskelstammzellen, die zuvor durch eine minimalinvasive Biopsie einem Tier entnommen und vermehrt 
wurden. In den Tanks ist es für die Zellen so, als wären sie im Körper eines Tieres. Es ist genauso warm und es gibt die richtigen Nährstoffe. Dementsprechend verhalten sie sich wie im Körper: Sie differenzieren sich in Muskelzellen, teilen und mehren sich. Anschließend verbinden sie sich zu Muskelfasern. Damit sich das Ganze im Mund »fleischig« anfühlt, werden die Muskelfasern (zumindest momentan) wie richtige Muskeln durch mechanische und elektrische Impulse trainiert. Auf diese Weise ziehen sie sich zusammen und entspannen sich, werden also gewissermaßen stärker. Klassisches Fleisch besteht jedoch nicht nur aus Muskelmasse, sondern auch aus Fett. Das ist, in erhöhten Mengen verspeist, nicht gesund, schmeckt den meisten aber. Um also den Geschmack tierischen Fleischs zu treffen, muss es hinzugegeben werden. Es darf kein tierisches Fett sein, da das Endprodukt vegan sein soll. Also nimmt man pflanzliche Fettsäuren oder lässt die Fettsäuren von Hefezellen herstellen, die so verändert wurden, dass sie tierische Fettsäurefabriken werden. Das solchermaßen biotechnologisch produzierte Fett unterscheidet sich letztlich nicht vom biologischen Original.

Hier wie bei den Muskelzellen gilt: Das Verfahren ist unnatürlich, das Endprodukt dagegen ist es nicht. Es besteht aus den gleichen Zellen wie das tierische Produkt. Allein der Ort, wo sich die Zellen vermehrten, nämlich nicht in einem Körper (in vivo), sondern außerhalb von ihm (in vitro), ist neu. In den Niederlanden wurde bereits in den 1990ern erforscht, wie Muskelfleisch im Labor hergestellt werden könnte, um die Lebensmittelsicherheit $\mathrm{zu}$ erhöhen und Umweltschäden verringern zu können. 2013 wurde der erste in vitro-Burger unter der Leitung von Mark Post (Post 2012) von der Universität Maastricht öffentlich in London verkostet. Er enthielt eine BurgerFrikadelle aus zellulärem Rindfleisch. Seitdem ist eine Menge passiert und diverse Start-ups haben mit weiteren Erfolgen auf sich aufmerksam gemacht: 2016 war Memphis Meats das erste Unternehmen, das zelluläre Fleischbällchen herstellte. 2017 präsentierte Memphis Meats außerdem erstmals zelluläres Geflügelfleisch (Huhn und Ente). Im gleichen Jahr machte Finless Foods Thunfischfleisch aus Zellen, was schon deswegen interessant ist, weil Fischfleisch aus dem Labor, anders als natürlicher Fisch, nicht mit Mikroplastik kontaminiert ist (Smith et al. 2018). 2018 präsentierte New Age Meats die weltweit erste in vitro-Schweinewurst. Ebenfalls 2018 produzierte das israelische Startup-Unternehmen Aleph Farms unter der Leitung von Didier Toubia das erste im Labor kultivierte Steak aus Rindfleisch. Das war ein besonderer Moment, denn ein tierfreies Steak zu kreieren, war bis dato das schwierigste Projekt. Nicht nur, dass ein Steak wie ein Steak aussehen muss - und dazu genügt 
es nicht Zellen so zusammenzupressen, wie man es bei Fleisch- und Fischbällchen, Frikadellen oder Würsten machen kann. Die Zellen müssen in die Höhe, auf ess- und genießbaren Strukturen wachsen. Zudem bestehen Steaks zu unterschiedlichen Anteilen aus Muskel-, Fett- und Bindegewebe und dann ziehen sich durch ein Steak auch noch Blutadern. Man muss folglich vier Zelltypen in einer 3D-Struktur kultivieren und dazu bringen, dass sie zusammenwachsen. Aleph Farms hat dieses Kunststück vollbracht. 2019 folgten die nächsten Premieren: Shiok Meats aus Singapur führte zellulär gewonnenes Fleisch von Schrimps vor. Fleisch von Hummern und Krebsen planen die beiden Gründerinnen Sanhya Siram und Ka Yi Ling von Shiok Meats ebenfalls, ohne die Meerestiere im Tank zu kultivieren. Im gleichen Jahr verkostete Wild Type das weltweit erste zellulär gewonnene Lachsfleisch in Portland.

Diese Start-ups demonstrieren, dass der Geist aus der Flasche entwichen ist. Er wird nicht mehr in sie zurückkehren, zumal die klassische Fleischproduktion ein immenses Risiko für die Entwicklung der Menschheit ist und sich überdies jedes Jahr neue Teams zusammenfinden, um tierische Produkte im Labor herzustellen. Die Start-ups demonstrieren auch, dass die zellbasierte Produktion von Fleisch bereits im kleinen Maßstab funktioniert. Allerdings ist der Weg von kleinen Produktionsmengen im Labor in die Großproduktion eines jeden zellulär hergestellten Produktes mit Hindernissen bestückt und nimmt etwa zehn Jahre in Anspruch.

Wie ist die Ökobilanz des zellulären Fleisches im Vergleich zum tierischen? Während der weltweite Viehbestand das globale Ökosystem und die menschliche Zivilisation gefährdet, hat das in vitro-Verfahren eine Schwachstelle - nämlich den Energieverbrauch, der notwendig ist, um die Tanks auf $37 \mathrm{Grad}$ Celsius zu erwärmen und warm zu halten. Wird die benötigte Energie mittels fossiler Energieträger gewonnen, ist die Klimabilanz negativ. Stammt die Energie jedoch von Wind und Sonne, oder später einmal aus Fusionskraftwerken, ist die Bilanz entsprechend besser (Lynch/Pierrehumbert 2019; Mattick et al. 2015). Die beste Umwelt- und Ethikbilanz nutzt jedoch nichts, wenn das zelluläre Produkt vom Konsumenten verschmäht wird. Wie steht es also um die Akzeptanz von zellulärem Fleisch?

Gut. Und auch dies spricht dafür, dass hier ein neuer Trend entsteht. In Indien, China und den USA durchgeführte repräsentative Umfragen, zeigen, dass die Mehrheit der Befragten in jedem der drei Länder, in denen zusammen fast drei Milliarden Menschen leben, zellulär hergestelltes Fleisch bevorzugen würden, sofern es nicht teurer als das tierische ist. Im Allgemeinen war die Akzeptanz bei höher gebildeten Stadtbewohner*innen am höchsten. 
In den USA waren nur 26 Prozent der Befragten der Ansicht, sie würden das neue Fleisch definitiv nicht in Erwägung ziehen, in Indien waren es elf und in China sogar nur sieben Prozent (Bryant et al. 2019). Auch in Deutschland deuten erste Umfragen darauf hin, dass eine Akzeptanz für zelluläres Fleisch vorhanden ist. So war in einer Umfrage die Mehrheit der Befragten der Meinung, dass für sie in vitro produziertes Fleisch eine akzeptable Alternative darstelle (Böhm et al. 2018).

Berücksichtigt man, dass offenbar nur ein Viertel der Mitglieder einer Grundgesamtheit erforderlich sind, damit sich eine neue Norm in der Gruppe durchsetzen kann, wird die zelluläre Revolution realistisch: Centolaet et al. konnten in verschiedenen Experimenten zeigen, dass ein Schwellenwert von 25 Prozent erreicht sein muss, damit sich in einer Gruppe oder Gesellschaft eine Einstellung oder Verhaltensweise ändert. Dieser Schwellenwert scheint damit zu sein, was man gerne als »kritische Masse« bezeichnet. Sobald dieser Schwellenwert erreicht wurde, änderte sich in den Experimenten die Gruppendynamik und die Mehrheit der Gruppe übernahm die neue Norm. Dies war in Experimenten sogar dann der Fall, wenn Belohnungen für die Beibehaltung der etablierten Verhaltensweise ausgesetzt und schließlich erhöht wurden (Centolaet et al. 2018).

Folglich kann angenommen werden, dass die gesellschaftliche Verbraucherakzeptanz für in vitro-Produkte rasch zunimmt, sobald der genannte Schwellenwert in der jeweiligen Gesellschaft erreicht ist - und dies ist er in den großen Volkswirtschaften offenbar bereits. Allerdings darf das kultivierte Fleisch nicht teurer sein, und schlechter schmecken als das tierische darf es auch nicht. Können die Hersteller dies leisten, dürfte die Revolution schnell gehen, da viele Verbraucher Fleisch nun ohne schlechtes Gewissen konsumieren können. Umgekehrt müssen sich jene, die am Verzehr tierischen Fleischs festhalten, zunehmend unangenehme Fragen gefallen lassen.

Wenn die Akzeptanz für zellulär hergestelltes Fleisch schon in der Gegenwart hinreichend groß ist, kann dies für einen Megatrend sprechen, der das 21. Jahrhundert prägen wird. Dies umso mehr, da zelluläres Fleisch zugleich als eine Art Akzeptanzbeschaffer für andere in vitro-Produkte fungiert - und von ihnen gibt es weitere. Zum Beispiel Insektenfleisch, Leder oder Milchprodukte. 


\section{Planet ohne Weiden: Teil II}

Im Sommer 2019 verkostete das Start-up Perfect Day das erste aus zellulärer Milch gewonnene Produkt: Eiscreme. Milch wird das erste einer neuen Generation post-landwirtschaftlicher Lebensmittel sein, das auf den Markt kommt (siehe perfectdayfoods.com).

Möchte man nachvollziehen, wie Kuhmilch ohne Kühe hergestellt werden kann, muss man Kuhmilch unter die Lupe nehmen. Obwohl sie das Wunder vollbringt, dass Babys wachsen und sich entwickeln, ist Milch nicht sehr kompliziert. Kuhmilch besteht zu 88 Prozent aus Wasser, zu drei Prozent aus sechs verschiedenen Proteinen (wobei Casein den mit Abstand größten Anteil ausmacht), zu vier Prozent aus acht Fettsäuren und zu fünf Prozent aus Kohlenhydraten. Hat man alle Zutaten beisammen, mixt man sie - erhält man Milch. Milch, die identisch mit dem ist, was aus dem Euter einer Kuh gezapft wird (abzüglich der Hormonzusätze, Steroid- und Antibiotikarückstände, die in Kuhmilch aus der Massentierhaltung enthalten sind). Hat man die Milch aus ihren einzelnen Bestandteilen zusammengesetzt, kann man sie trinken oder in alle möglichen Milchprodukte verarbeiten.

Die Kunst besteht nun darin, die Proteine und Fettsäuren der Milch in vitro herzustellen. Hierzu kann man Mikroorganismen wie Hefezellen so umprogrammieren, dass sie das jeweils gewünschte Produkt herstellen. Dazu benötigen die Hefezellen die genetischen Informationen, die sie zur Herstellung der Proteine und Fette benötigen. Diese Informationen kann man aus der DNA von Kuhzellen ablesen und in die DNA der Hefen integrieren. Die solchermaßen genetisch veränderten Mikroorganismen kommen in einen Tank, wo sie ideale Bedingungen (Wärme und Zucker) vorfinden, sich teilen und z.B. Casein oder andere Milcheiweiße herstellen. Das bedeutet: Die Mikroorganismen, die sozusagen zu Proteinfabriken umgebaut wurden, sind genetisch verändert worden. Aber das Endprodukt, die Milchproteine und damit auch die Milch (und der Käse), ist frei von genetischen Veränderungen. Auf diese selbe Weise lassen sich auch die einzelnen Fettsäuren der Milch produzieren. Letztlich lässt sich tierfreie Milch einfacher als tierfreies Fleisch herstellen.

Ethisch spricht jedes Argument für den Umstieg auf in-vitro-Milch, weshalb sie in den letzten Jahren vermehrt mit dem Begriff "Clean Milk« bezeichnet wird (Milburn 2018). Aber auch bei ihr stellt sich die Frage nach der Ökobilanz. Da die großtechnische Produktion tierfreier Milch noch aussteht, lassen sich hierzu bislang nur vorläufige Aussagen treffen. Da Kühe aber gro- 
ße Mengen Trinkwasser konsumieren, Treibhausgase emittieren und Landflächen für ihr Futter konsumieren, sollte das in vitro-Verfahren mit deutlich weniger Umweltbelastungen einhergehen. Ein erster Vergleich zwischen Kuhmilch und in vitro-Milch ergab, dass sowohl der Carbon Footprint, der Water Footprint sowie der Land Footprint erheblich günstiger für das synthetische Verfahren ausfallen (Steer 2015).

Um die heimische Viehindustrie zu schützen, könnten Regierungen die Zulassung der in vitro-Produkte erschweren. Da aber vergleichsweise wenige Arbeitnehmer*innen in dieser Branche angestellt sind und keine volkswirtschaftlichen Einbußen durch die Umstellung auf das neue Verfahren drohen, dieses aber globalökologische Vorteile mit sich bringt, sollte keine vernünftige Regierung solche Schritte erwägen (konservative Regierungen sind für ökologisch unvernünftige Entscheidungen allerdings anfällig). Gleichwohl braut sich Widerstand aus der Viehindustrie zusammen. Die Argumentationsstrategie läuft auf die Behauptung hinaus, Fleisch von Tieren sei natürlich und darum gesund, indes »Fake Meat « ungesund sein muss. ${ }^{1}$ Nun sind weder die Massentierhaltung noch das Vieh natürlich. Letzteres ist ein Zucht- bzw. Kunstprodukt, das nach den Vorlieben des Profits gestaltet wurde und oft mit Hormonen und Antibiotika aufgepäppelt wird. Und gesund ist vor allem der Verzicht auf Fleisch - speziell auf tierisches (IARC 2015).

Da die Produktion umweltunabhängig erfolgt, ist die Erzeugung von und die Versorgung mit »Clean Milk« auch bei Umweltbedingungen gesichert, die für Kühe eine (zu) große Belastung sind. Selbst für ein von der Viehwirtschaft geprägtes Land wie Neuseeland lassen sich keine objektiv plausiblen Gründe für die Beibehaltung der hergebrachten Viehindustrie aufführen (Dixon 2019).

\section{Planet ohne Felder}

Vertical Farming steht für eine Landwirtschaft ohne Land und sollte sie einmal für alle Nutzpflanzen konsequent verwirklicht werden können, könnte

2019 schaltet das »Center for Consumer Freedom « eine populistische Kampagne gegen pflanzliches »Fleisch« in überregionalen US-Zeitungen und auf cleanfoodfacts.com. Das Center bestand aus zwei Personen (!), deren »Präsident« zugleich Präsident der rechtskonservativen Werbeagentur Berman and Company war, die zwei weitere Mitarbeiter*innen beschäftigte. 
sie die Erde langfristig von ihren Feldern befreien. Eine Fläche, die mindestens vom Kap Hoorn bis zum Rio Grande reicht, könnte wieder ein Lebensraum für viele Arten werden. Eine andere Revolution geht noch einen Schritt weiter, denn sie kommt nicht nur ohne Land, sondern zusätzlich ohne Pflanzen aus. Sie ist der zweite Teil der Post-Landwirtschaftlichen Revolution. Deren erster Teil kann die Weideflächen abschaffen, ihr zweiter die Ackerflächen. Denn nicht nur tierische Produkte können zellulär hergestellt werden, sondern auch pflanzliche.

In der Cultured Meat-Szene kursiert ein Zitat von Winston Churchill, das dieser 1931 in seinem Essay >Fifty Years Hence « niederschrieb (Churchill 1931). In diesem nahm Churchill die große Leitidee des tierlosen tierischen Fleischs vorweg: »Wir werden der Absurdität entkommen, ein ganzes Huhn zu züchten, um die Brust oder den Flügel zu essen, indem wir diese Teile separat in einem geeigneten Medium züchten. "Churchill's Prognose beginnt sich rund hundert Jahre später zu bewahrheiten. Warum jedoch nicht auch auf Pflanzen anwenden, was er in Bezug auf das Huhn vorhergesagt hat: Warum nicht der Absurdität entkommen, eine ganze Ölpalme oder Kartoffelpflanze zu züchten, nur um deren Früchte ernten zu können? Warum sollte man die Früchte nicht separat in einem geeigneten Medium hervorbringen können?

Und warum könnte es überhaupt Sinn machen, auch pflanzliche Produkte zellulär herzustellen? Zum einen sind die von der Landwirtschaft, ihrem Flächen-, Wasser- und Düngerverbrauch verursachten Schäden hochproblematisch. Zudem gelangen durch sie große Mengen an Pflanzen-, Pilz- und Insektengiften in die Umwelt. Diese Vorgänge befördern den Klimawandel, die Ausbreitung sauerstoffarmer Zonen in den Meeren sowie den Rückgang der Biodiversität.

Der landwirtschaftliche Bio-Anbau ist keine Rettung, da er größere Flächen beansprucht. Obendrein gefährdet der Klimawandel die sichere Versorgung mit Ackerfrüchten. Das alles spricht gegen den klassischen Ackerbau, aber auch für vertikale Farmen, doch sind ihr Bau und Betrieb (noch) teuer und das spricht eben gegen sie.

Der Ausgangspunkt ist erneut die Stammzelle. Jede Pflanze hat Stammzellen, die sich (a) teilen und mehr Zellen ihrer Art erzeugen und sich (b) in jeden Gewebetyp der jeweiligen Pflanze verwandeln können - z.B. in die Zellen der Kartoffelknolle. Diese besteht aus Zellen nur eines Zelltyps, die Stärke speichern. Stammzellen der Kartoffel werden also vermehrt und in die Zellen der Kartoffelknolle umgewandelt. Damit die Stammzellen wissen, in welchen Zelltypus sie sich vermehren sollen, benötigen sie eine Anweisung. In 
der Pflanze erhalten sie diese durch bestimmte Signalstoffe. Dabei handelt es sich um bestimmte Hormone und Proteine. In den letzten Jahren wurden sie entschlüsselt (Kolachevskaya et al. 2018: Signalhormone; Hannapel et al. 2017; Dutt 2017: Signalproteine) und gibt man sie den Stammzellen ins Nährmedium, beginnen sie sich wie gewünscht zu differenzieren. Die Stammzellen der Reis-, Soja- oder Maispflanze hören auf andere Signale, aber auch sie hören auf Signale, die aus Hormonen und Proteinen bestehen. Auch bei ihnen lässt sich das zelluläre Verfahren anwenden.

Die Schale kann dabei allerdings nicht hergestellt werden, da sie aus anderem Material besteht. Da die Schale der Kartoffel aber keine Nährstoffe, sondern das Gift Solanin enthält, um Fraßfeinde abzuwehren, ist das sogar ein Vorteil. Man kann die so gewonnen Knollenzellen essen, man kann ihnen aber auch die gespeicherte Stärke entnehmen, um sie als Industriestärke z.B. in der Produktion von Biokunststoffen einzusetzen.

Selbst Fruchtfleisch kann zellulär gewonnen werden, da es seinerseits nur aus Zellen besteht. Ein Team der Universität Helsinki um Heiko Rischer zerkleinerte diverse Beerenpflanzen. Daraufhin bildete sich an den Schnittstellen der Pflanzen Kallusgewebe, dass sich sehr gut vermehren lässt. Die Kallihaufen untersuchte das Team anschließend nach Zellen, die eine fruchtfleischartige Morphologie (z.B. Farbe) aufwiesen. Diese wurden extrahiert und in einer Standardnährlösung vermehrt. Das Endprodukt war ein Beerenfruchtmus aus Fruchtfleischzellen. Das Zellmaterial aller Beerenarten ähnelte farblich den frischen Originalbeeren. Der Clou aber waren die Nährwerte, denn sie waren im Vergleich mit den Nährwerten pflanzlicher Beeren entweder gleich oder deutlich besser (Nordlund et al. 2018).

Neben pflanzlichen Lebensmitteln lassen sich weitere pflanzliche Produkte ohne nennenswerten Flächenverbrauch herstellen - z.B. Palmöl. Ungefähr 22 Prozent der eisfreien Landflächen der Erde sind für die Forstwirtschaft reserviert (IPCC 2019: 4). In der Regel handelt es sich dabei um Monokulturen, die speziell in tropischen Regionen, in denen die Biodiversität besonders hoch ist, ein großes ökologisches Problem sind. Auf ausgedehnten Flächen in Indonesien, Malaysia und Kolumbien wurde tropischer Wald abgebrannt und in Plantagen für die Ölpalme umgewandelt. Von ihren Früchten wird Palmöl extrahiert und dieses Öl ist das weltweit am meisten genutzte pflanzliche Öl. Denn die Ölpalme kann auf Böden gedeihen, auf denen andere Nutzpflanzen nicht wachsen können. Sie liefert den höchsten Ertrag pro Hektar unter allen Ölpflanzen - fast fünfmal so viel Öl pro Hektar wie Raps, fast sechsmal so viel wie Sonnenblumen und mehr als achtmal so viel wie Sojabohnen. 
Die Substituierung von Palmöl würde folglich erheblich mehr Fläche bzw. Lebensraum für viele Arten benötigen (Tullis 2019). Es wäre darum ökologisch sehr bedeutsam, Palmöl ohne Ölpalmen zu gewinnen.

Seit Jahren wird daran geforscht, die Lipide der Ölpalme von Hefe- oder Algenzellen herstellen zu lassen. Manche dieser Versuche waren dabei in der Sache erfolgreich, aber ökonomisch noch ineffizient. Die eingesetzten Mikroorganismen konnten Palmöl herstellen, doch mit Kosten von über fünf Dollar pro kg (im Vergleich dazu kostet Palmöl ca. 50 Cent pro kg). Denn die Ölpalme ist nicht nur sehr ergiebig, ihr Anbau und die Ernte ihrer Früchte sind außerdem sehr billig. Die Integration der ökologischen Kosten in den Marktpreis des Palmöls würde dieses zwar sehr teuer machen, doch ist eine solche Maßnahme nicht in Sicht.

Masri et al. (2019) konnten die Produktion pflanzlicher Öle durch Hefezellen nun so optimieren, dass sie auf Kosten von immerhin 1,60 Dollar pro kg kommen. Dazu schleusten sie den Hefezellen die gewünschten LipidGene ein und setzten sie anschließend auf Diät, indem sie ihnen während des Wachstums Stickstoff und Phosphat entzogen. Darauf reagierte die Hefe, indem sie Fette als Notvorrat einlagerte. Normalerweise besteht eine Hefezelle aus rund 20 Prozent Fett, während der Diät aber aus bis zu 80 Prozent. Obendrein konnten Masri et al. mit den Hefen verschiedene pflanzliche Öle und Fischöl erzeugen, indem sie die Einzeller mit Essigsäure und verschiedenen Zuckern fütterten. Je nach Zuckerart produziert die Hefe anschließend ein anderes Fettsäureprofil - ganzjährig und von gleichbleibend hoher Qualität. Darüber hinaus waren die $\mathrm{CO}_{2}$-Emissionen bei der Hefeölherstellung niedriger als bei der konventionellen Palmölherstellung, ferner musste kein tropischer Lebensraum zerstört werden.

Auch hier steht die großtechnische Produktion noch aus, doch ist zu erwarten, dass sie in den 2020ern ökonomisch konkurrenzfähig realisiert wird. Bislang schätzen die Biotechnolog*innen, dass sie in einem Tank mit 10.000 Liter Volumen täglich $108 \mathrm{~kg}$ und jährlich fast $40.000 \mathrm{~kg}$ pflanzliches Öl herstellen können (Masri et al. 2019).

Damit besteht langfristig die Option, Ökosystemen weite Acker- und Plantagenflächen und damit Lebensraum für viele Tier- und Pflanzenarten zurückzugeben. Ausgerechnet im Anthropozän könnte damit die von Menschen genutzte Fläche auf der Erde schrumpfen, obwohl die Zahl der Menschen steigt. 


\section{Von vegan zu cegan}

Die vegane Ernährungsweise gilt derzeit zu Recht als die umweltverträglichste. Aber auch sie greift noch in Ökosysteme ein, benötigt sie doch große Flächen für den Anbau der Pflanzen. Eine maximale ökologische Entlastung geht nur mit minimalinvasiven ökologischen Eingriffen einher und in dieser Hinsicht kann der Veganismus übertroffen werden. Die Postlandwirtschaftliche Revolution gewährt die Möglichkeit, pflanzliche und tierische Produkte ohne Pflanzen und Tiere konsumieren zu können. Dadurch tut sich eine völlig neue Ernährungsweise auf, die den in der menschlichen Geschichte bisher bekannten künftig hinzuzufügen ist: In der menschlichen Geschichte dominierten bislang vor allem der carnivore, dann der vegetarische Ernährungsstil. In den letzten Jahren wurde in manchen Ländern zudem der Veganismus populär. Die auf zelluläre Verfahren basierende Produktion von Lebensmitteln ermöglicht nun einen Ernährungsstil, der cegan genannt werden kann. Während sich das »veg« in vegan von Vegetation bzw. Pflanzen ableitet, leitet sich das »ce« in cegan von Cell bzw. Zelle ab.

\section{Schluss}

Die Postlandwirtschaftliche Revolution vollzieht sich wie die Industrielle und Digitale Revolution nicht über Nacht. Absehbar ist jedoch, dass die Post-Landwirtschaftliche Revolution eine Disruption für die Viehindustrie und Agrarkonzerne sein wird. Diese verdienen viel Geld mit dem Verkauf von Schlachtfleisch, Dünger, Insekten- und Unkrautvernichtungsmitteln und Saatgut. Diese Produkte werden mit der zellulären Revolution an ökonomischer Bedeutung verlieren. Die betroffenen Konzerne werden sich wehren. Sie gilt es jedoch nicht zu retten, sondern eine Welt, die im Sterben liegt. Nicht zuletzt können cegane Verfahren auch für die Raumfahrt von großem Interesse sein. Denn die Besiedelung des Sonnensystems sollte ein weiterer Megatrend im 21. Jahrhundert werden. 
Abb. 1: Die Abbildung zeigt weltweite Ernährungsstile und ihren Einfluss auf das globale Ökosystem. Je größer die Fläche des jeweiligen Feldes ist, desto mehr wird die Umwelt durch den jeweiligen Ernährungsstil belastet. Die größten negativen Einwirkungen gehen vom carnivoren Ernährungsstil aus, den der Verzehr von tierischem Fleisch (inkl. Fisch und Meeresfrüchte), von tierischen Milchprodukten und Eiern kennzeichnet. Der vegetarische Stil verzichtet aufden Konsum von Tieren, die auf dem Land oder im Wasser leben. Veganer tun dies ebenfalls und nehmen zusätzlich keine tierischen Milchprodukte und Eier zu sich, weshalb Veganer nicht auf die Viehwirtschaft angewiesen sind. Ein ceganer Ernährungsstil würde Lebensmittel bevorzugen, die zellulär hergestellt sind. Die cegane Ernährungsweise kann äußerlich sogar der carnivoren gleichen, da auch Fleisch und Milch konsumiert werden können. Diese stammen jedoch nicht von Tieren. Auch die pflanzlichen Produkte der Ceganer"innen stammen nicht von Pflanzen, weshalb eine konsequente cegane Ernährung ohne die klassische Vieh-und Landwirtschaft auskommt.

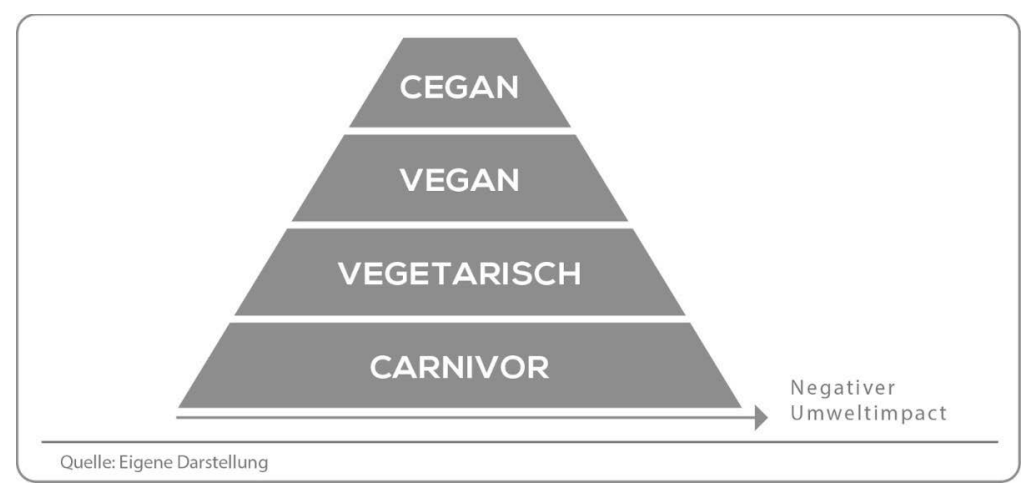

Quelle: Eigene Darstellung.

\section{Literatur}

Barnosky, Anthony/Hadly, Elizabeth A., Bascompte, Jordi/Berlow, Eric L./Brown, James H./Fortelis, Mikael/Getz, Wayne M./Harte, John/Hastings, Alan/Marquet, Pablo A./Martinez, Neo D./Mooers, Arne/Roopnarine, Peter/Vermeij, Geerat/Williams, John W./Gillespie, Rosemary/Kitzes, Justin/Marshall, Charles/Matzke, Nicholas/Mindell, David P./Revilla, Eloy/Smith, Adam B. (2012): "Approaching a state shift in Earth's biosphere«, in: nature 486, S. 52-58. https://doi.org/10.1038/nature11018 
Beresford-Jones, David/Arce, Susana/Whaley, Oliver/Chepstow-Iusty, Alex (2009): »The Role of Prosopis in Ecological and Landscape Change in the Samaca Basin, Lower Ica Valley, South Coast Peru from the Early Horizon to the Late Intermediate Period «, in: Latin American Antiquity 20, S. 303-332. https://doi.org/10.1017/S1045663500002650

Billman, Brian/Lambert, Patricia Marie/Leonard, Banks (2000): „Cannibalism, Warfare, and Drought in the Mesa Verde Region during the Twelfth Century A.D.«, in: American Antiquity 1, S. 145-178. https://doi.org/10. 2307/2694812

Böhm, Inge/Ferrari, Arianna/Woll, Silvia (2018): »Visions of in vitro meat among experts and stakeholders in Germany«, in: NanoEthics 12, S. 211224. https://doi.org/10.1007/s11569-018-0330-0

Bryant, Christopher/Szejda, Keri/Parekh, Nishant/Desphande, Varun/Tse, Brian (2019): »A Survey of Consumer Perceptions of Plant-Based and Clean Meat in the USA, India, and China.", in: Frontiers in Sustainable Food Systems 3. https://doi.org/10.3389/fsufs.2019.00011

Centolaet Damon/Becker, Joshua/Brackbill, Devon/Baronchelli, Andrea (2018): »Experimental evidence for tipping points in social convention «, in: Science 360, 6393, S. 1116-1119. https://doi.org/10.1126/science.aas 8827

Churchill, Winston (1931): »Fifty years hence«, in: The Strand Magazine 488.

Coghlan, Andy (2009): »Clearing oasis trees felled ancient Peru civilization«, in: New Scientist 204, 2733, S. 16. https://doi.org/10.1016/S02624079(09)62909-2

Dixon, Hugh (2019): Synthetic food: should NZ worry? Online verfügbar unter: https://berl.co.nz/research/synthetic-food-should-nz-worry. Zuletzt aufgerufen am 20.01.2020.

Dutt, Som/Manjul, Anshul/Raigond, Pinky/Singh, Brajesh/Siddappa, Sundaresha/Bhardwaj, Vinay/Kawar, Prashant/Patil, Virupaksh/Kardile, Hemant (2017): "Key players associated with tuberization in potato«, in: Critical Reviews in Biotechnology 7, S. 942-957. https://doi.org/10.1080/ 07388551.2016 .1274876

Hannapel, D.J. et al. (2017): »The Multiple Signals That Control Tuber Formation«, in: Plant Physiology 174, S. 845-856. https://doi.org/10.1104/pp.17. 00272

Hunt, T. L. et al. (2006): »Ancient DNA of the Pacific rat (Rattus exulans) from Rapa Nui (Easter Island)«, in: Journal of Archaeological Science 33, S. 15361540. https://doi.org/10.1016/j.jas.2006.02.006 
Intergovernmental Panel on Climate Change (IPCC) (2019): Climate change and land. Summary for policy makers. Online verfügbar unter: https://www.ipcc.ch/site/assets/uploads/2019/08/4.-SPM_Approved_ Microsite_FINAL.pdf. Zuletzt aufgerufen am 20.01.2020.

Intergovernmental Platform on Biodiversity and Ecosystem Services (IPBES) (2019): Summary for policymakers of the global assessment report on biodiversity and ecosystem services of the Intergovernmental SciencePolicy Platform on Biodiversity and Ecosystem Services. Online verfügbar unter: https://ipbes.net/sites/default/files/ipbes_7_10_add.1_en_1.pdf. Zuletzt aufgerufen am 20.01.2020.

International Agency for Research on Cancer (IARC) (2015): Q\&A on the carcinogenicity of the consumption of red meat and processed meat. Online verfügbar unter: https://www.iarc.fr/wp-content/uploads/2018/07/ Monographs-QA_Voli14.pdf. Zuletzt aufgerufen am 06.02.2020.

Kaneda, Toshiko/Haub, Carl (2018): How many people have ever lived on Earth? Online verfügbar unter: https:/www.prb.org/howmanypeople haveeverlivedonearth/. Zuletzt aufgerufen am 20.01.2020.

Kennett, Douglas J./Breitenbach, Sebastian F. M./Aquino, Valorie V./Asmerom, Yemane/Awe, Jaime/Baldini, James U. L./Bartlein, Patrick/Culleton, Brendan J./Ebert, Claire/Jazwa, Christopher/Macri, Martha J./Marwan, Norbert/Polyak, Victor/Prufer, Keith M./Ridley, Harriet E./Sodemann, Harald/Winterhalder, Bruce/Haug, Gerald H. (2012): »Development and Disintegration of Maya Political Systems in Response to Climate Change«, in: Science 338, 6108, S. 788-791. https://doi.org/10.1126/science. 1226299

Kohler, Timothy A./Matthews, Matthews H. (1988): »Long-Term Anasazi Land Use and Forest Reduction«, in: American Antiquity 3, S. 537-564. https:// doi.org/10.2307/281216

Kolachevskaya, Oksana O./Sergeeva, Lidiya I./Getman, Irina A./Lomin, Sergey N./Savelieva, Ekaterina M./Romanov, Georgy A. (2018): »Core features of the hormonal status in in vitro grown potato plants«, in: Plant Signaling \& Behavior 13, 5. https://doi.org/10.1080/15592324.2018.1467697 Lynch, John/Pierrehumbert, Raymond (2019): »Climate Impacts of Cultured Meat and Beef Cattle«, in: Frontiers in Sustainable Food Systems 3. https://doi.org/10.3389/fsufs.2019.00005

Masri, Mahmoud A./Garbe, Daniel/Mehlmer, Norbert/Brück, Thomas B. (2019): "A sustainable, high-performance process for the economic production of waste-free microbial oils that can replace plant-based equiv- 
alents«, in: Energy \& Environmental Science 9. https://doi.org/10.1039/ C9EEOO210C

Mattick, Carolyn S./Ladnis, Amy E./Allenby, Braden R./Genovese, Nicholas J. (2015): »Anticipatory life cycle analysis of in vitro biomass cultivation for cultured meat production in the United States «, in: Environmental Science \& Technology 49, S. 11941-11949. https://doi.org/10.1021/acs.est. 5 bo1614

Milburn, Josh (2018):»Death-Free Dairy? The Ethics of Clean Milk«, in: Journal of Agricultural and Environmental Ethics 2, S. 261-279. https://doi.org/10. 1007/s10806-018-9723-x

Nordlund, Emilia/Lille, Martina/Silventoinen, Pia/Nygren, Heli/SeppänenLaasko, Tuulikki/Mikkelson, Atte/Aura, Anna-Maria/Heiniö, RaijaLiisa/Nohynek, Liisa/Puupponen-Pimiä, Riita/Rischer, Heiko (2018): »Plant cells as food - A concept taking shape«, in: Food Research International 107, S. 297-305. https://doi.org/10.1016/j.foodres.2018.02.045

Poore, Joseph/Nemecek, Thomas (2018): »Reducing food's environmental impacts through producers and consumers", in: Science 360, 6392, S. 987992. https://doi.org/10.1126/science.aaq0216

Post, Mark J. (2012): „Cultured meat from stem cells«, in: Meat Science 3, S. 297-301. https://doi.org/10.1016/j.meatsci.2012.04.008

Ramankutty, Navin/Evan, Amato/Monfreda, Chad/Foley, Jonathan (2010): Global Agricultural Lands: Pastures, 2000. Palisades, NY: NASA Socioeconomic Data and Applications Center (SEDAC). https://doi.org/10.7927/ H47HiGGR.

Robinson, Timothy P./Wint, G. R. William/Conchedda, Guilia/Boeckel, Thomas P. van/Ercoli, Valentina/Palamara, Elisa/Cinardi, Giuseppina/ D'Aietti, Laura/Hay, Simon I./Gilbert, Marius (2014): »Mapping the Global Distribution of Livestock«, in: PLoS ONE, 5, e96084. https://doi.org/10. 1371/journal.pone.0096084

Smith, Madeleine/Lover, David C./Rochmann, Chelsea M./Neff, Roni A. (2018): "Microplastics in Seafood and the Implications for Human Health«, in: Current Environmental Health Reports 3, S. 375-386. https:// doi.org/10.1007/s40572-018-0206-Z

Spengler, Oswald (1995) [1923]: Der Untergang des Abendlandes. München: dtv.

Steer, Mark (2015). A comparison of land, water and energy use between conventional and yeast-derived dairy products: An initial analysis. Report. 
Online verfügbar unter: www.animalfreemilk.com/files/PD-LCA.pdf. Zuletzt aufgerufen am 30.01.2020.

Steffen, Will/Richardson, Katherine/Rockström, Johan/Cornell, Sarah/Fetzer, Ingo/Bennet, Elena/Biggs, Reinette/Carpenter, Stephen/Vries, Wim/Witt, Cynthia de/Folke, Carl/Gerten, Dieter/Heinke, Jens/Persson, Linn/Ramanathan, Veerabhadran/Reyers, Belinda/Sörlin, Sverker (2015): »Planetary Boundaries: Guiding human development on a changing Planet «, in: Science 347, 6223. https://doi.org/10.1126/science.1259855

Stengel, Oliver (2020): Ohne Vieh und Acker. München: oekom.

Stengel, Oliver/Looy, Alexander van/Wallaschkowski, Stephan (Hg.) (2017): Digitalzeitalter - Digitalgesellschaft. Wiesbaden: Springer VS. https://doi. org/10.1007/978-3-658-16509-3

Stramma, Lothar/Schmidtko, Sunke (2019): Global evidence of ocean deoxygenation, in: Dan Laffoley/John Baxter (Hg.), Ocean deoxygenation: Everyone's problem, Gland: IUCN. https://doi.org/10.2305/IUCN.CH.2019.13.en

Tullis, Paul (2019): How the world got hooked on palm oil. Online verfügbar unter: https://www.theguardian.com/news/2019/feb/19/palmoil-ingredient-biscuits-shampoo-environmental. Zuletzt aufgerufen am 23.01.2020. 



\section{Empowerment in Transformations-Arenen ${ }^{1}$ Berücksichtigung sozialer Disparitäten im Energiewendeprozess}

Anna Nora Freier, Stephanie Lübke, Katharina Schrot und Jenny Zorn

\section{Einleitung}

Megatrends werden gemeinhin als positiv konnotierte Entwicklungen gerahmt. Soziale Disparitäten fallen nicht in diese Kategorie positiver $\mathrm{Zu}$ kunftserzählungen, sondern gelten als weltweite Problemlage. Die Maßstäbe für soziale Disparitäten sind unter anderem Verteilungsgerechtigkeit und Chancengleichheit. Sie adressieren auf der einen Seite die Ungleichverteilung (knapper) Ressourcen, die für ein "gutes Leben« benötigt werden, auf der anderen Seite die Chancen, die eigene Position hinsichtlich des Zugriffs auf jene Ressourcen zu verbessern. Bestimmte Ausprägungen sozialer Merkmale zum Beispiel Alter, Bildungsstand, Geschlecht oder ethnische Zugehörigkeit - machen das Auftreten sozialer Ungleichheiten wahrscheinlicher.

Gleichzeitig werden Chancengleichheit und Mitbestimmung als Zentrum demokratischer Politik und als Werte an sich verstanden. Der eigenen Stimme Gehör zu verleihen ist ohne Einflussfaktoren wie Geld, Macht, Prestige oder Bildung abseits von Wahlen aber kein einfaches Unterfangen. Die theoretische Möglichkeit, am politischen Gestaltungsprozess teilzuhaben, geht mit der Praktikabilität einer solchen Teilhabe für prekäre Milieus und bildungsferne Schichten nicht konform: Fehlende Bildung erschwert den

1 Dieser Beitrag ist im Rahmen mehrerer Forschungsprojekte des Virtuellen Instituts »Transformation - Energiewende NRW« entstanden. Das Projekt »EnerChange: Spaltung in NRW überwinden - Energiewende für alle kommunizieren « ebenso wie das Projekt »EnerUrb - Urbanisierung: Energiewende in NRW im Spannungsfeld von Stadt und Land « ist unter Zuwendung des Ministeriums für Wirtschaft, Innovation, Digitalisierung und Energie des Landes Nordrhein-Westfalen (MWIDE) durchgeführt worden. 
Zugang durch fehlendes Wissen - sei es inhaltlich oder formal; fehlendes Einkommen verringert die Mobilitätsmöglichkeiten oder Zeitkontingente, die notwendig sind, um an Gestaltungsprozessen teilhaben zu können.

Die Energiewende ist ein aktuelles Thema, das (1) politisch als Gemeinschaftswerk gerahmt wird und (2) Auswirkungen auch auf sozial schwache Haushalte haben wird. So könnte bei vulnerablen Bevölkerungsgruppen die Energiewende beispielsweise durch die Erhöhung der Strompreise das Entstehen von Energiearmut begünstigen. Insofern sollten sozial benachteiligte Gruppen nicht nur aufgrund einer idealistischen Vorstellung eines Gemeinsamen, sondern auch darum beteiligt werden, weil die Energiewende direkte Auswirkungen auf ihre soziale Situation haben könnte. In dieser Hinsicht bedarf es, von politischer Seite aus betrachtet, nicht nur passiv der Akzeptanz der Bevölkerung, sondern die Bürger*innen sollten aktiv den Prozess der Energiewende mitgestalten können. Dazu bieten sich sogenannte Empowerment-Konzepte an, deren Ziel die Partizipation von ansonsten außenstehenden Gruppierungen ist.

In diesem Beitrag wird veranschaulicht, wie Empowerment-Konzepte in transformativer Forschung zum Thema Energiewende und Nachhaltigkeit genutzt werden können. Dazu wird zunächst verdeutlicht, was die Rolle von Empowerment und das Ziel der Anwendung von Empowerment-Konzepten in Energiewende- und Nachhaltigkeitsprozessen sind. Am Beispiel von zwei Empowerment-Konzepten - dem Partizipativen Reallabor und dem Citizen Engagement Prozess - und einem Empowerment-Instrument - dem Escape Room -, die im Rahmen von Projekten des »Virtuellen Instituts - Transformation Energiewende NRW« zur Anwendung gebracht wurden, wird veranschaulicht, wie Empowerment in transformativen Forschungskontexten eingesetzt werden kann.

Empowerment - Brückenschläge zur Partizipation

Ursprünglich aus dem Bereich der Bürgerrechtsbewegung in den USA stammend, hat das Empowerment-Konzept weltweit in unterschiedlichen Disziplinen und Berufsfeldern - im deutschsprachigen Raum vor allem in der Sozialen Arbeit, im Gesundheitswesen, in Entwicklungsarbeit und in der Antirassismusarbeit - eine starke Aufmerksamkeit erfahren und eine bunte Vielfalt von Praxisprojekten und Methoden stimuliert (vgl. Herriger 1991/2014; Keupp 1992; Stark 1996; Böll Stiftung 2013; bpb 2014). So facettenreich wie seine Anwendung, so ist auch das Begriffsverständnis von 
Empowerment ${ }^{2}$. Allen Definitionsangeboten gemeinsam ist jedoch, dass sie normativ gerahmt, »werthaltige« mutmachende Prozesse der Selbstbemächtigung, Selbstbefähigung, Stärkung von sozialer, politischer, ökonomischer, spiritueller Eigenmacht und Autonomie einer Community oder Person (vgl. z.B. Alinsky 1973; Freyth/Alinsky 1985) beschreiben. Als Arbeitsansatz zielt Empowerment auf die professionelle Förderung und (Wieder-)Herstellung von Selbstbestimmung, Selbstgestaltung und Handlungsmächtigkeit in Situationen des Mangels, gesellschaftlicher Benachteiligung oder Ausgrenzung (Rassismus, Klassismus, Sexismus, Heternormativität u.ä.; vgl. Herriger 2014). Empowerment als transitiven, also professionell unterstützenden Prozess $\mathrm{zu}$ betreiben und jene Erfahrungen anzuregen und $\mathrm{zu}$ fördern, in denen sich Menschen (wieder) ihrer individuellen und kollektiven Ressourcen bewusstwerden und sie zu nutzen lernen, erscheint gerade auch in Arenen der Nachhaltigkeit besonders bedeutsam: Auf der Ebene der Selbstaktivität und -veränderung ebenso wie in der kollektiven Dimension, im sozialen und im politischen Engagement von Bürger*innen.

So ist die "Große Transformation« zur nachhaltigen Gesellschaft - soweit soziale und politische Partizipation von Bürger*innen zentrale Prinzipien für ihr Gelingen bilden (vgl. WBGU 2011) - ebenfalls vor jene "großen« Herausforderungen und Entwicklungstendenzen gestellt, mit denen gegenwärtig die repräsentative Demokratie konfrontiert ist: Die aktive politische Teilhabe nimmt kontinuierlich ab und ist nicht sozial repräsentativ (vgl. Decker 2013). Bildungsstand, Arbeitslosigkeit und Kaufkraft beeinflussen empirisch nachweislich maßgeblich das konventionelle (verfasste, gesetzlich garantierte und geregelte) politische Partizipationsverhalten wie z.B. die Teilnahme an Wahlen, die Mitarbeit in Parteien oder an Petitionen. Die Ausübung von informellen politischen Betätigungsformen (z.B. Bürgerinitiativen, Demonstrationen) ist dabei mutmaßlich noch einmal mehr durch Milieu-, Ethnizitätsund Gender-Konstruktionen, Alter und Ability geprägt (vgl. z.B. Klatt/Walter 2011; Böll Stiftung 2013). Die Konsequenz davon ist, dass meist diejenigen Bürger*innen partizipieren, die ohnehin bereits politisch aktiver, da artiku-

2 Ebenso wie der Begriff der Bürgerbeteiligung stellt auch Empowerment ein catch-allKonzept, einen Sammelbegriff für unterschiedliche Konzepte mit einer großen Bandbreite an möglichen Bedeutungsinhalten dar, die vom Anspruch auf die Neustrukturierung von Machtverhältnissen zur Ermächtigung einer Selbstentfaltung reichen (siehe z.B. Herriger 2014). 
lationswilliger und -fähiger hinsichtlich der Durchsetzung ihrer Interessen sind (vgl. Schäfer/Schoen 2013: 95).

Es ist davon auszugehen, dass sich dieses Problem der sogenannten sozialen Selektivität im Bereich des Umwelt- und Klimaschutzes in verschärfter Art und Weise stellt. So werden hier oftmals eine Reihe hochkomplexer Themen und lokal begrenzte Projekte verhandelt, die nicht gleichermaßen interessieren, Lebenslagen betreffen oder denen in der Alltagswirklichkeit Priorität zugeschrieben werden. Einen sichtbaren Ausdruck finden diese spezifischen Problemlagen beispielsweise in Phänomenen wie »Nimby « ${ }^{3}$, lautstark vorgetragenen Partikularinteressen oder dem sogenannten Beteiligungsparadoxon (vgl. u.a. Reinert/Sinnig 1997; Radtke/Kersting 2018): Erst mit zunehmendem Konkretisierungsgrad von Infrastrukturprojekten steigt öffentliches Interesse und bürgerschaftlicher Mitsprachebedarf, dem stehen - zum fortgeschrittenen Zeitpunkt in Planungs- und Entscheidungsverfahren - oftmals jedoch keine formale, adäquate partizipative Mitgestaltungsmöglichkeit mehr gegenüber.

Es bleibt eine Kluft zwischen dem in empirischen Studien anhaltend dokumentierten Beteiligungswillen bzw. Wunsch nach verstärkter politischer Mitsprache und der Beteiligungspraxis (vgl. Lietzmann 2016; Mehr Demokratie 2020). Dieser Zielkonflikt zwischen politischer (Un-)Gleichheit in Partizipationsinteressen, -bereitschaft und -ausübung und dem Ruf nach mehr Beteiligung, stellt eine wesentliche Herausforderung dar (vgl. Schäfer/Schoen 2013). Unter Einbezug der Befunde, dass den gesteigerten Partizipationsansprüchen zudem oftmals kein handlungspraktisches Wissen zum Wie gegenübersteht (Leggewie/Schneider 2009) und dass Gefühle von politischer Einflusslosigkeit, Apathie oder Deprivation (vgl. Kersting 2008) entscheidende Hürden bilden, ergeben sich klare Zielsetzungen für eine transformative und transitive Perspektive.

3 Das englischsprachige Akronym »Nimby« steht für Not in my backyard (»Nicht in meinem Hinterhof«) und bezeichnet das Phänomen, dass trotz hoher abstrakter Unterstützung von z.B. Technologien oder Infrastruktur-Projekten und dem Wunsch, deren Vorteile zu nutzen, im Falle direkter Betroffenheit im eigenen Umfeld eine Ablehnung bis hin zum lautstarken Protest formuliert wird. Die Tatsache, dass das Phänomen auch als Bezeichnung für Personen verwendet wird, die eben gegen solche Projekte protestieren, ist bei der Vermittlung und der Suche nach Lösungswegen sicherlich nicht gewinnbringend. Dieser Problematik kann an dieser Stelle aber leider nicht weiter nachgegangen werden. 


\section{Das Partizipative Reallabor}

\section{Modell und Methodik}

Das Partizipative Reallabor stellt einen handlungspraktischen Ansatz von Empowerment zur und in der politischen Beteiligung an der »Großen Transformation zur Nachhaltigkeit« dar. Als eine innovative Forschungsmethode im Sinne von Citizen Science und ein partizipatives Instrument zur dialogischen Bürgerbeteiligung an der Gestaltung der Energiewende, wurde diese Methode im Forschungsverbund des Virtuellen Instituts »Transformation - Energiewende NRW « entwickelt und in der Praxis mehrfach erprobt. Grundlegend für diese Vorgehensweise ist die Annahme, dass die Energiewende immer zugleich auch komplexe gesellschaftliche Wandlungsprozesse erfordert, die als solche der sozial-räumlichen Kontextualisierung und der partizipativen Gestaltung bedürfen.

Dem Reallabor-Ansatz folgend sollen jene Transformationsprozesse gezielt angestoßen und auf experimentelle Art und Weise sowohl wissenschaftliche als auch gesellschaftliche Lernprozesse in einem räumlich abgegrenzten gesellschaftlichen Kontext gefördert werden (vgl. z.B. Schneidewind/SingerBrodowski 2014; Parodi et al. 2016; Ukowitz 2017; Wuppertal Institut 2019). Normativität, Nachhaltigkeitsorientierung, Lokalität, Transdisziplinarität und Transformativität bilden dabei wesentliche Prinzipien; ebenso, dass ein ethisch gut begründetes, gesellschaftlich legitimiertes Ziel verfolgt wird, das am gesellschaftlichen Gemeinwohl (in Gegenwart wie Zukunft) orientiert ist (vgl. Giulio/Defila 2018).

Für die Partizipative Reallabor-Variante ist zudem eine stark bürgerschaftliche Ausrichtung maßgebend. Ziel ist die systematisch inklusive und sozial-integrative Beteiligung der unorganisierten Bürgergesellschaft als Konsultative (vgl. Leggewie/Nanz 2016). Dafür sieht sie ein fallbezogenes Dialogverfahren zu einer konkreten Problemstellung in Transformationskontexten vor. Das Prozessdesign knüpft dafür unmittelbar an die Methodik der Planungszelle an (vgl. Dienel 2002, Lietzmann 2014; vgl. Lietzmann/DankwartKammoun/Freier 2017). Dieses spezifische Format wurde Anfang der 1970er Jahre als Instrument zur informellen Beteiligung von zufallsausgewählten Bürger*innen an politischen Planungs- und Entscheidungsprozessen an der Bergischen Universität Wuppertal entwickelt und ist seitdem vielfach, auch international, auf kommunaler, regionaler und nationaler Ebene z.B. als Bürgerrat, Citizens' Assembly oder Citizens' Jury umgesetzt worden (vgl. z.B. 
Buchstein 2009/2012; Fishkin 2013; Suiter/Farrell/O'Malley 2016; Lindell et al. 2017).

Mit einem mehrtägigen Beteiligungsprozess schafft das Partizipative Reallabor einen geschützten Partizipations-Raum zur Mitwirkung und Mitgestaltung im Lebensumfeld. In deliberativen ${ }^{4}$ Meinungsbildungs-, Aushandlungs- und Priorisierungsprozessen erarbeiten die im Losverfahren ausgewählten Bürger*innen ein orientierungsgebendes, sozial innovatives Transformationswissen mit lokaler Passform. Entlang eines thematisch strukturierten Arbeitsprogramms werden plurale Sachinformationen $\mathrm{zu}$ politischen, wirtschaftlichen, ökonomischen, zivilgesellschaftlichen und wissenschaftlichen Rahmenbedingungen ebenso in das Verfahren integriert wie auch empirische Evidenzen $\mathrm{zu}$ tatsächlichen Wirkungen, zu Ungewissem und $\mathrm{zu}$ Kontroversen. Ergebnis ist ein Bürgergutachten, das die bürgerschaftlichen Leitlinien, Empfehlungen und Stellungnahmen in ihrer Entstehung differenziert und im Hinblick auf ihre »soziale Robustheit«, d.h. ihre gesellschaftliche Zustimmungsfähigkeit (Nowotny/Scott/Gibbons 2004) ${ }^{5}$ transparent, nachvollziehbar und kompakt zusammenfasst.

Empowerment-Prozesse - individuelle, gruppenbezogene und strukturelle - spielen dabei auf verschiedenen Ebenen eine zentrale Rolle; sie bilden einen integralen Bestandteil von methodischem Design und Verfahrensgestaltung ebenso wie auch im Rahmen der konkreten Konzeption und Durchführung.

Das deliberative Demokratiemodell beschreibt eine auf den Austausch von Argumenten angelegte Form der Entscheidungsfindung unter Gleichberechtigten. Das bessere Argument soll die Entscheidungen prägen und zu besseren Entschlüssen führen, weil - im Idealfall - alle Argumente gegeneinander abgewogen werden und eine Einigung auf die beste Lösung möglich ist. Damit dies gelingt, werden an diesen Prozess hohe Anforderungen gestellt (vgl. Habermas 1991; für einen Überblick siehe Schmidt 2019). »Wissenschaft neu denken« fordern Nowotny et al. in ihrer grundlegenden Kritik. Im Kontrast zur traditionellen Form hierarchischer, disziplinärer, homogener und akademischer Wissenschaft sei die zeitgenössische Produktion wissenschaftlichen Wissens durch Kontextabhängigkeit, Heterogenität, Komplexität und Transdisziplinarität geprägt. Der Ausdruck des »sozial robusten Wissens « bringt dies zum Ausdruck. So produziertes Wissen sei zuverlässig, wenn es wirkmächtig Eingang in Gesellschaften zu findet. 


\section{Empowerment im Partizipativen Reallabor: Normative Rahmung}

Aus emanzipatorischen, legitimatorischen sowie auch aus Gründen gesteigerter Effektivität ${ }^{6}$ soll mit der Beteiligungsmethode eine größtmöglich inklusive und repräsentative politische Beteiligung der Bürgergesellschaft erreicht werden.

Aus der Intention der Inklusion gilt das Empowerment im Kontext des Partizipativen Reallabors nicht speziell für besonders benachteiligte Gruppen und Milieus ${ }^{7}$. Vielmehr wird ein weites Verständnis von Empowerment mit partizipatorischer Passform zugrunde gelegt. Explizit im Fokus steht die soziostrukturellen, individualistischen und verfahrensbezogenen Erklärungsansätze für Nicht-Beteiligung (vgl. Rohr et al. 2017) mit verschiedenen Methoden und Instrumenten $\mathrm{zu}$ reflektieren. Ziel ist, ein individuelles sowie kollektives Wollen und Können zur Beteiligung anzuregen, zu unterstützen und zu fördern. Ein besonderes Augenmerk liegt dafür auch auf den inzwischen weitverbreiteten Gefühlen von politischer Einflusslosigkeit, Deprivation oder Apathie (vgl. Kersting 2008). Über den ausschließlichen Einbezug besonders benachteiligter Gruppen und Milieus und der damit verbundenen Gefahr einer Verstärkung bestehender Stigmatisierungen hinaus (vgl. z.B. Gohde-Ahrens 2013), werden sie adressiert mit dem Ziel, Zuversicht in individuelle und kollektive Ressourcen zu stärken und die (Wieder-)Aneignung von politischen Handlungskompetenzen und einer aktiven Bürgerrolle zu ermöglichen. Im Kontext der Transformation des Energiesystems heißt dies, partizipative Arenen zu eröffnen, in welchen sich der/die Einzelne als aktiven »Klima-Citoyen« (vgl. Müller et al. 2016) erlebt und auch die mit ihr verbundenen alltagsweltlichen Herausforderungen und Interessen-, Wert- und Zielkonflikte artikuliert und solidarisch, in kollektiver Aktion, aktiv bewältigt.

Die normativen Grundlagen basieren damit auch auf einer ressourcenund stärkenorientierten Betrachtung von unorganisierter Bürgergesellschaft.

Zur normativen Rahmung und den demokratietheoretischen Implikationen dieser Beteiligungsmethode siehe z.B.; Pateman 1970; Giddens 1988; Habermas 1991; Dryzek 1996; Benhabib 2000; Barber 2003; Buchstein/Jörke 2003; Rosanvallon 2018; zur angestrebten Legitimität durch Repräsentativität siehe z.B. Sarcinelli, 2011; Lietzmann 2014 sowie zur angestrebten Ergebnisqualität durch Multiperspektivität z.B. Kersting, 2008; Alcántara et al., 2014.

7 Anders hier die »klassischen «Zugänge zum Empowerment vor allem im deutschsprachigen Raum, die ihr Augenmerk vor allem auf besondere Zielgruppen und ihre Aktivierung legen (siehe Herriger 1991/2014). 
Gerade auch bei hochkomplexen Problemstellungen werden Bürger*innen als solche jenseits von Funktionsträgerschaft oder direkter Betroffenheit mit einbezogen. In ihrem Alltagswissen, ihren Erfahrungen und Kompetenzen ebenso wie auch in ihren Anforderungen und Wünschen werden sie in der Rolle von kompetenten (energie-)politischen Akteuren wahrgenommen, beteiligt, und in den Mittelpunkt gesellschaftlicher Lern- und Transformationsprozesse gestellt. Lokales, bürgerschaftliches und externes Fachwissen werden dabei nicht als Gegenpole verstanden, sondern als Schnittmenge. Das produktive Verhältnis von »Bürgerwissen« und technologischen, ökonomischen, ökologischen, politischen und administrativen "Funktionswissen«, so die Annahme, eröffnet lokal angepasste und, im Sinne Nowotnys, "sozial robuste« Innovationen.

Dieses Vertrauen in die individuellen und kollektiven Ressourcen der Menschen, über hochkomplexe Zusammenhänge und auch über Belastungen und Zumutungen in der Großen Transformation zur Nachhaltigkeit in produktiver, gemeinwohlorientierter Weise zu verhandeln, ist Leitmotiv des Partizipativen Reallabors und seiner Empowerment-Praxis.

\section{Empowerment zur Partizipation}

Das Partizipative Reallabor zielt darauf ab, eine »mini public«, eine »kleine« Öffentlichkeit in ihrer soziodemographischen Vielschichtigkeit zu beteiligen, die nahezu dem repräsentativen Querschnitt der Stadt- bzw. Gemeindebevölkerung entspricht. Mit der Abbildung der Heterogenität von Stadtgesellschaft(en) und ihrer Multiperspektivität ist maßgeblich die Qualität und Akzeptanz der erarbeiteten Lösungswege im Bürgergutachten verbunden (vgl. Sarcinelli 2011; Lietzmann 2014). Dieses umfassende Inklusionsziel stellt wiederum hohe Anforderungen an die akteurs- als auch verfahrensbezogene Empowerment-Praxis des Partizipativen Reallabors.

Das methodische Design des Bürgerbeteiligungsmodells »Partizipatives Reallabor « reflektiert und berücksichtigt die sozio-ökonomische und soziokulturelle Selektivität von Partizipation (vgl. Lipset 1960; van Deth 1990; Verba et al. 1995; Jacobs/Skocpol 2005; Steinbrecher 2009; Decker 2013) - konzeptionell durch die Gestaltung eines aufsuchenden, qualifizierten aleatorischen Auswahl- und Einladungsverfahrens.

Im Kontrast zu so genannten selbstselektiven Partizipationsverfahren, die frei zugänglich sind, wird hier die Möglichkeit zur Teilnahme per Los bestimmt. Die Zufallsauswahl hat sich international als zuverlässige Metho- 
Abb. 1: Gründe für die Nicht-Beteiligung; eigene Darstellung nach IPG (vgl. Rohr et al. 2017 45)

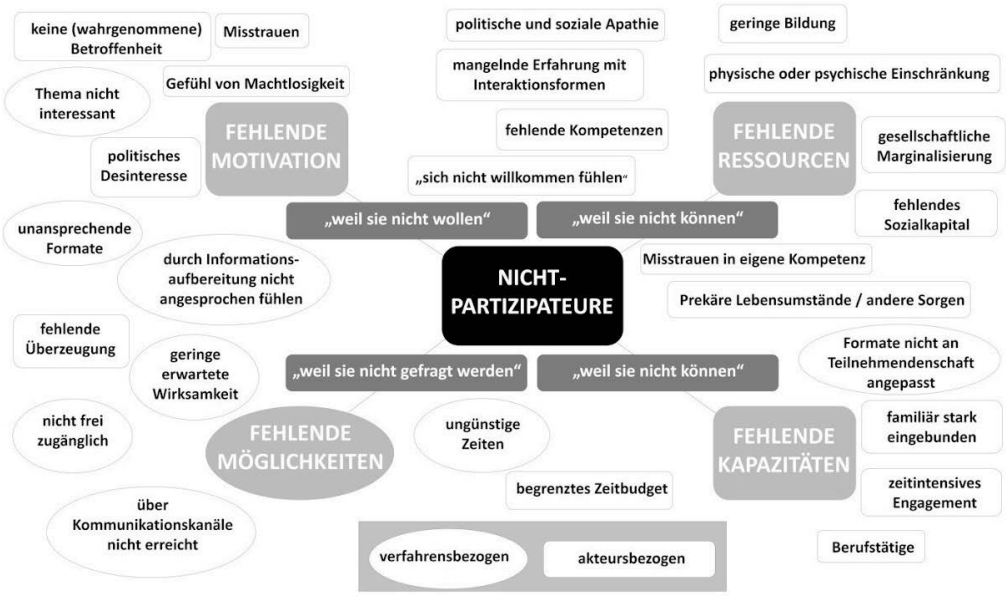

de der aktiven Rekrutierung in lokalen, regionalen und nationalen Kontexten bewährt (vgl. z.B. Dienel 2002; Reybrouck 2016; Fishkin 2013; Suiter/Farrell/O'Malley 2016; Lindell et al. 2017). Dies gilt für diejenigen Bürger*innen, die ansonsten fernbleiben, in besonderer Weise; bietet doch der äußere Impuls zur Partizipation, der höchstpersönliche und zeitlich befristete Auftrag per Los, die Möglichkeit, sich als potentiell aktive* $r$ politische* $r$ Akteur*in (wieder) zu erfahren.

Die Verfahrensgestaltung berücksichtigt zudem ressourcenbasierende Exklusionsfaktoren von partizipationsbereiten Bürgergruppen. Bereits mit der Einladung zur Teilnahme an der Partizipation sind verschiedene niedrigschwellige inklusive Angebote zum Abbau von Beteiligungsbarrieren verbunden. Dem punktuellen, mehrtägigen Beteiligungsformat entsprechend, richten sie sich vor allem auf die Schaffung einer finanziellen, beruflichen und familiären Vereinbarkeit. Neben dem Erhalt eines finanziellen Teilnehmendenbetrags, wird Arbeitnehmenden beispielsweise eine bezahlte Freistellung von der Arbeit (Bildungsarbeit) ermöglicht; Selbstständige erhalten wiederum eine Ausfallentschädigung. Das aktive Angebot von Kinderbetreuung und Pflege Angehöriger, Fahrtdiensten und Übersetzungsleistungen bzw. Sprachmittler*innen erfolgt ebenfalls mit der Einladung. Die vielfältigen individuellen 
Bedarfe (z.B. Fahrdienste, Hunde-Sitting) werden je im persönlichen Gespräch vereinbart, was sich im Abgleich mit der Verfahrensevaluation als sehr bedeutsam erweist. Eine Begleitforschung gewährleistet, dass die per Los ausgewählten Teilnehmenden nahezu dem repräsentativen Querschnitt der Stadt- bzw. Gemeindebevölkerung entsprechen (vgl. Bürgergutachten des $\operatorname{IDPF}^{8}$ ).

In einem weiteren Schritt erfolgt eine qualifizierte Zufallsauswahl. Mit ihr werden gezielt diejenigen Bürger*innen im Verfahren integriert, die aufgrund von sozialen Konstrukten - Zuschreibungs- und Ausgrenzungspraxen wie Klasse, Ethnizität, Gender, Alter, Religion, Sexualität und Disability strukturell benachteiligt sind. Zur Organisation erweisen sich hier zum Teil zielgruppenspezifische Kooperationen als sinnvoll, so z.B. mit sozialpsychologischen Institutionen, um Menschen $\mathrm{zu}$ erreichen, die über keinen festen Wohnsitz verfügen. In Abhängigkeit von der konkreten Verfahrensausrichtung kann das Zufallsverfahren auch variiert und z.B. nach ortsbzw. stadtteilbezogener und/oder sozialstrukturell gebotener Gruppierung stratifiziert, d.h. geschichtet, durchgeführt werden.

\section{Empowerment-Prozesse im Partizipativen Reallabor}

Auf diese Weise wird in dem mehrtägigen Verfahren eine »mini public«, eine »bunte Mischung« an Bürger*innen mit ihrer Multiperspektivität integriert. In getrennten Gruppen (mit einer festen Teilnehmendenanzahl von je 25 Personen) erarbeiten sie das Bürgergutachten zu einer konkreten Fragestellung in Transformationskontexten. Die Grundlage bildet ein nach methodischen Gesichtspunkten präzise strukturiertes Arbeitsprogramm. Es sieht die Gliederung in etwa 14 Arbeitseinheiten vor, die eine Behandlung verschiedener Transformationsaspekte nach einem festen und zeitlich exakt bemessenen Ablauf ermöglicht (Informations-, Deliberations- und Abstimmungsphase) ${ }^{9}$.

Eine Evaluation der Teilnehmendenschaft nach verschiedenen sozio-ökonomischen Merkmalen gibt Auskunft darüber, inwiefern der idealtypische Anspruch im Abgleich mit statistischen Bevölkerungsdaten (Zensus) erreicht wurde. Die Transparenz über die Teilnehmendenzusammensetzung schafft einen Überblick über die hiernach im Verfahren repräsentierten Meinungen und Interessen sowie denjenigen, die zum Beispiel über zielgruppenspezifische Formate gesondert zu beteiligen sind.

9 Siehez.B. Bürgergutachten Zeche Westerholt 2020: 13 unter www.idpf.eu/portfolio/zukunfts-zeche 
Ergebnis des mehrtägigen Prozesses ist das Bürgergutachten, das die bürgerschaftlichen Prioritätensetzungen zu jeder Arbeitseinheit in Form von thematisch differenzierten Leitlinien, Empfehlungen und Stellungnahmen transparent, nachvollziehbar und kompakt zusammenfasst. Das Verfahren arbeitet dabei zwingend ergebnisoffen und wird von einem strikt neutralen und auch mit Blick auf soziodemographische Merkmale gemischt besetztem Moderatorenteam begleitet.

Abb. 2: Ablauf Partizipatives Reallabor; eigene Darstellung des IDPF

\section{Partizipatives Reallabor}

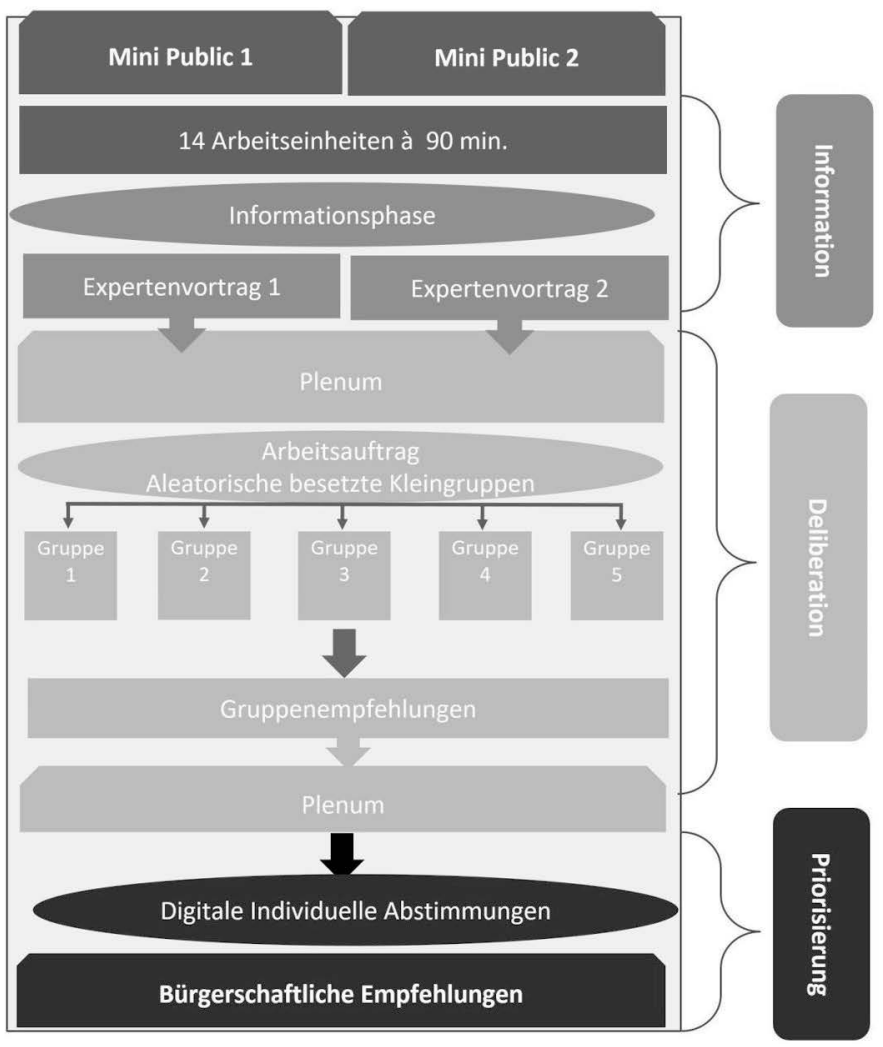


In dem stufenweisen Partizipationsprozess werden von den erweiterten Formen der Wissensproduktion über die deliberativen Aushandlungs-, Verständigungs- und Priorisierungsprozesse schrittweise PartizipationsKompetenzen entdeckt, entfaltet und eingebracht. In Verbindung mit der Zuerkennung und Ausübung von werthaltigen Aufgaben werden kognitives Wissen, affektiv-moralische Einstellungen sowie eine demokratiekompetente Bürgerschaftlichkeit z.B. durch den Aufbau von Selbstwirksamkeit(-serwartungen), sozialem Vertrauen und kommunikativen Fähigkeiten angeregt (vgl. Bandura 1997; Lüttringhaus 2000; Himmelmann 2001; Fung/Wright 2003). Damit sind aufs Engste Erfahrungen von Empowerment auf verschiedenen Ebenen verknüpft.

Aktivierung zur individuellen und kollektiven Wissensproduktion

Der Erwerb von unterschiedlichem, pluralem Fachwissen stellt einen grundlegenden Schritt im Empowerment-Prozess im Kontext des Partizipativen Reallabors dar. Wie auch die parlamentarischen Gremien der Stadträte, Landund Bundestage in ihren Anhörungen und Ausschüssen, so bedürfen auch die Bürger*innen Fachwissen für ihre Gutachtertätigkeit.

$\mathrm{Zu}$ jedem einzelnen Themenbereich werden Fakten- und Sachwissen durch Vorträge verschiedener Expert*innen verständlich aufbereitet und aufgebaut. Mit ihnen wird das Interesse am Thema geweckt, gesteigert und fundiert. Abgebildet wird dabei ausdrücklich die Heterogenität, Komplexität und Transdisziplinarität von Wissen, empirischen Evidenzen zu tatsächlichen Wirkungen, zu Ungewissem und zu Kontroversen (vgl. Nowotny/Scott/Gibbons 2004). Das lokale, bürgerschaftliche Wissen und Problemlösungspotential bildet dabei keinen Gegenpol zum externen Fach-Wissen; es wird daneben voll inhaltlich anerkannt.

Als Expertise wird es in der nächsten Phase des Beteiligungsprozesses einem mehrstufigen deliberativen Meinungsbildungs-, Austausch- und Verhandlungsprozess - unmittelbar einbezogen. Bei diesem Dialogprozess steht der Austausch individueller Perspektiven, Wünsche und Interessen mit dem Ziel einer kollektiven Meinungsbildung im Mittelpunkt. Aktiviert wird dieser wesentliche Schritt im Verfahren durch ausgewählte, themenzentrierte Impulsgeber. In Form von offenen Orientierungsfragen akzentuieren sie die deklarative (das Was) und prozedurale Seite (das Wie) sowie die Relevanz von ökologischen Transformationsprozessen vor dem Hintergrund der eigenen bürgerschaftlichen und lokalen Alltagsperspektiven. Als Arbeitsaufträge 
schaffen sie eine klare Ziel-und Rahmensetzung und regen einen gegenseitigen Lernprozess im Sinne sowohl einer individuellen als auch kollaborativen Wissenskonstruktion und -produktion an.

Dafür werden im Verfahren in einem ersten Schritt plurale DeliberationsRäume etabliert. Mit konkretem Auftrag und zeitlich befristet erfolgen dort Meinungsbildungs-, Austausch- und Verhandlungsprozesse in nach dem $\mathrm{Zu}$ fallsprinzip besetzten Kleingruppen. Mit dieser Art der Verfahrensgestaltung sind verschiedene Empowerment-Ziele und Strategien verknüpft:

- Das Kleingruppenformat: Mit ihm wird reflektiert, dass sich - gerade auch in Abhängigkeit von der sozialen Prägung - nicht jede Person gleichermaßen wohlfühlt, in größeren Gruppen zu sprechen, über persönliche Erfahrungen zu berichten oder auch eine abweichende Meinung zu formulieren. Um zu vermeiden, dass in diesen Zusammenhängen gerade marginalisierte Gruppenzugehörige mit Schweigen oder Zustimmung reagieren, wird mit der Kleingruppe eine spezielle Situation produziert: In ihr sollen im Sinne eines "geschützten« Raums, frei und offen persönliche Erfahrungen erzählt und Wissensressourcen ausgetauscht werden (vgl. Yiğit/Can 2009).

- Das Zufallsprinzip: Die immer wieder neue Besetzung der Kleingruppen nach dem Losverfahren wirkt der Herausbildung von Meinungsführerschaften sowie der Abbildung und Reproduktion bestehender Machtverhältnisse in den partizipativen Prozessen entgegen; unterstützt wird dies durch den zeitlichen Rahmen, der ein Ausufern der Diskussionen gerade durch geübte Rhetoriker verhindern soll.

- Der konkrete Arbeitsauftrag: Mit ihm verbunden ist eine Form des Empowerments, die die Fähigkeit sowohl zur individuellen als auch kollektiven Reflexion ohne Vorbehalt reizt. Sie erstreckt sich zum einen auf die eigenen Erfahrungen, die vor dem Hintergrund des Fachwissens kontextualisiert und idealerweise mit diesem neu verknüpft werden. Ebenso wird ein kritisches Verständnis für die eigene Rolle und die soziale Prägung in dem Austausch mit anderen, in dem auch Unterschiedlichkeiten erkannt werden, angeregt. Zum anderen erstreckt sich die Reflexion auch auf die eigenen Bedürfnisse, Fähigkeiten und Potentiale; mit dem Auftrag, gruppenbezogene Ziele, Strategien und Handlungsmöglichkeiten für die Zukunft $\mathrm{zu}$ formulieren, werden diese individuellen und kollektiven Ressourcen (wieder) erkannt oder neu entdeckt. Allein der Bewusstseinsanstieg, die 
kritische Reflexion des herrschenden Status quo an sich, ist Teil der Politisierung.

Konflikt - Kooperation - Konsens

Kooperation und Konsens ist die Methode und Haltung zur Erstellung der bürgerschaftlichen Leitlinien, Empfehlungen und Stellungnahmen. Sie sind als Gruppen-Entscheidung - erst im Kleingruppenformat, dann im Plenum - zu formulieren und im Anschluss individuell $\mathrm{zu}$ priorisieren. Auf diese Weise entsteht ein »sozial robustes« Konsenskonzept, welches alle Teilnehmenden auch im Hinblick auf ihre verschiedenen sozio-ökonomischen und sozio-kulturellen Hintergründe mittragen können und wollen. Dies setzen ein Erkennen, Formulieren und Abwägen eigener Bedürfnisse gegenüber Gemeinwohl-Interessen voraus; dem Austragen und Aushandeln von teils bewussten, teils latenten Konflikten kommt in diesen verständigungsorientierten Prozessen dabei eine zentrale Bedeutung zu. So treffen gerade auch sozial-ökologische Transformationsprozesse in ihrer Vielfältigkeit ob als Grundsatzentscheidungen, im Hinblick auf einzelne Maßnahmen, in Gestalt von regionalen Konzepten oder aber als konkrete Umsetzungen vor Ort - immer auch auf offene oder hinhaltend formulierte Interessen-, Wertund Ziel-Konflikte. Das Ausbleiben von artikuliertem Konflikt kann nicht als Befürwortung interpretiert werden, sondern kann z.B. aufgrund der Abweichung von der herrschenden Meinung oder dominanten diskursiven Wahrheiten bewusst oder unbewusst verschwiegen werden. Dieses differenzierte Bild von Befürwortung und Ablehnung jenseits einfacher LabelingKonzepte (vgl. Schweizer-Ries 2008) angeleitet erkennbar und benennbar werden zu lassen, steht explizit im Fokus der deliberativen Prozesse. Erst auf diese Weise lassen sich mögliche Kompensationen für unabänderliche (»systemische«) Benachteiligungen, sozio-kulturelle, sozio-demographische, milieuspezifische, lokalspezifische Differenzmuster sowie einzelner Interessen im sozialen Miteinander erkunden und wiederum aushandeln (»Bridging the Gap«). Über die Ausgestaltung der verständigungsorientierten Prioritätensetzung im Bürgergutachten findet dieser Kompromiss eine transparente Abwägung und Begründung. Für das Gelingen des Conflict-Mappings und für das Bridging the Gap sind vor allem zentral:

- Ergebnisoffenheit und Fairness des Verfahrens: Eine nicht zu unterschätzende Rolle spielt bei diesen Prozessen die Moderation. Sie sorgt für eine 
faire Einforderung von Diskussionsdisziplin und -zeit. Dem voraus geht eine sorgfältige und kompetente Verfahrenskonzeption. Durch die transparente Zielsetzung und einem hohen Grad an transparenter Formalisierung durch über zeitlich exakt definierte, sich wiederholende Methodensets wird eine spezielle, klare und verlässliche Situation für die Teilnehmenden mit Konsequenzen für die Art der Meinungsbildungs- und Aushandlungsprozesse kreiert.

- Geschützte Deliberations-Räume: Sie erlauben eine hohe Gemeinschaftlichkeit und sind durch einen respektvollen, wertschätzenden Umgang in Vielfalt und Unterschiedlichkeit geprägt. Dies ermöglicht geteilte Erfahrung von Zugewandtheit und Solidarität: Der Akt des Sprechens und des Gehört-Werdens in den eigenen Bedürfnissen, Anforderungen und Wünschen ebenso wie in der eigenen Kritik und Skepsis, bilden einen wesentlichen Schritt für das Empowerment, gerade auch von denjenen Bürger*innen, die unter »normalen« Umständen nicht um ihre Meinung gefragt werden. Die Erfahrung, in Bedürfnissen und Ängsten nicht allein $\mathrm{zu}$ sein, verstanden und ernst genommen $\mathrm{zu}$ werden, erweist sich als besonders bedeutsam. Dies schafft auch eine Solidarisierung mit Menschen anderer sozialer Prägung, die das Überdenken eigener Positionen explizit anregt und fördert.

- Thematische Strukturierung: Die Verhandlungsbereiche sowie auch der konkrete Einbezug von entsprechendem Fachwissen erfordern eine sorgsame Konzeptionierung. Für das Conflict-Mapping ist die Transparenz über die Planungs-und Entscheidungsprozesse durch den Einbezug der pluralen politischen, wirtschaftlichen, ökonomischen, zivilgesellschaftlichen und wissenschaftlichen Rahmenbedingungen, aber auch der empirischen Evidenzen zu tatsächlichen Wirkungen, zu Ungewissem und zu Kontroversen zentral (vgl. Nowotny 1999; Lietzmann 2017).

\section{Begeisterung für die Macht Miteinander: Das Bürgergutachten}

Das Partizipative Reallabor sieht ebenfalls eine "machtbewusste« Form des Empowerments vor (vgl. Weber 2009), die sich in Form des Bürgergutachtens manifestiert. Mit diesem eindeutigen Mandat zielt es auf die Begeisterung für die und das Vertrauen in jene Kollektivmacht, die Hannah Arendt (1970) als »Macht miteinander« formuliert. Diese drückt sich aus als etwas grundsätzlich Produktives und Gestalterisches, als gesellschaftliches Vermögen, durch 
Abb. 3: Ablaufkooperativer Aushandlung im Partizipativen Reallabor; eigene Darstellung des IDPF

\section{Partizipatives Reallabor}

Aleatorik, Inklusion, Reprāsentativität
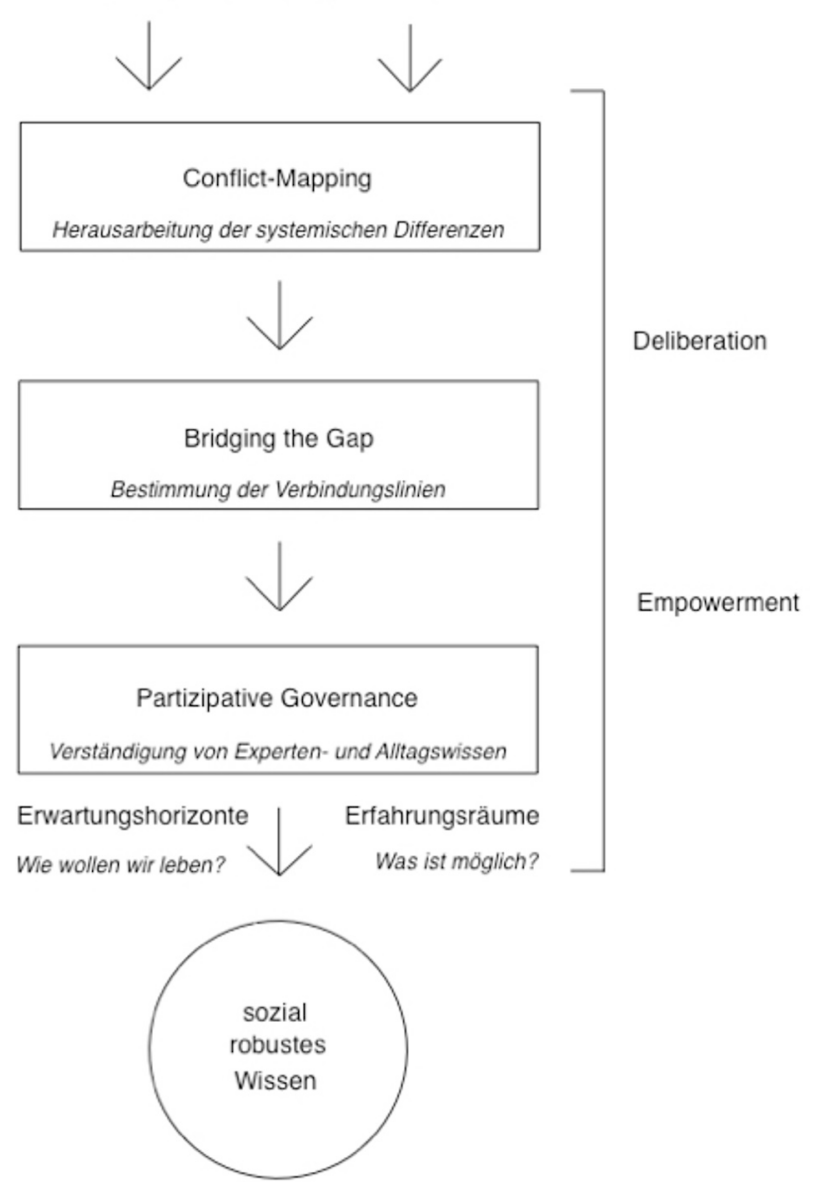

den Zusammenschluss von Menschen im Politischen. In Form von kollektiven Denk- und Handlungsprozessen zugunsten des Gemeinwesens und in der So- 
lidarität füreinander konstituiert, erhält diese Form von Macht im politischen Nahraum ihre Legitimation über den Konsens, die Erreichung eines Einvernehmens in der Gruppe. Dieser Konsens findet im Partizipativen Reallabor seinen Ausdruck und seine Publizität im Bürgergutachten. Die Ergebnisse werden so kompakt und transparent an Politik und Verwaltung rückgebunden ebenso wie auch der Kompromiss als Prozess, in seiner Abwägung und Begründung differenziert dargestellt wird. Als gut begründete und durch Repräsentativität und Multiperspektivität politisch breit legitimierte Leitlinien, Stellungnahmen und Handlungsempfehlungen nehmen sie - im Sinne einer der Konsultativen (Leggewie/Nanz 2016) - Einfluss auf die politische Planung und Umsetzung von Transformationspolitik (vgl. z.B. Sarcinelli 2011; Lietzmann 2014; Schüwer et al. 2020).

\section{Citizen Engagement Prozess}

Der Citizen Engagement Prozess ist ein nach der Methode CIVISTI (Citizens Visions on Science, Technology and Innovation) angewandtes EmpowermentKonzept, das durch die Sozialforschungsstelle der TU Dortmund im Rahmen des Projektes "EnerChange« zur Anwendung gebracht wurde und nachfolgend als beispielhafte Durchführung skizziert wird. Das Konzept eignet sich dazu, Wünsche und Bedürfnisse von ausgewählten Zielgruppen herauszustellen. Außerdem wird der Zielgruppe der eigene Stellenwert im jeweiligen Thema, beispielsweise zur erfolgreichen Umsetzung einer gerechten Energiewende, verdeutlicht. Durch einen direkten Austausch mit Expert"innen und Vertreter"innen der regulativen Ebene wird dargelegt, dass die Bedarfe der ausgewählten Zielgruppe ernst genommen werden. Die Ergebnisse ermöglichen darüber hinaus eine Erarbeitung von weiteren Empfehlungen für Forschung- und Förderpolitik.

\section{Methodik: Woher kommt »CIVIST|« und was zeichnet diese Methode aus?}

Die Methode CIVISTI wurde im gleichnamigen EU-Forschungsprojekt in den Jahren 2008-2011 entwickelt und erprobt und in diesem Beispiel als Beteiligungsmethode ausgewählt, da das Wissen verschiedener Gruppen integriert betrachtet wird. Der Schwerpunkt wird dabei auf Partizipation mit klar getrennten Rollen von Bürger*innen und Expert*innen gesetzt (vgl. Gudowsky et al. 2012). Ziel des EU-Projekts war es, neue, relevante Themen für die For- 
schung und Entwicklungspolitik der EU durch Konsultation der Bürger*innen in sieben europäischen Ländern (Dänemark, Österreich, Flandern/Belgien, Finnland, Malta, Bulgarien, Ungarn) zu identifizieren. Das CIVISTIProjekt deckte die Zukunftsvisionen der europäischen Bürger*innen auf und wandelte diese in relevante langfristige Wissenschafts-, Technologie- und Innovationsthemen um (vgl. Jacobi et al. 2011).

CIVISTI als qualitative Beteiligungsmethode basiert auf der Annahme, dass die Entwicklung einer Forschungsagenda, die die sozialen und gesellschaftlichen Bedürfnisse und Anliegen berücksichtigt, durch den Einbezug von Bürger*innen profitiert. Bürger*innen werden als Träger*innen dieser gesellschaftlichen Anliegen gesehen, deren Erwartungen und Anliegen durch die Verwendung der Methode in den Vordergrund gestellt werden. Außerdem zielt die Methode darauf ab, die verschiedenen Akteursgruppen zusammenzubringen und zwischen ihnen zu vermitteln (vgl. Jacobi et al. 2011). Den Bürger*innen kommt dabei eine besondere Bedeutung zu, da sie auf der Basis von aufeinander abgestimmten Prozessschritten beratend und bewertend agieren.

Abb. 4: CIVISTI-Prozessschritte; eigene Darstellung nach Jacobi et al. 2011 9f.

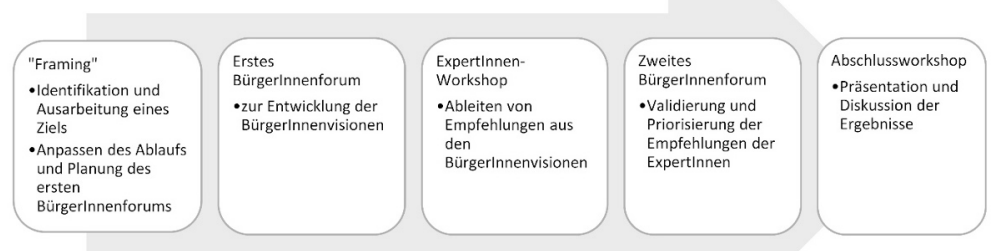




\section{Anwendungsbeispiel: das Projekt "EnerChange " des Virtuellen Instituts "Transformation - Energiewende NRW «}

Das Projekt »EnerChange: Spaltung in NRW überwinden - Energiewende für alle kommunizieren« wurde im Rahmen des Virtuellen Instituts (VI) »Transformation - Energiewende NRW « durchgeführt. Unter der Prämisse, dass die Energiewende nur als Gemeinschaftswerk gelingen kann, lautete die zentrale Projektforschungsfrage: Wie können Individuen und Gesellschaft auf Basis der Strukturwandelprozesse, von denen das Ruhrgebiet stark betroffen ist, mit in die neue Energie-Gesellschaft genommen werden? Dabei setzte das Projekt den Fokus der Untersuchung auf die Zielgruppe der sozial Benachteiligten, die sich häufig als »Verlierer*innen« der Energiewende sehen. Gemeinsam wurde mit Bürger*innen an Visionen zur Energiewende in NRW gearbeitet, die ihre persönliche Lebenswelt berücksichtigen, und daraus mithilfe von Expert*innen Politikempfehlungen ableiteten.

Die qualitative Beteiligungsmethode »CIVISTI: Citizen Visions on Science, Technology and Innovation « wurde von der Sozialforschungsstelle der TU Dortmund, die die Leitung des Projektesinnehatte, als geeignete Methode im Projekt »EnerChange« ausgewählt, da das Wissen verschiedener Gruppen integriert betrachtet wird und der Schwerpunkt auf Partizipation mit klar getrennten Rollen von Bürger*innen und Expert*innen gesetzt wird (vgl. Gudowsky et al. 2012). Die konzeptionelle Ausarbeitung im Projektrahmen erfolgte aus dargestellten Gründen auf Basis des Konzepts der Bürgerbeteiligungsmethode CIVISTI. Wichtige konzeptionelle Schritte als Vorbereitung für den Beteiligungsprozess bildeten die Adaption der Beteiligungsmethode CIVISTI für den Prozess auf NRW-Ebene sowie die Übersetzung und Nachvollziehbarkeit von Konzept und Ablauf für alle beteiligten Projektpartner und Bürger*innen. Die detaillierte Planung des Ablaufs des ersten Bürgerforums war für die weitere Bearbeitung des Arbeitspakets von besonderer Bedeutung, da in dieser ersten Veranstaltung die Visionen erarbeitet wurden, die die Basis für die weitere Arbeit gebildet haben. Darauf aufbauend wurde das weitere Konzept der Beteiligungsmethode an den Projektkontext angepasst und strukturiert.

Die qualitative Beteiligungsmethode ermöglicht es, das Wissen und die Bedürfnisse sozial benachteiligter Gruppen herauszustellen, um dann weitere Empfehlungen für Forschungs- und Förderpolitik abzuleiten. Die Umsetzung von Bürgerforen ermöglicht es, langfristige Visionen, Bedürfnisse und Sorgen der Bürger*innen herauszustellen (vgl. Jacobi et al. 2011). 


\section{Adaption und Anpassung der Methode für das Projekt}

Abb. 5: Adaption der Methode für das Projekt »EnerChange«: Ablauf des Beteiligungsprozesses; eigene Darstellung

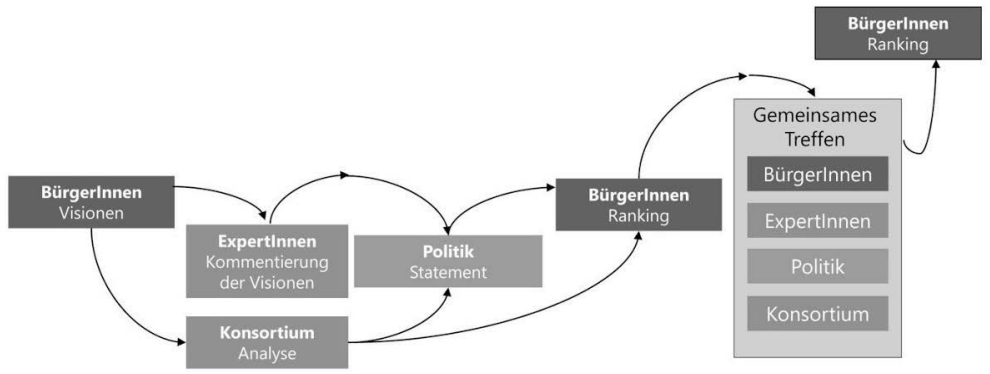

Der Prozess (vgl. Abb. 5) startete mit der Identifizierung von Wünschen und Bedürfnissen der Zielgruppe (z.B.: Welche Bedarfe sehen Sie für sich in der Umsetzung einer "gerechten Energiewende«?) in einem zweitägigen Workshop mit 25 Bürger*innen. Die Teilnehmer*innen formulierten Wünsche und Bedürfnisse für die nahe und fernere Zukunft (10-40 Jahre), aus denen Expert*innen Empfehlungen generieren konnten. Die Wünsche und Bedürfnisse konnten an dieser Stelle z.B. in Forschungsschwerpunkte für die Zukunft umgewandelt werden. Diese Empfehlungen wurden in einem nächsten Schritt mit der regulativen Ebene diskutiert und in einem anschließenden 2. Bürgerforum (mit den gleichen Teilnehmer*innen) an die Zielgruppe zur Bewertung zurückgegeben, um aufgezeigte Prioritäten der Expert*innen und Politikvertreter*innen in einer weiteren Runde der Bürgerbeteiligung überprüfen, bestätigen und ergänzen zu lassen. In einem abschließenden Workshop mit allen Beteiligten (Bürger*innen, Expert*innen, Politikvertreter*innen) wurden die aufbereiteten Ergebnisse vorgestellt und diskutiert. Ziel dieses Workshops war es, den Teilnehmer*innen aufzuzeigen, welche Wünsche und Bedarfe in welcher Form in die politische Forschungsagenda einfließen können. Die letztendliche Auswahl der wichtigsten Empfehlungen wurden von den Teilnehmer*innen der Bürgerforen eigenständig getroffen. Die Ergebnisse wurden vom Forschungskonsortium für relevante Stakeholder und Entscheidungsträger*innen in Form von Empfehlungen aufbereitet. 
Ziel des Prozesses war es darüber hinaus, der Zielgruppe der sozial Benachteiligten den eigenen Stellenwert zur erfolgreichen Umsetzung einer gerechten Energiewende zu verdeutlichen. Durch einen direkten Austausch mit Expert*innen und der politischen Ebene wurde dargelegt, dass die Wünsche und Bedürfnisse dieser Zielgruppe ernst genommen werden. Darüber hinaus sollten neue geeignete, zielgruppenspezifische Kommunikationsformen aufzeigen, wie und in welcher Form die Ansprache seitens Expert*innen und Stakeholdern gelingen kann, damit sozial benachteiligte Gruppen in die Prozesse der Umsetzung der Energiewende eingebunden werden können.

Die Methode wurde außerdem im Rahmen von »EnerChange« ergänzt, indem die regulative Ebene in den Prozess eingebunden wurde. Die Einbindung wurde für sinnvoll erachtet, damit der Mehrwert und Nutzen der Teilnahme für die Zielgruppe sowie die politische Wirksamkeit deutlich gemacht werden konnten.

Abb. 6: Zeitlicher AblaufCitizen Engagement Prozess im Projekt »EnerChange«; eigene Darstellung

\begin{tabular}{|c|c|}
\hline Zeit & Aktivität \\
\hline März - Mai 2018 & $\begin{array}{l}\text { Absprachen zum Ablauf des gesamten Citizen Engagement } \\
\text { Prozesses und des ersten BürgerInnenforums mit Partnern }\end{array}$ \\
\hline April - Mai 2018 & $\begin{array}{l}\text { Rekrutierung von TeilnehmerInnen für das } \\
\text { BürgerInnenforum und Übersetzung der Arbeitsmaterialien }\end{array}$ \\
\hline Juni 2018 & $\begin{array}{l}\text { Erstes Bürgerlnnenforum (1,5-tägig, 2. und 9. Juni 2018) } \\
\text { Aufbereiten der ausgewählten Visionen und Rückkoppeln } \\
\text { mit den TeilnehmerInnen }\end{array}$ \\
\hline Oktober 2018 & $\begin{array}{l}\text { Zwei Fachgespräche mit ExpertInnen zu den ausgewählten } \\
\text { Visionen aus dem ersten BürgerInnenforum (24. und } 30 . \\
\text { Oktober 2018) }\end{array}$ \\
\hline Januar 2019 & $\begin{array}{l}\text { Zweites BürgerInnenforum (19. Januar 2019) } \\
\text { Weitergabe der ExpertInnenmeinungen zu den Visionen } \\
\text { und der Ergebnisse des Fachgesprächs an BürgerInnen und } \\
\text { Bestätigung oder Korrektur der Visionen. }\end{array}$ \\
\hline März/April 2019 & $\begin{array}{l}\text { Abschlussworkshop mit allen Stakeholdern (BürgerInnen, } \\
\text { Expertlnnen und Konsortium; 4. April 2019) } \\
\text { Präsentation der Ergebnisse }\end{array}$ \\
\hline
\end{tabular}




\section{Umsetzung der Methode im Rahmen des Projekts "EnerChange"}

Mithilfe der auf den Projektkontext angepassten CIVISTI-Methode wurde im Rahmen von »EnerChange« ein Beteiligungsprozess durchgeführt, der die Zielgruppe der sozial Benachteiligten in den Fokus rückte. Die Akquise von Teilnehmer*innen für die geplanten Bürgerforen wurde mithilfe von bestehenden Kontakten zu Intermediären durchgeführt. Außerdem wurde die Methode an den Kontext und die Zeitplanung des Projekts angepasst (siehe Abb. 6). Essentiell für den Erfolg der Methode und die Erarbeitung von Ergebnissen war die Beteiligung desselben Teilnehmerkreises bei beiden Bürgerforen, da, aufbauend auf den Ergebnissen der ersten Veranstaltung, bei der zweiten Veranstaltung daran weitergearbeitet wurde.

\section{Erstes Bürgerforum}

Die inhaltliche Gestaltung des ersten Bürgerforums startete mit der Formulierung von Visionen zum Thema Energiewende der Zielgruppe in einem zweitätigen Workshop. Dabei wurden folgende Aspekte abgefragt:

- Was ist die Energiewende für Sie?

- Hoffnungen und Träume sowie Ängste und Sorgen in der Vergangenheit (Blick in die Vergangenheit)

- Blick in die Zukunft: Welche Hoffnungen und Träume, Ängste und Sorgen haben Sie? (Zukunft in 10-40 Jahren)

Auf der Basis der Diskussionsergebnisse vom »Blick in die Zukunft« entschieden sich die Bürger*innen für einzelne Aspekte, die daraufhin als »grobe« Visionen ausgearbeitet wurden. Dazu wurde für jede Vision eine kurze Beschreibung von etwa 10 Zeilen geschrieben sowie ein passender Titel für die Vision gefunden.

In einem abschließenden Auswahlprozess konnten die Bürger*innen aus insgesamt 14 groben Visionen die für sie vier wichtigsten auswählen. Die Teilnehmer*innen formulierten zur Beantwortung dieser Fragen ihre Wünsche und Visionen für die nahe und fernere Zukunft. Das Ergebnis des ersten Bürgerforums waren vier ausgearbeitete Visionen, die die Grundlage für den weiteren Prozess geboten haben: ॥Umwelt- und Naturschutz als Schulfach«, »Energieerzeugung für Jedermann«, »Das Ende der Machtlosigkeit« und »Sei Teil der Zukunft, die du dir wünschst«. 


\section{Sicht der Expert*innen}

Anschließend an das erste Bürgerforum und basierend auf den Themenschwerpunkten der vier ausgewählten und ausgearbeiteten Visionen wurden relevante Expert*innen recherchiert und in einem nächsten Schritt kontaktiert. Gleichzeitig wurden die Termine inhaltlich vorbereitet. Statt eines vorgesehenen Workshops, wurden zwei Termine in Form von Fachgesprächen umgesetzt. Die inhaltliche Gestaltung der Fachgespräche orientierte sich an der Diskussion von folgenden Leitfragen:

- Ist die Vision umsetzbar? Welche Umsetzungsansätze gibt es bereits?

- Was fehlt in der Vision?

Die Visionen wurden von Expert*innen hinsichtlich ihrer Umsetzbarkeit bewertet und vor allem positive Ansatzpunkte herausgestellt.

\section{Zweites Bürgerforum}

Die inhaltliche Gestaltung des zweiten Bürgerforums startete mit der Zusammenfassung der Ergebnisse des ersten Bürgerforums, um den Teilnehmer*innen die ausgewählten vier Visionen erneut ins Gedächtnis zu rufen und sie auf die interaktive Arbeitsphase und Diskussion vorzubereiten. Ziel des zweiten Bürgerforums war es, die Einschätzungen und Empfehlungen aus den im Oktober durchgeführten Fachgesprächen mit Expert*innen an die Zielgruppe zurückzugeben und in einer weiteren Runde der Bürgerbeteiligung überprüfen, bestätigen und ergänzen zu lassen.

Um eine aktive Beteiligung aller Teilnehmer*innen $\mathrm{zu}$ gewährleisten, wurde die Vorstellung der Expert*innenmeinungen und Kommentierung sowie die anschließende Diskussion in Form eines World Cafés gestaltet, bei dem jede Vision an einem Thementisch behandelt wurde und die Teilnehmer*innen in kleinen Gruppen von Tisch zu Tisch wechseln konnten.

\section{Abschlussworkshop}

In einem abschließenden Workshop, zu dem alle Beteiligten des Beteiligungsprozesses (Bürger*innen, Expert*innen, Konsortium) eingeladen wurden, wurden die aufbereiteten Ergebnisse vorgestellt und diskutiert. Ziel dieses Workshops war es, den Teilnehmer*innen aufzuzeigen, welche Wünsche und Bedarfe in welcher Form in die politische Forschungsagenda einfließen können. Die letztendliche Auswahl der wichtigsten Empfehlungen 
wurden von den Teilnehmer*innen der Bürgerforen getroffen. Die Ergebnisse wurden für relevante Stakeholder*innen und Entscheidungsträger*innen in Form von Empfehlungen aufbereitet.

\section{Schlussfolgerungen}

Eine Analyse der Strukturwandelprozesse im Ruhrgebiet und die Fallstudie der englischen Stadt Plymouth, die Entwicklung einer nicht-textbasierten Kommunikationsstrategie in Form eines Do-It-Yourself Escape Rooms (siehe weiter unten) sowie computergestützte Experimente zur Verhaltensökonomik haben im Rahmen des Projekts »EnerChange« die Ergebnisse der Zusammenarbeit mit den Bürger*innen ergänzt und zur Genese einiger zentraler Aussagen geführt.

Die Zielgruppe der sozial Benachteiligten beschäftigt sich nicht mit großen und für sie ungreifbaren Themen wie Nachhaltigkeit oder Klimaschutz, sondern mit kleineren Dimensionen ihres alltäglichen Lebens. Dabei geht es für diese Personen oftmals um die Frage, wie Geld gespart werden kann, damit sie ihren Alltag besser und mit mehr finanziellen Möglichkeiten bewältigen können. Dabei haben sie mitunter »das große Ganze« im Hinterkopf und sind dann doppelt stolz, wenn sie zusätzlich zum individuellen Erfolg, beispielsweise der Rückzahlung eines Betrags nach der Stromabrechnung, auch noch etwas für die Umwelt getan haben.

Die Erarbeitung der Visionen und die Auseinandersetzung mit den Themen wie Klimaschutz, Energiewende und Nachhaltigkeit haben im Laufe des Prozesses gezeigt, dass es offensichtliche Diskrepanzen gibt zwischen dem, was die Bürger*innen sich wünschen und daher annehmen, dass es dazu noch keine Umsetzungsaktivitäten gibt, und dem, was es (in Ansätzen) schon gibt und in diesen Bereichen schon umgesetzt wird. Diese Lücke lässt sich sicherlich auf das fehlende Wissen bei der Zielgruppe zurückführen und lässt die Frage offen, ob es hier eine fehlerhafte Kommunikation von Informationen gibt.

Die Vermittlung von Informationen und richtigen Verhaltensweisen spielt bei der Erreichung der Zielgruppe der sozial Benachteiligten eine wichtige Rolle. Hier wurde vermehrt der Wunsch nach positiven Vorbildern genannt. Diese Vorbilder sollen einerseits Personen sein (Personen des öffentlichen Lebens, die in Social Media Werbung machen, Politiker*innen, aber auch Personen des alltäglichen Lebens, mit denen man/Kinder in ständigem Kontakt steht/stehen - z.B. geschultes Personal an Schulen), aber auch Gebäude. Hier 
lautete der Wunsch, dass vor allem öffentliche Gebäude mit gutem Beispiel vorangehen und so zeigen sollen, wie eine alternative Stromgewinnung möglich ist.

Die Methode CIVISTI ist anwendbar auf andere Themen und Kontexte, vor allem dann, wenn eine eher schwierig zu erreichende bzw. schwer mitzunehmende Zielgruppe eingebunden werden soll. Hier legt die Methode einen besonderen Fokus auf die Bürger*innen und misst ihnen eine zentrale Rolle zu. Dies ermöglicht eine enge Zusammenarbeit mit den teilnehmenden Bürger*innen. Außerdem sorgt der gleichbleibende Personenkreis dafür, dass sich über den Zeitraum der Durchführung ein Kennenlernen aller Beteiligten und ein damit verbundenes Gruppengefühl einstellen kann.

Der Einbezug der regulativen Ebene in den Ablauf der Methodik ist eine sinnvolle Erweiterung, um die entwickelten Visionen und Expert*innenkommentare aus Sicht von Entscheidungsträger*innen bewerten zu lassen und deren Inhalte zu diskutieren. So können Empfehlungen, basierend auf den Ergebnissen des Prozesses, zielgruppengerecht ausgearbeitet und formuliert werden.

\section{Escape Rooms als Baustein im Empowerment-Prozess}

Empowerment-Prozesse wie der Citizen Engagement Prozess lassen Spielraum zur Anpassung an die Ziele und jeweiligen Bedürfnisse der Gruppe. Trotz des Rahmenkonzepts lassen sich verschiedene Methoden und Bausteine zum Empowerment kombinieren und in den Prozess integrieren. Da zum Empowerment im Bereich Nachhaltigkeit auch die Wissensvermittlung gehört, also die Art und Weise, wie Wissen über nachhaltiges Handeln auf der einen Seite vermittelt und auf der anderen Seite erlernt wird, hat sich das Kulturwissenschaftliche Institut Essen (KWI) im Rahmen des Projekts »EnerChange« diesem Themenkomplex gewidmet. Die Forschungsfrage lautete: Mit welcher Kommunikationsstrategie kann man Wissen über nachhaltiges Handeln so vermitteln, dass es für möglichst viele Personen verständlich und eingängig ist?

\section{Das Problem der Kommunikation von nachhaltigkeitsbezogenem Wissen}

Nachhaltigkeit und nachhaltiges Handeln ist längst kein gesellschaftliches Randthema mehr. In Zeitschriften, Sachbüchern, Radio- und Fernsehsendun- 
gen wird darübergeschrieben und gesprochen, was jede* $r$ Einzelne tun kann, um nachhaltiger zu leben. Von Mülltrennung und dem Einkauf regionaler Produkte über die Nutzung öffentlicher Verkehrsmittel und innovativer Mobilitätsformen bis hin zum Einsparen von Strom, Wärmeenergie und Wasser wird kein Bereich des täglichen Lebens ausgelassen. Doch obwohl das Thema im Mittelpunkt der medialen Aufmerksamkeit steht, tangiert es kaum die individuellen Handlungen. Wenn man über die Kenntnis verfügt, welche Handlungsoption »besser« wäre, sich aber nicht dementsprechend verhält, so spricht man von einer Lücke zwischen Denken und Handeln, der intentionbehaviour gap (wahlweise auch mind-oder attitute-behaviour gap genannt): Die Menschen wissen, was sie tun sollten, um nachhaltig zu leben, setzen dieses Wissen aber nicht in die Praxis um. Die Gründe dafür sind vielfältig und noch lange nicht hinreichend geklärt. Eine Auswertung verschiedener Studien zu diesem Phänomen findet sich bei Hassan, Shiu und Shaw (2016).

Ein anderes, zeitlich vorgelagertes Problem ist aber, das Wissen überhaupt erst so zu vermitteln, dass es das Potential hat, handlungsleitend zu sein. In vielen Fällen wird auf textbasierten Kommunikationsstrategien wie Ratgeberliteratur und Broschüren, rein visuellen Darstellungsformen wie Internetvideos, Plakate, Fernsehsendungen oder Vorführungen wie in Seminaren zurückgegriffen. Diese Kommunikationsstrategien sind für das Erlernen von nachhaltigem Handeln, einer Handlungskompetenz also, problematisch, weil durch sie Wissen vermittelt wird, das handlungsanleitend sein soll, aber von den Rezipient"innen im Prozess des Erlernens selbst keine Handlung verlangt. Sie nehmen das Wissen passiv auf, wenden es aber erst nach dem Lernprozess an.

Unter der Prämisse, dass möglichst viele Menschen die Kompetenz zum nachhaltigen Handeln erlangen sollen, muss die Kommunikationsstrategie verschiedene Kriterien erfüllen. Sie muss erstens für möglichst viele Personen leicht verständlich sein, also auch bildungsbenachteiligte Menschen mitdenken, was unter anderem beinhaltet, dass die Strategie nicht zu textlastig und komplex sein darf. Sie sollte zweitens möglichst auf theoretische Ausführungen verzichten und das Erlernen des Handlungswissens in der Praxis selbst erfahrbar machen. Drittens sollte sich die Kommunikationsstrategie nicht nur der Argumentation, sondern auch der emotionalen Erfahrung bedienen, beispielsweise dadurch, dass die Bürger*innen in eine Geschichte involviert werden, in der sie selbst eine Rolle spielen. 


\section{Escape Rooms als Kommunikationsstrategie}

Im Projekt »EnerChange« haben die Mitarbeiterinnen des KWIs eine Kommunikationsstrategie entwickelt, die diesen Kriterien genügen soll. Um Handlungswissen zum Thema Nachhaltigkeit learning by doing zu erlernen und um eine erfahrbare Geschichte zu entwickeln, hat sich das KWI für den Kommunikationstyp Spiel entschieden. Lernspiele haben den Vorteil, dass sich die Lernenden selbst die Lösungen erarbeiten müssen. Spielbasierte Ansätze haben außerdem ein hohes Potential, auf viel Text verzichten zu können und die Spieler"innen emotional für die Thematik zu erreichen.

Es gibt bereits zahlreiche Spiele zum Thema Nachhaltigkeit, die als Zielgruppe explizit Schüler*innen nennen und dazu geeignet sind, das theoretisch bereits erlernte Wissen in einem Spiel abzufragen oder umzusetzen (beispielsweise »Das Energie- und Klimaquiz« oder »Wirtschaft und Energie«). Auch Gesellschaftsspiele in Form von Brett- oder Kartenspielen gibt es bereits zu Themen der Nachhaltigkeit. Diese weisen aber eine recht hohe Komplexität auf (beispielsweise die Spiele »Lobby« und "Bougoni«) oder verbleiben weitgehend abstrakt (beispielsweise die Spiele »Prosperity« oder »Planet Steam«).

Deshalb hat sich das KWI dazu entschlossen, einen relativ neuen Spieletypus für die Vermittlung nachhaltigkeitsbezogenen Wissens zu wählen: den Escape Room. Escape Rooms, oder allgemeiner: Escape Games, eignen sich besonders als Kommunikationsstrategie, weil die Regeln schnell zu erklären sind und der Aspekt des learning by doing hier sehr stark ist. Der Escape Room bedarf keiner Vorkenntnisse und die emotionale Ebene wird aufgrund des Settings stark angesprochen.

Escape Rooms sind auf Grundlage von Computerspielen 2007 in Japan als Live Escape Games entstanden und heute aus der kommerziellen Spielelandschaft nicht mehr wegzudenken. Auch in Deutschland boomen Escape Rooms, sodass es in jeder größeren Stadt Angebote gibt, Escape Rooms zu besuchen. Das Grundprinzip ist immer dasselbe: Man muss Aufgaben und Rätsel lösen, um in einer gewissen vorgegebenen Zeit aus dem Raum entkommen, einen Mordfall aufzudecken oder ähnliches. Dazu gibt es einzelne Rätsel, die entweder nur mit einem anderen Rätselteil gelöst werden können, zu einem nächsten Rätsel führen oder ein Teil des Metarätsels ausmachen, das zum Schluss gelöst werden muss (vgl. Villar Lama 2018). In den meisten Fällen gibt es eine"n Spielleiter"in außerhalb des Raumes, der die Gruppe über Kameras beobachten kann und Tipps über einen Monitor oder einen Laut- 
sprecher gibt, wenn er oder sie merkt, dass die Gruppe mit den Rätseln nicht weiterkommt. Escape Rooms erfordern aufgrund der Vielzahl an Rätseloptionen Teamwork. Die Spielergruppe muss gemeinsam knobeln, um den Raum zu lösen und - in den meisten Fällen - den Schlüssel zu finden, um dem Raum zu entkommen. Escape Rooms werden in Deutschland, anders als in den USA, bisher kaum in Bildungszusammenhängen eingesetzt. ${ }^{10}$ Sie eignen sich jedoch in besonderer Weise zur Abstraktionsreduktion und lassen Inhalte emotional erfahrbar werden.

\section{"Noch zu retten...?« Ein Do-It-Yourself Escape Room zum Thema Nachhaltigkeit im Alltag}

Im Rahmen des Projekts »EnerChange« wurde ein Escape Room zum Thema Nachhaltigkeit im Alltag entwickelt, der die Besonderheit aufweist, dass man ihn - nach dem Prinzip Do It Yourself - in jedem Raum mit einer büroähnlichen Grundausstattung mit nur wenigen Materialen - und daher kostengünstig - selbst aufbauen kann. Die meisten Materialien, die man dazu benötigt, stehen auf der Website www.vi-transformation.de/escaperoom zum Download bereit. Zusätzlich werden Materialien benötigt, die bereits vorhanden sind, die man sich leihen kann oder die kostengünstig zu erwerben sind wie beispielsweise Fahrrad- oder Vorhängeschlösser, Schnellhefter, eine Steckdosenleiste oder eine nicht zu öffnende Spardose.

$\mathrm{Da}$ es nicht in jedem Raum möglich oder zu kostenintensiv ist, Kameras anzubringen, hat sich das Projektteam des KWIs dazu entschlossen, eine hilfestellungleistende Person mit in die Geschichte $\mathrm{zu}$ integrieren, sodass es ihr möglich ist, während des Spiels mit im Raum zu sein. Des Weiteren geht es in der Rahmengeschichte des Escape Rooms nicht darum, dem Raum zu entkommen, da das Einschließen von Personengruppen in vielen Bildungskontexten nicht möglich bzw. nicht erwünscht ist.

10 Ein Beispiel für einen in Deutschland entstandenen Escape Room im Bildungskontext ist »Auf Mikas Spuren«, der im Rahmen der Demokratielabore von Open Knowledge Foundation Deutschland e.V. (2018) entwickelt wurde. Hier können die Teilnehmenden das Thema »Populismus« spielerisch erarbeiten. Ole Jantschek (06.11.2019) hat die Vorteile von Escape Rooms im bildungspolitischen Kontext auf der Website der Bundeszentrale für politische Bildung zusammengefasst; online verfügbar unter: https://www.bpb.de/lernen/digitale-bildung/werkstatt/299698/ole-jantschek.Zuletzt aufgerufen am 25.02.2020. 
Eine weitere Besonderheit des Escape Rooms besteht darin, dass die Rahmengeschichte zwar immer die Gleiche ist, die Nachhaltigkeitsthemen und die dazugehörigen Rätsel aber variieren können. So gibt es Module zu den Themen (1) Strom, (2) Müll und Recycling, (3) Wärme sowie (4) Mobilität und Urlaub, bei denen je andere Aufgaben gestellt werden, wodurch die Spieler*innen themenspezifisch im direkten Umgang Nachhaltigkeit im Alltag erlernen können.

\section{Die Story}

"Die Spieler*innen haben einen Termin bei Doro Blitz. Sie ist eine Expertin auf dem Gebiet der Nachhaltigkeit und die Spieler*innen sind ihr Team. Doch als die Gruppe das Büro von Doro betritt, ist sie nicht da, sondern nur ENNY, ihr menschlicher Computer. ENNY hat keine guten Nachrichten für die Gruppe. Das Team ist Doros letzte Hoffnung ...« (Virtuelles Institut »Transformation - Energiewende NRW« 2019: 5)

Doro, die Nachhaltigkeitsexpertin, ist krank. Sie verwandelt sich ab und an in eine andere Person, die alles zerstört, was mit Nachhaltigkeit zu tun hat. Aber Doro hat eine Formel für ein Mittel gefunden, das sie retten kann. Sie hat die Formel in ihrem Büro versteckt und alles unnachhaltig gemacht, damit sich diese andere Person nicht dafür interessiert. Das Spielerteam muss nun den Raum nachhaltig gestalten bzw. nichtnachhaltige Strukturen aufdecken, um an die Formel zu gelangen. ENNY, der menschliche Computer, hilft ihnen dabei, wenn er das Gefühl hat, dass die Gruppe nicht weiterkommt.

Je nachdem, welche Module von der Spielleitung gewählt wurden, müssen die Spieler*innen nun verschiedene Aufgaben lösen, um an die Formel zu gelangen. Dadurch erlernen sie spielerisch nachhaltiges Wissen, wie beispielsweise wieviel Strom und Geld man mit einer abschaltbaren Steckdosenleiste sparen kann, welche Verkehrsmittel nachhaltig sind, was die besten Tipps für nachhaltiges Heizen sind oder wie Müll richtig getrennt wird. Der Escape Room ist geeignet für Spieler*innen ab 14 Jahren - ganz egal, welcher Bildungskontext vorliegt.

\section{Escape Rooms als Puzzleteil für Empowerment-Konzepte}

Die Standardaufgabe, die auch namensgebend für den Escape Room ist, ist die Flucht aus einem Raum. An einem Ort gefangen zu sein, dem man durch das Lösen von Aufgaben und Rätseln entkommen muss, klingt zunächst nicht 
oder nur in sehr zynischer Weise nach Empowerment. Tatsächlich kann der Escape Room aber ein Empowerment-Baustein in dreifacher Weise sein: Erstens steht er für diejenigen zur Verfügung, die bestimmte Personengruppen empowern möchten. Zweitens kann er im Bildungskontext dafür sorgen, dass die Spieler*innen dazu ermächtigt werden, bestimmtes Handlungswissen in ihren Alltag einzubauen, weil sie dieses bereits schon einmal aktiv im Spiel angewendet haben. Im Projekt »EnerChange« kam außerdem noch die Ebene hinzu, dass die Personen, die am Citizen-Engagement- Prozess teilgenommen haben, die Entwicklung des Spiels durch die Wahl der Rahmengeschichte und die Rätselvarianten, die zur Auswahl gestellt wurden, beeinflussen konnten. Insofern wäre drittens auch für ein Langzeitprojekt eine gemeinsame Entwicklung eines Escape Rooms als Empowerment-Methode denkbar.

\section{Fazit und Ausblick}

Transformative Forschung lebt von der Wechselwirkung zwischen Beobachtung von gesellschaftlich relevanten Prozessen und Veränderungen, die sie selbst mit hervorruft. Es ist nicht ohne Sinn, dass in diesem Kontext z.B. von Reallaboren gesprochen werden kann, da in Laboren die Veränderungen eines Systems unter selbst geschaffenen Bedingungen und Veränderungen von Bedingungen beobachtet werden. Im Fall der vorgestellten Konzepte hat sich die Anwendung als fruchtbar erwiesen dahingehend, dass (1) durch die Perspektive von Empowerment auch die sozialen Gruppen in den Fokus geraten, die sonst im Energiewendeprozess wenig beachtet werden, (2) durch die Anwendung von Empowerment-Konzepten eben diese soziostrukturell benachteiligten Gruppen in das Gemeinschaftswerk Energiewende integriert wurden und Output für die Energiewende von diesen Gruppen generiert werden konnte und (3) die Empowerment-Konzepte durch die Anwendung selbst für den Energiewendeprozess evaluiert werden können. Letzteres wurde in diesem Aufsatz dargestellt. Die Ergebnisse lassen sich wie folgt zusammenfassen.

Empowerment-Konzepte sind für die transformative Forschung $\mathrm{zu}$ Energiewende- und Nachhaltigkeitsthemen besonders gut geeignet, um einen sozial ausgleichenden egalitären Zugang zu diesen politisch hochaktuellen Themen zu finden. Für die Umsetzung der Energiewende ist der Einbezug aller Bevölkerungsgruppen und Milieus grundlegend, da sie (1) ideell als Gemeinschaftswerk verstanden wird und (2) der Einbezug die Gefahr minimiert, dass Proteste die Umsetzung gefährden oder die sozialen 
Ungleichheiten vertieft werden. Empowerment-Konzepte eignen sich daher unserer Ansicht nach, um dem Megatrend Soziale Disparitäten im Energiewendeprozess und breiter - in der Großen Transformation hin zu mehr Nachhaltigkeit - reflektiert zu begegnen.

Soll Partizipation - auch in der Transformation zur Nachhaltigkeit - nicht bloß instrumentell verstanden werden, sondern als ein wesentliches Prinzip und Voraussetzung für ihre erfolgreiche Umsetzung, muss Empowerment als Leitsatz zur Gestaltung von Partizipationsprozessen anerkannt und in seiner politischen Dimension als zentrale Strategie Einzug in Theorie und Praxis erhalten. Hierdurch eröffnet sich nicht zuletzt ein enormes Potential auch für ökologische Transformationsprozesse, die auf diese Weise ebenfalls »empowered« werden können.

Wie in diesem Artikel skizziert, wird bereits auch durch punktuelle Bürgerbeteiligung in Nachhaltigkeits-Arenen ein politisches Empowerment auf verschiedene Art und Weise gefördert. Bestmöglich reichen diese Empowerment-Erfahrungen über den konkreten Fall hinaus und stoßen Veränderungen im Privaten und im Politischen gleichermaßen an. Inwieweit aber individuelles und kollektives Empowerment sich gegenseitig verstärken und inwieweit auch bei erneuten Partizipationsmöglichkeiten und -erlebnissen auf die in der punktuellen Partizipation erfahrene Ermutigung und Begeisterung zur politischen Teilhabe und zur Macht zurückgegriffen werden kann, ist empirisch unklar. Hierzu bedarf es eines geeigneten, idealerweise Mixed-Method-Designs, welches die Wirkungen von Empowerment im Kontext punktueller, informeller Bürgerbeteiligung angemessen erfassen und so auch empirisch Aufschluss geben kann über geeignete Möglichkeiten der Verschränkung und Verstärkung; sowohl im Kontext von Partizipation als auch im Hinblick auf die Interaktionen zwischen individueller, gruppenbezogener und struktureller Empowerment-Ebene.

Aber auch eine politiktheoretische Reflexion und Fundierung der Empowerment-Diskurse erscheint äußerst fruchtbar. Neben einer »machtbewussten« Vertiefung von Empowerment (vgl. Alinsky 1973; Freyth/Alinsky 1985) durch theoretische Zugänge z.B. von Arendt (1970) oder Foucault (1994), sind es vor allem auch Ansätze der partizipatorischen Demokratietheorie (insbesondere Fung/Wright 2001), mit denen sich ein »Empowerment mit emanzipatorischem Anspruch « weiter konzeptualisieren ließe. Insbesondere im Hinblick auf die Interaktion individueller und kollektiver Ressourcenerschließung und -ausübung - und in Vertiefung des Agency-Konzepts - ließen sich dabei für die transitive Praxis-Perspektive wertvolle Impulse $\mathrm{zu}$ ihrer 
Förderung erhalten. Diese Verknüpfung eröffnet weitreichende Perspektiven auf die (Re-)Politisierung des Empowerment-Diskurses und umgekehrt. In Verbindung mit dem Solidaritätsbegriff (z.B. Bayertz 1998; Habermas 1991; Wildt 2007; Brunkhorst 1997), aber auch im Rekurs auf die Anerkennungstheorie (Honneth 1992) ließen sich dabei ebenfalls neue Zugänge für die Förderung einer »demokratischen Polarisierung« (Habermas 2016) eröffnen und »Mut« für die Begeisterung für die Macht und die Mitgestaltung der »Großen Transformation« als politische Grundhaltung schöpfen.

\section{Literatur}

Alcántara, Sophia/Kuhn, Rainer/Renn, Ortwin/Back, Nicolas/Böhm, Birgit/Dienel, Hans-Liudger/Ullrich, Peter/Schröder, Carolin/Walk, Heike (2014): DELIKAT - Fachdialoge Deliberative Demokratie: Analyse partizipativer Verfahren für den Transformationsprozess, Dessau-Roßlau: Umweltbundesamt. Online verfügbar unter: www.umweltbundesamt.de/publikationen/delikat-fachdialoge-deliberative-demokratie-analyse. Zuletzt aufgerufen am 28.02.2020.

Alinsky, Saul D. (1973): Leidenschaft für den Nächsten. Strategien und Methoden der Gemeinwesenarbeit, Gelnhausen/Berlin: Burckardthaus-Verlag.

Bundeszentrale für politische Bildung (bpb) (Hg.) (2014): Aus Politik und Zeitgeschichte (APuZ) 13-14. Rassismus und Diskriminierung.

Arendt, Hannah (1960): Vita activa oder Vom tätigen Leben, Stuttgart: Kohlhammer.

Arendt, Hannah (1970): Macht und Gewalt, München: Piper.

Bandura, Albert (1997): Self Efficacy. The Exercise of Control, New York: W H Freeman/Times Books/Henry Holt \& Co.

Barber, Benjamin R. (2003): Strong Democracy. Participatory Politics for a New Age, Berkeley/Los Angeles/London: University of California Press.

Bayertz, Kurt (Hg.) (1998): Solidarität. Begriff und Problem, Berlin: Suhrkamp. Benhabib, Seyla (1996): Kulturelle Vielfalt und demokratische Gleichheit. Politische Partizipation im Zeitalter der Globalisierung (Horkheimer Vorlesungen), Frankfurt a.M.: Fischer.

Böll Stiftung (2013): Dossier Empowerment. MID Redaktion, Berlin. Online verfügbar unter https://heimatkunde.boell.de/sites/default/files/dossier_ empowerment.pdf. Zuletzt aufgerufen am 28.02.2020.

Brunkhorst, Hauke (1997): Solidarität unter Fremden, Frankfurt a.M.: Fischer. 
Buchstein, Hubertus/Jörke, Dirk (2003): »Das Unbehagen an der Demokratietheorie«, in: Leviathan 31, S. 470-495. https://doi.org/10.1007/s11578-0030027-8

Buchstein, Hubertus (2009): Demokratie und Lotterie - Das Los als politisches Entscheidungsinstrument von der Antike bis zur EU, Frankfurt/New York: campus.

Buchstein, Hubertus (2012): »Wählen, Losen und politische Gerechtigkeit Plädoyer für einen >demokratisch-deliberativen pouvoir neutre«, in: Zeitschrift für Politikwissenschaften 22 (3), S. 384-403. https://doi.org/10.5771/ 1430-6387-2012-3-395

Decker, Frank (2013): Wenn die Populisten kommen. Beiträge zum Zustand der Demokratie und des Parteiensystems, Heidelberg: Springer VS. https://doi.org/10.1007/978-3-658-00802-4

Deth, Jan W. van (1990): »Politische Partizipation« In: Kaina, Viktoria/Römmele, Andrea (Hg.): Politische Soziologie. Ein Studienbuch, Heidelberg: Springer VS, S. 141-161. https://doi.org/10.1007/978-3-531-91422-0_6

Giulio, Antonietta di/Defila, Rico (2018): Transdisziplinär und transformativ forschen. Eine Methodensammlung, Wiesbaden: Springer VS. https://doi. org/10.1007/978-3-658-21530-9

Dienel, Peter C. (2002): Die Planungszelle. Der Bürger als Chance, Wiesbaden: Springer VS. https://doi.org/10.1007/978-3-322-80842-4

Dryzek, John S. (1996): »Political Inclusion and the Dynamics of Democratization«, in: American Political Science Review 90 (1), S. 475-487. https://doi. org/10.2307/2082603

Fishkin, James S. (2013): »Deliberation by the People Themselves: Entry Points for the Public Voice«, in: Election Law Journal: Rules, Politics, and Policy 12 (4), S. 490-507. https://doi.org/10.1089/elj.2013.0200

Freyth, Marianne/Alinsky, Saul D. (1985): Eine amerikanische Theorie der Praxis. Studien zur Politikwissenschaft Band 4, Münster: LIT.

Foucault, Michel (1994): Überwachen und Strafen. Die Geburt des Gefängnisses, Frankfurt a.M.: Suhrkamp.

Fung, Archon/Wright, Erik O. (2001): »Deepening Democracy: Innovations in Empowered Participatory Governance«, in: Politics \& Society 29, S. 5-41. https://doi.org/10.1177/0032329201029001002

Fung, Archon/Wright, Erik O. (2003): Deepening Democracy. Institutional Innovations in Empowered Participatory Governance, The Real Utopias Project IV, London/New York: Verso. 
Giddens, Anthony (1988): Die Konstitution der Gesellschaft. Grundzüge einer Theorie der Strukturation, Frankfurt a.M./New York: campus.

Gohde-Ahrens, Rixa (2013): Partizipation und soziale Inklusion aus der Quartierssicht - ein Blick nach Hamburg, in: eNewsletter Bürgerbeteiligung Nr. 1/2013. Online verfügbar unter: https://www.netzwerkbuergerbeteiligung.de/fileadmin/Inhalte/PDF-Dokumente/newsletter_ beitraege/nwbb_beitrag_gohde_ahrens_130320.pdf. Zuletzt aufgerufen am 20.02.2020.

Gudowsky, Niklas/Peissl, Walter/Sotoudeh, Mahshid/Bechtold, Ulrike (2012): »Forward-looking activities: incorporating citizens' visions«, in: Poiesis \& Praxis 9, S. 101-123. https://doi.org/10.1007/s10202-012-0121-6

Habermas, Jürgen (1991): Erläuterungen zur Diskursethik. Frankfurt a.M.: Suhrkamp.

Habermas, Jürgen (2016): »Für eine demokratische Polarisierung. Wie man dem Rechtspopulismus den Boden entzieht«, in: Blätter für deutsche und internationale Politik 11, S. 35-42. Online verfügbar unter: https://www. blaetter.de/archiv/jahrgaenge/2016/november/fuer-eine-demokratischepolarisierung. Zuletzt aufgerufen am 13.02.2020.

Hassan, Louise M./Shiu, Edward/Shaw, Deirdre (2016): »Who Says There is an Intention-Behaviour Gap? Assessing the Empirical Evidence of an Intention-Behaviour Gap in Ethical Consumption«, in: Journal of Business Ethics 136 (2), S. 219-236. https://doi.org/10.1007/s10551-014-2440-0

Herriger, Norbert (1991): »Empowerment. Annäherungen an ein neues Fortschrittsprogramm der sozialen Arbeit«, in: Neue Praxis 4, S. 221-229.

Herriger, Norbert (2014): Empowerment in der Sozialen Arbeit. Eine Einführung, Stuttgart: Kohlhammer.

Himmelmann, Gerhard (2001): Demokratie-Lernen: als Lebens-, Gesellschafts- und Herrschaftsform. Ein Lehr- und Studienbuch, Schwalbach/Taunus: Wochenschau-Verlag.

Honneth, Axel (1992): Kampf um Anerkennung. Zur moralischen Grammatik sozialer Konflikte, Berlin: Suhrkamp.

Jacobi, Anders/Andersen, Ida/Rask, Mikko/Lanckriet, Anouk/Cruyce, Els van den/Damme, Lieve van/Warrington, Brian/Damianova, Zoya/Bakonyi, Eszter/Sotoudeh, Mashid/Peissl, Walter (2011): CIVISTI Collaborative project on Blue Sky Research on Emerging Issues Affecting European S\&T. D 3.3 Final Project Report. Online verfügbar unter: www.civisti.org/files/images/Civisti_Final_Report.pdf. Zuletzt aufgerufen am 13.02.2020. 
Jacobs, Lawrence R./Skocpol, Theda (Hg.) (2005): Inequality and American Democracy. What We Know and What We Need to Learn, New York: Russell Sage Foundation.

Jantschek, Ole (2019): »Gemeinsam rätseln und demokratisch entscheiden: Escape Games in der politischen Bildung«, 06.11.2019. Online verfügbar unter: https://www.bpb.de/lernen/digitale-bildung/werkstatt/299699/ gemeinsam-raetseln-und-demokratisch-entscheiden-escape-gamesin-der-politischen-bildung. Zuletzt aufgerufen am 16.01.2020.

Kersting, Norbert (2008): Innovative Partizipation: Legitimation, Machtkontrolle und Transformation. Eine Einführung, in: Norbert Kersting (Hg.), Politische Beteiligung, Wiesbaden: VS Verlag für Sozialwissenschaften, S. 11-39. https://doi.org/10.1007/978-3-531-91071-0_1

Keupp, Heiner (1992): »Gesundheitsförderung und psychische Gesundheit. Lebenssouveränität und Empowerment.«, in: Psychomed 4, S. 244-250.

Klatt, Johanna/Walter, Franz (2011): Entbehrliche der Bürgergesellschaft? Sozial Benachteiligte und Engagement, Bielefeld: transcript. https://oi. org/10.14361/transcript.9783839417898

Leggewie, Claus/Schneider, Jan (2009): »Demokratie in Gefahr? Wie man Beteiligungskompetenz zurückerobert «, in: Sozialwissenschaftlicher Fachinformationsdienst soFid, Politische Soziologie 2, S. 35-46. Online verfügbar unter: https://www.ssoar.info/ssoar/handle/document/20610. Zuletzt aufgerufen am 20.02.2020.

Leggewie, Claus/Nanz, Patricia (2016): Die Konsultative. Mehr Demokratie durch Bürgerbeteiligung, Berlin: Klaus Wagenbach.

Lietzmann, Hans J. (2016): »Die Demokratisierung der Repräsentation. Dialogische Politik als neue Form der repräsentativen Demokratie«, in: Manuela Glaab (Hg.), Politik mit Bürgern - Politik für Bürger. Praxis und Perspektiven einer neuen Beteiligungskultur, Wiesbaden: Springer VS, S. 4158. https://doi.org/10.1007/978-3-658-12984-2_3

Lietzmann, Hans J. (2017): »Representation in Postnational European Context«, in: Kari Palonen/Claudia Wiesner, Conceptual Changes in European Politics. Essex: ECPR.

Lietzmann, Hans J./Dienel, Hans-Liudger/Vergne, Antoine/Franz, Kerstin/Fuhrmann, Raban (Hg.) (2014): Die Qualität von Bürgerbeteiligungsverfahren - Evaluation und Sicherung von Standards am Beispiel von Planungszellen und Bürgergutachten, München: oekom.

Lietzmann, Hans J./Dankwart-Kammoun, Saskia/Freier, Nora (2017): »Das partizipative Reallabor. Gestalten Bürger ihre Energiewende?«, in: Jörg 
Sommer (Hg.), Kursbuch Bürgerbeteiligung 2, Berlin: Deutsche Umweltstiftung, S. 487-505.

Lindell, Marina/Bächtiger, André/Grönlund, Kimmo/Herne, Kaisa/Setälä, Maija/Wyss, Dominik (2017): What drives the Polarization and Moderation of Opinions? Evidence from a Finnish Citizen Deliberation Experiment on Immigration, in: European Journal of Political Research 56, S. 2345. https://doi.org/10.1111/1475-6765.12162

Lipset, Seymour M. (1960): Political Man. The Social Bases of Politics, New York: Doubleday.

Lüttringhaus, Maria (2000): Partizipation in benachteiligten Stadtteilen: Planung am >bunten Tisch`statt >grünem Tisch««. In: Susanne Elsen/Heinz A. Ries/Nikolas Löns/Hans G. Homfeldt (Hg.), Sozialen Wandel gestalten - Lernen für die Zivilgesellschaft, Neuwied: Hermann Luchterhand, S. 213-229.

Mehr Demokratie (2020), Umfrage Ostdeutschland. Bürgerräte und Volksentscheide wirken der Politikverdrossenheit in Ostdeutschland entgegen. Online verfügbar unter: https://www.buergerrat.de/presse/umfrage-ostdeutschland/. Zuletzt aufgerufen am 28.02.2020.

Müller, Ria/Hildebrand, Jan/Rubik, Frieder/Rode, Diana/Söldner, Sigrid/ Bietz, Sabine (2016): Der Weg zum Klimabürger. Kommunale Unterstützungsmöglichkeiten, Strategien und Methoden. Empfehlungen aus dem Forschungsprojekt Klima-Citoyen. Online verfügbar unter: https:// www.ioew.de/fileadmin/user_upload/BILDER_und_Downloaddateien/ Publikationen/2016/Klima-Citoyen_Wegweiser_Klimabuerger.pdf. Z Zuletzt aufgerufen am 20.02.2020.

Nowotny, Helga (1999): Es ist so. Es könnte auch anders sein, Frankfurt a.M.: Suhrkamp.

Nowotny, Helga/Scott, Peter/Gibbons, Michael (2004): Wissenschaft neu denken. Wissen und Öffentlichkeit in einem Zeitalter der Ungewißheit, Weilerswist: Velbrück.

Open Knowledge Foundation Deutschland e.V. (2018): Auf Mikas Spuren. Online verfügbar unter: https://demokratielabore.de/workshops/auf-mikasspuren/. Zuletzt aufgerufen am 16.01.2020.

Pateman, Carole (1970): Participation and Democratic Theory, Cambridge: Cambridge University Press. https://doi.org/10.1017/CBO9780511720444

Parodi, Oiver/Albiez, Marius/Beecroft, Richard/Meyer-Soylu, Sarah/Quint, Alexandra/Seebacher, Andreas/Trenks, Helena/Waitz, Colette (2016): »Das 
Konzept »Reallabor« schärfen. Ein Zwischenruf des Reallabor 131: KIT findet Stadt«, in: GAIA 25 (4), S. 284-285. https://doi.org/10.14512/gaia.25.4.11 Radke, Jörg/Kerstin, Norbert (Hg.) (2018): Energietransformation: Energiewende. Politikwissenschaftliche Perspektiven, Wiesbaden: Springer Fachmedien Wiesbaden.

Reinert, Adrian/Sinnig, Heidi (1997): »Mobilisierung der Kompetenz der Bürgerinnen und Bürger. Das Bürgergutachten ÜSTRA zum öffentlichen Nahverkehr in Hannover«, in: Theo Bühler (Hg.): Bürgerbeteiligung und Demokratie vor Ort, Bonn: Stiftung Mitarbeit, S. 143-157.

Reybrouck, David van (2016): Gegen Wahlen. Warum Abstimmen nicht demokratisch ist, Göttingen: Wallstein.

Rohr, Jascha/Ehlert, Hanna/Möller, Benjamin/Hörster, Sonja/Hoppe, Marie (2017): Impulse zur Bürgerbeteiligung vor allem unter Inklusionsaspekten - empirische Befragungen, dialogische Auswertungen, Synthese praxistauglicher Empfehlungen zu Beteiligungsprozessen, Forschungsbericht; herausgegeben im Auftrag des Umweltbundesamts. Online verfügbar unter: https://www.umweltbundesamt.de/sites/default/files/ medien/1410/publikationen/2017-05-08_texte_36-2017_impulse-buergerbeteiligung_0.pdf Zuletzt aufgerufen am 16.01.2020.

Rosanvallon, Pierre (2018): Die gute Regierung, Berlin: Suhrkamp.

Sarcinelli, Ulrich (2011): Politische Kommunikation in Deutschland. Medien und Politikvermittlung im demokratischen System, Heidelberg: Springer VS.

Schäfer, Armin/Schoen, Harald (2013): »Mehr Demokratie, aber nur für wenige? Der Zielkonflikt zwischen mehr Beteiligung und politischer Gleichheit.«, in: Leviathan 41(1), S. 94-120. https://doi.org/10.5771/0340-04252013-1-94

Schmidt, Manfred G. (2019): »Beteiligungsfreundliche Demokratietheorie: Partizipation und Deliberation.«, in: Manfred G. Schmidt, Demokratietheorien. Eine Einführung, Wiesbaden: Springer VS, S. 227-244. https:// doi.org/10.1007/978-3-658-25839-9_15

Schneidewind, Uwe/Singer-Brodowski, Mandy (2014): Transformative Wissenschaft. Klimawandel im deutschen Wissenschafts- und Hochschulsystem, Marburg: Metropolis-Verlag.

Schüwer, Dietmar/Warburg, Annalena/Buchholz, Martin/Freier, Anna Nora/ Jentsch, Andrej/Pauschinger, Thomas/Verheyen, Othmar/Witte-Humperdinck, Nicolas (2020): LowEx Herten: Innovative interkommunale Wärmeversorgung für die >Neue Zeche Westerholt< in Herten/Gelsenkir- 
chen, Wuppertal: Wuppertal Institut für Klima, Umwelt, Energie. Online verfügbar unter: https://epub.wupperinst.org/files/7461/7461_LowEx_ Herten.pdf. Zuletzt aufgerufen am 15.02.2020.

Schweizer-Ries, Petra (2008): „Umweltpsychologie«, in: Karin Sternberg/Manfred Amelang (Hg.), Psychologen im Beruf. Anforderungen, Chancen und Perspektiven, Stuttgart: Kohlhammer, S. 282-298.

Schweizer-Ries, Petra (2013): »Akzeptanz für Klimaschutzmaßnahmen - ein Rahmenmodell für den kommunalen Klimaschutz«, in: Petra SchweizerRies/Jan Hildebrand/Irina Rau (Hg.), Klimaschutz \& Energienachhaltigkeit: Die Energiewende als sozialwissenschaftliche Herausforderung, Saarbrücken: Universaar, S. 19-37.

Stark, Wolfgang (1996): Empowerment. Neue Handlungsstrategien in der psychosozialen Praxis, Freiburg i.Br.: Lambertus.

Steinbrecher, Markus (2009): Politische Partizipation in Deutschland, BadenBaden: Nomos. https://doi.org/10.5771/9783845212838

Suiter, Jane/Farrell, David M./O'Malley, Eoin (2016): »When do deliberative citizens change their opinions? Evidence from an Irish citizens' assembly«, in: International Political Science Review 37 (2). https://doi.org/10. 1177/0192512114544068

Ukowitz, Martina (2017): Transdisziplinäre Forschung in Reallaboren. Ein Plädoyer für Einheit in der Vielfalt, in: GAIA 26 (1), S. 9-12. https://doi.org/10. 14512/gaia.26.1.4

Verba, Sidney/Schlozman, Kay L./Brady, Henry E. (1995): Voice and Equality. Civic Voluntarism in American Politics, Cambridge: Harvard University Press.

Villar Lama, Arsenio (2018): »Millennial Leisure and Toursim: The Rise of Escape Rooms «, in: Cuadernos de Turismo 41, S. 743-746. Online verfügbar unter: https://digitum.um.es/xmlui/bitstream/10201/58487/2/3271811123751-1-PB.pdf. Zuletzt aufgerufen am 09.01.2020.

Virtuelles Institut »Transformation - Energiewende NRW« (2019): »Noch zu retten ...? - Ein Do-It-Yourself Escape Room zum Thema Nachhaltigkeit im Alltag, Essen. Online verfügbar unter: https://www.vitransformation.de/escaperoom/. Zuletzt aufgerufen am 25.02.2020.

Weber, Joachim (2009): »Begeisterung für die Macht als politische Grundhaltung. Ein Gegenentwurf zur deutschen Rezeption von Empowerment«, in: WIDERSPRÜCHE 30 (112), S. 7-22.

Wildt, Andreas (2007): "Solidarität als Strukturbegriff politisch-sozialer Gerechtigkeit«, in: Jahrbuch für Christliche Sozialwissenschaf- 
ten 48: Solidarität, S. 39-60. Online verfügbar unter: https://www.unimuenster.de/Ejournals/index.php/jcsw/article/view/81. Zuletzt aufgerufen am 20.02.2020.

Wissenschaftlicher Beirat der Bundesregierung für Globale Umweltveränderungen (WBGU) (Hg.) (2011): Welt im Wandel: Gesellschaftsvertrag für eine Große Transformation, Berlin.

Wuppertal Institut (2019): »Reallabore erhöhen Sichtbarkeit - Karlsruher Institut für Technologie, Wuppertal Institut, Leuphana Universität Lüneburg und Ecornet gründen »Netzwerk Reallabore der Nachhaltigkeit«, 11.04.2019. Online verfügbar unter: https://wupperinst.org/a/wi/a/s/ad/ 4706. Zuletzt aufgerufen am 05.02.2020.

Yiğit, Nuran/Can, Halil (2009): »Die Überwindung der Ohn-Macht. Politische Bildungs- und Empowerment-Arbeit gegen Rassismus in People of Color-Räumen - das Beispiel der Projektinitiative HAKRA«, in: Gabi Elverich/Annita Kalpaka/Karin Reindlmeier (Hg.), Spurensicherung. Reflexion von Bildungsarbeit in der Einwanderungsgesellschaft, Frankfurt a.M.: Unrast, S. 167-193. 



\section{Die Energiewende als Werk ausgewählter Gemeinschaften? ${ }^{1}$ Zur sozialen Exklusivität von Energiegenossenschaften}

Emily Drewing und Sabrina Glanz

\section{Energiewende für alle?}

2011 wurde in Deutschland die Energiewende als politisches Programm beschlossen. Essentieller Bestandteil ist - neben der Steigerung der Energieeffizienz etwa durch Sanierungsmaßnahmen und der Optimierung industrieller Prozesse - die Erhöhung des Anteils von Energie aus erneuerbaren Quellen am Gesamtenergieverbrauch. Für den Ausbau der erneuerbaren Energien spielen in Deutschland kleine, private Akteur*innen eine zentrale Rolle: fast die Hälfte der Anlagen zur Erzeugung erneuerbarer Energie befand sich noch vor wenigen Jahren im Besitz von Bürger*innen - zum Beispiel Eigenheimbesitzer*innen mit Photovoltaikanlagen, Landwirt*innen, Bürgerwindparks und Energiegenossenschaften. Nach aktuellen Zahlen halten Bürger*innen, ihre Zusammenschlüsse und Landwirt*innen immerhin noch 42 Prozent der bundesweit installierten Leistung zur erneuerbaren Stromerzeugung in ihrem Besitz (vgl. AEE 2018; Blömer et al. 2015: 3ff.). 2012 befanden sich rund neun Prozent der installierten Leistung erneuerbarer Energien im Besitz von Bürgerenergiegesellschaften (vgl. trend:research/Leuphana Universität Lüneburg 2013: 42) ${ }^{2}$.

Eine frühere Version dieses Aufsatzes wurde im Rahmen des 27. Wissenschaftlichen

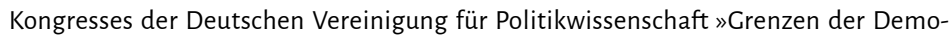
kratie/Frontiers of Democracy « im Panel »Energie! Ökonomische und demokratische Herausforderungen« vorgestellt. Wir danken allen Teilnehmer*innen für den spannenden Austausch und ihre konstruktiven Hinweise.

2 In der Regel werden Daten zur Eigentümerverteilung bei der installierten Leistung erneuerbarer Energien nicht nach Untergruppen der Bürgerenergie (dies sind pri- 
Unter Energiegenossenschaften werden im Folgenden »alle solchen eingetragenen Genossenschaften, deren Hauptzweck darin besteht, Aktivitäten im Energiesektor durchzuführen - ohne Beschränkung auf bestimmte Wertschöpfungsstufen, d.h. entlang der gesamten Wertschöpfungskette« (Holstenkamp 2012: 7) verstanden. Wenngleich sie tatsächlich nur für einen kleinen Anteil der Stromproduktion in Deutschland verantwortlich zeichnen ${ }^{3}$, sind sie seit einigen Jahren nicht nur ein beliebter Forschungsgegenstand, sondern auch fester Bestandteil von Diskursen rund um die Energiewende. Grund für diese Aufmerksamkeit dürfte das bis noch vor wenigen Jahren außerordentlich dynamische Gründungsgeschehen sein. Die Zunahme von Unternehmensgründungen in genossenschaftlicher Rechtsform nach der Novellierung des Genossenschaftsgesetzes 2006 basiert insbesondere auf der vermehrten Entstehung von Energiegenossenschaften (vgl. Poppen 2015: 6).

Energiegenossenschaften werden diskutiert als kollektive Bestrebungen von Bürger*innen, die sich für ein alternatives Energiesystem einsetzen:

»Die Cenossenschaft ist durch ihre Governance nah am Ideal dessen, was man sich gemeinhin unter Bürger-Energiewende vorstellen mag. Die Genossenschaft steht in der öffentlichen Wahrnehmung als Gegenpol zu Investororientierten Firmen, die mit der Energiewende ihren Gewinn maximieren möchten« (Kahla et al. 2017: 24; ähnlich etwa auch Kropp 2017: 309).

Weiterhin gelten sie als Anzeichen eines »Revivals« von Genossenschaften und ihren Werten (im Sinne einer Re-Aneignung öffentlicher Güter durch lokale und regionale Nutzergemeinschaften) sowie vor dem Hintergrund des aktuellen Zinsniveaus auch als attraktive Anlagemöglichkeit, da sich über die Zeichnung von Genossenschaftsanteilen noch vor wenigen Jahren aufgrund der hohen Einspeisevergütung für erneuerbar erzeugten Strom verlässliche Renditen realisieren ließen (vgl. Elsen 2012; kritisch u.a. Chatalova/Valentinov 2014; Martens 2015).

vate Hauseigentümer*innen, Landwirt*innen, Bürgerenergiegesellschaften und rein finanzielle Beteiligungen an Erneuerbare-Energie-Projekten) aufgeschlüsselt; dementsprechend können an dieser Stelle keine aktuelleren Daten aufgeführt werden. Im Jahr 2014 produzierten Energiegenossenschaften 0,58 Prozent des Stroms aus erneuerbaren Energien (der Anteil energiegenossenschaftlich produzierten Stroms am insgesamt in Deutschland produzierten Strom ist naturgemäß noch geringer) (Müller et al. 2015: 98). 
Im öffentlichen Diskurs, aber auch in kritischen (teils populär-)wissenschaftlichen Befassungen werden bestimmte Bevölkerungsgruppen im Kontext der Energiewende als benachteiligt, mitunter gar als »Verlierer*innen« beschrieben (vgl. z.B. Diermann 2019; Frondel/Sommer 2014; Frondel et al. 2012; Kreutzfeldt 2014). Das folgende Zitat fasst die Beobachtungen, die jener Diagnose zugrunde liegen, pointiert zusammen:

»Ärmere Bevölkerungsschichten tragen überproportional die Lasten grüner Stromproduktion [...] und können nicht einmal zum Ausgleich von der Eigenproduktion grüner Energie oder den Chancen grüner Investments profitieren.« (Heindl et al. 2014: 509).

Tatsächlich finanziert sich die Energiewende, die bislang vor allem strombasiert stattfindet, unter anderem über die EEG-Umlage ${ }^{4}$, die einen Bestandteil des Strompreises darstellt. Während energieintensive Unternehmen eine Befreiung von selbiger Umlage beantragen können, um ihre Wettbewerbsfähigkeit nicht zu gefährden, vermögen Privatpersonen ihren Strompreis nur über den Verbrauch zu regulieren. Indes macht der für den konsumierten Strom zu entrichtende Arbeitspreis nur etwa die Hälfte des Gesamtstrompreises für Privathaushalte aus (vgl. Bundesnetzagentur 2020b), sodass sich die finanziell entlastende Wirkung stromsparenden Verbrauchsverhaltens in Grenzen hält. Zudem handelt es sich bei Strom um ein Gut, dass sich schwerlich substituieren lässt. Anbieterwechsel verschaffen nur begrenzt Abhilfe und gerade ärmere Haushalte leben oftmals in Gebäuden von schlechtem Sanierungsstand. Einen Umzug und/oder den Austausch älterer, energieintensiver Elektrogeräte können sie sich mitunter nicht leisten (vgl. Heindl et al. 2014).

Vor dem Hintergrund der immerhin diskursmächtig als »Megatrends« verhandelten Entwicklungen I. des Anstiegs sozio-ökonomischer Disparitäten, II. einer alle Gesellschafts- und Lebensbereiche allmählich durchdringenden Neo-Ökologie sowie III. eines sich stetig vollziehenden Gender Shifts (deren empirische Ausprägungen freilich weit weniger eindeutig sind, als es ihre bezeichnenderweise gleichsam diffusen und eingängigen Charakterisierungen durch Zukunftsinstitut und Z_punkt ${ }^{5}$ suggerieren $^{6}$ ) kommt

4 Zur EEG-Umlage vgl. Bundesnetzagentur (2020a).

5 Die Ausführungen des Zukunftsinstituts finden sich unter https://www.zukunftsinstitut.de/dossier/megatrends/, die der »Foresight Company«Z_punkt unter https://www. z-punkt.de/themen/artikel/megatrends, zuletzt aufgerufen am 21.01.2020.

6 Zur Kritik populärwissenschaftlicher Befassungen mit Zukunft siehe die Aufsätze von Rust, Schweiger/Schecke und Zorn/Schweiger in diesem Band. 
Energiegenossenschaften eine interessante Rolle zu. Erstens werden Energiegenossenschaften als eine Möglichkeit betrachtet, Bevölkerungsgruppen, die bislang nicht zum Kreis derjenigen gehören, die von der Energiewende profitieren, (finanziell) daran zu beteiligen ${ }^{7}$ (vgl. Viardot 2013). Jener Potentialzuschreibung zugrunde liegt eine althergebrachte Vorstellung von Genossenschaften, der zufolge sie (primär) gemeinwohlorientiert agieren und als Zusammenschluss von sozial "Schwachen« denselben $\mathrm{zu}$ gemeinsamer Stärke verhelfen. In dieser Lesart, die aktuell wieder an Popularität gewinnt, sind Genossenschaften geeignet, soziale Ungleichheiten durch gemeinschaftliche Selbsthilfe der Benachteiligten zu verringern (vgl. Elsen/Walk 2016; Klemisch/Maron 2010). Zweitens sei das kollektive Aktivwerden im Bereich der erneuerbaren Energieerzeugung Ausdruck des Bestrebens, die Energiewende ganzheitlich voranzubringen, sowie Anreiz und Mahnung, den eigenen Umgang mit Energie zu reflektieren und darüber zu einem insgesamt nachhaltigeren Lebensstil zu finden (vgl. z.B. Überlegungen bei Leggewie 2013: 19ff.; empirisch Bauwens/Devine-Wright 2018, Bauwens 2016; für eine umfassende Zusammenschau der Debatten zu Demokratie, Engagement/Partizipation und Bürgerenergie vgl. Radtke 2016). Drittens verweisen empirische Befunde auf Gender-Unterschiede sowohl hinsichtlich des (energiewendeassoziierten) Elektrizitätsarmutsrisikos (vgl. Bleckmann et al. 2016; Bontrup/Marquardt 2014) als auch in Bezug auf den individuellen Umgang mit Energiefragen (vgl. z.B. Dunphy et al. 2017; Offenberger 2016; Tjørring 2016) sowie die Beteiligung in Bürgerenergiegesellschaften (Radtke 2016: 297ff., 424ff.).

\section{Bestandsaufnahme: Energiegenossenschaften in Deutschland}

Je nach zugrunde gelegter Definition variiert die Anzahl der Energiegenossenschaften in den Jahren 2013 bis 2017 zwischen 889 und 1.024 (vgl. Debor 2014: 5f.; Kahla et al. 2017: 25; Müller et al. 2015: 99; Poppen 2015: 2ff.).

7 Gleichzeitig wird durchaus anerkannt, dass zur Zeichnung von Anteilen Kapital benötigt wird, über welches Einkommensschwächere nicht notwendigerweise verfügen bzw. das sie zu investieren nicht bereit sind. Folglich gelten einschlägige Beteiligungsmöglichkeiten gemeinhin keineswegs als Allheilmittel im Kontext sozialer Gerechtigkeitsfragen der Energiewende, werden aber wiederholt als ein (potentielles) Instrument aufgebracht, um derlei Ungleichgewichte zu nivellieren (vgl. z.B. Heindl et al. 2014: 509; Kopatz 2013: 54). 
Auf den Internetseiten der »Bundesgeschäftsstelle Energiegenossenschaften« ist aktuell die Rede von 860 Energiegenossenschaften ${ }^{8}$, die beim Deutschen Genossenschafts- und Raiffeisenverband organisiert sind.

Ab 2007 lässt sich ein deutlicher Anstieg von Energiegenossenschaften beobachten, zwischen 2009 und 2013 kann von einem regelrechten "Neugründungsboom« gesprochen werden. Ende 2014 waren etwa 11 Prozent aller in Deutschland eingetragenen Genossenschaften Energiegenossenschaften (Müller/Holstenkamp 2015: 9). Die jährlichen Neueintragungsraten in den Genossenschaftsregistern erreichten ihren Höhepunkt 2011, der Gründungsboom endete etwa Mitte des Jahres 2013 (vgl. Müller/Holstenkamp 2015: 8f.).

Die meisten Energiegenossenschaften verfolgen Aktivitäten im Feld der erneuerbaren Energien. Häufig sind Erzeugung und Verkauf von regenerativ erzeugter Energie. 87 Prozent der produzierenden Energiegenossenschaften setzen dabei auf eine einzige Energiequelle (vgl. Debor 2014: 9ff.). Zumeist handelt es sich um Strom aus Photovoltaikanlagen. Zudem gibt es gerade im ländlichen Raum einige Genossenschaften, die - zuweilen über eigene Netze - durch Biomasse generierte Wärme vertreiben. Demgegenüber stellen etwa der Betrieb von Windenergieanlagen und eigenen Stromnetzen seltenere Geschäftsmodelle dar (vgl. Kahla et al. 2017: 29). Für die Überzahl der Photovoltaik-Genossenschaften werden mehrere Gründe angenommen; der wichtigste besteht darin, dass es sich um ein vergleichsweise einfaches Geschäftsmodell handelt, das bei geringem Risiko Know-how, Kapital und Engagement in vergleichsweise überschaubarem Ausmaß erfordert und nach Anfangsinvestitionen verlässliche (da gesetzlich garantierte) Renditen generiert (vgl. z.B. Debor 2014: 11f.). Für den Gründungsboom waren denn auch vor allem Energiegenossenschaften, deren Geschäftsmodell in der vergüteten Einspeisung von Photovoltaikstrom bestand, verantwortlich ${ }^{9}$. In jüngerer Zeit ist eine Diversifizierung der Geschäftsfelder zu beobachten. Wenngleich

Online verfügbar unter https://www.genossenschaften.de/bundesgesch-ftsstelle-energiegenossenschaften.Zuletzt aufgerufen am 21.01.2020.

9 Diese Strategie trägt heute nicht mehr. Vereinzelt gibt es noch Energiegenossenschaften, die das klassische Einspeisemodell umsetzen. In der Regel besitzen sie ein größeres Portfolio von älteren Anlagen, die noch für einige Jahre die deutlich höheren Einspeisevergütungen, gesetzlich garantiert nach früheren EEG-Versionen, erhalten. So können sie die geringen Erträge der neuen Anlagen mit den deutlich höheren der alten verrechnen und in der Folge dennoch eine für ihre Mitglieder akzeptable Renditenausschüttung realisieren. 
die Gründungsdynamik früherer Jahre zum Erliegen gekommen ist, wächst die Zahl der Energiegenossenschaften weiterhin (vgl. Kahla et al. 2017: 25ff.).

Ergebnisse zu sozio-ökonomischen Merkmalen von Mitgliedern zeigen, dass es bislang eher bestimmte Gruppen - und nicht ein breiter Querschnitt der Bevölkerung - sind, die sich in Energiegenossenschaften beteiligen. So sind etwa 80 Prozent der Mitglieder männlichen Geschlechts und fast 90 Prozent älter als 35 Jahre (knapp 42 Prozent sind älter als 55 Jahre). Gut die Hälfte der Mitglieder verfügt über einen Universitätsabschluss und nur 11 Prozent verdienen weniger als 1.500 Euro im Monat - über 70 Prozent verfügen hingegen über ein monatliches Bruttoeinkommen von über 2.500 Euro (vgl. Yildiz et al. 2015: 64).

\section{Forschungsinteresse und Vorgehen}

Betrachtet man einerseits die Potentiale, die Energiegenossenschaften als »Vehikel par excellence für die Bürgerbeteiligung an der Energiewende« (Müller et al. 2015: 97) zugeschrieben werden, und andererseits den aktuellen Forschungsstand insbesondere zu (sozio-ökonomischen) Merkmalen der Mitglieder, lässt sich eine Diskrepanz feststellen. Grundsätzlich ist die Eignung von Energiegenossenschaften, (mehr) Menschen (finanziell) an der Energiewende zu beteiligen, indes nicht von der Hand zu weisen. Vor diesem Hintergrund widmet sich der vorliegende Beitrag der Suche nach möglichen Gründen für die verstärkte Beteiligung bestimmter Bevölkerungsgruppen.

Im Ruhrgebiet, das als ehemalige Bergbauregion und Sitz großer Energieunternehmen einen besonderen historischen Bezug zu Energiethemen und damit assoziierten strukturellen Wandelsprozessen aufweist, konnten Vertreter*innen ${ }^{10}$ von 15 der dort ansässigen Energiegenossenschaften sowie Perso-

10 Befragt wurden Personen, die in der jeweiligen Energiegenossenschaft zum Interviewzeitpunkt eine leitende Position innehatten und/oder maßgeblich an der Cründung beteiligt waren. Wenn möglich, wurden mehrere Vertreter*innen derselben Energiegenossenschaft (einzeln) interviewt. Da die Befragten ihre Funktion in der Energiegenossenschaft ehrenamtlich ausübten und sich die Gewinnung von Interviewpartner*innen grundsätzlich aufwändig und langwierig gestaltete, konnte jedoch meist nur eine Person pro Energiegenossenschaft interviewt werden. 
nen, die eine Energiegenossenschaft gründen möchten, für teilstandardisierte Interviews gewonnen werden. ${ }^{11}$

Die Interviews wurden aufgezeichnet und transkribiert. Die Auswertung erfolgte gemäß der Methode des »thematischen Codierens« nach Hopf (vgl. Hopf/Schmidt 1993). Das Verfahren eignet sich zur Überprüfung bestehender Theorien und Vorannahmen ebenso wie zur Erweiterung dieser anhand von Erkenntnissen aus der Analyse von Einzelfällen. Es wird also nicht jegliches Vorwissen zurückgestellt; gleichzeitig ermöglicht die Offenheit des Verfahrens den Gewinn neuer Erkenntnisse. Die Auswertungskategorien ergaben sich im Wesentlichen aus dem Leitfaden sowie aus Theorie und Forschungsstand; mitunter auch aus dem Material selbst, anhand dessen sie stetig überprüft und bei Bedarf modifiziert wurden (vgl. Kuckartz 2010: 85ff.).

\section{Intersektionalität und die Theorie der Gendered Organizations}

Um das Inklusivitätspotential von Energiegenossenschaften in einer diversen Gesellschaft zu untersuchen, bietet sich das Konzept der Intersektionalität an. Geprägt durch die amerikanische Juristin Kimberlé Crenshaw (1989) mit der Forderung, Geschlecht nicht isoliert, sondern in Verflechtung mit class und race $\mathrm{zu}$ betrachten, hat die »Intersektionalitätsdebatte« innerhalb der Sozialstrukturtheorie seit den 1990ern einen innovativen Beitrag geleistet die Entwicklung einer neuen theoretischen Konzeption multipler Benachteiligungen. Im Zentrum intersektionaler Untersuchungen steht die Überlagerung und Kreuzung von auf sozialstrukturelle Merkmale zurückzuführenden Effekten (vgl. Großmann 2017: 62; Lenz 2010: 158f.). Dieser Ansatz unterscheidet sich von bisherigen Perspektiven auf soziale Ungleichheit, in denen durch die einfache Addition sozialstruktureller Merkmale - wie Ethnizität, Bildung, Einkommen und Geschlecht - die Interaktionen zwischen den Merkmalen unerkannt blieben. Der Ansatz ermöglicht die Berücksichtigung von Heterogenitäten innerhalb von Gruppen sowie Homogenitäten zwischen Gruppen. Dabei besteht der Anspruch, soziale Kategorien nicht zu essentialisieren und festzusetzen, sondern als dynamisch und kontextabhängig zu betrachten (vgl. Eberherr 2014: 380). 
Mechanismen der Exklusion, die in Energiegenossenschaften als Organisationen wirken, lassen sich mithilfe der Theorie der Gendered Organizations (vgl. Acker 1990) in den Blick nehmen. Mit Gender als zentraler Analysekategorie wird, in Abgrenzung zu anderen Ansätzen der Organisationsforschung, angenommen, dass Organisationen nicht (gender-)neutral sind, sondern soziale Wirklichkeit und gesellschaftliche Machtverhältnisse repräsentieren. Durch häufig unsichtbare organisationale Prozesse und Strukturen ist Gender in die organisationale Substruktur eingebettet und wird dort reproduziert (vgl. ebd.: 146ff.). In der Weiterentwicklung der Theorie integriert Acker den intersektionalen Ansatz durch die Erweiterung der Analyse von gender regimes um inequality regimes (vgl. Acker 2012: 219).

Die organisationale Substruktur wird durch die Einteilung in vier analytische Kategorien präzisiert (vgl. Acker 1990; Acker 2012):

1 In Organisationsprozessen und -strukturen - gemeint ist vor allem die Arbeitsteilung, spiegelt sich die gesellschaftliche Einteilung in bezahlte und unbezahlte (Care) Arbeit wider. Dementsprechend sind in Führungsund Entscheidungspositionen überwiegend Männer vertreten, während sich Frauen vor allem auf den unteren Ebenen von Organisationen finden.

2 In der Organisationskultur ist Geschlecht in Symbolen, Bildern und Werten verankert - so zum Beispiel in Vorstellungen über Unterschiede zwischen den Geschlechtern, über die eine je nach Geschlecht unterschiedliche Definition von angemessenem Verhalten oder, allgemeiner, über die Bedeutung von (Un-)Gleichheit.

3 Interaktionen in der Organisation werden sowohl auf formeller wie auf informeller Ebene von Gender beeinflusst. So schlagen sich z.B. stereotype Vorstellungen von Geschlechterrollen in Umgangsformen und Kommunikationsweisen nieder.

4 Geschlechtsidentitäten führen auf der Subjektebene zu bestimmten Vorstellungen und Verhaltensweisen der Organisationsmitglieder.

Durch Verknüpfung des Intersektionalitätsansatzes mit der Theorie der Gendered Organizations lassen sich Energiegenossenschaften als Organisationen aus einer intersektionalen Perspektive in den Blick nehmen. Dies bietet die Möglichkeit, die Interaktionsmechanismen ungleichheitsrelevanter Kategorien in Organisationen zu untersuchen. Die Kategorien werden im vorliegenden Beitrag in der Annahme, dass sie sozial konstruiert und nicht aus sich selbst heraus relevant sind, angewendet. Dementsprechend sind sie über- 
wiegend empirisch induktiv-bestimmt, wobei sich Gender, Migration, Alter, finanzieller Hintergrund und Bildungshintergrund als die zentralen Kategorien herausstellten.

\section{Ergebnisse}

\section{Mitgliederstrukturen der untersuchten Energiegenossenschaften}

Im Rahmen der vorliegenden Arbeit wurden keine statistischen Daten zur Mitgliederstruktur erhoben (zu den methodischen Fallstricken solcher Erhebungen vgl. Poppen 2015). Die Befragten wurden im Rahmen der Interviews auf die soziostrukturelle Zusammensetzung der Mitglieder ihrer Energiegenossenschaft angesprochen. Nach Einschätzung der meisten Befragten ${ }^{12}$ zeichnen sich die sozio-ökonomischen Mitgliederstrukturen durch einen hohen Anteil an Älteren, Höhergebildeten (oft Akademiker*innen) und Besserverdienenden aus. Zudem sind häufig Personen, die eher »technischen« Professionen nachgehen, Selbstständige und Landwirt*innen vertreten. Dieses Muster zeigte sich sogar in einer Energiegenossenschaft, die explizit mit dem Ziel gegründet worden war, Menschen ohne eigene (Dach-)Flächen und/oder hohes, frei verfügbares Vermögen die (finanzielle) Teilhabe an der Energiewende zu ermöglichen:

»da ging es ja um Bürgerengagement für eine nachhaltige Energieversorgung auch derer, die im Prinzip kein eigenes Dach haben, weil sie zur Miete wohnen [...] Das war also Grundidee, auch Teilhabe zu ermöglichen [...] Aber wir haben relativ wenig, die, also das quasi als Motivation haben. [...] der ganz überwiegende Teil [.] wohnt in selbstgenutzten Immobilien, die auch Eigentum sind.

I: Haben die zum Teil selber auch vielleicht eine PV-Anlage?

-Ja, ja, klar.«

Aus einer Mehrzahl der Energiegenossenschaften wurde von einem überwiegenden Männeranteil berichtet, wenngleich dies nicht bei allen der Fall war. Ein höherer Anteil an weiblichen Mitgliedern bestand nach Einschätzung der

12 Die folgenden Ergebnisse basieren auf der Auswertung von Interviews mit 19 Befragten. 16 waren männlichen, 3 weiblichen Geschlechts. 
Interviewten zum Befragungszeitpunkt indes in keiner der untersuchten Energiegenossenschaften, auch die befragten »Gründungswilligen« waren ausschließlich männlichen Geschlechts. Im Gespräch konnte keine*r der Interviewten von mindestens einem Mitglied mit Migrationshintergrund in der eigenen Genossenschaft berichten. Auch jüngere Mitglieder kommen nur in wenigen Energiegenossenschaften vor. Häufig war eher die Rede von fehlendem »Nachwuchs«, der mit Blick auf die Überalterung der Mitglieder und auch der Führungsriegen durchaus problematisiert wurde.

\section{Energiegenossenschaften als Gendered Organizations}

Analytisch wird im Folgenden häufig zwischen Energiegenossenschaften, die von anderen Organisationen gegründet wurden ${ }^{13}$, und Energiegenossenschaften, die aus Bürger"inneninitiativen hervorgegangen sind, unterschieden, da sich im Zuge der Auswertung herausstellte, dass sich die Prozesse, Organisationskulturen, Interaktionen und Identitäten zwischen diesen beiden Genossenschaftstypen meist grundlegend unterscheiden.

\section{Organisationsprozesse und -strukturen}

In Energiegenossenschaften, die von anderen Organisationen gegründet wurden, werden Vorstand und Aufsichtsrat in der Regel mit Führungspersonal aus eben diesen Organisationen besetzt. Mehr noch: oft sind die Posten in der Energiegenossenschaft (informell) an eine bestimmte Funktion in den an der Gründung beteiligten Unternehmen und/oder Institutionen gebunden. Im Falle einer Beförderung oder des Ausscheidens aus einer der gründenden Organisationen wird die entsprechende genossenschaftliche Führungsposition mit dem/der Nachfolger*in in der Organisation besetzt. So kommt es zwar in mehrjährigen Abständen zu Wechseln in der energiegenossenschaftlichen Führung; jedoch nicht in Folge ergebnisoffener Wahlen im Zuge der jährlichen Mitgliederversammlungen. Entsprechend nehmen insbesondere diejenigen Funktionär*innen, die mit der operativen Geschäftsführung der Energiegenossenschaft betraut und meist dem mittleren Management in einer der an der Gründung beteiligten Organisationen

13 Oft werden Energiegenossenschaften durch Cenossenschaftsbanken, Kommunen und (kommunale) Energieversorgungsunternehmen gegründet, wobei es vielfach zu Kooperationen mehrerer Organisationen kommt. Dieser Typus findet sich insbesondere in ländlicheren Räumen. 
zuzuordnen sind, ihre damit assoziierten Aufgaben als Bestandteil ihrer Tätigkeit in einer der beteiligten Organisationen wahr: »also Motiv [für die Leitung der Energiegenossenschaft] ist, dass ich sozusagen meinem Arbeitgeber helfe, dieses Instrument Genossenschaft als Kundenbindung auch erfolgreich darzustellen." Zwar beschreiben sie ihre Arbeit für die Energiegenossenschaft als ehrenamtlich, erledigen diese jedoch zumeist innerhalb ihrer bezahlten Arbeitszeit und unter Nutzung von Infrastrukturen und Netzwerken des Unternehmens oder der Institution, bei dem/der sie angestellt sind. Die energiegenossenschaftlichen Führungsriegen werden bis auf wenige Ausnahmen durch Männer besetzt - vermutlich, da Frauen in Führungspositionen und technischen Berufsfeldern (etwa auch im Energiesektor) unterrepräsentiert sind (vgl. IRENA 2019: 29f.).

Die Mitgliedschaft in diesen Genossenschaften wird oft zunächst Kund*innen und Mitarbeiter*innen der an der Gründung beteiligten Organisationen angeboten. Zur Mitgliederwerbung werden interne Verteiler und Informationsmaterialien, die in Filialen ausgelegt werden, sowie aktive Ansprache potentieller Mitglieder etwa im Zuge von Kundengesprächen genutzt. Weiterhin wird in der Regel in der (lokalen) Presse über die Energiegenossenschaft berichtet, wodurch weitere mögliche Interessent*innen erreicht werden können. Da mehrere der untersuchten Energiegenossenschaften unter Mitwirkung von Energieversorgungsunternehmen gegründet wurden, erklärt sich die Überrepräsentation männlicher Mitglieder möglicherweise zum Teil durch den geringen Frauenanteil in technischen Berufen.

Weiterhin gibt es »bottom-up«-initiierte Energiegenossenschaften, die durch kleine Gruppen (in der Regel bestehend aus drei bis sechs Personen) unter erheblichem Zeitaufwand gegründet werden. In diesen »Gründungszirkeln«, die sehr intensiv zusammenarbeiten, kommen Frauen nur vereinzelt vor. Da die Gründer*innen in erheblichem Umfang unbezahlte Arbeit in die Gründung der Energiegenossenschaft investieren, bilden sie nach Abschluss des Gründungsverfahrens in der Regel die genossenschaftliche Führung. Ihr zeitintensives Engagement für die Energiegenossenschaft bringt ihnen hohes Ansehen unter den Mitgliedern ein; oft werden sie als unentbehrlich für das Ent- und Bestehen der Genossenschaft wahrgenommen. Entsprechend kommt es kaum zu Wechseln an der energiegenossenschaftlichen Spitze.

Weitere Mitstreiter*innen werden in der Regel durch persönliche Ansprache von Familienmitgliedern sowie von Bekannten und Freund*innen der Gründer*innen gewonnen. Dabei erfolgt durchaus eine Selektion besonders geeigneter Personen, wie das folgende Zitat verdeutlicht: 
»also ich würde jetzt beispielsweise nicht meinen Nachbarn [...] ansprechen [...] mit dem mich vielleicht irgendwie so unsere Freude am Fußball verbindet, weil ich glaube, der würde da, in so eine Ceschichte nicht [...] so reinpassen«.

In den meisten Energiegenossenschaften beiden Typs kommt es - oft schon wenige Wochen nach erfolgter Gründung - zu einem Aufnahmestopp für neue Mitglieder. In der Regel wurden zu diesem Zeitpunkt bereits genügend Mitglieder gefunden, um den Eigenkapitalbedarf für anvisierte Projekte $\mathrm{zu}$ decken. ${ }^{14}$

\section{Organisationskultur}

Genossenschaften, die von anderen Organisationen gegründet werden, bleiben jenen über ihr Führungspersonal eng verbunden. Vor diesem Hintergrund verwundert es nicht, dass sie nach herkömmlichen unternehmerischen Logiken »rational«, profitorientiert und risikominimierend geführt werden. Da die leitenden Positionen mit Personen besetzt sind, die sich für ihre Entscheidungen in Bezug auf die Energiegenossenschaft nicht nur auf der jährlichen Mitgliederversammlung, sondern in erster Linie gegenüber den involvierten Organisationen (ihren Arbeitgebern) rechtfertigen und sowohl ihr eigenes als auch das gute Ansehen mindestens zweier Organisationen (nämlich der gründenden Organisation und der Energiegenossenschaft) vor Ort sichern müssen, sind innovative (und damit potentiell risikobehaftete) Projekte oder auch nur Schritte, die die Höhe der jährlichen Renditeausschüttung an die Mitglieder vermindern könnten, selten zu beobachten. Strategien, die Energiegenossenschaft für weitere Mitglieder zu öffnen oder sogar eine Diversifizierung der Mitglieder zu fördern, spielen in diesen Genossenschaften kaum eine Rolle. Ihre Organisationskultur wird stark geprägt durch die enge Anbindung an mindestens eine der gründenden Organisationen. Jenen gelten sie eher als Mittel zum Zweck, um etwa durch eine attraktive Anlageoption mit ideellem Mehrwert die Kundenbindung an eines der involvierten Unternehmen zu fördern oder die gelingende Zusammenarbeit der beteiligten Organisationen zu symbolisieren. Die interviewten Führungskräfte be-

14 Da viele Energiegenossenschaften vor den Absenkungen der Einspeisevergütungen für Solarstrom, die im Zuge mehrerer Novellierungen des EEG erfolgten, gegründet wurden, taugt ihr bewährtes Ceschäftsmodell nicht für weitere Projekte. In der Folge werden in vielen Energiegenossenschaften keine neuen Projekte realisiert-und somit auch keine weiteren Mitglieder aufgenommen. 
schreiben diese eher instrumentelle Sicht von Energiegenossenschaften weitgehend offen und wertfrei. Mehrere Interviewpartner*innen erklärten, dass ihre Energiegenossenschaft dem oft von Energiegenossenschaften gezeichneten Bild als »Bürgerorganisationen« ein Stück weit widerspricht. Gefragt nach der Rolle von Energiegenossenschaften im Kontext der Energiewende, erklärte eine Befragte:

»Letztendlich treffen für mich in der Energiegenossenschaft zwei Aspekte aufeinander, einmal der Klimaschutz mit der Möglichkeit viele Menschen zu beteiligen und ein Sprachrohr zu sein und die andere Seite sind Renditegesichtspunkte oder Profit [...] da muss man vielleicht auch gucken welche Energiegenossenschaft, es gibt da ja viele Varianten. Die Form, die wir hier haben, die ist aus meiner Sicht in Klammern leider kein Treiber [der Energiewende], wenn man ganz ehrlich ist«

Nur eine Minderheit der Führungspositionen in Energiegenossenschaften, die durch andere Organisationen gegründet werden, wird von Frauen besetzt. Entsprechend kann keine Aussage darüber getroffen werden, inwieweit die beobachteten Unterschiede in der Motivation und im Führungsverhalten auf das soziale Geschlecht zurückzuführen sind (wenngleich eine Befragte sie darauf attribuiert, s. das nächstfolgende Zitat). Bei den betreffenden weiblichen Interviewten entstand indes ob des Umfangs, den sie diesen Themen im Gespräch selbst einräumten, und den von ihnen geäußerten Einstellungen der Eindruck, dass sie dem Klima- und Umweltschutz mehr Relevanz zuschreiben als die männlichen Befragten ${ }^{15}$. Mehr noch: sie bemühten sich, diese Werte in ihrer täglichen Arbeit und auch im Rahmen ihrer Rolle in der Energiegenossenschaft umzusetzen. Dies stand nicht immer im Einklang mit den unmittelbaren (Rendite-)Interessen der sie beschäftigenden Organisationen, sodass sie mitunter eigene Strategien entwickelten - etwa um die betreffenden Themen in der sie beschäftigenden Organisation auf die Agenda zu setzen.

»Wenn Sie gerade jetzt im Klimaschutz in einer Organisation arbeiten, dann haben Sie die Möglichkeit, die Organisation oder die Wirtschaft ein Stück

15 Diese ob der geringen Fallzahl eher als anekdotisch einzustufende Beobachtung wird durch Ergebnisse einer quantitativen Befragung mehrerer tausend Mitglieder von Bürgerenergiegesellschaften in Deutschland gestützt: »Für Frauen ist der RenditeGesichtspunkt offensichtlich unwichtig, die Zustimmungswerte bei ökologischer Motivation sind entsprechend sehr viel höher als bei Männern.«(Radtke 2016: 314) 
weit voranzubringen. [...] Klimaschutz müsste uns ja alle angehen. [...] Ich muss das schon sehr vorsichtig machen, sodass es quasi nicht so auffällt oder dass es immer auch die Interessen des anderen berührt. [...] Das kann ich besser als wie so'n U-Boot, ja, als eine ältere Frau mit lange Jahre Erfahrung«

In »bottom-up«-initiierten Energiegenossenschaften bestimmen die Einstellungen der Gründer*innen die Organisationskultur. Sie sehen die Energiegenossenschaft oftmals nicht nur als einen Weg, die Energiewende voranzubringen, sondern als Mittel, um »die Gesellschaft« im Ganzen zu verbessern.

»ich sehe also [...] wenn die Mütter ihre Kinder abholen, dann wollen die Kinder den Müttern was erzählen und die Mutter läuft da neben dem Kind und guckt nur noch auf ihr Handy. Und das ist Standard. [...] Diese Individualisierung der Gesellschaft hat extrem durch die Nutzung solcher Medien zugenommen und zwar explosionsartig, dass wir kaum hinterherkommen. [...] Aber was verloren geht, ist [.] klare Regeln und Erziehung für Kinder. Und wie soll das dann vernünftig weitergehen, wenn da was fehlt? Also die Schulen tun einen großen Anteil daran, das zu machen. Sie haben mich gefragt, warum, ob wir, was wir überhaupt wollen als Bürgerenergiegenossenschaft? I: Was ist die Rolle in diesem-

Was ist die Rolle? Die Rolle ist einfach diese Keimzelle, also lokal die Menschen dort abzuholen, wo sie jetzt gerade sind.«

Die Normativität ihrer Werthaltungen dient der Abgrenzung der Genossenschaft gegenüber Personen(-gruppen) und auch Organisationen, die ihren Standards nicht genügen. Mitunter erwecken sie den Eindruck, sich als (moralisch) überlegen (und damit als prädestiniert, andere $\mathrm{zu}$ erziehen und $\mathrm{zu}$ belehren) wahrzunehmen:

»ich glaube, meine Einschätzung liegt in vielen Dingen schon sehr richtig [...] gerade in dem Bereich was unsere Cesellschaft angeht, darum auch meine Kritik daran [...] ich wage mich auch in Bereiche, wo ich nur noch aus Lebenserfahrung spreche, aber nicht unbedingt wissenschaftlich fundiert das nachgearbeitet oder vorgearbeitet habe. Aber dadurch, dass ich ja schon ein bisschen Lebenserfahrung habe, kann ich das auch sehr locker machen [...] da fehlt ein Erklärbär, der mal sagt, was da eigentlich los ist«

Ohne, dass dem stets eine solche Intention zugrunde läge, wirken diese Sichtweise und das oft fehlende Verständnis für Menschen in anderen Lebenssituationen mitunter exklusiv, wenn etwa die für die Mitgliedschaft notwen- 
digerweise zu zeichnenden Genossenschaftsanteile vergleichsweise hoch angesetzt werden, weil angenommen wird, nur dann ließen sich Wertekongruenz, eine hohe Identifikation der Mitglieder mit der Energiegenossenschaft und eine entsprechende Bereitschaft, sich darin $\mathrm{zu}$ engagieren, sicherstellen: "Wenn Sie überlegen, dass so ein scheiß Golf vernünftig ausgestattet, schon fast 30.000 Euro kostet [...] dann sind 10.000 Euro für eine Bürgerenergiegenossenschaft nicht viel«. Man sucht Mitglieder, »die Gemeinwohl denken« und »so eine gesunde Kapitaldecke haben für sich, dass sie sagen >ich kann da ohne Not mitmachen««. Der Anspruch, breite Bevölkerungsschichten an der Energiewende zu beteiligen, besteht auch bei diesen »bottom-up«Energiegenossenschaften oft nicht: »Wir wollen jetzt auch nicht unbedingt eine Massengenossenschaft sein.«

\section{Interaktionen in der Organisation}

Mit Blick auf die Interaktionen in den Energiegenossenschaften ist vor allem die Art des Kontaktknüpfens - die Form des Netzwerkens - relevant. Dies gilt sowohl für den Gründungsprozess als auch für die Mitgliederrekrutierung. So spielt für beide Formen die Gewinnung von Mitgliedern aus der Familie eine wichtige Rolle. Gerade Ehepaare treten einer Energiegenossenschaft oft gemeinsam bei, wobei die Initiative in der Regel vom Ehemann ausgeht. Vereinzelt wurde von jüngeren Mitgliedern berichtet, die ihre Anteile meist von ihren Eltern oder Großeltern geschenkt bekommen hatten und mit Erreichen des (jungen) Erwachsenenalters beginnen, sich für die Energiegenossenschaft zu interessieren. Eine befragte Vorstandsvorsitzende beschrieb die Mitgliederstruktur ihrer Genossenschaft folgendermaßen:

»Ich würde die vom Milieu her auch [.] Mittelschicht, dieses [.] es gibt ja diese Milieus, Sinus-Milieus und da ist eben so diese gehobene Mittelschicht. Und die Jüngeren kommen schon aus denen, die da so die nachhaltigen Crundwerte haben, die anderen sind auch mit ihren Dingen beschäftigt [...] also so

[...] diese Mischung aus grün-schwarz, die stimmt schon.«

Aus »bottom-up«-Genossenschaften wurde oft berichtet, dass sich die Initiator*innen vor ihrer Zusammenarbeit im (kleinen) Gründungskreis bereits bekannt waren. Sofern es sich nicht um langjährige Freundschaften handelte, kannten sie sich von Demonstrationen und anderen klimaschutzassoziierten Veranstaltungen sowie über gemeinsame Kontakte. Wiederholt war die Rede von einer "Szene«, aus der Energiegenossenschaften Mitstreiter*innen gewinnen. 
Die Gründung von Energiegenossenschaften durch andere Organisationen kam oft über Kontakte zwischen den Führungsriegen der involvierten Institutionen und Unternehmen zustande (»dann haben wir den Bürgermeister kontaktiert, dann sind wir dem Kollegen, sind wir dort [zum Besuch einer anderen Energiegenossenschaft hin] gefahren, haben uns dort informiert und dann kam so Anfang 2009: >so, das machen wir«!«). Durch informelle Verabredungen wurden in vielen Fällen schon vor der Gründung gemeinsame Projekte geplant, persönliche Bekanntschaften stellten das nötige Vertrauen sicher. Soweit dies aus dem erhobenen Material und den öffentlich zugänglichen Informationen ersichtlich ist, handelte es sich bei den Involvierten zumeist um Männer, was wir zum einen auf die geringe Anzahl an Frauen in leitenden Positionen, zum anderen auf das männlich konnotierte Themenfeld »Energie« zurückführen.

Die zentrale Rolle sozialer Netzwerke - ob beruflicher oder persönlicher Art - stellt ein exklusives Moment für Gruppen dar, die aus verschiedenen Gründen nicht Teil dieser Netzwerke sind. Auch eine Mitgliedschaft birgt jedoch, anders als möglicherweise ob des für die genossenschaftliche Rechtsform so kennzeichnenden Demokratieprinzips zu erwarten wäre, häufig nur wenige Möglichkeiten der Mitgestaltung. In Genossenschaften, die von anderen Organisationen gegründet wurden, haben die jährlichen Mitgliederversammlungen eine primär legitimierende Funktion. Vereinzelt kommt es zu Anregungen aus der Mitgliederschaft, die, sofern sie dem Professionalitätsanspruch des Vorstands genügen, geprüft werden; jedoch entscheiden die Mitglieder in der Regel nicht über die nächsten Schritte der Energiegenossenschaft. Auf die Frage, ob er sich die Zusammenarbeit mit Arbeitsgruppen aus der Mitgliederschaft vorstellen könne, entgegnete ein Interviewter:

»ich stell' es mir auch teilweise schwierig vor, ja, das dann so professionell zu managen, dass man diese Arbeitsgruppen, ja, vernünftig einbindet, dass auch was, dass es auch einen vernünftigen Output gibt, der dann auch wirklich, ja, nutzbar ist und kurzfristig nutzbar ist [...] Wenn es dann hinterher dazu führt, dass es eigentlich mehr Aufwand als Nutzen bringt, dann, ja, muss man sich die Frage stellen, will man das wirklich?«

In »bottom-up«-Energiegenossenschaften kommt es kaum zu Kontroversen. Aufgrund ihres hohen Engagements gelten die Vorstands- und Aufsichtsratsmitglieder vielen als unentbehrlich und genießen großen Respekt. Weiterhin verhindert die überwiegende Einigkeit der Mitglieder über ihre Werte und Ziele oft das Aufkommen von Konflikten. 


\section{Gendered Identities}

Wie oben bereits angeführt wurde, kam es zur Gründung von Energiegenossenschaften durch bestehende Organisationen oft durch Personen in höheren Positionen - etwa Bankdirektor*innen oder auch Bürgermeister*innen. Recht häufig wird die Mitgliedschaft in einer solchen Energiegenossenschaft von den Mitgliedern als Investition gesehen. Darin liegt mit Blick auf den gender investment gap möglicherweise eine weitere Ursache der Überrepräsentation von Männern; zudem ist die Rolle des »Investors« traditionell eher männlich geprägt (vgl. Balachandra et al. 2017; Barber/Odean 2001; Booth/Nolen 2012; Charness/Gneezy 2012).

Vertreter*innen der »bottom-up«-Energiegenossenschaften beschrieben sich und ihre Mitstreiter*innen als Höhergebildete, Intellektuelle, Unternehmer*innen sowie als finanziell abgesichert. Um sich mit den Themen, die durch die Energiegenossenschaft bearbeitet werden, wirklich auseinandersetzen zu können, werden diese persönlichen Voraussetzungen als notwendig erachtet. Weiterhin wird, wie bereits erwähnt, eine »Szene« angenommen, die sich Energiegenossenschaften besonders zugehörig fühlt. Diese Szene zeichnet sich insbesondere durch sozialen und ökologischen Aktivismus aus und kann, wenn nicht bereits über Bekanntschaftsnetzwerke, so doch zumindest durch Flyer »an den entsprechenden Stellen von Kneipen bis Bioläden« erreicht werden:

»das sind meistens Leute, die schon bis über die Ohren in vielen anderen Projekten engagiert sind [...] denen es nicht egal ist, wie sie leben, die soziales Engagement haben oder auch generell engagierte Leute sind. Das erkennt man schon, wenn man die Leute schon anguckt, sieht man schon, dass die, ja, [...] dieser ökologischen Szene angehören«

Mehrere Befragte aus Energiegenossenschaften beider Typen äußerten sich zu möglichen Gründen der (offenbar) nicht existenten Beteiligung von Menschen mit Migrationshintergrund. Zumeist wurden die Gründe in (vermeintlichen) Merkmalen der nicht repräsentierten Bevölkerungsgruppen ${ }^{16}$ gesehen, vereinzelt wurden fehlende bzw. unpassende Ansprachestrategien auf Seiten der Energiegenossenschaft angeführt.

\footnotetext{
16 Mehrfach wurden kulturelle Aspekte (geringe Bedeutung von erneuerbaren Energien und Klimaschutz in den »Herkunftsländern«), niedriger Bildungsgrad und geringes Einkommen/Vermögen genannt.
} 
»Vielleicht haben die auch [...] andere Probleme [.] so? [...] In der Regel kommen die ja- wenige Länder haben Energie so hoch auf dem Programm wie in Deutschland, die Nordischen vielleicht.«

»[D]as sind die Akademiker, die sich damit auseinandersetzen, da setzt sich kein Migrant mit auseinander. Oder wir haben da keinen türkischstämmigen Anteilseigner. Für die ist das ganz weit weg. Ich glaub' auch nicht, dass die so [...] umweltbewusst sind, dass sie sagen, >da liegt mir sehr viel dran<. Wenn Sie sehen, wie viele Nachbarstaaten sich noch auf Atomkraft stürzen. [...] warum macht Griechenland nicht Photovoltaik ohne Ende? Die könnten doch Stromexporteur werden. Die haben doch tolle Sonnenstunden. [...] aber da passiert auch nichts. [.] Da sind wir in Deutschland sind wir ja vielbedeutend weiter und eigentlich ist ja unser Land gar nicht dafür prädestiniert, die Sonne einzufangen, das ist ja Spanien, Portugal, Südfrankreich, aber die setzen auch alle auf Atomkraft. Also Griechen, gerade Griechenland, verstehe ich nicht. Dass die nicht sagen, >komm', da gehen wir mal in die Offensive und werden Stromexporteur<. Die haben ja nix, ihr Olivenöl und [.] ihre Zucchinis und weiß ich was nicht."

Eine Frau, die die Gründung einer Energiegenossenschaft maßgeblich vorangebracht hat, wird von einem Mitstreiter charakterisiert als "schon eine Macherin, kann man nicht anders sagen, hat neben der Genossenschaft noch ein eigenes Unternehmen gegründet«. In den meisten Interviews wird Unternehmertum indes eher mit männlichen Personen in Verbindung gebracht und als wichtige Voraussetzung des energiegenossenschaftlichen Erfolgs skizziert (»was ich sehr gut finde an unserer Energiegenossenschaft ist, dass da sehr sehr viele Unternehmer dabei sind«).

Obgleich viele Mitglieder von Energiegenossenschaften kaum Berührungspunkte mit den konkreten Projekten haben und es zumeist einzelne sind, die sich im Detail in die energietechnischen Sachverhalte einarbeiten oder diese Kompetenz im Beruf erworben haben, werden Energiegenossenschaften insbesondere von Interviewten aus »bottomup«-Gründungskontexten als Organisationen mit einem hohen Bezug zu »Technik« und erneuerbaren Energien beschrieben. Dies wurde mehrfach angeführt, um den höheren Anteil männlicher Mitglieder zu erklären. Demgegenüber engagierten sich Frauen eher in Sportvereinen oder in der solidarischen Landwirtschaft (»Ernährung ist Frauensache«). 
»Das ist bei uns eben nicht, dass wir Vorbehalte hätten, aber das sind möglicherweise Themen, die nicht so interessieren und vielleicht ist es auch die Herangehensweise und die Strategie [.] für mich ticken ja Frauen und Männer schon unterschiedlich und die Frauen gehen ja ganz anders ran. Man kann das nicht pauschalieren, aber ich denke da, da ist was dran, die Frau führt ganz anders und dass das irgendwie nicht so passt.«

Die wenigen Frauen, die in »bottom-up«-Energiegenossenschaften eine tragende Rolle spielen, scheinen unter besonderer Beobachtung ihrer männlichen Mitstreiter zu stehen. So beschreibt ein Befragter zunächst eine Aktive: »die macht das aber sehr [...] pausbackig, also sehr lieb [...] Da fehlt aber [...] so das Peppige«. Eine weitere Mitstreiterin, die, gemessen am Umfang ihres Engagements und der Intensität, mit der sie ihre Überzeugungen lebt, eigentlich gut zur »energiegenossenschaftlichen Identität « passen müsste, charakterisiert er folgendermaßen:

»wenn Sie mit der reden, dann kriegen Sie Gehirnwäsche [...] die ist [...] echte Überzeugungstäterin, dass es schon manchmal weh tut und ist auch da anstrengend. [...] es ist halt ein sehr spezieller Mensch, ja, hat den richtigen Mann an ihrer Seite, [...] der hat die Ruhe weg. Und dann ist das der gute Gegenpol, dass das funktioniert, aber der unterstützt sie zu 1000 Prozent und dann passt das auch.«

\section{Diskussion und Ausblick}

Wie sich anhand des empirischen Materials zeigt, ist für den Zugang zu Energiegenossenschaften - ob als Gründer*in oder Mitglied, ob in »bottomup«-initiierten oder durch andere Organisationen gegründeten Energiegenossenschaften - die Zugehörigkeit zu bestimmten sozialen Netzwerken entscheidend. Diese können informeller Natur sein (Familie, Freunde, Bekannte), sich aus beruflichen Kontexten oder aus persönlichem Engagement - etwa in Klima- und Umweltschutzbelangen - ergeben. Oft spielen auch mehrere Faktoren eine Rolle. Die entscheidenden Netzwerke waren eher von Männern dominiert. Sofern Frauen involviert waren, erhielten sie Zugang oft über Männer oder aufgrund ihrer Position in einer Organisation, die an der Gründung einer Energiegenossenschaft beteiligt war.

Aus intersektionaler Perspektive kann festgehalten werden, dass neben dem Geschlecht weitere Variablen eine wichtige Rolle in der Frage spielen, 
wer Mitglied in einer Energiegenossenschaft wird, eine solche gründet - und wer nicht. Menschen mit höheren Einkommen oder Vermögen verfügen über Kapital, das sie zur Zeichnung von Anteilen entbehren können, und haben überdies ein Interesse an attraktiven Anlagemöglichkeiten. Oft handelt es sich dabei um Höherqualifizierte, die sich im Klima- und Umweltschutz engagieren. Der für eine aktiv-gestaltende Zugehörigkeit zu einer Energiegenossenschaft respektive zu deren Vorstand in der Regel nötige erhebliche Umfang an freier Zeit lässt sich nur von Personen aufbringen, die keiner bezahlten Arbeit nachgehen oder es sich leisten können, die Arbeitszeit zu reduzieren und auch nicht intensiv in andere Formen der Arbeit eingebunden sind. Hier spielt offensichtlich auch das Alter eine Rolle: traditionelle Geschlechterrollen und identitäten sind insbesondere in älteren Generationen, die aktuell in Energiegenossenschaften überrepräsentiert sind, verbreitet (vgl. Backes 2010, Lück 2005). Würde in der nachberuflichen Phase die Haus- und Fürsorgearbeit gleichmäßiger aufgeteilt, verfügten Frauen über mehr Zeit, um sich (unter anderem) in Energiegenossenschaften einzubringen. Die wenigen Beispiele von Frauen, die sich in »bottom-up«-initiierten Energiegenossenschaften in führenden Funktionen einbringen, zeigen, dass sie es im gleichen zeitlichen Ausmaß wie ihre männlichen Mitstreiter tun; in den durch Organisationen initiierten Energiegenossenschaften sogar tendenziell in einem darüberhinausgehenden größeren Umfang. In der oben angeführten Beschreibung einer als »überengagiert « charakterisierten Frau, die der Führungsriege einer Energiegenossenschaft angehört, wird auch ihr Mann erwähnt, der ihr intensives Involviertsein vollends unterstützt (und zumindest aus Sicht des Befragten, der von dieser Beziehung berichtet, damit auch ein Stück weit erst ermöglicht).

Eine direkte Absicht, bestimmte Bevölkerungsgruppen auszuschließen, kann nicht konstatiert werden. Eher kam es durch die Strategien der Mitstreiter"innen- und Mitgliedergewinnung sowie durch Bedingungen der Mitgliedschaft (wie etwa hochpreisige Genossenschaftsanteile) zu indirekter Exklusion. Allerdings war gerade mit Blick auf die Höhe des Genossenschaftsanteils mehreren Interviewpartner*innen bewusst, dass die Mitgliedschaft in ihrer Genossenschaft für bestimmte Gruppen nicht in Frage kommen würde. Ebenso geschah die Bevorzugung von Mitgliedern aufgrund ihrer Lebensstile und Werthaltungen in dem Wissen, dass eben nicht "alle« diese Anforderungen erfüllen.

Vielfach hatten die Befragten schlicht nicht den Anspruch, in den Mitgliederstrukturen ihrer Energiegenossenschaft einen breiten Querschnitt der 
Bevölkerung abzubilden. Entsprechend wurde auch kaum von Bemühungen, bislang unterrepräsentierte Gruppen als potentielle Mitglieder anzusprechen, berichtet. Im Interview erklärten mehrere Befragte, es handle sich um eine interessante Frage, über die man einmal nachdenken könnte; einen dringenden Handlungsbedarf sähen sie jedoch nicht - auch, da es bislang immer gelungen sei, genügend Interessent*innen für die zu zeichnenden Summen zu gewinnen. Das genossenschaftliche Handeln wird also bei den meisten Energiegenossenschaften (wie für deutsche Genossenschaften typisch und sogar gesetzlich vorgeschrieben) primär durch die Maßgabe der Mitgliederförderung und nicht durch "gemeinwohlförderliche« Zielsetzungen bestimmt. Aktuell handelt es sich bei Energiegenossenschaften jedenfalls nicht (ausschließlich) um Bürgerorganisationen, die darauf abzielen, gemeinschaftlich ein alternatives Energiesystem aufzubauen - wenngleich es sicher einige Beispiele gibt, die darauf hinarbeiten. ${ }^{17}$

Über den deutschen Kontext hinaus Implikationen für Energiegenossenschaften auch in anderen Ländern $\mathrm{zu}$ formulieren, wird durch die spezifischen Rahmenbedingungen erschwert. Traditionell sind Genossenschaften in Deutschland eher mittelständisch-konservativ geprägt und trugen, wie Erdmann (1997) gezeigt hat, historisch zumindest im ländlichen Kontext oft eher zur Konsolidierung bestehender Machtverhältnisse und Ungleichheiten als zu deren Abschaffung bei, da beispielsweise Führungspositionen mit Personen, die vor Ort bereits ein hohes Ansehen und großen Einfluss genossen, besetzt wurden. Genossenschaften im Allgemeinen und Energiegenossenschaften im Besonderen sind mit Blick auf ihre Prinzipien vielversprechende Instrumente zur Selbsthilfe und -ermächtigung. Die genossenschaftliche Idee allein vermag jedoch soziale Ungleichheiten nicht aufzuheben, und die genossenschaftliche Hülle schützt nicht davor, dass dieselben innerhalb der Organisation reproduziert werden. Wenngleich genossenschaftliche Organisationsweisen auch in vielfältigen Kontexten jenseits des "globalen Nordens" wertvolle Anregungen bergen können, handelt es sich gewiss nicht um ein Allheilmittel.

17 Schon die in diesem Beitrag beschriebene Vielfalt trotz kleiner Fallzahl deutet auf eine sehr heterogene energiegenossenschaftliche Landschaft hin und es ist gut möglich, dass es in Deutschland (und in der Zwischenzeit auch im Ruhrgebiet) bereits Energiegenossenschaften gibt, die sich bemühen, gesellschaftliche Diversität in ihren Mitgliederstrukturen abzubilden. 
Bei der Interpretation der vorliegenden Ergebnisse ist ferner zu berücksichtigen, dass sie mittels einer explorativen, qualitativen Vorgehensweise erhoben und ausgewertet wurden. Sie beruhen auf Einschätzungen von Personen, die in der jeweiligen Energiegenossenschaft eine zentrale Position (etwa Mitglied des Vorstands oder Aufsichtsrats und/oder Gründungsmitglied) innehaben. Daraus dürfte in vielen Fällen das Bemühen erwachsen, die eigene Organisation möglichst positiv darzustellen. Weiterhin handelt es sich bei ihren Aussagen nicht um die Ergebnisse eigener Auswertungen von Mitgliederdaten, sondern um subjektive Eindrücke. Die hier beschriebenen Beobachtungen sind also mehr als Indizien, als mögliche Ausgangspunkte weiterer Studien zu werten. Welchen Anteil welche Determinanten sozialer Ungleichheit an der Exklusivität von Energiegenossenschaften haben und ob es weitere Faktoren oder Mechanismen gibt, die hier eine Rolle spielen, ist im Rahmen weiterer Untersuchungen festzustellen.

\section{Literatur}

Acker, Joan (1990): »Hierarchies, Jobs, Bodies: A Theory of Gendered Organizations«, in: Gender and Society 4 (2), S. 139-158. https://doi.org/10.1177/ 089124390004002002

Acker, Joan (2012): »Gendered Organizations and Intersectionality: Problems and Possibilities«, in: Equality, Diversity and Inclusion: An International Journal 31 (3), S. 214-224. https://doi.org/10.1108/02610151211209072

Agentur für Erneuerbare Energien (AEE) (2018): Bürgerenergie bleibt Schlüssel für erfolgreiche Energiewende. Online verfügbar unter: https:// www.unendlich-viel-energie.de/buergerenergie-bleibt-schluesselfuer-erfolgreiche-energiewende. Zuletzt aufgerufen am 03.02.2020.

Backes, Gertrud (2010): »Alter(n)«, in: Ruth Becker/Beate Kortendiek (Hg.), Handbuch Frauen- und Geschlechterforschung. Theorie, Methoden, Empirie, Wiesbaden: Springer Fachmedien, S. 454-460. https://doi.org/10. 1007/978-3-531-92041-2_54

Balachandra, Lakshmi/Briggs, Tony/Eddleston, Kim/Brush, Candida (2017): "Don't Pitch Like a Girl!: How Gender Stereotypes Influence Investor Decisions «, in: Entrepreneurship Theory and Practice 43 (1), S. 116-137. https:// doi.org/10.1177\%2F1042258717728028 
Barber, Brad M./Odean, Terrance (2001): »Boys will be Boys: Gender, Overconfidence, and Common Stock Investment «, in: The Quarterly Journal of Economics 116 (1), S. 261-292. https://doi.org/10.1162/003355301556400

Bauwens, Thomas (2016): "Explaining the diversity of motivations behind community renewable energy«, in: Energy Policy 93, S. 278-290. https:// doi.org/10.1016/j.enpol.2016.03.017

Bauwens, Thomas/Devine-Wright, Patrick (2018): »Positive energies? An empirical study of community energy participation and attitudes to renewable energy«, in: Energy Policy 118, S. 612-625. https://doi.org/10.1016/j. enpol.2018.03.062

Bleckmann, Lisa/Luschei, Frank/Schreiner, Nadine/Strünck, Christoph (2016): Energiearmut als neues soziales Risiko? Eine empirische Analyse als Basis für existenzsichernde Sozialpolitik. Abschlussbericht über das von der Hans-Böckler-Stiftung geförderte Projekt Nr. 2013-654-4. Universität Siegen. Siegen.

Blömer, Sebastian/Pehnt, Martin/Rechsteiner, Eva (2015): Energiesparen in Bürgerhand. Vom Modellprojekt zum Standbein der Energiewende von unten. Heidelberg: Institut für Energie- und Umweltforschung (ifeu).

Bontrup, Heinz J./Marquardt, Ralf M. (2014): Verteilungskonflikte infolge der Energiewende: Elektrizitätsarmut. Hannover/Lüdinghausen.

Booth, Alison L./Nolen, Patrick (2012): »Gender Differences in Risk Behaviour: Does Nurture Matter?«, in: The Economic Journal 122 (558), S. F56-F78. https://doi.org/10.1111/j.1468-0297.2011.02480.x

Bundesnetzagentur (2020a): EEG-Umlage. Was ist die EEG-Umlage und wie funktioniert sie? Online verfügbar unter: https://www.bundesnetz agentur.de/SharedDocs/FAQs/DE/Sachgebiete/Energie/Verbraucher/ Energielexikon/EEGUmlage.html. Zuletzt aufgerufen am 03.02.2020.

Bundesnetzagentur (2020b): Informationen $\mathrm{zu}$ Strom- und Gaspreisen für Haushaltskunden. Wie setzt sich der Strompreis zusammen? Online verfügbar unter: https://www.bundesnetzagentur.de/DE/Sachgebiete/ ElektrizitaetundGas/Verbraucher/PreiseRechnTarife/preiseund Rechnungen-node.html. Zuletzt aufgerufen am 20.01.2020.

Charness, Gary/Gneezy, Uri (2012): »Strong Evidence for Gender Differences in Risk Taking «, in: Journal of Economic Behavior \& Organization 83 (1), S. 50-58.

Chatalova, Lioudmila/Valentinov, Vladislav (2014): »Die zwielichtige Rolle der Energie-Genossenschaften«, in: Zeit Online, 20.04.2014. Online verfügbar 
unter: https://www.zeit.de/wissen/umwelt/2014-04/energie genossenschaften-rendite. Zuletzt aufgerufen am 30.01.2020.

Crenshaw, Kimberle (1989): Demarginalizing the Intersection of Race and Sex: A Black Feminist Critique of Antidiscrimination Doctrine, Feminist Theory and Antiracist Politics. University of Chicago Legal Forum: Vol. 1989: Iss. 1, Article 8.

Debor, Sarah (2014): The Socio-Economic Power of Renewable Energy Production Cooperatives in Germany. Results of an Empirial Assessment. Wuppertal Papers 187. Wuppertal: Wuppertal Institute for Climate, Environment and Energy.

Diermann, Ralph (2019): »Missionarisches Predigen hilft nicht«, in: Spiegel Online, 20.01.2019. Online verfügbar unter https://www.spiegel.de/ wissenschaft/natur/energiewende-es-wird-auch-verlierer-geben-a1248615.html. Zuletzt aufgerufen am 30.01.2020.

Dunphy, Niall/Revez, Alexandra/Gaffney, Christine/Lennon, Breffní/Ramis Aguilo, Ariadna/Morrissey, John/Axon, Stephen (2017): Intersectional Analysis of Energy Practices. Irland: University College Cork. Online verfügbar unter: www.entrust-h2020.eu/wp-content/uploads/2018/03/D3.2Intersectional-Analysis-of-energy-practices_release.pdf. Zuletzt aufgerufen am 05.02.2020.

Eberherr, Helga (2014): »Intersektionalität aus der Organisationsperspektive«, in: Maria Funder (Hg.), Gender Cage revisited. Handbuch zur Organisations- und Geschlechterforschung, Baden-Baden: Nomos, S. 369-387. https://doi.org/10.5771/9783845244617-369

Elsen, Susanne (2012):»Genossenschaften als Organisationen der sozialen Innovation und nachhaltigen Entwicklung«, in: Gerald Beck/Cordula Kropp (Hg.), Gesellschaft innovativ. Wer sind die Akteure?, Wiesbaden: Springer VS, S. 85-102. https://doi.org/10.1007/978-3-531-94135-6_5

Elsen, Susanne/Walk, Heike (2016): »Genossenschaften und Zivilgesellschaft: Historische Dynamiken und zukunftsfähige Potenziale einer ökosozialen Transformation«, in: Forschungsjournal Soziale Bewegungen 29 (3), S. 60 72. https://doi.org/10.1515/fisb-2016-0226

Erdmann, Gero (1997): Diesseits der Theorie. Partizipation, Demokratie und ländliche Genossenschaftsbewegung in Deutschland. Freiburger Schriften zur Politikwissenschaft Bd. 6. Freiburg: Arnold-BergstraesserInstitut.

Frondel, Manuel/Schmidt, Christoph M./aus dem Moore, Nils/Bruckner, Thomas/Kondziella, Henrik/Krawinkel, Holger/Goldammer, Kathrin (2012): 
Energiewende und steigende Strompreise: Wer trägt die Hauptlast der hohen Kosten? ifo Schnelldienst 65 (17), S. 3-8.

Frondel, Manuel/Sommer, Stephan (2014): Energiekostenbelastung privater Haushalte. Das EEG als sozialpolitische Zeitbombe?, RWI Materialien 81, Essen. https://doi.org/10.1007/BF03373078

Großmann, Katrin (2017): »Energiearmut als multiple Deprivation vor dem Hintergrund diskriminierender Systeme«, in: Katrin Großmann/André Schaffrin/Christian Smigiel (Hg.), Energie und soziale Ungleichheit. Zur gesellschaftlichen Dimension der Energiewende in Deutschland und $\mathrm{Eu}$ ropa, Wiesbaden: Springer VS, S. 55-76. https://doi.org/10.1007/978-3658-11723-8_2

Heindl, Peter/Schüßler, Rudolf; Löschel, Andreas (2014): »Ist die Energiewende sozial gerecht?«, in: Wirtschaftsdienst 94 (7), S. 508-514. https://doi. org/10.1007/s10273-014-1705-7

Holstenkamp, Lars (2012): »Ansätze einer Systematisierung von Energiegenossenschaften«, in: Arbeitspapierreihe Wirtschaft \& Recht (11).

Hopf, Christel/Schmidt, Christiane (1993): Zum Verhältnis von innerfamilialen sozialen Erfahrungen, Persönlichkeitsentwicklung und politischen Orientierungen. Dokumentation und Erörterung des methodischen Vorgehens in einer Studie zu diesem Thema. Hildesheim. Online verfügbar unter: https:/www.ssoar.info/ssoar/bitstream/handle/document/45614/ ssoar-1993-hopf_et_al-Zum_Verhaltnis_von_innerfamilialen_sozialen.pdf ?sequence=1\&isAllowed=y\&lnkname=ssoar-1993-hopf_et_alZum_Verhaltnis_von_innerfamilialen_sozialen.pdf. Zuletzt aufgerufen am 22.01.2020.

International Renewable Energy Agency (IRENA) (2019): Renewable Energy: A Gender Perspective. Abu Dhabi. Online verfügbar unter: https://irena. org/-/media/Files/IRENA/Agency/Publication/2019/Jan/IRENA_Gender_ perspective_2019.pdf. Zuletzt aufgerufen am 05.02.2020.

Kahla, Franziska/Holstenkamp, Lars/Müller, Jakob R./Degenhart, Heinrich (2017): Entwicklung und Stand von Bürgerenergiegesellschaften und Energiegenossenschaften in Deutschland. Arbeitspapierreihe Wirtschaft \& Recht 27. Lüneburg: Leuphana Universität. Online verfügbar unter: https://www.buendnis-buergerenergie.de/fileadmin/user_ upload/wpbl27_BEG-Stand_Entwicklungen.pdf. Zuletzt aufgerufen am 04.02.2020.

Klemisch, Herbert/Maron, Helene (2010): »Genossenschaftliche Lösungsansätze zur Sicherung der kommunalen Daseinsvorsorge«, in: Zeitschrift 
für das gesamte Genossenschaftswesen 60 (1), S. 3-13. https://doi.org/10. 1515/zfgg-2010-0102

Kopatz, Michael (Hg.) (2013): Energiewende. Aber fair! Wie sich die Energiezukunft sozial tragfähig gestalten lässt. München: oekom.

Kreutzfeldt, Malte (2014): Das Strompreis-Komplott. Warum die Energiekosten wirklich steigen und wer dafür bezahlt. München: Knaur.

Kropp, Cordula (2017): »Forschung zu sozialen Innovationen am Scheideweg«, in: GAIA 26 (4), S. 309-312. https://doi.org/10.14512/gaia.26.4.5

Kuckartz, Udo (2010): Einführung in die computergestützte Analyse qualitativer Daten. Wiesbaden: Springer VS. https://doi.org/10.1007/978-3-53192126-6

Leggewie, Claus (2013): »Die Stadt als Gemeingut?«, in: Bund Deutscher Architekten (Hg.), Stadtquartiere sanieren: Sozial Ökologisch Ästhetisch, Berlin. S. 16-21.

Lenz, Ilse (2010): »Intersektionalität «, in: Ruth Becker/Beate Kortendiek (Hg.): Handbuch Frauen- und Geschlechterforschung. Theorie, Methoden, Empirie. Wiesbaden: Springer Fachmedien, S. 158-165. https://doi.org/10. 1007/978-3-531-92041-2_19

Lück, Detlev (2005): Cross-national comparison of gender role attitudes and their impact on women's life courses. Globalife Working Paper 67. Bamberg: Otto-Friedrich-Universität Bamberg.

Martens, Holger (2015): "Anders Wirtschaften - genossenschaftliche Selbsthilfe«, in: Aus Politik und Zeitgeschichte 65 (35-37), S. 40-46.

Müller, Jakob R./Dorniok, Daniel/Flieger, Burghard/Holstenkamp, Lars/Mey, Franziska/Radtke, Jörg (2015): »Energiegenossenschaften - das Erfolgsmodell braucht neue Dynamik«, in: GAIA 24 (2), S. 96-101. https://doi. org/10.14512/gaia.24.2.7

Müller, Jakob R./Holstenkamp, Lars (2015): Zum Stand von Energiegenossenschaften in Deutschland. Aktualisierter Überblick über Zahlen und Entwicklungen zum 31.12.2014. Arbeitspapiere Wirtschaft \& Recht 20. Lüneburg: Leuphana Universität.

Offenberger, Ursula (2016): Geschlecht und Gemütlichkeit: Paarentscheidungen über das beheizte Zuhause. Reihe Qualitative Soziologie 21. Oldenburg: De Gruyter.

Poppen, Silvia (2015): Energiegenossenschaften und deren Mitglieder - Erste Ergebnisse einer empirischen Untersuchung. Arbeitspapiere des Instituts für Genossenschaftswesen der Westfälischen Wilhelms-Universität Münster 157. Münster: Westfälische Wilhelms-Universität Münster. On- 
line verfügbar unter: https://www.econstor.eu/bitstream/10419/123711/1/ 841415013.pdf. Zuletzt aufgerufen am: 11.02.2020.

Radtke, Jörg (2016): Bürgerenergie in Deutschland. Partizipation zwischen Gemeinwohl und Rendite. Wiesbaden: Springer. https://doi.org/10.1007/ 978-3-658-14626-9

Tjørring, Lise (2016): »We forgot half of the population! The significance of gender in Danish energy renovation projects«, in: Energy Research \& Social Science 22, S. 115-124. https://doi.org/10.1016/j.erss.2016.08.008 trend:research/Leuphana Universität Lüneburg (2013): Definition und Marktanalyse von Bürgerenergie in Deutschland. Bremen/Lüneburg: trend:research $\mathrm{GmbH}$, Leuphana Universität Lüneburg. Online verfügbar unter: https://www.buendnis-buergerenergie.de/fileadmin/user_up load/downloads/Studien/Studie_Definition_und_Marktanalyse_von_ Buergerenergie_in_Deutschland_BBEn.pdf. Zuletzt aufgerufen am 20.01.2020.

Viardot, Eric (2013): »The role of cooperatives in overcoming the barriers to adoption of renewable energy«, in: Energy Policy 63, S. 756-764. https:// doi.org/10.1016/j.enpol.2013.08.034

Yildiz, Özgür/Rommel, Jens/Debor, Sarah/Holstenkamp, Lars/Mey, Franziska/Müller, Jakob R./Radtke, Jörg/Rognli, Judith (2015): »Renewable energy cooperatives as gatekeepers or facilitators? Recent developments in Germany and a multidisciplinary research agenda«, in: Energy Research \& Social Science 6, S. 59-73. https://doi.org/10.1016/j.erss.2014.12.001 



\section{Zwischen Wettbewerbsfähigkeit und Versorgungssicherheit \\ Wie wird der Megatrend »Digitalisierung « im Lichte einer umzusetzenden Energiewende in der Industrie diskursiv verhandelt?}

Sonja Knobbe, Marius Beckamp und Ann-Kristin Götz

\section{Einleitung}

Das Thema Digitalisierung beherrscht die Wirtschaft - nicht nur im Rahmen der Industrie-4.0-Debatte. Diskutiert werden neben den vielversprechenden technologischen Möglichkeiten der Zukunft auch Auswirkungen über rein technisch-ökonomische Angelegenheiten hinaus: Beispielsweise die Zukunft der Arbeitswelt oder Datensicherheit. Es ist gar die Rede von einer digitalen Transformation der gesamten Wirtschaft und Gesellschaft (Fricke/Thiessen 2016: 6; Lütkenhorst 2018: 6). Entsprechend werden die Digitalisierung sowie damit verbundene Phänomene auch zu den großen Megatrends unserer Zeit gezählt (Grömling/Haß 2009: 47ff.; Z_punkt GmbH 2019; Zukunftsinstitut $\mathrm{GmbH}$ o.J.).

Auch mit dem Großprojekt der Energiewende scheinen digitale Technologien in enger Verbindung zu stehen: 2016 führte die Bundesregierung in Deutschland das Gesetz zur Digitalisierung der Energiewende ein; teilweise wird die Digitalisierung gar als notwendige Voraussetzung einer erfolgreichen Energiewende gesehen (Bindel 2018: 2; VDI Zentrum Ressourceneffizienz 2017: 10). 
Diese Studie ${ }^{1}$ untersucht, wie die Industrie die Themen Digitalisierung und Energiewende diskutiert bzw. ob die Einschätzung, dass der Wandel des Energiesystems notwendig mit einer digitalen Transformation einhergehen muss, auch von der industriellen Praxis geteilt wird. Betrachtungsraum ist Deutschland, mit Schwerpunkt auf Nordrhein-Westfalen. Deutschland wird als eines der Vorreiter-Länder für eine politisch forcierte Energiewende verhandelt (Haas 2016: 22); NRW als bevölkerungs- und industriereichstes Bundesland mit dem höchsten Energieverbrauch (Statistische Ämter des Bundes und der Länder 2016-2019) ist zudem von besonderem Interesse, wenn es darum geht, die industrielle Perspektive der Energiewende in den Blick zu nehmen.

Als $\mathrm{zu}$ betrachtende Branchen wurden die Chemieindustrie, die Metallindustrie und der Maschinenbau ausgewählt: Diese Branchen weisen in der Produktion einen vergleichsweise hohen Stromverbrauch auf (IT.NRW 2016) und sind somit als besonders relevant einzuordnen. Weiterhin gelten sie als überproportional digitalisiert (Bertenrath et al. 2017), etwa aufgrund der in den Branchen eingesetzten Technologien, der Nutzungsintensität des digitalen Angebots oder auch ihres eigenen digitalen Angebotsspektrums.

Die Energiewende stellt die Industrie vor diverse Herausforderungen: Einerseits benötigen energieintensive Industriezweige Versorgungsicherheit, andererseits besteht die Gefahr, dass der Wandel des Energiesystems höhere Energiekosten verursacht und somit die internationale Wettbewerbsfähigkeit der Unternehmen beeinträchtigt. Im Rahmen einer Transformation des Energiesystems ist es jedoch zu kurz gegriffen, Unternehmen nur als passive Akteur*innen in der Energiewende zu betrachten: Durch die zunehmende Bedeutung Erneuerbarer Energien gibt es einen Paradigmenwechsel von »verbrauchsorientierter Erzeugung hin zum erzeugungsorientierten Verbrauch « (Doleski 2016: 8).

Unternehmen als Energienachfragende können die Energiewende daher nicht nur durch gesteigerte Effizienz unterstützen, sondern im Rahmen einer flexiblen Energienachfrage auch aktiv zum Gelingen der Energiewen-

1 Die Analyse wurde im Rahmen des Forschungsprojektes »EnerDigit - Energiewende und Digitalisierung zwischen Dezentralität und Zentralität: Regionale und unternehmenskulturelle Perspektiven « erstellt, welches durch das Ministerium für Wirtschaft, Innovation, Digitalisierung und Energie des Landes Nordrhein-Westfalen gefördert und durch das Institut Arbeit und Technik in Celsenkirchen, das Kulturwissenschaftliche Institut in Essen und das Wuppertal Institut durchgeführt wurde. 
de beitragen, indem sie das Stromnetz durch die Abnahme von Überschüssen oder eine reduzierte Nachfrage im Fall von Unterspeisungen stabilisieren (dena 2010). Insbesondere die Grundstoffindustrie birgt hohe Potentiale für eine flexiblere Energienachfrage (Seidl/Schenuit/Teichmann 2016; Ausfelder/Seitz/Roon 2018; Vallentin et al. 2016; EnergieAgentur NRW 2016; Buber et al. 2013; dena 2010).

Dennoch finden sich in der Praxis wenige Unternehmen, die eine marktoder netzdienliche Flexibilisierung ihrer Produktionsprozesse implementieren, wie z.B. ein Blick auf den geringen Anteil der industriellen Lasten als Anbieter von Systemdienstleistungen zeigt: Für die Abschaltung von Lasten (gemäß $\mathrm{AbLaV}^{2}$ ) sind deutschlandweit 18 Lasten präqualifiziert (BNetzA/Bundeskartellamt 2019) und auch der Anteil an präqualifizierten Lasten für die Regelenergie ist gering (Tennet et al. 2018). Seitens der Unternehmen fehlt es weiterhin aktuell an Bewusstsein für die Flexibilisierung ihrer Produktion als Beitrag zur Energiewende (Scharl/Praktiknjo 2019). Auch im Rahmen von Interviews mit sieben Expert*innen im Bereich der Energiewirtschaft ${ }^{3}$ stellte sich heraus, dass eine Flexibilisierung der Produktion zwar technologisch möglich ist, aktuell jedoch wenig umgesetzt wird: Einerseits fehlt es in Unternehmen an Informationen bezüglich des Energieverbrauchs der einzelnen Anlagen, andererseits gibt es im Bereich der Netzentgelte Anreize für eine möglichst gleichförmige Energienachfrage.

Da Deutschland als einstigem Vorreiter-Land der Energiewende immer wieder vorgeworfen wird, den Anschluss verpasst zu haben und nun auf dem Weg in eine nachhaltige Energiewelt hinterherzuhinken (Handelsblatt 2019; World Economic Forum 2018), lohnt es sich, das Engagement der Industrie als größte Energieverbraucherin zur Umsetzung der Energiewende mittels digitaler Technologien genauer zu betrachten.

2 Verordnung über Vereinbarungen zu abschaltbaren Lasten (AbLaV).

3 Cesprochen wurde mit Wissenschaftler ${ }^{*}$ innen aus der Energiewirtschaft sowie Praxisexpert"innen aus Energieversorgungsunternehmen. 


\section{Vorgehen und Methode}

\section{Sampling}

Um für die Industrie relevant erscheinende Themen herauszufiltern, bildet eine Inhaltsanalyse der entsprechenden Branchenkommunikation ein geeignetes Instrument. Brancheneigene Pressemitteilungen erlauben es, differenziert die jeweiligen Schwerpunktsetzungen und Strategien abzuleiten, ohne dass dabei der mediale Filter und/oder die Sichtweisen anderer Interessensgruppen einfließen. Pressemitteilungen sind im Allgemeinen subjektiv verfasst und sollen das eigene Image positiv beeinflussen. Daher nehmen interessengeleitete Forderungen mehr Raum ein als objektive, darstellende Elemente. Darüber hinaus ist der Blick auf einzelne Unternehmen schwierig, da die Branche als Einheit verhandelt wird. Es ist allerdings davon auszugehen, dass branchenübergreifende Verbände gebündelte Positionen vertreten, die auch von der Mehrheit einzelner Unternehmen der jeweiligen Branche befürwortet werden.

Darüber hinaus können nicht nur aus explizit verhandelten Themen, sondern auch aus öffentlich relevanten, aber im Branchendiskurs ausgelassenen Inhalten erste Schlüsse gezogen werden. Die Themen Digitalisierung der Energiewende wie auch Digitalisierung und Energiewende jeweils im Einzelnen sind Themen von großem politischen Interesse, sodass zu vermuten ist, dass nicht zuletzt zum Zwecke der eigenen Imagepflege über Aktivitäten in diesem $\mathrm{Zu}$ sammenhang berichtet wird. Relevante Aktivitäten innerhalb der Branchen sollten daher hier Erwähnung finden. Auch ohne, dass Rückschlüsse auf einzelne Unternehmen gezogen werden können, sollte daher sichtbar werden, ob die Themen Digitalisierung und Energiewende in den Branchen insgesamt von Relevanz sind, wie sie begriffen und verhandelt werden.

Der Inhaltsanalyse liegt ein Datenkorpus bestehend aus Pressemitteilungen der jeweiligen Branchen zugrunde. Dazu wurden die online veröffentlichten Pressemitteilungen der Branchenverbände (VCI, BVM, VDMA), Gewerkschaften (IG BCE, IG Metall) sowie Arbeitgeberverbände (BAVC, Gesamtmetall) betrachtet. ${ }^{4}$ Dies soll eine möglichst umfassende Abbildung der Perspektiven innerhalb der Industrie (Arbeitnehmer*innen, Arbeitgeber*innen, weitere Akteur*innen) ermöglichen. Neben deutschlandweiten Verbänden wer- 
den die regionalen Bezirksverbände in NRW betrachtet, um exemplarisch die Situation im industriereichsten Bundesland mit einzubeziehen. Alle Pressemitteilungen, die nach dem o1. Januar 2011 veröffentlicht wurden, wurden für die Analyse in Betracht gezogen (insofern das Online-Archiv der Verbände bis dahin zurückreicht). Dieses Datum ist gewählt, weil das Jahr 2011 aufgrund des Atom-Moratoriums nach Fukushima als Wendepunkt der politisch gesteuerten Energiewende gilt (Gochermann 2016: 41ff.). Zudem fand die Bezeichnung >Industrie 4.0< als Dreh- und Angelpunkt der Debatte um die Digitalisierung der Wirtschaft ihren Ursprung in diesem Jahr, als das Konzept öffentlichkeitswirksam auf der Hannover Messe vorgestellt wurde (Paul 2016).

Der Datenkorpus wurde mithilfe einer Stichwortsuche in den OnlineArchiven der genannten Verbände erstellt; gesucht wurde nach den Stichworten »Energie« und »Digital« in den angegebenen Titeln und (wo vorhanden) in den Anriss- bzw. Teaser-Texten der archivierten Pressemitteilungen. Die Stichworte für die Suche wurden allgemein gehalten, um auf diese Weise ein möglichst umfassendes Themenspektrum im Datenkorpus abbilden zu können. So werden auch Begriffe wie Energiewende, Energiepolitik, Energiespeicher, Digitalisierung, Digitale Technologien, Digitaler Wandel usw. erfasst. Der Datenkorpus wurde im März 2019 erstellt; jüngere Pressemitteilungen finden keine Berücksichtigung. ${ }^{5}$

Insgesamt wurde ein Datenkorpus von 199 Pressemitteilungen (PM) erstellt, wovon ein Großteil (140, 70,4 \%) der Branche Chemie zuzuordnen ist. Die restlichen beziehen sich auf die Verbände, die die Metallbranche und/oder den Maschinenbau repräsentieren. Rund die Hälfte des Materials (48,2\%) stammt aus gewerkschaftlicher Quelle; Branchenverbände sind mit 38,7\% vertreten, Arbeitgeberverbände haben 12,6\% der analysierten Pressemitteilungen verfasst. ${ }^{6}$

Ferner wurden folgende Ausschlussregeln definiert:

- Pressemitteilungen der IC BCE werden ausgeschlossen, insofern sie das Schlagwort `Energie` ausschließlich im ausgeschriebenen Eigennamen (IC Bergbau, Chemie, Energie) beinhalten.

- Pressemitteilungen der IG Metall werden ausgeschlossen, insofern sie das Schlagwort > Digital < ausschließlich im Hinweis > Digitale Pressemappe< beinhalten.

6 Eine Pressemitteilung im Datenkorpus wurde gemeinsam von einem Arbeitgeberverband und einer Gewerkschaft verfasst, diese macht die fehlenden 0,5\% aus. 
Abb. 1: Übersicht Datenkorpus; eigene Darstellung

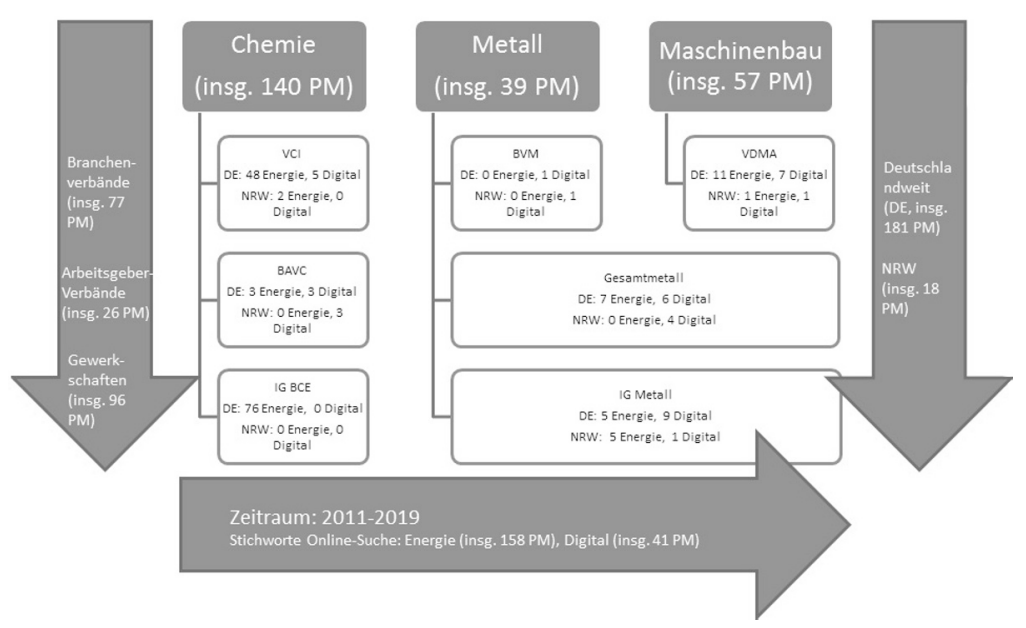

\section{Methodisches Vorgehen}

Die Daten wurden nach Branche, Herkunftsverband und Datum aufbereitet und anschließend einer computergestützten, qualitativ-strukturierenden Inhaltsanalyse (Mayring 2015) mit MAXQDA unterzogen. Dabei wurden deduktiv themenbezogene Kategorien gebildet, um die Digitalisierungs- und Energiewendebezogenen Forderungen und Strategien wie auch die zugrunde liegenden Argumentationsstränge herauszuarbeiten, welche dann jeweils induktiv tiefergehend systematisiert wurden. Dies jeweils für die beiden Themen im Einzelnen wie auch gesondert für die Textbausteine, die Energiewende und Digitalisierung im Zusammenhang betrachten. ${ }^{7}$

Die Kategorisierung der gefundenen Phänomene erfolgt zwangsläufig idealtypisch und es sind sicherlich Schnittmengen zu verzeichnen. Dennoch

7 Das Material wurde zudem einer Zweitcodierung unterzogen. Die IntercoderÜbereinstimmung in Bezug auf das Vorhandensein der jeweiligen Argumente, Forderungen und Strategien (107 verschiedene Codes und Subcodes) liegt hier im Durchschnitt bei 93,71\% ( $\mathrm{K}=0,94)$, weshalb der Untersuchung eine überaus hohe IntercoderReliabilität zugeschrieben werden kann. 
eignet sich die vorgenommene Einteilung dazu, einen Überblick über die wesentlichen Argumentationsmuster und Einstellungen $\mathrm{zu}$ geben, die in den industriellen Verbänden zu verzeichnen sind. Vorläufige Ergebnisse sowie das methodische Vorgehen wurden im Rahmen eines Workshops mit Expert*innen aus Wissenschaft und Praxis diskutiert.

\section{Ergebnisse}

Ganz allgemein lässt sich zunächst festhalten, dass die Energiewende, gerade im Zusammenhang mit der industriellen Energieversorgung, sehr viel umfassender diskutiert wird als die Digitalisierung. Während $123(61,8 \%)$ der untersuchten Pressemitteilungen das Schlagwort »Energiewende« aufweisen (von 158 (79,4\%), die sich mit Energie generell auseinandersetzen), widmen sich $41(20,6 \%)$ digitalen Inhalten. Nur 10 Pressemitteilungen (5\%) setzen die beiden Themen Energie und Digitales in einen Zusammenhang.

Abb. 2: Datenkorpus sortiert nach Such-Schlagworten; eigene Darstellung

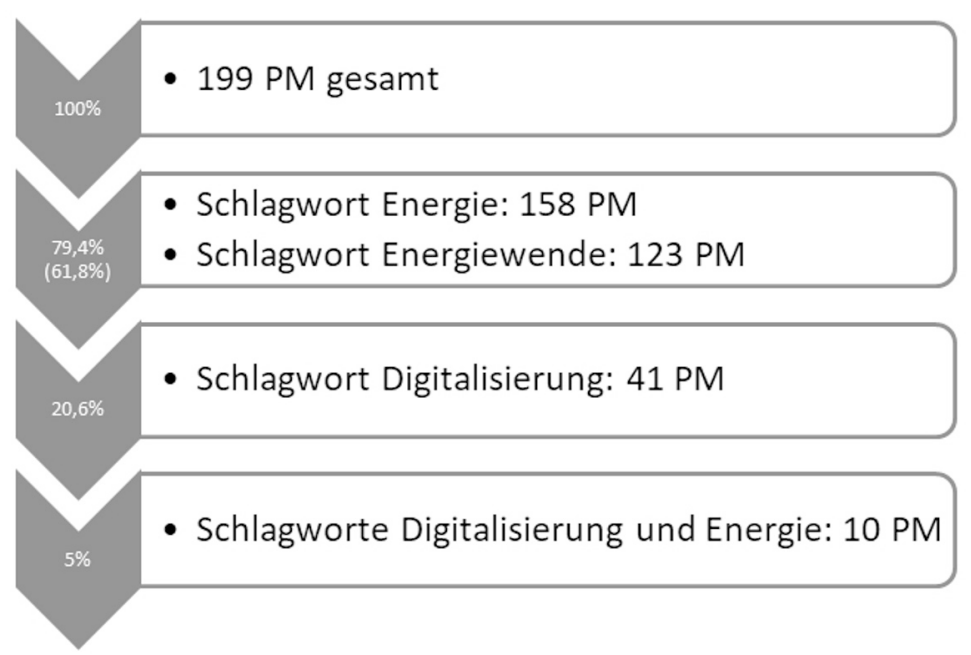

Im Folgenden wird dargestellt, wie die einzelnen Themen jeweils qualitativ behandelt werden: 


\section{Digitalisierung}

\section{Der Begriff der Digitalisierung}

Der Begriff der Digitalisierung ist - wie wohl alle Begriffe, die komplexe sozioökonomische Prozesse beschreiben - eher unspezifischer Natur. Dies ist primär darauf zurückzuführen, dass er häufig ohne Definition verwendet wird und in diesen Fällen vermutlich ungefähr salles, was mit Computern und dem Internet zu tun hat umschreiben soll. Werden in der Forschung Definitionen vorangeschickt, so stimmen die verschiedenen Studien diesbezüglich selten überein (Mertens/Barbian/Baier 2017: 35ff.).

Die hier analysierten Branchen verwenden den Begriff der Digitalisierung in einem sehr umfassenden Sinne: Es werden nicht nur technologische Prozesse und die digitale Aufbereitung von Daten darunter verstanden, sondern auch die Nutzung dieser Daten und deren sozioökonomische Auswirkungen, seien es eine gesteigerte Effizienz oder gesellschaftliche Effekte, zum Beispiel aufgrund der zunehmenden Relevanz von Smartphones (Paul 2016: 4f.; Werning et al. 2017: 6f.). Der transformative Charakter einer so verstandenen Digitalisierung wird deutlich, etwa wenn von einer »Welt im Umbruch« (VCI 2017) die Rede ist und auf gesamtgesellschaftliche Konsequenzen digitaler Veränderungsprozesse hingewiesen wird. Besonders im Vordergrund stehen die ökonomischen Veränderungen in Form von neuen Möglichkeiten für die Industrie durch neue Geschäftsmodelle oder Umstrukturierungen. Damit ist die Industrie einig mit der Forschung, die disruptive Veränderungen für die Wirtschafts- und Industriewelt durch die gebündelte Anwendung digitaler Technologien erwartet (Lütkenhorst 2018: 6). Diese ökonomischen Veränderungen gilt es zu gestalten, was auch in den Forderungen der Industrie deutlich wird:

\section{Zentrale Forderungen}

Im Vordergrund steht für die Industrie das Thema Mensch und Arbeit. Hier werden vielfach Forderungen nach (Weiter-)Bildung in digitalen Anwendungen und der Ausbildung von Fachkräften laut. Diese richten sich an die Politik wie auch an andere Stakeholder, wie Universitäten und Ausbildungsbetriebe. Weiterhin wird die Wahrung sozialer Sicherheit für die Arbeitnehmenden gefordert. Hier stehen der Erhalt von Arbeitsplätzen und ein verlässliches Arbeitsumfeld im Vordergrund sowie die Schaffung von Sicherheit am Arbeitsplatz angesichts der neuen Herausforderungen. 
Ebenso spielt für die Industrie die technologische Weiterentwicklung eine Rolle, um so aktiv den Prozess der Digitalisierung mitgestalten zu können. In diesem Rahmen ist die an die Politik gerichtete Forderung zu nennen, den Ausbau einer digitalen Infrastruktur (Breitbandausbau) voranzutreiben. Ferner werden Gelder für die Innovations- und Forschungsförderung erbeten, um die digitale Weiterentwicklung aktiv vorantreiben zu können. Das Thema Datenschutz und -sicherheit spielt eine untergeordnete Rolle, insofern sich nur eine Pressemitteilung im Datenkorpus überhaupt darauf bezieht.

Nicht zuletzt wird immer wieder auf die Relevanz der Erhaltung und Förderung von unternehmerischer Flexibilität und sektorübergreifender Kooperation sowie von Akzeptanz und Mitbestimmung hingewiesen. Aus industrieller Perspektive gilt es, Bedarfe eigenverantwortlich und flexibel verfolgen zu können, dies aber in praxisnaher Zusammenarbeit mit verschiedenen Sektoren wie auch außerwirtschaftlichen Interessensgruppen. Partizipative Elemente werden dabei als Erfolgsvoraussetzung einer gelingenden Digitalisierung beschrieben und umfassen vor allem gewerkschaftliche Mitbestimmung, gesellschaftliche Akzeptanz sowie intra- und intersektorale Kooperation auf nationaler wie globaler Ebene.

\section{Bilder der Digitalisierung}

Die Forderungen werden primär durch bestimmte Bilder gestützt, die die Industrie von der Digitalisierung zeichnet. Hier lassen sich drei Kategorien ausmachen: Zunächst das weitgehend neutrale Bild einer transformativen Digitalisierung, wonach es sich auf die umfassenden Effekte derselben vorzubereiten gilt. Hier finden insbesondere die ohnehin stattfindenden Veränderungen, auf die es sich unter dem Stichwort der Zukunftsfähigkeit vorzubereiten gilt, Erwähnung.

Neben dieser neutralen Bewertung findet sich aber ebenfalls häufig ein negativ gezeichnetes Bild einer risikobehafteten Digitalisierung, wonach es sich nicht weiterzuentwickeln, sondern vor mit der Digitalisierung verbundenen Risiken abzusichern gilt. Hier werden die Digitalisierung und die damit verbundenen Kosten als Druck von außen gezeichnet, der die Wettbewerbsfähigkeit und somit den Erhalt der betroffenen Unternehmen bedrohe. Die damit verbundene Argumentationslinie betont die Angewiesenheit der Unternehmen auf Unterstützung, um sich und auch die Arbeitnehmenden vor den auf die Digitalisierung zurückgehenden Gefahren - wie etwa einem immensen Kostenanstieg oder auch einer zunehmenden sozialen Spaltung - zu schützen. 
Weiterhin findet sich ein positiv konnotiertes Bild, welches die Chancen der Digitalisierung betont. In diesem Kontext werden dann neue Möglichkeiten für Produkte, Geschäftsmodelle und andere Innovationen angeführt, welche Produktivitäts- und Gewinnzuwächse versprechen.

\section{Strategische Maßnahmen}

Was aber tun die Branchen nun konkret, um sich auf die digitale Transformation vorzubereiten? Hier konnten drei zentrale Antworten herausgelesen werden. Zumeist wird auf Weiterbildungsmaßnahmen zur Sicherung von Fachkräften Bezug genommen. Häufig wird auf die in den Branchen bereits etablierte Nutzung digitaler Lösungen sowie auf dialogische und kooperative Maßnahmen verwiesen, wie etwa auf die Einrichtung von Netzwerken und Plattformen oder die Durchführung von Tagungen. Hier wird deutlich, dass die Industrie partizipative Ansprüche an die Digitalisierung durchaus ernst nimmt und vielfältige Maßnahmen einleitet, um den branchenübergreifenden Austausch zu fördern.

\section{Energiewende}

\section{Der Begriff der Energiewende}

Ebenso wie hinsichtlich der Digitalisierung wird die Komplexität und Reichweite der Energiewende regelmäßig hervorgehoben. Weniger wird aber in diesem Zusammenhang auf konkrete Maßnahmen und Prozesse verwiesen, sondern vielmehr auf die staatliche Verantwortung, die Umstellung des Energiesystems regulativ anzuleiten.

\section{Zentrale Forderungen}

Das Bild einer komplexen, durch den Staat zu verantwortenden Energiewende spiegelt sich auch in den im Datenkorpus artikulierten Forderungen wider: Ein Großteil derselben ist direkt an die politischen Regulierungsinstanzen gerichtet. Häufig zu finden ist die Forderung nach einer ganzheitlichen politischen Energiewende-Strategie, die einen langfristigen, konsistenten und verlässlichen politischen Rahmen und so den Unternehmen Planungssicherheit bietet. Neben dem Ausgleich von Zielkonflikten gelte es, das Ziel der sozialen Gerechtigkeit nicht aus dem Blick zu verlieren; dies in Bezug auf die Absicherung der Beschäftigten, aber auch auf einkommensschwache Haushalte und Strukturwandelregionen. Darüber hinaus wird eine Abstimmung der Strategie auf europäischer und internationaler Ebene gefordert wie auch die par- 
tizipative Integration verschiedener Interessensgruppen. So ist die Rede von einer »Energiepolitik aus einem Guss « (IG BCE 2012) oder einer »Klimapolitik mit Weitblick« (VCI 2013), »die Wettbewerbsfähigkeit, Arbeitsplätze und Klimapolitik in einen sinnvollen Einklang bringt « (IG BCE 2014).

Über die strategisch-politische Ebene hinaus wird vor allem an den Staat appelliert, die Industrie zu unterstützen, um den Herausforderungen der Energiewende überhaupt standhalten zu können. Diese Forderungen manifestieren sich primär kostenseitig, insofern eine Senkung der Energiepreise oder eine Verhinderung von Mehrbelastungen der Industrie durch eine Erhöhung der EEG-Umlage oder verschärfte Klimaziele gefordert werden. Neben Aspekten einer »bezahlbaren Energiewende« (VCI 2014) findet sich oft die Forderung nach einer sicheren Energieversorgung, worin sich die Skepsis gegenüber dem derzeitigen Potential der Erneuerbaren Energien ausdrückt, die Energiebedarfe der Industrie zu decken. In diesem Zusammenhang wird auch der Wunsch geäußert, konventionelle und nicht regenerative Energiequellen, insbesondere Braunkohle, zumindest als »Brückentechnologie« (IG BCE 2016) vorerst beizubehalten. Übergreifend geht es der Industrie darum, die eigene Wettbewerbsfähigkeit zu sichern, die sie bedroht sieht.

Im Vergleich zu den beiden anderen Forderungskategorien seltener wird ein tatsächliches Vorantreiben der Energiewende gefordert, vor allem in Form von Investitionen in den Ausbau Erneuerbarer Energien, in Netze sowie in Forschung und Entwicklung.

\section{Argumentationslinien}

Grundlage für die Forderungen bilden im Wesentlichen vier verschiedene Argumentationslinien. Am prominentesten ist die ökonomische Argumentation vertreten. In dieser Linie wird primär kostenfokussiert argumentiert; der Erhalt industrieller Betriebe bis hin zu ganzen Industriezweigen wird von der Erfüllung der geforderten Maßnahmen abhängig gemacht. Die Gefahr wird zumeist in den Kosten der Energiewende bzw. in einem durch Energiewendebezogene Maßnahmen verursachten Anstieg der Energiepreise gesehen. Neben den Kosten wird (häufig auch im Verbund) der Erhalt von Versorgungssicherheit, (internationaler) Wettbewerbsfähigkeit und Investitionssicherheit in Frage gestellt oder es wird die technische Umsetzbarkeit politischer Regularien seitens der Industrie angezweifelt. Prinzipiell wird ein Drohszenario aufgebaut, wonach die Abwanderung oder der Zerfall der Industrie in Aussicht gestellt wird, was nicht zuletzt auch einen Verlust von Arbeitsplätzen 
bedeuten würde. Selten wird diese Argumentationslinie auch positiv gewendet, insofern die Energiewende nicht als Gefahr, sondern als Chance dargestellt wird, die der Industrie Wachstumsmöglichkeiten bietet und somit auch neue Arbeitsplätze schafft.

Eine weitere Argumentationslinie lässt sich unter dem Stichwort ökologische Argumentation zusammenfassen, insofern hier nicht der Erhalt der Industrie, sondern eine gelingende Energiewende als das zentrale Ziel definiert wird. In diesem Kontext wird auf die Klimaschutz- oder Energiesparziele der Regierung verwiesen oder auch auf eine Unausweichlichkeit der Energiewende in Reaktion auf die Nuklearkatastrophe von Fukushima, um die eigenen Forderungen zu begründen. Der Einsatz neuer Technologien oder der Ausbau der Infrastruktur (vor allem des Stromnetzes) gilt hier als notwendige Voraussetzung zur Erreichung dieser Ziele. Ebenso werden auch die eigenen Forderungen als die effizienteren Maßnahmen beschrieben, die Klimaziele $\mathrm{zu}$ erreichen. Oder aber die Industrie verweist auf andere Maßnahmen, beispielsweise die Gebäudesanierung, die effizientere Lösungen darstellen würden als (zusätzliche) Belastungen des industriellen Stromverbrauches.

In diesem Zusammenhang gibt es jedoch auch die umgekehrte Argumentationsweise, in der die Industrie sich selbst als Grundlage für eine gelingende Energiewende darstellt. So werden beispielsweise eigene Produkte als notwendige Grundlage für eine höhere Energieeffizienz in der Produktion dargestellt. Eine weitere Argumentation, die ebenfalls indirekt der ökologischen Linie zugeordnet werden kann, ist die Ablehnung politischer Maßnahmen, die kostenintensiv aber wenig vielversprechend sind. Hier wird nicht das Ziel der Energiewende selbst, sondern bestimmte Maßnahmen im Hinblick auf dieses Ziel in Frage gestellt.

Die dritte zentrale Argumentationslinie ist die normative Argumentation, die sich auf normative Aussagen jenseits des ökologischen Kontextes beruft. Hier stehen allgemeine Gerechtigkeitsaspekte sowie Bürger*innen und Energie-Konsument*innen jenseits der Industrie und deren Kosten sowie soziale Sicherheit im Fokus. Bezüglich der Gerechtigkeitsaspekte wird darauf verwiesen, dass die Kosten der Energiewende auf alle Betroffenen in einem gerechten Verhältnis aufzuteilen seien und nicht nur der Industrie aufgebürdet werden könnten. Ebenso wird darum gebeten, an Stelle neuer Forderungen bereits vollbrachte Leistungen auch anzuerkennen. Aber auch im Hinblick auf andere gesellschaftliche Stakeholder wird angebracht, dass deren Meinungen und Wünsche aufzunehmen seien, um so das Ziel der (gesamt)gesellschaftlichen Akzeptanz zu erreichen. In diesem Kontext 
wird auch immer wieder auf Umfrageergebnisse oder die Stimmung in der Bevölkerung verwiesen.

Eine vierte Linie ist die der politischen Argumentation. Hier geht es primär um strategisch-konsistenzorientierte Aspekte. Es gelte, soziale, ökologische wie auch ökonomische Ziele in Einklang zu bringen und so ein regulatives Chaos zu vermeiden. Politische Vorgaben sollen auf Machbarkeit und Rechtskonformität geprüft werden und die wahrgenommene Realitätsferne bestehender Vorgaben wird kritisiert.

Tab. 1: Zentrale Energiewende-bezogene Argumentationslinien im Überblick; eigene Darstellung

\begin{tabular}{|l|l|}
\hline Argumentationslinie & Ziel \\
\hline Ökonomisch & Erhalt der Industrie \\
\hline Ökologisch & Celingende Energiewende \\
\hline Normativ & Cerechtigkeit, soziale Sicherheit \\
\hline Politisch & Strategische Konsistenz \\
\hline
\end{tabular}

\section{Strategische Beiträge}

Bezüglich eigener strategischer Beiträge verweist die Industrie zumeist auf Aktivitäten in der Zukunft, führt diese aber nur selten konkret aus, zum Beispiel indem genaue Zahlen und/oder Zeithorizonte bzw. Umsetzungsfristen genannt werden. Zumeist geht es um Absichtserklärungen, weitere Einsparmaßnahmen oder Forschungsvorhaben durchzuführen bzw. neue Technologien einzusetzen. Darüber hinaus wird häufig auf kommunikative Arbeit, zum Beispiel über die Etablierung von Netzwerken, Forschungskreisen, Beratungs- und Austauschorganen gesetzt. Konferenzen und andere Austausch-Plattformen spielen in dieser Hinsicht eine vorgeordnete Rolle.

Auch vergangene Aktivitäten werden herangezogen, um aufzuzeigen, dass bereits Schritte in Richtung der Umsetzung einer Energiewende erfolgt sind. Diesbezüglich wird dann auf bereits umgesetzte Effizienzmaßnahmen oder Forschungsprojekte verwiesen. Ebenso wird die Klimafreundlichkeit bzw. die Relevanz der eigenen Produkte für die Energiewende hervorgehoben. Darüber hinaus muss angemerkt werden, dass sich der eigene EnergiewendeBeitrag in den untersuchten Pressemitteilungen auch durchaus nicht selten auf bloße Bekenntnisse beläuft: Unterstützung für die Energiewende im Allgemeinen wird erklärt, konkrete Maßnahmen werden jedoch nicht genannt. 
Übergreifend kann festgehalten werden, dass konkrete strategische Vorhaben nicht auszumachen waren.

\section{Energiewende im Vergleich zur Digitalisierung}

Insgesamt fällt auf, dass die Energiewende durch die Industrie anders behandelt wird als die Digitalisierung. Obwohl es sich bei beiden um gesamtgesellschaftliche und komplexe Vorgänge mit großer Reichweite handelt, wird in Bezug auf die Digitalisierung tendenziell auf die Eigeninitiative der Industrie gesetzt, während bezüglich der Energiewende die Handlungsverantwortung auf politischer Ebene verortet wird. Darüber hinaus wird die Digitalisierung im Mittel sehr viel positiver oder zumindest neutral bewertet, während im Hinblick auf die Energiewende die Hervorhebung der Risiken für die Industrie mit Abstand am prominentesten vertreten ist.

Bei einer Sortierung nach Branchen fällt auf, dass für die Chemie das Thema Energie von sehr viel größerer Wichtigkeit zu sein scheint als die Digitalisierung - zumindest lässt die Anzahl der veröffentlichten Pressemitteilungen zum Thema darauf schließen:

Abb. 3: Datenkorpus nach Branchen; eigene Darstellung

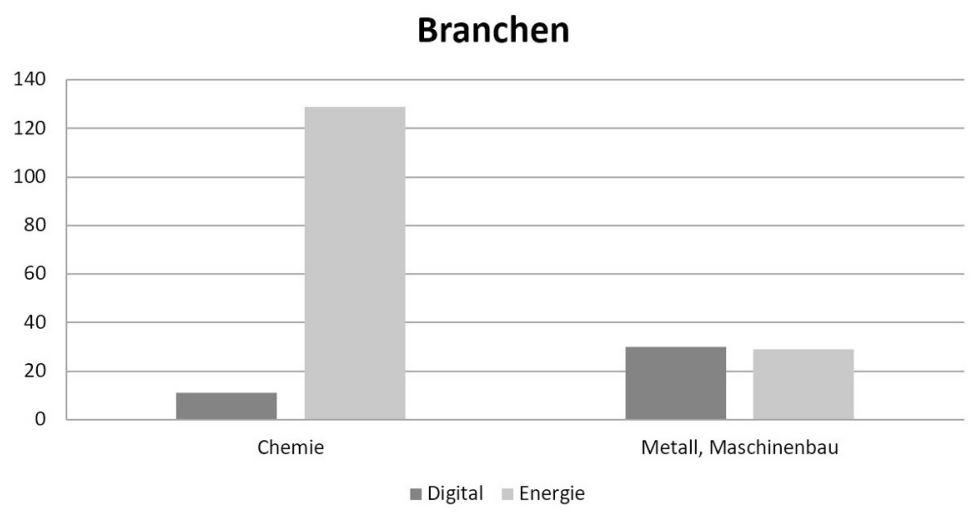

Zwar fällt die Chemie unter die energieintensiven Branchen, da dies jedoch auch auf die Metallindustrie zutrifft (IT.NRW 2016), kann der hohe Stromverbrauch nicht der einzige Grund für die hohe Presseaktivität der Chemie im Bereich Energie sein. Hier wird vermutlich auch eine Verzerrung 
der Daten sichtbar, insofern die IG BCE, die den Großteil der Daten seitens der Branche Chemie stellt, als Gewerkschaft neben der Chemie zusätzlich den Bergbau und die Energiewirtschaft vertritt und somit auch darüber eine besondere Nähe zu energiepolitischen Themen aufweist.

Inhaltlich lässt sich festhalten, dass in den Verbänden, die die Chemie vertreten, bezogen auf die Energiewende die Forderung nach einer konsistenten politischen Strategie überdurchschnittlich stark vertreten ist. Darüber hinaus wird die Energiewende im Vergleich zu den anderen Branchen hier auch häufiger als Gefahr für die deutsche Wirtschaft eingeschätzt. Die kostenfokussierte, ökonomische Argumentation taucht im Datenmaterial, welches durch Verbände der Chemie verfasst wurde, vergleichsweise häufig auf. In den Branchen Metall und Maschinenbau ${ }^{8}$ hingegen fällt auf, dass im Vergleich zur Energiewende eher die Digitalisierung als Risiko beschrieben wird.

Sortiert man das Datenmaterial nach regionalen Aspekten, fällt auf, dass sich die Landesverbände in NRW primär mit dem Thema der Digitalisierung beschäftigen. Der Befund lässt darauf schließen, dass energiepolitische Themen eher auf Bundesebene verhandelt werden (vgl. hierzu auch Trost/Büttgen/Geringhoff 2016: 12). In Verbindung mit der Digitalisierung wurde das Thema Energiewende von den NRW-Landesverbänden gar nicht behandelt.

\section{Digitalisierung und Energiewende}

Auch insgesamt gesehen werden die Prozesse Digitalisierung und Energiewende vergleichsweise selten im Zusammenhang betrachtet bzw. Querverbindungen zwischen beiden hergestellt. So gibt es kaum Material im Datenkorpus, welches darauf schließen lässt, dass die Industrie die Herausforderung der Energiewende digital lösen möchte. Die wenigen Pressemitteilungen zum Thema stammen primär von den jeweiligen Branchenverbänden; in den Arbeitgeberverbänden und Gewerkschaften taucht eine Verbindung der beiden Themen kaum auf.

Primär wird diese Verbindung über die Annahme geschaffen, dass die Digitalisierung neue Möglichkeiten für die Energiewende eröffne und somit eine

8 Die Branchen Metall und Maschinenbau werden nicht getrennt betrachtet, da sie sowohl gewerkschaftlich (IC Metall) als auch durch den Arbeitgeberverband (Gesamtmetall) gemeinsam repräsentiert werden. Eine branchenspezifische Analyse wäre dementsprechend nur anhand der Branchenverbände (BVM, VDMA) möglich, was aufgrund des begrenzten Datenmaterials wenig aussagekräftig wäre. 
"Chance für mehr Nachhaltigkeit« (VCI 2018) darstelle. In diesem Zusammenhang verspricht sich die Industrie von der digitalen Vernetzung neue Geschäftsmodelle mit einer höheren Energie- und Ressourceneffizienz. Alternativ werden Energiewende und Digitalisierung auch als parallel auftretende Erscheinungen unseres Zeitalters begriffen, die nicht zuletzt aufgrund ihrer jeweils großen Komplexität oft auch Verbindungen aufweisen bzw. die gleichen Bereiche der Gesellschaft betreffen.

Gefordert wird in diesem Zusammenhang primär die Entwicklung einer politischen Strategie. So solle der Staat zusätzliche Forschung und Entwicklung finanzieren, um auf diese Weise Investitionen und neue Geschäftsmodelle für die Industrie zu ermöglichen. Weiterhin gelte es aber auch, eine gesellschaftliche Debatte zwischen den verschiedenen Stakeholdern zu etablieren, um so ein "grundlegendes Verständnis in der Gesellschaft« (VCI 2017) zu fördern und »gemeinsam Lösungsansätze zu diskutieren« (VDMA 2019). Die Branchen stehen hier noch am Anfang, insofern auch in strategischer Hinsicht erst einmal davon gesprochen wird, Lösungen zu entwickeln und die benötigten Daten dafür zusammen zu tragen. Die Ausgangsvermutung, wonach technische Potentiale und aktuell bestehende Möglichkeiten, zum Beispiel die Einführung flexibler, netzdienlicher Produktionsprozesse nicht genutzt werden, kann also insofern als bestätigt gelten, als dass derartige Verknüpfungen im Datenmaterial nicht auszumachen waren.

\section{Veränderungen innerhalb des Untersuchungszeitraums}

Im Zeitverlauf lässt sich erkennen, dass die Industrie sich mit dem Thema Energie und besonders mit der Digitalisierung zunehmend auseinandersetzt. Während vor 2015 nur eine Pressemitteilung mit dem Stichwort "Digital« auftaucht, wird das Thema erst ab hier stärker aufgegriffen. Ab 2018 ist noch einmal ein bedeutender Anstieg zu verzeichnen. Eine mögliche Erklärung für diesen Anstieg ist, dass digitale Inhalte in diesem Jahr verstärkt durch die Bundes- und Landesregierungen aufgegriffen werden, die dann in den Pressemitteilungen thematisiert werden. Das Bundesministerium für Wirtschaft und Energie verabschiedete beispielsweise im November 2018 die Umsetzungsstrategie »Digitalisierung gestalten « (BMWE 2019) und auch das NRW-Ministerium für Wirtschaft, Innovation, Digitalisierung und Energie etablierte ab 2017 die Initiative »Digitale Wirtschaft NRW« (MWIDE NRW 2019). 
Abb. 4: Anzahl der Pressemitteilungen im Datenkorpus 2011- 2018;

eigene Darstellung

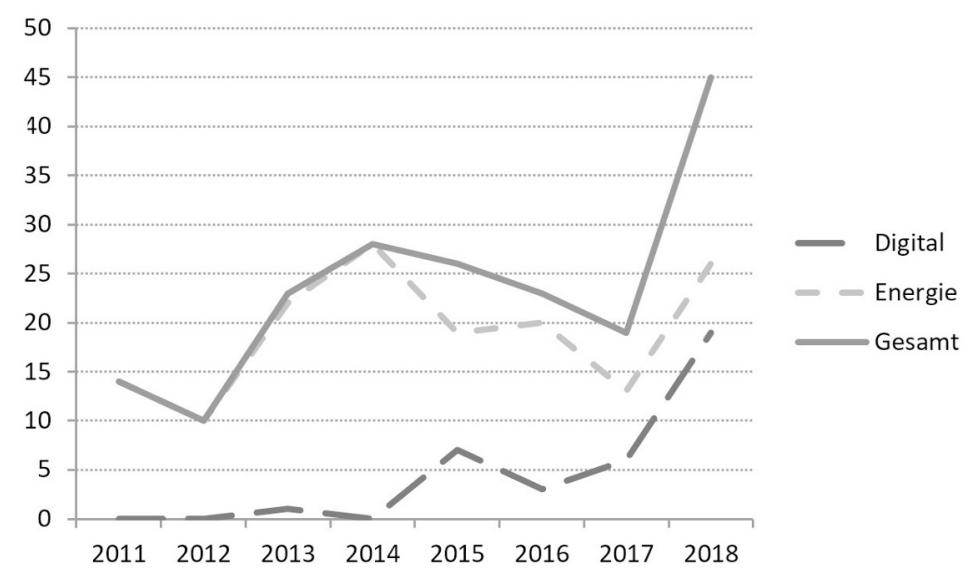

Abb. 5: Anzahl der Pressemitteilungen im Datenkorpus o1 /2011-01/2019 nach Quartalen; eigene Darstellung

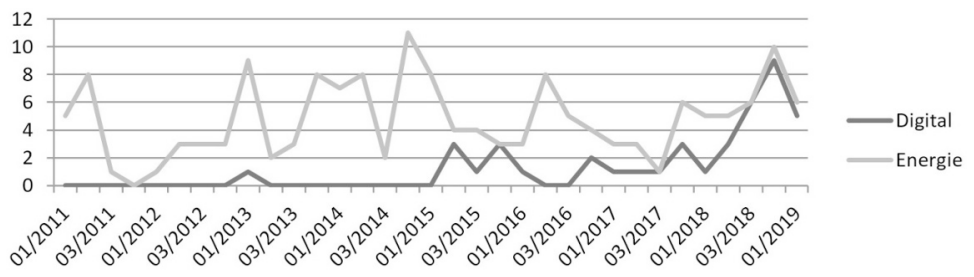

Die Anzahl der Pressemitteilungen, die sich mit dem Thema Energie auseinandersetzt, unterliegt im Zeitablauf großen Schwankungen, was insbesondere in der chronologischen Darstellung nach Quartalen deutlich wird (Abb. 5). Diese Schwankungen können als Indiz für den viel beklagten »allgemeinen Wirrwarr (IG BCE 2012) und fehlende »langfristig stabile Rahmenbedingungen « (VDMA 2018) in der Gesetzgebung gedeutet werden. Denn tatsächlich weisen die Spitzenwerte in der Graphik, also die Quartale, in denen mit sie- 
ben oder mehr vergleichsweise viele Pressemitteilungen veröffentlicht wurden, auffallende Zusammenhänge zu den Reformen in der deutschen Energiegesetzgebung im Untersuchungszeitraum auf, so z.B. zum Beschluss der Energiewende angesichts der Nuklearkatastrophe in Fukushima im Jahr 2011 oder zu den EEG-Reformen 2014 und $2016 .^{9}$

\section{Fazit und Ausblick}

Die Ergebnisse verdeutlichen, dass die energieintensive Industrie, vertreten durch die Branchen Chemie und Metall, sowie der Maschinenbau die Prozesse Digitalisierung und Energiewende als Herausforderungen mit transformativem Charakter erkannt haben. Die Industrie teilt also die Einschätzung, dass es sich sowohl bei der Digitalisierung als auch bei der Energiewende um langfristige und tiefgreifende Prozesse mit gesamtgesellschaftlicher Reichweite handelt. Entgegen der anfänglich getroffenen Annahme zur Erstellung des Datenkorpus allerdings führte die Einführung des Begriffs >Industrie 4.0< nicht zu einem verstärkten Diskurs über die Digitalisierung; Pressemitteilungen, die sich mit digitalen Themen auseinandersetzen, sind erst ab 2015 überhaupt und $a b 2018$ in verstärktem Maße im Datenmaterial zu finden.

Trotz einer grundlegend ähnlichen Einschätzung der beiden Megathemen sind jedoch wesentliche Unterschiede zu verzeichnen, was den Umgang mit den Themen betrifft: So wird die Digitalisierung in ihren Auswirkungen vornehmlich positiv bis neutral eingeschätzt. Es werden dementsprechend eher die Chancen oder der transformative Charakter der Digitalisierung diskutiert, wonach man diesen Prozess notwendig mitgehen muss, um den Anschluss an gesamtgesellschaftliche und somit auch ökonomische Entwicklungen nicht zu verpassen. Das in den Pressemitteilungen gezeichnete Bild der Energiewende ist hingegen tendenziell eher negativ behaftet. So wird die Energiewende an sich bzw. die Notwendigkeit ihrer Durchführung zwar nicht in Frage gestellt, aber dennoch wird sie von der Industrie als mit großen Risiken verbundene Herausforderung gesehen, die nur mithilfe politischer Unterstützung geschultert werden kann. Dies zeigt sich nicht zuletzt daran, dass ausgetragenen Tarifverhandlungen der IC BCE. 
die Pressemitteilungen, die sich mit der Energiewende befassen, zum Großteil politische Instrumente wie etwa energierechtliche Änderungen diskutieren.

Beide Themen im Zusammenhang werden auffallend selten diskutiert. Zwar werden digitale Mittel als Chance begriffen, die Energiewende schneller und effizienter umzusetzen - an konkreten Handlungsansätzen mangelt es jedoch bislang. Die Flexibilisierung von Lasten als möglicher Beitrag zur Energiewende wurde im Datenmaterial nicht erwähnt. Dementsprechend scheint dem Thema zumindest auf Branchenebene noch keine zentrale Bedeutung zuzukommen, obwohl es auch als eine Möglichkeit gesehen werden könnte, den viel diskutierten Herausforderungen der Energiewende, wie z.B. den hohen Strompreisen und der in Frage gestellten Energie-Versorgungssicherheit zu begegnen.

Selbstverständlich muss hier auch wieder beachtet werden, dass ein Blick auf branchenweite Pressemitteilungen nicht bedeutet, dass innerhalb einzelner Unternehmen nicht an diesen Themen gearbeitet wird. Weiterhin könnte eine Erweiterung der Suchbegriffe um die Stichworte Industrie 4.0 und Flexibilisierung ggf. weitere Ergebnisse bringen, gerade im Hinblick auf konkrete Handlungsansätze wie die Flexibilisierung industrieller Lasten. Auch eine repräsentative Untersuchung einzelner Unternehmen würde sich hier für weitere Forschung anbieten. Das vordergründige Ziel dieser Analyse war jedoch eine erste Einordnung dahingehend, inwiefern Energiewende und Digitalisierung innerhalb der Pressemitteilungen von Branchenverbänden verhandelt werden.

\section{Literatur}

Ausfelder, Florian/Seitz, Antje/Roon, Serafin von (2018): Flexibilitätsoptionen in der Grundstoffindustrie. Methodik, Potenziale, Hemmnisse, Frankfurt a.M.: DECHEMA, Gesellschaft für Chemische Technik und Biotechnologie.

Bertenrath, Roman/Fritsch, Manuel/Lichtblau, Karl/Schleiermacher, Thomas (2017): Digitale Wirtschaft Nordrhein-Westfalen. Studie im Auftrag der Initiative Digitale Wirtschaft NRW des Ministeriums für Wirtschaft, Energie, Industrie, Mittelstand und Handwerk des Landes NordrheinWestfalen, Hg. v. IW Consult GmbH. Köln.

Bindel, Ralf (2018): »Der digitale Wandel«, in: factorY (1), S. 2-3. 
Buber, Tim/Gruber, Anna/Klobasa, Marian/Roon, Serafin von (2013): »Lastmanagement für Systemdienstleistungen und zur Reduktion der Spitzenlast« in: Vierteljahrshefte zur Wirtschaftsforschung 82, S. 89-106. https:// doi.org/10.3790/vjh.82.3.89

Bundesministerium für Wirtschaft und Energie (BMWE) (2015): Ein Strommarkt für die Energiewende. Ergebnispapier des Bundesministeriums für Wirtschaft und Energie, Berlin: Weißbuch.

Bundesministerium für Wirtschaft und Energie (BMWE) (2019): Digitalisierung. Den digitalen Wandel gestalten. Online verfügbar unter: https:// www.bmwi.de/Redaktion/DE/Dossier/digitalisierung.html. Zuletzt aufgerufen am 26.08.2019.

Bundesnetzagentur/Bundeskartellamt (2019): Monitoringbericht 2018, Bonn.

Deutsche Energie-Agentur (dena) (2010): dena-Netzstudie II. Integration erneuerbarer Energien in die deutsche Stromversorgung im Zeitraum 2015 - 2020 mit Ausblick auf 2025, Berlin.

Doleski, Oliver D. (2016): Utility 4.0. Transformation vom Versorgungs- zum digitalen Energiedienstleistungsunternehmen, Wiesbaden: Springer.

EnergieAgentur.NRW (2016): Lastmanagement in Nordrhein-Westfalen: Potenziale, Hemmnisse, Handlungsoptionen, Düsseldorf.

Fricke, Hauke/Thiessen, Thomas (2016): Mittelstand im Wandel - Wie ein Unternehmen seinen digitalen Reifegrad ermitteln kann, Berlin: BSP Business School Berlin GmbH.

Gochermann, Josef (2016): Expedition Energiewende, 1. Auflage, Wiesbaden: Springer Spektrum. https://doi.org/10.1007/978-3-658-09852-0_1

Grömling, Michael/Haß, Hans-Joachim (2009): Globale Megatrends und Perspektiven der deutschen Industrie (= IW-Analysen, Nr. 47), Köln: Deutscher Instituts-Verlag.

Haas, Tobias (2016): Die politische Ökonomie der Energiewende. Dissertation. Unter Mitarbeit von Achim Brunnengräber (Energiepolitik und Klimaschutz).

Handelsblatt (2019): Saubere Revolution ohne Deutschland? Interview mit Daniel Kray. Hg. v. Handelsblatt. Online verfügbar unter: https:// veranstaltungen.handelsblatt.com/energie/saubere-revolution-ohnedeutschland-pc/. Zuletzt aufgerufen am 26.03.2019.

IG Bergbau, Chemie und Energie (IG BCE) (2012): »IG BCE fordert Energiepolitik aus einem Guss. Monitoringbericht Energiewende." Online verfügbar unter: https://www.igbce.de/presse/pressearchiv/xvi-19-12-2012- 
monitoringbericht-energiewende/19452?back=true\&highlightTerms=. Zuletzt aufgerufen am 15.07.2019.

IG Bergbau, Chemie und Energie (IG BCE) (2014): »Ermutigendes Signal für Neuorientierung der Energiepolitik. Vassiliadis begrüßt Vorstoß des Wirtschaftsministers.« Online verfügbar unter: https://www.igbce.de/ themen/energie/xviii-53-neuorientierung-energiepolitik/90654?back=. Zuletzt aufgerufen am 26.08.2019.

IG Bergbau, Chemie und Energie (IG BCE) (2016): »Jetzt Tempo machen.« Online verfügbar unter: https://www.igbce.de/presse/pressearchiv/xx-25vattenfall-verkauf/131810?back=true\&highlightTerms=. Zuletzt aufgerufen am 15.07.2019.

IT.NRW (2016): Erhebung über die Energieverwendung der Betriebe des Verarb. Gewerbes sowie des Bergbaus und der Gewinnung von Steinen und Erden, Nordrhein-Westfalen.

Lütkenhorst, Wilfried (2018): Creating wealth without labour? Emerging contours of a new techno-economic landscape, in: DIE Discussion Paper 11. https://doi.org/10.23661/dp11.2018

Mayring, Philipp (2015): Qualitative Inhaltsanalyse. Grundlagen und Techniken, Weinheim: Beltz. https://doi.org/10.1007/978-3-531-18939-0_38

Mertens, Peter/Barbian, Dina/Baier, Stephan (2017): Digitalisierung und Industrie 4.0 - eine Relativierung, Wiesbaden: Springer. https://doi.org/10. 1007/978-3-658-19632-5

Ministerium für Wirtschaft, Innovation, Digitalisierung und Energie des Landes Nordrhein-Westfalen (MWIDE) (2019): »Digitale Wirtschaft«. Online verfügbar unter: https://www.wirtschaft.nrw/digitale-wirtschaft. Zuletzt aufgerufen am 26.08.2019.

Paul, Hansjürgen (2016): »Industrie 4.o. Annäherung an ein Konzept.«, in: IAT - Forschung aktuell 5, S. 1-21.

Scharl, Stefan/Praktiknjo, Aaron (2019): »The Role of a Digital Industry 4.0 in a Renewable Energy System«, in: Int J Energy Res 43, S. 3891-3904. https:// doi.org/10.1002/er.4462

Seidl, Hannes/Schenuit, Carolin/Teichmann, Mario (2016): Roadmap Demand Side Management. Industrielles Lastmanagement für ein zukunftsfähiges Energiesystem. Schlussfolgerungen aus dem Pilotprojekt DSM Bayern, Berlin: Deutsche Energie-Agentur.

Statistische Ämter des Bundes und der Länder (2016-2019): Umweltökonomische Gesamtrechnungen der Länder. Primärenergieverbrauch. Gemeinsames Statistikportal. 
Tennet/50 Hertz/Transnet BW/Amprion (2018): »Präqualifizierte Leistung in Deutschland«. Online verfügbar unter: www.regelleistung.net/ext/download/pq_capacity. Zuletzt aufgerufen am 12.11.2018.

Trimet Aluminium SE (2018): Geschäftsbericht 2017/18. Online verfügbar unter: https:/www.trimet.eu/de/geschaeftsberichte/trimetgeschaeftsbericht-2018.pdf. Zuletzt aufgerufen am 13.02.2020.

Trost, Esther/Büttgen, Alexandra/Geringhoff, Lisa (2016): »Deutungsmuster der Energiewende auf lokaler Ebene. Erste Ergebnisse aus NRW.«, in: KWI-Working Paper 04.

Vallentin, Daniel/Wehnert, Timon/Mölter, Helena/Best, Ben (2016): Klimaschutz- und Energiewende-Innovationen in der (energieintensiven) Industrie. Fallstudien zur Entstehung innovativer Prozesse und Produkte, Wuppertal: Wuppertal Institut.

VDI Zentrum Ressourceneffizienz (Hg.) (2017): Ressourceneffizienz durch Industrie 4.0. Potenziale für KMU des verarbeitenden Gewerbes. Paderborn.

Verband der Chemischen Industrie e.V. (VCI) (2013): »Europa braucht Klimapolitik mit Weitblick«. Online verfügbar unter: https:/www.vci.de/ presse/pressemitteilungen/2013-03-27-europa-braucht-klimapolitikmit-weitblick-vci.jsp. Zuletzt aufgerufen am 15.07.2019.

Verband der Chemischen Industrie e.V. (VCI) (2014): »Verlorenes Jahr für den Chemie-Mittelstand. Mittelständische Unternehmen sehen Kostenprobleme der Energiewende weiter ungelöst.«. Online verfügbar unter: https://www.vci.de/presse/pressemitteilungen/2014-11-18verlorenes-jahr-fur-den-chemie-mittelstand-vci.jsp. Zuletzt aufgerufen am 15.07.2019.

Verband der Chemischen Industrie e.V. (VCI) (2017): »Chemie 4.0: Innovationen für eine Welt im Umbruch«. Online verfügbar unter: https://www. vci.de/presse/pressemitteilungen/chemie-4-punkt-o-innovationenfuer-eine-welt-im-umbruch-studie-von-deloitte-und-vci.jsp. Zuletzt aufgerufen am 10.07.2019.

Verband der Chemischen Industrie e.V. (VCI) (2018): »Wie Nachhaltigkeit gelingt. Zukunft der Chemie- und Pharma-Industrie.«. Online verfügbar unter: https://www.vci.de/presse/pressemitteilungen/wienachhaltigkeit-gelingt-zukunft-der-chemie-und-pharma-industrie.jsp. Zuletzt aufgerufen am 15.07.2019.

Verband Deutscher Maschinen- und Anlagenbau (VDMA) (2018): »Klimaziele und Energiewende sind nur mit Power-to-X erreichbar«. Online verfügbar 
unter: https://www.vdma.org/v2viewer/-/v2article/render/27599230. Zuletzt aufgerufen am 28.08.2019.

Verband Deutscher Maschinen- und Anlagenbau (VDMA) (2019): »Industriemotorenbranche setzt auf Power-to-X und synthetische Kraftstoffe«. Online verfügbar unter: https://www.vdma.org/v2viewer/-/v2article/render/ 29361617. Zuletzt aufgerufen am 24.07.2019.

Verordnung $\mathrm{zu}$ abschaltbaren Lasten vom 16. August 2016 (BGBl. I S. 1984), die zuletzt durch Artikel 9 des Gesetzes vom 22. Dezember 2016 (BGBl. I S. 3106) geändert worden ist.

Werning, Elena/Lentz, Patrick/Wittberg, Volker/Sandoval, Charlotte/Lupp, Nathalie/Fechner, Sandra (2017): Studie Digitalisierungsindex bei KMU in NRW. Ergebnisse des Digitalisierungsstands in den Branchen Industrie, Handwerk und industrienahe Leistungen, Bielefeld: Fachhochschule des Mittelstands (FHM).

World Economic Forum (2018): Fostering Effective Energy Transition. A Fact-Based Framework to Support Decision-Making. With analytical support from McKinsey \& Company. Online verfügbar unter: http:// reports.weforum.org/fostering-effective-energy-transition-2018/foreword/?code=wr123. Zuletzt aufgerufen am 26.03.2019.

Z_punkt GmbH (2019): »Der neue Blick auf die globale Dynamik des Wandels«. Online verfügbar unter: www.z-punkt.de/de/themen/artikel/megatrends. Zuletzt aufgerufen am 09.07.2019.

Zukunftsinstitut GmbH (o.J.): »Megatrends«. Online verfügbar unter: https:// www.zukunftsinstitut.de/dossier/megatrends/. Zuletzt aufgerufen am 09.07.2019. 



\section{Megatrends und die Energiewende in NRW aus Sicht kommunaler Akteure ${ }^{1}$ Einblicke in eine NRW-Gemeindestudie}

Juliane Lunge, Benjamin Best und Katja Witte

Dass Megatrends das Leben in den Städten und Gemeinden sowie die Umsetzung der Energiewende in NRW maßgeblich beeinflussen, ist nicht von der Hand zu weisen. Aber bei Entscheidungsträger*innen in Verwaltung, Politik und Wirtschaft auf kommunaler Ebene klafft eine Lücke zwischen Wissen über Megatrends und umsetzungsorientierter Reaktionen auf diese. Dabei sind die Handlungsmöglichkeiten an der Schnittstelle von Energiewende und Megatrends umfassend - und noch lange nicht ausgeschöpft.

\section{Globale Megatrends und die Energiewende auf kommunaler Ebene}

Globale Megatrends gelten als schwerwiegende Veränderungen, die sich jetzt und in den kommenden Dekaden auf den Zustand unserer Umwelt, Wirtschaft und Gesellschaft auswirken (Hajkowicz 2012: 2). Auch die Energiewende, als eine der größten Transformationsherausforderungen unserer Zeit, wird maßgeblich von äußeren Einflüssen geprägt. In der Transitionstheorie werden langfristige Megatrends auf der landscape Ebene verortet (Geels et al. 2016: 896). Gleichzeitig bestimmt die deutsche Energiewende allerdings auch maßgeblich globale, nationale und lokale Prozesse. Diese Wechselwirkungen zwischen Megatrends und Energiewende zu erfassen ist essentiell, um »sehr

1 Dieser Beitrag ist im Rahmen des Forschungsprojektes »EnerTrend: Systemische Analyse von Wechselwirkungen der Energiewende in NRW mit zentralen Megatrends « entstanden. Die Zuwendung für dieses Projekt stammt vom Ministerium für Wirtschaft, Innovation, Digitalisierung und Energie des Landes Nordrhein-Westfalen (MWIDE). 
früh mögliche Konfliktfelder zu erkennen und gegenzusteuern oder Synergieeffekte zu identifizieren, deren Aufgreifen beschleunigend wirken kann.« (Fischedick 2016: 48).

Es gibt unterschiedliche Ansichten darüber, welche Megatrends existieren bzw. je nach räumlichen und politischen Kontextbedingungen relevant sind. So stellt das Zukunftsinstitut in ihrer »Megatrend-Map « eine Vielzahl globaler Trends und Subtrends mit Verflechtungen untereinander dar (Zukunftsinstitut o.J.). Das Virtuelle Institut (VI) »Transformation Energiewende NRW« veröffentlichte in der Studie »Megatrends unserer Zeit « im Rahmen des Projekts EnerTrend ${ }^{2}$ eine Auswahl von Trends, welche die Vielschichtigkeit der Megatrends andeuten und starke Anknüpfungspunkte an kommunale Governance aufweisen und deren Überschneidungen, die sie mit der Energiewende haben (Virtuelles Institut 2019).

Trotz unterschiedlicher Ansichten darüber, welche Megatrends sowohl aktuell sind als auch zukünftig relevant sein werden, herrscht in der Literatur Einigkeit, dass Megatrends langfristige und fundamentale Veränderungen in sämtlichen Lebensbereichen entfalten werden. Durch diese vorhergesagte »Garantie« von Veränderungen ergeben sich einerseits Risiken, wenn die Auswirkungen eines Megatrends unvorbereitete Gesellschaften treffen, als auch Chancen, wenn sich strategisch auf Megatrends eingestellt wird. Dies beschreibt auch John Naisbitt, der den Begriff Megatrend maßgeblich in seinem Buch »Megatrends« von 1982 geprägt hat, in einem Interview: »Megatrends zeichnen ein Bild zukünftiger Entwicklungen. Also können sie als Wegweiser für langfristige strategische Planungen dienen« (Naisbitt 2015: 3). Dementsprechend ist es von Bedeutung, dass Akteure in Entscheidungspositionen sich mit dem aktuellen Stand der Megatrendentwicklung auseinandersetzen. Da globale Megatrends wie beispielsweise die Digitalisierung oder Demographischer Wandel Auswirkungen auf sämtliche Bereiche einer Gesellschaft haben, stehen dementsprechend viele Akteure auf den unterschiedlichen Entscheidungsebenen vor der Aufgabe sich mit den Trends auseinanderzusetzen. Dazu zählen neben Politik auch Wirtschaft, Verwaltung, Zivilgesellschaft oder auch Nichtregierungsorganisationen.

Der Fokus des Beitrags in der Auseinandersetzung mit der Schnittstelle zwischen globalen Megatrends und der Energiewende liegt auf der kom- 
munalen Ebene. Unter dem Begriff der Kommune werden nach Bogumil und Holtkamp (2013) sowohl die Gemeinden, kreisfreien Städte, kreisangehörigen Städte und die Landkreise gefasst. Die kommunale Ebene ist die unterste politische Ebene, welche im deutschen politischen System über ein grundgesetzlich garantiertes Selbstverwaltungsrecht (beispielsweise über die Organisations-, Finanz- und Satzungshoheit im Gemeindegebiet) verfügt. Darüber hinaus werden auf kommunaler Ebene wesentliche politische Entscheidungen getroffen, die eine unmittelbare Wirkung auf die Lebensumstände der Bürger*innen haben, beispielsweise in den Bereichen Versorgung (z.B. Einkaufsmöglichkeiten), Fürsorge (z.B. Pflege- und Betreuungseinrichtungen) und Raumplanung (z.B. Verkehrswegeplanung). Durch die geringe Distanz auf lokaler Ebene entstehen unmittelbare Mitwirkungsmöglichkeiten für Bürger*innen (Bogumil/Holtkamp 2013: 8): »die lokale Ebene ist also die Ebene umfassender Mitwirkungsmöglichkeiten.« (Ebd.) Auch der Wissenschaftliche Beirat der Bundesregierung Globale Umweltveränderungen (WBGU) (2016) schreibt den Städten als »weltweit wichtigste Siedlungs- und Lebensräume der Menschheit « eine zentrale Rolle, im Sinne ihrer Bedeutung etwa als politische Steuerungszentralen und kreative Entfaltungsräume zu. So wird gesagt: »Städte sind Orte, in denen sich globaler Wandel niederschlägt und vorangetrieben wird: Städte und ihre Bevölkerung sind gleichzeitig Treiber und Betroffene globalen Wandels.«(S. 43). Gegenwärtig zeichnet sich $\mathrm{ab}$, dass es in Städten viele unterschiedliche Wege zur Umsetzung globaler nachhaltiger Entwicklungsziele (SDGs) gibt. Aufgrund der unmittelbaren Relevanz für das Leben der Bürger*innen sowie ihrer umfassenden Aufgabenbereiche, stehen Entscheidungsträger*innen auf dieser Ebene hier im Fokus der Betrachtung.

Für ihre Arbeit in den Städten und Gemeinden in Nordrhein-Westfalen sind globale Megatrends von immenser Bedeutung - das zeigt sich bereits heute anhand der Trends Demographischer Wandel und Digitalisierung. Zukünftig werden die verschiedenen Megatrends für das Leben und Handeln in den Städten und Gemeinden in NRW weiter an Bedeutung gewinnen, etwa in Fragen nach neuen, individualisierbaren Arbeitsformen oder wachsenden sozialen Ungleichheiten. Dabei stellen sich die Fragen, ob kommunale Entscheidungsträger*innen globale Megatrends in ihrer Gemeindearbeit wahrnehmen, wie ihr Wissensstand in Hinblick auf Megatrends ist, ob bereits heute Maßnahmen in dem Kontext auf den Weg gebracht werden und wie sie ihre Handlungsmöglichkeiten in den Kommunen einschätzen. Diese The- 
men wurden im Rahmen des VI-Projekts EnerTrend im Jahr 2019 mithilfe einer NRW-Gemeindestudie adressiert.

Dieser Beitrag soll:

- darlegen, wie ausgewählte kommunale Akteure in NRW bestimmte Megatrends wahrnehmen, ob eine Notwendigkeit zur Reaktion auf die Trends erkannt wird und wie eine solche aussehen kann;

- aufzeigen, wo Treiber und Hemmnisse im Umgang mit globalen Megatrends und der Energiewende bei kommunalen Akteuren liegen können.

\section{Einblick in die NRW-Gemeindestudie: Methodik und Vorgehen}

Den Kern der explorativ angelegten Gemeindestudie bilden eine quantitative Online-Befragung und 24 qualitative Interviews. Beide Methoden legen einen inhaltlichen Fokus auf folgende vier Megatrends:

- Digitalisierung

- Soziale Disparitäten

- Übernutzung natürlicher Ressourcen

- Urbanisierung und Demographischer Wandel

Die Megatrends wurden auf Basis einer umfangreichen Literaturrecherche ausgewählt. Kriterien zur Auswahl waren eine möglichst hohe allgemeine Verständlichkeit des Megatrends, ein aus der Literatur ableitbarer Zusammenhang zur Energiewende sowie eine angenommene Relevanz für Kommunen in NRW. Eine Festlegung auf ausgewählte Trends ermöglicht eine Vergleichbarkeit der jeweiligen Antworten innerhalb der einzelnen Megatrends sowie über die verschiedenen Megatrends hinaus. Die Interviews boten die Möglichkeit, mithilfe von offenen Fragestellungen weitere Megatrends neben den vier ausgewählten in die Analyse mit aufzunehmen.

Das methodische Vorgehen der Gemeindestudie folgt einem »Mixed Methods «-Ansatz (nach Baur/Blasius 2014), um einerseits sowohl der Komplexität des Themas Energiewende und Megatrends gerecht zu werden als auch verschiedene Forschungserkenntnisse zu ermöglichen. Durch die NRW-weit angelegte Online-Befragung soll einerseits eine flächendeckende und vergleichende Status-Quo-Analyse in den Städten und Gemeinden NRWs ermög- 
licht werden. Die qualitativen Interviews ermöglichen andererseits eine tiefenscharfe Analyse ausgewählter Kommunen, wodurch mögliche Erklärungszusammenhänge erkannt werden können.

Die Einladung zur Teilnahme an der Online-Befragung visierte alle 396 Städte und Gemeinden in NRW an. Um eine möglichst große Überschneidung mit den vier Megatrends und der Energiewende zu gewährleisten, wurde die Ebene der technisch ausgerichteten Dezernatsleitungen, zumeist Umwelt- oder Versorgungsdezernate, angesprochen. Es wurde jedoch in der Ansprache ausdrücklich darauf hingewiesen, dass die Befragung von Personen aus unterschiedlichen Fachdezernaten innerhalb einer Kommune beantwortet werden kann, um möglichst die gesamte Breite der behandelten Themen abfragen zu können. Die entsprechenden E-Mail-Adressen der Umweltdezernatsleitungen der 396 Kommunen NRWs wurden über die Homepages der Städte und Gemeinden identifiziert - mit sehr wenigen Ausnahmen. Die Einladung zur Teilnahme an der Befragung wurde in Summe an 374 E-Mail-Adressen geschickt.

Die Fragen $\mathrm{zu}$ den vier ausgewählten Megatrends in der OnlineBefragung folgten diesen Analysedimensionen: Wahrnehmung, Wissen, Einstellungen, Verhalten und Handlungskapazitäten. Ziel der Befragung war es, einen Überblick und Vergleich zwischen Kommunen in NRW und zwischen den Megatrends und deren Wechselwirkungen zur Energiewende ermöglichen. Von den 374 angefragten Städten und Gemeinden haben 64 an der Befragung teilgenommen. Dadurch ergibt sich eine Rücklaufquote von knapp über $17 \%$, dies entspricht bezogen auf die Gesamtzahl der in NRW ansässigen Städte und Gemeinden einem Anteil von $16 \%$. Eine generelle Übertragbarkeit der Ergebnisse auf alle Städte und Gemeinden in NRW ist damit nicht leistbar, d.h. Repräsentativität ist nicht gewährleistet. Jedoch zeigen die Ergebnisse Tendenzen auf und bieten einen wertvollen Ausgangspunk für die Diskussion über kommunale Akteure und deren Sichtweise auf globale Megatrends und die Energiewende in NRW.

Zur Diskussion der Ergebnisse der Online-Befragung sowie um praxisorientierte Einblicke in die Zusammenhänge globaler Megatrends und der Energiewende zu bekommen, wurden im Anschluss an die Online-Befragung 24 qualitative Interviews mit kommunalen Entscheidungsträger*innen geführt. Sie kamen aus den Bereichen Verwaltung (z.B. Bauamt oder Klimaschutzmanagement), Politik (z.B. Bürgermeister*innen) und Wirtschaft (z.B. Leitungspersonen aus Stadtwerken). Dafür wurde ein semistrukturierter Leitfaden entwickelt. Die Auswahl der Städte und Gemeinden erfolgte anhand von 
Interessensbekundungen im Nachgang an die Online-Befragung sowie einer Desktop-Recherche, um Städte und Gemeinden zu identifizieren, welche sich in besonderem Maße mit einem der ausgewählten Megatrends oder der Energiewende auseinandersetzen. Um darüber hinaus ein vielfältiges Bild der Städte und Gemeinden in NRW und deren Umgang mit gesellschaftlichen Megatrends zu erhalten, wurden Kommunen unterschiedlicher siedlungsstruktureller Prägung ausgewählt: ländlich, teilweise städtisch und überwiegend städtisch.

\section{Wahrnehmung von Megatrends im Zusammenhang mit der Energiewende in den Gemeinden}

Die Ergebnisse aus Online-Befragung und Interviews zeigen: der Großteil der befragten kommunalen Akteure aus NRW nimmt die vier abgefragten Megatrends als relevant wahr. Es wird deutlich, dass die Trends sowohl als in sich bedeutsame Entwicklungen als auch im Zusammenhang mit der Energiewende wahrgenommen werden. Dabei sind jedoch starke Unterschiede sowohl in der Auffassung der unterschiedlichen Trends als auch in der Betroffenheit zwischen befragten Städten und Gemeinden in NRW erkennbar.

Besonders starke Zustimmung erhalten in diesem Sinne die Megatrends Digitalisierung und Demographischer Wandel (siehe Abb. 1). Bei beiden Trends ist die Mehrheit der befragten kommunalen Entscheidungsträger*innen der Meinung, dass diese bereits jetzt wie auch in Zukunft für die Städte und Gemeinden eine wichtige Rolle spielen. Im Themenfeld Demographischer Wandel werden insbesondere Veränderungen in der Bevölkerungsstruktur, wie Veränderungen in der Altersstruktur der Bevölkerung, als Herausforderung für die Gemeindeentwicklung allgemein genannt. Im Zusammenhang mit der Energiewende erkennen die Befragten die Spannungsfelder des Wohngebäudebereiches sowie der Versorgungsinfrastruktur, hierbei vor allem der Bereich Mobilität. Während Veränderungen in der Bevölkerungsstruktur die Städte und Gemeinden in NRW seit jeher prägen, ist das Thema Digitalisierung in den letzten Jahren verstärkt aufgekommen. Aus den Interviews wird deutlich: Der Digitalisierung wird von den Gemeinden mehrheitlich eine impulsgebende Rolle für die Energiewende zugeschrieben, wie etwa eine stärkere Flexibilisierung des Energiesystems. Bedenken über Risiken, wie im Bereich Datenschutz, werden nur vereinzelt genannt. Im Allgemeinen werden in den Interviews häufig die Digitalisierung von Verwal- 
Abb. 1: Zustimmung (»absolut« und »stark«vs. »absolut nicht« und »kaum«) zu der aktuellen Wahrnehmung der Trends Soziale Disparitäten, Übernutzung natürlicher Ressourcen, Digitalisierung, Demographischer Wandel im Rahmen der OnlineBefragung ( $n=64)$; eigene Darstellung

Dieser Trend ist in unserer Kommune aktuell ein Thema

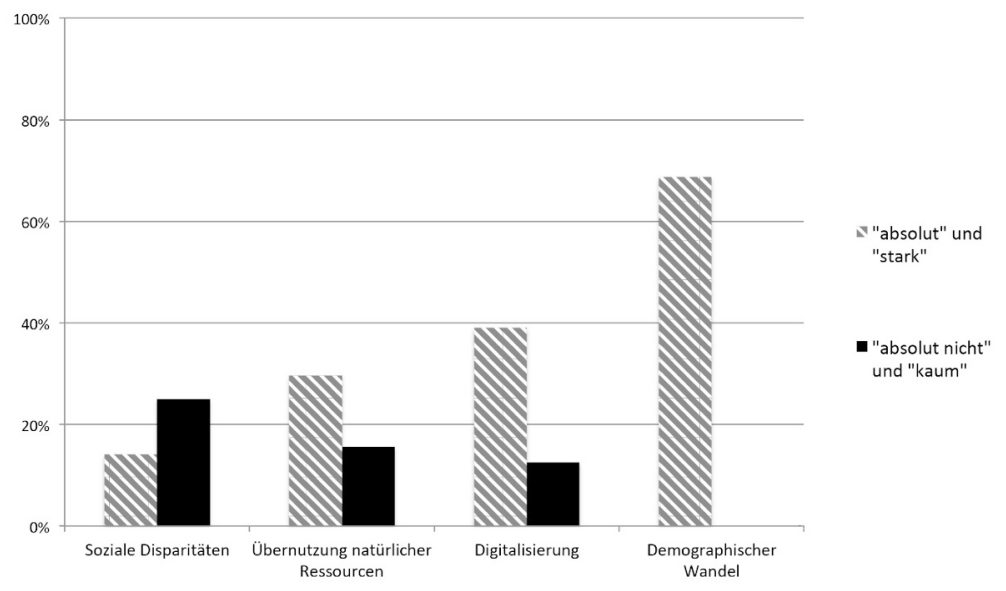

tungsabläufen sowie der Breitbandausbau als geläufige Schwerpunktthemen genannt.

In der Wahrnehmung der Trends Soziale Disparitäten und Übernutzung natürlicher Ressourcen gibt es größere Diskrepanzen zwischen den Befragten (siehe Abb. 1). Soziale Disparitäten werden stark abhängig von der siedlungsstrukturellen Prägung der Städte und Gemeinden eingeschätzt - hier sind insbesondere Unterschiede zwischen ländlich und städtisch geprägten Räumen von Bedeutung. So geben mehrere Interviewpartner*innen an, aufgrund eines starken sozialen Zusammenhalts und einer wirtschaftlich stabil aufgestellten Bevölkerung in der eigenen, ländlich geprägten Gemeinde, kaum Berührungspunkte mit sozialen Ungleichheiten beobachten zu können. Dies ist für NRW nicht überraschend, da das Bundesland grundsätzlich von starken, sozio-ökonomischen Unterschieden geprägt ist (Ministerium für Arbeit, Integration und Soziales des Landes Nordrhein-Westfalen 2016). Im Hinblick auf die Energiewende werden - sowohl in der Online-Befragung als auch in 
den Interviews - tendenziell negative sozio-ökonomische Auswirkungen wie Verteilungswirkungen zuungunsten heute bereits armer Haushalte erwartet.

Der Trend der Übernutzung natürlicher Ressourcen wird durch die befragten Akteure sehr unterschiedlich eingeschätzt; es lässt sich kein eindeutiges Bild zeichnen, wie lokale Akteure die Brücke zwischen global wirkenden Dynamiken und der Betroffenheit und Bedeutung auf lokaler Ebene schlagen. Das liegt insbesondere daran, dass in den befragten Städten und Gemeinden kaum Ansätze bekannt sind, die über klassische Abfallwirtschaft hinausgehen. In den Interviews wird häufig eine individuelle Dimension eingebracht und das Thema Übernutzung natürlicher Ressourcen auf private Entscheidungen wie sparsames Verhalten, Vermeidung und Verwertung von Lebensmittelresten und Reparaturinitiativen gemünzt. Handlungs- und Eingriffsmöglichkeiten auf kommunaler Ebene werden dagegen kaum wahrgenommen.

Aus den Ergebnissen lässt sich ganz allgemein ableiten, dass den befragten kommunalen Akteuren die globalen Megatrends bewusst sind und sie sich in ihrem Arbeitsalltag bereits damit auseinandersetzen. Dies ist eine Grundvoraussetzung für die notwendige Beschäftigung mit Zukunftsthemen wie der Energiewende, um für eine nachhaltige Entwicklung der Städte und Gemeinden in NRW sorgen zu können:

»Für die erfolgreiche Umsetzung der Energiewende ist es von essentieller Bedeutung, die Zusammenhänge mit Megatrends und die wechselseitigen Abhängigkeiten besser zu durchdringen.« (Fischedick 2016: 51)

\section{Strategien für die Umsetzung von Maßnahmen und Projekten}

Wenn davon ausgegangen werden kann, dass sich das Fachpersonal in einem Großteil der Städte und Gemeinden in NRW bereits bewusst mit globalen Megatrends auseinandersetzt, stellt sich die Anschlussfrage nach den Folgen für politisches und administratives Handeln. Gibt es Strategien und Maßnahmen, welche die Schnittstelle zwischen einem oder mehreren Megatrends und der Energiewende adressieren? Auf diese Frage antworteten unsere Interviewpartner*innen sehr unterschiedlich: Einzelne Städte und Gemeinden sind bereits aktiv in der Maßnahmenumsetzung, einige haben erste Ansätze geplant und andere haben weder Maßnahmen umgesetzt noch geplant. Die folgende Liste zeigt einen Auszug der Antworten auf Maßnahmen und Strate- 
gien, welche im Spannungsfeld Megatrends und Energiewende angewendet werden:

- Kooperationen mit Institutionen, welche sich bereits intensiv mit bestimmten Themen beschäftigen. Hierzu zählen beispielsweise karitative Einrichtungen, die sich auf soziale Themen fokussieren oder Stadtwerke, Verbraucherzentralen und regionale Netzwerke, die sich mit den Themen nachhaltige Mobilität oder Stromversorgung beschäftigen.

- Die proaktive, finanzielle oder organisatorische Unterstützung von »bottom-up«-Bürgerinitiativen, wie Bürger-Energiegenossenschaften, Repair Cafés oder Unverpackt-Läden.

- Maßnahmen zur gezielten Information, Beratung und (Weiter-)Bildung, wie etwa Bildungsinitiativen an Schulen und Kitas oder Projekte zur Weiterbildung von Langzeitarbeitslosen zu Energieberater*innen.

- Maßnahmen innerhalb der Gemeindeverwaltung (z.B. Digitalisierung von Verwaltungsprozessen oder Umstellung des Fuhrparks auf E-Mobilität).

- Infrastrukturelle Maßnahmen in den Städten und Gemeinden vor Ort (z.B. Bau neuer Radverkehrsstrecken zur Stärkung von klimafreundlicher Mobilität oder die Umstellung auf digitale Kataster unter der Beteiligung von Bürger*innen).

- Das Akquirieren von Fördermitteln, etwa durch KfW-Förderlinien oder (Klimaschutz- und Energiewende-)Förderprogramme auf EU- und Bundesebene.

- Die Entwicklung von eigenen Strategien und Leitbildern innerhalb der Gemeindeverwaltung (wie »Demographiestrategie« oder »Klimaanpassungsstrategie«).

- Forschungsprojekte durch Kooperationen mit Hochschulen, beispielsweise zur Aufstellung eines Handlungsplans zum Erreichen von Energieautarkie.

Häufig meistern Gemeindeverwaltungen diese Maßnahmen in Form von freiwilligen Zusatzaufgaben, da es nur sehr begrenzte Handlungsmöglichkeiten bei diesen Schnittstellenthemen gibt. Dieser Fakt wurde in der OnlineBefragung und in den Interviews häufig bemängelt. Als Grund wurde vor allem der Mangel an finanziellen und personellen Ressourcen benannt (siehe Abb. 2). Daher ist es für einige der befragten Kommunen unverzichtbar mit externen Organisationen zu kooperieren, welche sich bereits mit den relevanten Themen beschäftigen (z.B. Angebote der Verbraucherzentralen zur 
Energieberatung oder karitative Träger für Teil- und Tausch-Angebote), sowie auch das Einwerben von Fördermitteln, mithilfe derer die zur Verfügung stehenden Haushaltsmittel ergänzt werden. Andererseits geben in der OnlineBefragung aber auch knapp ein Viertel der Befragten an, ausreichend (finanzielle) Ressourcen zur Verfügung zu haben, um auf die Auswirkungen der Megatrends reagieren zu können.

Abb. 2: Zustimmung im Rahmen der Online-Befragung (»absolut »stark" und »eher« vs. "absolut nicht«kaum « und »eher nicht«) zu den in den Gemeinden zur Verfügung stehenden Ressourcen im finanziellen und personellen Bereich sowie in Eigeninitiativen von Bürger*innen ( $n=64)$; eigene Darstellung

Diese Ressourcen stehen unserer Kommune zur Verfügung

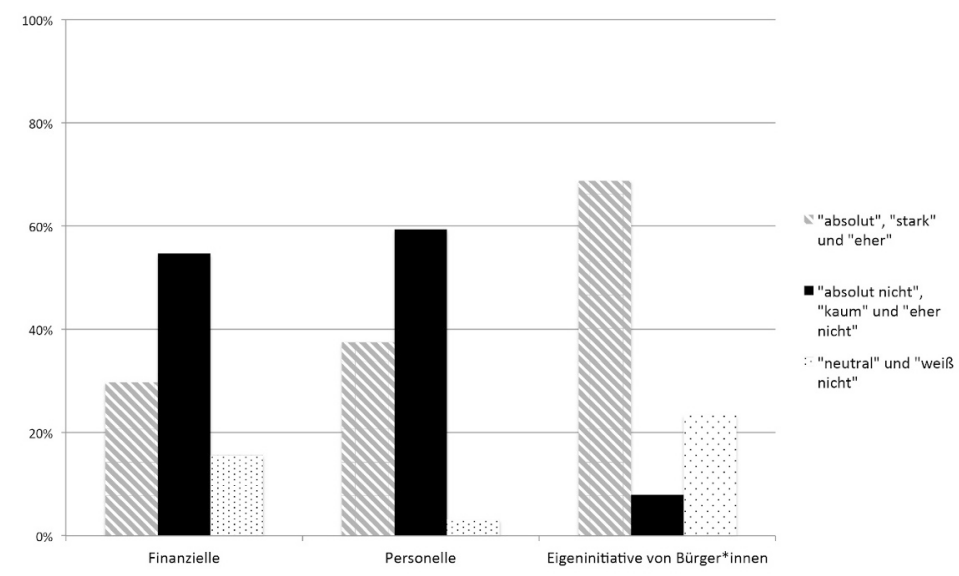

Nicht nur die wahrgenommene Relevanz der Trends, auch die Handlungsmöglichkeiten, Megatrends im Zusammenhang mit der Energiewende aktiv anzugehen, sind demnach in den Städten und Gemeinden in NRW unterschiedlich stark ausgeprägt. Wir sehen sie sowohl als eine Funktion der (siedlungs-)strukturellen und wirtschaftlichen Rahmenbedingungen der Städte als auch der Innovations- und Kooperationskultur in der Gemeinde. Es kann angenommen werden, dass ein Großteil der Städte und Gemein- 
den in NRW noch keine umfassenden Strategien zur Bewältigung globaler Megatrends im Zusammenhang mit der Energiewende entwickelt hat.

\section{Diskussion: Treiber und Hemmnisse im Umgang mit Megatrends und der Energiewende}

Globale Megatrends werden in wirtschaftlichen Organisationen bereits zur strategischen Entscheidungsfindung und Entwicklung genutzt (Retief et al. 2016: 52). Sie sind auch für das Handeln von Entscheidungsträger*innen auf kommunaler Ebene relevant. Es ist notwendig, dass die Kommunen megatrendbezogene Entwicklungen erkennen und ihr Handeln und ihre Entscheidungen darauf abstimmen. Da Megatrends sich bereits heute auswirken, sollte dies umgehend geschehen.

Aus den Ergebnissen der Gemeindestudie kann eine Tendenz zu einer Lücke zwischen dem Wissen und dem Handeln in Bezug auf die Energiewende abgeleitet werden. Es wurde deutlich, dass es vielen Kommunen zum konkreten Handeln auf kommunaler Ebene häufig an notwendigen Voraussetzungen fehlt. Es wird angenommen, dass sich kommunale Akteure in NRW der Wechselwirkungen zwischen der Energiewende und den einzelnen Megatrends bewusst sind. Eine proaktive und strategische Gestaltung dieser Wechselwirkungen erfolgt zum Großteil bisher nicht. Es sind jedoch vereinzelt Handlungsansätze vorhanden. Schwierige siedlungsstrukturelle und ökonomische Voraussetzungen allein können dies aber nicht erklären. Vielmehr werden auch komplexe Themen meist in etablierten administrativen Systemgrenzen angegangen, sprich von kommunalen Entscheidungsträger*innen innerhalb der in ihren Ressorts vorhandenen Möglichkeiten (z.B. demographische Entwicklung und Siedlungsentwicklung). Die Notwendigkeit übergreifende Lösungsansätze verbunden mit der Fragestellung der Energiewende zu entwickeln wird erkannt; solche Ansätze kommen aber nur vereinzelt bis gar nicht in die praktische Umsetzung. Dies wird z.B. anhand des Themenkomplexes Smart City deutlich: einige Interviewpartner*innen haben sich bereits in der Theorie damit beschäftigt, in ihren Kommunen die Themen Mobilität und Energie digital miteinander zu vernetzen (etwa in Smart City Konzepten). So wird auch das Konfliktfeld Wohnen im Alter/Wohnen für Familien und Energieeffizienz bereits als wichtiges Handlungsfeld erkannt, jedoch nur vereinzelt in integrativen und energieeffizienten Wohnprojekten angegangen. 
Wir gehen davon aus, dass neue Herausforderungen, wie sie an den vielfältigen Schnittstellen zwischen der Energiewende und globalen Megatrends vorkommen, neben klassischen Steuerungsansätzen neue Formen der Steuerung - sogenannte Governance-Formen - benötigen. Unter neuen Governance Formen werden Steuerungsformen verstanden, welche sich von der Annahme distanzieren, dass der Staat alleine von oben herab Gesellschaft steuert (Government) (Mann 2017: 16). Governance-Formen lassen sich als »Selbstregulierungsprozess verstehen, der nicht >von außen ‘ auf >die Gesellschaft< wirkt, sondern Ausdruck der gesellschaftlichen Willensbildung- und Steuerungsprozesse ist [...].« (Ebd.) Zentral in der Auseinandersetzung mit Megatrends auf kommunaler Ebene sind die Akteure (»Spieler« im Governance-System (ebd.: 18)). Governance muss eine Vielzahl und Heterogenität von Akteuren berücksichtigen, die in (politischen) Entscheidungsprozessen involviert sind. Der Wissenschaftliche Beirat der Bundesregierung Globale Umweltveränderungen (WBGU) beschreibt Urbane Governance, also die Steuerung in städtischen Räumen, als bestehend aus den Handlungen hoheitlicher und nichthoheitlicher Akteure und Institutionen mit dem Ziel, die gemeinsamen Angelegenheiten einer Stadt zu organisieren (WBGU 2016: 102). Auch hier wird ein Fokus auf die Pluralität von beteiligten Akteuren gelegt, wobei mit hoheitlichen Akteuren solche der Verwaltung und Politik verstanden werden und mit nicht-hoheitlichen Akteuren solche der Zivilgesellschaft und Wirtschaft.

In der Literatur wird angenommen, dass eine effiziente Problemlösung innerhalb der genannten Spannungsfelder nur durch die vertikale und horizontale Integration der verschiedenen Ebenen erfolgen kann (multi-level Governance) (Mann 2017: 19). Mit diesen Ebenen werden sowohl die verschiedenen politisch-territorialen Ebenen (lokal, regional, national, global) (ebd.) als auch die zahlreichen Akteure (hoheitlich und nicht-hoheitlich) (WBGU 2016: 107) angesprochen. Dies ist insbesondere von Bedeutung bei den Themen Megatrends und Energiewende, welche auf alle Bereiche und Ressorts der kommunalen Ebene Einflüsse haben und darüber hinaus stetig an Komplexität zunehmen. Multi-level Governance kann beispielsweise durch eine stärkere Dezentralisierung, Etablierung nicht-hierarchischer Strukturen oder die Einbindung nicht-öffentlicher Akteure eingeführt werden (Mann 2017: 19). Derartige Governance-Formen existieren bereits in Modellprojekten, zum Beispiel in der InnovationCity Ruhr - Modellstadt Bottrop, in Form von Experimenten mit einer Vielzahl an Beteiligungsarten für die Zivilgesellschaft, darunter Beteiligungsveranstaltungen, Vorträge, Workshops, Informations- und kostenlose Beratungsangebote (Best/Roose 2014). Das Leipziger Projekt »Mach's lei- 
ser « setzte in der Beteiligung beim Lärmschutzplan der Stadt neben einer öffentlichen Möglichkeit zur Bewertung von Maßnahmen durch Bürger*innen auch auf die subjektive Wahrnehmung der Lärmprobleme in der Stadt sowie auf die Durchführung einer Planungszelle zur Entwicklung neuer Maßnahmen (WBGU 2016: 347-348). Die Projekte sind gelungene Beispiele für neue Governance-Formen; sie stellen jedoch (noch) nicht den Normalfall kommunaler Selbstverwaltung dar.

Als Hemmnisse für die praktische Umsetzung von Maßnahmen an der Schnittstelle Megatrends und Energiewende werden in einigen Interviews der Gemeindestudie mangelnde personelle Ressourcen genannt. Außerdem besteht ein hoher Kommunikationsaufwand von energiewendespezifischen Querschnittsthemen, die Anknüpfungspunkte in einer Vielzahl der kommunalen Ressorts haben. Insbesondere die befragten Klimaschutzmanager*innen berichten von dieser schwierigen Aufgabe. Hier sei, nach Aussage aus den Interviews, Verhandlungsgeschick gefragt, solche übergreifenden Themen in die alltägliche Pflichtarbeit der verschiedenen Ressorts unterzubringen und zu verknüpfen:

»Erfolgreiche Netzwerkarbeit hausintern bedeutet, dass man vor allen Dingen die Kolleg*innen mithilfe der Querschnittsbereiche entlasten sollte, ihnen Arbeit abnehmen sollte. Die Bereitschaft von Verwaltungseinheiten zusätzliche Arbeiten zu erledigen ist nach wie vor gering. Dies bedeutet auch, dass man wissen muss, was die Kolleg*innen tun. (Auszug aus einem Interviewtranskript)

Starre Hierarchien und Verwaltungsstrukturen können auch Gründe für fehlende Umsetzungsprojekte sein. Diesbezüglich sollte betont werden, dass Kooperation und Co-Produktion zwischen heterogenen urbanen Akteuren nicht mit der Effizienzorientierung administrativer Arbeitsteilung übereinstimmen. Mann (2017) nennt als Hemmnisse fehlender langfristiger Strategien bei Kooperationen (S. 31) die strikte sektorale Ausrichtung auf politisch-administrativer Ebene, Pfadabhängigkeiten und Ressortdenken, die eine Koordination und Kooperation zwischen einzelnen Zuständigkeitsbereichen und Entscheidungskompetenzen erschweren (S. 33), sowie Machtkämpfe und Interessenskonflikte (S. 34).

In den Interviews wurden außerdem fehlende finanzielle Möglichkeiten genannt. Dadurch können Planungs- und Durchführungsprozesse entweder zeitlich verzögert werden oder gar nicht erst stattfinden. Als Folge, wenn bestimmte megatrendbezogene Themen nicht direkt von den Kommunen an- 
gegangen werden können, nennen die Interviewpartner*innen wiederholt, dass Projekte schlichtweg "unter den Tisch fallen würden « oder an privatwirtschaftliche Unternehmen oder karitative Institutionen abgegeben werden müssen. Häufig seien dies Projekte an der Schnittstelle Soziale Disparitäten und Energiewende wie etwa Energiesparberatungen für wirtschaftlich schwache Haushalte oder Angebote zum Teilen und Tauschen.

Erfolgsfaktoren für die Implementierung neuer Governance-Formen sind nach Mann (2017: 32) motivierte und kreative kommunale Akteure, die es verstehen übergreifende Lösungsansätze zu kommunizieren und in die Umsetzung zu bringen. Darüber hinaus haben bürgerschaftliche Initiativen einen hohen Stellenwert in neuen Governance-Formen (ebd.). Auch die in unserer Gemeindestudie befragten kommunalen Entscheidungsträger*innen stimmen mehrheitlich darin überein, dass Bürger*innen eine zentrale Rolle in der Mitgestaltung aktueller Themen, wie der Energiewende oder globaler Megatrends, einnehmen (siehe auch Abb. 2):

»Die Energiewende kann nur von unten gewinnen und muss eine gesellschaftliche Bewegung werden« (Interviewzitat).

Sowohl kommunale Entscheidungsträger*innen als auch einzelne Bürger*innen und Unternehmen (vor allem Start-ups) können als Pioniere des Wandels oder Change Agents eine zentrale Rolle als Erfolgsfaktoren spielen. Indem sie als aktive und visionäre Einzelpersonen Initiativen initiieren und andere für ihre Ideen begeistern (Mann 2017: 32), sind sie hilfreich für die erfolgreiche Umsetzung von Maßnahmen. Neben den Einzelpersonen braucht es dafür auch Freiräume zum Experimentieren und Selbstverwaltungsstrukturen, welche die Vernetzung von Akteuren ermöglichen (ebd.); etwa so wie es in den Beispielen in Bottrop und Leipzig gemacht wurde (s.o.).

Um komplexe globale Entwicklungen wie Megatrends und die Energiewende auf kommunaler Ebene erfolgreich anzugehen, können neue Governance-Formen hilfreich sein. Selbstverständlich muss in jeder Stadt oder Gemeinde in NRW die spezifischen Kontextbedingungen für die Auswirkungen der Trends und das Zusammenspiel mit der Energiewende dabei in Betracht gezogen werden. Ein zentraler Begriff, welcher sowohl in der Literatur als auch in unserer Gemeindestudie genannt wurde, ist: Partizipation. Kommunale Akteure sollten offen dafür sein, Strukturen für systemübergreifende Zusammenarbeit auf kommunaler Ebene zu schaffen, hierarchische Strukturen »aufzuweichen« und den Einbezug von Bürger*innen zu stärken. Wenn Gemeinden die globalen nachhaltigen Entwicklungsziele (SDG's) um- 
setzen wollen, müssen hoheitliche Akteure der Zivilgesellschaft, Wirtschaft und Wissenschaft verantwortliche Rollen in der Stadtgestaltung übertragen.

Über Lösungsansätze auf rein kommunaler Ebene hinaus müssen jedoch auch die Rahmenbedingungen auf den übergeordneten Ebenen stimmen; wie auch einzelne Interviewteilnehmer*innen betonen. Demnach könnten Änderungen in ordnungsrechtlichen Rahmenbedingungen dafür sorgen, dass Digitalisierungsprozesse beschleunigt werden können oder beispielsweise durch Änderungen im Baugesetzbuch die Voraussetzungen für die Umsetzung energetischer Maßnahmen geschaffen werden.

\section{Literatur}

Baur, Nina/Blasius, Jörg (Hg.) (2014): Handbuch Methoden der empirischen Sozialforschung, Wiesbaden: Springer VS. https://doi.org/10.1007/978-3531-18939-0

Best, Benjamin/Roose, Ilka (2014): »Ich fahr kein Bus! Bottroper Bürgerinnen und Bürger im Zentrum sozial-ökologischer Transformationsprozesse«, in: pnd online II (2014), S. 2-12.

Bogumil, Jörg/Holtkamp, Lars (2013): Kommunalpolitik und Kommunalverwaltung: eine praxisorientierte Einführung, Bonn: bpb.

Fischedick, Manfred (2016): »Energiewende im Kontext von Megatrends«, in: Neue Energie 26 (2016), 7, S. 48-51.

Geels, Frank/Kern, Florian/Fuchs, Gerhard/Hinderer, Nele/Kungl, Gregor/Mylan, Josephine/Neukirch, Mario/Wassermann, Sandra (2016): »The enactment of socio-technical transition pathways: A reformulated typology and a comparative multi-level analysis of the German and UK lowcarbon electricity transitions (1990-2014)«, in: Research Policy 45/4, S. 896913. https://doi.org/10.1016/j.respol.2016.01.015

Hajkowicz, Stefan (2012): Our Future World: Global megatrends that will change the way we live. The 2012 Revision, Clayton South: CSIRO PUB, Australia.

Mann, Carsten (2017): »Governance von Gesellschaftstransformation: Konzeptionelle Überlegungen und eine Momentaufnahme politischer Initiativen und Maßnahmen in Deutschland«, in: Jana Rückert-John/Martina Schäfer (Hg.), Governance für eine Gesellschaftstransformation: Herausforderungen des Wandels in Richtung nachhaltige Entwicklung, Wiesbaden: Springer VS, S. 13-43. https://doi.org/10.1007/978-3-658-16560-4_1 
Ministerium für Arbeit, Integration und Soziales des Landes NordrheinWestfalen (2016): Sozialbericht NRW 2016. Armuts- und Reichtumsbericht. Analyse-Maßnahmen-Ergebnisse, Düsseldorf.

Naisbitt, John (2015): »Der Horizont reicht meist nur bis zum nächsten Wahltag«, in: Aus Politik und Zeitgeschichte (APuZ) 65 (31-32), S. 3-6.

Retief, Francois/Bond, Alan/Morrison-Saunders, Angus/King, Nicholas (2016): »Global megatrends and their implication for environmental assessment practice«, in: Environmental Impact Assessment Review 61, S. 52-60. https://doi.org/10.1016/j.eiar.2016.07.002

Virtuelles Institut »Transformation Energiewende NRW« (2019): Megatrends unserer Zeit. Online verfügbar unter: https://www.vi-transformation.de/ output-uebersicht-und-arbeitsdefinitionen-einzelner-megatrends-desenertrend-projektes/. Zuletzt aufgerufen am 25.06.2019.

Wissenschaftlicher Beirat der Bundesregierung Globale Umweltveränderungen (WBGU) (2016): Der Umzug der Menschheit: Die transformative Kraft der Städte, Berlin: WBGU.

Zukunftsinstitut (o.J.): Megatrends Map. Online verfügbar unter: https:// www.zukunftsinstitut.de/artikel/die-megatrend-map/. Zuletzt aufgerufen am 31.01.2020. 


\title{
Urbane nachhaltige Entwicklung am Beispiel von Initiativen in Gießen und Essen ${ }^{1}$
}

Abeer Abdulnabi Ali

\author{
»Our struggle for global sustainability \\ will be won or lost in cities.« \\ (Ban Ki-moon)
}

\section{Einleitung}

Weltweit erhalten Städte immer mehr Aufmerksamkeit, denn seit 2007 lebt die Mehrheit der Menschen in Städten (UN DESA 2014: 7). Innerhalb von 64 Jahren stieg die urbane Bevölkerung 2014 weltweit auf $54 \%$ an und bis 2050 wird prognostiziert, dass diese $66 \%$ betragen wird (ebd.). Diese Veränderung in der menschlichen Siedlungsgeschichte wird auch als "urbane Wende« (Schubert/Stratmann 2008: 2963) bezeichnet. Der Wendepunkt rückt Städte weltweit als relevanteste Lebensräume der Menschheit ins Augenmerk der Wirtschaft, Politik, sowie Wissenschaft und Gesellschaft (WBGU 2016: 43). Trotz ihrer steigenden Anzahl und Größe nehmen Städte nur ungefähr zwei Prozent der totalen Landmasse ein. Jedoch werden $70 \%$ des BIPs, über $60 \%$ des Energieverbrauchs, $70 \%$ der Treibhausgasemissionen und $70 \%$ des $\mathrm{Ab}$ falls in Städten erzeugt (UN-Habitat 3 2016). Die genannten Veränderungen in den letzten Dekaden lassen sich als Megatrend Urbanisierung zusammenfassen. Weiterhin wird die Relevanz der Städte durch das Eingangszitat des ehemaligen Generalsekretärs der Vereinten Nationen, Ban Ki-moon, für die Umsetzung einer nachhaltigen Entwicklung betont (UNRIC 2015). 2017. Ein besonderer Dank gilt den Gutachtern Prof. Dr. Andreas Dittmann und Dr. Steven Engler. 
Internationale Organisationen, darunter die Vereinten Nationen, sowie nationale und lokale Regierungen haben einen speziellen Fokus auf soziale, infrastrukturelle und ökologische Krisen gelegt (Brenner 2018: 2). Zudem wurden universelle Ziele in der globalen Agenda für nachhaltige Entwicklung formuliert. Diese Sustainable Development Goals (SDGs) wurden 2015 von den Vereinigten Nationen beschlossen und gelten für alle Staaten der Welt (Martens/Obenland 2016: 9). Eines der 17 SDGs wurde für alle Städte weltweit formuliert: das SDG 11 (»Make cities and human settlements inclusive, safe, resilient and sustainable« (UN 2020)). Weltweit steigt das Umweltbewusstsein der Menschen und in den Städten lassen sich einige Veränderungen verzeichnen (Krehl 2015: 7). Es wurden diverse Initiativen in ökologischen, ökonomischen und sozialen Bereichen gegründet. Jedoch macht sich das gesteigerte Bewusstsein noch kaum in der Verringerung des zusätzlichen anthropogenen Treibhauseffekts bemerkbar (Kappas 2016: 97ff.).

Um der Anhäufung der Probleme, wie den Verlust von Ökosystemleistungen und biologischer Vielfalt, Bodendegradation und Desertifikation, der Verstärkung des Klimawandels und damit seiner Auswirkungen, zu entgegnen, ist ein Wandel in den Lebensstilen und Handlungen erforderlich. Die Probleme werden oftmals global betrachtet, wobei sich hierbei immer die Frage stellt, wie das Bestehen des Menschen für Gegenwart und Zukunft gewährleistet werden kann. Weiterhin ist festzustellen, dass es Anstrengungen auf der regionalen und lokalen Ebene bedarf, um Nachhaltigkeit zu erzielen. Nachhaltige Entwicklung auf der lokalen und kommunalen Ebene ist somit zu einem neuen Forschungs- und Akteursfeld geworden (Nischwitz/Molitor/Rohne 2002: 1).

Wenn das Ziel nachhaltige Entwicklung ist, müssen die industrialisierten Staaten eine leitende Rolle bei der Lösungsfindung einnehmen. Obgleich Staaten wie China und Indien ebenfalls miteinbezogen werden sollten, müssen die Veränderungen zuerst in den weiterentwickelten Staaten angegangen werden (Grin/Rotmans/Schot 2010: 2). Als wirtschaftlich hochpotenter Staat steht Deutschland dabei in einer besonderen Verantwortung (Schuster 2013: 13f.). Deutschland gehört zu den Staaten in der europäischen Union, die im Zuge der Globalisierung überproportional profitiert haben, während die Umweltbelastungen und Ausbeutung der natürlichen Ressourcen zunehmend in Staaten des globalen Südens verlagert wurden (Schütz/Moll/Bringezu 2004: 4).

Vor dem beschriebenen Hintergrund wurden Initiativen der Transition Town, Urbanen Gärten, Critical Mass und das Stadtradeln ausgewählt, um 
die Umsetzung von Nachhaltigkeit auf lokaler Ebene durch bürgerschaftliches Engagement zu untersuchen. In Deutschland ist nachhaltige Stadtentwicklung in der kommunalen Politik ein wichtiger Faktor (Walcha 1999: 3). Jedoch ist die Umsetzung aufgrund der Differenziertheit der Städte (Löw 2013: 9) komplex. Ein Kernaspekt der Nachhaltigkeitsforschung beschäftigt sich mit der Fragestellung, wie sich Maßnahmen zur Förderung der Nachhaltigkeit gesellschaftlich akzeptabel gestalten lassen (Kopatz 1999: 9). Für den vorliegenden Beitrag wurde folglich die Forschungsfrage: Wie kann die Umsetzung von Nach haltigkeit aufder lokalen Ebene durch Initiativen verbessert werden, gewählt.

\section{Methodische Vorgehensweise}

Für eine Analyse der Nachhaltigkeit auf der lokalen Ebene wurden die Städte Gießen, mittelgroße Stadt in Hessen an der Lahn und Essen, Großstadt in Nordrhein-Westfalen im Zentrum des Ruhrgebiets untersucht. Es wurden zwei unterschiedliche städtische Räume ausgewählt, da diese hinsichtlich der Rahmenbedingungen, wie Infrastruktur oder Demographie auf unterschiedlicher Weise von Nachhaltigkeitsstrategien beeinflusst werden. Für den Vergleich wurden die Parameter Fläche, Bevölkerungszahl und Durchschnittsalter herangezogen (Tab. 1).

Tab. 1: Vergleich der Untersuchungsgebiete Gießen und Essen

\begin{tabular}{|l|l|l|}
\hline & Gießen & Essen \\
\hline Fläche & $72,56 \mathrm{~km}^{2}$ & $210,3 \mathrm{~km}$ \\
\hline Bevölkerungszahl & 84.677 Einwohner & 589.579 Einwohner \\
\hline Durchschnittsalter & 39 Jahren & 44,6 Jahren \\
\hline
\end{tabular}

(Eigene Darstellung mithilfe von Bertelsmann Stiftung 2011; Gießen 2017a, b, c; Essen 2017a)

Ein weiterer wichtiger Faktor ist der Anteil an Studierenden. Gießen hat mit 37.000 Studierenden die höchste Studierendendichte in Deutschland (Gießen 2017c). Die Universität Duisburg-Essen hat 43.089 Studierende, von denen etwa die Hälfte in Essen wohnt (Essen 2017b). Darüber hinaus war Essen »Grüne Hauptstadt Europas 2017«. Dieser Titel soll einen Wandel der ehemaligen Kohle- und Stahlstadt zu einer nachhaltigen Stadt im Ruhrgebiet aufzeigen (Essengreen.Capital 2017). 
Für die Bearbeitung des Forschungsgegenstands wurden Methoden der qualitativen empirischen Sozialforschung angewandt. Qualitative Forschung verfolgt das Ziel, durch gezielte Untersuchungen die Prozesse zu verstehen, durch welche die soziale Wirklichkeit konstruiert wird (Lamnek/Krell 2016: 44). Es wurden zehn explorative Experten- bzw. Tiefeninterviews geführt. Die Reichweite der Erkenntnisse, die durch die Interviews gewonnen wurden, sind lokal, zeitlich sowie situativ begrenzt (Flick 2012: 22f.). Diese Interviewform wurde gewählt, um das Handlungsfeld der Expert*innen als Repräsentant*innen einer Gruppe zu untersuchen (Flick 2012: 214). Dabei wurden Expert*innen aus den gewählten Initiativen in Gießen und in Essen befragt. Als Initiativen für nachhaltige Mobilität wurde die »bottom-up«-gegründete Critical Mass in Gießen und das »top-down«-gesteuerte Stadtradeln in Essen untersucht. Ziel war es die persönlichen Erfahrungen und Eindrücke in der Stadt sowie den Einfluss der Initiativen in Bezug auf nachhaltige Stadtentwicklung zu erfassen. Darüber hinaus wurden auch Geographen in den Untersuchungsgebieten und ein Politiker zu der Thematik befragt, um durch ihr fachliches Wissen einen externen Einblick auf die Umsetzung von Nachhaltigkeit vor Ort $\mathrm{zu}$ generieren.

Als Bewertungs- und Interpretationsmethode im Rahmen der Bachelorarbeit wurde eine SWOT-Analyse für die Initiativen durchgeführt. Die SWOTAnalyse steht für »Strenghts« (Stärken), »Weaknesses« (Schwächen), »Opportunities« (Chancen) und »Threats« (Risiken) eines bestimmten Vorhabens. Damit können Schlüsselfaktoren, die für eine Zielerreichung bedeutend sind, identifiziert werden (Fürst/Scholles 2008: 505). Der vorliegende Beitrag beleuchtet die wichtigsten Ergebnisse der ausgewählten Kriterien Partizipation und Engagement, Unterstützung durch Stadt und Politik, Zeit und Problembewusstsein von Bürger*innen. Dadurch wurden Handlungsperspektiven entwickelt, um die Umsetzung von Nachhaltigkeit auf der lokalen Ebene effektiv und effizient zu verbessern.

\section{Nachhaltigkeit auf der lokalen Ebene}

Die innerstädtischen und suburbanen Aktivitäten, ob es wirtschaftliche oder soziale sind, haben Auswirkungen auf das Umland, entfernte Regionen und Ökosysteme, sowie auf planetare Systeme (Satterthwaite 2003: 77). Der UNHabitat Bericht »Urbanization and Development: Emerging Futures« (2016) erläutert, dass die Urbanisierung in vielen Aspekten bislang nicht nachhal- 
tig verlaufen ist. In der Planung wurde beispielsweise das Auto als Transportmittel bevorzugt eingebracht, welches den Bedarf an Energie erhöht und zur globalen Erderwärmung beiträgt (siehe dazu auch den Beitrag von Canzler/Knie in diesem Band). Aus gesellschaftlicher Perspektive hat die Urbanisierung mehrere Formen von Ungleichheit, Ausgrenzung und Mangel verursacht. Ökonomisch gesehen ist die Urbanisierung nicht nachhaltig verlaufen, da die Arbeitslosigkeit, besonders bei jungen Menschen weit verbreitet ist und instabile niedrigbezahlte Arbeitsplätze, sowie informelle Aktivitäten Ungleichheit fördern und Lebensqualität senken (UN-Habitat 2016: 5). Um diesen urbanen Herausforderungen $\mathrm{zu}$ begegnen, erfordert es Handlungen sowohl in ökologischen, ökonomischen als auch in sozialen Bereichen. Hierbei ist Nachhaltigkeit der Dreh- und Angelpunkt.

Nachhaltigkeit ist zum populären Buzzword geworden. Ökonom*innen, Politiker*innen und viele andere Akteur*innen haben das Trendwort bereits in ihren Lebensläufen integriert (Pufé 2012: 6). Doch ist unklar, ob jedem bekannt ist, was der Begriff bedeutet (Grober 2013: 14). Er ist überaus mehrdeutig und man kann nur schwer eine universelle Definition formulieren, da er in unterschiedlichen Zusammenhängen eine wichtige Rolle besetzt (Michelsen/Adomßent 2014: 1; Grober 2013: 16).

Während der Begriff Nachhaltigkeit einen Zustand beschreibt, stellt die nachhaltige Entwicklung ein dynamisches Konzept dar. Für die vorliegende Arbeit wird die Definition aus dem Brundtland-Bericht »Our Common Future« (1987) verwendet:

"Sustainable Development is development that meets the needs of the present without compromising the ability of future generations to meet their own needs.« (WCED 1987:43)

Die Ziele der nachhaltigen Entwicklung können nur erreicht werden, wenn der Schutz der Natur in die Entwicklungsvorgänge integriert, die Bedürfnisse der Umwelt für Gegenwart und Zukunft angepasst, sowie die Bekämpfung der Armut als grundlegende Maßnahmen in den Vordergrund gestellt werden (Steen 1994: 84). Diese Herausforderungen gilt es gemeinsam zu bewältigen.

1992 fand in Rio de Janeiro die Weltkonferenz der Vereinigten Nationen statt, an der 178 Staaten teilgenommen haben. Zum ersten Mal hatten sich die Staats- und Regierungschefs versammelt, um gemeinsam Strategien aufzustellen, die dem Umweltschutz und der Bekämpfung der Armut mittels nachhaltiger Entwicklung dienen (Schuster 2013: 11). Nachhaltige Entwicklung sollte sowohl in den Themen der Umwelt als auch der Entwicklung welt- 
weit Verbindlichkeit beanspruchen. Um das Ziel einer nachhaltigen Entwicklung zu erreichen wurden im Laufe der Geschichte mehrere Berichte verfasst und internationale Konferenzen der UN gehalten. Dabei lässt sich ein zunehmendes Verständnis für die Bedeutung der Nachhaltigkeit bis hin zur Ausprägung als Leitbild und Bindeglied für die Bereiche Umwelt und Fortschritt beobachten.

Immer mehr Menschen in immer mehr städtischen Regionen sind von den Problemen und Herausforderungen einer nicht-nachhaltigen Gesellschaft betroffen. Wenn ein Wandel hin zur nachhaltigen Entwicklung erreicht werden soll, sind Handlungen der Gesellschaft vor Ort umzusetzen. Anhand der ausgewählten Initiativen werden Handlungsfelder für die Einführung nachhaltiger Stadtentwicklung auf der lokalen Ebene analysiert.

\section{Transition Town}

Die Transition Town Initiative hat ihren Ursprung in Kinsale (Irland). Es wurde ein Plan zur Senkung des Energieverbrauchs entwickelt, um die Unabhängigkeit der Stadt vom Erdöl zu erreichen (Krehl 2015: 15). 2005 gründete Rob Hopkins die Bürgerinitiative »Transition Town Totnes«. Sie wurde zum Leitbild für viele weitere Initiativen weltweit. Innerhalb weniger Jahre breitete sich die Idee in Großbritannien, Europa, Australien, über Nord- und Südamerika bis nach Japan, Indien und Südafrika aus (Maschkowski/Wanner 2014: 1).

Die »bottom-up«-Initiative Transition Town beinhaltet eine Reihe von verschiedenen Aktivitäten, darunter Urbane Gärten, Repair-Cafés, solidarische Landwirtschaft und Lastenfahrräder (Transition Town Gießen 2017). Getragen und beeinflusst werden die Projekte von Bürger*innen (WBGU 2011: 256). Um die globalen Herausforderungen zu bewältigen, soll die lokale ökonomische, zivilgesellschaftliche und individuelle Resilienz gestärkt werden. Dabei sollen Selbstversorgung, lokal erzeugte Produkte und erneuerbare Energien gefördert werden (Krehl 2015: 16).

Es zeigt sich, dass die Initiative der Transition Town in der Großstadt Essen viel Engagement und Partizipation von Seiten der Bürger*innen erfährt und dadurch sehr aktiv ist. Als Großstadt hat Essen im Vergleich zu Gießen etwa fünfmal so viele Einwohner. Zudem liegt Essen im Ruhrgebiet, dem größten Agglomerationsraum Deutschlands (WBGU 2016: 278). In der mittelgroßen Stadt Gießen mangelt es an engagierten Bürger*innen. In Essen gehört die Initiative zum Runden UmweltTisch und durch ihre Funktion als Dach für andere Bewegungen ist sie mit den Urbanen Gärten, die dort 
gegründet wurden, dem Repair-Café und einigen anderen vernetzt. Auch in Gießen hat die Bewegung eine Dachfunktion für Initiativen. Dennoch liegt ein schwaches Netzwerk vor und die Initiative ist »ein Geisterschiff [...], was online noch sehr präsent ist, was die Leute auch so sehr gut wahrnehmen. Aber hinter den Kulissen passiert gar nichts mehr«, erklärte der Experte aus der Transition Town in Gießen. Hopkins ist hierbei der Ansicht, dass es die Beteiligung einer möglichst großen Öffentlichkeit erfordert, um Wandel zu schaffen (Hopkins 2010: 120). Eine wesentliche Schwäche, welche ein existentielles Risiko darstellt, ist die fehlende Partizipation für die Initiative in Gießen. Ohne das Engagement und den Einsatz der Bürger*innen können innerhalb der Transition Town keine Projekte in der Region organisiert werden. Gründe für das Auftreten dieses Risikos könnten Blockaden gegenüber Veränderungen sein. Darunter zählen persönliche Hindernisse, wie die Ablehnung des Verzichts, von Sparsamkeit oder das Aufgeben von bekannten Lebensstilen (Krehl 2015: 13).

Als weiteres Kriterium wurde der Zeitaspekt innerhalb der Analyse betrachtet. In Essen wird das Kriterium von den befragten Expert*innen nicht erwähnt. Hingegen erweist sich mangelnde Zeit als große Schwäche für die Transition Town in Gießen. Der Faktor Zeit wird häufig mit fehlender finanzieller Unterstützung verknüpft. In beiden Städten stellt dies für die Initiative ein Risiko dar. Da sich in Essen relativ viele Personen beteiligen, die finanziell flexibler sind als Studierende, ist das Problem weniger stark ausgeprägt als in Gießen. Gleichwohl erklärten die Befragten aus der Initiative in Essen, dass sich etwas in der Arbeitswelt ändern muss, da sich die »[...] Welt nicht im Ehrenamt verändern« lässt. In der Universitätsstadt Gießen, werden solche Initiativen häufig von Studierenden getragen, denen es an Zeit mangelt, wenn sie noch nebenbei arbeiten. Zeit ist bei Aufbau und Entwicklung einer solchen Initiative ein wichtiger Aspekt (Hopkins 2014: 85). Dennoch ist $\mathrm{zu}$ beachten, dass die Transition Town vor allem in Gießen eine sehr junge Bewegung ist, da sie erst 2015 ins Leben gerufen wurde. Da es in der mittelgroßen Stadt allerdings an Engagierten fehlt, die Zeit und Geld haben, um Energie, Ideen und Aktivitäten in die Bewegung zu stecken, ist die Initiative zurückgefallen. Dabei zeigt sich wieder, dass solche Initiativen »Pioniere« sind und »[...] so schön »bottom-up«-Prozesse sind, man braucht irgendwie Leute, die die Moderation und alles drum herum in die Hand nehmen [...]", so der Befragte. Dennoch braucht es solche Bewegungen, als Impuls- und Ideengeber, um einen Ansporn und ein gesellschaftliches Klima zu schaffen, erläuterte der interviewte Politiker. 
Das Problembewusstsein der Bürger"innen bezüglich der Notwendigkeit für die Transformation zu nachhaltigen Lebensstilen, stellt für die Transition Town in beiden Untersuchungsgebieten ein Risiko dar. In Essen haben die interviewten Experten wahrgenommen, dass es »einen großen Anteil von diesen Leuten mit den Scheuklappen, die so sehr in ihrem Alltag stecken [...] und ja vielleicht auch Angst vor Veränderungen haben« gibt. Dennoch kann das Kriterium zu einer Chance umgewandelt werden, wenn Strategien eingesetzt werden, die das Bewusstsein stärken. Hierbei ist zu beachten, dass eine Steigerung der Partizipation an lokalen Initiativen erst erreicht wird, wenn sich ein Großteil der Bürger*innen bewusst ist, dass der Wandel zu einer dauerhaft tragfähig handelnden Gesellschaft notwendig ist (Krehl 2015: 30).

Durch Kooperation mit Stadt und Politik kann die Bekanntheit der Initiative gefördert und dadurch die Anzahl der Teilnehmer*innen erhöht werden. Hinsichtlich dessen erwies sich der Titel »Grüne Hauptstadt Europas 2017«als Chance für die Initiative in Essen, da sich Möglichkeiten ergeben, die Transition Town zu präsentieren und »[...] einer breiten Öffentlichkeit zugänglich zu machen« betonte der Stadtforscher. Auch für die Transition Town in Gießen kann der Entschluss gefasst werden, dass es die Unterstützung durch lokale und kommunale städtische sowie politische Akteure benötigt. Durch ihre Maßnahmen und die Zusammenarbeit der Transition Town mit diesen Akteuren, kann die Initiative in Gießen wiederbelebt werden, da nur somit eine breite Öffentlichkeit auf die Bewegung aufmerksam gemacht werden kann (Maschkowski/Wanner 2014: 9). Denn meist mangelt es solchen Initiativen und Projekten, die von unten gesteuert werden, an Aufmerksamkeit und Reichweite (Hossain 2016: 977).

Durch die hohe Fluktuation der Studierenden in Gießen konnte die Initiative bislang kaum Erfolge erzielen. Dennoch können auch aus dem ständigen Wechseln der Studierenden Potentiale genutzt werden. Sollten die Studierenden im Sinne der Nachhaltigkeit positive Verhaltensweisen und Ideen in Gießen gesammelt haben, könnten sie diese an einem anderen Ort einsetzen und weiterentwickeln. Die Studie »Umweltbewusstsein in Deutschland 2016« zeigt, dass ein hohes Problembewusstsein gegenüber Umwelt- und Klimaproblematiken und eine Offenheit für nachhaltigere Wirtschafts- und Lebensweisen besteht, jedoch ist das Engagement der Industrie, der Bundesregierung sowie der Bürger*innen noch sehr gering (BMUB 2017). Das Gefühl von Verantwortung und die damit verbundene Bereitschaft zum Handeln wird meist nur für das Umland getragen. Denn erst Ereignisse in der eigenen Stadt bzw. 
Gemeinde verschärfen die Motivation etwas zu unternehmen. Gleichwohl ein Gefühl der Betroffenheit geschaffen ist, wird aufgrund einer sogenannten Partizipationsbarriere nicht immer direkt gehandelt (Kopatz 1999: 45). Diese gilt es mithilfe von gezielten Maßnahmen zu durchbrechen. Folglich lässt sich die Problematik nicht auf Gießen und Essen beschränken, sondern erweist sich als ubiquitäre Herausforderung.

\section{Urbane Gärten}

Begonnen hat die Bewegung der Urbanen Gärten (engl.: »Urban Gardening«) in den späten 1960er und 1970er Jahren in New York City, USA, als eine Verschlechterung der wirtschaftlichen Lage in Städten das Interesse für urbane Grünflächen geweckt hat. Besonders Nahrungsmittel- und Ressourcenkrisen sowie klimabedingte Desertifizierung von Agrarflächen steigern das Interesse an lokalen und biologischen Lebensmitteln (Müller 2012: 24). Durch bürgerschaftliches Engagement wurden Gemeinschaftsgärten auf freien Flächen realisiert. Da die vorherigen Freiflächen oftmals von Drogendealern und anderen Kriminellen verwendet wurden, haben die Gärten somit unter anderem die Möglichkeit für gesellschaftliche Veränderungen geschaffen (Saldivar-Tanaka/Krasny 2004: 399). Durch die kontinuierliche Arbeit und öffentliche Sichtbarkeit wurde aus vielen Einzelaktionen eine weltweite Bewegung (von der Haide et al. 2012: 267). Der Fokus bei dieser Untersuchung wurde auf die Gemeinschaftsgärten (engl.: »community gardens«) gesetzt. Rosol (2006) definiert Gemeinschaftsgärten als

»[...] gemeinschaftlich und durch freiwilliges Engagement geschaffene und betriebene Gärten, Crünanlagen und Parks mit Ausrichtung auf eine allgemeine Öffentlichkeit.«(Rosol 2006: 7)

Demnach sind es Gärten auf städtischen Freiflächen, die von Bürger*innen organisiert und geführt werden sowie für jeden zugänglich sind.

Die Auswertung der Interviews mit den Expert*innen aus den Urbanen Gärten in Essen und Gießen hat gezeigt, dass sich die Initiativen nur in wenigen Aspekten unterscheiden. Das Kriterium Engagement und Partizipation stellt für die Urbanen Gärten in beiden Städten eine zentrale Schwäche dar. In Essen gibt es mehr Urbane Gärten als in Gießen und sie werden sowohl von der Stadt durch die "Grüne Hauptstadt«, als auch von der Transition Town unterstützt. Obgleich es mehr Leute gibt, die sich engagieren, ist »die größte Schwierigkeit [...] Mitgärtner zu finden. Also genügend Aktive [...]«, erklärte die interviewte Akteurin aus Essen. In Gießen scheitern solche Projekte oft- 
mals, laut dem Interviewten aus den Urbanen Gärten, denn »[...] viele Leute, viele jüngere Leute, die nach Gießen kommen zögern immer dabei, wenn es darum geht sich [...] zu engagieren oder [...] Verantwortung zu übernehmen [...]«. Die Aussage des Experten zeigt zudem, dass die junge, studentisch geprägte Gesellschaft in Gießen ein Risiko für die Initiative darstellt. Dieses Problem wird weiterhin durch die offene Struktur der Gärten gefördert, wodurch das Risiko entsteht, dass sich niemand verantwortlich fühlt. Als Folge lastet ein Großteil der Arbeit auf den wenigen, die sich engagieren (Gehrke 2012:3). Zusätzlich kann eine solche Initiative laut der Studie von Leigh Holland (2004) $\mathrm{zu}$ community gardens, nur langfristig bestehen, wenn gute Organisation und Partizipation vorhanden sind, sowie durch einen regelmäßigen Beitrag neuer Ideen, welche die Entwicklungen in der Gesellschaft reflektieren (Rosol 2006: 207).

Der Zeitaspekt wurde wiederum nur in Gießen als Schwäche dargestellt, denn der Interviewte erklärte, dass »[...] die meisten Leute [...] nicht mehr die Zeit [haben,] sich da so reinzuknien und so viel $\mathrm{zu}$ machen und daran scheitert [...] sehr viel«. Im Wesentlichen sind es die Akteur*innen, die über mehr Zeit verfügen, die sich in den Gemeinschaftsgärten engagieren (Rosol 2006: 212). Ähnlich, wie bereits bei der Transition Town erläutert, hängt das Problem des Zeitmangels bei dieser Initiative oftmals mit der finanziellen Unterstützung zusammen.

Problembewusstsein könnte sich in beiden Städten als Chance für die Initiativen herausbilden, da die Entwicklung immer weiter in Richtung regionaler und lokaler Lebensmittel geht (Kögl/Tietze 2010: 10). Wie bereits bei der Transition Town aufgeführt, gibt es Partizipationsbarrieren, die es aufzubrechen gilt. Weiterhin bedarf es beim Gärtnern an viel Arbeit, wodurch von einigen das Argument »wieso hier das Gemüse großziehen, wenn ich bei [Discountern] für 49 Cent irgendwie meine Paprika kaufen kann« vorgebracht wird, berichtete der interviewte Geograph aus Gießen. Es zeigt sich, dass fehlende Spürbarkeit der Auswirkungen des individuellen Handelns ein Hindernis für Veränderungen in den Lebensweisen darstellt. Auf der einen Seite gibt es einen Teil der Gesellschaft, der Bio-Produkte einkauft und vielleicht sogar lokal Lebensmittel anbaut. Auf der anderen Seite findet sich jedoch eine Gesellschaft, »die das Kilo Gehacktes für 3,99 nur gerne hätte«, obgleich sie um die Konsequenzen weiß, erläuterte der Geograph aus Essen. Weiterhin führte der Experte aus, dass die gesellschaftliche Aufgabe darin besteht alle Gesellschaftsgruppen zu einem umweltbewussten Handeln zu bringen und »[...] einen nachhaltigen gesellschaftlichen Konsens« zu schaffen. Folglich müssen 
Information und Umweltbildung in alle Lebensbereiche diffundieren, um die Anzahl der Beteiligten zu erhöhen.

\section{Critical Mass}

Eines der zentralen Merkmale einer modernen europäischen Gesellschaft ist Mobilität. Um Mobilität nachhaltig zu gestalten, müssen die lokal spürbaren negativen Auswirkungen des Verkehrs, unter anderem Treibhausgase, Luftverschmutzung und Lärm, deutlich reduziert werden (Butzin/Terstriep/Welschhoff 2013: 2). Die Problematiken, welche durch den motorisierten Individualverkehr verursacht werden, sind überwiegend in Städten spürbar - somit in den Regionen, in denen alternative Verkehrskonzepte am ehesten umgesetzt werden können (Zimmer/Hülsmann/Havers 2014: 11). Das Mobilitätsverhalten muss folglich nachhaltig verändert werden.

Eine Lösung für die Problematiken bietet der Radverkehr. Er hat mit dem Fußgängerverkehr die höchsten Umweltentlastungspotentiale, da er weder Emissionen noch Lärm verursacht und wenig Flächen verbraucht (Zimmer/Hülsmann/Havers 2014: 19). Zusätzlich fördert Fahrradfahren die Gesundheit (Garrard/Rissel/Bauman 2012: 31). Fahrradfahren kann somit den Wandel zu einer nachhaltig handelnden Gesellschaft vorantreiben. Dennoch hat der Radverkehr in vielen deutschen Städten noch nicht den Stellenwert erhalten, den er benötigt, um sich zu entwickeln (Zimmer/Hülsmann/Havers 2014: 20). Die jährlichen Pro-Kopf-Ausgaben für den Radverkehr in den sechs größten Städten in Deutschland und in Amsterdam und Kopenhagen von 2018 verdeutlichen dies. In Stuttgart, Berlin und Frankfurt sind es vier bis fünf Euro, in Köln und Hamburg knapp drei Euro, wohingegen die Ausgaben in Amsterdam elf Euro und in Kopenhagen circa 36 Euro betragen (statista 2020).

Eine Initiative, welche die Aufmerksamkeit auf das Radfahren gelenkt hat, ist Critical Mass. Sie begann 1992 in San Francisco als sich Fahrradfahrer während der Rushhour des letzten Freitags jeden Monats getroffen haben, um in der Öffentlichkeit gemeinsam Fahrrad zu fahren. Damit verbreiteten sie die Botschaft: »Wir behindern nicht Verkehr, wir sind Verkehr! « und verlangten nach Gleichberechtigung des Fahrrads. Die Bewegung sieht sich einerseits als Party und andererseits als Demonstration. Durch die Critical Mass wurde eine große Anzahl von Radfahrern mobilisiert, massive Kontroversen erzeugt und dazu beigetragen die öffentliche Auffassung zum Fahrrad weltweit zu verbessern (Furness 2007: 299). Auch diese Bewegung hat sich global verbrei- 
tet. Als Gegenbewegung zur Automobilität zeigt die Critical Mass eine Methode, um die Rolle des Fahrrads in einer zunehmend motorisierten Welt zu stärken und die Nutzung des öffentlichen Raumes zu thematisieren (Furness 2007: 300).

Die Untersuchungen der Critical Mass in Gießen haben gezeigt, dass es anders als bei den zuvor untersuchten Initiativen - nicht an Teilnehmer*innen mangelt. Critical Mass lebt mehr von Partizipation als von Engagement. Durch die Freiheit der Teilnehmer*innen die Veranstaltung individuell zu interpretieren, ergibt sich die Chance, eine große Anzahl von Bürger*innen zur Teilnahme zu motivieren (Furness 2010: 136f.). Die Teilnehmer*innen machen aus unterschiedlichen Gründen an der Bewegung mit, sei es, um die globalen Umweltgesetze zu ändern, die Prioritäten des lokalen und regionalen Transportes zu stärken oder einfach nur um gemeinschaftlich Fahrrad zu fahren (Blickstein/Hanson 2001: 361). Da "gerade in Gießen [...] eigentlich alles fußläufig erreichbar ist, [ist man] mit dem Fahrrad [...] hier super aufgestellı", berichtete der Critical Mass-Aktivist. Zusätzlich kann der hohe Studierendenanteil in Gießen eine Chance für die Critical Mass ergeben, da die neue Bewegung besonders junge Leute anspricht. Romanowska, Okraszewska und Jamroz (2019: 2) erläutern zudem, dass Studierende eine hohe Bereitschaft aufzeigen, neue Ideen anzunehmen und Lebensgewohnheiten zu verändern.

Eine Unterstützung von Stadt und Politik erfährt die Initiative nicht direkt. Zu Beginn wurde die Bewegung von der Polizei begleitet, um den Schutz der Fahrradfahrer*innen zu gewährleisten. Vom Experten der Critical Mass war die Bewertung hierzu, dass es »[...] eigentlich ganz nett war, um dem Ganzen [...] eine Seriosität zu geben«. Der Faktor Zeit spielt für diese Initiative nur eine untergeordnete Rolle, da ein Treffen nur einmal im Monat stattfindet.

Als Chance für die Initiative erweist sich das gestiegene Umweltbewusstsein in der Gesellschaft, resultierend aus der Verschlechterung der Umwelt durch anthropogene Ausbeutung und Umweltverschmutzung (Chen/Tsai 2016: 958). Die Einstellung zum Auto und ihre Anforderungen an Dienstleistungen der Mobilität zeigen eine Veränderung, die weg vom motorisierten Verkehr hin zu anderen Transportmitteln führt. Unterstützt wird dieser Wandel von den Zielen der Bundesregierung, die eine Reduzierung von 40 Mio. Tonnen $\mathrm{CO}_{2}$-Emissionen anstrebt (Kabitzke et al. 2011: 6). Eine weitere Chance bildet die Förderung des Bewusstseins für den öffentlichen Raum, wodurch die Stadtbewohner*innen angeregt werden, sich zu fragen, »[...] wie viel Platz hat eigentlich [...] das stehende Auto? Wie viel Platz hat das fahrende Auto? Und was nimmt der Fahrradfahrer auf sich in Gießen, weil 
er keinen Fahrradweg [...] hat?«, erklärte der Critical Mass-Experte. Auch Furness (2007) geht auf das Potential der Erschaffung eines sozialen Raumes ein, in dem die Normen des motorisierten Verkehrs hinterfragt werden und die Bürger*innen die Möglichkeit haben, sich von Angesicht zu Angesicht zu unterhalten (Furness 2007: 307f.). Folglich ergibt sich durch die Critical Mass eine indirekte Maßnahme, die ein Bewusstsein für den Wandel schafft.

Amy Stork plädiert für einen radikalen Flügel damit ein gesellschaftlicher Wandel erreicht werden kann. Sie argumentiert, dass die Auffassung von Critical Mass als radikale Bewegung, die Errichtung eines Fahrradwegs zu einem vernünftigen Entschluss macht. Hingegen in Regionen ohne Critical Mass erscheint ein Fahrradweg radikal (Furness 2010: 138). Somit kann der Druck durch Fahrradaktivisten gegenüber der Stadt und Politik dazu beitragen die Ziele zu erreichen.

Zusätzlich ergibt sich die Chance, dass Nachhaltigkeit und nachhaltige Lebensstile lokal durch die Bewegung der Critical Mass gefördert werden. Durch die Förderung des Radverkehrs werden mehr Stadtbewohner*innen zum Fahrradfahren motiviert. Infolgedessen kann sich die urbane Lebensqualität durch die oben genannten Vorteile des Radverkehrs verbessern. Der Erfolg der Bewegung ist jedoch schwer zu messen, da ein formales Ziel und eine spezifische Agenda fehlen (Blickstein/Hanson 2001: 359).

\section{Stadtradeln}

Eine andere Initiative, um nachhaltige Mobilität durch das Radfahren zu fördern, ist das Stadtradeln. Diese wird im Gegensatz zu den vorangegangenen Initiativen, »top-down« gesteuert und organisiert. Entwickelt wurde das Stadtradeln 2008 vom Klima-Bündnis, dem größten Netzwerk von Städten, Gemeinden und Landkreisen, welches sich dem Klimaschutz widmet. Obgleich das Motto des Stadtradelns »Radeln für ein gutes Klima« lautet, soll damit nicht nur Klimaschutz betrieben, sondern auch der Radverkehr gefördert werden. Es kann jedes Jahr von allen Kommunen in Deutschland an 21 zusammenhängenden Tagen im Zeitraum vom o1. Mai bis zum 30. September durchgeführt werden. Somit bietet diese Aktion den Kommunen eine Maßnahme, welche einfach zu realisieren ist und nur einen geringen Aufwand und Mittel bedarf, um nachhaltige Mobilität aktiv voranzutreiben (Klima-Bündnis 2017: 3).

Die Ziele des Stadtradelns reichen von der Sensibilisierung der Bürger*innen für die Nutzung des Fahrrads im Alltag bis hin zu verstärkter 
Implementierung von Themen zur Fahrradnutzung und der Planung des Radverkehrs in die kommunalen Parlamente. Beim ersten Stadtradeln 2008 haben 1.813 Radfahrer*innen teilgenommen, davon 264 Mitglieder aus 23 Stadtparlamenten. Zusammen haben sie 340.523,11 Kilometer mit dem Fahrrad zurückgelegt und somit über 68 Tonnen $\mathrm{CO}_{2}$ einsparen können (Stadtradeln 2017). Vergleichsweise werden durch einen Hin- und Rückflug von Düsseldorf nach Südafrika circa sechs Tonnen $\mathrm{CO}_{2}$ pro Person emittiert (atmosfair 2020).

Trotz der gestiegenen Anzahl an Teilnehmer*innen hat sich das Engagement und die Partizipation als Schwäche herausgestellt, denn die interviewte Expertin für das Stadtradeln hat in den letzten Jahren festgestellt, »[...] dass es zunehmend schwieriger wird, die Leute zu motivieren teilzunehmen und [...] Kilometer aufzuschreiben«. Chancen könnten jedoch entstehen, wenn Engagement für und Partizipation an der Initiative des Stadtradelns steigen. Wie bereits bei der Critical Mass aufgeführt, kann die erhöhte Teilnehmerzahl der Radfahrer*innen zu einer verbesserten städtischen Lebensqualität durch nachhaltige Mobilität führen. Anders als bei der Critical Mass, motiviert das Stadtradeln die Bürger*innen besonders durch den Wettbewerbscharakter. Dadurch kann sich das Konzept als Chance erweisen, die Partizipation der Fahrradaktivist*innen und wettbewerbsbegeisterten Bürger*innen $\mathrm{zu}$ fördern. 2017 war in Essen ein Wachstum der Begeisterung für das Radfahren zu erkennen (Klimaagentur Essen 2017). Dies könnte sich als Chance herausstellen, um in den nächsten Jahren weiterhin Erfolge zu erzielen. Die Partizipation von Firmen, Vereinen, Kirchen, Institutionen, Schulen sowie anderen Initiativen, Nachbarschaften und Fahrradliebhaber*innen könnten dazu beitragen, dass das Fahrrad als Alltagsverkehrsmittel gefördert wird. Durch Mundpropaganda und Nachahmungen im sozialen Umfeld ergibt sich die Chance einer stärkeren Verbreitung durch die Teilnehmer*innen (Wolsing/Ahaus/Behr 2015: 31f.). Obgleich die Beteiligung einiger Akteure gesteigert wird, kann fehlende Teilnahmebereitschaft bei der jüngeren Bevölkerung zu einem Risiko für die Initiative werden. Die Stadtradeln-Expertin erläuterte, dass in Essen die Versuche Schulen, Jugend- und Freizeiteinrichtung für das Stadtradeln zu motivieren scheiterten. Dabei besteht das Risiko, dass die Möglichkeit des Stadtradelns als Initiative für nachhaltige Mobilität von jungen Leuten nicht genutzt wird.

Die Unterstützung durch Stadt und Politik bietet vielseitige Chancen für das Stadtradeln, da es im Gegensatz zu den vorher untersuchten Initiativen einem »top-down«-Ansatz entspringt. Zum einen ergeben sich viele Vernet- 
zungen zu Einrichtungen und Ämtern. Zum anderen kann die Vernetzung mit der kommunalen Politik dazu beitragen, dass die Vorbildfunktion andere zum Radfahren ermutigt. Außerdem wird durch das Stadtradeln aktiv von Stadt und Politik nachhaltige Mobilität gefördert. Die Aufforderung der Politiker*innen an der Initiative teilzunehmen kann sich als Möglichkeit erweisen, um ein Feedback zur Verkehrsinfrastruktur zu erhalten. Dieses kann genutzt werden, um die Radwege und die Verkehrssituation für die Radfahrer*innen $\mathrm{zu}$ verbessern.

Weiterhin leiten sich Chancen aus der »top-down«-gesteuerten Organisationstruktur der Initiative ab. In Essen ist das Stadtradeln ein zentrales Projekt der Klimawerkstatt. Zudem wird die Verbreitung des Themas Radverkehr durch die Berichterstattung politischer Akteure in regionalen Medien gefördert (Wolsing/Ahaus/Behr 2015:32). Folglich wird verstärkt langfristig Einfluss auf die Stadtentwicklung und den Klimaschutz durch den Popularitätsanstieg des Fahrrads als Transportmittel genommen. Während der ökologische Beitrag anhand $\mathrm{CO}_{2}$-Einsparungen messbar ist, stellt der relativ kurze Zeitraum von drei Wochen eine Begrenzung für den Einfluss und Beitrag der Initiative für Nachhaltigkeit im urbanen Raum dar.

Das Stadtradeln im Ruhrgebiet, dem größten Agglomerationsraum Deutschlands, ermöglicht eine Vernetzung der Radverkehrsförderung über die Stadtgrenzen hinaus und kann dazu beitragen, dass die Region als fahrradfreundlich wahrgenommen wird (Wolsing/Ahaus/Behr 2015: 32). Die dichte Siedlungsstruktur in Essen und des gesamten Ruhrgebiets ergeben die Chance einer Stadt der kurzen Wege, wodurch der Wandel zu einem umweltfreundlichen, nachhaltigen Verkehrsmittel ermöglicht wird (Cramer 2014: 14).

\section{Handlungsperspektiven}

Den gezeigten Herausforderungen innerhalb der Untersuchungen gilt es mittels unterschiedlicher Maßnahmen entgegenzuwirken. »Bottom-up«-Ansätze werden hauptsächlich durch ehrenamtliche Partizipation getragen, weshalb dieses Kriterium entscheidend für den Erfolg der Initiativen ist. Da die Transition Town in Gießen aufgrund fehlender Partizipation zurzeit nur noch als Informationsplattform im Internet existiert, bräuchte es zunächst Freiwillige, die ein Event veranstalten, welches die lokale Gesellschaft wieder auf die Initiative aufmerksam macht. Um dieses Risiko abzubauen und das Engage- 
ment in der Transition Town zu steigern, werden positive Beispiele und Vorbilder, Druck zur Veränderung, machbare und kleine Schritte sowie viele kleine Lösungen und eine erhöhte Informationsdurchlässigkeit benötigt (Krehl 2015: 14; Maschkowski/Wanner 2014: 3). Weiterhin reicht es nicht, Formulierungen für die Zukunft zu haben; es braucht greifbare und anschauliche Umsetzungsbeispiele der Ideen (Welzer 2011: 40f.). Genau diese Visualisierung wird durch die untersuchten Initiativen ermöglicht. Darüber hinaus gilt es, die Umweltbildung hin zu einer nachhaltigen Gesellschaft so früh wie möglich in den Kindergärten und Schulen zu verankern.

Die Stadtradeln-Expertin betonte hierfür ein Beispiel im Bereich Mobilität:

»Das hat natürlich auch gerade im Kindesalter was mit Vorbildverhalten zu tun, wenn die Kinder immer mit dem Auto zur Schule gefahren werden und die Eltern selbst nicht Fahrrad fahren, dann werden die Kinder auch nicht unbedingt den Spaß dran haben [...]

Dadurch zeigt sich, dass es nicht nur eine Aufgabe der Bildungsinstitute ist, sondern auch die Eltern Verantwortung für eine nachhaltige Mobilität tragen.

Damit die »bottom-up«-gesteuerten Initiativen langfristig erhalten bleiben und im urbanen Raum nachhaltige Entwicklung umsetzen können, braucht es die Unterstützung städtischer und politischer Akteure. Die Akteure der höheren Ebenen müssen die Notwendigkeit einer nachhaltigen Entwicklung in den Städten verbreiten. Unterstützungen durch Stadt und Politik verhelfen den Bewegungen in Essen zu einer Teilnehmersteigerung. Aufgrund von mangelnder Beteiligung in Gießen kann die Unterstützung durch städtische und politische Akteure die Transition Town effektiv wiederbeleben und zu mehr Teilnehmer*innen verhelfen. Mögliche Maßnahmen, um Nachhaltigkeit auf der lokalen Ebene zu fördern, können Belohnungen sein. Beispielsweise können Preise für Aktivist*innen verliehen werden, die sich in einem gewissen Zeitraum besonders im ökologischen, ökonomischen und sozialen Sinne nachhaltig verhalten haben. Diese Fördermöglichkeit wird bereits bei der »top-down«-Initiative des Stadtradelns eingesetzt und kann für Transition Town, Urbane Gärten und Critical Mass sowie für weitere nachhaltige Bewegungen genutzt werden. Dadurch kann es gelingen, die Motivation zu steigern und somit die Teilnehmerzahl zu erhöhen.

Das Merkmal der kurzen Wege in Gießen können die Beteiligten der Initiativen nutzen, um über ihre Aktivitäten verstärkt $\mathrm{zu}$ informieren und dadurch die Partizipation zu fördern. In Essen muss die zukunftsfähige Ent- 
wicklung gezielt innerhalb der einzelnen Stadtregionen eingebracht werden. Aus langfristiger Sicht müssen sich die Initiativen stärker mit den unterschiedlichen Bereichen vernetzen, damit Nachhaltigkeit breiter in die Gesellschaft durchdringen kann. Um dem Risiko des Zeitmangels zu begegnen, müssten bezahlte Stellen geschaffen werden. Dies, so der Experte aus den Urbanen Gärten in Gießen, sei jedoch eine Wunschvorstellung.

\section{Fazit und Ausblick}

Das Wachstum der Weltbevölkerung und die voranschreitende Urbanisierung haben erhebliche Auswirkungen auf das Ökosystem Erde, das Wirtschaften und die Politik. Durch unterschiedliche Berichte, Studien und Konferenzen wurden Handlungsempfehlungen und Maßnahmen entwickelt, um nachhaltige Entwicklung zu erreichen. Auch wenn das Umweltbewusstsein der Menschen größer als jemals zuvor ist, schreiten die Zerstörung der Umwelt und Verhaltensweisen, die soziale Ungleichheiten fördern, weiter voran. Bei der Umsetzung einer nachhaltigen Entwicklung hat sich der Fokus von der global politischen zur nationalen bis hin zur lokalen Ebene gewandelt.

Die lokalen Dimensionen der Nachhaltigkeit konnten durch die ausgewählten Initiativen, der Transition Town, Urbanen Gärten, Critical Mass und Stadtradeln in Gießen und Essen dargelegt werden. Gießen und Essen unterscheiden sich in Bezug auf Rahmenbedingungen, wie Infrastruktur oder Demographie. Hinsichtlich der Stadtgröße ergibt sich ein Vorteil für Gießen, da Informationen die Menschen schneller erreichen. Daher verläuft die Umsetzung von Nachhaltigkeitsstrategien unterschiedlich. Bei der Umsetzung der Nachhaltigkeitsziele innerhalb der untersuchten Initiativen ergeben sich Herausforderungen in ökologischen, ökonomischen und sozialen Problembereichen. Partizipationsbarrieren der Bürgerinnen und Bürger müssen überwunden und Motivationen generiert werden. Diese Barrieren sind tief in der Gesellschaft verwurzelt. Eine Schlüsselrolle besetzt dabei die Politik. Sie muss sich verstärkt für Nachhaltigkeit in ihrem Einflussgebiet einsetzen. Der Vergleich zwischen Gießen und Essen hat gezeigt, dass Initiativen besonders in Kommunen, in denen Stadt und Politik aufgrund von Projekten, wie »Grüne Hauptstadt 2017 « hohes Interesse an Nachhaltigkeit zeigen, erfolgreich sind. In Essen bekommen Bewegungen, wie die Gemeinschaftsgärten stärkere Unterstützung. Zusätzlich ist es schwer den ökologischen, ökonomischen und 
sozialen Beitrag der Initiativen zu messen, da der Anteil der engagierten Bevölkerung bislang noch zu gering ist und es sehr junge Initiativen sind.

Wie Amy Stork zur Critical Mass oder Al Gore ${ }^{2}$ generell fordern, muss in den Denkweisen der Menschen die Lücke zwischen persönlicher Einstellung und aktivem Handeln überwunden werden. Jedoch geschieht Wandel oft erst, wenn Probleme eskalieren oder Menschen selbst aktiv betroffen sind. Damit es $\mathrm{zu}$ dieser Eskalation nicht kommt, müssen die Herausforderungen und Problematiken global abgebaut und die Nachhaltigkeit lokal umgesetzt werden. Als Prozess kann nachhaltige Entwicklung nicht zeitlich festgelegt werden, sondern muss allmählich und fortlaufend entwickelt, geplant und vor Ort durchgeführt werden. Dabei kann und sollte jeder seinen Teil leisten, um den Wandel zu einer nachhaltig handelnden Gesellschaft zu erreichen. Die untersuchten Initiativen in den urbanen Räumen zeigen dabei beispielhaft, wie lokal die ökologische, ökonomische und soziale nachhaltige Entwicklung in den Städten vorangetrieben wird.

\section{Literatur}

$\mathrm{Al}$ Gore (2017): Informationen $\mathrm{zu} \mathrm{Al}$ Gore. Online verfügbar unter: https:// www.algore.com/about. Zuletzt aufgerufen am 14.09.2017.

Atmosfair (2020): $\mathrm{CO}_{2}$-Fußabdruck berechnen. Online verfügbar unter: https:/www.atmosfair.de/de/kompensieren/flug. Zuletzt aufgerufen am 13.02.2020.

Bertelsmann Stiftung (2011): Demographischer Wandel. Online verfügbar unter: https://www.wegweiser-kommune.de/statistik/essen+demogra phischer-wandel+2012-2014+tabelle. Zuletzt aufgerufen am 11.09.2017.

Blickstein, Susan/Hanson, Susan (2001): »Critical mass: forging a politics of sustainable mobility in the information age«, in: Transportation 28 , S. 347-362. https://doi.org/10.1023/A:1011829701914

Brenner, Neil (2018): »Debating planetary urbanization: For an engaged pluralism«, in: Environment and Planning D: Society and Space, S. 1-21.

Bundesministerium für Umwelt, Naturschutz, Bau und Reaktorsicherheit (BMUB) (Hg.) (2017): Umweltbewusstsein in Deutschland 2016 - Er-

2 Co-Nobelpreisträger mit dem IPCC 2007 für die Informierung der Weltbevölkerung über die Bedrohungen des Klimawandels und ehemaliger Vizepräsident der USA (AI Gore 2017). 
gebnisse einer repräsentativen Bevölkerungsumfrage. Online verfügbar unter: https://www.umweltbundesamt.de/sites/default/files/medien/ 376/publikationen/umweltbewusstsein_deutschland_2016_bf.pdf. Zuletzt aufgerufen am 11.09.2017.

Butzin, Anna/Terstriep, Judith/Welschhoff, Jessica (2013): »Nachhaltige Mobilität durch soziale Innovationen!? Verkehrsprobleme und alternative Ansätze zu ihrer Lösung«, in: Forschung Aktuell 2, Institut Arbeit und Technik (IAT), Gelsenkirchen.

Chen, Chung-Ling/Tsai, Chen-Hao (2016): »Marine environmental awareness among university students in Taiwan: a potential signal for sustainability of the oceans«, in: Environmental Education Research 22, S. 958-977. https://doi.org/10.1080/13504622.2015.1054266

Cramer, Michael (2014): »Wie mobil sind wir in der Stadt von morgen? - Forderungen aus Brüssel«, in: Thomas J. Mager (Hg.), »Mobilität für die Stadt der Zukunft« - Kommt nach der Energie- und die Verkehrswende?, Köln: KSV-Verlag, S. 11-16.

Essen (2017a): Bevölkerungsstand 30.06.2017. Online verfügbar unter: https:// www.essen.de/rathaus/statistik/Statistik_Bevoelkerung.de.html. Zuletzt aufgerufen am 11.09.2017.

Essen (2017b): Daten und Fakten. Online verfügbar unter: https://www.unidue.de/imperia/md/content/dokumente/datenundfakten/ daten_und_fakten.pdf. Zuletzt aufgerufen am 11.09.2017.

Essengreen.Capital (2017): Essen 17, Grüne Hauptstadt Europas. Online verfügbar unter: https://www.essengreen.capital/startseite_essen_egc.de. html. Zuletzt aufgerufen am 05.09.2017.

Flick, Uwe (2012): Qualitative Sozialforschung - Eine Einführung, Hamburg: Rowohlt.

Furness, Zack (2007): „Critical mass, urban space and velomobility«, in: Mobilities 2, S. 299-319. https://doi.org/10.1080/17450100701381607

Furness, Zack (2010): „Critical mass rides against car culture«, in: Jesús Ilundáin-Agurruza/Michael W. Austin (Hg.), Cycling Philosophy for Everyone. A Philosophical Tour de Force, Malden: Wiley-Blackwell, S. 134145. https://doi.org/10.1002/9781444324679.ch13

Fürst, Dietrich/Scholles, Frank (2008): Handbuch Theorien und Methoden der Raum- und Umweltplanung, Dortmund: Dorothea Rohn.

Garrard, Jan/Rissel, Chris/Bauman, Adrian (2012): »Health benefits of cycling", in: John Pucher/Ralph Buehler (Hg.), City cycling, Cambridge, Massachusetts: MIT Press, S. 31-56. 
Gehrhe, Janna (2012): Urban Gardening. Wie die Gärten in die Stadt zurückkehren. Impuls. Stadt-Land-Fläche, NABU. Online verfügbar unter: https://www.nabu.de/downloads/NABU-Impuls-StadtLandFlaeche/ NABU-Impuls_SLF_102012.pdf. Zuletzt aufgerufen am 11.09.2017.

Gießen (2017a): Lage und Verkehr. Online verfügbar unter: https:/www. giessen.de/Wirtschaft/Wirtschaftsstandort/Lage_und_Verkehr/. Zuletzt aufgerufen am 11.09.2017.

Gießen (2017b): Zahlen und Fakten. Online verfügbar unter: https://www. giessen.de/Wirtschaft/Wirtschaftsstandort/Zahlen_und_Fakten/. Zuletzt aufgerufen am 11.09.2017.

Gießen (2017c): Gießen in Zahlen. Kennzahlen 2017/2018. Online verfügbar unter: https://www.giessen.de/media/custom/684_5261_1.PDF?144967 8904. Zuletzt aufgerufen am 11.09.2017.

Grin, John/Rotmans, Jan/Schot, Johan (2010): »Introduction: From Presistent Problems to System Innovations and Transitions«, in: John Grin/Jan Rotmans/Johan Schot (Hg.), Transition to Sustainable Development: new directions in the study of long term transformative change, New York, London: Routledge, S. 1-10. https://doi.org/10.4324/9780203856598

Grober, Ulrich (2013): Die Entdeckung der Nachhaltigkeit - Kulturgeschichte eines Begriffs, München: Antje Kunstmann.

Hopkins, Robert (2014): Einfach. Jetzt. Machen!: Wie wir unsere Zukunft selbst in die Hand nehmen, München: oekom.

Hopkins, Robert John (2010): »Localisation and Resilience at the local level: the case of Transition Town Totnes«. (Dissertation, University of Plymouth, School of Geography, Earth and Environmental Science, Faculty of Science and Technology).

Hossain, Mokter (2016): „Grassroots innovation: A systematic review of two decades of research«, in: Journal of Cleaner Production 137, S. 973-981. https://doi.org/10.1016/j.jclepro.2016.07.140

Kappas, Martin (2016): »Klimawandel und Gesundheit«, in: Tamcke, Martin (Hg.): Armut und Gesundheit. Göttingen International Health Network (GIHN), Institut für Ökumenische Theologie, Georg-August-Universität Göttingen, S. 95-118.

Klima-Bündnis (2017): Stadtradeln - Kampagnenkonzept. Online verfügbar unter: https://www.stadtradeln.de/fileadmin/stadtradeln_responsive/ Dateien_und_Dokumente/allgemeingueltig/stadtradeln_konzept_2017. pdf. Zuletzt aufgerufen am 07.06.2017. 
Klimaagentur Essen (2017): Essener STADTRADELN so erfolgreich wie noch nie. Nicht nur die Grüne Hauptstadt Europas - Essen 2017 bricht alle Rekorde. Online verfügbar unter: https:/www.essen.de/meldungen/ pressemeldung_1089070.de.html. Zuletzt aufgerufen am 05.09.2017.

Kögl, Hans/Tietze, Jana (2010): »Regionale Erzeugung, Verarbeitung und Vermarktung von Lebensmitteln«, Forschungsbericht, Professur für Landwirtschaftliche Betriebslehre und Management, Universität Rostock.

Köhler, Thomas/Abraham, Jonas (2015): »Eine andere Stadtpolitik ist möglich - In Transition Towns sorgen Nachbarschaftshilfe, alternative Versorgungskonzepte und Stadtgärten für Nachhaltigkeit und Zusammenhalt«, in: Le Monde diplomatique (Hg.), Atlas der Globalisierung - Weniger wird mehr, Berlin, S. 138-139.

Kopatz, Michael (1999): Lokale Nachhaltigkeit: vom internationalen Diskurs zur Umsetzung in Kommunen, Oldenburg: BIS.

Krehl, Stefan (2015): »Transition Town Initiativen im deutschsprachigen Raum: Ein systematischer Überblick über Vorkommen, Schwerpunkte und Einfluss auf die Energiewende vor Ort«, in: Wirtschaftswissenschaftliche Schriften, Reihe: Ökonomie der Nachhaltigkeit, Ernst-AbbeHochschule Jena, Fachbereich Betriebswirtschaft 03.

Kuckartz, Udo/Rädiker, Stefan (2014): »Datenaufbereitung und Datenbereinigung in der qualitativen Sozialforschung«, in: Nina Baur/Jörg Blasius (Hg.), Handbuch Methoden der empirischen Sozialforschung, Wiesbaden: Springer Fachmedien, S. 383-396. https://doi.org/10.1007/978-3-53118939-0_27

Lamnek, Siegfried/Krell, Claudia (2016): Qualitative Sozialforschung, Weinheim, Basel: Beltz.

Löw, Martina (2013): Soziologie der Städte, Berlin: Suhrkamp.

Martens, Jens/Obenland, Wolfgang (2016): Die 2030-Agenda: Globale Zukunftsziele für nachhaltige Entwicklung, Global Policy Forum/terre des hommes (Hg.), Bonn, S. 1-18.

Maschkowski, Gesa/Wanner, Matthias (2014): »Die Transition-Town-Bewegung: Empowerment für die große Transformation?«, in: PND-online 2, S. 60-71.

Michelsen, Gerd/Adomßent, Maik (2014): »Nachhaltige Entwicklung: Hintergründe und Zusammenhänge«, in: Harald Heinrichs/Gerd Michelsen (Hg.), Nachhaltigkeitswissenschaften, Berlin, Heidelberg: Springer, S. 359. https://doi.org/10.1007/978-3-662-44643-0_1 
Müller, Christa (2012): »Urban Gardening. Grüne Signatur neuer urbaner Zivilisation«, in: Christa Müller (Hg.), Urban Gardening. Über die Rückkehr der Gärten in die Stadt, München: oekom, S. 22-53.

Nischwitz, Guido/Molitor, Reimar/Rohne, Silvia (2002): »Local und Regional Governance für eine nachhaltige Entwicklung«, in: Schriftenreihe des IÖW 161.

Pucher, John/Buehler, Ralf (2012): »Introduction: Cycling for Sustainable Transport«, in: John Pucher/Ralf Buehler (Hg.), City cycling, Cambridge, Massachusetts: MIT Press, S. 1-8. https://doi.org/10.7551/mitpress/9434. 001.0001

Pufé, Iris (2012): Nachhaltigkeitsmanagement, Kamiske, Gerd (Hg.), München: Carl Hanser.

Rana, Masud Parves (2009): »Sustainable city in the global North and South: goal or principle?«, in: Management of Environmental Quality: An International Journal 20, S. 506-521. https://doi.org/10.1108/14777830910981195

Romanowska, Aleksandra/Okraszewska, Romanika/Jamroz, Kazimierz (2019): »A Study of Transport Behaviour of Academic Communities«, in: sustainability 11, S. 1-18. https://doi.org/10.3390/su11133519

Rosol, Marit (2006): Gemeinschaftsgarten in Berlin: Eine qualitative Untersuchung zu Potenzialen und Risiken bürgerschaftlichen Engagements im Grünflächenbereich vor dem Hintergrund des Wandels von Staat und Planung (Doctoral dissertation, Zugl.: Berlin, Humboldt-Univ., Diss., 2006).

Saldivar-Tanaka, Laura/Krasny, Marianne E. (2004): »Culturing community development, neighbourhood open space, and civic agriculture: The case of Latino community gardens in New York City«, in: Agriculture and human values 21, S. 399-412. https://doi.org/10.1023/B:AHUM.0000047207. 57128.a5

Satterthwaite, David (2003): »The links between poverty and the environment in urban areas of Africa, Asia, and Latin America«, in: The Annals of the American Academy of Political and Social Science 590, S. 73-92. https:// doi.org/10.1177/0002716203257095

Schubert, Herbert/Stratmann, Bernhard (2008): "Naturalisierung des Städtischen: Perspektiven der Stadtsoziologie«, in: Karl-Siedbert Rehberg/Deutsche Gesellschaft für Soziologie (DGS) (Hg.), Die Natur der Gesellschaft: Verhandlungen des 33. Kongresses der Deutschen Gesellschaft für Soziologie in Kassel 2006, Frankfurt a.M.: campus, S. 2961-2967. 
Schuster, Wolfgang (2013): Nachhaltige Städte - Lebensräume der Zukunft. Kompendium für eine nachhaltige Entwicklung der Stadt Stuttgart, München: oekom.

Schütz, Helmut/Moll, Stephan/Bringezu, Stefan (2004): »Globalisation and the shifting environmental burden: material trade flows of the European Union; which globalisation is sustainable?«, Wuppertal Papers.

Stadtradeln (2017): Ergebnisse 2008. Online verfügbar unter: https://www. stadtradeln.de/ergebnisse2008/. Zuletzt aufgerufen am 19.08.2017.

Statista (2020): Jährliche Ausgaben je Einwohner für den Radverkehr in sechs deutschen Städten sowie in Amsterdam und in Kopenhagen 2018. Online verfügbar unter: https:/de.statista.com/statistik/daten/studie/909259/ umfrage/jaehrliche-ausgaben-je-einwohner-fuer-den-radverkehrin-deutschen-staedten/. Zuletzt aufgerufen am 27.01.2020.

Steen, Uwe (1994): »Kultur als Quelle einer ökologisch nachhaltigen Entwicklung«, in: Siawuch Amini/Eckhard Baum/Hans-Joachim Glauner(Hg.), Nachhaltigkeit der Entwicklung ländlicher Regionen Afrikas, Asiens und Lateinamerikas, in: Journal of Agriculture in the Tropics and Subtropics, Der Tropenlandwirt 52, Witzhausen: Selbstverlag des Verbandes der Tropenlandwirte, S. 81-102.

Swiaczny, Frank/Schulz, Reiner (2009): »Wachstum der Weltbevölkerung und nachhaltige Tragfähigkeit«, in: Journal für Verbraucherschutz und Lebensmittelsicherheit 4, S. 136-144. https://doi.org/10.1007/s00003-0090477-2

Transition Town Gießen (2017): Gruppen und Projekte. Online verfügbar unter: www.ttgiessen.de/gruppen_und_projekte. Zuletzt aufgerufen am 17.08.2017.

United Nations Department of Economic and Social Affairs (UN DESA) (2014): World Urbanization Prospects: The 2014 Revision. Final Report (ST/ESA/SER.A/366), New York.

UN-Habitat - United Nations Human Settlements Programme (2016): Urbanization and development: emerging futures; world cities report 2016. Nairobi: UN-Habitat.

UN-Habitat 3 (2016): The New Urban Agenda. Online verfügbar unter: http:// habitat3.org/the-new-urban-agenda/. Zuletzt aufgerufen am 20.03.2018. United Nations (UN) (2020): SDG 11. Online verfügbar unter: https:// sustainabledevelopment.un.org/sdg11. Zuletzt aufgerufen am 11.02.2020. United Nations Regional Information Centre (UNRIC) (2015): Zitat von Ban Ki-moon. Online unter: https://archive.unric.org/en/latest-un-buzz/ 
29754-our-struggle-for-global-sustainability-will-be-won-or-lost-incities. Zuletzt aufgerufen am 18.02.2020.

Haide, Ella von der/Halder, Severin/Jahnke, Julia/Mees, Carolin (2012): „Guerilla Gardening und andere politische Gartenbewegungen. Eine globale Perspektive«, in: Christa Müller (Hg.), Urban Gardening. Über die Rückkehr der Gärten in die Stadt, München: oekom, S. 266-278.

Walcha, Henning (1999): »Materialien für die Arbeit vor Ort. Nachhaltige Stadtentwicklung«, in: Konrad-Adenauer-Stiftung, Kommunalpolitik 11.

Welzer, Harald (2011): Mentale Infrastrukturen: Wie das Wachstum in die Welt und in die Seelen kam, Heinrich-Böll-Stiftung (Hg.), Schriftreihe zur Ökologie 14.

Wissenschaftlicher Beirat der Bundesregierung Globale Umweltveränderungen (WBGU) (2011): Welt im Wandel: Gesellschaftsvertrag für eine Große Transformation, Berlin.

Wissenschaftlicher Beirat der Bundesregierung Globale Umweltveränderungen (WBGU) (2016): Der Umzug der Menschheit: Die transformative Kraft der Städte, Berlin.

Wolsing, Dagmar/Ahaus, Björn/Behr, Friederike (2015): »KLIMA| WERK| STADT| ESSEN als Change Agency für neue Klimakulturen «, in: Hermann-Josef Wagner/Christina Sager (Hg.), Wettbewerb »Energieeffiziente Stadt«, Bd. 5: Kommunikation und Partizipation, Münster: LIT, S. 27-36.

World Commission on Environment and Development (WCED) (1987): Our common future, Oxford, New York: Oxford University Press.

Zimmer, Wiebke/Hülsmann, Friederike/Havers, Kirsten (2014): Stadt der Zukunft Lebenswerte Innenstädte durch emissionsfreien Verkehr, ÖkoInstitut Working Paper 4. 


\section{Transformation von unten gestalten Das Fachgeschäft für Stadtwandel als Ort des sozial-ökologischen Wandels im Quartier}

Martina Nies und Björn Ahaus

Megatrends finden stets ihre Entsprechung im Lokalen. So wird etwa die Energiewende - neben der Rahmensetzung durch den Gesetzgeber - von Menschen vor Ort gestaltet. Ebenso werden soziale und kulturelle Disparitäten zunächst vor Ort sichtbar und vor Ort werden sie zu einer Herausforderung für die Wege des Umgangs, die gefunden werden müssen. Dabei ist die Suche nach Lösungen keineswegs allein hoheitliche Aufgabe von Politik und Verwaltung. Städte verändern sich auf zwei Wegen: »top-down« durch veränderte Politikansätze und entsprechende Planungen und »bottom-up« durch Verhaltensänderungen von Menschen sowie durch zivilgesellschaftliche Initiativen und Projekte. Ohne die Menschen vor Ort geht es nicht. Protestbewegungen wie Stuttgart 21 und Fridays for Future sowie das wachsende Interesse an Projekten und Realexperimenten (Burkhardt/Schupp 2019), die einen positiven Einfluss auf die Lebensqualität und die nachhaltige Entwicklung des städtischen Raums entwickeln, zeugen davon, dass Menschen sich aktiv in die Entwicklung »ihrer « Stadt einbringen wollen. Sie wollen Einfluss nehmen, mitgestalten und mitbestimmen, in welche Richtung es geht. Auf den Wunsch nach direkter Mitbestimmung reagieren Politik und Verwaltung seit einiger Zeit in Form neuer Beteiligungs- und Partizipationsformate (Bürgerworkshops, Onlineforen usw.), in denen Bürger*innen jenseits rechtlich fixierter Verfahren ihre Meinungen, Einschätzungen und Wünsche äußern können. Abseits dieser von oben initiierten Foren ist vielerorts zu beobachten, dass die Zivilgesellschaft spontan neue Initiativen bildet und die Gestaltung ihres Lebensraums zunehmend selbst in die Hand nimmt. Auffällige Beispiele sind die im Jahr 2015 deutschlandweit unzähligen entstandenen Initiativen zur Flüchtlingshilfe (vgl. Gesemann/Seidel/Mayer 2019), die Bildung von Orts- 
gruppen von großen Bewegungen, wie Fridays for Future, oder auch Transition Town sowie Initiativen zur Initiierung von Bürgerbegehren für mehr Radverkehr (»Radentscheid«) oder eine soziale Wohnbaupolitik. Diese Gruppen bilden sich spontan, sind offen für Engagement in unterschiedlicher Intensität und Dauer, setzen auf breite Kollaboration, kooperieren häufig mit alteingesessenen Vereinen und Verbänden und schaffen so Netzwerke. Nachhaltige urbane Entwicklung ist damit ein Megatrend, der vor Ort gestaltet wird. Dabei meint »vor Ort« nicht unbedingt die gesamte Stadt, sondern bezieht sich vielmehr auf den Raum vor der eigenen Haustür, den direkten Lebensraum im Quartier.

Der vorliegende Bericht stellt das Fachgeschäft für Stadtwandel in Essen-Holsterhausen als einen dieser neuen Orte des Engagements vor. Das Kooperationsprojekt der Initiative für Nachhaltigkeit, dem Runden Tisch Holsterhausen und dem Standpunkt e.V. ist 2018 als Keimzelle für sozialökologischen Wandel gegründet worden. Mit seinem Angebot und seinen Aktivitäten werden drei zentrale Themen miteinander verbunden: Lebendige Nachbarschaft, sozialökologischer Wandel und interkulturelle Gemeinschaft. Im Folgenden werden zunächst die wesentlichen gesellschaftlichen Entwicklungstrends skizziert, die den Grundstein für die Gründung, das Verständnis und auch den Erfolg des Fachgeschäfts für Stadtwandel bilden. Danach folgen eine Beschreibung des Projektes und seiner Entstehung, sowie die Einordnung in den Stadtteil. Abschließend wird seine Bedeutung im Kontext sozialer Innovationen sowie seine bisherige Entwicklung diskutiert ${ }^{1}$. Unsere Perspektive ist die wissenschaftlich geschulter Praktiker*innen, die den Entwicklungsprozess des Fachgeschäfts unter anderem vor dem Hintergrund gesellschaftlicher Herausforderungen reflektieren.

\section{Transformation braucht Engagement, Engagement braucht Raum}

Eine demokratische Gesellschaft, die wirksame und akzeptierte Lösungen für die Herausforderungen von Megatrends wie Klimawandel, nachhaltige urbane Entwicklung und Umgang mit sozialen und kulturellen Disparitäten finden will, braucht Menschen, die Verantwortung nicht nur für sich selbst, son-

1 Wir verstehen soziale Innovationen im Anschluss an Howaldt et al. als »intentionale Neukonfiguration sozialer Praktiken« (Howaldt et al. 2014: 12f). Vgl. hierzu auch Ahaus 2019 (S. 24ff.). 
dern auch für die Gestaltung der Gesellschaft jetzt und für zukünftige Generationen übernehmen. Dafür müssen die Menschen spüren und erleben, dass und wie sie in der Gesellschaft wirksam sein können.

Deutschland hat eine lange Tradition des freiwilligen und zivilgesellschaftlichen Engagements ${ }^{2}$. Nahezu jede* $r$ Zweite in Deutschland engagiert sich ehrenamtlich in einem Verein. Neben dem kontinuierlichen dauerhaften Engagement in etablierten Institutionen der Kirche, der Wohlfahrt oder in Vereinen hat sich aus den sozialen und umweltpolitischen Bewegungen der 1970er Jahre ein neues freiwilliges Engagement entwickelt, das flexibler, selbstorganisiert und stärker projektgetrieben, also zeitlich begrenzt ist (vgl. Beher/Liebig/Rauschenbach 1999). Traditionelle Institutionen wie die Kirchen verlieren an Bindungskraft und Mitgliedern. Sie hinterlassen eine Lücke, die Platz bietet und Bedarfe schafft für neue Formen des sozialen Zusammenlebens. Eine weitere Begründung für den »Strukturwandel des Ehrenamts« (Beher/Liebig/Rauschenbach 1999; Hacket/Mutz 2002) ist in der Form der Organisation moderner Arbeit zu suchen: Menschen wechseln häufiger den Arbeitsplatz und oftmals auch den Wohnort. Damit werden soziale Beziehungen im Arbeitskontext oberflächlich und das Bedürfnis nach sozialer Zugehörigkeit und Gemeinschaft muss außerhalb der Erwerbsarbeit bedient werden. Zudem führt die erhöhte Fluktuation im Job zu einem stärkeren Kommen und Gehen im bürgerschaftlichen Engagement (Burkhardt/Schupp 2019; Denner 2014).

Darüber hinaus sind der Wunsch, sich als Teil einer Gemeinschaft zu fühlen, aber auch die Befriedigung alltäglicher Bedürfnisse in einem sozialen Netzwerk, wie z.B. nachbarschaftliche Hilfe, Versorgung von Kindern und alten Menschen, Versorgung mit Lebensmitteln, häufig Motivation für Engagement. Als soziales Wesen braucht der Mensch soziale Netzwerke für ein gu-

2 Die Begriffe Ehrenamt, zivilgesellschaftliches und freiwilliges Engagement kommen in diesem Artikel gleichermaßen zur Anwendung, da im Fachgeschäft für Stadtwandel sämtliche Ausprägungen zum Tragen kommen. Also Ehrenamt im Sinne unentgeltlicher Tätigkeiten in einer formalisierten Struktur zum Wohle Dritter (wie z.B. einem Verband oder Verein, vgl. Stricker 2011) und zivilgesellschaftliches Engagement im Sinne freiwilliger, gemeinwohlorientierter, nicht auf materiellen Gewinn abzielender, gemeinschaftlicher Tätigkeiten, die im (teil-)öffentlichen Raum stattfinden (vgl. Priller 2011: 35). Der Begriff freiwilliges Engagement betont, dass eine Tätigkeit freiwillig und ohne oder nur gegen geringe Aufwandsentschädigung ausgeführt wird (Simonson et al. 2017: 37). Er schließt auch Tätigkeiten im privaten Raum ein (z.B. Unterstützung einer Nachbarin beim Einkaufen). 
tes Leben. Soziale Beziehungen brauchen Zeit und - insbesondere für die Begegnung mit anderen Kulturen und Lebensentwürfen - Begegnungsräume. Fehlen Anlässe und/oder Orte der Begegnung und des Miteinanders, steigt das Potential von Argwohn, Vereinsamung und Isolierung (Landmann et al. 2017). Lebendige Quartiere entstehen durch ein aktives Leben im öffentlichen Raum und ein Interesse für dessen Gestaltung (vgl. Berding/Bukow 2020).

Gemeinschaftsbildende soziale Innovationen wie offene, soziokratisch organisierte Nachbarschaftsprojekte in städtischen Quartieren können diese Wünsche bedienen. Sie bieten gerade einer aufgeklärten Bürgerschaft, die ihre Interessen selbstorganisiert in die kommunale Entwicklung einbringen will, eine Basis und einen Nährboden für Aushandlungsprozesse und gemeinschaftlich organisierte Aktionen. Gerade im Ruhrgebiet mit seiner korporatistischen Tradition, wo lange galt und zum Teil noch gilt »der Zechendirektor bzw. die Politik wird es schon regeln«, wird vielen Bürgerinnen zunehmend klar, dass sie die Geschicke ihrer Kommune mitgestalten können und zum Teil auch müssen. Antrieb sind nicht zuletzt kommunale Schuldenkrisen und daraus resultierende Einschränkungen der Handlungsfähigkeit der öffentlichen Hand sowie überalterte Räte, die radikale Änderungen scheuen. Die Gestaltung des Lebensraums und die Beantwortung der Frage, wie wir leben wollen, wird nicht mehr vertrauensvoll Politik und Verwaltung überlassen. Denn deren Antworten unterwerfen sich in der Wahrnehmung Vieler primär dem Diktat der Wirtschaft und dem Postulat des Wirtschaftswachstums als Wohlstandsbringer, während gleichzeitig die Folgen nicht-nachhaltiger Politikansätze wie der Klimawandel und soziale Disparitäten auch vor Ort in den Kommunen sichtbar und spürbar werden. Vielmehr nehmen Bürgerinnen es selbst in die Hand, Ziele zu formulieren und Strategien zu entwickeln. Dazu gehört auch die Festlegung, welche Aufgaben von wem geleistet werden können und sollen, damit der vorsorgende Sozialstaat weiterhin existieren kann und Ehrenamt nicht mit hoheitlichen Aufgaben der Daseinsvorsorge überfrachtet wird, wie es derzeit zum Teil in ländlichen Gegenden passiert (vgl. Kummel/Nadler 2018).

In Zeiten kommunaler Finanzkrisen, Abbau von Personal und Finanzmitteln für Aufgaben der Daseinsvorsorge, Investitionsstau und überalterter Stadträte sehen viele Menschen Chancen und Bedarfe sich einzubringen, um ihre eigenen Vorstellungen von Stadtentwicklung und einer zukunftsgerechten, enkeltauglichen Gestaltung ihrer Lebenswelt geltend zu machen und ihre Beiträge dazu zu realisieren. Diese Besinnung auf die Wahrnehmung eigener Interessen und demokratischer Pflichten ist auch dringend notwen- 
dig. Der Blick auf die durchschnittliche Wahlbeteiligung bei den Kommunalwahlen 2014 in NRW verdeutlicht eindrücklich, dass die lokale Demokratie in einer Krise steckt. Wenn weniger als $50 \%$ der Bürger*innen ihr Wahlrecht wahrnehmen und somit mehr als die Hälfte auf der ihnen räumlich und lebensweltlich am nächsten liegenden politischen Ebene nicht mehr aktiv entscheiden, ist dies ein deutliches Warnsignal. Demokratie braucht interessierte Bürger*innen, die sich aktiv einbringen - an der Urne oder durch eigenes Engagement.

Die Ebenen, auf der Menschen unterschiedlicher Couleur ungezwungen zusammenkommen, sich austauschen, gemeinsam aktiv werden und unterstützen, sind die eigene Nachbarschaft und das Quartier, also der direkte Lebensraum vor der eigenen Haustür. Damit die sozial-ökologische Transformation im Quartier stattfinden kann, braucht es flexible Räume, die frei zugänglich sind, unabhängig vom sozialen Status. In den Quartieren fehlt es heute aber gerade an diesen Orten. Regelmäßige Besuche von Cafés und Restaurants sind nicht Jede" $r$ möglich und bieten auch nicht die Offenheit und Inspiration eines kreativen Raums. Räume zur Förderung der sozialökologischen Transformation ermöglichen Engagement in unterschiedlichen Ausprägungen und Intensitäten, schaffen niederschwellige Angebote, die auch für Menschen zugänglich sind, die noch keine Berührung mit diesen Themen hatten, oder sich bisher bewusst davon fernhalten. Sie ermöglichen, Selbstwirksamkeit zu erfahren, Fähigkeiten zur Kooperation zu üben und im Rahmen von Kollaborationen zu entfalten. Damit bieten sie einen Gegenpol zu der von Richard Sennett so genannten »Dequalifizierung« der Kooperation durch moderne Gesellschaften:

»Menschen verlieren die Fähigkeit, mit hartnäckigen Unterschieden umzugehen, wenn materielle Ungleichheit sie isoliert und kurzzeitige Arbeitsverhältnisse ihre sozialen Kontakte oberflächlich werden lassen und Angst vor dem Anderen auslösen. Wir sind dabei, die für eine komplexe Gesellschaft unerlässliche Kooperationsfähigkeit einzubüßen«(Sennett 2019: 22).

Stadtwandel braucht soziale Innovationen. Und die Diffusion sozialer Innovationen für eine sozial-ökologische Transformation braucht Begegnungen, Verbindungen, Kooperation und Kollaboration. Dafür braucht es Räume für Macher"innen und Möglichkeiten. 


\section{Das Fachgeschäft für Stadtwandel}

Ein Ort, der unserer Beobachtung nach, Selbstwirksamkeit erfahrbar macht und Möglichkeiten für Kooperation und Kollaboration bietet, ist das Fachgeschäft für Stadtwandel im Essener Stadtteil Holsterhausen.

Essen-Holsterhausen ist ein für das Ruhrgebiet typischer mittelständischer Stadtteil: Soziokulturell durchmischt hat er vor allem Wohnfunktion mit Gewerbegebieten an der Peripherie. Ein großer Arbeitgeber ist das Klinikum Essen, das im Randgebiet gewissermaßen ein Stadtteil im Stadtteil ist. Die Gemarkenstraße, eine ehemals florierende Einkaufsstraße, ist gezeichnet von hohen Ladenleerständen, die meist von Discountern und Schnellimbissen geschlossen werden. Einmal in der Woche findet hier ein Wochenmarkt statt, für den die Straße gesperrt wird. Inhabergeführter Fachhandel zieht nur vereinzelt nach. Holsterhausen hat eine recht hohe Verkehrsbelastung: durch die direkte Nachbarschaft zur Innenstadt durchzieht ein dichtes Straßennetz mit mehreren Hauptverkehrsadern den Stadtteil, zudem teilt die Autobahn A40 Holsterhausen. Trotz fast völliger Abwesenheit von Fahrradinfrastruktur ist der Fahrradanteil am Modal Split, d.h. der Verteilung der zurückgelegten Wege auf die verschiedenen Verkehrsmittel, aufgrund der urbanen, zentralen Lage einer der höchsten in der Stadt. Mit über 26.600 Einwohnerinnen auf $2,98 \mathrm{~km}^{2}$ ist Holsterhausen einer der am dichtesten besiedelten und bebauten Orte in Essen (Amt für Statistik und Wahlen der Stadt Essen 2019). Zudem prägt seine Geschichte als Lebensort für Flüchtlinge und Migrantinnen den Stadtteil. Holsterhausen war nach dem Zweiten Weltkrieg eines der größten Wiederaufbaugebiete Westdeutschlands und Zielort vieler Flüchtlinge aus dem Osten. Der Anteil der Bewohnerinnen ohne deutschen Pass ist mit 20,1\% etwas höher als der Essener Durchschnitt (16,8\%), während sich beim Anteil von Bürgerinnen mit doppelter Staatsbürgerschaft weniger $\mathrm{Ab}$ weichungen zeigen (9,8\% in Holsterhausen und 10,3\% in Essen). In der Nähe des Fachgeschäfts für Stadtwandel befindet sich das Flüchtlingsdorf Papestraße. Mit einem Durchschnittsalter von 41,7 Jahren sind die Holsterhauser etwas jünger als der gesamtstädtische Durchschnitt (44,1 Jahre). Dabei liegt der Anteil der unter 18-Jährigen bei 12,7\% (16,1\% in Essen) und der Anteil der mindestens 65 -Jährigen bei $17,2 \%$ (21,5\% in Essen). Holsterhausen verfügt über eine positive Bevölkerungsbilanz. Die Arbeitslosenquote ist mit 7,7\% niedriger als in der Gesamtstadt (8,2 \%) und 16,6\% (gesamtstädtisch 17,9\%) der Anwohner*innen beziehen existenzsichernde Leistungen außerhalb von Einrichtungen (vgl. Amt für Statistik und Wahlen der Stadt Essen 2019). 
Das Fachgeschäft für Stadtwandel konnte in einem leerstehenden Ladenlokal in der Gemarkenstraße, der Haupteinkaufsstraße des Stadtteils, eingerichtet werden. Getragen wird das Fachgeschäft für Stadtwandel von der Initiative für Nachhaltigkeit e.V., dem Runden Tisch Holsterhausen und dem Standpunkt e.V. ${ }^{3}$. Begonnen hat alles mit acht Menschen aus der Transition Town-Bewegung (siehe hierzu auch Abdulnabi Ali in diesem Band) und der Initiative für Nachhaltigkeit e.V., die beim Parking Day 2017 die Idee eines eigenen Ladenlokals entwickelten. Angetrieben wurden sie von dem Gedanken, einen Nachbarschaftsort zu eröffnen, an dem sich die Engagierten und Bewohnerinnen Holsterhausens begegnen, austauschen und kollaborativ Projekte, Aktionen und Angebote schaffen können. Entstehen sollte eine Keimzelle für den sozialökologischen Wandel im Stadtteil und darüber hinaus, die neue Impulse für eine zukunftsfähige Gesellschaft in kultureller Vielfalt gibt.

Während dieses Team noch in der Ideenentwicklung steckte, war die lokale Initiative »Runder Tisch Holsterhausen« schon lange zum Thema Integration im Stadtteil aktiv. An dem Bündnis sind Institutionen, Kirchengemeinden, Vereine und in Arbeitskreisen organisierte, ehrenamtliche Helfer*innen beteiligt. Im Fachgeschäft engagieren sich die Arbeitskreise »Klamotte« und »Kommunikation«. Für die Kleiderkammer suchte die Initiative neue Räumlichkeiten. Daraus ist im Fachgeschäft der nun für alle Besucher*innen geöffnete Second-Hand-Bereich »die Klamotte« entstanden. Ebenso auf der Suche nach neuen Räumlichkeiten war der Standpunkt e.V., der ein bestehendes Werkstattcafé in das neue Projekt eingebracht hat. Standpunkt e.V. ist ein gemeinnütziger Verein mit Sitz in Essen, in dem spezialisierte Fachkräfte wie Therapeut*innen und Sozialpädagog*innen zusammen mit Künstler*innen, Unternehmer*innen, Freund*innen und weiteren Interessierten sozialer Ungerechtigkeit entgegenwirken wollen. Konkret werden praxisorientierte Projekte entwickelt, die Kindern und Jugendlichen aus allen Bevölkerungsgruppen Zugang zu Bildung, zu Kultur, sinnvoller Alltags- und Freizeitgestaltung und damit zu sozialem Miteinander geben. Die Trägerschaft für das Fachgeschäft für Stadtwandel hat die Initiative für Nachhaltigkeit e.V. übernommen. Unter dem Dach der Initiative finden sich verschiedene Menschen zusammen, die in unterschiedlichen Themenfeldern die nachhaltige Entwicklung in der Stadt Essen und im Ruhrgebiet fördern und vorantreiben 
wollen. Sie kümmert sich um alle administrativen Belange im Fachgeschäft für Stadtwandel und entwickelt die Veranstaltungen und Angebote zu sozialökologischen Themen.

Nachdem sich das neu entstandene Team für das jetzige etwa 250 qm große Ladenlokal entschieden hatte und mit dem Umbau begann, kamen kontinuierlich weitere Mitstreiter*innen hinzu. Durch die öffentlich gestaltete Bauphase im Herbst 2018, während der die Öffentlichkeit stets zur Mitarbeit eingeladen wurde, sowie im Zuge der Eröffnung im Dezember 2018 wurden immer mehr Einwohner*innen auf das Projekt aufmerksam. Sie bereichern seitdem das Kernteam und ermöglichen den Betrieb des Fachgeschäfts für Stadtwandel. Mittlerweile engagieren sich über fünfzig Menschen ehrenamtlich dafür, dass das Fachgeschäft für Stadtwandel an fünf Tagen pro Woche jeweils fünf Stunden für Besucher*innen öffnet und an den Vormittagen, abends und an den Wochenenden regelmäßige Angebote von Bürger*innen für Bürger*innen sowie Beratungen für Menschen mit und ohne Migrationshintergrund stattfinden können. Bemerkenswert ist an dieser Stelle, dass Gruppen, die bereits viele Jahre zu einem Thema zusammengearbeitet haben, mit Engagierten aus anderen Kontexten zusammenkommen, sich öffnen und gemeinsam neue Strukturen finden.

Kern des Ansatzes ist, unkommerziellen Freiraum für Begegnungen, Austausch und neue Ideen zur Verfügung zu stellen. Frei nach dem Motto »Wenn man nix tut, tut sich auch nix « wird eigener Einsatz gefordert und gefördert. Die zentralen Ziele des Fachgeschäfts für Stadtwandel bestehen darin, 1. einen Nährboden für eine lebendige Nachbarschaft zu bieten, die den sozialökologischen Wandel gestaltet, 2. die nachhaltige Entwicklung des Stadtteils voranzubringen und 3. Interkulturalität im Quartier zu leben.

Die Menschen, die in das Fachgeschäft kommen, sollen den Freiraum nutzen, sich ausprobieren, experimentieren, neue Erfahrungen sammeln, Kontakte knüpfen und vor allem: sich als (Mit-)Gestalter*innen der Stadtgesellschaft wahrnehmen. Die verschiedenen Angebote des Fachgeschäfts bieten Menschen eine Möglichkeit, sich einzubringen und sich als wertvoll und wirksam wahrzunehmen. Zudem kommen sie mit unterschiedlichen Lebenskonzepten und insbesondere mit nachhaltigen Alltagspraktiken in Berührung. Das stößt Reflektionen eigener Konzepte und Verhaltensmuster an, schafft Offenheit und Toleranz und lädt ein, nachhaltige Alltagspraktiken nachzuahmen und auszutesten. Durch die soziokulturelle Mischung des Teams und der Besucher*innen steht jede*r immer wieder vor der Aufgabe, sich mit anderen Meinungen und Denkmustern auseinander zu setzen. Dies erfor- 
dert vor allem Ambiguitätstoleranz (vgl. Frenkel-Brunswik 1949; Krappmann 1969; Müller-Christ/Weßling 2007; Bauer 2011), also die Fähigkeit, Unterschiede und Widersprüche wahrzunehmen, zu akzeptieren und dennoch eine gemeinsame Sprache und einen Weg zur Zusammenarbeit zu finden.

So wurde im ersten Jahr seines Bestehens der Raum mit unterschiedlichen Angeboten rund um Nachhaltigkeit, Gemeinschaft und Interkulturalität gefüllt. Aufgeteilt wurde der Raum in einen Cafébereich, der bei kostenlosen Getränken und gespendetem Kuchen zum Verweilen und Austausch einlädt. In einem Geschenkekoffer finden unterschiedlichste Alltagsgegenstände neue Besitzer*innen. In der Wandelbibliothek kann in Büchern und Zeitschriften über unterschiedliche Themen der sozialökologischen Transformation gestöbert werden. Im Werkstattbereich werden regelmäßig Selbsthilfewerkstätten zur Fahrradreparatur und Nähkurse angeboten. Für die Kinder gibt es eine Spielecke und lokale Künstler*innen können ihre Werke auf einer Ausstellungsfläche präsentieren. In der »Klamotte« werden Kleiderspenden angenommen und zu einem kleinen Preis verkauft. In der Küche wird bei Veranstaltungen vegetarisch und vegan gekocht. Aus dem Fairteiler von Foodsharing ${ }^{4}$ kann sich jede*r gerettete Lebensmittel nehmen. Lastenräder, Fahrradanhänger und ein Solarmodul werden kostenlos verliehen. Dazu gibt es Veranstaltungen zu Nachhaltigkeit, Integration und Demokratie sowie Beratung von und für Menschen mit und ohne Migrationshintergrund.

Vieles entsteht aus Eigeninitiative von Besucher"innen und Akteur*innen der Zivilgesellschaft. So nutzen auch einige Essener Initiativen wie die Organisator*innen der Gemeinschaftsgärten von Transition Town, die Ortsgruppe von Extinction Rebellion, Arbeitsgruppen des NABU und viele andere den Raum für ihre regelmäßigen Arbeitstreffen oder zur Vorbereitung von Aktionen. Anstehende Termine und Aktionen werden online über die Webseite und die Facebookseite des Fachgeschäfts für Stadtwandel angekündigt sowie offline über einen wöchentlich aktualisierten Kalender im Schaufenster. Grundlegende Bedingung für Angebote ist, dass sie kostenlos und für alle zugänglich sind. Kursleiter*innen, Betreuer*innen oder Kunst- und Kulturschaffende dürfen um eine Spende für ihren Aufwand bitten.

Foodsharing ist ein Netzwerk von Freiwilligen, die Lebensmittel aus Betrieben abholen, die noch essbar, aber nicht mehr verkäuflich sind und weggeschmissen würden. Die Verteilung der geretteten Lebensmittel erfolgt u.a. über öffentlich zugängliche »Fairteiler«, die meist aus einem Kühlschrank und Regalsystemen bestehen (siehe www.foodsharing.de. Zuletzt aufgerufen am 28.02.2020). 
Zentral ist, dass jede* $r$ teilhaben kann und keine wirtschaftlichen Interessen verfolgt werden. Grundlage für das kollaborative Miteinander ist eine eher soziokratische Organisation ohne strenge Hierarchien. Das Zusammenwirken basiert demnach auf einer konsensorientierten und co-kreativen Arbeitsweise, die es ermöglicht, dass unterschiedliche Perspektiven sichtbar werden und ein gegenseitiges Verständnis entwickelt wird. Einmal in der Woche findet ein Jour fixe statt, der allen Interessierten offensteht. In ein bis eineinhalb Stunden werden nach festgelegtem Muster die wichtigsten Anliegen und Themen der zurückliegenden sowie der kommenden Woche, Anfragen, Termine, Anschaffungen und Aufgaben besprochen. Der Jour fixe stellt das Gremium dar, in dem neue Ideen vorgestellt werden und nach dem Prinzip des systemischen Konsensierens (vgl. Paulus/Schrotta/Visotschnig 2013) darüber entschieden wird, ob eine Idee aufgenommen wird, ein Termin stattfindet oder wahrgenommen wird, welche Aktivitäten geplant werden, und wer sich in welcher Intensität daran beteiligt. Durch dieses Vorgehen soll sichergestellt werden, dass Viele gleichzeitig Verantwortung übernehmen, Selbstwirksamkeit erfahren und persönlich wachsen.

Im ersten Jahr hat sich das Fachgeschäft für Stadtwandel als ein Freiraum, Anker und eine Plattform für die Nachbarinnen und Stadtbewohnerinnen entwickelt. Über verschiedene Formate werden immer wieder Informationsund Mitmach-Impulse gesetzt, die Reflektionen und Experimente im eigenen Alltag anstoßen. Durch die Kooperation und Verbindung mit Initiativen und Aktiven, die für den sozialökologischen Wandel und das Miteinander in kultureller Vielfalt eintreten, existiert ein sich stetig erweiterndes Netzwerk mit gewichtiger Stimme in der Stadt. Zudem bestehen gute Verbindungen zu kommunalen Einrichtungen, Verwaltung und Politik. Die Stabsstelle Integration des Bezirks Essen-West hat sich unbürokratisch an Renovierungskosten beteiligt und stellt Mittel für Grundkosten, wie Miete und integrative Projekte zur Verfügung. Weitere Mittel werden über den Verkauf der SecondHand-Kleidung über Spenden und über die Akquise von Projektförderungen generiert.

Wie lange der Freiraum durch das Fachgeschäft für Stadtwandel offengehalten werden soll, haben die Nachbarinnen selbst in der Hand: So lange es Menschen gibt, die den Raum zum Ausprobieren und Mitmachen, zum Geben und Nehmen offenhalten, so lange kann das Fachgeschäft für Stadtwandel existieren. Genauso bestimmt das Engagement der Menschen, welche Themen und Schwerpunkte im Fachgeschäft behandelt werden. 


\section{Das Fachgeschäft als soziale Innovation für Stadtwandel}

Durch seine Lage in einem Ladenlokal der Haupteinkaufsstraße des Quartiers ist das Nachbarschaftsprojekt in der Lebenswelt der Menschen angesiedelt und kann an alltägliche Routinen und Lebensstile andocken. Inhaltliche Angebote werden niederschwellig vermittelt und mit Grundbedürfnissen verbunden, wie Ernährung, Gemeinschaft, Mobilität, Kultur, Kleidung usw. Damit wird ein einfacher Zugang zu neuen, komplexen Themen gewährleistet. Die Menschen, die im Fachgeschäft zusammenkommen, werden als Expertinnen oder »Fachverkäufer*innen« für ihre eigene Lebenswelt wahrgenommen. Diese bringen sie auf verschiedene Art in das Projekt ein, sodass gegenseitiges Lernen stattfinden kann (Trumann o.J.). Auf diese Weise werden Beiträge zu einer niederschwelligen und partizipativen Stadtentwicklung ermöglicht (vgl. Behr/Ahaus 2016). Dabei ist das Fachgeschäft für Stadtwandel nicht monothematisch angelegt, sondern ein »Gemischtwarenladen der Nachhaltigkeit«. Die Palette reicht von Nachhaltigkeit im Alltag (Mobilität, Ernährung, Müllvermeidung usw.) über demokratisches und interkulturelles Miteinander bis hin zu Kollaboration und neuen Formen der Entscheidungsfindung. Besucher*innen werden einerseits durch thematische Angebote angelockt, kommen aber auch ohne Anlass vorbei, wollen eigene Ideen entwickeln oder sich einfach nur aufhalten und unterhalten. Die günstige und zentrale Lage in der Einkaufsstraße und das große Schaufenster bringen Passant"innen dazu, stehen zu bleiben und neugierig einzukehren. Das Fachgeschäft unterstützt die Entwicklung neuer Ideen und Angebote mit Methoden und Ressourcen etwa für Workshops. Es ist daneben ein Begegnungsort, der einen geschützten Rahmen bietet und neutraler Ort ist. Die räumliche Nähe der vielfältigen sozial-ökologischen Ideen und Angebote unter einem Dach und Begegnungen im Laden fördern Spill-over-Effekte zwischen verschieden Themen. Die Verbindung von face-to-face-Kommunikation, Öffentlichkeitsarbeit und Nutzung digitaler "sozialer Medien« schafft Reichweite und hilft über den Stadtteil hinaus zu wirken. So wird mit Teilnahme an Wettbewerben sogar eine bundesweite Öffentlichkeit erreicht ${ }^{5}$.

Somit verbindet der Quartiersansatz des Projektes »bottom-up« und »top-down«. Einerseits wird die Ortsverbundenheit von Menschen genutzt, 
gestärkt oder auch erst geschaffen (»Place Attachment« vgl. u.a. Giuliani 2003; Manzo/Devine-Wright 2018). Andererseits ist das Projekt durch seine Ausstrahlungskraft über den Stadtteil hinaus im kommunalen Setting groß genug, um auch von »top«, also von kommunalen und politischen Vertreterinnen, als wichtige Instanz in und für die Stadtgesellschaft wahrgenommen $\mathrm{zu}$ werden.

Schließlich kann das Fachgeschäft für Stadtwandel auch als dauerhafter Experimentierraum oder Reallabor betrachtet werden, in dem offene Innovationen möglich sind. Menschen können (Frei-)Räume nutzen für Ideen, die nicht vorgegeben oder bereits von anderen angedacht sind. Es gibt Veranstaltungs- und Projektangebote, aber auch viel Offenheit in den Themen. Bürger*innen können Anschluss zu vielfältigen Netzwerken finden, die hier zusammenkommen oder sich überlappen.

Damit wird deutlich, dass im Fachgeschäft für Stadtwandel unterschiedliche Typen von Akteur*innen zusammenkommen, die für die Entwicklung und Verbreitung von sozialen Innovationen der Nachhaltigkeit bedeutsam sind, wie Agent*innen des Wandels (Change Agents), Promotor*innen, Early Adopter usw. (Ahaus 2019). In sozial-ökologischen Veränderungsprozessen lassen sich verschiedene Promotor*innen-Rollen beschreiben (Kristof 2010 \& 2017), welche sich auch im vorliegenden Projekt wiederfinden. So können die beteiligten Vereine und Vertreter*innen dieser als Ideengeber*innen bzw. Fachpromotor*innen beschrieben werden. Sie besetzen aber auch die Rollen von Prozesspromotor*innen, die den Prozess der Gründung und Eröffnung des Ladens vorantreiben, sowie von Beziehungspromotor*innen, die die Netzwerke aufspannen und entwickeln. In dieser Rolle tragen Akteur*innen zur Bildung von Sozialkapital bei, es werden Netzwerke etabliert und gepflegt. Machtpromotor*innen sind hier insbesondere Stiftungen und die Stadtverwaltung, die Fördermittel für den Betrieb des Fachgeschäftes und für seine Projekte bereitstellen (vgl. auch Ahaus 2019).

Damit kann das Fachgeschäft zugleich als soziale Innovation und als Katalysator für soziale Innovationen beschrieben werden.

\section{Zur Nachahmung empfohlen? - Ein vorläufiges Fazit}

Nachbarschaftsprojekte wie das hier vorgestellte Fachgeschäft für Stadtwandel in Essen-Holsterhausen bieten vielfältige Chancen, das kooperative En- 
gagement für die Demokratie zu stärken und gemeinschaftlich gesellschaftlichen Herausforderungen zu begegnen.

Sie bieten niederschwellige Partizipationsangebote, die an den alltäglichen Grundbedürfnissen in der Lebenswelt von Bürger*innen ansetzen, und eröffnen den vielfältigen Interessen im Quartier und der Stadtgesellschaft einen Kristallisationsraum. Hier können individuelle und kollektive Selbstwirksamkeitserfahrungen gemacht werden und so auch grundlegende Fähigkeiten für eine lebendige Demokratie eingeübt werden. Selbst aktiv zu werden, eigene Interessen zu definieren, wahrzunehmen und zu verfolgen sowie Konflikte auszuhandeln, sind Basis solcher Projekte und zugleich ein offenes Angebot an die Öffentlichkeit. Langfristig fördern solche Projekte, dass sich die Menschen im Stadtteil und darüber hinaus zu einem lebendigen Netzwerk verbinden, welches sich gegenseitig unterstützt, interkulturelle Vielfalt schätzt und gemeinsam die nachhaltige Entwicklung des Stadtteils und der Stadt gestaltet und vorantreibt.

Damit wirken Projekte wie das Fachgeschäft für Stadtwandel der von Richard Sennett mahnend skizzierten Dequalifizierung der Kooperation von modernen Gesellschaften entgegen und bilden einen Gegenpol zur globalisierungsbedingten kulturellen Homogenisierung. Vielfalt wird gelebt, Unterschiede begegnen einander und ergebnisoffene Dialoge, die gegenseitiges Verständnis geben und Raum für gemeinsame Experimente öffnen, werden täglich geübt.

Durch das Wesen eines offenen Experimentierfeldes werden Veränderungen sowohl auf individueller als auch auf gemeinschaftlicher Ebene angestoßen: Individuen können sich neu ausprobieren und Fähigkeiten, Überzeugungen und Grenzen neu entdecken. Die sich hier aufspannenden Netzwerke können ungezwungen kollektive Prozesse organisieren, unterschiedliche Wege der Kooperation und Kollaboration ausprobieren.

Der materielle und sozial konstruierte Raum erzeugt ein Wir-Gefühl, das nicht eindimensional, sondern vielfältig ist, dessen Rahmen nicht festgelegt und dessen Intensität individuell ist. Wie tief sich jemand in das »Wir« einbringt, bleibt persönliche Entscheidung. Als Orte, die offen sind für jeden Menschen, unabhängig von seiner Motivation und den Grad des Engagements, bedienen solche Projekte ein Stück weit die Sehnsucht nach dem ungezwungenen »Dorf in der Stadt«.

Dabei darf nicht übersehen werden, dass es enorme Anstrengungen von allen Beteiligten erfordert, den Raum offen $\mathrm{zu}$ halten. Die ehrenamtlichen »Raumbewahrer*innen", die konstant und mit hohem Eigenengagement mit- 
wirken, sind besonders gefordert. Auf persönlicher Ebene braucht es Dialogfähigkeiten, Empathie im Sinne einer Neugier für den Menschen an sich (vgl. Sennett 2019: 29ff.) und Ambiguitätstoleranz. Strukturell müssen Regeln gefunden werden, die genug Halt geben und zugleich eine Offenheit, die erlaubt, dass sie immer wieder hinterfragt und bedarfsgerecht angepasst werden. Eine weitere Herausforderung ist die Balance zwischen Flexibilität und Zuverlässigkeit: Damit ein Raum wie das Fachgeschäft für Stadtwandel von der Öffentlichkeit wahrgenommen und angenommen wird, muss er verlässliche Öffnungszeiten und Angebote bieten. Auch die freiwillig Engagierten brauchen ein gewisses Maß an Zuverlässigkeit und Verbindlichkeit, zugleich aber auch Flexibilität für sich. Sich zu engagieren ist eine ständig neue Entscheidung, die mehr oder weniger bewusst mit jedem Tag des Engagements getroffen wird. In einem Team, das ausschließlich aus freiwillig Engagierten besteht, ist der Spagat zwischen (gefühltem) Fremdanspruch auf Verfügbarkeit und Anwesenheit sowie eigenem Wunsch nach Flexibilität besonders herausfordernd. Hier braucht es Achtsamkeit für Grenzen: die eigene Fähigkeit, persönliche Grenzen im Blick zu behalten und sich nicht selbst zu überfordern, sowie die Achtsamkeit im Team, dass Einzelne nicht überbeansprucht werden oder sich überfordern.

Zur Unterstützung hat das Team einen Supervisions-Prozess mit erfahrenen Coaches angestoßen. Solche Teamprozesse sind für manche Ehrenamtliche eine ungewohnte und neue Erfahrung. Einige lassen sich dennoch darauf ein, andere sehen den Nutzen nicht und möchten sich »einfach nur engagieren«. Insgesamt wurde der Prozess von denen, die sich eingelassen haben, als wertvolle Erfahrung erlebt, die neue Begegnungen untereinander ermöglicht. Es hat sich gezeigt, dass es für das sehr aktive raumbewahrende Team wichtig ist, außerhalb des freiwilligen Engagements Gelegenheiten für gemeinsame Aktionen und Begegnungen untereinander zu schaffen.

Nachdem das Fachgeschäft für Stadtwandel im Dezember 2019 seinen ersten Geburtstag gefeiert hat, kann als Zwischenfazit festgehalten werden: Das Projekt etabliert sich als Keimzelle für sozialökologischen Wandel, die die Akteure im Stadtteil und darüber hinaus zusammenbringt. Es soll auch langfristig als hierarchiearmer Freiraum von Bürger*innen für Bürger*innen zur Verfügung stehen, die gemeinsame Aktionen und Projekte für einen lebenswerten Stadtteil planen und umsetzen und die Transformation zu einem nachhaltigen Quartier selbst gestalten. Es soll weiterhin Begegnungsraum sein, in dem generationen- und kulturübergreifend Vielfalt, achtsames Miteinander und eine respektvolle Diskussionskultur gelebt werden. 
Weiterhin zeigt das Interesse aus anderen Stadtteilen und Städten, dass es Vorbild für weitere Fachgeschäfte an anderen Orten sein kann. Dabei ist eine exakte Übertragung nicht möglich. Denn das Fachgeschäft für Stadtwandel, wie es sich aktuell darstellt, ist vor allem geprägt durch die Personen, die es beleben, ihre individuellen und kollektiven Lernprozesse und die Situationen und Rahmenbedingungen im Stadtteil und in der Stadt. Übertragen werden kann aber die Grundidee und ein Baukasten mit verschiedenen Elementen, die auch in anderen Städten und Stadtteilen Anwendung finden können. Das Projekt selbst hat das Rad nicht in jeglicher Hinsicht neu erfunden, sondern mit einer Menge Mut, Offenheit und Experimentierfreude einen Raum geschaffen, Angebote entwickelt und verschiedene Akteur*innen, existente Projekte und sozial-ökologische Handlungsansätze im lokalen Kontext in einen gemeinsamen Rahmen gebracht. Genauso müssen individuell der lokale Kontext, die Akteursnetzwerke und die sozio-demographischen Rahmenbedingungen für ähnliche Projekte betrachtet werden, um passgenaue Experimentierfelder für ähnliche Projekte an anderen Orten $\mathrm{zu}$ entwickeln und die erforderlichen Ressourcen zu organisieren.

\section{Literatur}

Ahaus, Björn (2019): Urbane Agent_innen des Wandels für soziale Innovationen der Nachhaltigkeit: eine qualitative Studie über Eigenschaften, Rollen und Netzwerke von Promotor_innen der Bürgergesellschaft im zentralen Ruhrgebiet, Stuttgart. Online verfügbar unter: https://elib. uni-stuttgart.de/bitstream/11682/10519/5/Druckfassung-Diss_Ahaus_ 20190729.pdf. Zuletzt aufgerufen am 06.02.2020.

Amt für Statistik und Wahlen der Stadt Essen (2019): Ein Blick auf ... Stadtteile in Essen Holsterhausen 2018, 10/2019, Essen.

Bauer, Thomas (2011): Die Kultur der Ambiguität - Eine andere Geschichte des Islam, 5. Auflage, Berlin: Verlag der Weltreligionen im Insel Verlag.

Beher, Karin/Liebig, Reinhard/Rauschenbach, Thomas (1999): Das Ehrenamt in empirischen Studien - ein sekundäranalytischer Vergleich, Schriftreihe des Bundesministeriums für Familie, Senioren, Frauen und Jugend, Band 163, 2. Auflage, Stuttgart: W. Kohlhammer.

Behr, Friederike/Ahaus, Björn (2016): ")Lebensweltbezug< im kommunalen Klimaschutz - Ein Plädoyer für eine gemeinsame Maßnahmenentwicklung in den Stadtteilen.«, in: Alexander J. Schmidt (Hg.), Reihe Ergebnis- 
se aus dem Projekt Klima-Initiative Essen für Wissenschaft und Praxis 9, Essen.

Berding, Nina/Bukow, Wolf-Dietrich (Hg.) (2020): Die Zukunft gehört dem urbanen Quartier. Wiesbaden: Springer VS. https://doi.org/10.1007/9783-658-27830-4

Burkhardt, Luise/Schupp, Jürgen (2019): »Wachsendes ehrenamtliches Engagement: Generation der 68er häufiger auch nach dem Renteneintritt aktiv«, in: DIW Wochenbericht 86 (42), Berlin, S. 766-773.

Denner, Gabriele (Hg.) (2014): Hoffnungsträger, nicht Lückenbüßer. Ehrenamtliche in der Kirche. Ostfildern: Schwabenverlag.

Frenkel-Brunswik, Else (1949): »Intolerance of Ambiguity as an Emotional and Perceptual Personality Variable«, in: Journal of Personality 18, S. 108-143. https://doi.org/10.1111/j.1467-6494.1949.tbo1236.x

Gesemann, Frank/Seidel, Alexander/Mayer, Margit (2019): Entwicklung und Nachhaltigkeit von Willkommensinitiativen, vhw-Schriftenreihe 13, 2019, Berlin.

Giuliani, Maria Vittoria (2003): »Theory of attachment and place attachment «, in: Mirilia Bonnes/Terence Lee/Marino Bonaiuto (Hg.), Psychological theories for environmental issues, Aldershot: Ashgate, S. 137-170.

Hacket, Anne/Mutz, Gerd (2002): »Empirische Befunde zum bürgerschaftlichen Engagement«, in: Bundeszentrale für politische Bildung (bpb) (Hg.), Aus Politik und Zeitgeschichte (APuZ) 9, Bonn, S. 39-46.

Howaldt, Jürgen/Kopp, Ralf/Schwarz, Michael (2014): Zur Theorie sozialer Innovationen. Tardes vernachlässigter Beitrag zur Entwicklung einer soziologischen Innovationstheorie, 1. Auflage, Weinheim/Basel: Beltz Juventa.

Krappmann, Lothar (1969): Soziologische Dimensionen der Identität. Strukturelle Bedingungen für die Teilnahme an Interaktionsprozessen, Stuttgart: Klett-Cotta.

Kristof, Kora (2010): Models of Change. Einführung und Verbreitung sozialer Innovationen und gesellschaftlicher Veränderungen in transdisziplinärer Perspektive, Zugl.: Habil.-Schrift, 2006, Zürich: vdfHochschulverlag. Online verfügbar unter: www.vdf.ethz.ch/info/showDetails.asp?isbnNr=3315. Zuletzt aufgerufen am 24.01.2020.

Kristof, Kora (2017): „Change Agents in gesellschaftlichen Veränderungsprozessen«, in: Julia-Lena Reinermann/Frederike Behr (Hg.), Die Experimentalstadt. Kreativität und die kulturelle Dimension der Nachhaltigkeit, Wiesbaden: VS Verlag für Sozialwissenschaften, S. 165-179. https://doi. org/10.1007/978-3-658-14981-9_9 
Kummel, Olivia/Nadler, Robert (2018): Die Grenzen des Ehrenamts, in: Informationen zur Raumentwicklung 6, S. 102-111.

Landmann, Helen/Aydin, Anna Lisa/Dick, Rolf van/Klocke, Ulrich (2017): »Die Kontakthypothese: Wie Kontakt Vorurteile reduzieren und die Integration Geflüchteter fördern kann«, in: Das In-Mind Magazin 9 (3). Online verfügbar unter: http://de.in-mind.org/article/die-kontakthypothesewie-kontakt-vorurteile-reduzieren-und-die-integration-gefluechteter.

Zuletzt aufgerufen am 26.02.2020.

Manzo, Lynne C./Devine-Wright, Patrick (2018): »Place Attachment«, in: Linda Steg/Judith I. M. de Groot (Hg.), Environmental Psychology: An Introduction, 2. Auflage, Hoboken/NJ: John Wiley \& Sons, S. 135-143. https:// doi.org/10.1002/9781119241072.ch14

Müller-Christ, Georg/Weßling, Gudrun (2007): Widerspruchsbewältigung, Ambivalenz- und Ambiguitätstoleranz. Eine modellhafte Verknüpfung, in: Georg Müller-Christ/Lars Arndt/Ina Ehnert (Hg.), Nachhaltigkeit und Widersprüche: Eine Managementperspektive, Berlin: LIT.

Paulus, Georg/Schrotta, Siegfried/Visotschnig, Erich (2013): Systemisches Konsensieren. Der Schlüssel zum gemeinsamen Erfolg, Holzkirchen: Danke-Verlag.

Priller, Eckhard (2011): »Dynamik, Struktur und Wandel der Engagementforschung. Rückblick, Tendenzen und Anforderungen«, in: Eckhard Priller/Mareike Alscher/Dietmar Dathe/Rudolf Speth (Hg.), Zivilengagement. Herausforderungen für Gesellschaft, Politik und Wissenschaft, Berlin: LIT, S. 11-40.

Sennett, Richard (2019): Zusammenarbeit - Was unsere Gesellschaft zusammenhält, 5. Auflage, München: Carl Hanser.

Simonson, Julia/Vogel, Claudia/Zieglemann, Jochen/Tesch-Römer, Clemens (2017): »Einleitung: Freiwilliges Engagement in Deutschland«, in: Julia Simonson/Claudia Vogel/Clemens Tesch-Römer (Hg.), Freiwilliges Engagement in Deutschland - Der Deutsche Freiwilligensurvey 2014, Wiesbaden: Springer VS, S. 31-49. https://doi.org/10.1007/978-3-658-12644-5

Stricker, Michael (2011): »Ehrenamt«, in: Thomas Olk/Birger Hartnuß (Hg.), Handbuch Bürgerschaftliches Engagement, Weinheim: Beltz Juventa, S. 163-171.

Truman, Jana (2020): »Unperfekthaus, Impact Hub und Fachgeschäft - drei Beispiele für informelle >Häuser der Erwachsenenbildung in der Stadt Essen«, in: Bernd Käpplinger (Hg.), Neue Häuser der Erwachsenenbildung, Berlin: Peter Lang. 



\section{Biographische Angaben zu den Beiträger*innen}

Abeer Abdulnabi Ali, geb. 1994, ist wissenschaftliche Mitarbeiterin in der AG Gesellschaft und Nachhaltigkeit im Wandel am Geographischen Institut der Ruhr-Universität Bochum und arbeitet im BMBF-geförderten Projekt > JaCLab - Integrated Regional Climate Lab North Jakarta and Port (JaC-Lab)<.

Björn Ahaus, geb. 1977, Dr. rer. pol., ist wissenschaftlicher Mitarbeiter am Kulturwissenschaftlichen Institut in Essen und einer der Initiatoren des Fachgeschäfts für Stadtwandel. Er arbeitet am KWI im Forschungsbereich Partizipationskultur und hat zu Urbanen Agent*innen des Wandels für soziale Innovationen der Nachhaltigkeit promoviert.

Marius Beckamp, geb. 1990, ist wissenschaftlicher Mitarbeiter im Forschungsschwerpunkt Raumkapital des Instituts Arbeit und Technik, Gelsenkirchen. Er forscht derzeit zu Fragestellungen an der Schnittstelle von nachhaltiger Stadtentwicklung und Energiewende.

Benjamin Best, Dr. rer. pol., ist wissenschaftlicher Mitarbeiter im Forschungsbereich Strukturwandel und Innovation am Wuppertal Institut für Klima, Umwelt, Energie und leitet eine BMBF-Nachwuchsforschergruppe zum Thema Energiesuffizienz. Er interessiert sich für partizipative Governance, Reallabore, alternative Wohlstandsmodelle und die Tiefsee.

Weert Canzler, geb. 1960, Dr. phil. habil, leitet zusammen mit Andreas Knie die Forschungsgruppe Digitale Mobilität und gesellschaftliche Differenzierung im Wissenschaftszentrum Berlin für Sozialforschung (WZB).

Emily Drewing, geb. 1991, ist wissenschaftliche Mitarbeiterin am Seminar für Sozialwissenschaften der Universität Siegen sowie am Geographischen 
Institut der Ruhr-Universität Bochum. Aktuell forscht sie zur Umsetzung der Energiewende vor dem Hintergrund zeitgenössischer Großentwicklungen (Megatrends) und zunehmender Konflikte (etwa beim Stromnetz- und Windenergieausbau). Sie promoviert an der sozialwissenschaftlichen Fakultät der Ruhr-Universität Bochum zur sozialen Exklusivität von Energiegenossenschaften.

Steven Engler, geb. 1985, Dr. rer. nat., ist Leiter der AG Gesellschaft und Nachhaltigkeit im Wandel am Geographischen Institut der Ruhr-Universität Bochum. Er forscht derzeit zu nachhaltiger Ernährung und multifunktionaler Hafennutzung in Jakarta, Indonesien.

Anna Nora Freier, Sozialwissenschaftlerin, ist wissenschaftliche Mitarbeiterin am Institut für Demokratie- und Partizipationsforschung der Bergischen Universität Wuppertal. Dort ist sie in Lehre und Forschung mit aktuellen Fragestellungen der politischen Partizipation sowie mit der Gestaltung und Durchführung von Bürger*innenbeteiligungen in der Energiewende befasst.

Sabrina Glanz, geb. 1988, ist Promotionsstudentin und wissenschaftliche Mitarbeiterin am Lehrstuhl für Allgemeine Soziologie, Arbeit und Wirtschaft an der Ruhr-Universität Bochum. Dort beschäftigt sie sich mit Energiepraktiken in privaten Haushalten und der gesellschaftlichen Wahrnehmung von Technologien im Kontext der Energiewende.

Edgar Göll, geb. 1957, Dr., ist Sozialwissenschaftler und Forschungsleiter des Clusters >Zukunftsforschung und Partizipation`im IZT - Institut für Zukunftsstudien und Technologiebewertung in Berlin. Seine Arbeitsschwerpunkte sind Konzepte und Methoden der Zukunftsforschung, Nachhaltige Entwicklung und Transformation. Er ist Dozent im Masterstudiengang >Zukunftsforschungく am Institut Futur der FU Berlin und Boardmitglied im supranationalen Netzwerk Zukunftsforschung.

Ann-Kristin Götz, geb. 1993, ist wissenschaftliche Hilfskraft am CERES der Ruhr-Universität Bochum. Sie studiert Religionswissenschaft im Master und arbeitet derzeit zur Ökonomisierung des Sozialen und deren Einfluss auf die Gegenwartsreligiosität. 
Julia Janik, geb. 1993, ist studentische Hilfskraft in der AG Gesellschaft und Nachhaltigkeit im Wandel am Geographischen Institut der Ruhr-Universität Bochum. Sie arbeitet dort im Projekt >EnerTrend - Systemische Analyse von Wechselwirkung der Energiewende in NRW mit zentralen Megatrends< (MWIDE) und dem internationalen BMBF-geförderten Projekt >JaC-Lab Integrated Regional Climate Lab North Jakarta and Port«.

Andreas Knie, geb. 1960, Prof. Dr. phil. habil., ist Professor für Soziologie an der Technischen Universität Berlin und leitet zusammen mit Weert Canzler die Forschungsgruppe Digitale Mobilität und gesellschaftliche Differenzierung im Wissenschaftszentrum Berlin für Sozialforschung (WZB).

Sonja Knobbe, geb. 1986, ist wissenschaftliche Mitarbeiterin im Forschungsbereich Partizipationskultur am Kulturwissenschaftlichen Institut in Essen. Derzeit forscht sie zur Demokratisierung eines nachhaltigen Strukturwandels und zur philosophischen Begriffsklärung ökonomischer Praktiken.

Olaf Kühne, geb. 1973, Prof. Dr. Dr., forscht und lehrt als Professor für Stadtund Regionalentwicklung an der Eberhard Karls Universität Tübingen. Seine Forschungsschwerpunkte umfassen Landschaftstheorie, soziale Akzeptanz von Landschaftsveränderungen, Nachhaltige Entwicklung, Transformationsprozesse in Ostmittel- und Osteuropa, Regionalentwicklung sowie Stadtund Landschaftsökologie.

Claus Leggewie, geb. 1950, Prof. Dr., ist Politikwissenschaftler und Inhaber der Ludwig Börne-Professur an der Justus-Liebig-Universität Giessen. Leggewie ist Mitherausgeber der Blätter für deutsche und internationale Politik. Seine Forschungsschwerpunkte sind intra- und transdisziplinäre Zusammenhänge zu Themen der Klima- und Interkultur.

Stephanie Lübke, Dipl. Umweltwiss., ist wissenschaftliche Mitarbeiterin an der Sozialforschungsstelle (sfs) der TU Dortmund im Forschungsbereich Transformative Governance in Stadt und Region. Der Fokus ihrer wissenschaftlichen Arbeit sind soziale Innovationen im Kontext der Energiewende, Transformationsprozesse nachhaltiger Entwicklung sowie innovative Beteiligungsund Umsetzungsstrukturen im Bereich Klimaanpassung. 
Juliane Lunge, M.A., ist wissenschaftliche Mitarbeiterin am Wuppertal Institut für Klima, Umwelt, Energie im Forschungsbereich Strukturwandel und Innovation. Ihre Arbeitsschwerpunkte liegen in partizipativen Projekten der nachhaltigen Stadtentwicklung sowie im Bereich Governance der Energiewende auf lokaler und regionaler Ebene.

Martina Nies, geb. 1980, hat als Diplom-Geographin über zehn Jahre zu lokalen und regionalen Strategien im Umgang mit dem Klimawandel und der Energiewende sowie $\mathrm{zu}$ individuellem Umwelthandeln und Klimaschutz geforscht. Ein besonderer Fokus ihrer Arbeit liegt auf Methoden der transdisziplinären Zusammenarbeit. Aktuell arbeitet sie zu zivilgesellschaftlichem Empowerment in der transformativen Stadt- und Gesellschaftsentwicklung.

Jörg Radtke, geb. 1982, Dr. phil., ist wissenschaftlicher Mitarbeiter am Seminar für Politikwissenschaft der Universität Siegen. Er lehrt und forscht zur Energie- und Verkehrswende, Partizipation und Nachhaltigkeitspolitik. Er leitet derzeit ein Forschungsprojekt zu Online-Beteiligung und ist Teilprojektleiter in einem Projektforschungsverbund im Themenfeld Energiewende und Populismus.

Holger Rust, geb. 1946, Dr., war bis zu seinem Ruhestand Professor für Wirtschaftssoziologie an Universitäten des In- und Auslandes. Seit mehr als 25 Jahren ist er zudem Konzeptmanager und Scientific Consultant in politischen Institutionen und Unternehmen. Dortige Schwerpunkte umfassen Forschungsstrategien von Foresight-Projekten, Transformationsfolgen und Talent-Management. Holger Rust ist Autor von mehr als 30 Fach- und Sachbüchern und Publizist in renommierten Wirtschaftsmagazinen.

Nora Schecke, geb. 1989, ist wissenschaftliche Mitarbeiterin in der AG Gesellschaft und Nachhaltigkeit im Wandel am Geographischen Institut der RuhrUniversität Bochum. Zurzeit arbeitet Sie im Projekt >EnerTrend - Systemische Analyse von Wechselwirkungen der Energiewende in NRW mit zentralen Megatrends` (MWIDE) des Virtuellen Instituts (VI) >Transformation - Energiewende NRW<.

Katharina Schrot, Sozialwissenschaftlerin, ist wissenschaftliche Mitarbeiterin an der Sozialforschungsstelle (sfs) der TU Dortmund im Forschungsbereich Transformative Governance in Stadt und Region. Soziale Innovation 
im Bereich Nachhaltigkeit, Akteurskonstellationen in Multistakeholderprozessen und Innovationsdialoge im Kontext der Energiewende und zur breiteren Stärkung der regionalen Klimaanpassung bilden den Fokus ihrer Forschungsarbeit.

Stefan Schweiger, geb. 1980, ist Koordinator des BMBF-Projekts >Plastikbudget<, Teil des Koordinatorenteams des Virtuellen Instituts (VI) >Transformation- Energiewende NRW und des BMBF-Projekts >Integrated Regional Climate Lab North Jakarta and Port (JaC-Lab)< an der RuhrUniversität Bochum. Er forscht derzeit zu Narrativen der Nachhaltigkeit.

Oliver Stengel, geb. 1974, Dr. phil., ist Vertretungsprofessor für Nachhaltige Entwicklung an der Hochschule Bochum. Er forscht zu globalen ökologischen und gesellschaftlichen Transformationen.

Florian Weber, geb. 1983, Jun.-Prof. Dr. habil., forscht und lehrt als Juniorprofessor an der Universität des Saarlandes. Seine Forschungsschwerpunkte liegen in der Diskurs- und Landschaftsforschung, Border Studies, erneuerbaren Energien sowie Stadtpolitiken und Stadtentwicklungsprozessen im internationalen Vergleich.

Katja Witte, Dipl. Soz.-Wiss., ist Co-Leiterin des Forschungsbereichs Strukturwandel und Innovation am Wuppertal Institut für Klima, Umwelt, Energie. Sie arbeitet schwerpunktmäßig zu Fragestellungen der Energiewende aus nicht-technologischer Sicht sowie an Innovationsstrategien für eine erfolgreiche Umsetzung eines nachhaltigen Strukturwandels.

Matthias Wolf, geb. 1993, ist studentische Hilfskraft in der AG Gesellschaft und Nachhaltigkeit im Wandel am Geographischen Institut der Ruhr-Universität Bochum. Er arbeitet dort im Projekt >EnerTrend - Systemische Analyse von Wechselwirkungen der Energiewende in NRW mit zentralen Megatrends (MWIDE).

Jenny Zorn, geb. 1986, ist Co-Koordinatorin des Virtuellen Instituts >Transformation - Energiewende NRW in der AG Gesellschaft und Nachhaltigkeit im Wandel am Geographischen Institut der Ruhr-Universität Bochum. Sie forscht derzeit im Projekt >EnerTrend (MWIDE) zu den Wechselwirkungen 
zwischen der Energiewende und zentralen Megatrends unserer Zeit sowie in ihrer Dissertation zu philosophischen Implikationen der Energiewende. 


\section{Politikwissenschaft}
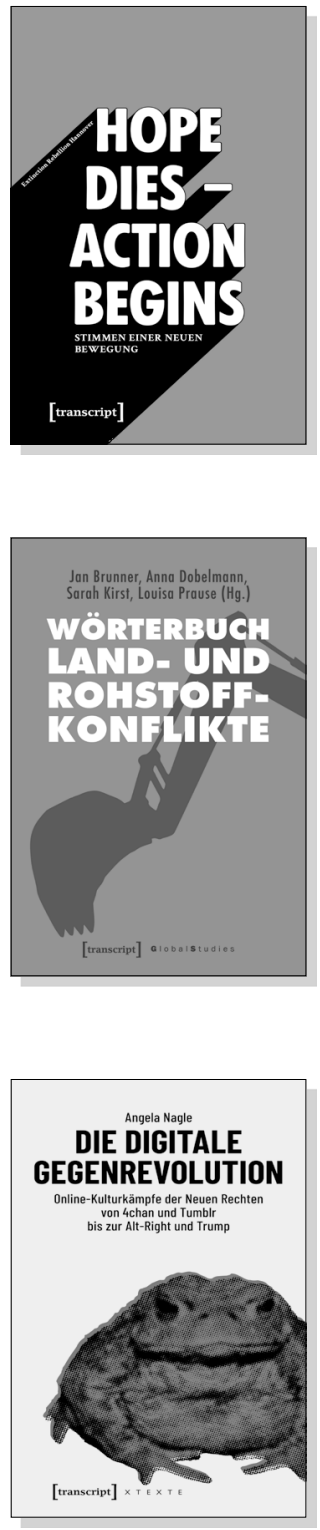

Extinction Rebellion Hannover

"Hope dies - Action begins":

\section{Stimmen einer neuen Bewegung}

2019,96 S., kart.

7,99 € (DE), 978-3-8376-5070-9

E-Book: kostenlos erhältlich als Open-Access-Publikation, ISBN 978-3-8394-5070-3

EPUB: kostenlos erhältlich als Open-Access-Publikation, ISBN 978-3-7328-5070-9

Jan Brunner, Anna Dobelmann,

Sarah Kirst, Louisa Prause (Hg.)

\section{Wörterbuch Land- und Rohstoffkonflikte}

2019, 326 S., kart., Dispersionsbindung, 1 SW-Abbildung 24,99€ (DE), 978-3-8376-4433-3

E-Book: 21,99 € (DE), ISBN 978-3-8394-4433-7

Angela Nagle

Die digitale Gegenrevolution

Online-Kulturkämpfe der Neuen Rechten

von 4chan und Tumblr bis zur Alt-Right und Trump

2018, 148 S., kart.

$19,99 €(D E), 978-3-8376-4397-8$

E-Book: 17,99 € (DE), ISBN 978-3-8394-4397-2

EPUB: $17,99 €$ (DE), ISBN 978-3-7328-4397-8 


\section{Politikwissenschaft}

Ines-Jacqueline Werkner Gerechter Frieden Das fortwährende Dilemma militärischer Gewalt

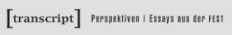

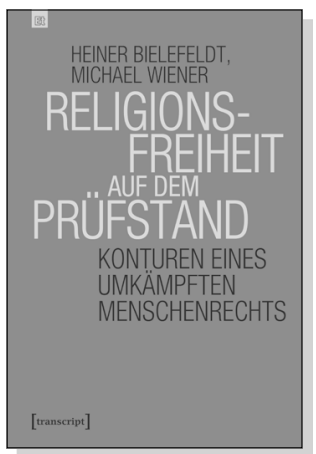

Judith Vey, Johanna Leinius, Ingmar Hagemann ( $\mathrm{Hg}$. )

Handbuch

Poststrukturalistische

Perspektiven auf

soziale Bewegungen

Ansätze, Methoden

und Forschungspraxis
Ines-Jacqueline Werkner

\section{Gerechter Frieden}

Das fortwährende Dilemma militärischer Gewalt

2018,106 S., kart.

$14,99 €$ (DE), 978-3-8376-4074-8

E-Book: 12,99 € (DE), ISBN 978-3-8394-4074-2

Heiner Bielefeldt, Michael Wiener

\section{Religionsfreiheit auf dem Prüfstand}

Konturen eines umkämpften Menschenrechts

Februar 2020, 278 S., kart.

$32,99 €(D E), 978-3-8376-4997-0$

E-Book: 32,99€ (DE), ISBN 978-3-8394-4997-4

Judith Vey, Johanna Leinius, Ingmar Hagemann (Hg.)

Handbuch Poststrukturalistische Perspektiven auf soziale Bewegungen

Ansätze, Methoden und Forschungspraxis

2019, 306 S., kart., Dispersionsbindung,

2 SW-Abbildungen, 2 Farbabbildungen

24,99€ (DE), 978-3-8376-4879-9

E-Book: kostenlos erhältlich als Open-Access-Publikation, ISBN 978-3-8394-4879-3 